\title{
Multifunctional, complex polymers for functional and stimuli-responsive coatings
}
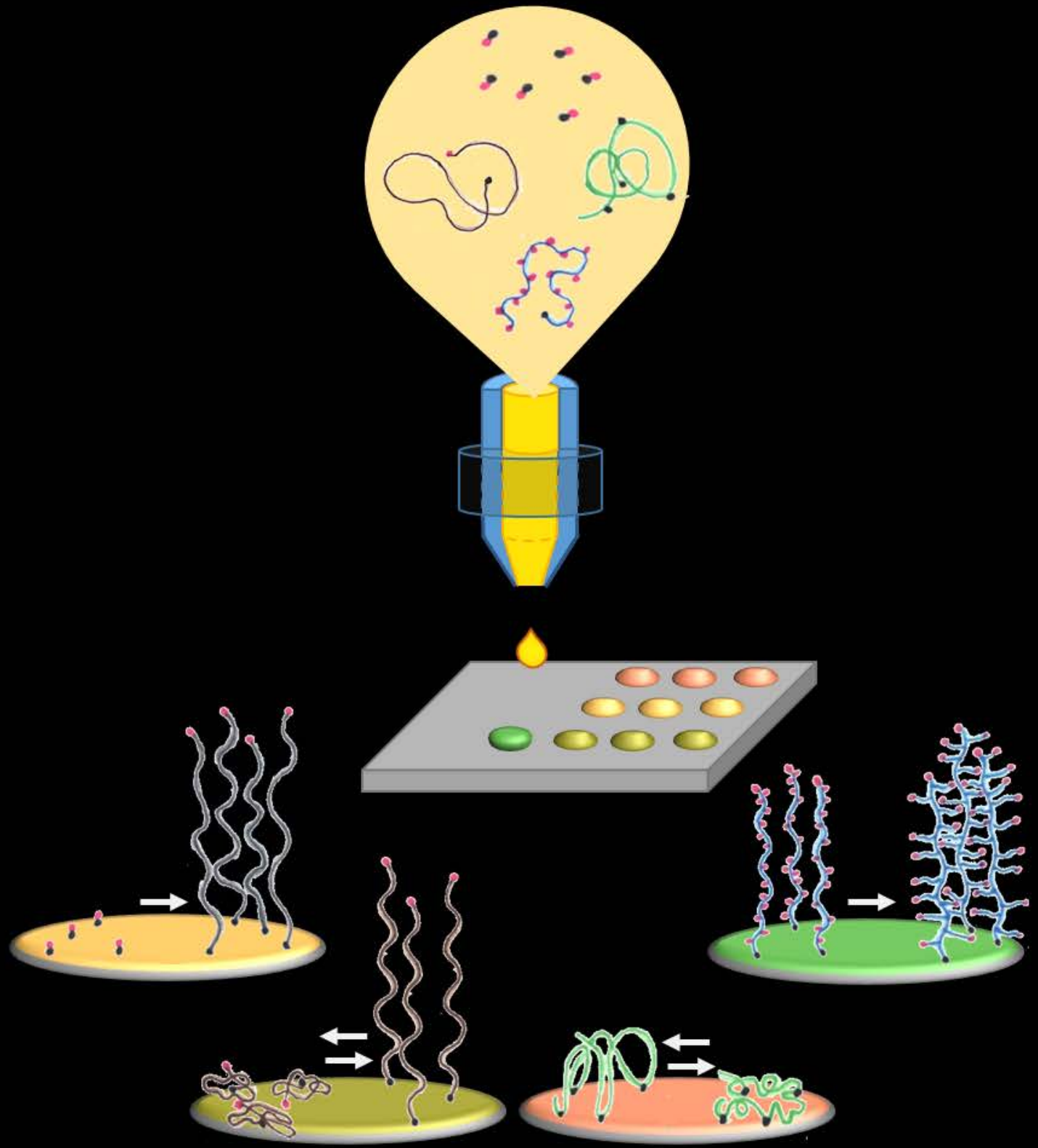

Marco Cirelli 


\section{MULTIFUNCTIONAL, COMPLEX POLYMERS FOR FUNCTIONAL AND STIMULI-RESPONSIVE COATINGS}

Marco Cirelli 



\title{
MULTIFUNCTIONAL, \\ COMPLEX POLYMERS FOR FUNCTIONAL AND STIMULI-RESPONSIVE COATINGS
}

\section{DISSERTATION}

\author{
to obtain \\ the degree of doctor at the University of Twente, \\ on the authority of the rector magnificus, \\ prof.dr. T. T. M. Palstra, \\ on account of the decision of the graduation committee, \\ to be publicly defended \\ on Friday, the $10^{\text {th }}$ of January 2020 at 16.45
}

\section{Marco Cirelli}

born on the $17^{\text {th }}$ June 1986

in Prato, Italy 
This dissertation has been approved by:

Promoter: Prof. dr. G. J. Vancso

Co-promoter: $\quad$ Prof. dr. R. Akkerman

Co-supervisor: Dr. J. Duvigneau

The work described in this Thesis was carried out at the Materials

Science and Technology of Polymers (MTP) group, MESA+ Institute for

Nanotechnology, Faculty of Science and Technology, and at the

Production Technology (PT) group, Faculty of Engineering Technology, University of Twente, the Netherlands.

This research was financially supported by the European Commission through the Marie Skłodowska-Curie initial training network: "Complex wetting phenomena" (MC-ITN-CoWet-607861) and by the MESA+ Institute for Nanotechnology.

Title: Multifunctional, complex polymers for functional and stimuliresponsive coatings

Copyright (C) Marco Cirelli, Enschede, the Netherlands, 2020

All rights reserved. No part of this publication may be reproduced by print, photocopy or any other means without the permission of the copyright owner.

Cover designed by Liliana Parcesepe, Prato, Italy

ISBN: 978-90-365-4931-8

DOI: $10.3990 / 1.9789036549318$ 


\section{Graduation Committee}

Chairman/secretary

Supervisors

Co-supervisor

Committee Members: prof.dr. J.L. Herek

prof.dr. G.J. Vancso

prof.dr.ir. R. Akkerman

dr. J. Duvigneau

prof.dr.ir. R.G.H. Lammertink

prof.dr. F.G. Mugele

prof.dr. R. Simonutti

prof.dr. X. Sui

prof.dr.ir. P. Jonkheijm 



\section{Contents}

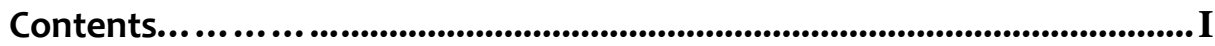

Chapter 1 General Introduction ...............................................................................1

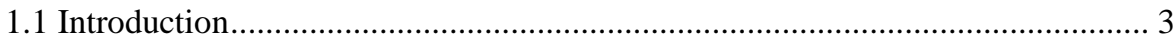

1.2 Concept of this Thesis.............................................................................. 4

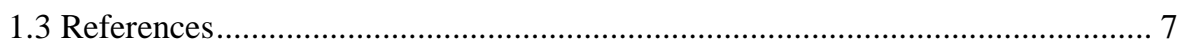

Chapter 2 Design and Engineering of Functional and Smart Surfaces using Polymers..................................................................................................9

2.1 Introduction: Polymer-based films coating ......................................................11

2.2 Synthesis of polymers with controlled molecular architectures..........................13

2.2.1 Controlled radical polymerization techniques..........................................14

2.2.2 Atom transfer radical polymerization.....................................................17

2.3 Surface engineering by polymer brushes. ......................................................18

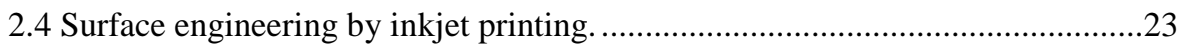

2.5 Anticorrosion polymer coatings ...................................................................26

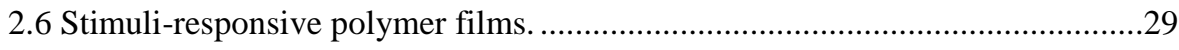

2.6.1 Temperature-responsive polymer films.....................................................30

2.6.2 Solvent-responsive polymer films........................................................

2.6.3 Redox-responsive polymer films.............................................................32

2.6.4 Multi-stimuli-responsive polymer films ...................................................35

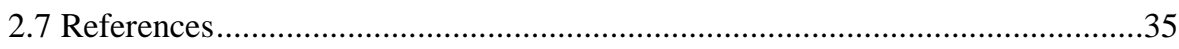

Chapter 3 Swelling and Collapse of PNIPAM Brushes in Response to

Temperature and Co-Non-Solvents Unveiled by Neutron

Reflectivity ...................................................................................................45

3.1 Introduction. 47 


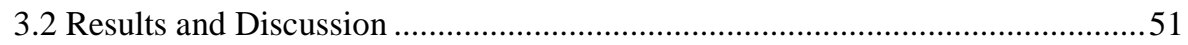

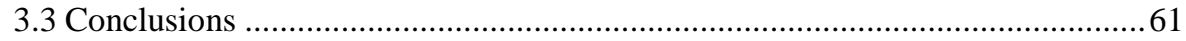

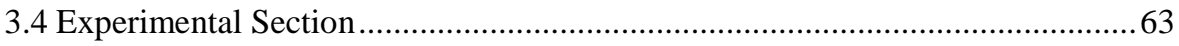

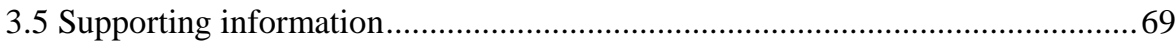

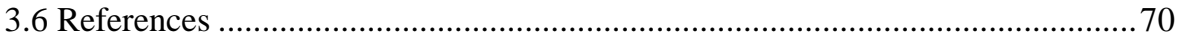

\section{Chapter 4 Tunable Friction by Employment of Co-Non-Solvency of} PNIPAM Brushes ......................................................................................... 73

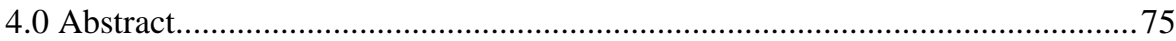

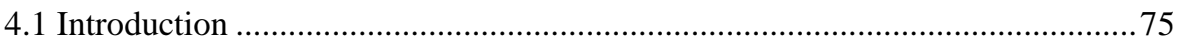

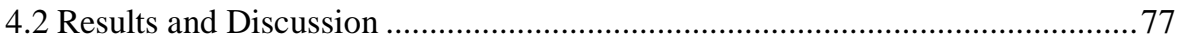

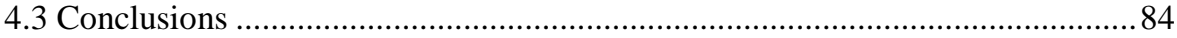

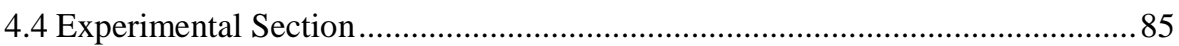

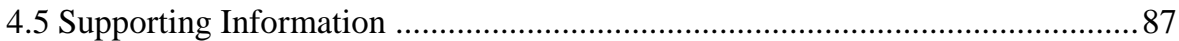

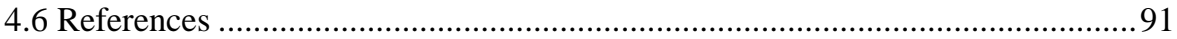

\section{Chapter 5 Effect of Lateral Deformation by Thermo-Responsive} Polymer Brushes on the Measured Friction Forces ...................... 95

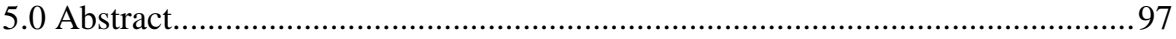

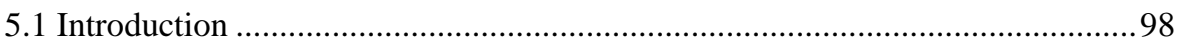

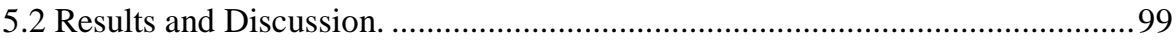

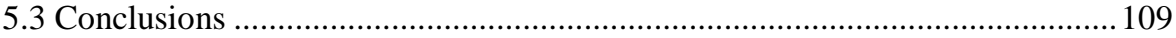

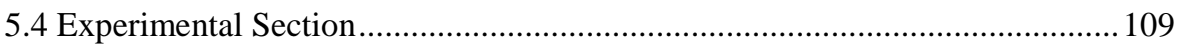

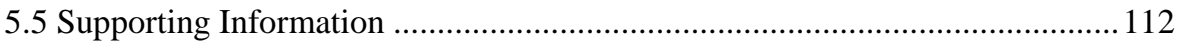

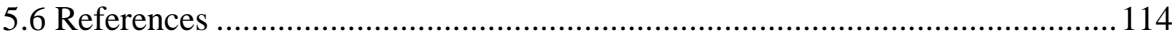

Chapter 6 Protective Coatings for Complex Aluminum Substrates ......... 119

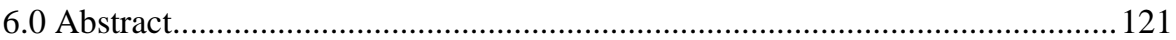

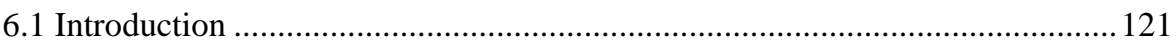

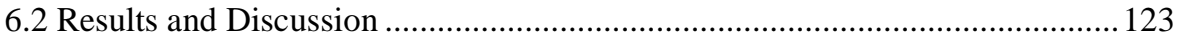




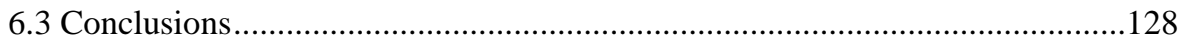

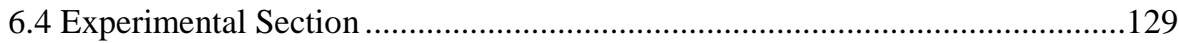

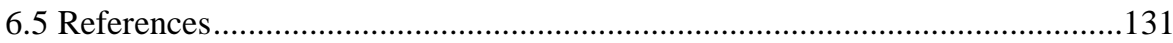

Chapter 7 Grafting-To and Grafting-From Approaches to Obtain Stimuli-Responsive Patterned Fluorescent Polymer Films by Combining ATRP and Inkjet Printing...................................133

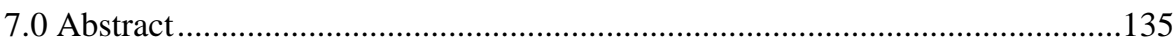

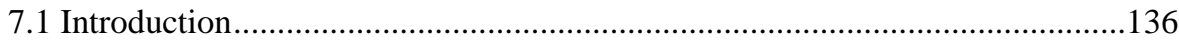

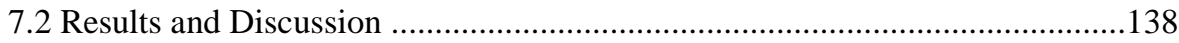

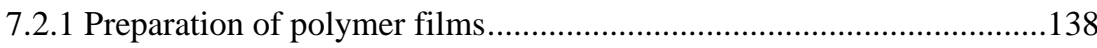

7.2.2 Co-solvency behavior of fluorescnet end-tethered PMMA grafts obtained both via GF and GT approaches in IPA-W mixtures ........................................144

7.2.3 Preparation of fluorescent patterns ….................................................147

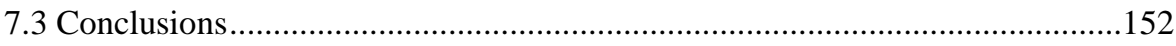

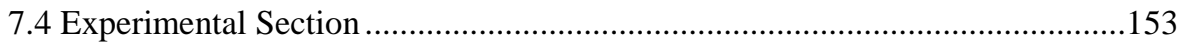

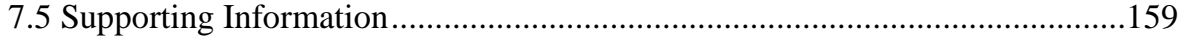

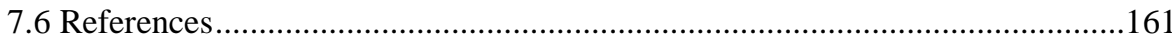

Chapter 8 Printing "Smart" Inks of Redox-Responsive Organometallic Polymers on MicroElectrode Arrays for Molecular Sensing .........................................................................167

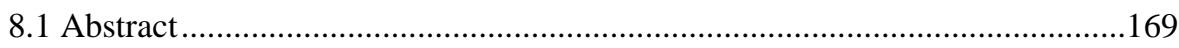

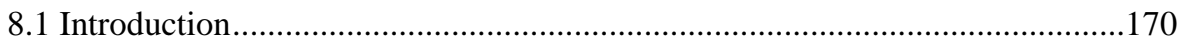

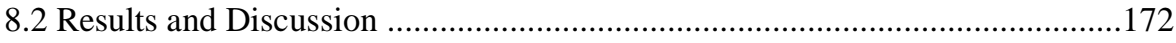

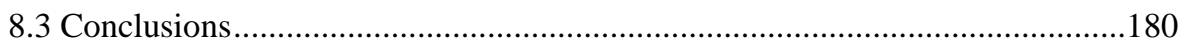

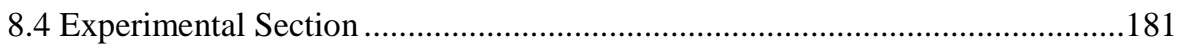

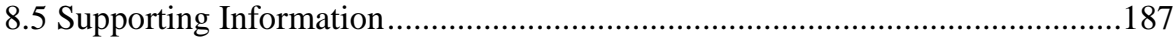

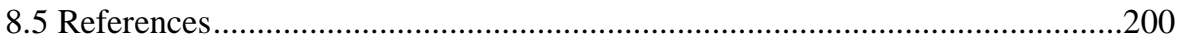


Chapter 9 Synthesis and Design of Bio-Inspired ATRP Functional Macro-Initiators Applicable to a Broad Range of Surface ......... 205

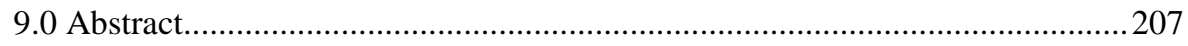

9.1 Mussel-inspired ATRP functional macro-initiator, ATRP-MIs........................207

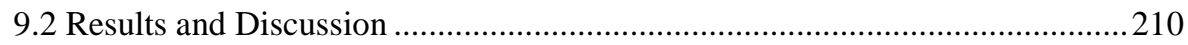

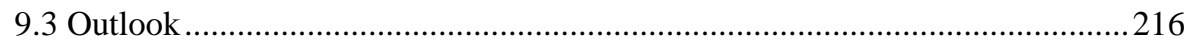

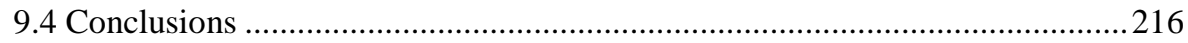

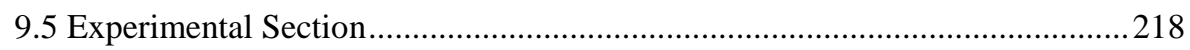

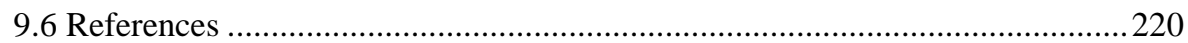

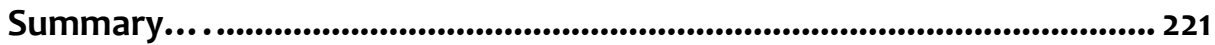

Samenvatting...................................................................................................225

Acknowledgements.................................................................................... 231

List of Publications ..................................................................................2235 
Chapter 1

General introduction 


\section{Contents}

Chapter 1 General introduction ...................................................................................... 1

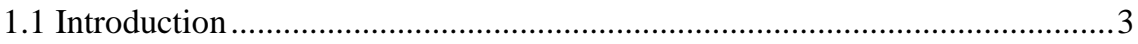

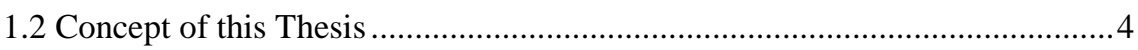

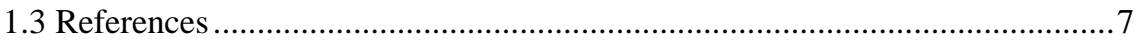




\subsection{Introduction}

As far as commercial applications are concerned, polymers are the most important class of synthetic materials because of their incredible range of physical, chemical, and biological properties. Polymer coatings have been largely studied for the development of functional, reactive, and stimuli-responsive films for biomedical, protective, decorative, adhesive applications. ${ }^{1-6}$ The surface properties of these layers depend on the intrinsic properties of the polymer, as well as on the interactions of the coating with the surrounding environment. Recent advances in macromolecular engineering have led to the development of molecular coatings capable to precisely tune the interfacial physical and chemical properties of a variety of metallic and non-metallic materials. ${ }^{7-11}$ Synthetic polymers can be prepared with the accurate design of every detail of the macromolecules topology, chain architecture, chemical composition, and functionality. ${ }^{8-10}$ Originally, only living anionic polymerization could be used to produce functional complex macromolecules and the "suppression" of the chain termination reaction could only be achieved at the expense of elimination of any impurities from the polymerization medium. ${ }^{12-13}$ Living polymerization was subsequently expanded in different strategies to form polymers such as reversible deactivation radical polymerization techniques (RDRP). ${ }^{8-10,13-14}$ In particular, atom transfer radical polymerization (ATRP) has been extensively studied and used as a versatile, cheaper, and robust technique to synthesize complex polymer systems. ${ }^{10,15-17}$ The conditions of the ATRP method, in comparison with the anionic polymerization, are milder (it is necessary only to work in oxygen-free environment), cheaper (because a small amount of catalyst is needed to achieve high control over the physical and chemical properties of the polymer), and a wider range of monomers can be employed. ${ }^{10,17}$ Moreover, the development of the ATRP methods allows the fabrication of interfaces/coatings capable to improve, or exapand the surface properties of a material. ${ }^{16}$ Several strategies have been developed to fabricate polymer film "brush" coatings grafted onto a surface including grafting-to and grafting-from approaches. ${ }^{1}, 10,18-20$ In the grafting-to approach, a pre-synthesized polymer is immobilized on the surface, ${ }^{19-20}$ while in the grafting-from approach the polymer chains grow from polymerization initiating sites on the surface. ${ }^{1,10,17-19}$ Depending on the physical and chemical properties of the immobilized polymer, the number of anchoring points, grafting density, topology, chemical composition, and functionality, the properties of the coating can be tuned and improved. ${ }^{3}$

Functional and reactive coatings are characterized by specific physical and chemical properties constant in the application conditions. ${ }^{21-22}$ Stimuli-responsive coatings, also called smart or intelligent materials, are capable to reversibly change their physical and/or chemical properties when exposed to a specific stimulus which dramatically affects the surface properties of a material. ${ }^{7}$ The changes in the physical and/or chemical surface properties can be induced by one or more stimuli. ${ }^{5-6}$ For all coatings, the key factor of the 
application is the effect of the interaction between the polymer coating and the surrounding environment on the peculiar properties of the coating. ${ }^{23-24}$ In order to understand structure-property relationships for further development and design of new functional and smart coatings, we have investigated the properties of the functional and stimuli-responsive coatings in complex fluids (e.g., alkaline corrosive aqueous solutions, solvent mixtures). We note that the coating layer can be also patterned. ${ }^{25-29}$ Various patterning methods have been developed to permit the fabrication of spatially controlled functional or stimuli-responsive coating patterns on various materials as sensing, cell adhesion, nanoelectronics. ${ }^{25,30}$ Inkjet printing technique is a well-studied and well-known deposition and patterning technique capable to deposit a variety of complex solutions on the material surfaces. ${ }^{31-34}$ This method requires no physical contact, no mask and no master are needed, and is efficient and cheap. ${ }^{31-34}$ The formulation of the complex fluid/ink is a pivotal factor for the inkjet printing process. ${ }^{33,35-36}$ The applicability of the process, as well as the quality of the polymer coating, are strongly affected by rheological/mechanical properties of these solutions which can be tuned and controlled by choosing the type of solvent, the solid content of the functional component, and presence of additives. ${ }^{33}, 35-36$ A better understanding of the relationship between the formulation of the ink and the deposition, spreading, and evaporation processes of the complex fluid on solid substrates was achieved in this Thesis.

In our studies, we have aimed at exploring the combination of inkjet printing, with various functional and smart coatings for metal and metal oxide surfaces. These coatings were obtained via various grafting to and grafting from approaches permitting the design and optimization of new coatings that could be used in chemical sensing, tribology control, drug delivery, and protection of metal surfaces.

\subsection{Concept of the Thesis}

This Thesis describes the preparation and characterization of tailored polymer coatings with various architectures and patterns developed for specific applications. Functional and stimuli-responsive coatings, including poly(methyl methacrylate) (PMMA) and poly(ferrocenylsilane) (PFS), were prepared via "grafting-from" and "grafting-to" approaches on various materials and were patterned via inkjet printing. Hereby, we demonstrate the synergy of these techniques to fabricate corrosion-resistant coatings for aluminum surfaces and devices for chemical sensing and fluorescent patterns. Furthermore, a deep understanding of the co-nonsolvent-induced and temperatureinduced conformational transition mechanisms of stimuli-responsive $\operatorname{poly}(\mathrm{N}$ isopropylacrylamide) (PNIPAM) brushes was achieved by investigating the distribution of the solvent molecules through the polymer film layer as well as via the analysis of the tribo-mechanical properties. 
In Chapter 2, an overview of the general topics addressed in this Thesis is provided. Firstly, the RDRP techniques are discussed focusing on the ATRP process. Then, the most relevant strategies to chemically graft polymer chains to a surface are discussed. A description and comparison between the physical and chemical properties of the classical coatings, characterized by multi anchoring points, and of the more sophisticated single end-tethered polymer chain brush coatings are provided. A general overview of the patterning techniques is presented focusing on the fundamentals and the principles of the inkjet printing method. Finally, various examples of protective functional (anticorrosion and anti-biofouling) polymer coatings and stimuli-responsive (temperature-, solvent, and redox-responsive) polymer coatings are introduced and discussed.

In Chapter 3, the temperature- and the solvent-responsiveness properties of single end-tethered PNIPAM layers with different grafting densities are investigated via neutron reflectivity (NR) measurements. The PNIPAM brush layers were synthesized via surfaceinitiated atom transfer radical polymerization (SI-ATRP) and the control over the grafting density was achieved controlling the ratio between active and inactive ATRP initiator compounds. Moreover, the PNIPAM layers were characterized via AFM and ellipsometry analysis. The "from the top" mechanism of co-nonsolvency conformational transition of PNIPAM brushes in 3:1 v:v water:ethanol mixture was demonstrated.

In Chapter 4, tunable friction by co-nonsolvency of PNIPAM brushes is investigated. This chapter explores the enhanced friction and dissipation of PNIPAM brushes due to the co-non-solvency effect. Both in water and in ethanol, low friction is obtained due to the high osmotic pressure of good solvents in the brush. However, in 10 vol.\% fraction of ethanol-water composition, a maximum friction is observed. The highest friction is about two orders of magnitude larger than the lowest friction.

In Chapter 5, the effect of lateral deformation on PNIPAM brushes is investigated via friction loops recorded using an atomic force microscopy-based lateral force microscopy. The measurement of friction between polymer brush coating and an AFM colloidal probe consists of different contributions to the frictional energy dissipation such as lateral bending and stretching of the polymer brushes. They are regulated by the mechanical/conformational properties of the polymer chains which are strongly dependent on the conformation of the polymer chains which depend on the temperature of the medium. In this work, the lateral deformation of the two PNIPAM layer with different thicknesses (thin layer with a dry thickness around $10 \mathrm{~nm}$ and the thick layer around $420 \mathrm{~nm}$ ) was analyzed below and above the lower critical solution temperature (LCST).

Chapter 6 describes the joint collaboration with Tanatex and Verosol, members of the ITN-MC-CoWet on the formulation and application of anti-corrosive polyurethanebased coatings for aluminum and its alloys. Various inks for a piezo-inkjet printing process were prepared and printed with different patterns on porous textile substrates. The 
anti-corrosion performance of the coatings was verified with a model alkaline dissolution experiment. The quality of the coated materials was investigated via optical, laser confocal and scanning electron microscopy.

In Chapter 7, the fabrication of solvent-responsive fluorescent patterns by inkjet printing combined with ATRP is reported. Firstly, the synthesis of a fluorescent and acid terminated telechelic PMMA-containing polymer chain is described. It is composed of ARGET-ATRP in solution, halogen exchange reaction and $\mathrm{Cu}$-catalyzed azide-alkyne cycloaddition (CuAAC) click chemistry to couple the fluorescent dye to the polymer chain. Then, the fabrication of reactive coatings on glass and silicon was achieved via vapor chemical deposition of (3-aminopropyl)triethoxysilane (APTES) followed by chemical coupling reaction between the acid extremity of the fluorescent-modified polymer and the amino-functionalized reactive surface via dip coating. Secondly, the stimuli-responsive fluorescent coatings were obtained combining chemical vapor deposition of APTES and SI-ARGET-ATRP of MMA. High grafting density single endtethered polymer chains were obtained. Then, the halogen exchange at the free end of the PMMA brushes with an azide moiety and the CuAAC click chemistry were performed to obtain the fluorescent coatings. Thirdly, the co-solvent-induced effect of the PMMAcontaining brush layers was investigated for both the fabrication approaches. Finally, inkjet printing was employed to fabricate the i) APTES-reactive patterns from where the telechelic PMMA polymer chains were immobilized (grafting-to approach) and ii) the active surface for the SI-ARGET-ATRP of MMA. A discussion of the comparison between the two approaches is presented.

In Chapter 8, the synthesis of a new redox-responsive poly(ferrocenylsilane) (PFS) with disulfide side groups is described and employed to fabricate a sensing device for a model analyte (e.g., ascorbic acid). A new random PFS copolymer was designed and synthesized to carry a tailored number of disulfide moieties able to covalently bind onto gold electrode surfaces. Various piezo inkjet printable inks were prepared and fully characterized. The best ink with respect to the inkjet printability properties was used to modify a microelectrode array (MEA) with different redox-active PFS-based pattens. The PFS-modified MEAs were tested as amperomerometric ascorbic acid sensing device demonstrating the enhanced sensitivity due to the PFS coating.

In Chapter 9, is an outlook chapter that is expected to inspire the synthesis of new stimuli-responsive polymers for more complex and sophisticated applications. A facile approach to fabricate ATRP macroinitiator containing catechol-based groups via free radical polymerization (FRP) is reported. Catechol-derivates have a strong affinity for a wide range of materials and can be considered an universal adhesive group. Firstly, we reported the synthesis of the catechol-based monomer, in particular dopamine methacrylamide (DOMA). Then the copolymerization of three components via FRP composed of DOMA as adhesive group, 2-(2-bromoisobutyryloxy)ethyl methacrylate 
(BIEM) as ATRP initiating group, and the methyl methacrylate (MMA) monomer as solubilizing agent. The activity of the ATRP moieties was confirmed by synthesizing molecular bottle brushes with poly(acrylonitrile) (PAN) side chains and then the adhesive property of the catechol-derivatives was tested by grafting the ATRP-macroinitiator on silicon oxide followed by the SI-ATRP of the AN.

\subsection{References}

1. Minko, S., Responsive polymer brushes. Journal of Macromolecular Science, Part C: Polymer Reviews 2006, 46 (4), 397-420.

2. Yang, W. J.; Neoh, K.-G.; Kang, E.-T.; Teo, S. L.-M.; Rittschof, D., Polymer brush coatings for combating marine biofouling. Progress in Polymer Science 2014, 39 (5), 1017-1042.

3. Liu, F.; Urban, M. W., Recent advances and challenges in designing stimuli-responsive polymers. Progress in Polymer Science 2010, 35 (1-2), 3-23.

4. Bawa, P.; Pillay, V.; Choonara, Y. E.; Du Toit, L. C., Stimuli-responsive polymers and their applications in drug delivery. Biomedical Materials 2009, 4 (2), 022001.

5. Cabane, E.; Zhang, X.; Langowska, K.; Palivan, C. G.; Meier, W., Stimuli-responsive polymers and their applications in nanomedicine. Biointerphases 2012, 7 (1), 9.

6. Mendes, P. M., Stimuli-responsive surfaces for bio-applications. Chemical Society Reviews 2008, 37 (11), 2512-2529.

7. Gil, E. S.; Hudson, S. M., Stimuli-reponsive polymers and their bioconjugates. Progress in Polymer Science 2004, 29 (12), 1173-1222.

8. Perrier, S., 50th Anniversary Perspective: RAFT Polymerization-A User Guide. Macromolecules 2017, 50 (19), 7433-7447.

9. Lee, H. A.; Ma, Y.; Zhou, F.; Hong, S.; Lee, H., Material-Independent Surface Chemistry beyond Polydopamine Coating. Accounts of Chemical Research 2019, 52 (3), 704-713.

10. Ribelli, T. G.; Lorandi, F.; Fantin, M.; Matyjaszewski, K., Atom transfer radical polymerization: billion times more active catalysts and new initiation systems. Macromolecular Rapid Communications 2019, 40 (1), 1800616.

11. Feng, X.; Zhang, K.; Hempenius, M. A.; Vancso, G. J., Organometallic polymers for electrode decoration in sensing applications. RSC Advances 2015, 5 (129), 106355-106376.

12. SZWARC, M., 'Living'polymers. Nature 1956, 178 (4543), 1168.

13. Grubbs, R. B.; Grubbs, R. H., 50th Anniversary Perspective: Living Polymerization-Emphasizing the Molecule in Macromolecules. Macromolecules 2017, 50 (18), 6979-6997.

14. Pan, X.; Fantin, M.; Yuan, F.; Matyjaszewski, K., Externally controlled atom transfer radical polymerization. Chemical Society Reviews 2018, 47 (14), 5457-5490.

15. Matyjaszewski, K.; Tsarevsky, N. V., Macromolecular Engineering by Atom Transfer Radical Polymerization. Journal of the American Chemical Society 2014, 136 (18), 6513-6533.

16. Kim, J.-B.; Huang, W.; Bruening, M. L.; Baker, G. L., Synthesis of Triblock Copolymer Brushes by Surface-Initiated Atom Transfer Radical Polymerization. Macromolecules 2002, 35 (14), 5410-5416.

17. Matyjaszewski, K.; Dong, H.; Jakubowski, W.; Pietrasik, J.; Kusumo, A., Grafting from surfaces for "everyone": ARGET ATRP in the presence of air. Langmuir 2007, 23 (8), 4528-4531.

18. Barbey, R. R. R., Polymer brushes via surface-initiated controlled radical polymerization: synthesis, characterization, properties, and applications. Chemical Reviews 2009, 109 (11), 5437-5527.

19. Minko, S., Grafting on solid surfaces:"Grafting to" and "grafting from" methods. In Polymer Surfaces and Interfaces, Springer: 2008; pp 215-234.

20. Zdyrko, B.; Luzinov, I., Polymer brushes by the "grafting to" method. Macromolecular Rapid Communications 2011, 32 (12), 859-869.

21. Nandivada, H.; Chen, H. Y.; Bondarenko, L.; Lahann, J., Reactive polymer coatings that "click". Angewandte Chemie International Edition 2006, 45 (20), 3360-3363.

22. Zhang, Z. P.; Rong, M. Z.; Zhang, M. Q., Polymer engineering based on reversible covalent chemistry: A promising innovative pathway towards new materials and new functionalities. Progress in Polymer Science 2018, 80, 39-93. 
23. Gelbart, W. M.; Ben-Shaul, A., The "new" science of "complex fluids". The Journal of Physical Chemistry 1996, 100 (31), 13169-13189.

24. Larson, R. G., The structure and rheology of complex fluids. Oxford university press New York: 1999; Vol. 150.

25. Chen, J.-K.; Chang, C.-J., Fabrications and applications of stimulus-responsive polymer films and patterns on surfaces: A review. Materials 2014, 7 (2), 805-875.

26. Chen, T.; Amin, I.; Jordan, R., Patterned polymer brushes. Chemical Society Reviews 2012, 41 (8), 32803296.

27. del Campo, A.; Arzt, E., Fabrication approaches for generating complex micro-and nanopatterns on polymeric surfaces. Chemical Reviews 2008, 108 (3), 911-945.

28. Geissler, M.; Xia, Y., Patterning: Principles and some new developments. Advanced Materials 2004, 16 (15), 1249-1269.

29. Lamping, S.; Buten, C.; Ravoo, B. J., Functionalization and Patterning of Self-Assembled Monolayers and Polymer Brushes Using Microcontact Chemistry. Accounts of Chemical Research 2019, 52 (5), 13361346.

30. Nie, Z.; Kumacheva, E., Patterning Surfaces with Functional Polymers. Nature Materials 2008, 7, 277.

31. Alamán, J.; Alicante, R.; Peña, J.; Sánchez-Somolinos, C., Inkjet printing of functional materials for optical and photonic applications. Materials 2016, 9 (11), 910.

32. De Gans, B. J.; Duineveld, P. C.; Schubert, U. S., Inkjet printing of polymers: state of the art and future developments. Advanced Materials 2004, 16 (3), 203-213.

33. Hoath, S. D., Fundamentals of inkjet printing: the science of inkjet and droplets. John Wiley \& Sons: 2016.

34. Tekin, E.; Smith, P. J.; Schubert, U. S., Inkjet printing as a deposition and patterning tool for polymers and inorganic particles. Soft Matter 2008, 4 (4), 703-713.

35. Derby, B., Inkjet printing of functional and structural materials: fluid property requirements, feature stability, and resolution. Annual Review of Materials Research 2010, 40, 395-414.

36. Magdassi, S., Ink requirements and formulations guidelines. The chemistry of inkjet inks. MAGDASSI, S. Eds 2010, 19-42. 
Chapter 2

Design and Engineering of Functional and Smart Surfaces using Polymers

In this chapter, the general concepts and most relevant developments concerning functional and smart coatings, controlled radical polymerization, ink jet printing, and polymer brushes are provided. 


\section{Contents}

\section{Chapter 2 Design and Engineering of Functional and Smart Surfaces} using Polymers .............................................................................................. 9

2.1 Introduction: Polymer-based film coatings ..... 11

2.2 Synthesis of polymers with controlled molecular architectures .................... 13

2.2.1 Controlled radical polymerization techniques ..................................... 14

2.2.2 Atom transfer radical polymerizations, ATRP ................................... 17

2.3 Surface engineering by polymer brushes ................................................ 18

2.4 Surface engineering by inkjet printing ....................................................23

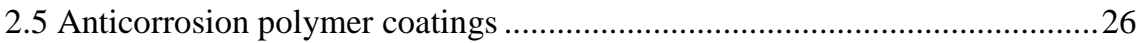

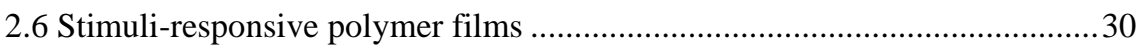

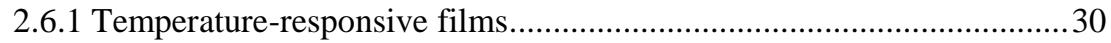

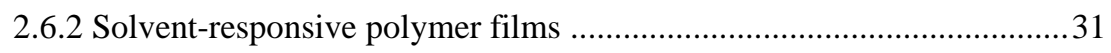

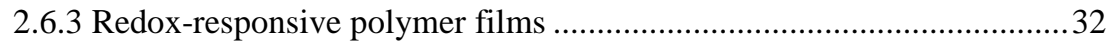

2.6.2 Multi-stimuli-responsive polymer films ............................................ 35

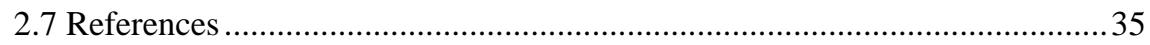




\subsection{Introduction: Polymer-based film coatings}

Functional and smart polymer-based coatings provide specific and/or sophisticated surface properties with minimal influence on the composition and the properties of the bulk materials. Therefore, they play an important role in a diverse range of application areas such as corrosion protection, medical, biomedical, separation, electronics, diagnostics, sensing, and tissue engineering. ${ }^{1-25}$ Following major developments in macromolecular synthesis and engineering, there is a continuously growing interest in the development and utilization of designer functional and smart coatings by scientists and industry. ${ }^{1-19,21-28}$

Polymer coatings can be divided into structural, functional, reactive, and smart. Structural coatings are capable to provide structural support to a material. Reactive coatings bear reactive groups that form strong and chemoselective coupling reactions with the surface, and can provide complementary options for the attachment of additional functions. ${ }^{13,19,29-31}$ Functional coatings exhibit specific physical intrinsic properties, e.g., optical, electrical, thermal or magnetic, which must be maintained during typical application conditions. ${ }^{13,15,19,30-34}$ Smart coatings are materials that are able to adapt their properties dynamically to respond to an external stimulus. ${ }^{1-12,14-18,21-25,35-39}$ These stimuliresponsive coatings possess one or more adaptive chemical or physical properties, which respond rapidly, reversibly, predictably, and in a controlled fashion to one or more applied stimuli, as is shown in Figure 2.1. The response to the applied stimuli may manifest itself a change in shape, surface characteristics, solubility, or it may lead to the formation of a molecular assembly/network or to a sol-to-gel transition. ${ }^{6,19,21,23,25}$

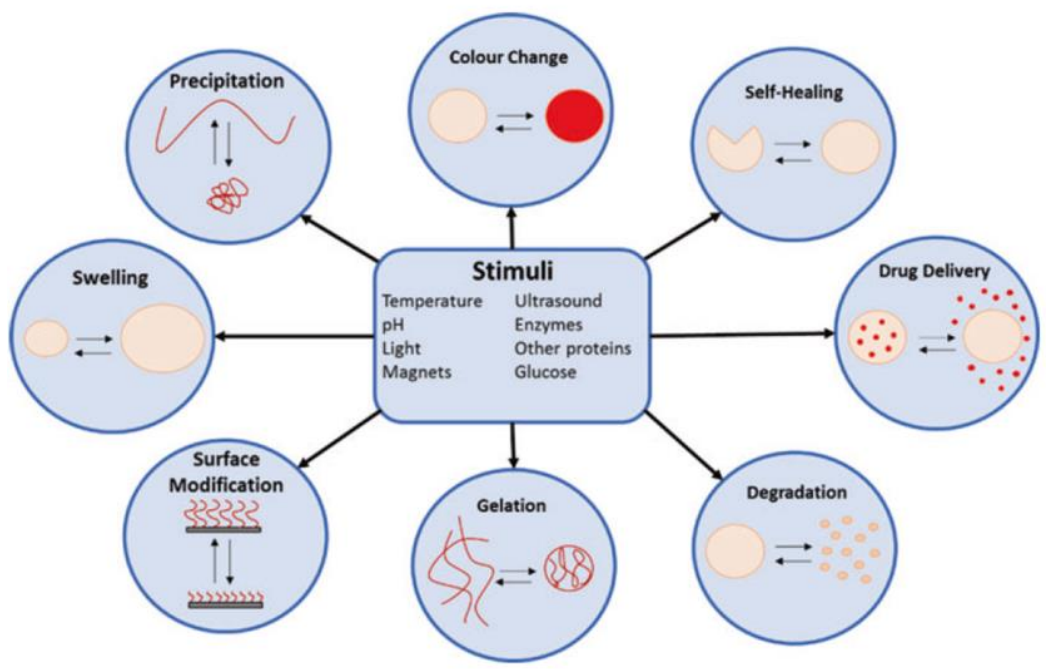

Fig. 2.1: Different stimuli may be used to cause changes in the chemical and/or physical properties of polymers resulting in a change of their bulk or surface polymer properties. Adapted from reference. ${ }^{25}$ 
Smart materials can respond to various stimuli such as i) physical e.g., temperature, light, mechanical force, magnetic field, and electrical fields, or ii) chemical e.g., $\mathrm{pH}$, ionic strength, solvent quality, and signaling chemical molecules, or iii) biological e.g., DNA and proteins. ${ }^{9}, 17,40$ From a chemical point of view, the nature of the monomers to constitute stimuli-responsive polymers plays an important role in the design of stimuliresponsive polymers. Figure $\mathbf{2 . 2}$ lists some of the smart molecules described in the literature. ${ }^{41}$

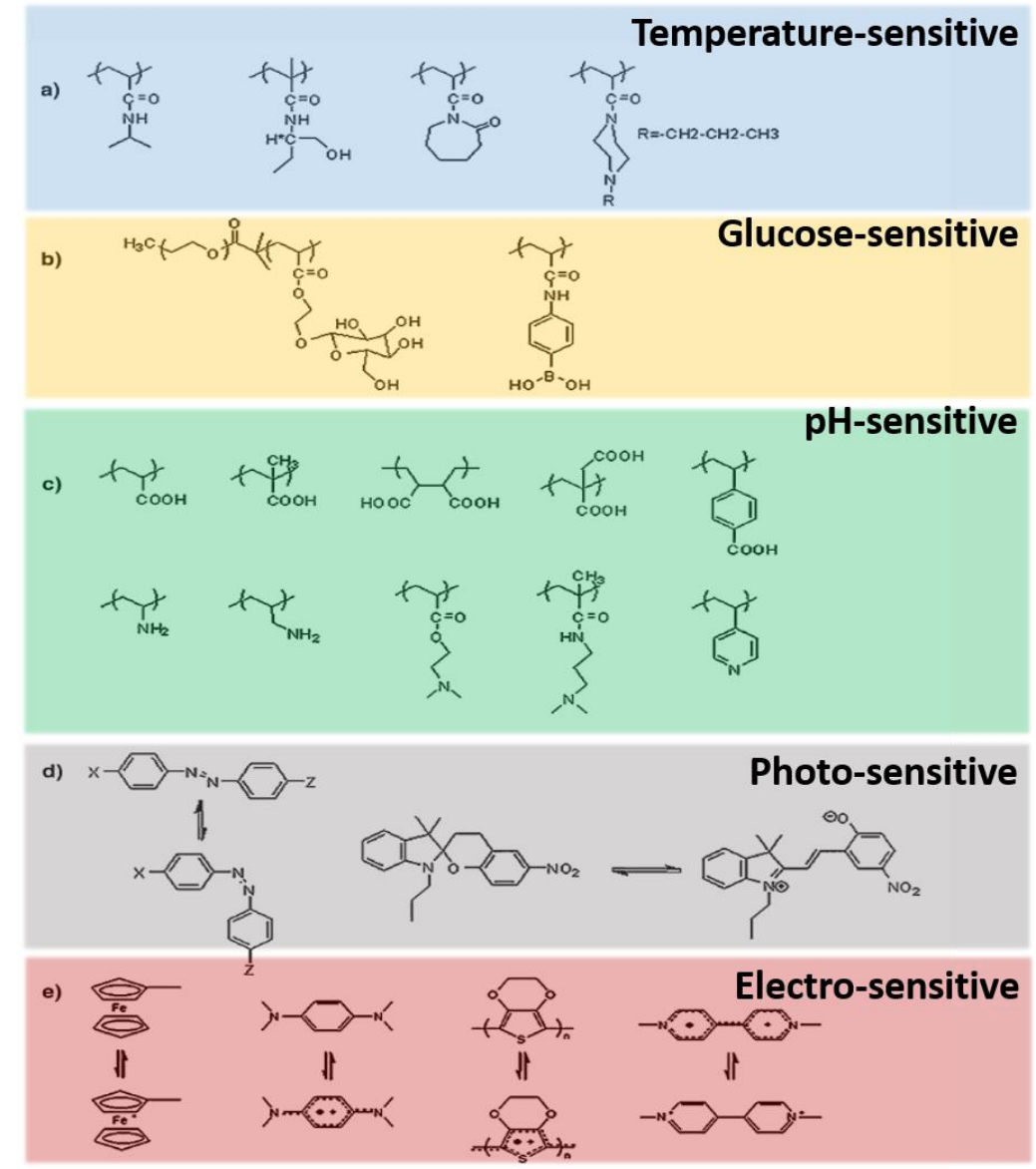

Fig. 2.2: Examples of molecules that are responsive to (a) temperature, (b) glucose concentration, (c) pH, (d) light and (e) electric field. Adapted from reference. ${ }^{9,41}$

From a physical point of view, the properties of stimuli-responsive polymers are strongly affected by their physical confinement and state, e.g. free chains in solution, network gels and surface grafts. ${ }^{2,}, 9,12,42-46$ In fact, the intrinsic properties, i.e. the response to an external stimulus, the speed of the transition, and the surface properties of functional and smart coatings (e.g., stiffness, wetting, adhesion, and friction) can be designed and tuned by controlling the physical constraints of the polymer chains. In fact, adding a constraint to polymer chains, like for single end surface-attached polymer 
brushes, the stimuli-responsiveness may change substantially depending on the distance from the surface and the freedom of movement of the polymer segments. This phenomenon is presented and discussed in detail in Section 2.3. Obviously, the fabrication of stimuli-responsive brush coatings with tailored responsive behavior requires unambiguously synthetic processes with good control over the macromolecular composition and architecture and therefore this is presented and discussed in more detail in the next section.

\subsection{Synthesis of polymers with controlled molecular architectures}

In 1956 the anionic polymerization of dienes and styrenes was the first successful example to synthesize well-defined macromolecules by so-called living polymerization reactions. ${ }^{47}$ The living nature of the reaction is explained by the absence of termination reactions, resulting in a constant concentration of growing polymer chains. ${ }^{47-48}$ Thus, living anionic polymerization allows the growth of uniform chain lengths as well as it provides control over chain compositions, end groups, and architectures. ${ }^{48}$ However, it requires very stringent conditions, i.e. high purity of the used reagents, as well as complete exclusion of moisture and air, which severely limits its widespread use. ${ }^{48}$

More recently, a number of essentially living radical polymerization methods were developed and introduced, i.e. the so-called reversible deactivation radical polymerization (RDRP) mechanism. ${ }^{27}$, 49-58 These polymerization methods conserve the important characteristics of living polymerization reactions, that is termination reactions are minimized allowing the synthesis of polymers with a narrow PDI and good control over chain-end functionalization. In addition, RDRP allows the synthesis of polymers with good control over the composition, architecture, functionality, and even molecular composites, as is shown in Figure 2.3. ${ }^{27}$ Furthermore, RDRP methods require cheaper catalysts, less stringent reaction conditions, and they have a high tolerance towards functional groups of common vinyl-based monomers compared to living anionic polymerization. ${ }^{27,48,50-58}$ This resulted in a continuously growing interest in utilizing corresponding chemistry at industrial scale and today the first successful commercial examples exist, such as Nanostrength ${ }^{\circledR} .{ }^{59}$ We have used RDRP chemistry in this research work, hence in the next section, the fundamental features of the main RDRP mechanisms are briefly introduced focusing on the atom transfer radical polymerization (ATRP). 


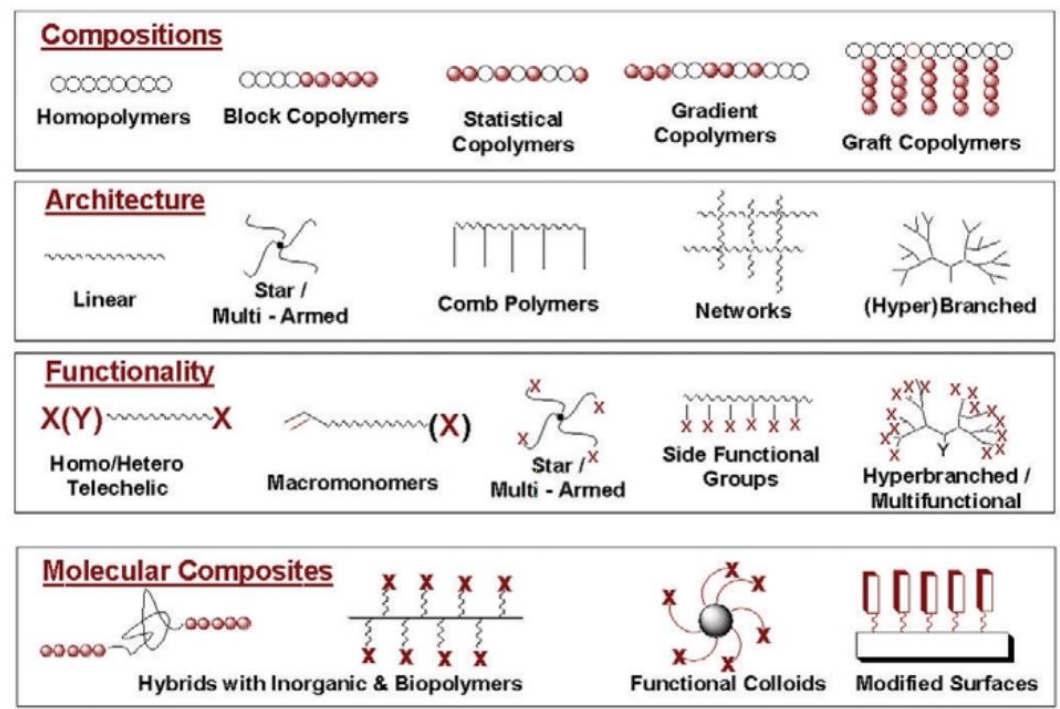

Fig. 2.3: Addressable polymer composition and architectures by RDRP processes. Modified from reference. ${ }^{27}$

\subsubsection{Controlled radical polymerization techniques}

The most frequently used RDRP techniques are nitroxide-mediated polymerization (NMP), ${ }^{56}$ atom transfer radical polymerization (ATRP), ${ }^{26,52-53,58,60}$ and reversible addition-fragmentation chain transfer radical polymerization (RAFT) ${ }^{57}$ These techniques were employed to synthesize homopolymers and copolymers with complex topologies, compositions and chain end functionalities with targeted molar mass, and narrow molar mass distributions. ${ }^{55,57-58}$ Their reaction mechanisms are shown in Figure 2.4. As is shown in Figure 2.4, the essence of a RDRP process is based on an equilibrium between active and dormant states of a growing polymer chain, which is controlled by a reversible activation-deactivation mechanism. ${ }^{52-54,61}$ In contrast to conventional free radical polymerization (FRP) reactions, in which the typical radical lifetime is about $1 \mathrm{~s}$ before termination events occur, in RDRP the active growing chains add to only a few monomers before being converted back to the dormant state. Thus, by alternating between short periods of activity and longer dormant periods the radical lifetime of the growing chain in RDRP is extended from seconds to days or even weeks. ${ }^{53,58}$ 


\section{A) Reversible Deactivation}
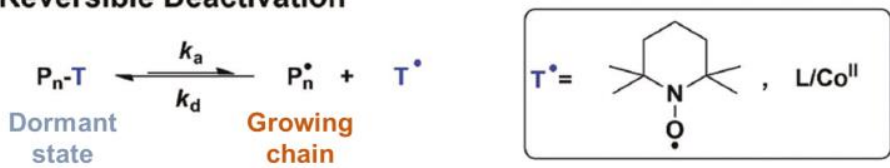

B) Catalytic Reversible Deactivation
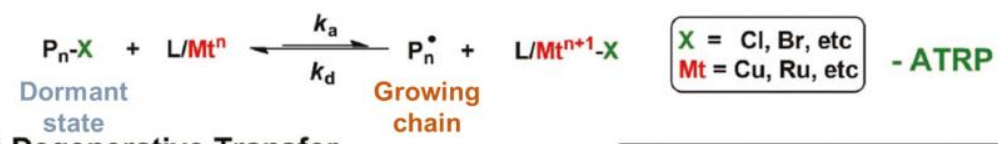

C) Degenerative Transfer
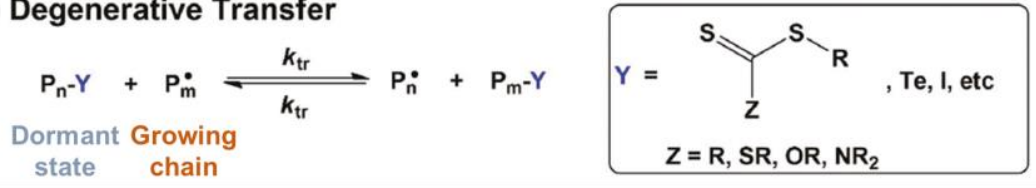

- RAFT

- TERP

- ITP

Fig. 2.4: General mechanisms for the most commonly used RDRP techniques. Modified from reference. ${ }^{58}$

In NMP and ATRP, the equilibrium between the active and the dormant states of the polymer chains is established through a mechanism of reversible termination of the propagating chain (Figure 2.4A and $\mathrm{B}$ ) in which the equilibrium strongly favors the dormant state through either nitroxide capping (NMP) or via a redox process with a metal halide salt (ATRP). This significantly reduces irreversible termination. RAFT, on the other hand, proceeds via a degenerative chain transfer process, in which the propagating species equilibrate with dormant species (Figure 2.4C). As the degenerative chain transfer does not create radicals, the reaction requires addition of an external source of radicals to maintain a constant rate of polymerization. This is performed commonly in the form of an azo initiator, such as 2,2'-Azobis(2-methylpropionitrile) (AIBN).

Table 2.1 summarizes a comparison of ATRP, NMP, and RAFT considering the range of monomers suitable for the specific polymerization, the typically required reaction conditions, the nature of transferable end groups/atoms, and necessary additives for the control of the polymerization kinetics. ${ }^{27}$ The results reported in this Thesis mainly relied on the use of ATRP for the synthesis of diverse brush architectures and therefore in the next section, we will introduce this technique and its evolution in more detail. 
Table 2.1: Comparison of ATRP, NMP, and RAFT polymerizations.

\begin{tabular}{|c|c|c|c|}
\hline & \multicolumn{3}{|c|}{ RDRP techniques } \\
\hline & NMP & ATRP & RAFT \\
\hline \multirow{2}{*}{$\begin{array}{l}\bar{\Phi} \\
\stackrel{\Xi}{0} \\
\stackrel{0}{0}\end{array}$} & $\begin{array}{l}\checkmark \text { Styrenes with TEMPO; } \\
\checkmark \text { Acrylates and acrylamides } \\
\quad \text { using new nitroxides }\end{array}$ & $\begin{array}{l}\checkmark \text { Nearly all monomers } \\
\text { with activated double } \\
\text { bonds }\end{array}$ & $\begin{array}{l}\checkmark \text { Nearly all monomers } \\
\text { with activated double } \\
\text { bonds }\end{array}$ \\
\hline & $\begin{array}{l}\downarrow \text { No vinyl acetate } \\
\downarrow \text { No methacylayes }\end{array}$ & $\downarrow$ No vinyl acetate & \\
\hline \multirow{2}{*}{ 苛 } & $\checkmark$ Waterborne systems & $\begin{array}{l}\checkmark \text { Waterborne systems } \\
\checkmark \text { Large range of } \\
\text { polymerization } \\
\text { temperature (from RT to } \\
150^{\circ} \mathrm{C} \text { ) }\end{array}$ & $\checkmark$ Waterborne systems \\
\hline & $\begin{array}{l}\downarrow \text { Elevated temperature } \\
\downarrow \text { Sensitive to oxygen }\end{array}$ & $\begin{array}{l}\downarrow \text { Sensitive to oxygen, } \\
\text { however new techniques } \\
\text { have been developed to } \\
\text { minimize it }\end{array}$ & $\begin{array}{l}\downarrow \text { Elevated temperature } \\
\text { for less active } \\
\text { monomers } \\
\downarrow \text { Sensitive to oxygen }\end{array}$ \\
\hline \multirow[b]{2}{*}{ 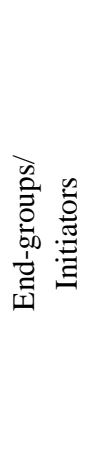 } & Alkoxyamines & Alkyl (pseudo)halides & $\begin{array}{l}\text { Dithioesters, iodides, and } \\
\text { methacrylates }\end{array}$ \\
\hline & $\begin{array}{l}\text { May act as stabilizer } \\
\text { Thermally unstable } \\
\text { Relatively expensive } \\
\text { For transformation: requires } \\
\text { radical chemistry }\end{array}$ & $\begin{array}{l}\text { Thermally and photostable } \\
\text { Inexpensive and available } \\
\text { For transformation: Sn, E, } \\
\text { or radical chemistry } \\
\text { Halogen exchange for } \\
\text { enhanced cross- } \\
\text { propagation }\end{array}$ & $\begin{array}{l}\text { Odor/color } \\
\text { Less thermally stable and } \\
\text { less photostable } \\
\text { Relatively expensive } \\
\text { For transformation: } \\
\text { radical chemistry }\end{array}$ \\
\hline 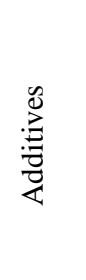 & $\begin{array}{l}\text { NMP may be accelerated with } \\
\text { acyl compounds }\end{array}$ & $\begin{array}{l}\text { Transition metal catalyst } \\
\text { (should be removed } \\
\text { and/or recycled) }\end{array}$ & $\begin{array}{l}\text { Conventional radical } \\
\text { initiator which may } \\
\text { decrease end } \\
\text { functionality or } \\
\text { produce too many new } \\
\text { chains }\end{array}$ \\
\hline
\end{tabular}




\subsubsection{Atom transfer radical polymerization, ATRP}

ATRP was independently reported by Mitsuo Sawamoto and coworkers ${ }^{62}$ and Krzysztof Matyjaszewski and coworkers ${ }^{60}$ in 1995 as a new, versatile and robust polymerization mechanism to prepare polymers by radical polymerization with control. As mentioned in the previous section, in ATRP an equilibrium between a small amount of growing free radicals and a large amount of dormant species, resulting from a smaller rate of activation compared to the rate of the deactivation, leads to the establishment of a persistent radical effect. ${ }^{63-64}$ Thus, the growth of the chains occurs in a stepwise fashion like for the living polymerizations. Generally, the main components of an ATRP process encompass the organic halide initiators (usually acyl halide derivates), a redox-active transition metal complex (e.g. copper-, ruthenium- or iron based) often coordinated by a nitrogen based ligand, and the monomer(s). ${ }^{53,58}$ Figure 2.5 shows the ATRP mechanism and the equilibrium reactions in more detail. The main ATRP equilibrium is between activation $\left(\mathrm{k}_{\mathrm{a}}\right)$ and deactivation $\left(\mathrm{k}_{\mathrm{d}}\right)$ of the growing chain established by the cleavage of the metal complex in its lower oxidation state $\left(\mathrm{Cu}^{\mathrm{I}} \mathbf{L}^{+}\right)$by the halogen atom from the iniator or the dormant polymer chain $\left(\mathbf{P}_{\mathbf{n}}-\mathbf{X}\right)$. After the extraction of the halogen atom from the iniator, or from the dormant polymer chain, the radical growing chain radical $\left(\mathbf{P}_{\mathbf{n}}{ }^{\bullet}\right)$ will propagate for a short period until the back-transfer process of the halogen atom occurs forming again the polymer chain in the dormant state. ${ }^{26,51-53,58}$

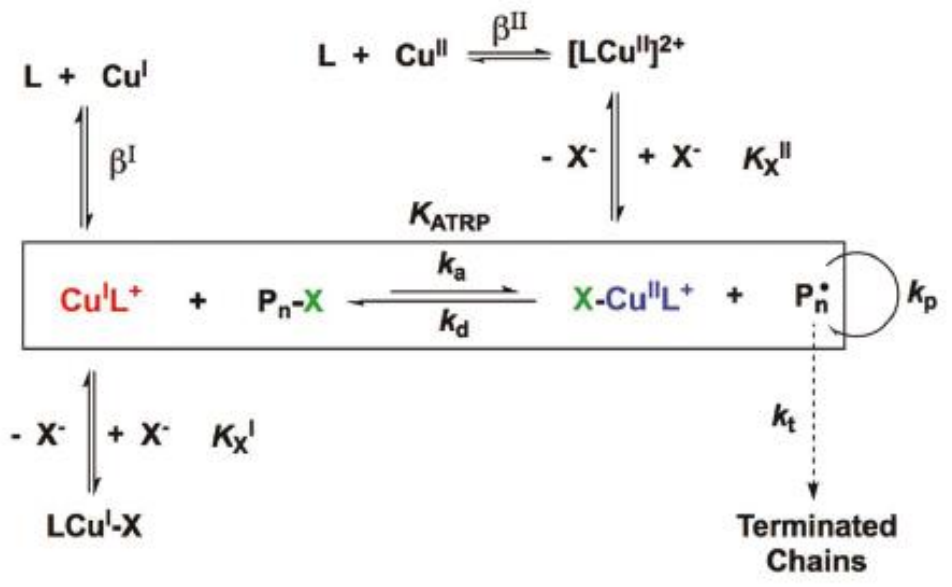

Fig. 2.5: General mechanism for ATRP. From references. ${ }^{53,58}$

As a result of the high tolerance of ATRP to functional groups present in monomers, such as hydroxy, amino, amido, ether, and ester groups, a variety of monomers, e.g., styrenes, (metha) acrylates, methacrylamides, vinyl pyridine, and acrylonitrile, have been successfully polymerized by ATRP. ${ }^{53,58}$

Few disadvantages of ATRP have so far limited the large scale industrial use, which are mainly related to the requirements of employing oxygen-free conditions and the high amount of copper catalyst required. These drawbacks result in upscaling issues and 
relatively high costs. Recently, new ATRP techniques were developed that are capable to continuously regenerate the catalyst, resulting in lower required amounts, and that require less stringent oxygen-free conditions, as is summarized in Figure 2.6. ${ }^{50,61,65-72}$ For example, Zhang and coworkers ${ }^{73}$ reported the synthesis of poly(styrene) and poly(4vinylpyridine) brushes grafted from cellulose nanocrystals via surface-initiated (SI) ATRP and surface-initiated activators regenerated by electron transfer ATRP (SIARGET-ATRP) for which the required amounts of $\mathrm{Cu}$ catalyst were of $2.000 \mathrm{ppm}$ and $25 \mathrm{ppm}$ with respect to the monomer concentration, respectively. ${ }^{73}$ Clearly from the amount of $\mathrm{Cu}$ catalyst, the SI-ARGET-ATRP was more environmentally friendly compared to the conventional SI-ATRP method. Interestingly, as a result of the higher propagation rate for SI-ARGET-ATRP the corresponding brushes prepared by the conventional SI-ATRP, leading to different film properties. ${ }^{73}$ In the next section, the grafting of polymer chains to solid surfaces and the subsequent impact on the surface properties is presented. We will, in particular, introduce single and multiple tethered polymer chain based coatings and the critical parameters that affect the physical and chemical properties of the resulting coatings.
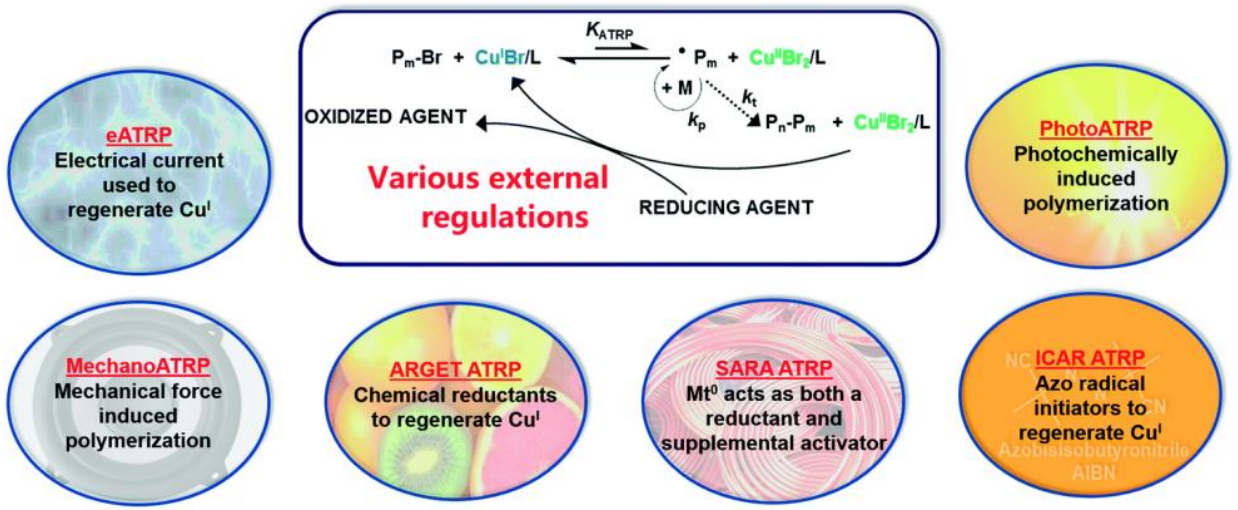

Fig. 2.6: New ATRP techniques capable of continuously regenerate the catalyst in various ways resulting in lower amounts of required catalyst and less stringent reaction conditions. From reference. ${ }^{72}$

\subsection{Surface engineering by polymer brushes}

Polymer brushes are among the most interesting polymer-based coatings to control wettability, friction, and adhesion of surfaces. ${ }^{20,24,74-75}$ There are two main approaches that can be employed to fabricate polymer film grafted coatings, i.e. "grafting-to" (GT) ${ }^{76-}$ 77 and "grafting-from" (GF). ${ }^{39,}$ 42, 44-45, 78-80 The GT approach consists of a chemical reaction between the reactive moieties of functionalized pre-synthesized polymers with surface-exposed reactive groups on the substrate. ${ }^{76-77}$ GF typically yields higher grafting densities compared to GT, which is ascribed to the relatively easy control over the surface-active initiator density and subsequent polymer growth in GF, while for GT steric hindrance by already grafted polymer chains limits the attachment of neighboring 
polymer chains in close proximity. ${ }^{42,} 44-45,76-78$ GT has the advantage that the presynthesized polymer chains are easy to characterize, which is not a trivial task for polymer chains prepared by the GF approach. ${ }^{42,44-45,76-78}$

Depending on the interaction of solvent molecules with polymer segments, we can distinguish three quality regimes for free polymer chains in solution, i.e. good (well swollen), poor (essentially collapsed) and theta (unperturbed, between good and poor) solvent conditions. ${ }^{81}$ For surface grafted polymers the mobility of the chain segments depends on the anchoring structure, the grafting density and the overall amount of the surface-bound polymer. ${ }^{9}, 21,25,39,52,82$ The grafting density, defined as the number of anchoring points per unit area, strongly affects the conformation of the polymer chains and as such the properties of the corresponding polymer films both in dry and wet conditions. ${ }^{83-87}$ If we consider single end-tethered polymer chains as the simplest case of surface grafted polymers, Figure 2.7 shows the three conformational regimes of single end-grafted polymer chains as a function of their grafting density, i.e. the collapsed coil, "mushroom-", and "brush"-like conformations. ${ }^{42,45,83,85,88}$

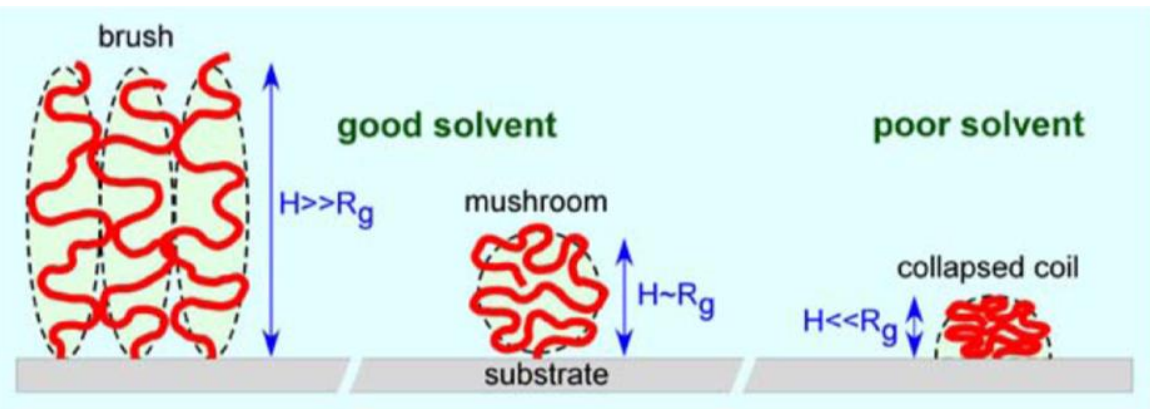

Fig. 2.7: Schematic representation of surface-immobilized polymers in brush, mushroom and collapsed coil conformation in a good or poor solvent. Adapted from references. ${ }^{83}$

In the mushroom regime in a good solvent, the polymer chains maximize the contact between the polymer segments and the solvent molecules while keeping chain stretching to a minimum, by adopting a conformation similar to that of a free polymer chain in a good solvent. Upon increasing the grafting density, the osmotic pressure among the chains increases as a result of the steric hindrance between polymer chains in close proximity to each other. This leads to stretching of the polymer chains in both a good solvent, as well as in the dry state. Thus, the wet thickness of the swollen polymer brush layer is larger than the radius of gyration of the swollen free polymer chain and of the polymer chain in the mushroom regime. ${ }^{89}$ In poor solvent conditions, the polymer chains tend to collapse by forming a collapsed globule conformation (or even place themselves flatly at the substrate in form of a "pancake" shaped molecular precipitate) in the mushroom regime, while upon increasing the grafting density the chains start to feel each other (strong steric hindrance/repulsion) resulting in the formation of collapsed aggregates..$^{39,90}$ 
Figure 2.8 shows different geometries of polymer films grafted on a surface depending on the number of anchoring points. In good solvent conditions, the physical and chemical properties of the grafted polymer chains is tunable by controlling the steric hindrance around the polymer chains, for instance by incorporation of loops, using cylindrical molecular grafts or branched architectures. ${ }^{83-87}$ This allows one to control properties like antifouling, lubricating or particle stabilization performance of a polymer film. $^{43,}$ 91-94 For example, Yakushiji and coworkers ${ }^{93}$ reported the effect of macromolecular architecture on the thermoresponsiveness of surface grafted $\operatorname{poly}(\mathrm{N}$ isopropylacrylamide) (PNIPAM) films. They showed that the lower critical solution temperature (LCST) of loop-grafted PNIPAM decreased significantly compared to PNIPAM singe end-tethered chains. This significant decrease in LCST was ascribed to the restricted segmental dynamics of the PNIPAM segments between the multiple anchoring points..$^{93}$

\section{Number of anchoring points}

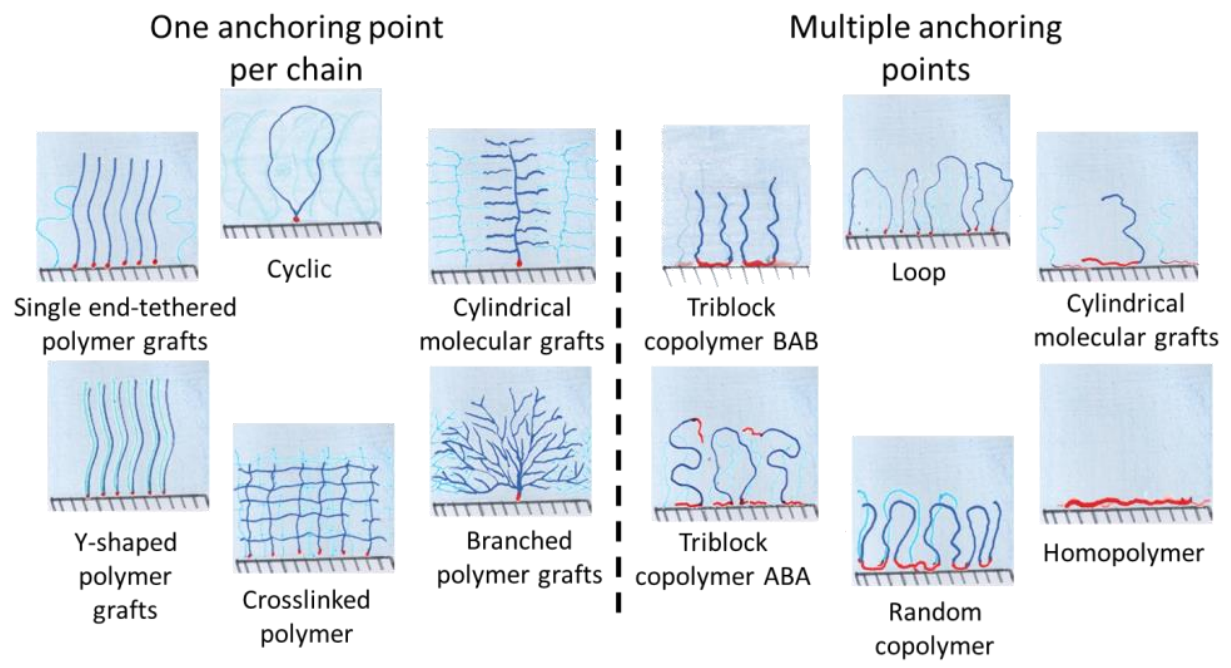

Fig. 2.8: Schematic representation of the different geometries of polymer films immobilized onto a substrates. On the left, the number of anchoring points per chains is one and on the right multiple anchor points per chain exist.

Next to homogenous surface engineering with polymer brushes one can readily obtain brush patterns and/or gradients by combining existing lithography approaches and controlled polymerization techniques. ${ }^{95}$ Recent advances in these fields opened new avenues for the preparation of advanced, sophisticated and more complex brush based applications at reduced cost and time. $.^{78,83,96-98} 99-102$

For a comprehensive overview of various patterning technologies, the interested reader is directed to Geissler et al. ${ }^{103}$ and Hill et al. ${ }^{104}$ Utilization of the well-known patterning techniques allows one to prepare brush patterns with lateral dimensions 
ranging from the macromolecular up to the macroscopic length scale. Figure 2.9 summarizes the most popular approaches to fabricate polymer brush patterns by surfaceinitiated polymerization. ${ }^{45}$, 105-108

As patterning was used in our research by employing inkjet printing, we provide a brief account of some representative examples. For example, switchable adhesive patterns were fabricated on gold by combining the synthesis of thermo-responsive PNIPAM brushes utilizing patterned, initiator self-assembled monolayers as was reported by Jones and co-workers. ${ }^{99}$ Similarly, Edmonson et al. ${ }^{109}$ and Emmerling et al. ${ }^{105}$ employed inkjet printing either to directly prepare initiator patterns ${ }^{109}$ or to selectively etch homogeneously deposited initiator layers for the subsequent SI-P of polymer brush patterns. ${ }^{105,109}$ Furthermore, recent progress in photoinduced RDRP methods opened a straightforward alternative route to photopatterning of polymer brushes by spatially controlled polymerization through a photomask. ${ }^{107-108}$

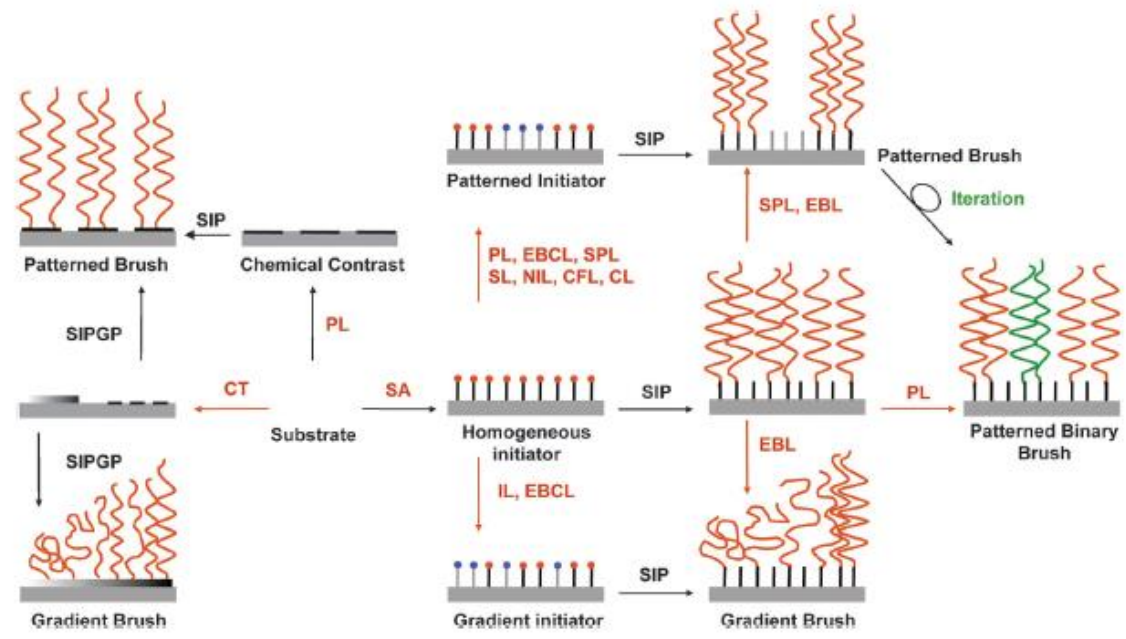

Fig. 2.9: Overview of common strategies used for the preparation of polymer brush patterns. (Abbreviations: SIPGP: self-initiated photografting and photopolymerization; SIP: surface-initiated polymerization; CT: carbon templating; PL: photolithography; SA: self-assembly; EBCL: electron beam chemical lithography; SPL: scanning-probe lithography; SL: soft lithography; NIL: nanoimprinting lithography; CFL: capillary force lithography; CL: colloidal lithography; IL: interference lithography; EBL: electron beam lithography). From reference. ${ }^{106}$

Furthermore, some applications, such as microfluidic devices, biological sensors, tissue engineering, and antifouling, require the fabrication of more complex coatings based on a gradient film. ${ }^{95}$ The gradient can be in physical and chemical coating properties, e.g. in polymer chain length, grafting density, functionality, composition or a combination of any of these parameters, as well as in the spatial dimensions, e.g., in lateral, transversal or orthogonal direction with respect to the direction of the surface. Figure 2.10 shows a few examples of gradient brush layers. ${ }^{83}$ 
l) Physical gradient

a)

b)

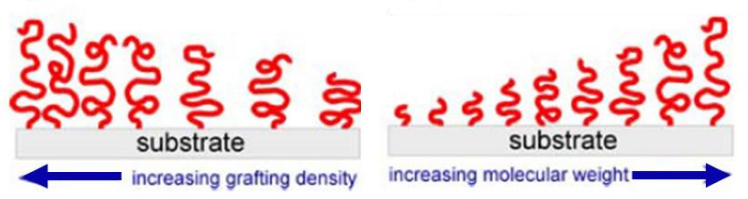

II) Chemical composition gradient

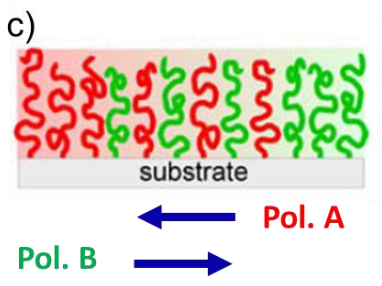

d)

e)
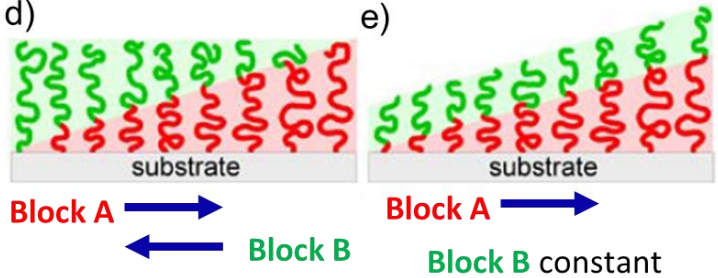

Fig. 2.10: Schematic representation of various gradient architectures of single end-anchored polymer chains. I) Physical and II) chemical composition gradients for polymer brush films. Physical gradient: a) orthogonal grafting density gradient and $b$ ) orthogonal polymer length gradient of a single end- tethered polymer chains. Chemical composition gradients: c) mixed homopolymer with orthogonal grafting density of the two homopolymers, d) grafted diblock copolymers with a constant total length of the polymer chains and an opposite gradient direction in polymer composition, and e) grafted diblock copolymer with a gradient in the polymer length for the first block and a constant length for the second block. Modified from reference. ${ }^{83}$

Among all the available techniques to create a gradient, SI-P is an interesting strategy that is capable of easily create gradients over the thickness of the film and/or in the surface chemistry (so-called gradient surfaces). The first to report an orthogonal film gradient was Chaudhury et al. ${ }^{97}$ They reported the fabrication of an uni-directional chemical gradient on a silicon substrate via the spatially controlled vapor deposition of selfassembled monolayers (SAMs) of a silane compound. With this method, the authors reported a first application of a surface tension gradient over a surface that was capable to make water run uphill. ${ }^{97} \mathrm{Wu}$ et al. ${ }^{110}$ reported the synthesis of a gradient film of single end-tethered polymer chains synthesized via SI-ATRP reporting and demonstrating the mushroom-to-brush transition crossover along the polymer film surface. ${ }^{110}$ Moreover, a gradient in the polymer brush length is readily obtained by simply varying the spatially controlled polymerization conditions (e.g. time, monomer concentration, temperature, solvent quality, etc.) as was reported by Zhang et al. ${ }^{83,111-112}$ Furthermore, really interesting gradient architectures were reported by Tomlinson et al. ${ }^{113}$ They reported a simple method to prepare molecular weight and composition gradients via the SI-ATRP of PHEMA and PMMA block copolymers with spatially controlled polymerization times for each block. ${ }^{113}$ Later the same authors reported the fabrication of gradient of block copolymers grafted on 2-D surfaces with an orthogonal variation of the lengths of both blocks. ${ }^{114}$ 


\subsection{Surface engineering by inkjet printing}

In several chapters of this Thesis (see Chapter 6, 7, and 8), we focus on the formulation of functional inks that are readily deposited by inkjet printing for surface functionalization, as well as on the fabrication of functional, reactive and stimuliresponsive patterns for electrochemical sensing applications. Therefore, this section will introduce inkjet printing as a tool for surface engineering in more detail.

In the $20^{\text {th }}$ century, industrial printing techniques tremendously evolved as deposition and patterning technique. This is ascribed to the fact that it is a contact-free deposition method that does not require the use of masks or masters and it allows spatially controlled, targeted delivery of inks. ${ }^{103,115-120}$ Furthermore, it is a versatile technique that can be used to functionalize any type of material, e.g liquid, rigid, planar surfaces as well as flexible, curved substrates, and soft or hard materials can all be employed. ${ }^{106-110,120}$ An overview of the properties and the challenges of inkjet printing is provided in Figure 2.11.

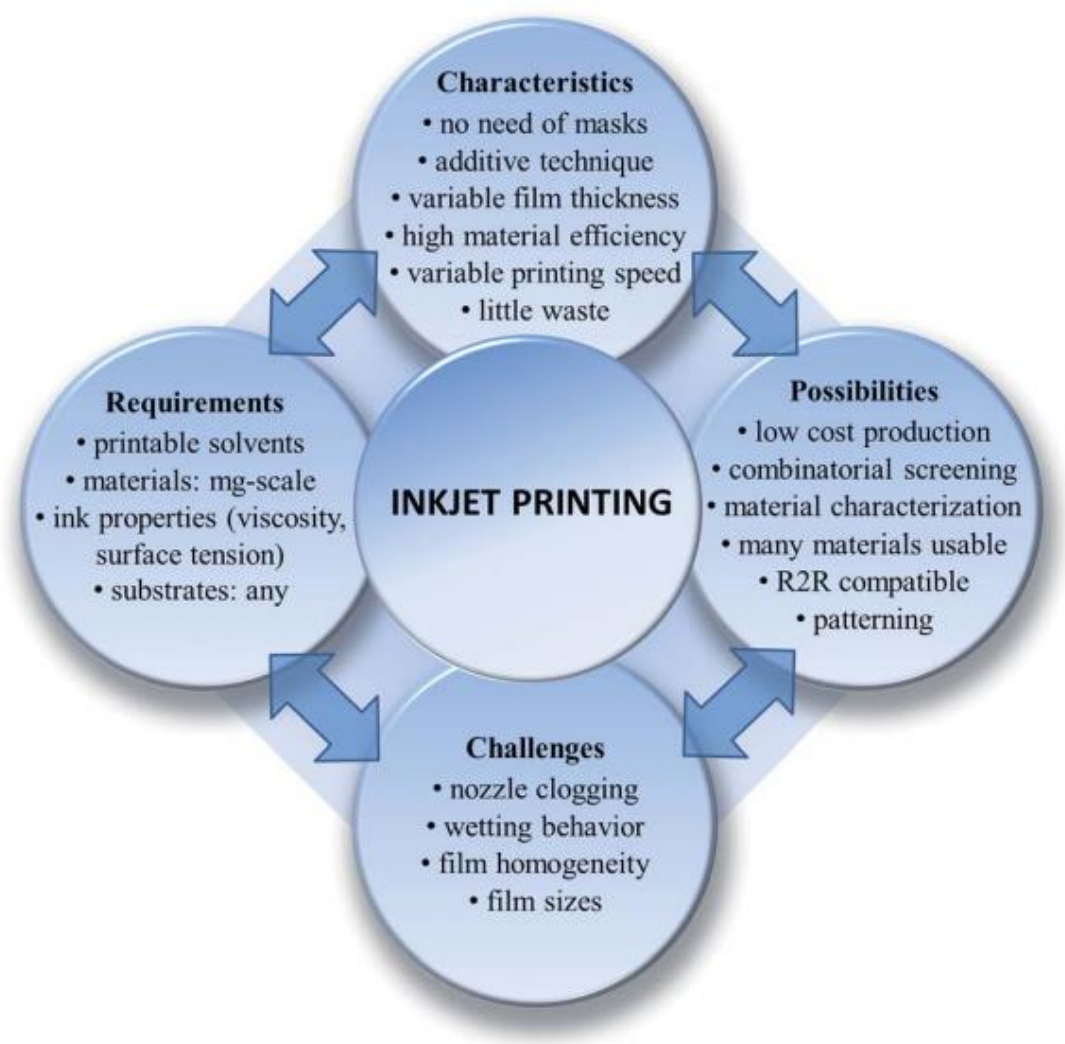

Fig. 2.11: Schematic representation of the characteristics, requirements, possibilities, and challenges of the inkjet printing process. From reference. ${ }^{121}$

Inkjet printing can be performed in continuous or in Drop-on-Demand (DoD) modes. In the continuous mode, the ink solution is pumped through a nozzle to form a liquid jet. This mode is mainly used in high-speed graphical applications since it allows high 
throughput production. In the DoD mode, an acoustic pulse generated by a piezoelectric or thermal trigger is used to generate and eject droplets from a reservoir through a nozzle to print with high placement accuracy. ${ }^{120}$ Employing the DoD mode minimizes the amount of ink used for the deposition (few tens of $\mathrm{pL}$ ) and as such it minimizes waste and consumption of the functional compound. Some requirements and recent challenges faced by inkjet printing processes are listed in Figure 2.11 and more in detail in Figure 2.12. ${ }^{116}$, $121-125$

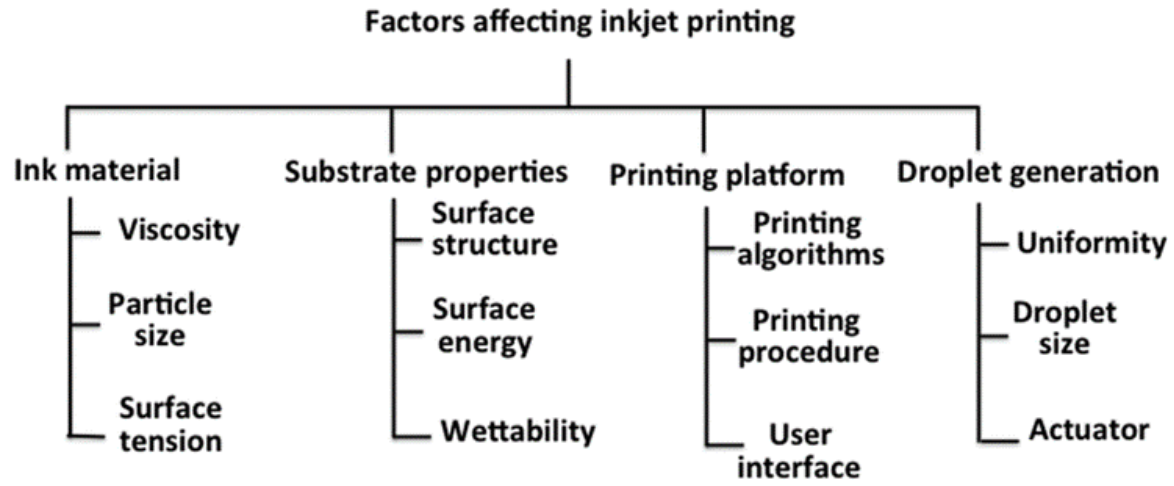

Figure 2.12: Factors affecting the inkjet printing process.

The ideal ink for functional and/or smart coatings would be of low cost, easy to prepare, store and jet, and would yield a homogenous coating layer after deposition and eventual post-processing. Depending on the inkjet printer system, there is a specific window for useable surface tension, viscosity, and density of the ink. ${ }^{121}$ The main parameter for inkjet printing is the ink chemistry and overall formulation because it strongly affects the printability/drop ejection of the ink, as well as the quality of the printed films. ${ }^{122-123,126}$ In terms of ink formulation, the choice of solvents is extremely important. Recently, there is a growing tendency to replace the traditional organic solvents with water due to the strict environmental regulations, and health and safety concerns. ${ }^{116,123,126-127}$ However, the development of suitable water-based inks for inkjet printing suffers from certain challenges (e.g. pinhole formation and lack of adhesion) in particular when the water-borne ink is not wetting properly the substrates, due to the difference between the surface energy of the material and the surface tension of the ink. ${ }^{122-}$ 123, 126 Furthermore, the rheological, surface tension, and the density are the main parameters which affect the performance of the inkjet process. The viscosity should be low enough to refill the nozzles in time but sufficient to prevent tailing with the formation of satellite droplets. The surface tension should be sufficient to avoid the flow of the ink through the nozzle, but enough to form spherical droplets.

In a piezo inkjet printer, the droplets are formed by the pressure impulse generated by the deformation of a piezoelectric element in the nozzle when a voltage is applied. In the simplest case, a trapezoidal voltage waveform is used for the droplet formation as is shown in Figure 2.13. 


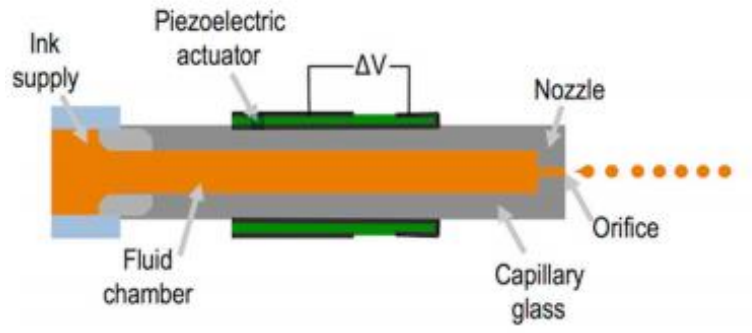

(a)

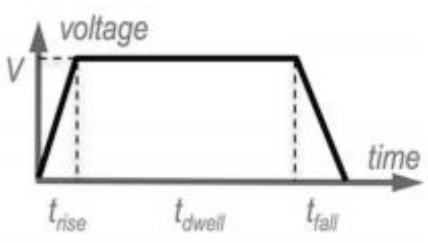

(b)

Figure 2.13: (a) A schematic structure of a piezoelectric nozzle for inkjet printing and (b) a trapezoidal voltage waveform used to generate the drops. From reference. ${ }^{120}$

The understanding of the droplet formation process is the key factor to control the drop deposition, through avoiding satellites, trajectory deviation, splashing, and tails, as is shown in Figure 2.14.

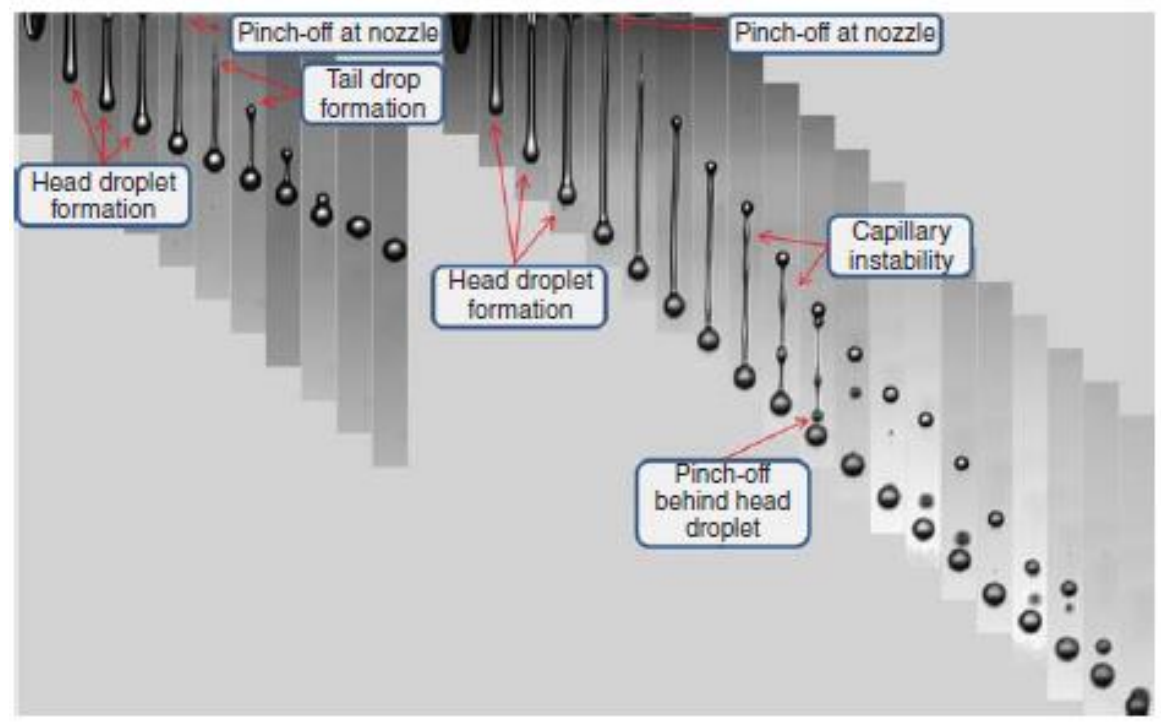

Fig. 2.14: Various jetting behavior of polymer-containing inks in DoD inkjet printing which can be obtained varying the rheological properties of the inks and the inkjet printing conditions. From reference. ${ }^{123}$

Demonstrating the readily applicability of inkjet printing as a versatile tool to coat and pattern substrates with polymer coatings, Edmonson and coworkers ${ }^{109}$ reported the deposition of a polyelectrolyte based macroinitiator that phase-separated in the dried printed droplet to form a sub-micrometer pattern of surface-active initiators ready for subsequent brush growth. ${ }^{109}$

Research is needed to better understand the interactions between the complex fluid and the surface, as well as the role of the surface morphology and surface chemistry. ${ }^{123}$ Despite the fact that the printed film size and thickness can be easily varied by changing 
the number of deposited droplets, the drying control of different film sizes is very complicated. ${ }^{121}$

\subsection{Anticorrosion polymer coatings}

In Chapter 6 the anti-corrosion performance of inkjet deposited polyurethane-based coatings on aluminum-metallized fabrics is presented and discussed. The performance of the protective coatings in alkaline environments is evaluated. In this section, the characteristics of aluminum corrosion and strategies to prevent it are provided, followed by a more in-depth overview of the use of complex fluids as promising protective coatings.

Aluminum (Al) and its alloys are known for the high strength-to-weight ratio. $\mathrm{Al}$ is an excellent heat and electrical conductor, highly reflective, ductile, nontoxic, and is therefore widely used in construction, chemical, food, electronics, transportation, aerospace, and packaging applications. ${ }^{128-129}$ However, due to the high chemical activity and potentially poor corrosion resistance the application of aluminum and its alloys is limited by environmental exposure, such as humidity and salty milieu. ${ }^{34,130-132}$

When exposed to oxygen in the air, aluminum surfaces develop a thin layer (5 to 10 $\mathrm{nm}$ thick) of aluminum oxide/alumina $\left(\mathrm{Al}_{2} \mathrm{O}_{3}\right)$ that provides a very good dry "selfprotective" corrosion barrier. However, when exposed to aqueous environments the aluminum oxide layer is vulnerable to $\mathrm{pH}$ - and salt-induced corrosion processes. ${ }^{34,130-132}$ In neutral and mildly acid solutions ( $\mathrm{pH} 4$ to 8 ), the aluminum oxide layer is quite stable. However, aluminum is an amphoteric material which dissolves in more acidic ( $\mathrm{pH}$ below 4) and more alkaline solutions ( $\mathrm{pH}$ above 8) forming $\mathrm{Al}^{3+}$ and $\mathrm{AlO}_{2}{ }^{-}$ions, respectively, as is shown in the Pourbaix diagram presented in Figure 2.15. ${ }^{132}$

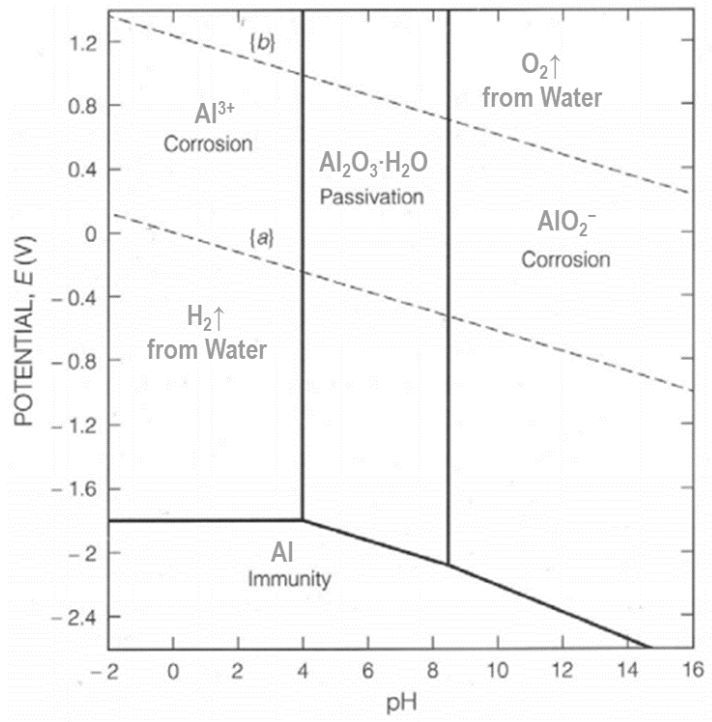

Fig. 2.15: Pourbaix diagram for aluminum in aqueous environments.From reference. ${ }^{132}$ 
Moreover, the presence of aggressive electrolytes (such as chloride and bromide anions) promote the electrochemical reactions, such as corrosion of the protective layer. ${ }^{133}$ Based on the appearance/morphological properties of the corroded metal surface, several forms of wet corrosion can be identified as is shown in Figure 2.16. ${ }^{33,134-135}$

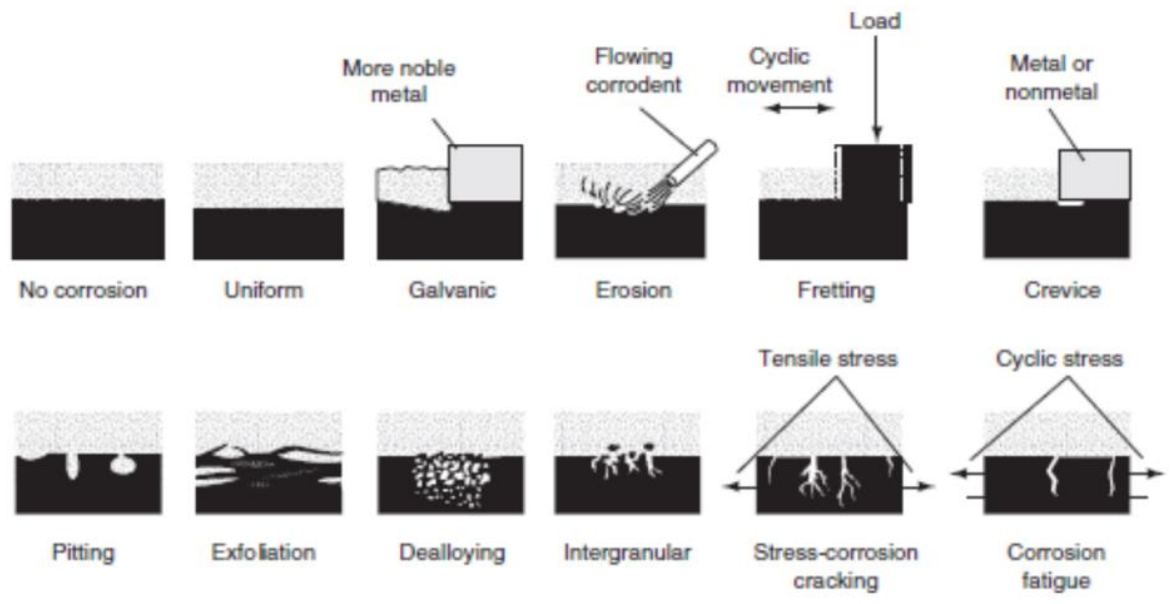

Fig. 2.16: Schematic summary of the various forms of wet corrosion. ${ }^{135}$

Among these processes, uniform corrosion of aluminum is the most dominant type of wet corrosion that occurs on aluminum in aggressive alkaline/acid conditions. This electrochemical process is diffusion-controlled and leads to the formation of nanoscale voids and hydrogen bubbles. ${ }^{34,130-132,136-137}$

Although the absolute prevention of corrosion is not possible, a coating system can be employed in order to delay or slow down the diffusion-controlled interfacial corrosion prosses of the exposed surface. ${ }^{22,32-34,133-135,138-139}$ The coatings can be divided into active or passive depending if the coating components interact with the corrosion environment and/or shifting the corrosion reaction. Various active and passive mechanisms of anticorrosion coatings are summarized in Figure 2.17. 15, 22, 33-34, 135, 140-141 
MODES OF CORROSION INHIBITION

BARRIER PROTECTION

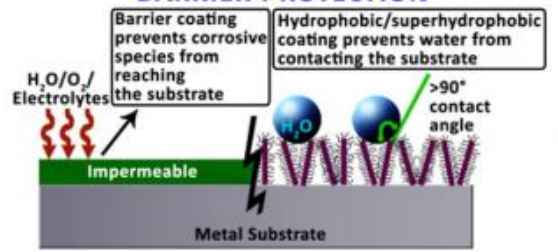

ANODIC PASSIVATION

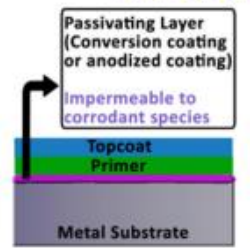

Rupture of
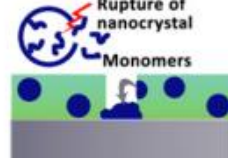

Metal Substrate
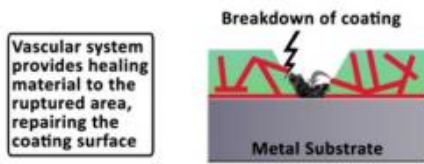

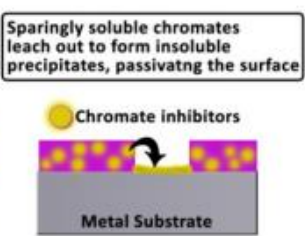

Metal Substrate

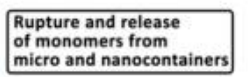

CATHOdic PROTECTION
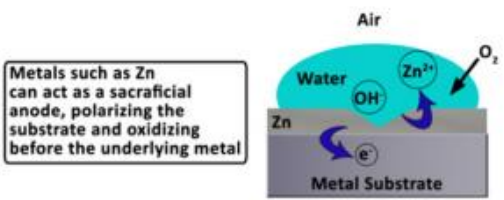

ACtIVE CORROSION INHIBITION

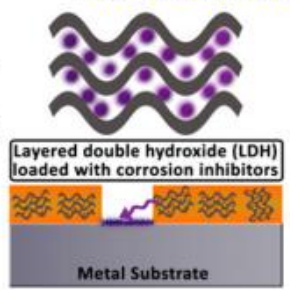
Strongly binding ligands
coordinate to the exposed
metal surface, inhibiting
further corrosion further corrosion

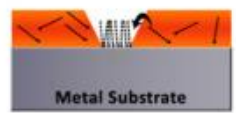

Metal Substrate

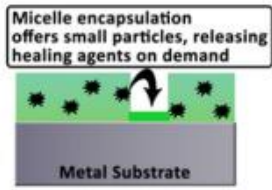

"Self-Healing" -

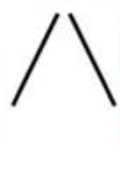

Sol-gel coating repairing

Capsules conatain sol-gel the void in the coating precursors such as alkoxyslilanes

Fig. 2.17: Overview of the different modes of corrosion inhibition, including barrier protection, cathodic protection, anodic passivation, active corrosion inhibition, and 'self-healing'. From reference. ${ }^{142}$

Many coating formulations still rely on toxic components like chromate-derivates as inhibiting compounds and on volatile organic compounds (VOCs) to help the drying process of the coating. ${ }^{34,127,130,139,143}$ The inhibiting pigments employed in anti-corrosion coatings are often inorganic, slightly water-soluble salts of which chromate-derivates are the most frequently used. This is ascribed to their outstanding anti-corrosion performance, i.e. they are capable to form a barrier against the aggressive electrolyte species by forming a bipolar bilayer as well as they are known to self-heal. ${ }^{140}$ However, chromate derivatives, in particular, hexavalent chromium species, have been banned because they are considered to be highly toxic. ${ }^{144}$

In recent years, the application of waterborne polymeric coatings combined with barrier and/or inhibitive pigments has been widely adopted as alternative approaches to chromate based coatings due to i) its ease of application, ii) good anti-corrosion performance, iii) lower toxicity compared to the inorganic alternatives, and iv) the fact that they are considered to be more environmentally friendly by avoiding harmful VOC emissions. ${ }^{15}$ However, there are still some differences between the water-borne and the solvent-based coatings in the anti-corrosion performance as well as in the chemical 
resistance, which demonstrates the need to further improve water-borne anti-corrosion coatings. ${ }^{33,145}$

The physical properties and protective performance of water-borne coating systems depend on the film formation mechanism, the choice of the binder and the type of corrosion inhibition. ${ }^{33}$ The presence of an active protection mechanism, like the addition of a corrosion inhibitor, represents an effective and reliable approach to obtain durable effective coatings. Common types of binders used in coatings are acrylic, alkyd, polyurethane, and epoxy-based polymers. ${ }^{34,139,146}$ Compared to epoxy based coating technology, the advantage of using polyurethane-based coatings is their excellent resistance to weathering and their self-healing behavior. ${ }^{147-153}$ However, the major drawbacks of most polyurethane-based coatings are their poor resistance towards mechanical strains and deformation, and their degradation at high temperatures (above $\left.110{ }^{\circ} \mathrm{C}\right) .{ }^{154}$ Recently, Noreen et al. ${ }^{149}$ have reviewed various formulations of environmentally friendly water-borne polyurethane-based coating systems and their application in the coating and paint industry. ${ }^{149}$ More recently, Zhou et al. ${ }^{148}$ reported the preparation and the application via inkjet printing of several water-borne polyurethanebased coatings from renewable resources. ${ }^{148}$ Furthermore, polyurethane-based coating systems can contain carbon derivatives, such as carbon nanotubes, graphene, and graphene oxide as anti-corrosion additives to organic coatings. ${ }^{150-153}$ For instance, Li et $a l .{ }^{152}$ reported that the anticorrosion properties of a polyurethane based coating was significantly enhanced by the addition of only $0.4 \mathrm{wt}$.\% of graphene oxide. This suprising anti-corrosion performance was ascribed to the extraordinary electrical and physical properties of the reduced graphene oxide. ${ }^{152}$

The replacement of the chromates as active inhibitor is a challenge and a large number of studies focused on the design and application of new corrosion prevention polymers as well as on finding new potential alternatives. ${ }^{139}$ Regarding the applications of inhibitors, we finally note that organic-based corrosion inhibitors are a promising and effective class of chromium-free alternatives. ${ }^{155}$ Other types of inhibitors encompass surfactants with hydrophilic and hydrophobic molecular moieties containing nitrogen, oxygen, sulfur and phosphorus atoms with lone electron pairs, triple bonds, and aromatic moieties. ${ }^{156-159}$ For example, straight chain aliphatic carboxylates posses good inhibition characteristics toward a number of metals, including aluminum. ${ }^{160}$

\subsection{Stimuli-responsive polymer films}

Inspired by stimuli-responsive biomacromolecular systems (such as proteins), stimuli-responsive polymer (SRP) materials have attracted wide interest in the past two decades. ${ }^{4,7,9-10,12,17,37-38}$ In this section, we will describe first the physical and chemical aspects of few "classical" stimuli-responsive films focusing on thermo-, solvent-, and redox- responsive materials that constitute the film platforms used throughout the work presented and discussed in this Thesis. 


\subsubsection{Temperature-responsive polymer films}

Temperature is the most widely used stimulus in smart polymer systems because a change of temperature is not only relatively easy to control but is also easily applicable. ${ }^{2}$, 161 This physical stimulus affects the equilibrium of conformations as well as the thermodynamical equilibrium of chemical reactions. Therefore, in this section we describe the temperature-induced phase transition of polymer chains and the temperaturetriggered reversible covalent bonding of dynamic self-healing films. Thermo-responsive behavior can be induced in a variety of settings, including in vivo, and potential benefits have been envisioned for a range of biologically relevant applications, including controlled drug delivery, bioseparation, filtration, and smart surfaces. ${ }^{2}$, 4, 6, 14, 25, 40, 161-163

Temperature-induced conformational transition in water is related to solution-phase diagrams, in particular to lower critical solution temperature (LCST) and upper critical solution temperature (UCST) behavior. A polymer with UCST exists in dissolved state in solution when the temperature is above the critical temperature, while a phase transition occurs when the temperature is below the critical temperature. Oppositely, the polymers with LCST are in solution when the temperature is below the critical temperature and precipitate when the temperature is above the critical temperature.

Poly $(N$-isopropylacrylamide) (PNIPAM) is a stimuli-responsive smart polymer that responds to a wide range of external stimuli, such as temperature, quality of the solvent, ionic strengths, and pressure. ${ }^{86-87,}$ 163-164 PNIPAM is the most studied temperatureresponsive polymer with a LCST (around $32{ }^{\circ} \mathrm{C}$ ) which is close to the physiological body temperature. ${ }^{86-87,163}$ Below LCST and at ambient pressure PNIPAM is soluble in water owing to the H-bonding interaction between the amide group and the water molecules. The polymer chains and solvent molecules are in one homogenous mixed phase. Above the LCST the inter-molecular H-bonding interactions dominate resulting in a coil-toglobule conformational phase transition. While free PNIPAM has an abrupt coil-toglobule transition at $32.5^{\circ} \mathrm{C}$, PNIPAM end-tethered in a brush regime displays a broad swollen-to-collapsed transition spanning as much as $25^{\circ} \mathrm{C} .{ }^{93}, 163$ Moreover, the LCST is further dependent on the chain length, tacticity, and incorporation of co-monomers, pressure or even the chemical nature of the end groups. ${ }^{2}{ }^{163}$ As previously mentioned, many factors can be used to tune the LCST value of PNIPAM films (see also section 2.3). ${ }^{84-87,93,165-166}$

Regarding surface engineering applications with PNIPAM, for example, Sun and coworkers $^{167}$ reported the reversible temperature-induced switching between superhydrophilicity and superhydrophobicity of silicon surfaces combining microgrooves and thermo-responsive films, as is shown in Figure 2.18. ${ }^{167}$ 
a)

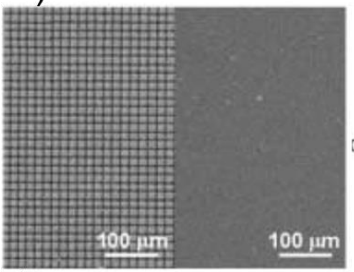

d) b)

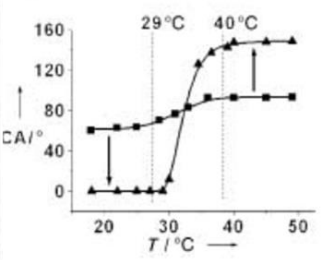

c)

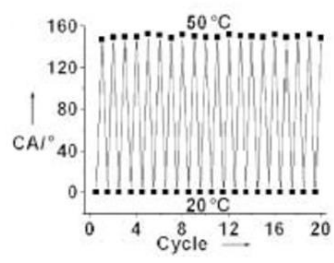

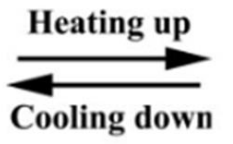
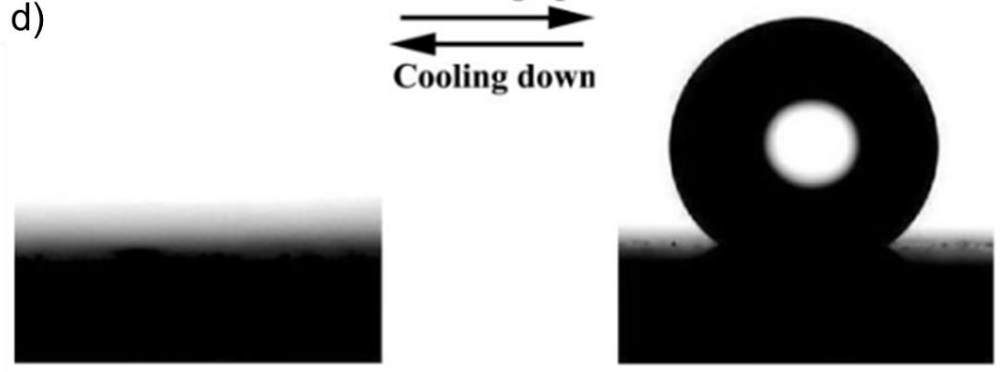

Fig. 2.18: Surface-roughness-enhanced wettability of a PNIPAM brush-modified surface. a) SEM images of a rough/structured substrate and a flat silicon surface. b) The temperature-induced transition of PNIPAMmodified flat and rough surfaces. c) Heating-cooling cycles of the switching between superhydrophilicity and superhydrophobicity of PNIPAM brushes grafted on rough silicon surfaces induced by the change in the temperature versus reversible. d) The water-drop profile of the superhydrophilicity and superhydrophobicity of PNIPAM brushes grafted on the rough silicon surfaces. Modified from reference. ${ }^{167}$

\subsubsection{Solvent-responsive polymer films}

The interaction and the affinity of a solvent with polymer segments can be controlled by the quality of the solvent mixtures. These can be used to trigger the conformational transition of the polymer chains (see also section 2.3). Two possible scenarios can be distinguished: the co-solvent effect that occurs when a polymer is insoluble in the pure solvents but dissolves in a mixture of the two solvents, and co-non-solvency behaviour when the polymer is completely miscible in both solvents but precipitates in a mixture of the two solvents.

As we discuss in a separate Chapter, PNIPAM has a co-non-solvent behavior in a mixture of water and alcohols such as methanol, ethanol or isopropanol. ${ }^{168-170}$ At room temperature, PNIPAM polymer chains are found to be swollen in water or one of these alcohols, while they become collapsed in a methanol-water mixture. The co-non-solvency behavior is affected by the physical constraints on the polymer chains; in fact, the conformational transition of PNIPAM chains occur in 10-20 vol.\% methanol when the polymer is free in solution, and in 30 vol.\% methanol in water for gels and brushes. ${ }^{171-173}$ The origin of co-non-solvency is still under debate, as shown in Figure 2.19. ${ }^{174-177}$

Irrespective of the exact explanation regarding its origin, the manifestation of the phenomenon of co-non-solvency is well established and has been widely observed for 
different combinations of polymers, solvents and co-solvents. ${ }^{172,}{ }^{178-181}$ Co-non- and cosolvency of polymer brushes is of interest $e$.g., for surfaces with tunable friction and adhesion properties in smart drug releasing devices, for the pick-up and release of nanoparticles, regulation of channel permeability in fluidics, as well as sensing devices. ${ }^{12}$, ${ }^{182-185}$ For instance, Sun et al. ${ }^{185}$ reported the fabrication of a microchip with a nanogelcontaining smart membrane capable to undergo a reversible swelling-shrinking volume transition to control the permeability of the membrane in response to variations in ethanol concentration. ${ }^{185}$

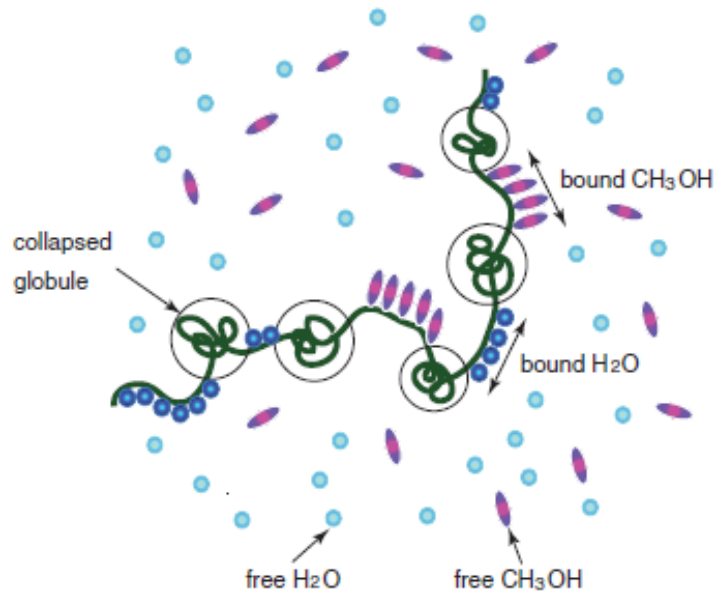

Fig. 2.19: Schematic representation of the competition between PNIPAM-water and PNIPAM-methanol hydrogen bonding. From reference. ${ }^{174}$

\subsubsection{Redox-responsive polymer films}

Redox-responsive polymers refer to macromolecules bearing redox-sensitive moieties that can change their oxidation state when a chemical or an electrochemical stimulus is applied. ${ }^{35-36,186-188}$ Due to the reversibility and easy external control of the redox process, these polymers are interesting for different applications covering the design of a number of electrochemical devices such as batteries, biosensors, electrochromic devices or biofuel cells, as is shown in Figure 2.20. ${ }^{35-36,186-188}$ 


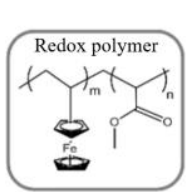

Drug Delivery Nanocapsules

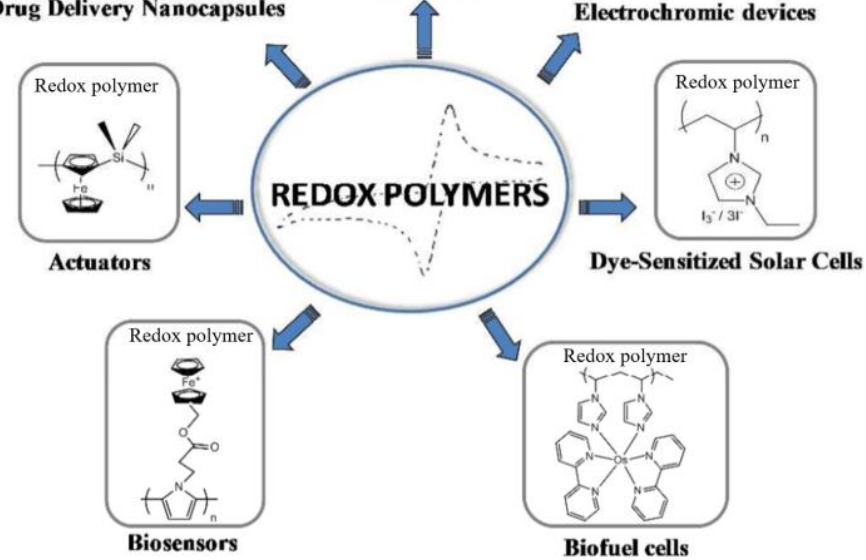

Fig. 2.20: Chemical structures of the most popular redox polymers for various applications. From reference. $^{188}$

Among redox-active materials, organometallic polymer bearing a transition metalbased group, such as ferrocene, have attracted interest due to their unique chemical and physical properties. ${ }^{35-36,186,188-190}$ Several different ferrocene-containing polymers have been synthesized, investigated, and used in electrocatalysis and electrochemical sensing. ${ }^{35,186-187}$ The ferrocene moieties can be positioned in the backbone of the polymer chain or in the side chains as a pedant group in various applications. Regarding pedant groups, for example Mazurowski et al. ${ }^{191}$ reported the fabrication of various electro-active stimulus-responsive architectures of single end-grafted polymer chains containing ferrocene moieties on crosslinked polystyrene particles via SI-ATRP as delivery systems, magnetic ceramic precursor, or as redox-responsive colloids, as is shown in Figure 2.21. ${ }^{191}$

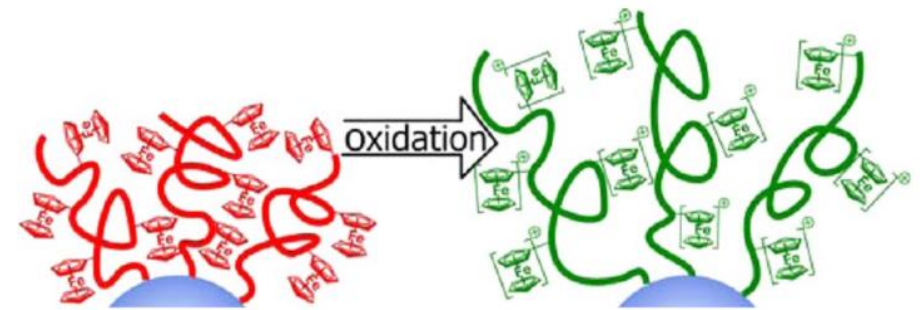

Fig. 2.21: Ferrocene-ferrocenium transition of the ferrocene-containing brushes as pendant side groups grafted on polystyrene particles. From reference. ${ }^{191}$

Polyferrocenylsilane polymers (PFSs) are an interesting class of materials that are composed of alternating ferrocene and alkylsiliane moieties in the backbone of the polymer chain. The ferrocene groups maintain their capability to be reversibly oxidized 
and reduced by chemical or electrochemical stimuli and the silane groups can be functionalized with other moieties conferring additional properties. ${ }^{36,186,192}$

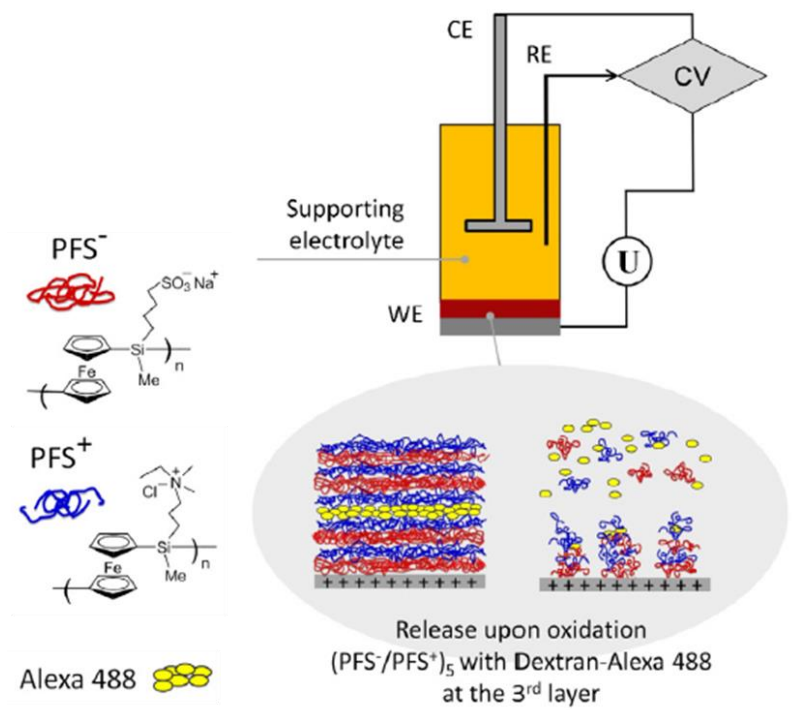

Fig. 2.22: Redox-controlled release of molecular payloads from a PFS-based composite multilayers. Modified from reference. ${ }^{197}$

PFSs can be incorporated into numerous polymer architectures as a homopolymer, block copolymers and random copolymers by using thermal, transition metal-catalyzed or living anionic ring-opening polymerization processes as extensively described by Hailes et al. ${ }^{187}$ Moreover, the chemical modification of the polymer allows tunability of the polymer properties via side group modification. Hempenius et al. ${ }^{193}$ have simplified the procedure to post-modified the PFSs by using halogenpropyl functionalized monomers (e.g. ([1]3-chloropropyl)methylsilaferrocenophane) which were largely used to introduce numerous groups as well as to prepare water-soluble PFSs (e.g. crosslinking moieties, anchoring moieties or functional/charged moieties). ${ }^{193-194}$ For example, Feng et al. ${ }^{195-196}$ reported the preparation of electrochemical sensing devices where the PFSs redox-active layer improved the sensitivity of the system to ascorbic acid as a model. The PFS layer was deposited via electrografting ${ }^{195}$ and Layer-by-Layer ${ }^{196}$ methods. The redox-responsiveness of the PFS based systems can also be used as drug delivery system as demonstrated and reported by Song et al. ${ }^{197}$

Furthermore, Espada et al. ${ }^{198}$ reported the exploit of PFS-based coating in optical fibers enhancing the sensitivity for a $\mathrm{NH}_{3}$ and $\mathrm{CO}_{2}$ gas sensor owing to its optical properties. ${ }^{199}$ The principle of operation of this optical-fiber device was based upon the changes in the transmitted optical signal caused by the exposure of the PFS-based film to redox-active gases, which changes the refractive index of the polymer layer. 


\subsubsection{Multi stimuli-responsive polymer films}

The right combination of responsive groups will allow the design of multi-functional polymers, which exhibits multifaceted behaviour when applying one or more external stimuli. ${ }^{18,21,41}$ For example, multi stimuli-responsive polymer coating layers can be obtained combining different stimuli-responsive polymer chains with various geometry and architectures.

For instance, mixed brush polymer brush coatings were reported by Vyas et al. ${ }^{200}$ They reported the preparation of dual-responsive mixed brushes containing polystyrene (PS) and poly(2-vinylpyridine) (P2VP) on silicon substrates. This coating was able to switch surface wettability by a reversible solvent-induced phase transition of the brush: toluene is a good solvent for PS and is a poor solvent for P2VP, while acidic water is a poor solvent for PS and a good solvent for the P2VP. Therefore, the PS swelled and the P2VP collapsed underneath when treated with toluene resulting in a hydrophobic surface. Vice versa, when the substrate was treated with acidic water, the P2VP chains solvated stretching away from the surface turning it in hydrophilic surface. ${ }^{200}$

Finally, it becomes even more interesting when more than two responsive groups constitute one polymer chain. This leads to different, sometimes unexpected, scenarios because the combinatorial effects of different stimuli-responsive groups can results in a more pronounced, or more complex, response of the overall polymer coating potentially for tailoring materials for new applications. ${ }^{18,21,201-202}$ For instance, Li et al. ${ }^{202}$ reported the development of smart interfaces for electrochemical sensing devices via the in-situ electrochemical induced free-radical polymerization process based on polymer brushes of PNIPAM, polyacrylic acid (PAA), or P(NIPAM-co-AA) that were responsive to temperature, ionic strength, and $\mathrm{pH}$ stimuli, independently or simultaneously. ${ }^{202}$

In conclusion, in this Chapter, we provided some insights into the broad range of scientific and technological challenges, into synthetic and materials chemistry-related issues, as well as the fundamentals of some applications to further enhance technologies related to surface engineering. Specific subject-related introductions will then be provided at the beginning of each Chapter for the different approaches and applications that have been tackled.

\subsection{References}

1. Ionov, L.; Minko, S.; Stamm, M.; Gohy, J.-F.; Jérôme, R.; Scholl, A., Reversible Chemical Patterning on Stimuli-Responsive Polymer Film: Environment-Responsive Lithography. Journal of the American Chemical Society 2003, 125 (27), 8302-8306.

2. Gil, E. S.; Hudson, S. M., Stimuli-reponsive polymers and their bioconjugates. Progress in polymer science 2004, 29 (12), 1173-1222.

3. Luzinov, I.; Minko, S.; Tsukruk, V. V., Adaptive and responsive surfaces through controlled reorganization of interfacial polymer layers. Progress in Polymer Science 2004, 29 (7), 635-698.

4. Mendes, P. M., Stimuli-responsive surfaces for bio-applications. Chemical Society Reviews 2008, 37 (11), 2512-2529. 
5. Nelson, A., Stimuli-responsive polymers: engineering interactions. Nature materials 2008, 7 (7), 523.

6. Bawa, P.; Pillay, V.; Choonara, Y. E.; Du Toit, L. C., Stimuli-responsive polymers and their applications in drug delivery. Biomedical materials 2009, 4 (2), 022001.

7. Tokarev, I.; Minko, S., Stimuli-responsive hydrogel thin films. Soft Matter 2009, 5 (3), 511-524.

8. Tokarev, I.; Motornov, M.; Minko, S., Molecular-engineered stimuli-responsive thin polymer film: a platform for the development of integrated multifunctional intelligent materials. Journal of Materials Chemistry 2009, 19 (38), 6932-6948.

9. Liu, F.; Urban, M. W., Recent advances and challenges in designing stimuli-responsive polymers. Progress in polymer science 2010, 35 (1-2), 3-23.

10. Lee, H.-i.; Pietrasik, J.; Sheiko, S. S.; Matyjaszewski, K., Stimuli-responsive molecular brushes. Progress in polymer science 2010, 35 (1-2), 24-44.

11. Roy, D.; Cambre, J. N.; Sumerlin, B. S., Future perspectives and recent advances in stimuli-responsive materials. Progress in Polymer Science 2010, 35 (1-2), 278-301.

12. Stuart, M. A. C.; Huck, W. T.; Genzer, J.; Müller, M.; Ober, C.; Stamm, M.; Sukhorukov, G. B.; Szleifer, I.; Tsukruk, V. V.; Urban, M., Emerging applications of stimuli-responsive polymer materials. Nature materials 2010, $9(2), 101$.

13. Yoon, J. A.; Kamada, J.; Koynov, K.; Mohin, J.; Nicolaÿ, R.; Zhang, Y.; Balazs, A. C.; Kowalewski, T.; Matyjaszewski, K., Self-healing polymer films based on thiol-disulfide exchange reactions and selfhealing kinetics measured using atomic force microscopy. Macromolecules 2011, 45 (1), 142-149.

14. Zhai, L., Stimuli-responsive polymer films. Chemical Society Reviews 2013, 42 (17), 7148-7160.

15. Montemor, M. F., Functional and smart coatings for corrosion protection: A review of recent advances. Surface and Coatings Technology 2014, 258, 17-37.

16. Chen, J.-K.; Chang, C.-J., Fabrications and applications of stimulus-responsive polymer films and patterns on surfaces: A review. Materials 2014, 7 (2), 805-875.

17. Ganesh, V. A.; Baji, A.; Ramakrishna, S., Smart functional polymers-a new route towards creating a sustainable environment. Rsc Advances 2014, 4 (95), 53352-53364.

18. Schattling, P.; Jochum, F. D.; Theato, P., Multi-stimuli responsive polymers-the all-in-one talents. Polymer Chemistry 2014, 5 (1), 25-36.

19. Wei, Q.; Haag, R., Universal polymer coatings and their representative biomedical applications. Materials Horizons 2015, 2 (6), 567-577.

20. Wu, Y.; Wei, Q.; Cai, M.; Zhou, F., Interfacial friction control. Advanced Materials Interfaces 2015, 2 (2), 1400392.

21. Cao, Z. Q.; Wang, G. J., Multi - Stimuli - Responsive Polymer Materials: Particles, Films, and Bulk Gels. The Chemical Record 2016, 16 (3), 1398-1435.

22. Ulaeto, S. B.; Rajan, R.; Pancrecious, J. K.; Rajan, T. P. D.; Pai, B. C., Developments in smart anticorrosive coatings with multifunctional characteristics. Progress in Organic Coatings 2017, 111, 294-314.

23. Zhang, Z. P.; Rong, M. Z.; Zhang, M. Q., Polymer engineering based on reversible covalent chemistry: A promising innovative pathway towards new materials and new functionalities. Progress in Polymer Science 2018, 80, 39-93.

24. Fujii, S.; Wanless, E. J.; Yusa, S.-i.; Webber, G. B.; Ishida, N., Stimulus-Responsive Soft Surface/Interface Toward Applications in Adhesion, Sensor and Biomaterial. In Stimuli-Responsive Dewetting/Wetting Smart Surfaces and Interfaces, Springer: 2018; pp 287-397.

25. Baldwin, E. T.; Wells, L. A., Stimuli-Responsive Polymers. Functional Biopolymers 2018, 1-24.

26. Matyjaszewski, K.; Tsarevsky, N. V., Macromolecular engineering by atom transfer radical polymerization. Journal of the American Chemical Society 2014, 136 (18), 6513-6533.

27. Braunecker, W. A.; Matyjaszewski, K., Controlled/living radical polymerization: Features, developments, and perspectives. Progress in polymer science 2007, 32 (1), 93-146.

28. Matyjaszewski, K., Polymer Chemistry: Current Status and Perspective. Chemistry International 2017, 39 (4), 7-11. 
29. Lahann, J., Reactive polymer coatings for biomimetic surface engineering. Chemical Engineering Communications 2006, 193 (11), 1457-1468.

30. Chung, C.-M.; Roh, Y.-S.; Cho, S.-Y.; Kim, J.-G., Crack healing in polymeric materials via photochemical [2+ 2] cycloaddition. Chemistry of Materials 2004, 16 (21), 3982-3984.

31. Nandivada, H.; Chen, H. Y.; Bondarenko, L.; Lahann, J., Reactive polymer coatings that "click". Angewandte Chemie International Edition 2006, 45 (20), 3360-3363.

32. Deshpande, P. P.; Jadhav, N. G.; Gelling, V. J.; Sazou, D., Conducting polymers for corrosion protection: a review. Journal of Coatings Technology and Research 2014, 11 (4), 473-494.

33. Sørensen, P. A.; Kiil, S.; Dam-Johansen, K.; Weinell, C. E., Anticorrosive coatings: a review. Journal of Coatings Technology and Research 2009, 6 (2), 135-176.

34. Twite, R.; Bierwagen, G. P., Review of alternatives to chromate for corrosion protection of aluminum aerospace alloys. Progress in organic coatings 1998, 33 (2), 91-100.

35. Feng, X.; Zhang, K.; Hempenius, M. A.; Vancso, G. J., Organometallic polymers for electrode decoration in sensing applications. RSC advances 2015, 5 (129), 106355-106376.

36. Giannotti, M.; Lv, H.; Ma, Y.; Steenvoorden, M.; Overweg, A.; Roerdink, M.; Hempenius, M. A.; Vancso, G. J., Stimulus responsive poly (ferrocenylsilanes): redox chemistry of iron in the main chain. Journal of inorganic and organometallic polymers and materials 2005, 15 (4), 527-540.

37. Maeda, T.; Otsuka, H.; Takahara, A., Dynamic covalent polymers: reorganizable polymers with dynamic covalent bonds. Progress in Polymer Science 2009, 34 (7), 581-604.

38. Urban, M. W., Stratification, stimuli-responsiveness, self-healing, and signaling in polymer networks. Progress in Polymer Science 2009, 34 (8), 679-687.

39. Minko, S., Responsive polymer brushes. Journal of Macromolecular Science, Part C: Polymer Reviews 2006, 46 (4), 397-420.

40. Cabane, E.; Zhang, X.; Langowska, K.; Palivan, C. G.; Meier, W., Stimuli-responsive polymers and their applications in nanomedicine. Biointerphases 2012, 7 (1), 9.

41. Xia, F.; Jiang, L., Bio - inspired, smart, multiscale interfacial materials. Advanced materials 2008, 20 (15), 2842-2858.

42. Barbey, R. R. R., Polymer brushes via surface-initiated controlled radical polymerization: synthesis, characterization, properties, and applications. Chemical Reviews 2009, 109 (11), 5437-5527.

43. Benetti, E. M.; Divandari, M.; Ramakrishna, S. N.; Morgese, G.; Yan, W.; Trachsel, L., Loops and Cycles at Surfaces: The Unique Properties of Topological Polymer Brushes. Chemistry-A European Journal 2017, 23 (51), 12433-12442.

44. Zhao, B.; Brittain, W. J., Polymer brushes: surface-immobilized macromolecules. Progress in Polymer Science 2000, 25 (5), 677-710.

45. Zoppe, J. O.; Ataman, N. C.; Mocny, P.; Wang, J.; Moraes, J.; Klok, H.-A., Surface-initiated controlled radical polymerization: state-of-the-art, opportunities, and challenges in surface and interface engineering with polymer brushes. Chemical reviews 2017, 117 (3), 1105-1318.

46. Korde, J. M.; Kandasubramanian, B., Fundamentals and effects of biomimicking stimuli-responsive polymers for engineering functions. Industrial \& Engineering Chemistry Research 2019, 58 (23), 97099757.

47. SZWARC, M., 'Living'polymers. Nature 1956, 178 (4543), 1168.

48. Hadjichristidis, N.; Pitsikalis, M.; Pispas, S.; Iatrou, H., Polymers with complex architecture by living anionic polymerization. Chemical reviews 2001, 101 (12), 3747-3792.

49. Grubbs, R. B.; Grubbs, R. H., 50th Anniversary Perspective: Living Polymerization-Emphasizing the Molecule in Macromolecules. Macromolecules 2017, 50 (18), 6979-6997.

50. Konkolewicz, D.; Wang, Y.; Zhong, M.; Krys, P.; Isse, A. A.; Gennaro, A.; Matyjaszewski, K., Reversible-deactivation radical polymerization in the presence of metallic copper. A critical assessment of the SARA ATRP and SET-LRP mechanisms. Macromolecules 2013, 46 (22), 8749-8772.

51. Liu, Y.; Klep, V.; Zdyrko, B.; Luzinov, I., Polymer grafting via ATRP initiated from macroinitiator synthesized on surface. Langmuir 2004, 20 (16), 6710-6718. 
52. Matyjaszewski, K., Advanced materials by atom transfer radical polymerization. Advanced Materials 2018, 30 (23), 1706441.

53. Matyjaszewski, K.; Xia, J., Atom transfer radical polymerization. Chemical reviews 2001, 101 (9), 29212990.

54. Wang, J.-S.; Matyjaszewski, K., Controlled/"living" radical polymerization. atom transfer radical polymerization in the presence of transition-metal complexes. Journal of the American Chemical Society 1995, 117 (20), 5614-5615.

55. Chiefari, J.; Chong, Y.; Ercole, F.; Krstina, J.; Jeffery, J.; Le, T. P.; Mayadunne, R. T.; Meijs, G. F.; Moad, C. L.; Moad, G., Living free-radical polymerization by reversible addition- fragmentation chain transfer: the RAFT process. Macromolecules 1998, 31 (16), 5559-5562.

56. Grubbs, R. B., Nitroxide-mediated radical polymerization: limitations and versatility. Polymer Reviews 2011, 51 (2), 104-137.

57. Perrier, S., 50th Anniversary Perspective: RAFT Polymerization-A User Guide. Macromolecules 2017, 50 (19), 7433-7447.

58. Ribelli, T. G.; Lorandi, F.; Fantin, M.; Matyjaszewski, K., Atom transfer radical polymerization: billion times more active catalysts and new initiation systems. Macromolecular rapid communications 2019, 40 (1), 1800616.

59. Gerard, P.; Couvreur, L.; Magnet, S.; Ness, J.; Schmidt, S., Controlled Architecture Polymers at Arkema: Synthesis, Morphology and Properties of All-Acrylic Block Copolymers. In Controlled/Living Radical Polymerization: Progress in RAFT, DT, NMP \& OMRP, American Chemical Society: 2009; Vol. 1024, pp 361-373.

60. Wang, J.-S.; Matyjaszewski, K., Controlled/" living" radical polymerization. atom transfer radical polymerization in the presence of transition-metal complexes. Journal of the American Chemical Society 1995, 117 (20), 5614-5615.

61. Min, K.; Gao, H.; Matyjaszewski, K., Use of ascorbic acid as reducing agent for synthesis of well-defined polymers by ARGET ATRP. Macromolecules 2007, 40 (6), 1789-1791.

62. Kato, M.; Kamigaito, M.; Sawamoto, M.; Higashimura, T., Polymerization of Methyl Methacrylate with the Carbon Tetrachloride/Dichlorotris- (triphenylphosphine)ruthenium(II)/Methylaluminum Bis(2,6-ditert-butylphenoxide) Initiating System: Possibility of Living Radical Polymerization. Macromolecules 1995, 28 (5), 1721-1723.

63. Fischer, H., The persistent radical effect in controlled radical polymerizations. Journal of Polymer Science Part A: Polymer Chemistry 1999, 37 (13), 1885-1901.

64. Fischer, H., The Persistent Radical Effect: A Principle for Selective Radical Reactions and Living Radical Polymerizations. Chemical Reviews 2001, 101 (12), 3581-3610.

65. Konkolewicz, D.; Magenau, A. J.; Averick, S. E.; Simakova, A.; He, H.; Matyjaszewski, K., ICAR ATRP with ppm Cu Catalyst in Water. Macromolecules 2012, 45 (11), 4461-4468.

66. Jakubowski, W.; Matyjaszewski, K., Activators regenerated by electron transfer for atom - transfer radical polymerization of (meth) acrylates and related block copolymers. Angewandte Chemie International Edition 2006, 45 (27), 4482-4486.

67. Konkolewicz, D.; Wang, Y.; Krys, P.; Zhong, M.; Isse, A. A.; Gennaro, A.; Matyjaszewski, K., SARA ATRP or SET-IRP. end of controversy? Polymer Chemistry 2014, 5 (15), 4396-4417.

68. Konkolewicz, D.; Schröder, K.; Buback, J.; Bernhard, S.; Matyjaszewski, K., Visible light and sunlight photoinduced ATRP with ppm of Cu catalyst. ACS Macro Letters 2012, 1 (10), 1219-1223.

69. Ribelli, T. G.; Konkolewicz, D.; Bernhard, S.; Matyjaszewski, K., How are radicals (re) generated in photochemical ATRP? Journal of the American Chemical Society 2014, 136 (38), 13303-13312.

70. Wang, Z.; Pan, X.; Li, L.; Fantin, M.; Yan, J.; Wang, Z.; Wang, Z.; Xia, H.; Matyjaszewski, K., Enhancing mechanically induced ATRP by promoting interfacial electron transfer from piezoelectric nanoparticles to Cu catalysts. Macromolecules 2017, 50 (20), 7940-7948.

71. Li, M.; Fromel, M.; Ranaweera, D.; Rocha, S.; Boyer, C.; Pester, C. W., SI-PET-RAFT: Surface-Initiated Photoinduced Electron Transfer-Reversible Addition-Fragmentation Chain Transfer Polymerization. ACS Macro Letters 2019, 8 (4), 374-380. 
72. Pan, X.; Fantin, M.; Yuan, F.; Matyjaszewski, K., Externally controlled atom transfer radical polymerization. Chemical Society Reviews 2018, 47 (14), 5457-5490.

73. Zhang, Z.; Wang, X.; Tam, K. C.; Sèbe, G., A comparative study on grafting polymers from cellulose nanocrystals via surface-initiated atom transfer radical polymerization (ATRP) and activator re-generated by electron transfer ATRP. Carbohydrate Polymers 2019, 205, 322-329.

74. De Beer, S.; Müser, M. H., Friction in (Im-) miscible polymer brush systems and the role of transverse polymer tilting. Macromolecules 2014, 47 (21), 7666-7673.

75. Jonas, A. M.; Hu, Z.; Glinel, K.; Huck, W. T., Chain entropy and wetting energy control the shape of nanopatterned polymer brushes. Macromolecules 2008, 41 (19), 6859-6863.

76. Minko, S., Grafting on solid surfaces:"Grafting to" and "grafting from" methods. In Polymer surfaces and interfaces, Springer: 2008; pp 215-234.

77. Zdyrko, B.; Luzinov, I., Polymer brushes by the "grafting to" method. Macromolecular rapid communications 2011, 32 (12), 859-869.

78. Edmondson, S.; Osborne, V. L.; Huck, W. T., Polymer brushes via surface-initiated polymerizations. Chemical society reviews 2004, 33 (1), 14-22.

79. Currie, E.; Norde, W.; Stuart, M. C., Tethered polymer chains: surface chemistry and their impact on colloidal and surface properties. Advances in colloid and interface science 2003, 100, 205-265.

80. Advincula, R. C., Polymer brushes. Encyclopedia of Polymer Science and Technology 2004.

81. Jonas, A. M.; Hu, Z.; Glinel, K.; Huck, W. T., Effect of nanoconfinement on the collapse transition of responsive polymer brushes. Nano letters 2008, 8 (11), 3819-3824.

82. Moh, L. C. H.; Losego, M. D.; Braun, P. V., Solvent Quality Effects on Scaling Behavior of Poly(methyl methacrylate) Brushes in the Moderate- and High-Density Regimes. Langmuir 2011, 27 (7), 3698-3702.

83. Bhat, R. R.; Tomlinson, M. R.; Wu, T.; Genzer, J., Surface-grafted polymer gradients: Formation, characterization, and applications. In Surface-initiated polymerization ii, Springer: 2006; pp 51-124.

84. Humphreys, B. A.; Prescott, S. W.; Murdoch, T. J.; Nelson, A.; Gilbert, E. P.; Webber, G. B.; Wanless, E. J., Influence of molecular weight on PNIPAM brush modified colloidal silica particles. Soft Matter 2019, 15 (1), 55-64.

85. Baulin, V. A.; Halperin, A., Signatures of a concentration - dependent flory $x$ parameter: Swelling and collapse of coils and brushes. Macromolecular theory and simulations 2003, 12 (8), 549-559.

86. Yim, H.; Kent, M.; Huber, D.; Satija, S.; Majewski, J.; Smith, G., Conformation of end-tethered PNIPAM chains in water and in acetone by neutron reflectivity. Macromolecules 2003, 36 (14), 5244-5251.

87. Yim, H.; Kent, M.; Mendez, S.; Balamurugan, S.; Balamurugan, S.; Lopez, G.; Satija, S., Temperaturedependent conformational change of PNIPAM grafted chains at high surface density in water. Macromolecules 2004, 37 (5), 1994-1997.

88. Kim, M.; Schmitt, S.; Choi, J.; Krutty, J.; Gopalan, P., From self-assembled monolayers to coatings: advances in the synthesis and nanobio applications of polymer brushes. Polymers 2015, 7 (7), 1346-1378.

89. Brown, H. R.; Char, K.; Deline, V., Enthalpy-driven swelling of a polymer brush. Macromolecules 1990, 23 (13), 3383-3385.

90. Huang, H.; Rankin, S. E.; Penn, L. S.; Quirk, R. P.; Cheong, T. H., Transition from mushroom to brush during formation of a tethered layer. Langmuir 2004, 20 (14), 5770-5775.

91. Morgese, G.; Trachsel, L.; Romio, M.; Divandari, M.; Ramakrishna, S. N.; Benetti, E. M., Topological Polymer Chemistry Enters Surface Science: Linear versus Cyclic Polymer Brushes. Angewandte Chemie International Edition 2016, 55 (50), 15583-15588.

92. Jung, Y.; Kim, J.-H.; Jang, W.-D., Linear and cyclic poly (2-isopropyl-2-oxazoline) s for fine control of thermoresponsiveness. European Polymer Journal 2017, 88, 605-612.

93. Yakushiji, T.; Sakai, K.; Kikuchi, A.; Aoyagi, T.; Sakurai, Y.; Okano, T., Graft architectural effects on thermoresponsive wettability changes of poly ( $\mathrm{N}$-isopropylacrylamide)-modified surfaces. Langmuir 1998, 14 (16), 4657-4662.

94. Hucknall, A.; Rangarajan, S.; Chilkoti, A., In pursuit of zero: polymer brushes that resist the adsorption of proteins. Advanced Materials 2009, 21 (23), 2441-2446. 
95. Lin, X.; He, Q.; Li, J., Complex polymer brush gradients based on nanolithography and surface-initiated polymerization. Chemical Society Reviews 2012, 41 (9), 3584-3593.

96. del Campo, A.; Arzt, E., Fabrication approaches for generating complex micro-and nanopatterns on polymeric surfaces. Chemical reviews 2008, 108 (3), 911-945.

97. Chaudhury, M. K.; Whitesides, G. M., How to make water run uphill. Science 1992, 256 (5063), 15391541 .

98. Zhang, T.; Benetti, E. M.; Jordan, R., Surface-Initiated Cu (0)-Mediated CRP for the Rapid and Controlled Synthesis of Quasi-3D Structured Polymer Brushes. ACS Publications: 2019.

99. Jones, D. M.; Smith, J. R.; Huck, W. T.; Alexander, C., Variable adhesion of micropatterned thermoresponsive polymer brushes: AFM investigations of poly ( $\mathrm{N}$ - isopropylacrylamide) brushes prepared by surface - initiated polymerizations. Advanced Materials 2002, 14 (16), 1130-1134.

100. Jhaveri, S. B.; Beinhoff, M.; Hawker, C. J.; Carter, K. R.; Sogah, D. Y., Chain-end functionalized nanopatterned polymer brushes grown via in situ nitroxide free radical exchange. ACS nano 2008, 2 (4), 719-727.

101. Benetti, E. M.; Zapotoczny, S.; Vancso, G. J., Tunable thermoresponsive polymeric platforms on gold by “photoiniferter" - based surface grafting. Advanced materials 2007, 19 (2), 268-271.

102. Sangermano, M.; Razza, N., Light induced grafting-from strategies as powerful tool for surface modification. eXPRESS Polymer Letters 2019, 13 (2), 135.

103. Geissler, M.; Xia, Y., Patterning: Principles and some new developments. Advanced Materials 2004, 16 (15), 1249-1269.

104. Hill, R. T.; Chilkoti, A., CHAPTER I. 2.13 SURFACE PATTERNING. In Biomaterials science: An introduction to materials in medicine, 2012; $\mathrm{p} 276$.

105. Emmerling, S. G.; Langer, L. B.; Pihan, S. A.; Lellig, P.; Gutmann, J. S., Patterning of a surface immobilized atrp initiator with an inkjet printer. Macromolecules 2010, 43 (11), 5033-5042.

106. Chen, T.; Amin, I.; Jordan, R., Patterned polymer brushes. Chemical Society Reviews 2012, 41 (8), 32803296.

107. Discekici, E. H.; Pester, C. W.; Treat, N. J.; Lawrence, J.; Mattson, K. M.; Narupai, B.; Toumayan, E. P.; Luo, Y.; McGrath, A. J.; Clark, P. G., Simple benchtop approach to polymer brush nanostructures using visible-light-mediated metal-free atom transfer radical polymerization. ACS Macro Letters 2016, 5 (2), 258-262.

108. Poelma, J. E.; Fors, B. P.; Meyers, G. F.; Kramer, J. W.; Hawker, C. J., Fabrication of complex three dimensional polymer brush nanostructures through light - mediated living radical polymerization. Angewandte Chemie International Edition 2013, 52 (27), 6844-6848.

109. Parry, A. V.; Straub, A. J.; Villar-Alvarez, E. M.; Phuengphol, T.; Nicoll, J. E.; Jordan, L. M.; Moore, K. L.; Taboada, P.; Yeates, S. G.; Edmondson, S., Submicron patterning of polymer Brushes: an unexpected discovery from inkjet printing of polyelectrolyte macroinitiators. Journal of the American Chemical Society 2016, 138 (29), 9009-9012.

110. Wu, T.; Efimenko, K.; Genzer, J., Combinatorial study of the mushroom-to-brush crossover in surface anchored polyacrylamide. Journal of the american chemical society 2002, 124 (32), 9394-9395.

111. Wu, T.; Efimenko, K.; Vlček, P.; Šubr, V.; Genzer, J., Formation and properties of anchored polymers with a gradual variation of grafting densities on flat substrates. Macromolecules 2003, 36 (7), 2448-2453.

112. Zhang, T.; Du, Y.; Müller, F.; Amin, I.; Jordan, R., Surface-initiated Cu (0) mediated controlled radical polymerization (SI-CuCRP) using a copper plate. Polymer Chemistry 2015, 6 (14), 2726-2733.

113. Tomlinson, M. R.; Genzer, J., Formation of surface-grafted copolymer brushes with continuous composition gradients. Chemical Communications 2003, (12), 1350-1351.

114. Tomlinson, M. R.; Genzer, J., Formation and properties of multivariant assemblies of surface-tethered diblock and triblock copolymers. Polymer 2008, 49 (22), 4837-4845.

115. Calvert, P. P. P., Inkjet printing for materials and devices. Chemistry of Materials 2001, 13 (10), 32993305.

116. De Gans, B. J.; Duineveld, P. C.; Schubert, U. S., Inkjet printing of polymers: state of the art and future developments. Advanced materials 2004, 16 (3), 203-213. 
117. Li, J.; Rossignol, F.; Macdonald, J., Inkjet printing for biosensor fabrication: combining chemistry and technology for advanced manufacturing. Lab on a Chip 2015, 15 (12), 2538-2558.

118. Moya, A.; Gabriel, G.; Villa, R.; del Campo, F. J., Inkjet-printed electrochemical sensors. Current Opinion in Electrochemistry 2017, 3 (1), 29-39.

119. Gonzalez-Macia, L.; Morrin, A.; Smyth, M. R.; Killard, A. J., Advanced printing and deposition methodologies for the fabrication of biosensors and biodevices. Analyst 2010, 135 (5), 845-867.

120. Alamán, J.; Alicante, R.; Peña, J.; Sánchez-Somolinos, C., Inkjet printing of functional materials for optical and photonic applications. Materials 2016, 9 (11), 910.

121. Teichler, A.; Perelaer, J.; Schubert, U. S., Inkjet printing of organic electronics-comparison of deposition techniques and state-of-the-art developments. Journal of Materials Chemistry C 2013, 1 (10), 1910-1925.

122. Derby, B., Inkjet printing of functional and structural materials: fluid property requirements, feature stability, and resolution. Annual Review of Materials Research 2010, 40, 395-414.

123. Hoath, S. D., Fundamentals of inkjet printing: the science of inkjet and droplets. John Wiley \& Sons: 2016.

124. Tekin, E.; Smith, P. J.; Schubert, U. S., Inkjet printing as a deposition and patterning tool for polymers and inorganic particles. Soft Matter 2008, 4 (4), 703-713.

125. Wijshoff, H., The dynamics of the piezo inkjet printhead operation. Physics reports 2010, 491 (4-5), 77 177.

126. Magdassi, S., Ink requirements and formulations guidelines. The chemistry of inkjet inks. MAGDASSI, S. Eds 2010, 19-42.

127. Meyer, T.; Boven, K.-H.; Günther, E.; Fejtl, M., Micro-electrode arrays in cardiac safety pharmacology. Drug Safety 2004, 27 (11), 763-772.

128. Davis, J. R., Corrosion of aluminum and aluminum alloys. Asm International: 1999.

129. Twite, R. L.; Bierwagen, G. P., Review of alternatives to chromate for corrosion protection of aluminum aerospace alloys. Progress in Organic Coatings 1998, 33 (2), 91-100.

130. Birbilis, N.; Hinton, B., Corrosion and corrosion protection of aluminium. In Fundamentals of Aluminium Metallurgy, Elsevier: 2011; pp 574-604.

131. Szklarska-Smialowska, Z., Pitting corrosion of aluminum. Corrosion science 1999, 41 (9), 1743-1767.

132. Pourbaix, M., Atlas of electrochemical equilibria in aqueous solution. NACE 1974, 307.

133. Qian, Y.; Li, Y.; Jungwirth, S.; Seely, N.; Fang, Y.; Shi, X., The Application of Anti-Corrosion Coating for Preserving the Value of Equipment Asset in Chloride-Laden Environments: A. Int. J. Electrochem. Sci 2015, 10, 10756-10780.

134. Almeida, E., Surface treatments and coatings for metals. A general overview. 1. Surface treatments, surface preparation, and the nature of coatings. Industrial \& engineering chemistry research 2001, 40 (1), 3-14.

135. Popoola, A.; Olorunniwo, O.; Ige, O., Corrosion resistance through the application of anti-corrosion coatings. In Developments in corrosion protection, IntechOpen: 2014.

136. Hebert, K. R.; Wu, H.; Gessmann, T.; Lynn, K., Positron annihilation spectroscopy study of interfacial defects formed by dissolution of aluminum in aqueous sodium hydroxide. Journal of the Electrochemical Society 2001, 148 (2), B92-B100.

137. Buckley, C.; Birnbaum, H.; Lin, J.; Spooner, S.; Bellmann, D.; Staron, P.; Udovic, T.; Hollar, E., Characterization of $\mathrm{H}$ defects in the aluminium-hydrogen system using small-angle scattering techniques. Journal of applied crystallography 2001, 34 (2), 119-129.

138. Metroke, T. L.; Parkhill, R. L.; Knobbe, E. T., Passivation of metal alloys using sol-gel-derived materials - a review. Progress in Organic Coatings 2001, 41 (4), 233-238.

139. Zhang, T.; Tang, D., Current research status of corrosion resistant coatings. Recent Patents on Corrosion Science 2009, 1, 1-5.

140. Nazeer, A. A.; Madkour, M., Potential use of smart coatings for corrosion protection of metals and alloys: A review. Journal of Molecular Liquids 2018, 253, 11-22. 
141. Schultze, J. W.; Lohrengel, M., Stability, reactivity and breakdown of passive films. Problems of recent and future research. Electrochimica Acta 2000, 45 (15-16), 2499-2513.

142. Dennis, R. V.; Patil, V.; Andrews, J. L.; Aldinger, J. P.; Yadav, G. D.; Banerjee, S., Hybrid nanostructured coatings for corrosion protection of base metals: a sustainability perspective. Materials Research Express 2015, 2 (3), 032001.

143. Kuznetsov, Y. I.; Thomas, J.; Mercer, A., Organic inhibitors of corrosion of metals. Springer Science \& Business Media: 1996.

144. End-of life vehicles. Official Journal of the European Communities 21/10/2000, L 269/34, 9.

145. Wegmann, A., Chemical resistance of waterborne epoxy/amine coatings. Progress in Organic Coatings 1997, 32 (1-4), 231-239.

146. Abdeen, D. H.; El Hachach, M.; Koc, M.; Atieh, M. A., A Review on the Corrosion Behaviour of Nanocoatings on Metallic Substrates. Materials 2019, 12 (2), 210.

147. Howarth, G., Polyurethanes, polyurethane dispersions and polyureas: Past, present and future. Surface coatings international part B: coatings transactions 2003, 86 (2), 111-118.

148. Zhou, X.; Li, Y.; Fang, C.; Li, S.; Cheng, Y.; Lei, W.; Meng, X., Recent advances in synthesis of waterborne polyurethane and their application in water-based ink: a review. Journal of Materials Science \& Technology 2015, 31 (7), 708-722

149. Noreen, A.; Zia, K. M.; Zuber, M.; Tabasum, S.; Saif, M. J., Recent trends in environmentally friendly water-borne polyurethane coatings: A review. Korean Journal of Chemical Engineering 2016, 33 (2), 388-400.

150. Song, D.; Yin, Z.; Liu, F.; Wan, H.; Gao, J.; Zhang, D.; Li, X., Effect of carbon nanotubes on the corrosion resistance of water-borne acrylic coatings. Progress in Organic Coatings 2017, 110, 182-186.

151. Li, Y.; Yang, Z.; Qiu, H.; Dai, Y.; Zheng, Q.; Li, J.; Yang, J., Self-aligned graphene as anticorrosive barrier in waterborne polyurethane composite coatings. Journal of Materials Chemistry A 2014, 2 (34), 14139-14145.

152. Li, J.; Cui, J.; Yang, J.; Li, Y.; Qiu, H.; Yang, J., Reinforcement of graphene and its derivatives on the anticorrosive properties of waterborne polyurethane coatings. Composites Science and Technology 2016, $129,30-37$

153. Mo, M.; Zhao, W.; Chen, Z.; Yu, Q.; Zeng, Z.; Wu, X.; Xue, Q., Excellent tribological and anti-corrosion performance of polyurethane composite coatings reinforced with functionalized graphene and graphene oxide nanosheets. Rsc Advances 2015, 5 (70), 56486-56497.

154. Le Gac, P.; Choqueuse, D.; Melot, D., Description and modeling of polyurethane hydrolysis used as thermal insulation in oil offshore conditions. Polymer Testing 2013, 32 (8), 1588-1593.

155. Brycki, B. E.; Kowalczyk, I. H.; Szulc, A.; Kaczerewska, O.; Pakiet, M., Organic Corrosion Inhibitors. In Corrosion Inhibitors, Principles and Recent Applications, IntechOpen: 2017.

156. Oguzie, E. E.; Li, Y.; Wang, S. G.; Wang, F., Understanding corrosion inhibition mechanismsexperimental and theoretical approach. Rsc Advances 2011, 1 (5), 866-873.

157. Du, Y. J.; Damron, M.; Tang, G.; Zheng, H.; Chu, C.-J.; Osborne, J. H., Inorganic/organic hybrid coatings for aircraft aluminum alloy substrates. Progress in organic coatings 2001, 41 (4), 226-232.

158. Xhanari, K.; Finšgar, M., Organic corrosion inhibitors for aluminium and its alloys in acid solutions: a review. RSC Advances 2016, 6 (67), 62833-62857.

159. Xhanari, K.; Finšgar, M., Organic corrosion inhibitors for aluminum and its alloys in chloride and alkaline solutions: a review. Arabian Journal of Chemistry 2016.

160. Hefter, G.; North, N.; Tan, S., Organic corrosion inhibitors in neutral solutions; part 1-inhibition of steel, copper, and aluminum by straight chain carboxylates. Corrosion 1997, 53 (8), 657-667.

161. Roy, D.; Brooks, W. L.; Sumerlin, B. S., New directions in thermoresponsive polymers. Chemical Society Reviews 2013, 42 (17), 7214-7243.

162. Ward, M. A.; Georgiou, T. K., Thermoresponsive polymers for biomedical applications. Polymers 2011, $3(3), 1215-1242$.

163. Halperin, A.; Kroeger, M.; Winnik, F. M., Poly (N - isopropylacrylamide) phase diagrams: fifty years of research. Angewandte Chemie International Edition 2015, 54 (51), 15342-15367. 
164. Heskins, M.; Guillet, J. E., Solution properties of poly (N-isopropylacrylamide). Journal of Macromolecular Science - Chemistry 1968, 2 (8), 1441-1455.

165. Plunkett, K. N.; Zhu, X.; Moore, J. S.; Leckband, D. E., PNIPAM chain collapse depends on the molecular weight and grafting density. Langmuir 2006, 22 (9), 4259-4266.

166. Varma, S.; Bureau, L.; Débarre, D., The Conformation of Thermoresponsive Polymer Brushes Probed by Optical Reflectivity. Langmuir 2016, 32 (13), 3152-3163.

167. Sun, T.; Wang, G.; Feng, L.; Liu, B.; Ma, Y.; Jiang, L.; Zhu, D., Reversible switching between superhydrophilicity and superhydrophobicity. Angewandte Chemie International Edition 2004, 43 (3), 357-360.

168. Mukherji, D.; Kremer, K., Coil-globule-coil transition of PNIPAm in aqueous methanol: Coupling allatom simulations to semi-grand canonical coarse-grained reservoir. Macromolecules 2013, 46 (22), 9158-9163.

169. Mukae, K.; Sakurai, M.; Sawamura, S.; Makino, K.; Kim, S. W.; Ueda, I.; Shirahama, K., Swelling of Poly(N-Isopropylacrylamide) Gels in Water-Alcohol (C1-C4) Mixed-Solvents. J Phys Chem-Us 1993, 97 (3), 737-741.

170. Yong, H.; Bittrich, E.; Uhlmann, P.; Fery, A.; Sommer, J.-U., Co-Nonsolvency Transition of Poly (Nisopropylacrylamide) Brushes in a Series of Binary Mixtures. Macromolecules 2019.

171. Winnik, F. M.; Ringsdorf, H.; Venzmer, J., Methanol-water as a co-nonsolvent system for poly (Nisopropylacrylamide). Macromolecules 1990, 23 (8), 2415-2416.

172. Scherzinger, C.; Schwarz, A.; Bardow, A.; Leonhard, K.; Richtering, W., Cononsolvency of poly-Nisopropyl acrylamide (PNIPAM): Microgels versus linear chains and macrogels. Current Opinion in Colloid \& Interface Science 2014, 19 (2), 84-94.

173. Zhu, P. W.; Napper, D. H., Coil-to-globule type transitions and swelling of poly(N-isopropylacrylamide) and poly(acrylamide) at latex interfaces in alcohol-water mixtures. Journal of Colloid and Interface Science 1996, 177 (2), 343-352.

174. Tanaka, F.; Koga, T.; Winnik, F. M., Temperature-responsive polymers in mixed solvents: competitive hydrogen bonds cause cononsolvency. Physical review letters 2008, 101 (2), 028302.

175. Liu, M. Z.; Bian, F. L.; Sheng, F. L., FTIR study on molecular structure of poly(N-isopropylacrylamide) in mixed solvent of methanol and water. Eur Polym J 2005, 41 (2), 283-291.

176. Mukherji, D.; Marques, C. M.; Kremer, K., Polymer collapse in miscible good solvents is a generic phenomenon driven by preferential adsorption. Nature communications 2014, 5, 4882.

177. Yong, H.; Bittrich, E.; Uhlmann, P.; Fery, A.; Sommer, J.-U., Co-Nonsolvency Transition of Poly(Nisopropylacrylamide) Brushes in a Series of Binary Mixtures. Macromolecules 2019, 52 (16), 6285-6293.

178. Chen, C.; Song, Y.; Wang, L., A Novel Sensor Based on Layer - by - Layer Hybridized Phosphomolybdate and Poly (ferrocenylsilane) on a Cysteamine Modified Gold Electrode. Electroanalysis: An International Journal Devoted to Fundamental and Practical Aspects of Electroanalysis 2008, 20 (23), 2543-2548.

179. Winnik, F. M.; Ottaviani, M. F.; Bossmann, S. H.; Pan, W.; Garcia-Garibay, M.; Turro, N. J., Cononsolvency of poly (N-isopropylacrylamide): a look at spin-labeled polymers in mixtures of water and tetrahydrofuran. Macromolecules 1993, 26 (17), 4577-4585.

180. Young, T. H.; Chuang, W. Y., Thermodynamic analysis on the cononsolvency of poly (vinyl alcohol) in water-DMSO mixtures through the ternary interaction parameter. Journal of Membrane Science 2002, 210 (2), 349-359.

181. Saunders, B. R.; Crowther, H. M.; Vincent, B., Poly[(methyl methacrylate)-co-(methacrylic acid)] microgel particles: Swelling control using $\mathrm{pH}$, cononsolvency, and osmotic deswelling. Macromolecules 1997, 30 (3), 482-487.

182. Chen, Q.; Kooij, E. S.; Sui, X.; Padberg, C. J.; Hempenius, M. A.; Schön, P. M.; Vancso, G. J., Collapse from the top: brushes of poly (N-isopropylacrylamide) in co-nonsolvent mixtures. Soft Matter 2014, 10 (17), 3134-3142.

183. Sui, X.; Chen, Q.; Hempenius, M. A.; Vancso, G. J., Probing the Collapse Dynamics of Poly (N isopropylacrylamide) Brushes by AFM: Effects of Co - nonsolvency and Grafting Densities. small 2011, 7 (10), 1440-1447. 
184. Espinosa-Marzal, R. M.; Nalam, P. C.; Bolisetty, S.; Spencer, N. D., Impact of solvation on equilibrium conformation of polymer brushes in solvent mixtures. Soft Matter 2013, 9 (15), 4045-4057.

185. Sun, Y.-M.; Wang, W.; Wei, Y.-Y.; Deng, N.-N.; Liu, Z.; Ju, X.-J.; Xie, R.; Chu, L.-Y., In situ fabrication of a temperature-and ethanol-responsive smart membrane in a microchip. Lab on a Chip 2014, 14 (14), 2418-2427.

186. Nguyen, P.; Gómez-Elipe, P.; Manners, I., Organometallic polymers with transition metals in the main chain. Chemical reviews 1999, 99 (6), 1515-1548.

187. Hailes, R. L.; Oliver, A. M.; Gwyther, J.; Whittell, G. R.; Manners, I., Polyferrocenylsilanes: synthesis, properties, and applications. Chemical Society Reviews 2016, 45 (19), 5358-5407.

188. Gracia, R.; Mecerreyes, D., Polymers with redox properties: materials for batteries, biosensors and more. Polymer Chemistry 2013, 4 (7), 2206-2214.

189. Nakahata, M.; Takashima, Y.; Yamaguchi, H.; Harada, A., Redox-responsive self-healing materials formed from host-guest polymers. Nature communications 2011, 2, 511.

190. Abd - El - Aziz, A. S., Organometallic polymers of the transition metals. Macromolecular rapid communications 2002, 23 (17), 995-1031.

191. Mazurowski, M.; Gallei, M.; Li, J.; Didzoleit, H.; Stühn, B.; Rehahn, M., Redox-responsive polymer brushes grafted from polystyrene nanoparticles by means of surface initiated atom transfer radical polymerization. Macromolecules 2012, 45 (22), 8970-8981.

192. Bellas, V.; Rehahn, M., Polyferrocenylsilane - Based Polymer Systems. Angewandte Chemie International Edition 2007, 46 (27), 5082-5104.

193. Hempenius, M. A.; Brito, F. F.; Vancso, G. J., Synthesis and characterization of anionic and cationic poly(ferrocenylsilane) polyelectrolytes. Macromolecules 2003, 36 (17), 6683-6688.

194. Zhang, K.; Feng, X.; Sui, X.; Hempenius, M. A.; Vancso, G. J., Breathing Pores on Command: Redox Responsive Spongy Membranes from Poly (ferrocenylsilane) s. Angewandte Chemie International Edition 2014, 53 (50), 13789-13793.

195. Feng, X.; Sui, X.; Hempenius, M. A.; Vancso, G. J., Electrografting of stimuli-responsive, redox active organometallic polymers to gold from ionic liquids. Journal of the American Chemical Society 2014, 136 (22), 7865-7868.

196. Feng, X.; Cumurcu, A.; Sui, X.; Song, J.; Hempenius, M. A.; Vancso, G. J., Covalent layer-by-layer assembly of redox-active polymer multilayers. Langmuir 2013, 29 (24), 7257-7265.

197. Song, J.; Jańczewski, D.; Ma, Y.; van Ingen, L.; Sim, C. E.; Goh, Q.; Xu, J.; Vancso, G. J., Electrochemically controlled release of molecular guests from redox responsive polymeric multilayers and devices. European polymer journal 2013, 49 (9), 2477-2484.

198. Espada, L. I.; Shadaram, M.; Robillard, J.; Pannell, K. H., Ferrocenylenesilylene polymers as coatings for tapered optical-fiber gas sensors. Journal of Inorganic and Organometallic Polymers 2000, 10 (4), 169-176.

199. Paquet, C.; Cyr, P. W.; Kumacheva, E.; Manners, I., Polyferrocenes: metallopolymers with tunable and high refractive indices. Chemical Communications 2004, (2), 234-235.

200. Vyas, M. K.; Schneider, K.; Nandan, B.; Stamm, M., Switching of friction by binary polymer brushes. Soft Matter 2008, 4 (5), 1024-1032.

201. Rahane, S. B.; Floyd, J. A.; Metters, A. T.; Kilbey, S. M., Swelling Behavior of Multiresponsive Poly (methacrylic acid) - block - - poly (N - isopropylacrylamide) Brushes Synthesized Using Surface Initiated Photoiniferter - Mediated Photopolymerization. Advanced Functional Materials 2008, 18 (8), 1232-1240.

202. Zhou, J.; Wang, G.; Hu, J.; Lu, X.; Li, J., Temperature, ionic strength and pH induced electrochemical switching of smart polymer interfaces. Chemical Communications 2006, (46), 4820-4822 
Chapter 3

Swelling and Collapse of PNIPAM Brushes in Response to Temperature and Co-Non-Solvents Unveiled by Neutron Reflectivity 


\section{Contents}

Chapter 3 Swelling and Collapse of PNIPAM Brushes in Response to Temperature and Co-Non-Solvents Unveiled by Neutron Reflectivity .......................................................................................... 45

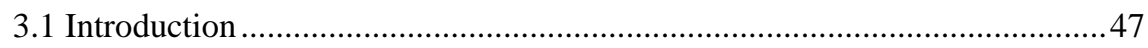

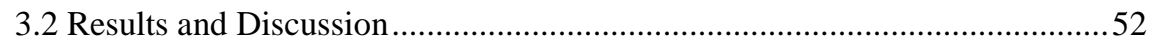

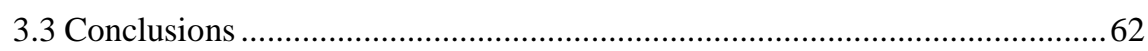

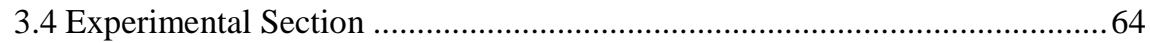

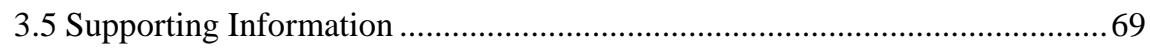

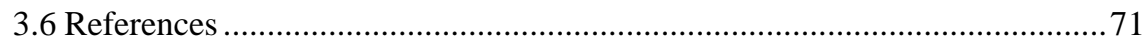




\subsection{Introduction}

The phase diagram and thermal responsive behavior of poly( $\mathrm{N}$-isopropyl acrylamide) (PNIPAM) in aqueous solutions have attracted tremendous interest since its discovery by Guillet and Heskins in 1968. ${ }^{1}$ PNIPAM and some derivatives show a lower critical solution (LCS) behavior in water at temperatures (LCST) near body temperature with little variation in LCST values in a broad composition range. ${ }^{2}$

While a molecular level explanation has already been offered in early work discussing LCST behavior, ${ }^{1}$ interest in PNIPAM has never seized to exist due to lack of full explanations of challenging details of the thermal phase behavior and the coil-toglobule transition, ${ }^{2-5}$ as well as due to the wide range of utilizing PNIPAM in biomedical technology, including drug release ${ }^{6}$ and responsive hydrogel applications. Numerous highly cited reviews have covered the state-of-the-art and captured the development of PNIPAM related research milestones. ${ }^{7-8}$

An interesting observation was reported by Winnik et al. ${ }^{9}$ in 1990 describing the effect of solvent mixtures consisting of water and methanol, on the LCST behavior. Inspired by a report obtained by Schild and Tirre ${ }^{10}$ they discovered a significant decrease of the LCST values with increasing amount of methanol (and other alcohols) in mixed solvents. The shape of the phase boundary in the phase diagram exhibited in mixed solvent systems of water and alcohol depended on the choice of the alcohol. ${ }^{5}$ The lowest LCST for methanol $(\mathrm{MeOH})$ was reported for the composition of 0.35 mole fraction, reaching a value of $-7.5^{\circ} \mathrm{C}$. This depression is followed by a steep elevation of LCST values with further increase of the $\mathrm{MeOH}$ concentration. ${ }^{9}$ Similar observations were simultaneously reported by Tirrell et al. ${ }^{10}$ with an added attempt to offer a theoretical explanation on the basis of the Flory Huggins (FH) theory. Comparisons were made between solution and hydrogel behavior and it was considered that perturbation of the $\mathrm{FH}$ $\chi$ parameter, reflecting an influence of water-MeOH complexes, causes the collapse by making this interaction with PNIPAM preferred instead of polymer-water bonds. This explanation however was rejected by the authors and instead formation of local contacts was proposed as the interaction relevant for the phase behavior causing LCST.

Confinement can not only be introduced by cross-linking in gels, but is also present in polymer brushes. ${ }^{11}$ Brushes are composed of surface grafted chains where usually one chain end is free and is closed to the free surface of the brush layer, while the other end (for linear polymers) is bound to a substrate surface. Brushes are interesting candidates for surface engineering, although challenges (in particular regarding scale up issues) remain. Concerning PNIPAM brush applications for surface engineering, our interest was captured by the question whether a gradient of collapsed and swollen PNIPAM brush layers can form when the free surface of a water swollen $n$ brush is exposed to a nonsolvent. We first monitored the collapse dynamics of PNIPAM brushes for different grafting densities with colloidal probe AFM indentation experiments by measuring 
increase of mechanical stiffness of the layers during collapse and collapse dynamics as a function of the grafting density. ${ }^{12}$ In subsequent studies, we investigated the reversible swelling and collapse in detail by ellipsometry thickness measurements in-situ, as well as AFM based friction and adherence experiments and assessed the variation of friction properties upon exposure to solvents and co-nonsolvent mixtures. ${ }^{13}$ We postulated a structural model assuming that brushes immersed in co-nonsolvents collapsed from the top layer first exposed to a non-solvent, while the brush interior remains partially hydrated. The layer structure was fitted by ellipsometry analysis and the results assuming this model were consistent with the observed variations in adhesion, friction and brush thickness upon exposure to non-solvents. However, for ellipsometry modelling, assumptions need to be made for a model to be fitted, as the ellipsometric response does not directly yield segment (and density) gradient distributions. In order to provide independent support for our layer model, and to gain further insights and confirmation for layered brush collapse behavior, we decided to observe brush collapse upon crossing the LCST by neutron reflectivity (NR) using co-nonsolvent mixtures. We monitored the formation of the collapsed top brush layer of PNIPAM, exposing the brush to water-short chain alcohol mixtures (water-methanol in $3: 1 \mathrm{v} / \mathrm{v}$ ratio). In order to inxrease the scattering contrast between the polymer and the background, and decrease incoherent background signal, deuterated $\left(\mathrm{D}_{2} \mathrm{O}\right)$ or partially deuterated $\left(\mathrm{D}_{2} \mathrm{O}-\mathrm{MeOH}\right.$ mixture $)$ solvents has been used. By fitting the reflectivity profiles, we measure the amount of trapped $\mathrm{D}_{2} \mathrm{O}$ in the brush as a function of temperature variation, or following exposure to $\mathrm{D}_{2} \mathrm{O}$ and $\mathrm{MeOH}$ mixtures (co-nonsolvent). We prepared a library of brush layers with different grafting densities (grafted from silicon) to enable us the study of the influence of molecular crowding (grafting density) on the layer collapse.

\section{Brush graft preparation and characterization}

We employed standard ATRP surface initiated polymerization procedures to prepare brush layers utilizing the "grafting from" procedure. ${ }^{12,}{ }^{14-15}$ The steps of surface modification and grafting are shown in Figure 3.1. In short, we used (3aminopropyl)triethoxysilane (APTES) functionalization, followed by coupling of the active initiator bromo isobutyrilbromide, BIBB, and 1-bromocarbonyl-1-methylethyl acetate, BMA, respectively. The ratio between the active and inactive BIBB/BMA was varied to obtain different grafting densities (see scheme on the right hand side of Figure 3.1). Further details of the surface functionalization process are shown in the Experimental Section. 


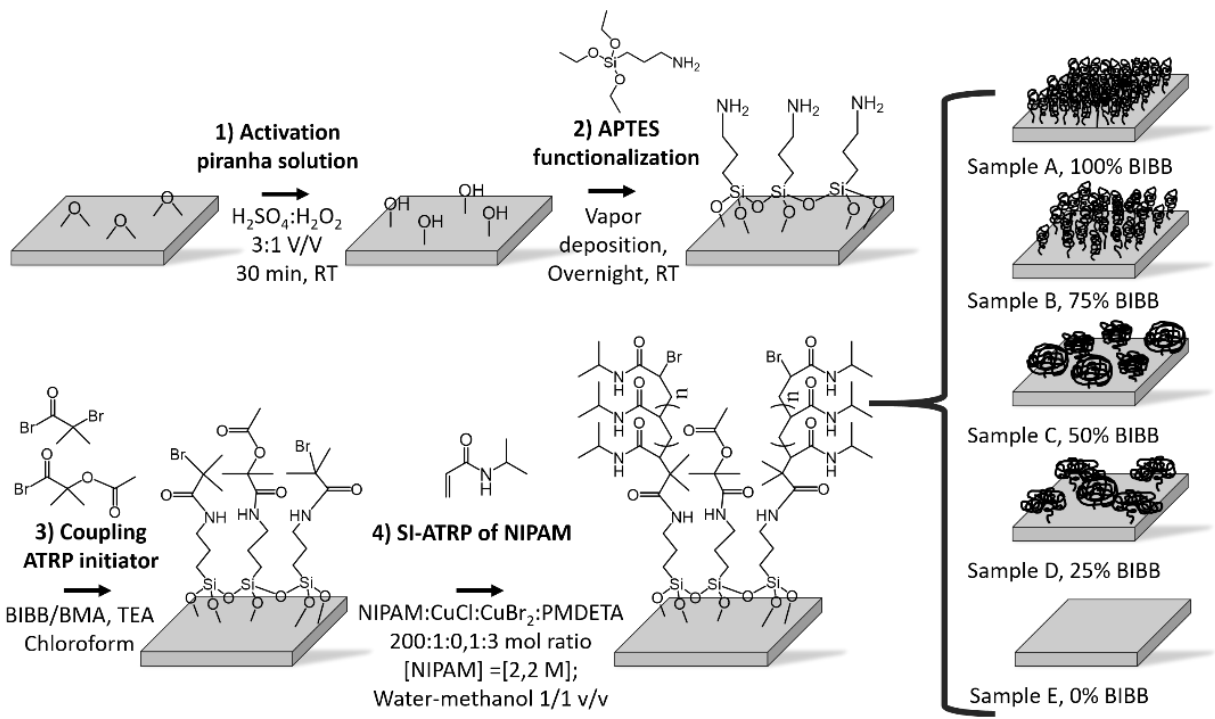

Figure 3.1: Preparation of the PNIPAM layers grafted on silicon surface: 1) cleaning and activation of the silicon surface via piranha treatment. 2) Immobilization of the ATRP initiator: a coupling agent on the surface via vapor deposition. 3) Coupling of the active ATRP initiators and inactive BMA, and 4) ATRP polymerization of NIPAM. The scheme on the right shows different grafting densities resulting from variations of the active initiator and inactive BMA.

Four different brushes (labeled from A to D) were prepared with different grafting density and thoroughly characterized. The objective was to use the same polymerization time (thus attempt to have the same molar mass) and only vary the initiator concentration. The dry and swollen thickness values, as well as the grafting densities obtained from AFM experiments (see Experimental Section) are shown for these brushes in Table 3.1. The grafting density values were determined using the relationship between dry and wet layer thickness values for PNIPAM according to the following relationship: ${ }^{16-17}$

$$
\mathrm{h}_{\mathrm{wet}} / \mathrm{h}_{\mathrm{dry}}=1.03 / \sigma^{2 / 3}
$$

Here $\sigma$ is the grafting density in chains $\cdot \AA^{-2}$ units.

Table 3.1: AFM investigation of the PNIPAM brush layers: dry and swollen thicknesses and estimated grafting density. The swelling ratio $\left(h_{\text {wet }}-h_{\text {dry }}\right) / h_{\text {wet }}$ and the grafting densities $h_{\text {wet }} / h_{\text {dry }}=1.03 / \sigma^{2 / 3}$ obtained from the AFM measurements are summarized in the table.

\begin{tabular}{|c|c|c|c|c|c|c|}
\hline $\begin{array}{c}\text { Wet state } \\
\text { in } \mathbf{D}_{\mathbf{2}} \mathbf{O}\end{array}$ & $\begin{array}{c}\text { Active } \\
\text { init. } \\
\text { mol\% }\end{array}$ & $\begin{array}{c}\AA \\
\text { dry }\end{array}$ & $\begin{array}{c}\mathbf{d}_{\text {wet }} \\
\AA\end{array}$ & $\begin{array}{c}\text { Swelling } \\
\text { ratio }\end{array}$ & $\begin{array}{c}\text { Grafting } \\
\text { density } \\
\text { Chains } \cdot \AA^{-2}\end{array}$ & $\begin{array}{c}\text { Units of } \\
\text { monomer } \\
\text { per chain }\end{array}$ \\
\hline Sample A & 100 & $630 \pm 10$ & $1460 \pm 70$ & 1.32 & 0.0030 & 680 \\
\hline Sample B & 75 & $390 \pm 30$ & $1410 \pm 140$ & 2.62 & 0.0015 & 1020 \\
\hline Sample C & 50 & $420 \pm 10$ & $1830 \pm 70$ & 3.36 & 0.0011 & 1600 \\
\hline Sample D & 25 & $420 \pm 10$ & $1210 \pm 100$ & 1.88 & 0.0021 & 700 \\
\hline
\end{tabular}




\section{Neutron reflectivity studies}

In neutron reflectivity NR (sometimes called reflectometry) the reflection of a beam of neutrons is measured from a flat surface as a function of the scattering vector $\mathrm{q}$ (momentum transfer vector) with a magnitude defined by the wavelength and angle of reflection of the neutrons. In simple reflectivity experiments we consider an ideally flat interface between medium " 0 " and medium " 1 " with neutrons hitting the interface with a wave vector $\mathbf{k}_{\mathbf{0}} ; \mathrm{k}_{0}=\left|\mathbf{k}_{\mathbf{0}}\right|=2 \pi / \lambda$; with $\lambda$ being the wavelength of the neutrons. The specular neutron reflectivity $\mathrm{R}$ is then defined for an ideal interface as follows using the reflection coefficient $\mathrm{r}_{0,1}$ for an ideal interface with the $\mathrm{n}$ wave amplitudes $a_{\text {out }}$ and $a_{i n}$ :

$r_{0,1}=\frac{a_{\text {out }}}{a_{\text {in }}}=\frac{k_{0}-k_{1}}{k_{0}+k_{1}}$

$\mathrm{R}=\left(\frac{k_{0}-k_{1}}{k_{0}+k_{1}}\right)^{2}$

For a single layer film with a thickness $\mathrm{d}_{1}$ (two interfaces, one homogeneous layer), this modifies to:

$\mathrm{R}=\frac{r_{0,1}^{2}+r_{1,2}^{2}+2 r_{0,1} r_{1,2} \cos \left(2 k_{1} d_{1}\right)}{1+r_{0,1}^{2} r_{1,2}^{2}+2 r_{0,1} r_{1,2} \cos \left(2 k_{1} d_{1}\right)}$

Such formulas can then be generalized for multilayers and used for fitting the experimental reflectivity profiles.

The reflectivity as a function of the z-component of the scattering vector

$\mathbf{q}_{\mathrm{z}}=\left|\mathbf{k}_{\text {out }}-\mathbf{k}_{\text {in }}\right|=2 \mathrm{k}_{0}\left(\sin \theta_{\text {out }}+\sin \theta_{\text {in }}\right)$

is measured and plotted in each of the Figures showing the directly observed experimental results. In general, from the specular reflectivity profiles, following fitting to the reflectivity formula chosen (like those displayed above), layer thickness values $d_{i}$, interfacial roughness and individual thickness values for multilayer films can be determined.

We were also interested in compositional information in the normal direction of the brush-substrate (in particular in segment gradients and solvent distribution). Such experiments could be attempted as for co-nonsolvency studies we can benefit from the strong contrast between $\mathrm{D}$ and $\mathrm{H}$ scattering. Additionally, for previously collapsed films using co-nonsolvents following drying, the determination of the remaining retained (heavy) water in the brush, and its spatial distribution within the brush, was also of interest. To obtain relevant information, we determined scattering length densities (SLD) and their depth dependence (as a function of $z$ values).

Scattering (and reflection) of a neutron plane wave is described using a "Fermi pseudopotential", which includes a parameter called neutron scattering length, $b_{i}$, to characterize the interaction of neutrons with the nucleus of the element " $i$ ". Its values are 
element-specific, depend on the nuclear spin, and can be found in standard tables. ${ }^{18}$ The power of neutron scattering in soft matter science is provided by the great contrast between $b_{i}$ values for $\mathrm{D}$ and $\mathrm{H}$, and also against the other elements of the periodic table. We make use of this contrast when we later selectively study the distribution of (heavy) water in the brush films.

As in neutron scattering and reflectivity the scattered (reflected) intensity depends on the nuclear density of the element " $i$ " within a given layer fitted e.g. to the specular reflectivity profile, it is convenient to consider continuous scattering length density (SLD) functions averaged over a given volume:

$\mathrm{SLD}=\rho(r)=\sum_{0}^{V} b_{i} N_{A} \delta / M_{V}$

where the average calculated over the volume $V, M_{V}$ is the molar mass, $\delta$ is the mass density, with Avogadro's number $N_{A}$. We summarize the calculated SLD values in the table below (Table 3.2) as provided by the fitting program "MOTOFIT"19 of Institute Laue Langevin used for $\mathrm{R}$ reflectivity profile evaluation.

Table 3.2: Calculated neutron scattering length densities (SLD or $\rho$ ) of the components used in neutron reflectivity measurements.

\begin{tabular}{|c|c|}
\hline & $\begin{array}{c}\text { SLD } \\
10^{-6} \AA\end{array}$ \\
\hline PNIPAM, $\mathrm{C}_{6} \mathrm{H}_{12 .} \mathrm{NO}$ & 0.44 \\
\hline D-PNIPAM, $\mathrm{C}_{6} \mathrm{H}_{9} \mathrm{D}_{2} \mathrm{NO}$ & 2.25 \\
\hline Water, $\mathrm{H}_{2} \mathrm{O}$ & -0.56 \\
\hline Deuterium oxide, $\mathrm{D}_{2} \mathrm{O}$ & 6.36 \\
\hline Methanol, $\mathrm{CH}_{3} \mathrm{OH}$ & -0.37 \\
\hline Silicon & 2.07 \\
\hline
\end{tabular}

Regarding the differences between hydrogenated and deuterated PNIPAM please see Figure S3.1 and S3.2 in the Supporting Information, discussing D-H exchange.

In the next section we first show our results based on experimental reflectivity curves and fitting to these data to gain insights for the different brush conformation scenarios studied (swelling, temperature collapse, co-nonsolvency collapse, re-swelling, solvents captured and retained in the films). We then provide a discussion describing the main findings, and conclusions. For a general introduction to NR see Torikai et al. ${ }^{18}$

\subsection{Results and discussion}

\section{Neutron reflectivity results}

In this section we first describe NR experiments ( $R$ vs. $q_{z}$ profiles) on dry brushes at RT (Figure 3.2). We then consider $\mathrm{D}_{2} \mathrm{O}$ swollen brushes at RT (Figure 3.3) and then 
display data obtained at the temperature of $40{ }^{\circ} \mathrm{C}$, thus heated to above the LCST (collapsed PNIPAM) (Figure 3.4). We cool down the system to below the LCST to test swelling reversibility at RT $\left(25{ }^{\circ} \mathrm{C}\right)$ (Figure 3.5). In Figure 3.6 we compare the reflectivity curves of the original swollen brush with the re-cooled swollen brush after heating to $40{ }^{\circ} \mathrm{C}$ to test (and establish) swelling reversibility. In Figure 3.7 we provide experimental data and their evaluation after exposing the brushes to a co-non-solvent system using a $\mathrm{D}_{2} \mathrm{O} / \mathrm{CH}_{3} \mathrm{OH}$ solution mixed in the ratio 3:1. Finally, in Figure 3.8 we display NR results and their evaluation for the brushes exposed to air, following a collapse response to the co-nonsolvent mixture (to check whether $\mathrm{D}_{2} \mathrm{O}$ remains retained in the films). We provide short interpretations for each Figure, and at the end we offer a general discussion. For each case the experimental geometry and fitting conditions are displayed in separate panels.

For dry (collapsed) brushes the specular reflectivity curves in panel (a) are vertically shifted by the values shown near the y axis (note the logarithmic scale, hence we used multiplicators of powers of 10) for better visibility. We note that we used similar shifting also in subsequent plots when several profiles are shown in one panel.

The film thickness values can be compared with data shown in Table 3.1 (determined by the AFM film scratch method). We note that the NR reflectivity fits gave somewhat higher values for the film thickness in this case. A direct comparison between AFM and NR is not possible, as the two methods measure different physical parameters. During NR experiments the top brush layer does not experience any mechanical stress (unlike in AFM) and the freely "dangling" chain ends could contribute to higher average film thickness values. Similar observations were made for free surfaces of amorphous thin films of bulk polymer, showing enhanced free volume and lowering of segment density at the free surface of polymeric solids. ${ }^{20}$ 
(a)

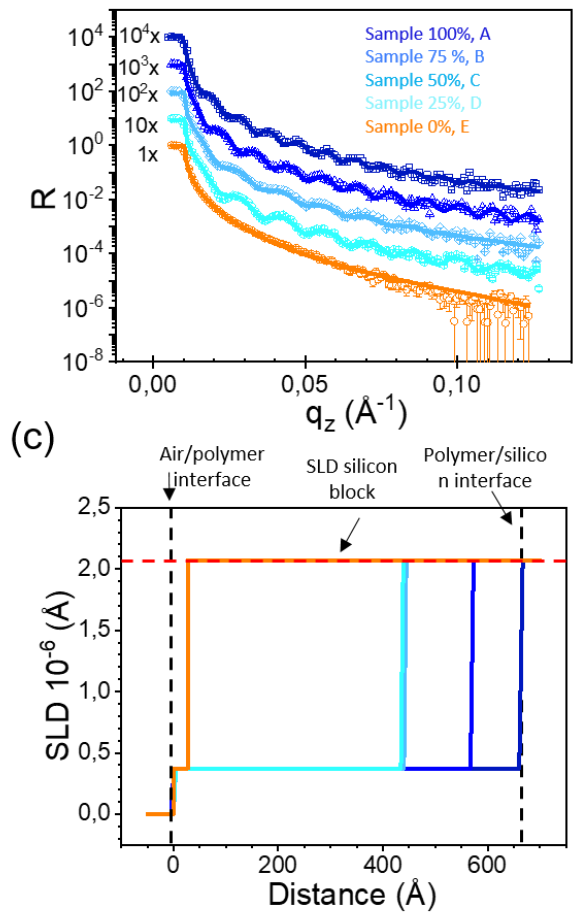

(b)

\section{Ambient Air}

$d=\infty, S L D=0$

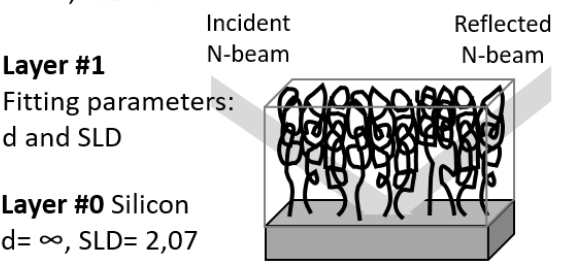

(d)

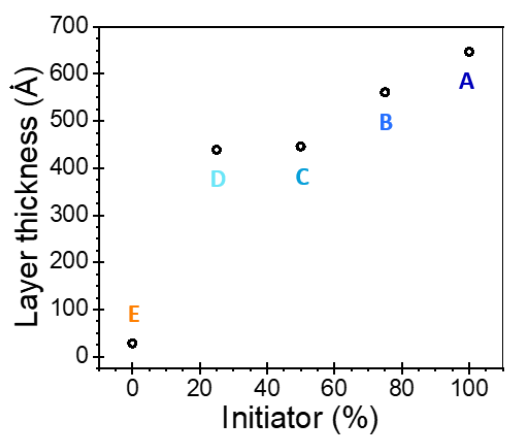

Fig. 3.2: NR measurements on dry (thus collapsed) PNIPAM brush layers with different grafting densities: (a) experimental reflectivity profiles (symbols) and the corresponding fitted specular reflectivity curves (lines). The curves are shifted vertically for clarify. Sample characteristics (initiator coverage, layer thickness, swelling ratio and estimated grafting density values are provided in Table 3.2. (b) Schematic representation of the experimental geometry used to fit the experimental reflectivity data (containing $\mathrm{SiO}_{2}$ surface / polymer layer / ambient air interfaces). The fitted scattering length densities (SLD) and the thickness values of the polymer layer as a function of the distance from the air interface are shown in (c) and (d), respectively. For example, in (c) the SLD profile Vs thickness graph of the sample A, the black dashed vertical lines indicate the location of the air/polymer and polymer/silicon interfaces, respectively. The red dashed horizontal line indicates the SLD of the silicon block.

The orange (bottom) reflectivity curve shows a monotonous decay with increasing scattering vector, corresponding to reflections from a single interface. The other four reflectivity profiles show interference fringes resulting from a one layer (two interface) system. The fits to the measured points are in excellent agreement with the measured points. Data fitting resulted in film thickness and scattering length density values as shown in panels (c) and (d).

For zero BIBB concentration the organic film (a combination of inactive BMA and APTES, see Figure 3.1) on the silicon substrate gives a small contribution to film thickness (note the non-zero BIBB/BMA ratio). The SLD values obtained from the fit show the same trend. We note that the distance values on the abscissa are measured from 
the air/film interface. At zero distance we see a jump from zero SLD value to ca. 0.4 (with a thickness of ca. $20 \AA$ ) which corresponds to the organic film (BMA and APTES) just mentioned. Then the large step to the value of 2.07 corresponds to reaching the silicon substrate. For specimens D, B and A this occurs at the thickness values shown in Panel (c). PNIPAM has a scattering length density value of 2.25 , which is not distinguishable from the BIBB + APTES organic film.

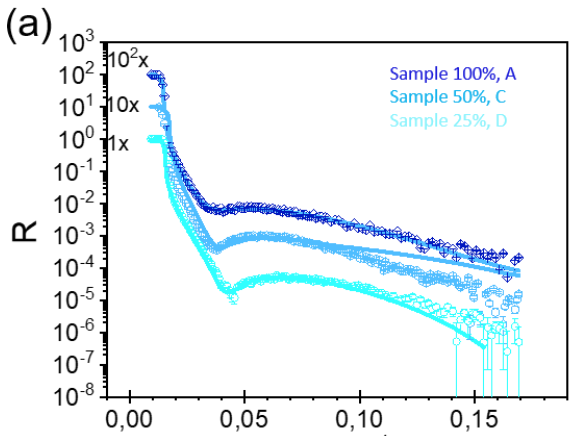

(b)

NR Model

(c)

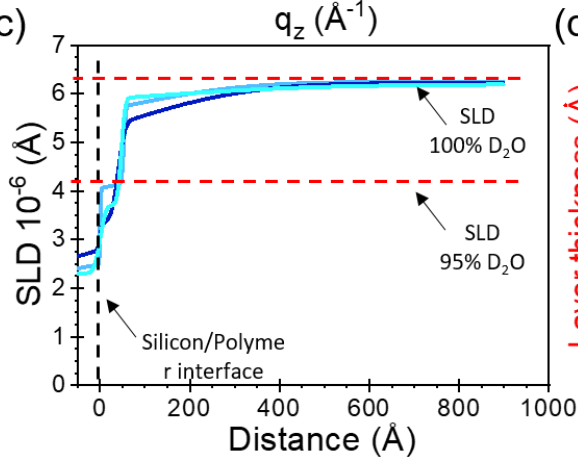

Layer \#0 Silicon

$\mathrm{d}=\infty, \mathrm{SLD}=2,07$

Layer \#1

Fitting parameters:

d and SLD

Ambient $\mathrm{D}_{2} \mathrm{O}$

$d=\infty, \operatorname{SLD}=6,3$ (d)

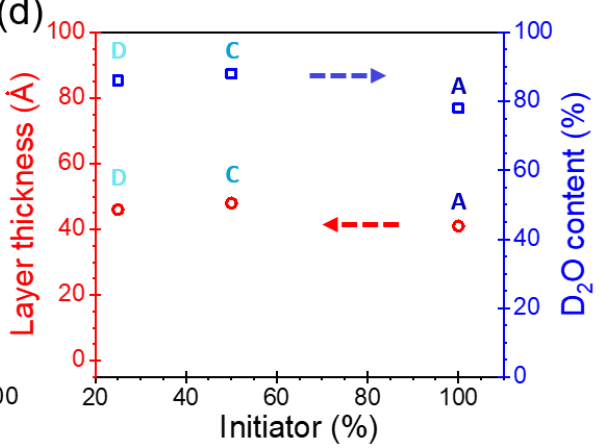

Fig. 3.3: NR measurements on $\mathrm{D}_{2} \mathrm{O}$ swollen PNIPAM brush layers with different grafting density at 25 ${ }^{\circ} \mathrm{C}$. (a) Experimental reflectivity profiles (symbols) and the corresponding fitted specular reflectivity curves (lines). The curves are shifted vertically for clarity. (b) Schematic representation of the experimental geometry used to fit the experimental reflectivity data (containing $\mathrm{SiO}_{2}$ surface / polymer-rich layer / $\mathrm{D}_{2} \mathrm{O}$-medium). Note that the entire film is immersed in $\mathrm{D}_{2} \mathrm{O}$, and the neutron beam enters from the back side of the silicon substrate. The scattering length densities (SLD) of the polymer layer as a function of the distance from the silicon interface are shown in (c). The black dashed vertical line indicates the location of the silicon/polymer interface. The red dashed horizontal lines indicate respectively the SLD values for the SLD of the polymer film containing $95 \%$ and $100 \%$ of $\mathrm{D}_{2} \mathrm{O}$. (d) Shows the calculated thickness and the $\mathrm{D}_{2} \mathrm{O}$ content in the polymer layer as a function of the grafting density.

The reflectivity profiles do not show distinct oscillations characteristic for welldefined films. We note that for higher scattering vector values the experimental noise increases as expected, but the fit quality becomes less good. This was to be expected due to less information included in the reflectivity data.

The SLD profiles fitted from these data exhibit a step increase from silicon (SLD = 2.07 to $4.110^{-6} \AA$ for $95 \% \mathrm{D}_{2} \mathrm{O}$ layer) with increasing distance from the substrate. A 
layer can be observed at the substrate exhibiting a slight variation with the initiator concentration. At higher distances the layers exhibit a further increase of swelling with increasing distance for all four grafting densities but in a gradual manner. In the SLD fits we do not see the (expected) full brush thickness with a sharp interface (lack of oscillating reflectivity profiles). This can either be due to high thickness values difficult to capture experimentally, or to a low segment density, and high concentration of $\mathrm{D}_{2} \mathrm{O}$ in the films, which masks the SLD of the polymer. In any case, it is evident from the SLD data that the brushes contain substantial amounts of solvent. The SLD profiles merge at high distances and asymptotically reach $100 \% \mathrm{D}_{2} \mathrm{O}$ for thicknesses higher that $500 \AA$. We interpret these observations as a result of the presence of a dense layer in the vicinity of the substrate which is terminated on the solvent side by a diffuse top swollen layer (possibly dangling chains at low segment density) in $\mathrm{D}_{2} \mathrm{O}$, as is shown in Figure 3.3, Panel (d). With increasing initiator content the segment crowding in the brush grafts increases (segment density increases) as indicated by the decreasing amount of solvent in the dense layers, as is shown in Figure 3.3, Panel (d).

Brush conformational variations upon heating, following passing the LCST and chain collapse, measured at $40{ }^{\circ} \mathrm{C}$ can clearly be seen in Figure 3.4 when compared with the results displayed in Figure 3.3. The scattering length density plots depicted in Figure 3.4. show again the presence of sharp interface, and good brush film definition, similar to the dry films initially measured (Figure 3.2). For all four grafting densities there is $\mathrm{D}_{2} \mathrm{O}$ retained in the films. SLD fits indicate the presence of two distinguishable diffuse layers. Close to the silicon substrate a dense layer can be seen, while in the vicinity of contact with the solvent, more solvent is present. The SLD fits show a somewhat unexpected and abrupt change between these two domains (probably due to fitting technical issues). The top layer thickness is estimated to vary around 40-50 $\AA$ and does not show variation with initiator density, as is shown in Figure 3.4, Panel (e). For the interface in contact with the solvent, there is a sharp jump from $80-85 \% \mathrm{D}_{2} \mathrm{O}$ to $100 \% \mathrm{D}_{2} \mathrm{O}$ indicating the presence of a well-defined polymer brush-solvent interface. The thickness of the compact first layer as a function of grafting density is shown in Figure 3.4, Panel (d). The thickness values here are comparable with the data obtained for the dry brush as shown in Figure 3.2. 
(a)

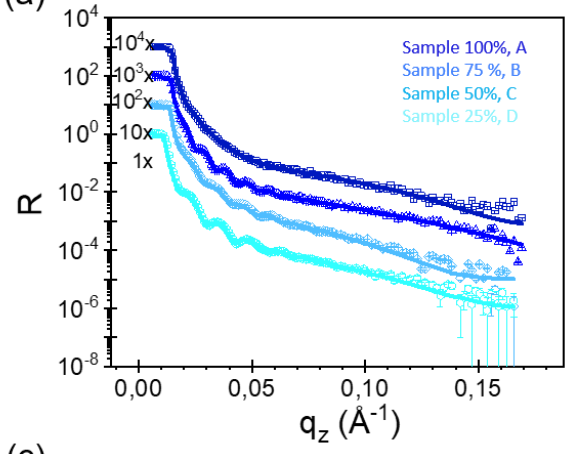

(c)

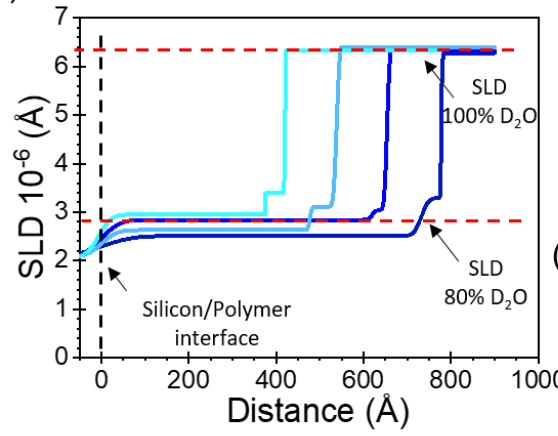

(b)

NR Model

Layer \#0 Silicon

Layer \#1 dense: $d$, SLD

Layer \#2 graded: $d$, SLD

Ambient $\mathrm{D}_{2} \mathrm{O}$

(d)
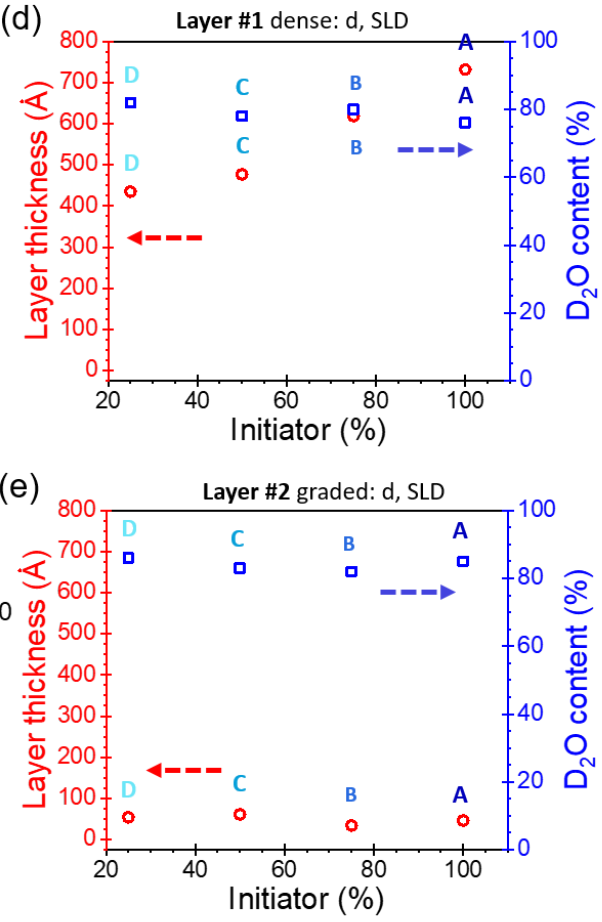

Fig. 3.4: NR measurements on Temperature-induced collapsed PNIPAM brush layers after swelling at room temperature, following heating to $40{ }^{\circ} \mathrm{C}$, to above the LCST. a) Experimental reflectivity profiles (symbols) and the corresponding fitted reflectivity curves (lines). The curves are shifted vertically for clarity. (b) Schematic representation of the experimental geometry used to fit the experimental reflectivity data (containing $\mathrm{SiO}_{2}$ surface / polymer-rich layer / $\mathrm{D}_{2} \mathrm{O}$-rich polymer layer / $\mathrm{D}_{2} \mathrm{O}$ bulk). (c) Obtained scattering length densities (SLD) as a function of the distance from the silicon interface. In (d) and (e) the calculated thickness and the $\mathrm{D}_{2} \mathrm{O}$ content values in the bottom dense and top SLD-gradient polymer layers as a function of the grafting density are depicted, respectively.

We display the main data in Table 3.3 below, allowing one to make direct comparisons for temperature and grafting density effects. 
Table 3.3: Comparison of the properties of the PNIPAM brush layer measured via NR as a function of the grafting density between the swollen and collapsed conformation of the PNIPAM brush layers. The conformational transition is here temperature-induced LCST.

\begin{tabular}{|c|c|c|c|c|c|c|c|c|c|c|}
\hline \multirow{2}{*}{\multicolumn{2}{|c|}{$\begin{array}{c}\text { Active } \\
\text { initiator } \\
\text { mol } \%\end{array}$}} & \multicolumn{3}{|c|}{ In $\mathrm{D}_{2} \mathrm{O}$ at $25^{\circ} \mathrm{C}$} & \multicolumn{6}{|c|}{ In $\mathrm{D}_{2} \mathrm{O}$ at $40{ }^{\circ} \mathrm{C}$} \\
\hline & & \multirow{2}{*}{$\begin{array}{l}\mathbf{d}_{\mathbf{1}} \\
\AA \\
\AA \\
\end{array}$} & \multirow{2}{*}{$\begin{array}{l}\text { SLD } \\
\frac{10^{-6} \AA}{2.61}\end{array}$} & \multirow{2}{*}{$\begin{array}{c}\mathbf{D}_{2} \mathbf{O} \\
\% \\
76\end{array}$} & \multirow{2}{*}{$\begin{array}{c}\mathbf{d}_{\mathbf{1}} \\
\AA \\
730\end{array}$} & \multirow{2}{*}{$\begin{array}{l}\text { SLD }_{1} \\
\frac{10^{-6} \AA}{2.51}\end{array}$} & \multirow{2}{*}{$\begin{array}{c}\begin{array}{c}\mathrm{D}_{2} \mathrm{O} \% \\
\text { in dense } \\
\text { layer }\end{array} \\
76\end{array}$} & \multirow{2}{*}{$\begin{array}{l}\mathbf{d}_{2} \\
\AA \\
46\end{array}$} & \multirow{2}{*}{$\begin{array}{l}\mathbf{S L D}_{2} \\
\frac{10^{-6} \AA}{3.29}\end{array}$} & \multirow{2}{*}{$\begin{array}{c}\begin{array}{c}\mathrm{D}_{2} \mathrm{O} \% \\
\text { in grad } \\
\text { layer }\end{array} \\
85\end{array}$} \\
\hline Sample A & 100 & & & & & & & & & \\
\hline Sample B & 75 & - & - & - & 620 & 2.84 & 80 & 34 & 3.05 & 82 \\
\hline Sample C & 50 & 48 & 3.63 & 87 & 480 & 2.62 & 78 & 61 & 3.11 & 83 \\
\hline Sample D & 25 & 46 & 3.44 & 85 & 440 & 2.94 & 82 & 54 & 3.37 & 86 \\
\hline
\end{tabular}

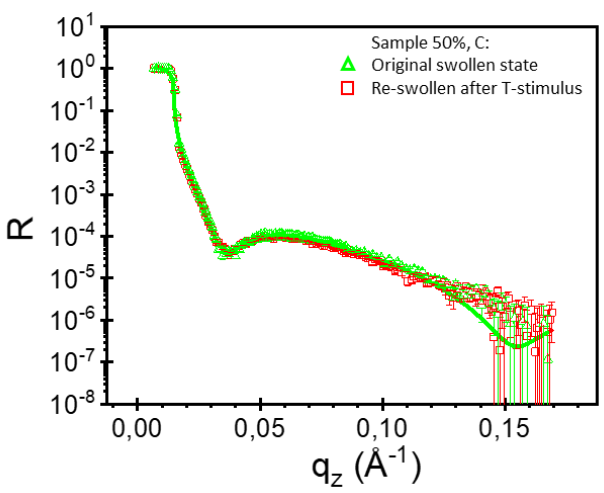

Fig. 3.5: Study of the temperature-switched conformational response of the PNIPAM. Comparison between the NR profiles obtained for specimen $\mathrm{C}$ in its swollen state (first time exposure to $\mathrm{D}_{2} \mathrm{O}$ ) and following passing the LCST to collapse, and re-swelling.

Following a detailed experimental study on our brush library comprising four layers, we tested the reversibility of the NR profiles on the specimen " $\mathrm{C}$ ". We compared specular reflectivity profiles obtained in the first swelling experiment, and following a temperature-induced collapse and re-swelling. As shown in Figure 3.5, both measured and fitted reflectivity curves for the two scenarios are indistinguishable, proving reproducibility.

Next we turn our attention to LCST transitions triggered by exposing the brush films to the co-nonsolvent mixtures of $\mathrm{D}_{2} \mathrm{O} / \mathrm{CH}_{3} \mathrm{OH}$ solution mixed in the ratio $3: 1$. This mole fraction value was chosen as the minimum of the LCST depression for $\mathrm{MeOH}-\mathrm{H}_{2} \mathrm{O}$ cononsolvent systems is found in this range (at $35 \mathrm{~mol} . \%$ ) at the value of $-7.5^{\circ} \mathrm{C} .{ }^{9}$ Three brushes, including 25, 50 and $100 \%$ initiator coverage (samples A, C and D) were investigated. The R profiles, the experimental geometry and the SLD profiles are shown in Figure 3.6. 
(a)

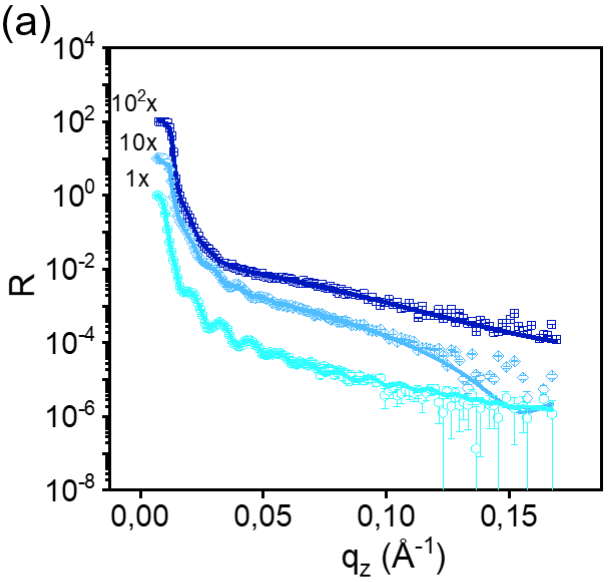

(b)

Layer \#0 Silicon Layer \#1 dense Layer \#2 graded Ambient $\mathrm{D}_{2} \mathrm{O} / \mathrm{CH}_{3} \mathrm{OH}$

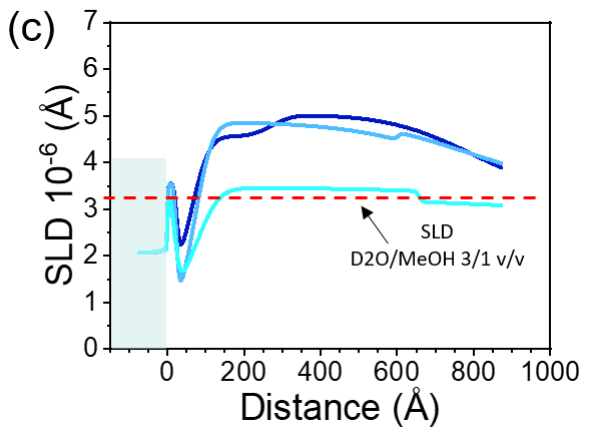

Fig. 3.6: NR measurements of collapsed PNIPAM brush layers in $\mathrm{D}_{2} \mathrm{O} / \mathrm{CH}_{3} \mathrm{OH}$ solution $3 / 1$ v/v: a) Experimental reflectivity profiles (symbols) and the corresponding fitted reflectivity curves (lines). The curves are shifted vertically for clarity. (b) Schematic representation of the model used to fit the experimental reflectivity data. (c) Scattering length densities (SLD) of the polymer layer as a function of the distance from the silicon interface. The silicon substrate is depicted by the light blue colored rectangle in the left bottom corner as background.

The NR measurements shown in Figure 3.6 were performed at RT $\left(25^{\circ} \mathrm{C}\right)$ where PNIPAM in pure water must exhibit a solubilized, swollen brush. However, interference maxima (in particular for samples $\mathrm{C}$ and D) are clearly visible in the NR patterns indicating the expected brush collapse due to immersion in co-non-solvent mixtures. From the SLD profiles it is clear that collapse and layer definition is not as well pronounced as it is for the T-collapsed PNIPAM layers at $40{ }^{\circ} \mathrm{C}$ (Figure 3.4). As shown by the SLD profiles, there is a thin $\mathrm{D}_{2} \mathrm{O}$ depletion layer near the Si interface, and a substantial amount of $\mathrm{D}_{2} \mathrm{O}$ in all three films. Interestingly, the SLD values for higher distances away from the substrate clearly decrease for all samples studied. This is a strong indication of a decreasing concentration of the solvent $\mathrm{D}_{2} \mathrm{O}$ moving towards the brush exterior within the collapsed brush. Instead of approaching the SLD of $\mathrm{D}_{2} \mathrm{O}$ we see lower $\mathrm{D}_{2} \mathrm{O}$ concentrations and a clear enhancement of the polymer segment concentration near the film interface in contact with the solvent. Additionally, substantial amounts of $\mathrm{D}_{2} \mathrm{O}$ are captured in the interior of the film. In other words, NR experiments provided further evidence, that collapse of PNIPAM brushes, when exposed to co-non-solvent mixtures, occurs first at the solvent-exposed interface, in agreement with our earlier reports. ${ }^{12-13}$

In the next set of experiments we tackled the question, what happens when we expose the films collapsed by the co-non-solvent mixture again to a solvent at room temperature (where we expect a fully swollen brush). Results are shown in Figure 3.7. 
(a)

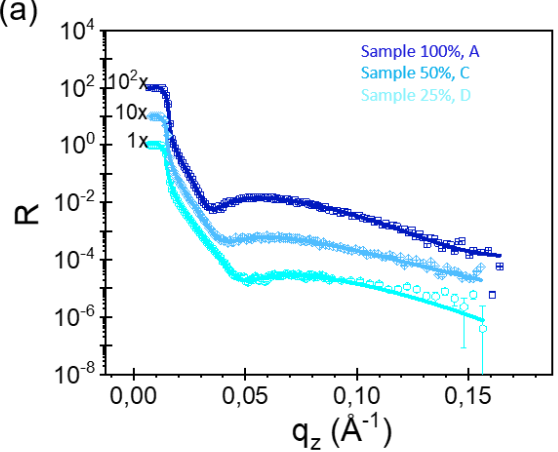

(c)

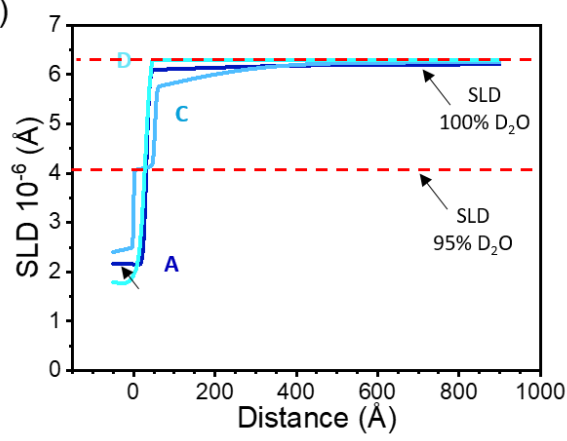

(b)

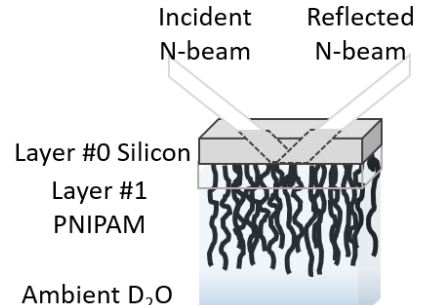

(d)

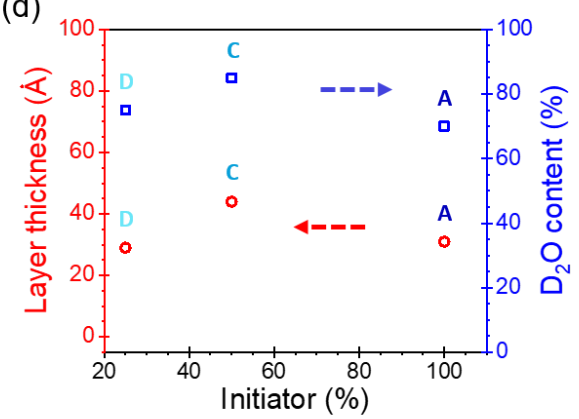

Fig. 3.7: NR measurements on re-swollen PNIPAM brush layers in $\mathrm{D}_{2} \mathrm{O}$ at $25{ }^{\circ} \mathrm{C}$ after co-non-solventinduced transition. (a) Experimental reflectivity profiles (symbols) and the corresponding fitted specular reflectivity curves are shown by lines. The curves are shifted vertically for clarity. (b) Schematic of the experimental geometry used in data fitting. (c) Scattering length densities (SLD) as a function of the distance from the silicon interface. In (d) the calculated thickness and the $\mathrm{D}_{2} \mathrm{O}$ content values of the polymer-rich layer as a function of the grafting density are depicted.

There are similarities between the SLD profiles for the re-swollen brush following co-non-solvency exposure, and the re-swollen brush following crossing the LCST for collapse, and then again for re-swelling by varying the temperature. Similar to Figure 3.3 the interference patterns disappear, the film layer definition becomes fuzzy, and the SLD profiles can be interpreted by an effective two layer model. Close to the substrate the SLDs show high polymer density, and then similar to the film re-swollen after T collapse, we observe an asymptotic approach of the SLD to effectively approach the $\mathrm{D}_{2} \mathrm{O}$ value at ca. $500 \AA$ distance from the substrate. There is however one striking difference between the two re-swollen films. Following exposure to co-non-solvents for collapse, and subsequent re-swelling, substantially more $\mathrm{D}_{2} \mathrm{O}$ remains retained in the films, as shown by the higher SLD values near the compactly packed-loose packed boundary (kink in the SLD-s both in Figure 3.3 Panel (c) and Figure 3.7. Panel (c).

In order to make a direct comparison of these two cases easier, we replotted in Figure 3.8 the SLD profiles and the modelled thickness values for the substrate-near dense layer, and the $\mathrm{D}_{2} \mathrm{O}$ content in the dense layer for the above discussed two cases of re-swollen brushes. The differences as described can clearly be seen. 
(a)

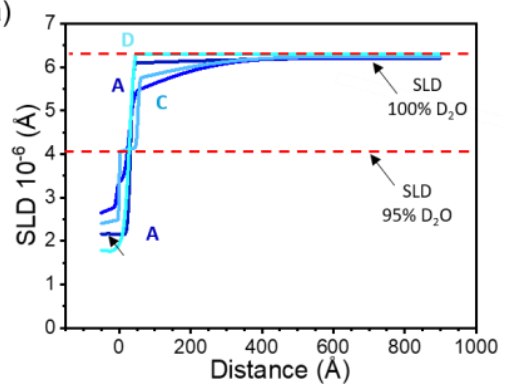

(b)

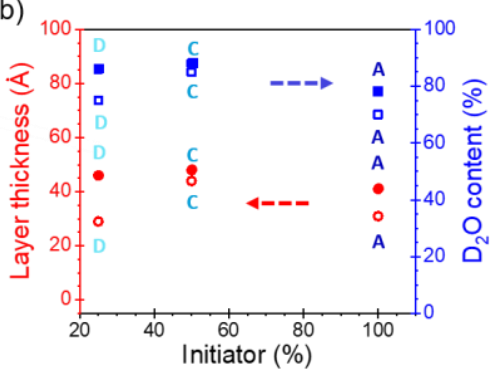

Fig. 3.8: Comparison between the re-swollen PNIPAM brush layers in $\mathrm{D}_{2} \mathrm{O}$ at $25{ }^{\circ} \mathrm{C}$ after temperatureinduced and after co-nonsolvent-induced conformational transitions. (a) Shows the scattering length densities (SLD) of the polymer layer as a function of the distance from the silicon interface. In b) the calculated thickness and the $\mathrm{D}_{2} \mathrm{O}$ content values of the bottom polymer dense layers are depicted as a function of the grafting density (the closed symbols and the open symbols are the swollen thickness and $\mathrm{D}_{2} \mathrm{O}$ content values before and after the temperature-induced and the co-non-solvent-induced transition).

Finally, we took the brush following co-non-solvent collapsed and dried in air. Brush in this film in principle should have the same conformation as the initial dry PNIPAM brush. The NR results, along with the SLD plots and estimated $\mathrm{D}_{2} \mathrm{O}$ content are shown in Figure 3.9.

(a)
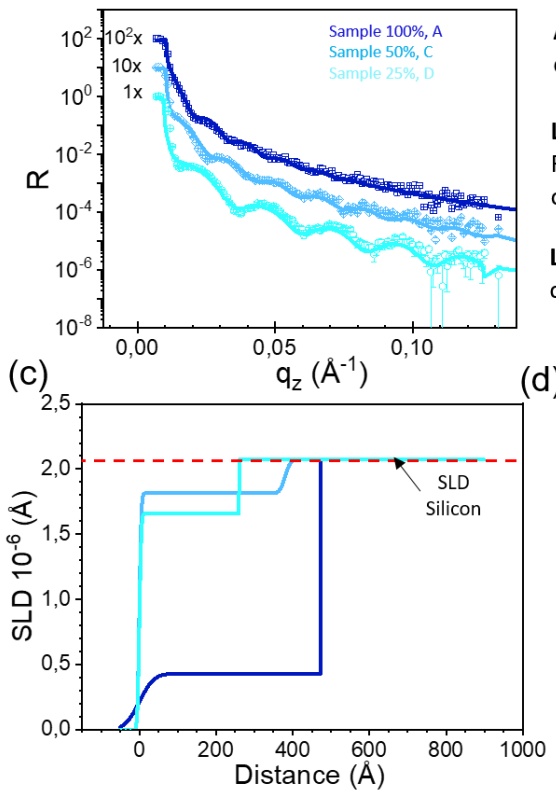

(b)
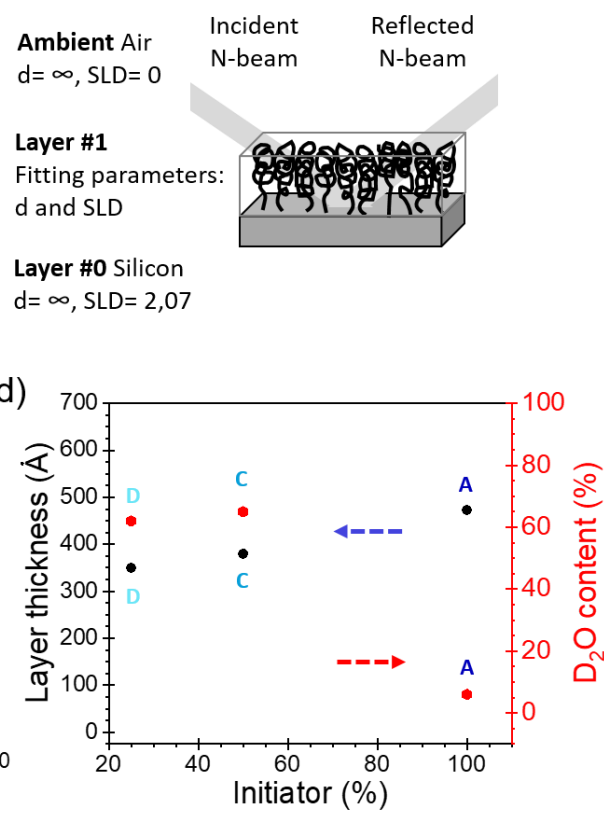

Fig. 3.9: NR profiles of films following co-non-solvent collapsed and dried in air. (a) Experimental reflectivity profiles (symbols) and the corresponding fitted reflectivity curves (lines). The curves are shifted vertically for clarity. (b) Schematic representation of the model used to fit the experimental reflectivity data 
(containing $\mathrm{SiO}_{2}$ surface/polymer layer/ambient air). (c) Scattering length densities (SLD) as a function of the distance from the air/polymer interface. In (d) the calculated thickness of the dry PNIPAM layers as a function of the grafting density are depicted.

According to the SLD fits the layer thickness of film A has a value of ca. $440 \AA$ (730 $\AA$ ), film C is of $400 \AA$ ( $480 \AA$ ) and D of $240 \AA$ (440 $\AA$ ) (in brackets the values of the initial dry film thickness are shown; see Table 3.3). We note that the initial film (prior to $\mathrm{T}$ and co-nonsolvent exposures) had a substantially higher film thickness for each cases. We speculate that the origin of this deviation might be related to some polymer degrafting of the brush chains during repeated swelling/collapse experiments, while we note that the agreement between experiments and fitted profiles is not as good as it was in the previous cases. ${ }^{21-23}$

\subsection{Conclusions}

We have prepared a library of thin PNIPAM brush films anchored on silicon surfaces with different grafting densities (grafted from silicon) over a range of and 0.0030 to 0.0011 chains $\cdot \AA^{-2}$ with, in good approximation, the same molar mass via SI-ATRP.

Neutron reflectivity was used to study the physical properties of the PNIPAM brush films with different grafting densities in temperature- and co-non-solvent-induced coilto-globule transition. The distribution of the solvent through the polymer films in the various conformations of the single end-tethered PNIPAM chins was investigated above and below the LCST, and in co-non-solvency conditions $\left(\mathrm{D}_{2} \mathrm{O}: \mathrm{CH}_{3} \mathrm{OH} 3: 1 \mathrm{v} / \mathrm{v}\right)$.

From neutron reflectivity (NR) experiments of the dry PNIPAM films, the thickness and the SLD values of the PNIPAM brush layers were estimated with a mono-layer model. The thicknesses of the PNIPAM layers were affected by the amount of the ATRPinitiator anchored on the silicon surface and the thickness increased by increasing the grafting density due to the steric hindrance between the neighbor chains. Moreover, from the measured SLD values of the PNIPAM brush layers $\left(3.74 * 10^{-7} \AA^{-2}\right)$, the chemical composition of the films was estimated to be $\left(\mathrm{C}_{6} \mathrm{H}_{13} \mathrm{NO}\right)_{n}$ which is in good agreement with the theoretical estimated composition of the polymer $\left(\mathrm{C}_{6} \mathrm{H}_{11} \mathrm{NO}\right)_{n}$.

Then, the physical properties of the PNIPAM brush films were explored in $\mathrm{D}_{2} \mathrm{O}$ at $25{ }^{\circ} \mathrm{C}$. We observed no sharp interphase between the swollen polymer and the $\mathrm{D}_{2} \mathrm{O}$ medium but only an interphase between the silicon surface and the swollen polymer-rich layer. The absence of the sharp interphase between polymer and $\mathrm{D}_{2} \mathrm{O}$ can be due to high thickness values difficult to capture experimentally, or to a low segment density, and high concentration of $\mathrm{D}_{2} \mathrm{O}$ in the films, which masks the SLD of the polymer. Regarding the bottom polymer-rich layer (at the interface with the silicon oxide surface) for all the different grafting density PNIPAM brushes was observed a homogeneous 40-50 A thick film containing approximately $20 \%$ of polymer. The thickness of this layer was independent on the amount of the initiator grafted to the surface. Thus, our PNIPAM brush layers in swollen state (in $\mathrm{D}_{2} \mathrm{O}$ at $25{ }^{\circ} \mathrm{C}$ ) were described with a two-layer model 
encompassing essentially two domains: a first polymer-rich domain at the silicon interface ( $80 \% \mathrm{D}_{2} \mathrm{O}$ amount in the layer and a thickness between $40-50 \AA$ thick), and a second $\mathrm{D}_{2} \mathrm{O}$-rich domain (containing more than $95 \%$ of $\mathrm{D}_{2} \mathrm{O}$ which reached the $\mathrm{D}_{2} \mathrm{O}$ SLD already at distances of $500 \AA$. The full swollen thickness of the PNIPAM brushes was not measurable because the polymer layer was too thick and the SLD of the polymer was masked by the high concentration of $\mathrm{D}_{2} \mathrm{O}$. Nevertheless, the swollen thickness of the PNIPAM brushes were measured to be thicker than $1000 \AA$ for all the grafting densities via AFM and ellypsometry measurements which elucidates the absence of distinguishable diffuse layers from the NR data.

As consequence of the temperature-induced conformational transition of the PNIPAM brushes (temperature was at $40^{\circ} \mathrm{C}$, far above the LCST of PNIPAM in $\mathrm{D}_{2} \mathrm{O}$ ), a sharp interface between the PNIPAM brush layer and the $\mathrm{D}_{2} \mathrm{O}$ solvent was observed. For all the grafting densities, two layers model (i.e., with two distinguishable diffuse layers) was used to fit the NR data: i) at the bottom, close to the silicon substrate, a dense polymer-rich layer and ii) at the top, at the polymer-solvent interface, a $\mathrm{D}_{2} \mathrm{O}$-rich layer. The thickness and the SLD values of the bottom layers varied with the grafting density, while the thickness and the SLD of the top layer did not show variation with the grafting density. The reversibility of the temperature-induced transition was confirmed.

When the swollen PNIPAM brushes were collapsed by exposing the films to the conon-solvent mixtures $\left(\mathrm{D}_{2} \mathrm{O}: \mathrm{CH}_{3} \mathrm{OH} 3: 1 \mathrm{v} / \mathrm{v}\right)$, interference maxima were clearly visible indicating the expected coil-to-globule transition of the PNIPAM chains.

A clear difference between the reflectivity profiles of the temperature-collapsed and the co-non-solvent-collapsed PNIPAM was noted. The reflectivity profile of the PNIPAM brush layers with three different grafting densities were different: the PNIPAM with the highest grafting density did not show the typical oscillations in the reflectivity curve indicating a $\mathrm{D}_{2} \mathrm{O}$-rich polymer film layer trapped between the PNIPAM brushes and confined by the silicon surface and the collapsed polymer film exposed to the co-nonsolvent mixture. At low grafting density, the sharp interface between the polymer and the medium was revealed from the reflectivity profiles. The $\mathrm{D}_{2} \mathrm{O}$ content in the polymer brush layer, as described by the SLD profiles, decrease of higher distance from the substrate. This phenomenon is due to the presence of a dense solvent-exposed collapsed PNIPAM layer which acted as a diffusion barrier of the $\mathrm{D}_{2} \mathrm{O}$ molecules into the medium. Also for these samples, a substantial amount of $\mathrm{D}_{2} \mathrm{O}$ is entrapped in the polymer layers but from the fitted SLD profile a lower concentration of $\mathrm{D}_{2} \mathrm{O}$ was present in the intermediate layer. This experiment provided further evidence, that collapse of PNIPAM brushes, when exposed to co-non-solvent mixtures, occurs first at the solvent-exposed interface and depending on the grafting density a different amount of $\mathrm{D}_{2} \mathrm{O}$ can be entrapped between the solid silicon surface and the collapsed polymer layer in contact with the co-nonsolvent mixture. 
The reversibility of the co-non-solvent-induced transition was verified, like for the temperature-induced transition. However, we note that after exposure to the co-nonsolvency mixture more $\mathrm{D}_{2} \mathrm{O}$ remains retained in the films.

From the NR measurements of the dry PNIPAM brushes after co-non-solventinduced conformational transition, a difference in the polymer layer thickness and of the SLD value were observed for all the grafting densities. We suggest that this phenomenon is due to the degrafting of the brush chains during the experiments.

Finally, we have analyzed the co-non-solvent-collapsed PNIPAM films and dried in air and the de-grafting of the polymer chains during the repeated swelling/collapse experiments was observed.

\subsection{Experimental section}

\section{Materials}

Substrates. Two different sizes of silicon substrates were used due to the technical requirements of the neutron reflectivity experiments: $(5 \times 5 \times 1) \mathrm{cm}$ and $(1 \times 1 \times 0.05) \mathrm{cm}$. The substrates were purchased by CrisTec GmbH (Germany) and Okmetic (Vantaa, Finland), respectively. Technical details are shown in Table 3.4.

Table 3.4: Technical requirements for the silicon samples used in this project.

\begin{tabular}{|c|c|c|}
\hline & Silicon oxide sample 1 & Silicon oxide sample 2 \\
\hline & used for N-Refl. & $\begin{array}{l}\text { used for CA, FTIR, AFM and } \\
\text { ellipsometry }\end{array}$ \\
\hline & CrysTec, Germany & Okmetic Oyj, Finland \\
\hline Orientation & $\left(\begin{array}{llll}1 & 0 & 0\end{array}\right)$ & $\left(\begin{array}{llll}1 & 0 & 0\end{array}\right)$ \\
\hline Tolerance & $<0.5^{\circ}$ & $<0.5^{\circ}$ \\
\hline Format & $50 \times 50 \mathrm{~mm}( \pm 0.2 \mathrm{~mm})$ & $\begin{array}{c}\text { Diameter } 100 \mathrm{~mm}( \pm 0.2 \mathrm{~mm}) \\
\text { Cut in square } 10 \times 10 \mathrm{~mm}\end{array}$ \\
\hline Thickness & $10 \mathrm{~mm}(+0.3 \mathrm{~mm})$ & $0.525 \mathrm{~mm}(+0.3 \mathrm{~mm})$ \\
\hline Flat & SEMI standard & SEMI standard \\
\hline $\begin{array}{c}\text { Type of conductivity, } \\
\text { Dopand }\end{array}$ & $\begin{array}{c}\text { n-Type, } \\
\text { Phosphorus }\end{array}$ & $\begin{array}{c}\text { n-Type, } \\
\text { Phosphorus }\end{array}$ \\
\hline Resistivity & $>1 \mathrm{Ohm} \cdot \mathrm{cm}$ & $>1 \mathrm{Ohm} \cdot \mathrm{cm}$ \\
\hline
\end{tabular}

Chemicals. Aminopropyl triethoxysilane (APTES), bromo isobutyrilbromide (BIBB), 1-bromocarbonyl-1-methylethyl acetate (BMA), trimethylamine (TEA), copper (II) bromide $\left(\mathrm{CuBr}_{2}\right)$, and $\left(\mathrm{N}, \mathrm{N}, \mathrm{N}^{\prime}, \mathrm{N}^{\prime}, \mathrm{N}^{\prime \prime}\right.$-pentamethyldiethylenetriamine) (PMDETA) were purchased from Sigma-Aldrich and used as received without purification. $N$ (isopropylacrylamide) (NIPAM) and Copper (I) chloride $(\mathrm{CuCl})$ were purchased from Sigma-Aldrich. NIPAM was purified twice by crystallization in toluene/ $n$-hexane mixture at $1 / 1 \mathrm{v} / \mathrm{v}$. The NIPAM was dissolved in the minimum volume possible of toluene at room temperature and crystallized overnight. The mixture was filtered, washed with $n$-hexane and dried under vacuum. $\mathrm{CuCl}$ was purified in concentrated acetic acid, filtered and 
washed with ethanol three times, followed by drying under vacuum at room temperature overnight. Deuterium oxide $\left(\mathrm{D}_{2} \mathrm{O}, 99.9 \%\right)$ was purchased from Cambridge Isotope Laboratories, methanol (absolute), chloroform (AR) and Milli-Q water (Advantage A-10, Millipore, Merk, Darmstadt, Germany) was used throughout the experiments.

\section{Synthesis of PNIPAM grafts with different grafting densities.}

Activation of the silicon samples. The silicon wafers were cleaned with piranha solution (i.e., concentrated $\mathrm{H}_{2} \mathrm{SO}_{4}$ : concentrated $\mathrm{H}_{2} \mathrm{O}_{2} 3: 1 \mathrm{v} / \mathrm{v}$ ) at room temperature for $30 \mathrm{~min}$. Then, the samples were rinsed extensively with water and ethanol, followed by drying in a nitrogen stream and immediately used for the next step.

Formation APTES self-assembly monolayer (SAM) on silicon surfaces. The clean silicon samples were placed in the desiccator around a vial containing approximately $0.1 \mathrm{ml}$ of ATRP initiator coupling agent APTES. The desiccator was evacuated for $5 \mathrm{~min}$ with a rotary vane pump and subsequently closed. The vapor deposition was allowed to proceed overnight. Then, the samples were rinsed with toluene, ethanol and water ensuring the complete removal of the unreacted silane, and dried in a nitrogen stream. The APTES-modified silicon samples were immediately used for the next step of coupling with reactive and unreactive ATRP initiators.

Formation various grafting densities of ATRP initiator on silicon surfaces. Five solutions containing $5.5 \mathrm{~mL}$ of TEA and $120 \mathrm{~mL}$ of chloroform were prepared in glass beaker (as summarized in Table 3.5) and subsequently, cooled in an ice bath. The APTES-modified samples were immersed into the solution and the solution was mixed under magnetic stirring. The ATRP initiator BIBB and the inert compounds BMA were added simultaneously and dropwise, under stirring in the right ratio. The molar ratio between the active and inert ATRP initiator agents, the complexant agent, and the solvent are summarized in Table 3.5.

Table 3.5: Experimental procedure for the coupling of the active and inactive ATRP initiator to the APTES-modified silicon surfaces.

\begin{tabular}{|c|c|c|c|c|c|}
\hline \multirow{2}{*}{ Name } & $\begin{array}{c}\text { Initiator molar } \\
\text { ratio }\end{array}$ & $\mathbf{V}_{\text {BIBB }}$ & $\mathbf{V}_{\text {BMA }}$ & Chloroform & TEA \\
\cline { 3 - 6 } & $\mathrm{mL}$ & $\mathrm{mL}$ & $\mathrm{mL}$ & $\mathrm{mL}$ \\
\hline Sample A & $100 \%$ & 3.0 & 0 & 120 & 5.5 \\
\hline Sample B & $75 \%$ & 2.25 & 0.75 & 120 & 5.5 \\
\hline Sample C & $50 \%$ & 1.5 & 1.5 & 120 & 5.5 \\
\hline Sample D & $25 \%$ & 0.75 & 2.25 & 120 & 5.5 \\
\hline Sample E & $0 \%$ & 0 & 3.0 & 120 & 5.5 \\
\hline
\end{tabular}

After the complete addition of the BIBB and BMA reagents, the flasks were warm up at room temperature and the coupling reaction was carried out for $1 \mathrm{~h}$ at room temperature. The samples were washed with chloroform, toluene, methanol and water, and dried in a stream of nitrogen. The ATRP-initiated silicon samples, with different grafting densities, were directly used for the SI-ATRP of NIPAM. 
Surface initiated-atom transfer radical polymerization (SI-ATRP) of $\mathrm{N}$ isopropylacrylamide (NIPAM). The SI-ATRP of NIPAM was performed under a nitrogen or argon atmosphere with a molar concentration of NIPAM of 2.2 $\mathrm{M}$ in a 50:50 water/methanol mixture, and an optimized ATRP recipe containing a molar ratio of NIPAM:CuCl:PMDETA:CuBr 2 of 200:1:3:0.1, as is summarized in Table 3.6. In separate flasks, each component of the polymerization system (i.e. the solvents, the monomer, the ligand, and the catalyst) were purged/bubbled with argon to assure the oxygen free atmosphere and the control over the ratio of the ATRP polymerization reagents. In detail, more than $200 \mathrm{ml}$ of water and methanol were placed in different flasks, as well as approximately $5 \mathrm{ml}$ of PMDETA which were stirred and bubbled with argon for $5 \mathrm{~h}$ and $10 \mathrm{~min}$, respectively. The NIPAM $(87.2 \mathrm{~g}, 770 \mathrm{mmol})$ was added and purged in a round bottom flask of $500 \mathrm{ml}$ with argon for more than 1 hour. The oxygen free solvents and ligand were added into the NIPAM flask in the right ratio as summarized in Table 3.6.

The $\mathrm{CuCl}$ (380.8 mg, $3.86 \mathrm{mmol}$ ) and the $\mathrm{CuBr}_{2}(86.2 \mathrm{mg}, 0.386 \mathrm{mmol})$ species were added and purged in a round bottom flask of $500 \mathrm{ml}$ with argon for more than 1 hour. The solvents were transferred into the monomer/ligand flask via a 3 times purged syringe in the right ratio. The solution was stirred under argon atmosphere until the solids were completely dissolved. Then, with a three times purged syringe, the solution monomer/ligand was transferred into the flask containing the catalyst system. The mixture was kept in argon atmosphere, stirred for three hours until the copper salts were completely dissolved.

Table 3.6: Experimental conditions for the SI-ATRP of NIPAM from silicon oxide surfaces.

\begin{tabular}{|c|c|c|c|c|c|c|c|}
\hline \multicolumn{2}{|c|}{ ATRP System } & \multicolumn{2}{|c|}{ Solvents } & Monomer & Ligand & $\mathbf{C u}^{\text {I }}$ & $\mathbf{C u}^{\text {II }}$ \\
\cline { 3 - 8 } & Methanol & Water & NIPAm & PMDETA & $\mathbf{C u C l}$ & $\mathbf{C u B r}_{\mathbf{2}}$ \\
\hline Conc. & $\mathrm{M}$ & & & 2.2 & 0.066 & & \\
\hline Volume & $\mathrm{ml}$ & 175 & 175 & - & 2.4 & - & - \\
\hline Weight & $\mathrm{mg}$ & - & - & 87200 & - & 380.6 & 86.2 \\
\hline Moles & $\mathrm{mmol}$ & 5147 & 9722 & 770 & 11.6 & 3.86 & 0.386 \\
\hline Ratio & & 1333 & 2500 & 199 & 3 & 1 & 0.1 \\
\hline
\end{tabular}

Due to the size of the silicon samples used for NR experiments, the ATRP polymerization of NIPAM was performed in a Petri dish in glove box purged with dry nitrogen gas to assure the oxygen free atmosphere. The ATRP initiated samples were placed in the glove box and purged with nitrogen gas for at least 1 hour. The ATRP solution was transferred into the petri disk containing the ATRP surface initiated samples and the polymerization was carried out for $30 \mathrm{~min}$.

The ATRP polymerization reaction was interrupted using abundant amount of ATRP quenching solution composed of $\mathrm{CuBr}_{2}(0.05 \mathrm{M})$ and PMDETA $(0.1 \mathrm{M})$ in Milli-Q water. The PNIPAM grafted silicon samples were washed in ethanol and dried in a stream of 
nitrogen. The samples were stored in nitrogen box and transported to Grenoble in one week for the neutron reflectivity experiments.

IR: $3400-3200 \mathrm{~cm}^{-1}(\mathrm{NH}), 1650$ and $1535 \mathrm{~cm}^{-1}$ (amide).

Contact angle: static water contact angles: piranha-cleaned silicon surface $<20^{\circ}$, APTES-modified silicon oxide $48 \pm 4^{\circ}$, ATRP-initiated surfaces $70-80^{\circ}$, and PNIPAM brush layer grafted on silicon $62 \pm 3^{\circ}$.

\section{Device and methods}

IR spectroscopy. An Apha Brucker FTIR spectrometer (Bruker Optik GmbH, Ettlingen, Germany) was employed in transmission mode. 512 scans were collected in a scan of frequency range of 4000-400 $\mathrm{cm}^{-1}$ and a resolution of $4 \mathrm{~cm}^{-1}$. The background spectra was obtained using a freshly piranha-cleaned silicon oxide substrate.

Contact angle. Static water contact angle measurements were performed by sessile drop technique using an optical contact angle device (OCA 15, Dataphysics, Germany) equipped with an electronic syringe unit and connected to a charge-coupled device (CDD) camera. The drop contour was fitted by Young-Laplace equation. At least three different measurements of each sample were performed using Milli-Q pure water.

${ }^{1}$ H NMR. ${ }^{1} \mathrm{H}$ NMR spectra were obtained on a Bruker Avance III $400 \mathrm{MHz}$ instrument at $400.1 \mathrm{MHz}$ and ${ }^{1} \mathrm{H}$ chemical shifts were based on the solvent residual signals.

Ellipsometer. Variable angle spectroscopic ellipsometer (VASE, J. A. Woollam) was used. Measurements were performed as a function of the photon energy in the range 0.8-4.0 eV (276-1550 nm) with a step size of $0.1 \mathrm{eV}$. The dry measurements of the PNIPAM brush layers were performed at various angles of incidence $\left(65^{\circ}, 70^{\circ}\right.$ and $\left.75^{\circ}\right)$ and fitted between 250-900 $\mathrm{nm}$. The wet measurements were performed at fixed angle $\left(63^{\circ}\right)$ using a dedicated custom-made temperature controlled custom-made liquid cell and fitted between 250-900 $\mathrm{nm}$. The ellipsometry spectra were analyzed employing the package CompleteEASE (Woollam), using bulk dielectric functions for silicon, silicon dioxide, air and water. The analysis of the brush layers was performed on the basis of the Cauchy models. Regarding the ellipsometry models, the dry films were considered as a single homogenous dense layer above the thin (16 ̊) silicon oxide and the bulk silicon layers. The thickness, A- and B-Cauchy parameters of the polymer layer were fitted between $250-1150 \mathrm{~nm}$. The properties of the swollen polymer films (in water with $\mathrm{T}<$ LCST) were estimated considering a single A-graded layer model (composed of 50 slices). The thickness, A-, B- Cauchy parameters and the A-gradient with 50 slices were fitted between 300-900 $\mathrm{nm}$ to the experimental data. The collapsed PNIPAM brush layers (water with $\mathrm{T}>\mathrm{LCST}$ ) were modelled as a single homogenous dense layer where the thickness, A and B-Cuachy parameters were fitted between 300-900 nm.

Atom force microscopy, AFM. The thickness and the topology of the PNIPAM brush layers grafted on the big silicon were measured using Agilent 5100 AFM 
instrument. The samples were gently scratched with Teflon tweezer to reveal the bare silicon wafer and measure the relative thickness of the polymer layer. The dry thicknesses of the polymer graft on the planar substrate were obtained by measuring the step height in intermittent-contact mode using silicon Olympus cantilevers with a spring constant of about $2 \mathrm{~N} / \mathrm{m}$ and a resonance frequency of $70 \mathrm{kHz}$ in air. The wet thicknesses of the polymer films were obtained by measuring the step height in water operating in contact mode with polystyrene colloids attached to soft silicon cantilevers $(6 \mathrm{~mm}$ diameter, $\mathrm{CP}$ CONT-PS, sQube, NanoAndMore, Germany) characterized by a spring constant of about $0.2 \mathrm{~N} / \mathrm{m}$. The spring constant of the cantilevers was estimated via thermal tune calibration method. ${ }^{24}$

Neutron reflectometer, NR. Neutron reflectometry provided information about the film thickness, roughness, scattering length density (SLD), density profile normal to the surface by measuring the reflected neutron intensity $(\mathrm{R})$ as a function of the incident angle $(\Delta \theta)$. The neutron reflectivity measurements were carried out with the superADAM (Advanced Diffractometer for Analysis of Materials) ${ }^{25-26}$ at the Institute Laue-Langevin (ILL, Grenoble, France) using a monochromatic beam with a wavelength $\lambda$ of $5.18 \AA$.

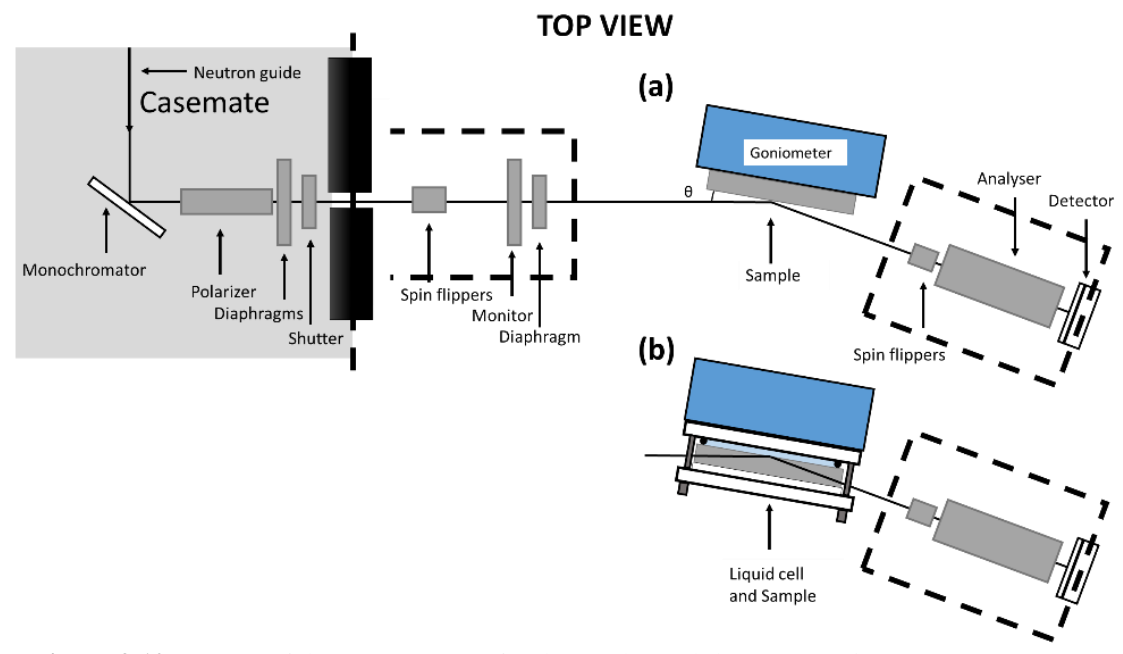

Figure 3.10: Layout of the SuperADAM for the (a) dry and (b) wet experiments.

Figure 3.10 and Figure 3.11 show the scheme of the superADAM reflectometer and the sample orientation for the dry and the "in liquid" measurements, respectively.

Dry samples have been measured in air, at the air/solid interface at room temperature, while the wet samples were measured in the liquid cell with different liquids (pure deuterium oxide and $33 \% \mathrm{v} / \mathrm{v}$ deuterium oxide-methanol mixture) and at different temperatures $\left(25\right.$ and $\left.40{ }^{\circ} \mathrm{C}\right)$. To ensure a total reflection of the neutron beam, the incident neutron path was going from air to the polymer brush layer to $\mathrm{SiO}_{2}$ to silicon for dry experiments. The liquid cell consisted of an O-ring made of poly(tetrafluoroethylene) (PFTE), a Teflon holder and two aluminum plates. The liquid "reservoir" is confined between the silicon surface and the aluminum plate, inside the O-ring, as is shown in the 
Figure 3.11. The temperature control was achieved by using an external thermostat-bath from which the water was continuously pumped to the cell. For the experiments in solution, the path was going from backside of the sample, i.e., from silicon to $\mathrm{SiO}_{2}$ to deuterated solvent-rich wet polymer brush layer to deuterated solvent. The isotopic contrast variation method was used to study the co-nonsolvent-induced transition. This method consists of using deuterated and hydrogenated solvent mixtures. By using the combination of hydrogenated and deuterated solvents, the measurement can give information about the distribution of the different solvent components with respect to the normal direction to the surface.

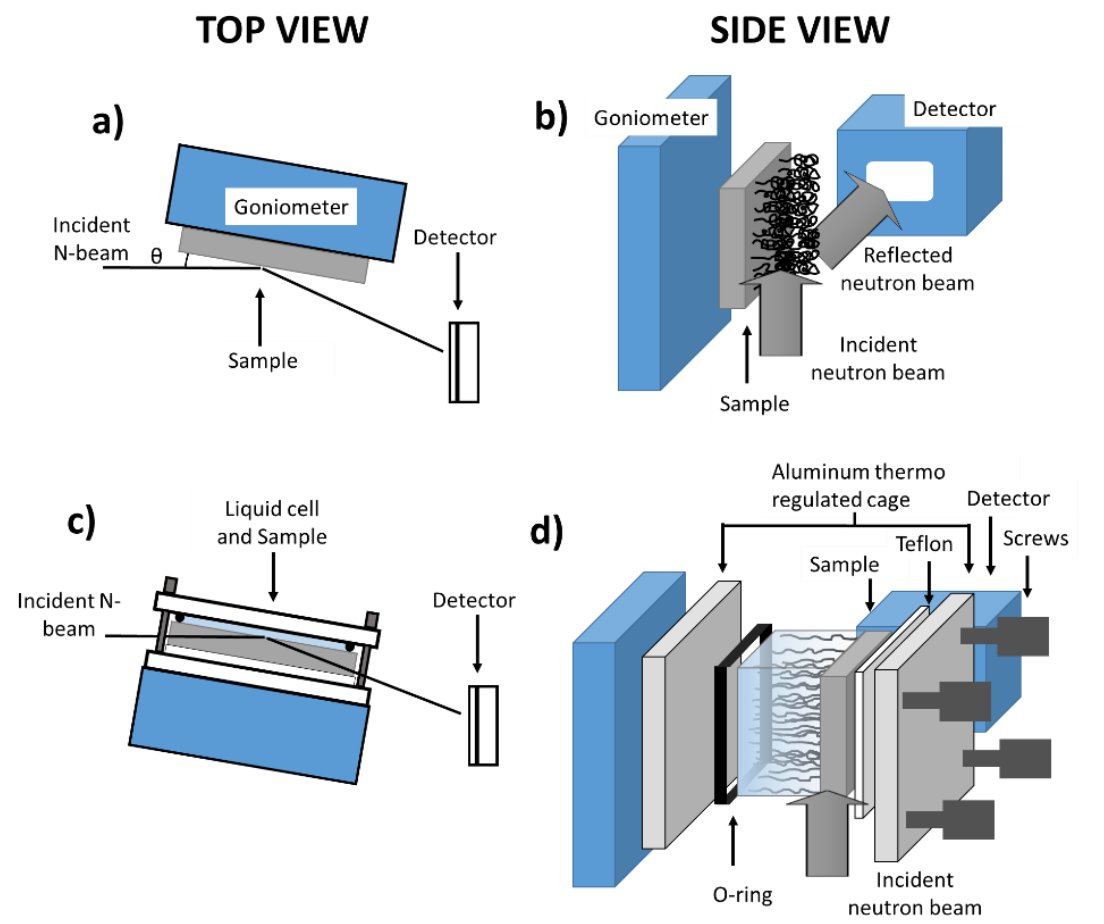

Figure 3.11: Characterization via NR measurements of the PNIPAM brush . a) top view and b) typical layout for the dry experiments. c) top view and layout for the liquid measurements. d) typical cell used for reflectivity measurements at the solid/liquid interface.

The SLD profile is directly related to the density and atomic composition normal to the surface. The SLD profiles were modelled by a stack of slabs; each slab was assigned an SLD, a thickness and a roughness described by a Debye-Waller factor. The volume density of intercalated molecules of each type of solvent through the polymer brush layers was obtained from the fitting of the NR data under the assumption of additivity of volumes.

Data were analysed using the MOTOFIT software. ${ }^{19}$ 


\subsection{Supporting Information}

In dorder to check whether any corrections for possible H-D exchange reactions are needed, the possible H-D exchange between the protons of the amide functional groups (N-H group) of PNIPAM and $\mathrm{D}_{2} \mathrm{O}$ was investigated. ${ }^{1} \mathrm{H}$ NMR measurements were performed at various time of dissolution of the polymer in the deuterated solvent at room temperature. Figure S3.1 shows a typical ${ }^{1} \mathrm{H}$ NMR spectra of amide of the PNIPAM freechains in $\mathrm{D}_{2} \mathrm{O}$ as a function of the time in solution. The ${ }^{1} \mathrm{H}$ NMR analysis allows to distinguish the proton signal of the amide (chemical shift at approximately $7.6 \mathrm{ppm}$, see Figure S3.1 and Figure S3.2). ${ }^{27}$ Complete H-D exchange was observed, in fact the intensity of the N-H peak disappeared completely after $60 \mathrm{~min}$, as is shown in Figure S3.2.

As a consequence of this experiment, a better fitting of the NR data can be obtained concerning the SLD and density profile evaluation.

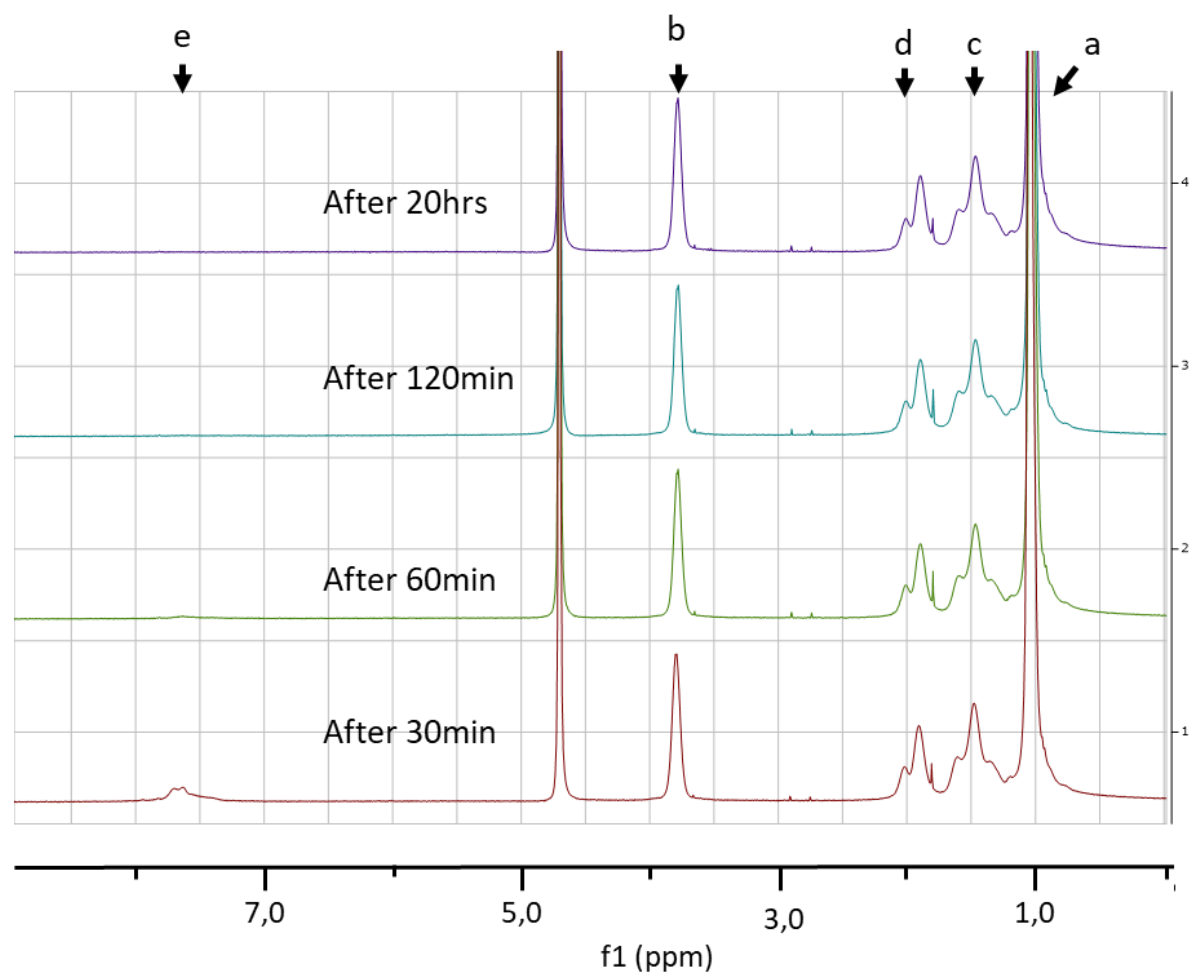

Figure S3.1: ${ }^{1} \mathrm{H}$ NMR analysis of the $\mathrm{H}-\mathrm{D}$ exchange for PNIPAM free-chain in $\mathrm{D}_{2} \mathrm{O}$ as a function of the time. Full ${ }^{1} \mathrm{H}$ NMR $\left(400 \mathrm{MHz}, \mathrm{CDCl}_{3}\right) \delta: 1.1\left(\mathrm{CH}_{3}, 6 \mathrm{H}\right) ; 1.5\left(\mathrm{CH}_{2}, 2 \mathrm{H}\right) ; 1.9(\mathrm{O}=\mathrm{CCH}, 1 \mathrm{H}) ; 3.8\left(\mathrm{CH}_{3} \mathrm{CH}, \mathrm{H}\right)$; $7.6(\mathrm{NH}, \mathrm{H})$. 


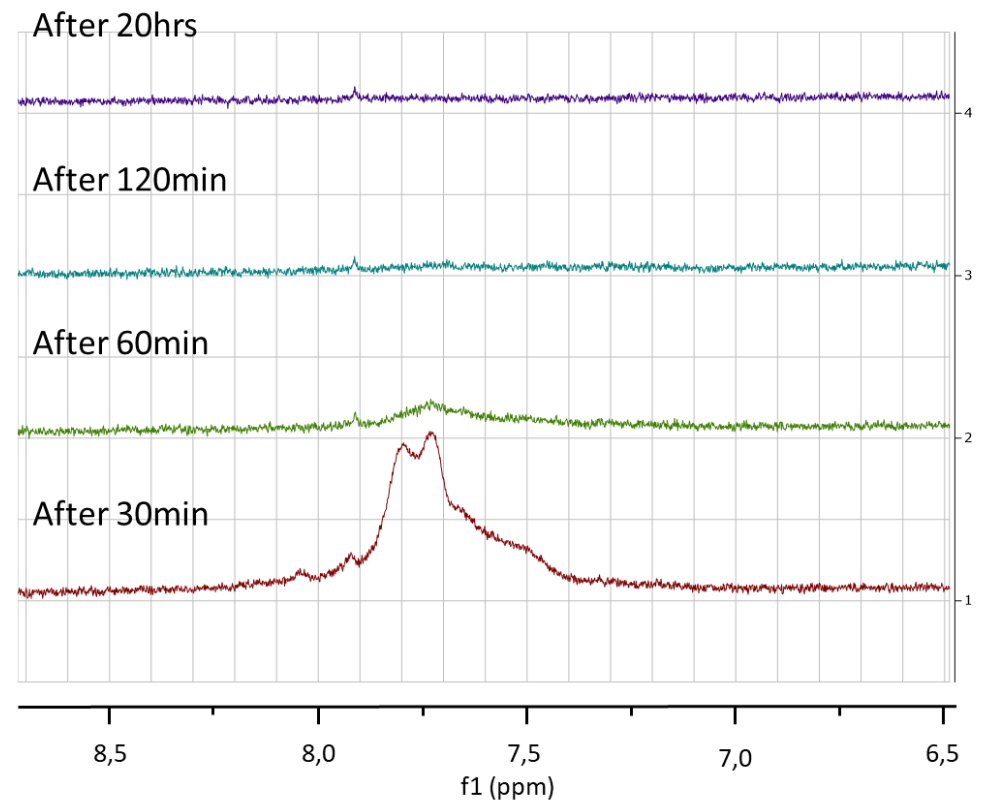

Figure S3.2: ${ }^{1} \mathrm{H}$ NMR analysis of the $\mathrm{H}-\mathrm{D}$ exchange for the amidic proton of PNIPAM in $\mathrm{D}_{2} \mathrm{O}$ as a function of the time.

\subsection{References}

1. Heskins, M.; Guillet, J. E., Solution properties of poly (N-isopropylacrylamide). Journal of Macromolecular Science - Chemistry 1968, 2 (8), 1441-1455.

2. Halperin, A.; Kroeger, M.; Winnik, F. M., Poly (N-isopropylacrylamide) phase diagrams: fifty years of research. Angewandte Chemie International Edition 2015, 54 (51), 15342-15367.

3. Fujishige, S.; Kubota, K.; Ando, I., Phase transition of aqueous solutions of poly (N-isopropylacrylamide) and poly (N-isopropylmethacrylamide). The Journal of Physical Chemistry 1989, 93 (8), 3311-3313.

4. Schild, H. G., Poly (N-isopropylacrylamide): experiment, theory and application. Progress in polymer science 1992, 17 (2), 163-249.

5. Yong, H.; Bittrich, E.; Uhlmann, P.; Fery, A.; Sommer, J.-U., Co-Nonsolvency Transition of Poly(Nisopropylacrylamide) Brushes in a Series of Binary Mixtures. Macromolecules 2019, 52 (16), 6285-6293.

6. Schmaljohann, D., Thermo-and pH-responsive polymers in drug delivery. Advanced drug delivery reviews 2006, 58 (15), 1655-1670.

7. De las Heras Alarcón, C.; Pennadam, S.; Alexander, C., Stimuli responsive polymers for biomedical applications. Chemical Society Reviews 2005, 34 (3), 276-285.

8. Karbarz, M.; Mackiewicz, M.; Kaniewska, K.; Marcisz, K.; Stojek, Z., Recent developments in design and functionalization of micro-and nanostructural environmentally-sensitive hydrogels based on $\mathrm{N}$ isopropylacrylamide. Applied Materials Today 2017, 9, 516-532.

9. Winnik, F. M.; Ringsdorf, H.; Venzmer, J., Methanol-water as a co-nonsolvent system for poly (Nisopropylacrylamide). Macromolecules 1990, 23 (8), 2415-2416.

10. Schild, H. G.; Muthukumar, M.; Tirrell, D. A., Cononsolvency in mixed aqueous solutions of poly (Nisopropylacrylamide). Macromolecules 1991, 24 (4), 948-952. 
11. Zoppe, J. O.; Ataman, N. C.; Mocny, P.; Wang, J.; Moraes, J.; Klok, H.-A., Surface-initiated controlled radical polymerization: state-of-the-art, opportunities, and challenges in surface and interface engineering with polymer brushes. Chemical reviews 2017, 117 (3), 1105-1318.

12. Sui, X.; Chen, Q.; Hempenius, M. A.; Vancso, G. J., Probing the Collapse Dynamics of Poly (Nisopropylacrylamide) Brushes by AFM: Effects of Co-nonsolvency and Grafting Densities. small 2011, 7 (10), 1440-1447.

13. Chen, Q.; Kooij, E. S.; Sui, X.; Padberg, C. J.; Hempenius, M. A.; Schön, P. M.; Vancso, G. J., Collapse from the top: brushes of poly (N-isopropylacrylamide) in co-nonsolvent mixtures. Soft Matter 2014, 10 (17), 3134-3142.

14. Li, L.; Zhu, Y.; Li, B.; Gao, C., Fabrication of Thermoresponsive Polymer Gradients for Study of Cell Adhesion and Detachment. Langmuir 2008, 24 (23), 13632-13639.

15. Ramakrishna, S. N.; Cirelli, M.; Kooij, E. S.; Klein Gunnewiek, M.; Benetti, E. M., Amplified Responsiveness of Multilayered Polymer Grafts: Synergy between Brushes and Hydrogels. Macromolecules 2015, 48 (19), 7106-7116.

16. Malham, I. B.; Bureau, L., Density effects on collapse, compression, and adhesion of thermoresponsive polymer brushes. Langmuir 2009, 26 (7), 4762-4768.

17. Wang, S.; Zhu, Y., Facile method to prepare smooth and homogeneous polymer brush surfaces of varied brush thickness and grafting density. Langmuir 2009, 25 (23), 13448-13455.

18. Imae, T.; Kanaya, T.; Furusaka, M.; Torikai, N., Neutrons in soft matter. John Wiley \& Sons: 2011.

19. Nelson, A., Co-refinement of multiple-contrast neutron/X-ray reflectivity data using MOTOFIT. Journal of applied crystallography 2006, 39 (2), 273-276.

20. Tomczak, N.; Vallée, R. A.; van Dijk, E. M.; Kuipers, L.; van Hulst, N. F.; Vancso, G. J., Segment dynamics in thin polystyrene films probed by single-molecule optics. Journal of the American Chemical Society 2004, 126 (15), 4748-4749.

21. Klok, H.-A.; Genzer, J., Expanding the Polymer Mechanochemistry Toolbox through Surface-Initiated Polymerization. ACS Macro Letters 2015, 4 (6), 636-639.

22. Wang, J.; Klok, H.-A., Swelling-Induced Chain Stretching Enhances Hydrolytic Degrafting of Hydrophobic Polymer Brushes in Organic Media. Angewandte Chemie International Edition 2019, 58 (29), 9989-9993.

23. Tugulu, S.; Klok, H.-A., Stability and nonfouling properties of poly (poly (ethylene glycol) methacrylate) brushes under cell culture conditions. Biomacromolecules 2008, 9 (3), 906-912.

24. Proksch, R.; Schäffer, T.; Cleveland, J.; Callahan, R.; Viani, M., Finite optical spot size and position corrections in thermal spring constant calibration. Nanotechnology 2004, 15 (9), 1344.

25. Vorobiev, A.; Devishvilli, A.; Palsson, G.; Rundlöf, H.; Johansson, N.; Olsson, A.; Dennison, A.; Wollf, M.; Giroud, B.; Aguettaz, O., Recent upgrade of the polarized neutron reflectometer Super ADAM. Neutron News 2015, 26 (3), 25-26.

26. Devishvili, A.; Zhernenkov, K.; Dennison, A. J.; Toperverg, B.; Wolff, M.; Hjörvarsson, B.; Zabel, H., SuperADAM: Upgraded polarized neutron reflectometer at the Institut Laue-Langevin. Review of Scientific Instruments 2013, 84 (2), 025112.

27. Scarpa, J. S.; Mueller, D. D.; Klotz, I. M., Slow hydrogen-deuterium exchange in a non-. alpha.-helical polyamide. Journal of the American Chemical Society 1967, 89 (24), 6024-6030. 
Chapter 4 Tunable Friction by Employment of
Co-Non-Solvency of PNIPAM Brushes

* This chapter has been published in: Yu Y., Cirelli M., Kieviet B.D., Kooij E.S., Vancso G. J., de Beer S.; Polymer. 2016, 102, 372-378. 


\section{Contents}

\section{Chapter 4 Tunable Friction by Employment of Co-Non-Solvency of} PNIPAM Brushes................................................................................... 73

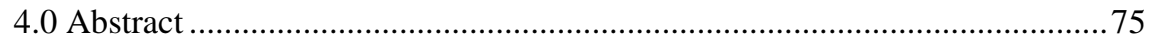

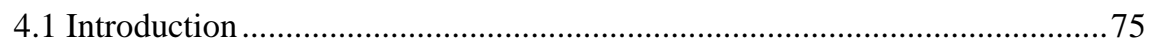

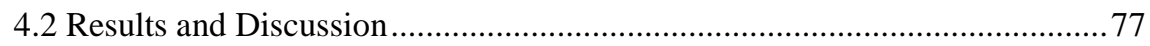

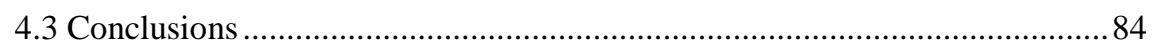

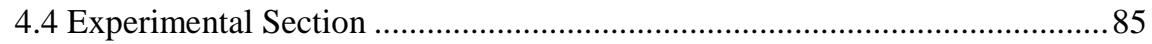

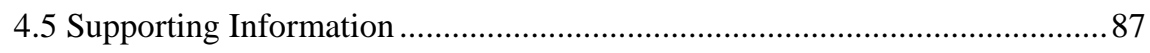

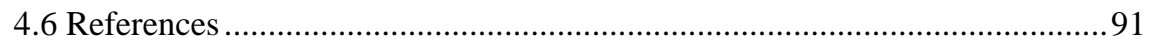




\subsection{Abstract}

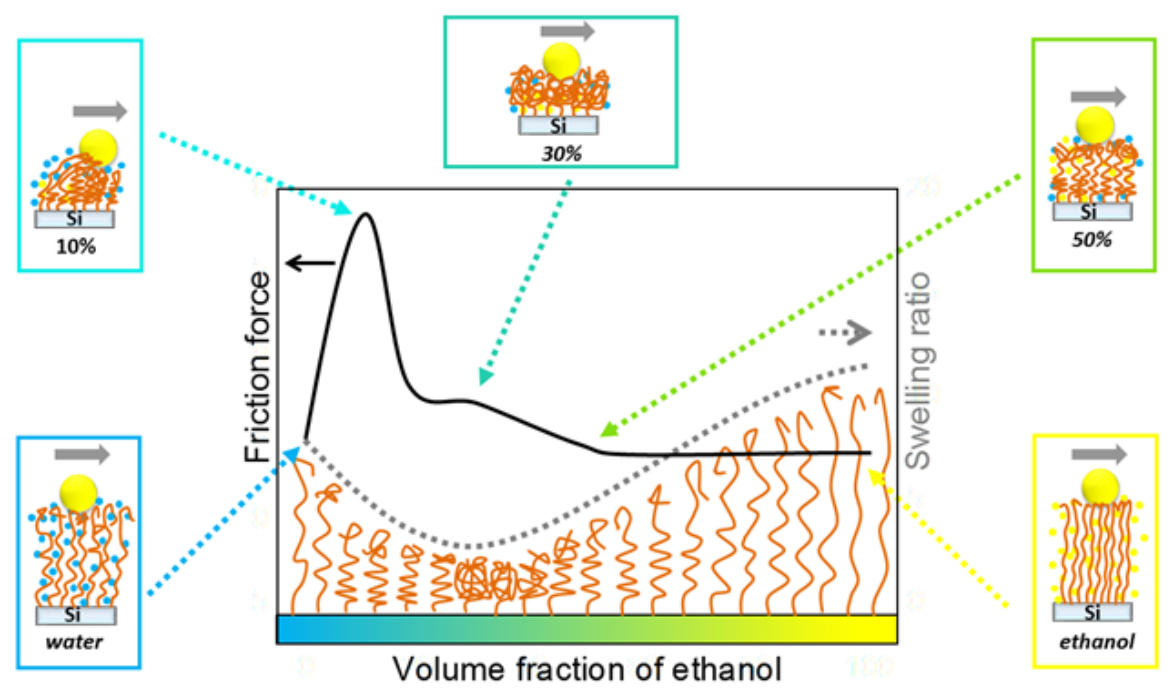

TOC Chapter 4: Tunable friction by co-non-solvency of the PNIPAM brush layer in an ethanol/water mixture. Friction force and swelling ratio of PNIPAM brush layers as a function of the solvent quality. The friction force was evalutated using AFM between the gold colloidal probe and the brush coating as a function of the solvent mixture.

We present a simple method to control the tribological properties of contacts between polymer brushes and a solid counter surface. We show, using atomic force microscopy (AFM) experiments, that the friction force, upon relative sliding of a $\operatorname{poly}(N$ isopropylacrylamide) (PNIPAM) brush and a gold colloid on an AFM cantilever, varies over two orders of magnitude when changing the composition of an ethanol-water mixture that solvates the brush. We achieve this large variation in friction via a co-nonsolvency effect: In pure water or ethanol, the PNIPAM brush is swollen and the friction is low, while in a 10-90 vol.\% ethanol-water mixture, the brush is partly collapsed and polymer-stretching causes the friction to be high.

\subsection{Introduction}

The friction between surfaces in relative motion is generally required to be either high or low: When walking, braking or picking up material, sufficiently high friction is of critical importance, while smooth sliding is preferred in systems where friction results in major costs due to energy-loss. However, for some advanced applications, it is necessary to be able to switch between high and low friction. Examples include robots that can walk on walls ${ }^{1}$ and smart tweezers. ${ }^{2}$ Polymer brushes provide a versatile platform for controlling surface properties ${ }^{3-6}$ including tribomechanical properties ${ }^{4,7-9}$ and, 
therefore, hold great potential for applications as surfaces with variable (switchable) friction. ${ }^{10-11}$

Polymer brushes can be created by end-anchoring polymers to a surface or interface at an adequately high density. ${ }^{12}$ When brushes are dry or kept in a poor solvent in their collapsed state, the polymers form a dense layer on the surface. The adhesion and friction between these dense polymer films and a solid counter-surface are generally high. ${ }^{13}$ When a polymer brush is kept in a good solvent, the polymers stretch out and absorb the solvent into the brush. Under these conditions, the friction and adhesion are typically low. ${ }^{14}$ By employing polymers that respond to an applied external stimulus (e.g. by changes in temperature, ${ }^{15}$ solvents, ${ }^{16} \mathrm{pH},{ }^{17}$ electric ${ }^{18}$ and magnetic field ${ }^{19}$ etc.), the effective solventquality can be readily changed such that friction and adhesion can be controlled. ${ }^{7,14,20-22}$ A particularly easy method to change the solvent quality is the employment of the socalled co-non-solvency of the polymers ${ }^{23-27}$ where a coil-to-globule transition of polymers can be triggered by simply adding a relatively small amount of co-solvent to the solvent.

When polymers are in a swollen, coiled state in pure solvent or co-solvent, but form a collapsed globule in a mixture of the two solvents, it is called a co-non-solvency effect. A well-studied example is poly( $N$-isopropylacrylamide) (PNIPAM) in a mixture of water and alcohols such as methanol, ethanol or isopropanol. ${ }^{28-29}$ At room temperature, PNIPAM polymers are found to be swollen in water or one of these alcohols, while the polymers are collapsed e.g. for 10-20 vol.\% methanol in a methanol-water mixture for polymers free in solution ${ }^{10}$ (at 30 vol.\% for gels and brushes ${ }^{24,30}$ ). The origin of co-nonsolvency is still under debate. ${ }^{25,31-32}$ Koga et al. ${ }^{25}$ proposed that co-non-solvency is a result of competitive hydrogen bonding where bonds between water and alcoholmolecules can be favored over hydrogen bonds between PNIPAM and the solventmolecules. In a different study, Sheng et al. ${ }^{31}$ conclude from their Fourier transform infrared spectroscopy experiments that the formation of "water-methanol clusters" $\left(\mathrm{H}_{2} \mathrm{O}\right)_{n}(\mathrm{MeOH})_{\mathrm{m}}$ of concentration-dependent size $(\mathrm{n}=1,2,3,4,5 ; \mathrm{m}=1)$ induces a coilglobule-coil transition. Depending on their size, these clusters create either good or poor solvent conditions for PNIPAM. Recently, Mukherji et al. ${ }^{33}$ reported that the FloryHuggins theory cannot explain the co-non-solvency mechanism. They show using coarsegrained molecular dynamics simulations ${ }^{32}$ that a possible explanation for the phenomenon might be that at low concentration of the better solvent, bridges are formed between parts of the PNIPAM polymer by the better solvent, which induces a collapse. Nevertheless, irrespective of the exact explanation for the origin, the manifestation of the phenomenon of co-non-solvency is well established and has been widely observed for different combinations of polymers, solvents and co-solvents. ${ }^{24,26-27,34-35}$ Moreover, it has been employed in the development of applications such as gating ${ }^{36}$ and in alcohol sensing. ${ }^{37}$ However, for tuning friction, it has been relatively unexplored. 
In this Chapter we show using friction force atomic force microscopy (AFM) experiments, that co-non-solvency of PNIPAM in ethanol-water mixtures can be used to tune friction over two orders of magnitude. In a recent publication Chen et al. ${ }^{14}$ reported on obtaining variable friction between PNIPAM and a silicon colloid using co-nonsolvency. They observe that, in a 50-50 vol.\% methanol-water mixture, the friction coefficient is approximately 4 times higher than in pure water. We built on these results and study the frictional response upon sliding a gold colloid over a PNIPAM brush at room temperature, while we systematically vary the relative amount of ethanol in ethanolwater mixtures. We find that for $10 \mathrm{vol} \%$ of ethanol-water mixture, the friction is the highest. At this concentration the friction is $120 \pm 10$ times higher than in pure ethanol. Surprisingly, we find that the concentration for the highest friction force does not coincide with the concentration of minimal brush swelling at $30 \mathrm{vol} . \%$ of ethanol-water mixture. Thus, the observed frictional response cannot be explained by brush-swelling alone. Instead, we show, using AFM measurements in which the colloid probe is approached towards and retracted from the surface, that the partly collapsed polymers in $10 \mathrm{vol} . \%$ ethanol-water mixture have become strongly stretched when the colloid is moved away from its initial contact with the brush. This can explain the enhanced frictional response found at 10 vol.\% of ethanol-water mixture. Because our results show that the friction can be varied over two orders of magnitude, we propose that PNIPAM in these solventco-solvent mixtures holds great potential for application in e.g. robots that can walk on walls or smart tweezers.

\subsection{Results and discussion}

Figure 4.1 shows in (a) a schematic representation of the effect of the solvent composition on the measured brush height and in (b) the measured swelling ratio of PNIPAM brushes as a function of ethanol-water mixture at various compositions. In the experiments, the brush height is measured by ellipsometry (triangles up and down) and by determining the vertical distance between brush-surface and the bare silicon surface using AFM (squares and circles), respectively. We cannot expect the swollen heights measured by the different techniques to be the same, since there is no well-defined height of a brush. Instead, the polymer-density gradually changes as a function of the distance from the substrate ${ }^{12}$ and, as discussed in more detail below, the different measurement techniques analyze different parts of this density distribution.

For the AFM measurements, we gently scratch off part of the brush by Teflon tweezers. Next, we inject the solvent and equilibrate the system for at least 10 minutes. Then, we determine the swollen height of the brush by i) imaging under a small normal load of $1.6 \mathrm{nN}$, ii) performing force distance measurements (FD) upon approach of the colloid towards the brush. In the latter, we compare the (still arbitrary) separation where the force starts to deviate from 0 to the height of the brush imaged at a known applied 
force. The difference in tip-surface separation between the two is added to the brush height. The swollen height is normalized by the dry brush height (close to $140 \mathrm{~nm}$ for the measurements shown in Figure 4.1 ${ }^{\dagger}$ to give the swelling ratio shown in Figure 4.1(b). We expect that we underestimate the brush height for the imaging method, as the brush is slightly compressed even under the lightest load applied. For the force distance measurements, we probably overestimate the brush height, because the force will increase as soon as the polymer density is high enough to bear a normal load, which can happen at larger distances than the brush height due to poly-dispersity. ${ }^{38}$ The difference in height for both measurements is most pronounced under good solvent conditions, where the brush stretches the most. This can be observed in pure ethanol, where the magnitude of the difference can be up to $250 \mathrm{~nm}$. The 'real' brush height will probably be between heights measured using imaging and the FD method.

Figure 4.1(b) also shows the swelling ratio measured with ellipsometry. A detailed explanation of the ellipsometry measurements can be found in the supporting information. In short, for the dry height measurements, the ellipsometry data is fitted to the traditional Cauchy model. For the swollen brush height measurements, we incorporate a gradual change in polymer density as a function of the distance from the surface. Such a gradual change is typically considered to be parabolic in the limit of dilute brushes, ${ }^{39-41}$ but approaches a step function for high density brushes ${ }^{47-48}$ and can become linear or even concave in the presence of polydispersity. ${ }^{49-50}$ Since we do not know the exact density profile of our brushes, we tested two different models to fit the ellipsometry data. Model 1 (single graded layer, SGL) effectively fits the refractive index - and thus the density to a power law function that can be tuned between concave and convex by changing the exponent. ${ }^{51}$ Model 2 (dense layer, DL), places this gradually changing refractive index on top of a layer of constant refractive index (density) close to the sample surface. ${ }^{14}$ Both models give comparable results, except in pure water where the single gradient layer seems to underestimate the swollen height by a factor 1.4 and at $60-70$ vol. $\%$ ethanol where the double layer model overestimates the swollen height by a factor 1.3-1.8. For each individual measurement (AFM or ellipsometry), the new solvent-composition is applied to a cleaned and dried brush surface (i.e. the solvent composition is not continuously varied). We tested that our results were independent of previous solvent conditions by stepwise decreasing the vol.\% ethanol after stepwise increasing it. Moreover, we found the same swelling ratios for all the tested samples within $15 \%$.

\footnotetext{
$\dagger$ Please note that the measurements on Figure 4.1 were performed on different samples than the sample used for GPC and therefore the dry height is also different.
} 


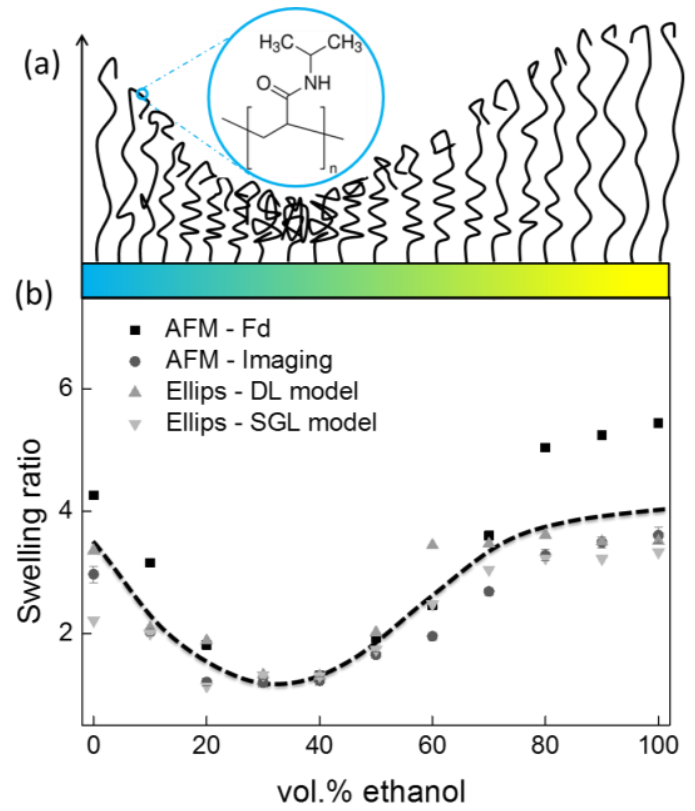

Fig. 4.1: Schematic of the co-non-solvency-induced conformational change of the PNIPAM brush (dry height $140 \mathrm{~nm}$ ) (a) and the swelling ratio of a PNIPAM brush as a function of the ethanol volume fraction in the ethanol-water mixture. (b) The swelling ratio is calculated from the swollen height of the brushes and is normalized by the dry brush height as measured by AFM force distance (FD, black squares) or imaging (dark gray circles) using a normal load that is kept as small as possible $(1.6 \mathrm{nN})$ over a scan size of $40 \mu \mathrm{m}$ at a scan rate of $1 \mathrm{~Hz}$ or by ellipsometry measurements using either the double layer model (DL, gray upward triangles) or the single gradient layer model (SGL, light gray downward triangles).

In pure water, we find that the swelling ratio is about 3, which is in agreement with the literature values reported for PNIPAM brushes with similar grafting densities. ${ }^{52-53}$ When adding a small amount of ethanol to the water (10 vol.\% ethanol in the ethanolwater mixture), the PNIPAM brush strongly shrinks to reach a swelling ratio of around 2. By further increasing the ethanol volume fraction, the brush further collapses. At 30 vol.\% ethanol, there is a minimum in the swelling ratio. Here the swelling ratio has, however, still a value of 1.25 . The observation that the system is still partly solvated at minimum swelling is in agreement with results obtained by others. Dalkas et al. ${ }^{54}$ measured the cloud point of PNIPAM in water-dioxane mixtures, and the point of minimum swelling is obtained around 30-40 vol.\% dioxane. Napper et al. ${ }^{30}$ find for PNIPAM on polystyrene latex particles that the minimum particle-diameter is obtained at $30 \mathrm{vol} \%$ ethanol-water mixture, and the swelling ratio is calculated to be roughly 1.5 . This is in direct agreement with our measurements. The presence of solvent in the collapsed brush is consistent with the bridging-model of Mukherji et al. ${ }^{32}$ where the better solvent is expected to be between the collapsed polymers. However, this remains speculative, since from our results we cannot tell the partition of water and ethanol within the brush. We note that the presence of solvent in a collapsed brush is not observed for a temperature-induced conformational 
change of a PNIPAM brush. ${ }^{55-56}$ For these systems, the swelling ratio has been found to be close to 1 above the lower critical solution temperature (LCST). This might indicate that the mechanism of collapse is different for a temperature-induced collapse compared to a co-non-solvency-induced collapse.

At a 40 vol.\% ethanol, the average swelling ratio is 1.3 and is slightly higher than at $30 \%$, which shows that the brush again absorbs more solvent molecules and re-swells. This is called the re-entrant phenomenon. ${ }^{31,54,57}$ By further increasing the ethanol content, the brush swells more until it reaches its maximum swelling ratio in 100 vol.\% ethanol. In pure ethanol, the swelling ratio is larger than 3.5, which is close to the height expected for fully stretched polymers and is more than $20 \%$ times higher than in pure water. This higher swelling ratio shows that ethanol is a better solvent for PNIPAM than water.

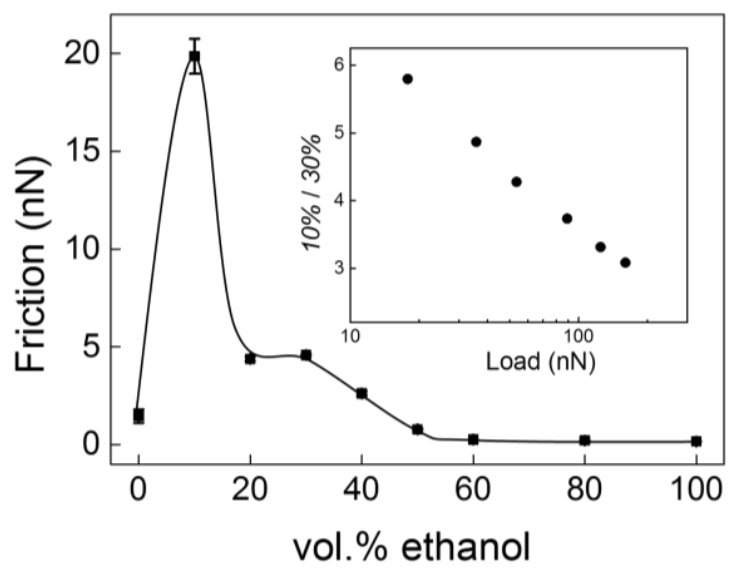

Fig. 4.2: Friction force between the PNIPAM brush (dry height $170 \mathrm{~nm}$ ) and a gold colloid as a function of ethanol volume fraction measured at a relative velocity of $10 \mu \mathrm{m} / \mathrm{s}$ under a normal load of $25 \mathrm{nN}$, while moving over a scan size of $20 \mu \mathrm{m}$. The error bars denote the standard error of the mean with a $95 \%$ confidence interval. For most data points the error bars are smaller than the symbol size. The inset shows the friction at 10 vol.\% ethanol normalized by the friction at $30 \mathrm{vol} . \%$ ethanol between a PS probe (radius $7 \mu \mathrm{m}$ ) and the PNIPAM brush (dry height $115 \mathrm{~nm}$ ) upon sliding at a velocity of $20 \mu \mathrm{m} / \mathrm{s}$ over a scan size of $20 \mu \mathrm{m}$.

So far, most studies on PNIPAM have focused on investigating the change in chemical or physical properties by comparing the system for two states, the swollen state and the collapsed state. ${ }^{14,58-61}$ In our recent publication, ${ }^{55}$ we show, however, that the tribomechanical properties of PNIPAM brushes change the strongest at the high temperature range of the coil-to-globule transition. In our previous work, ${ }^{55}$ we systematically vary the temperature and find that the friction just below the LCST is almost two orders of magnitude higher than the friction at lower or higher temperatures. Therefore, we expect that close to the coil-to-globule and globule-to-coil transition of PNIPAM in water ethanol mixtures, the friction will be high too.

Figure 4.2 shows the variation of friction force between the PNIPAM brush and a gold colloid as a function of the volume fraction of ethanol in the ethanol-water mixture. 
In water, the PNIPAM brush is swollen (see Figure 4.1) and the friction force is only 1.5 $\mathrm{nN}$ (Figure 4.2). When the brush is water-swollen, it is very lubricious. However, when a small amount of ethanol (10\%) is added, the friction force dramatically increases to about $21 \mathrm{nN}$, which is 14 times higher than the value observed in water. Such a strong variation of friction is also found for PNIPAM brushes in water close to the LCST. ${ }^{55}$ As shown in Figure 4.1 for a 10\% ethanol, the PNIPAM brush height decreases by $36 \%$ compared to the height in water and, thus, the polymer density is higher. This allows for forming more interaction-sites between the gold colloid and the PNIPAM brush. When sliding over the partially collapsed brush, the colloid can stretch the chains in the brush, which would enhance friction. A schematic representation of this postulated effect is shown in Figure 4.3. At the given solvent composition, the friction increases logarithmically with the velocity, which is consistent with the observations in recent molecular dynamics simulations using attractive polymer-surface interactions. ${ }^{62}$ In these simulations, it was shown that the dissipation upon sliding is caused by the formation and rupture of physical polymer-surface bonds, which causes instabilities and, thus yields a logarithmic dependence of the friction on the velocity.

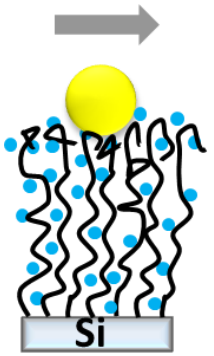

water

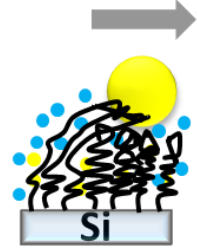

$10 \%$

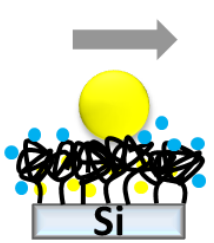

$30 \%$

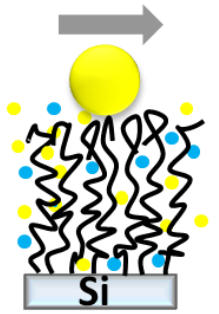

$50 \%$

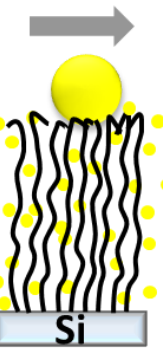

ethanol

Fig. 4.3: Schematic illustration of the proposed origin of friction between the gold colloid and the PNIPAM brush at various compositions of water (blue) and ethanol (yellow).

Upon further increase of 20 vol. \% ethanol, the PNIPAM brush-height shrinks by $40 \%$. However, the friction now decreases. The reason for this might be that the more strongly collapsed brush at 20 vol.\% ethanol resists stretching thus reduce the related energy dissipation, yielding a lower friction. The maximum collapse (minimum swelling ratio in Figure 4.1) occurs at 30 vol.\% ethanol-water mixture. In the maximally collapsed state, the friction is a $1.05 \pm 0.03$ times higher compared to the friction at $20 \mathrm{vol} . \%$. The mostly collapsed brush resists to be stretched due to the increased polymer-polymer interactions, and the friction is now mainly dominated by adherence interactions and viscoelastic deformations while sliding over the rough collapsed brush. Thus, a second, but smaller, maximum of friction is observed at this concentration.

When the ethanol volume fraction is further increased to $50 \%$ ethanol, the brush reswells. An effective lubricant layer is formed again and, as a result, the friction decreases 
to $0.8 \mathrm{nN}$. Chen et al. ${ }^{14}$ report that in a 50/50 (v/v) methanol/water solvent-co-solvent mixture, friction force between the PNIPAM brush and silicon colloid is 4.6 times than in water. In contrast, we find that in a 50 vol. $\%$ ethanol-water mixture the friction is lower than that in water. A possible explanation for this discrepancy might be that we measured at different temperatures. The solvation of PNIPAM brushes is very sensitive to the temperature, such that even a few degrees difference in temperature can alter the friction by orders of magnitude. ${ }^{55}$ Surprisingly, even though we have a re-entrance or globule-tocoil transition at 50 vol.\% ethanol, we do not observe a strong response in the friction. This asymmetry might indicate that the mechanism causing the coil-to-globule transition at $10 \mathrm{vol} . \%$ ethanol is different from the mechanism behind the globule-to-coil transition at $50 \mathrm{vol} . \%$ ethanol in the solvent mixture, which would be consistent with the bridgingby-the-better-solvent picture of Mukherji et al. ${ }^{32}$ However, an alternative explanation could be that the presence of ethanol disrupts the PNIPAM-gold interactions.

Above 60 vol.\% ethanol in the ethanol-water mixture, the friction is almost constant at a low value of $0.2 \mathrm{nN}$. Because alcohol is a better solvent for PNIPAM than water, ${ }^{16,63}$ the PNIPAM brushes will preferentially interact with ethanol molecules instead of gold. Moreover, the high osmotic pressure in the ethanol also reduces the contact area between colloid and brush. Thus, very low friction forces can be obtained. In pure ethanol, the friction force is as low as $0.16 \mathrm{nN}$, which is two orders of magnitude smaller than the friction in 10 vol.\% ethanol-water mixture. In pure ethanol, we find that the friction increases linearly with the velocity, which implies that the dissipation is dominated by hydrodynamic drag and not by polymer-colloid interactions.

The observed variation in the friction is strongest at low normal load values, as depicted in the inset of Figure 4.2. Upon increasing the normal load, the ratio of the friction force at 10 vol.\% ethanol compared to the friction at 30 vol.\% ethanol decreases logarithmically with the load. In the limit of very high loads, the friction will become independent of the solvent composition. It is, however, difficult to say at what load this will happen, since different dissipation mechanisms would dominate the friction at high normal loads.

Within a $15 \%$ uncertainty, we observe the same changes in friction as shown in Figure 4.2 by measuring 6 different samples using 4 different gold colloid probes. When a polystyrene colloid probe (colloid diameter $6 \mu \mathrm{m}$ ) is employed for the experiments, the variation in friction force reduces to a factor 72-95 (with variations around 25\%). This observation implies that the change in friction force depends on the solvent-mediated interaction between the colloid and the PNIPAM brush.

To study if polymer stretching could be at the origin of the enhanced frictional response at $10 \mathrm{vol} . \%$ ethanol-water mixture, we performed AFM experiments in which we measured the force on the colloid probe upon approach to and retract from the brush surface (displacement of $2 \mu \mathrm{m}$ at a velocity of $10 \mu \mathrm{m} / \mathrm{s}$ ). Figure 4.4 shows typical raw 
force-separation curves of a PNIPAM brush in various compositions of the ethanol-water mixture.

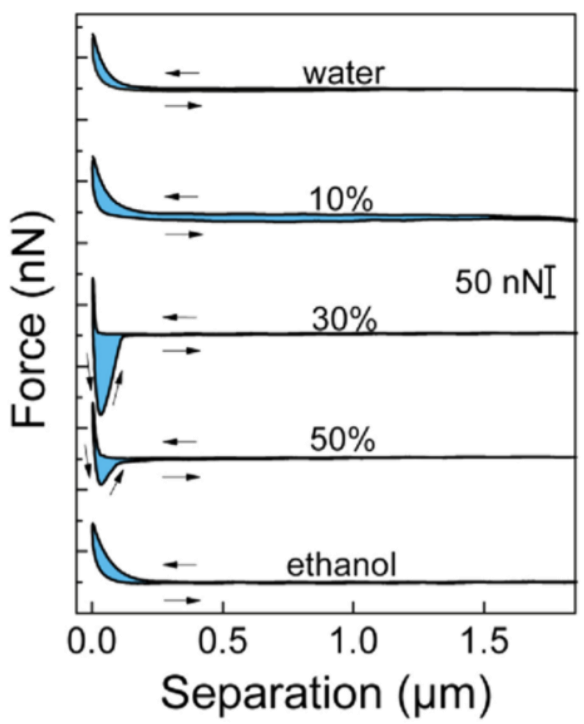

Fig. 4.4: Typical force versus separation curves at various ethanol volume fractions $(0,10,30,50,100 \%)$. The approach and retract velocity of the gold colloid to and from the PNIPAM brush (dry height $170 \mathrm{~nm}$ ) is 10 $\mu \mathrm{m} / \mathrm{s}$. The arrows denote the direction of motion. The difference in force between the approach and retract curves is colored blue to clarify the adhesive interaction. The scale bar equals $50 \mathrm{nN}$.

The maximum compression load was controlled to be $80 \mathrm{nN}$. As expected, in pure water we observe almost no adhesive interaction. When the brush is kept in a 10 vol.\% ethanol-water mixture, the adhesive interaction is clearly stronger compared to what we found in pure water. The maximum adhesion force at $10 \mathrm{vol} . \%$ ethanol is $6 \mathrm{nN}$, which is 3.4 times larger than the maximum adherence in water. Also, there is a pronounced longranged adhesive interaction that vanishes only at distances larger than $1.32 \pm 0.11 \mu \mathrm{m}$. As shown in our previous work on the adhesive interactions between PNIPAM and a gold colloid upon inducing a swelling transition using temperature, ${ }^{55}$ we attribute this longranged adhesive interaction to stretching of the partly collapsed polymers. Similar stretching of polymers has been observed. ${ }^{64-65}$ Upon moving the colloid in the lateral direction, this stretching of the brush-polymers can increase friction (shown in Figure 4.2). Stretching over such a large distance seems surprising considering our degree of polymerization, which is typically smaller than 2200 . However, our polydispersity index is high (PDI is 3-5), such that the longer chains in the brush can still stretch over distances around $1.3 \mu \mathrm{m}$. We note that such a high polydispersity index could indicate cross-linking in the brush. However, crosslinking would counteract polymer-stretching as is, therefore, probably not the main cause for our high PDI. As shown in the supporting information Figure S4.4, thinner brushes (20 nm dry height) show significantly less polymer 
stretching over only $650 \mathrm{~nm}$. For an 30 vol.\% ethanol-water mixture, the brush height is the smallest. At this concentration, the retract curve shows a sharp snap-off instability and a maximum adherence of $110 \mathrm{nN}$. No long-ranged adhesion is observed and thus chains would not become stretched. At a $50 \mathrm{vol} . \%$ ethanol volume fraction, the water-ethanol mixture solvates the brush such that the swelling ratio is 1.8 . The sharp snap-off instability and adherence $(40 \mathrm{nN})$ is less strong than for $30 \mathrm{vol} . \%$ ethanol. In pure ethanol, the snapoff instability has completely vanished and we observe no significant adhesion interaction.

Even though the swelling ratio is still 1.5 at maximum collapse of the brush at 30 vol.\% of ethanol-water mixture, we observe force curves that exhibit a sharp snap-off instability, which is typically only observed for fully collapsed brush. However, we note that in our experiments we probe the adherence between the colloid and the top of the brush. Therefore, we would like to argue that our results imply that the brush is collapsed at the top between 20-60 vol.\% of ethanol, which would be in agreement with the observation of Chen et al. ${ }^{14}$

\subsection{Conclusions}

In summary, we have shown that tunable friction is provided by co-non-solvency of a PNIPAM brush in ethanol-water mixtures. In pure water, the PNIPAM brush is swollen and acts as a lubricant layer. For 10 vol.\% ethanol-water mixture, the PNIPAM brush is partially collapsed and a large friction force is observed due to stretching of the partly collapsed brush chains when the colloid probe is moved away from the initial point of contact. The strongest collapse of the PNIPAM brush occurs in $30 \mathrm{vol} \%$ ethanol-water mixture. As expected, when the brush is in this state, the friction is higher than in pure water. Its value is, however, smaller than at $10 \mathrm{vol} \%$ ethanol, since the grafted chains do not stretch in this case during the sliding motion. At higher volume fractions of ethanol, the brush re-swells. At these solvent compositions, a lubricant layer is again formed. The friction in this case gradually decreases with increasing ethanol-content and reaches a minimum in pure ethanol. At this minimum, typical values of the friction force are two orders of magnitude smaller than the forces measured at $10 \mathrm{vol} . \%$ ethanol-water mixture. Our results show that friction can be tuned in a straightforward manner by varying the relative amount of ethanol. Such tunable friction by employing co-non-solvency of PNIPAM brush in ethanol-water mixtures has potential application in walking robots, and switchable tweezers. 


\subsection{Experimental section}

\section{Materials}

$N$-isopropylacrylamide (NIPAM, Aldrich, 97\%) was dissolved in toluene/hexane solution $(50 \% \mathrm{v} / \mathrm{v})$, and recrystallized twice in the refrigerator, then dried under vacuum for $48 \mathrm{~h}$ at room temperature. Copper (I) bromide ( $\mathrm{CuBr}$, Aldrich, 98\%) was purified by stirring in excessive acetic acid and filtered until the suspension solution is light grey, and dried under approximate vacuum conditions at room temperature overnight. $N, N, N^{\prime}, N^{\prime}, N^{\prime}$ pentamethyldiethylenetriamine (PMDETA) (98\%), (3-aminopropyl) triethoxysilane, 2bromo-2-methylpropionyl bromide (98\%), triethylamine (TEA, $\geq 99 \%$ ), copper (II) bromide ( $\geq 99 \%$ ), ethylenediaminetetraacetic acid tetrasodium salt dihydrate (EDTA, 99$102 \%)$ were purchased from Sigma-Aldrich, and used as received without purification. Methanol (absolute), sulfuric acid (95-97\%), tetrahydrofuran (THF), hexane and toluene (AR) were purchased from Biosolve, and hydrogen peroxide $\left(\mathrm{H}_{2} \mathrm{O}_{2}\right)$ and ethanol were purchased from Merck and used as received. MilliQ water was obtained from a MilliQ Advantage A 10 purification system (Millipore, Billerica, Ma, USA).

\section{Brush Preparation and Characterization}

The PNIPAM brushes were grafted from pieces of a silicon wafer $\left(1 \times 1 \mathrm{~cm}^{2}\right)$ via surface-initiated atom transfer radical polymerization (SI-ATRP). ${ }^{7}$ First, the silicon wafer was cleaned by Piranha solution $\left(\mathrm{H}_{2} \mathrm{SO}_{4}: \mathrm{H}_{2} \mathrm{O}_{2}=3: 1\right.$, v/v). (Caution: Piranha is very dangerous, extreme caution should be taken when handling it).

Next, a monolayer with an amine group ((3-aminopropyl)triethoxysilane) was attached through vapor deposition.

Then, the initiator was attached to the surface using 2-bromo-2-methylpropionyl bromide by a one-step replacement reaction. The NIPAM solution mixed with PMDETA, $\mathrm{CuBr}$ and $\mathrm{CuBr}_{2}$ was degassed and injected into a vial containing pieces of $\mathrm{Si}$ wafer substrates to conduct the ATRP polymerization at room temperature. Finally, specimens featuring the polymer brush were soaked in EDTA solution overnight to remove the copper, and dried with nitrogen. More details on the sample preparation and a movie showing the sample preparation procedure can be found in our preivous works. ${ }^{55,66}$ The grafting density and molar mass of the PNIPAM brush were estimated by the swelling ratio. ${ }^{4}$ For the specimens used the values of $0.45 \pm 0.2$ chains $/ \mathrm{nm}^{2}$ and $2.5 \pm 1.0 \times 10^{5}$ $\mathrm{g} / \mathrm{mol}$ were obtained, respectively. The absolute molar mass of PNIPAM brush-polymers from reference samples with a dry height of $120 \mathrm{~nm}$ was determined to be $M_{n}=1 \times 10^{5}$ $\mathrm{g} / \mathrm{mol}$ (typical PDI $=4$ for the detached chains) ${ }^{67}$ by gel permeation chromatography (GPC) in THF after detachment from the silicon substrate using $p$-toluene sulfonic acid. ${ }^{68}$

Contact angle. Static contact angle measurements were carried out on an optical contact angle device equipped with an electronic syringe unit (OCA15, Dataphysics, 
Germany). Contact angle values of the modified substrates at each reaction step were measured, and at least three samples were assessed for each step.

IR. Fourier transform infrared spectroscopy (FTIR) was employed to characterize the chemical composition of the polymer brush tethered on the silicon substrates. A Bruker Vertex 70v spectrometer was used to obtain the FTIR spectra with a spectral resolution of $4 \mathrm{~cm}^{-1}$. Background data was obtained by scanning a clean silicon wafer before sample measurement.

Ellipsometry. Swelling and co-non-solvency properties were investigated by measuring brush thickness using a Variable Angle Spectroscopic Ellipsometer (VASE, J. A. Woollam). Measurements were performed as a function of photon energy (in ambient: 0.8-4.0 eV, corresponding to a wavelength range of $276-1550 \mathrm{~nm}$; in ethanol-water mixture: $1.1-4 \mathrm{eV}$, i.e. $276-1127 \mathrm{~nm}$ ) with a step size of $0.1 \mathrm{eV}$. In liquid, the accessible range is limited due to absorption of infrared light. The experiments were performed at room temperature $\left(25^{\circ} \mathrm{C}\right)$ in air and in different compositions of ethanol/water mixtures, as outlined in the Supporting Information. A dedicated custom-built liquid cell was used, which enables optical access at a fixed angle of incidence of $63^{\circ}$. Spectra on dry samples were performed at three different angles of incidence $\left(65,70\right.$ and $\left.75^{\circ}\right)$. The detailed analysis of the spectra is shown in the Supporting Information.

AFM. AFM force measurements were conducted on a Multimode 8 AFM with a NanoScope V controller, a JV vertical engage scanner and a liquid cell (Bruker, San Barbara, CA). Polystyrene (PS) colloids attached to soft silicon cantilevers (6 $\mu \mathrm{m}$ diameter, CP-CONT-PS, sQube, NanoAndMore, Germany) were employed to determine the brush height at various ethanol-water compositions (typically $k=0.23 \mathrm{~N} / \mathrm{m} \pm 0.03$ ). Cantilevers with gold colloids (6 $\mu \mathrm{m}$ diameter, CP-FM-Au, sQube, NanoAndMore, Germany) were used to obtain the force curves and friction data. The deflection sensitivity and spring constant values (typical spring constants were in the range of $k=2.42 \mathrm{~N} / \mathrm{m} \pm$ 0.08) of the cantilevers featuring gold colloid probes were determined in liquid for each composition used. The friction was measured by sliding the gold colloid probe over the brush in the lateral direction (over $20 \mu \mathrm{m}$ at a velocity of $10 \mu \mathrm{m} / \mathrm{s}$ ), while measuring the torsional response of the cantilever. The torsional conversion factor (typically $S_{\theta}=1.42$ $x 10^{-7} \mathrm{~N} / \mathrm{V}$ ) was calibrated by using Vezenov's noncontact method. ${ }^{69}$ The measurements were repeated on 3 different positions on the sample, where we found the same forces within $10 \%$. Experiments were repeated on 3 different samples that were prepared in the same batch and 3 more samples prepared in a different batch. For all these samples we found qualitatively the same results (15\% deviations in swelling ratios, $25 \%$ in the friction and $50 \%$ in the adhesion (hysteresis)). 


\subsection{Supporting Information}

\section{Analysis of ellipsometry spectra}

For the determination of the optical constants and film thickness from ellipsometry spectra one needs to establish an optical model, which enables describing the experimental data in terms of actual physical parameters. The optical dispersion $(n)$ can be modelled with the Cauchy dispersion model:

$$
n(\lambda)=A+B / \lambda^{2}+C / \lambda^{4}
$$

where $n$ is the refractive index, and $A, B$ and $C$ are the Cauchy parameters which may be obtained from the model to the data; $\lambda$ is the wavelength in micrometers. The polymer film is considered to be a transparent "dielectric", with the assumption that the absorption coefficient is close to zero $(k=0)$ and consequently, the refractive index is a real quantity.

Silicon substrate and ambient. The optical properties of the substrate, consisting of bulk silicon with $2.0 \mathrm{~nm}$ of a native oxide, and the solvents (water and ethanol) were used as available in the CompleteEASE package (the refractive index at $600 \mathrm{~nm}$ of pure water and pure ethanol are 1.33 and 1.36 at $20{ }^{\circ} \mathrm{C}$, respectively). The refractive indexes of the various ethanol/water mixtures were obtained by considering them as sum of the product between the volume fraction and the pure refractive index of the single components; the extinction coefficients were considered to be zero.

Dry brush films. The experimental data obtained on dry films in air were fitted between 250-1150 $\mathrm{nm}$ with a model consisting of a single uniform Cauchy layer (representing the PNIPAM film) on top of the silicon substrate. The thickness $d$, and the Cauchy parameters $A$ and $B$ were used as fitting parameters; as the $C$ Cauchy parameter does not yield improved fitting results, it was set to 0 .

The PNIPAM film was considered to be uniform, and the roughness of the polymeric layer was neglected; the material density is distributed evenly within the volume of film and consequently there was no gradient in the optical refractive index.

Wet brush films. In liquid, the experimental data were fitted between 276-1127 nm with three different uniform layer models, each assuming the same silicon substrate (with native silicon oxide layer). The three models are schematically represented in Figure S4.1 and details are summarized below:

1. a dense Cauchy layer model, in which thickness $d$, and Cauchy parameters A and $\mathrm{B}$ were varied; the Cauchy parameter $\mathrm{C}$ was set to 0 .

2. a single gradient layer; the gradient in the optical density is described by an exponential decrease of $\mathrm{A}$, as a function of the distance from the substrate (layer composed of 50 slices). Only the A-parameter was considered, mainly for two reasons: in the Cauchy model of eq.1, the A parameter is the principal contributor of the refractive index and secondly, keeping the number of fitting parameters limited simplifies the analysis and the extraction of physical 
information. The thickness $d$, the Cauchy parameters $A$ and $B$, the percentage variation of the A-parameter $(\% A)$ and the exponent of the exponential equation for the decrease of A-parameter ( $\exp$ ) were fitted to the experimental data; the $\mathrm{C}$ Cauchy parameter was set to 0 .

3. a double layer model; on top of the silicon substrate, a dense uniform layer (represented by a thickness $\mathrm{d} 1$ and Cauchy parameters A and B) is followed by a second diluted layer with a gradient of the A parameter. The latter is represented by a linear decrease of the optical density as a function of the distance from the substrate (layer composed of 50 slices). The outermost linear gradient layer was modelled by the thickness $d_{2}$, Cauchy parameters $A$ the $B$ and the optical density variation $\% A$. For both layers, the Cauchy parameter was set to 0 .

Uniform

single dense

layer model
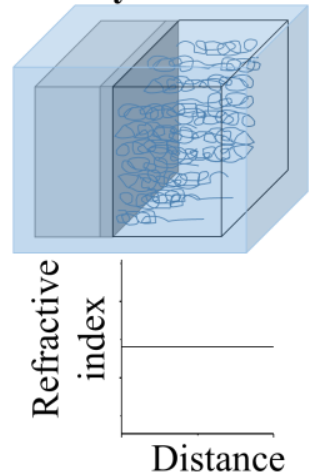

Fit

\begin{tabular}{c|c} 
Fit & d, A and B \\
Parameters & \\
\hline Fix & \\
parameters &
\end{tabular}

Single A-par graded layer model, exponential
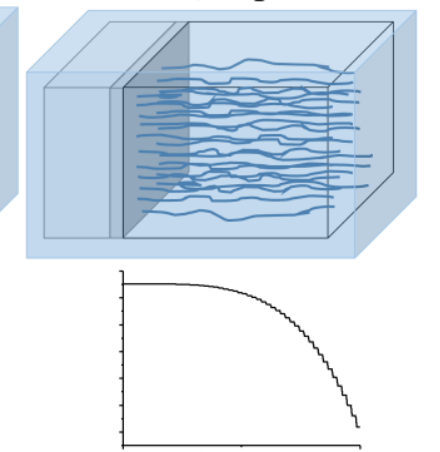

Distance

d, A, \%A, Exp. B

\section{Double layer model}

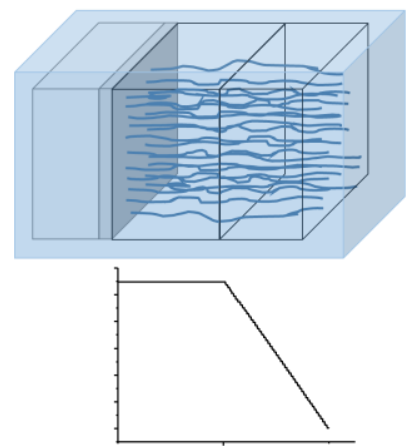

Distance

$d_{\# 1}, A_{\# 1}, B_{\# 1} ;$

$C=0$, thickness of silicon oxide $2.0 \mathrm{~nm}$

Fig. S4.1: Schematic representation of the ellipsometry models used for describing the optical properties of the polymer brushes in water.

Comparison between swollen in water and in ethanol. Upon immersion of the PNIPAM brush film, swelling is observed, as shown in Figure S4.2. The swelling behavior is different depending on whether the polymeric layer is immersed in pure water or in pure ethanol. Consequently, the change of the optical parameters for each composition of medium (ethanol-water mixtures) were considered. 

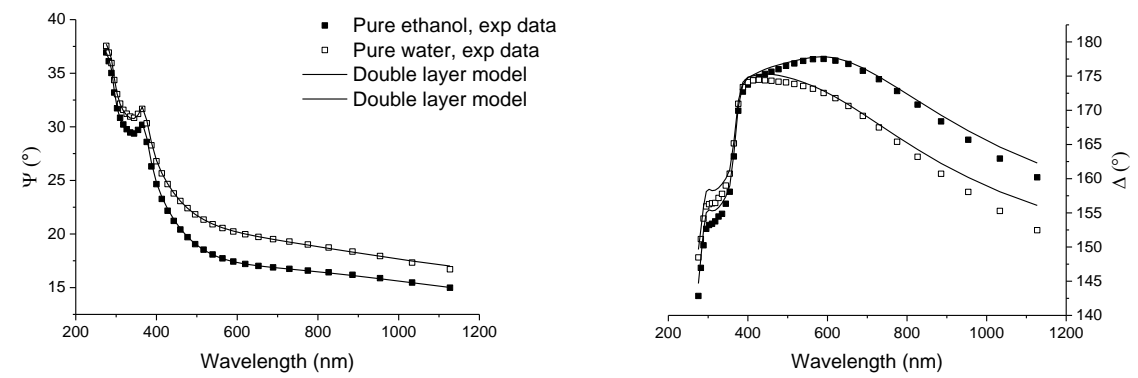

Fig. S4.2. In-situ ellipsometry spectra (symbols) and fit results (solid lines) for PNIPAM brush grafted on silicon swollen in water (open symbols) and in ethanol (filled symbols). For the fitting of the experimental data was used the double layer model described in the support information.

For all experimental data, the curves were first fitted using the single uniform layer, subsequently the exponential single gradient layer, and finally the double Cauchy layer model.

Figure S4.3 shows the dependence and the trend of the optical parameters on solvent composition at fixed room temperature. The change in $\Psi$ and $\Delta$ is evident between the volume ratio of 0.20 and 0.50 . The changes in $\Delta$ are more evident that the changes in $\Psi$.
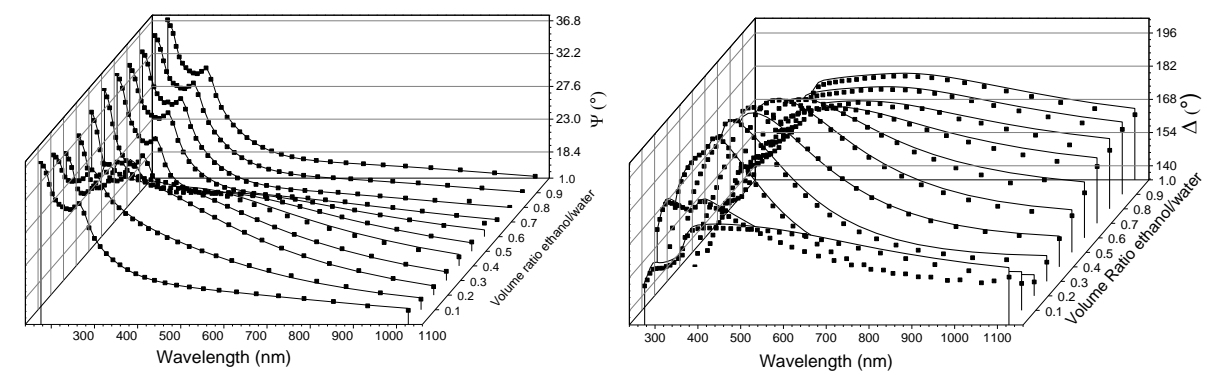

Fig. S4.3. Experimental ellipsometry spectra (symbols) and fit results (solid lines) for the in-situ PNIPAM films on silicon using the double layer model to describe the swollen state of the brushes between swollen in pure water and in volume ratio ethanol/water 0.1 . 
Table S4.1. Fit parameters using a double layer model for the optical modelling of PNIPAM brush layer in solvency and co-non-solvency. The sample was immersed in different volume ratio of ethanol-water mixture at room temperature.

\begin{tabular}{|c|c|c|c|c|c|c|c|c|c|c|c|c|}
\hline & & $0 \%$ & $10 \%$ & $20 \%$ & $30 \%$ & $40 \%$ & $50 \%$ & $60 \%$ & $70 \%$ & $80 \%$ & $90 \%$ & $100 \%$ \\
\hline $\begin{array}{r}\mathrm{T} \\
\text { thick } \\
\text { (n }\end{array}$ & & 396 & 247 & 222 & 156 & 154 & 238 & 406 & 409 & 426 & 413 & 414 \\
\hline & $\begin{array}{c}\mathrm{d}_{2} \\
\mathrm{~nm}\end{array}$ & 271 & 151 & 160 & - & - & 183 & 236 & 255 & 282 & 270 & 275 \\
\hline$\stackrel{\bar{\nu}}{\stackrel{0}{\circ}}$ & A & 1,312 & 1,360 & 1,405 & - & - & 1,415 & 1,370 & 1,372 & 1,372 & 1,373 & 1,371 \\
\hline 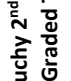 & $\begin{array}{c}\text { B } \\
\mu \mathrm{m}^{2}\end{array}$ & $\begin{array}{c}0,001 \\
73\end{array}$ & $\begin{array}{c}0,0003 \\
82\end{array}$ & $\begin{array}{c}0,0025 \\
9\end{array}$ & - & - & 0,00486 & $\begin{array}{c}0,0011 \\
5\end{array}$ & $\begin{array}{c}0,002 \\
44\end{array}$ & $\begin{array}{c}0,002 \\
57\end{array}$ & $\begin{array}{c}0,002 \\
24\end{array}$ & $\begin{array}{c}0,002 \\
32\end{array}$ \\
\hline & $\% \mathrm{~A}$ & $-2,42$ & $-5,73$ & $-13,26$ & - & - & $-1,86$ & $-3,15$ & $-2,61$ & $-2,17$ & $-2,04$ & $-2,04$ \\
\hline & Exp & 1 & 1 & 1 & - & - & 1 & 1 & 1 & 1 & 1 & 1 \\
\hline$\stackrel{\bar{\Xi}}{\gtrsim}$ & $\begin{array}{r}\mathrm{d}_{1} \\
\mathrm{~nm}\end{array}$ & 125 & 96 & 62 & 156 & 154 & 33 & 170 & 154 & 144 & 143 & 139 \\
\hline 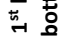 & A & 1,395 & 1,408 & 1,435 & 1,447 & 1,452 & 1,423 & 1,411 & 1,401 & 1,403 & 1,402 & 1,401 \\
\hline$\frac{5}{5}$ & B & 0,001 & 0,002 & 0,0025 & 0,0039 & 0,0041 & 0,000921 & 0,0035 & 0,004 & 0,002 & 0,002 & 0,002 \\
\hline & $\mu \mathrm{m}^{2}$ & 76 & 84 & 9 & 3 & 0 & 77 & 6 & 01 & 71 & 87 & 52 \\
\hline Amb & & $0 \%$ & $10 \%$ & $20 \%$ & $30 \%$ & $40 \%$ & $50 \%$ & $60 \%$ & $70 \%$ & $80 \%$ & $90 \%$ & $100 \%$ \\
\hline
\end{tabular}

In general, in the case of swollen PNIPAM brush films the double layer model provided the best description of the experimental data. This phenomena typically occurs when the PNIPAM film is immersed in solvent mixtures below 10 and above 60 vol.\% ethanol-water mixture.

In the other hand, the PNIPAM collapsed when the composition is between 2 and 50 vol.\% ethanol-water mixture. In this case, the dense uniform single layer model resulted in an adequate description of the experimental data. The fit parameter obtained with the double layer models are summarized respectively in the Table S4.1. 


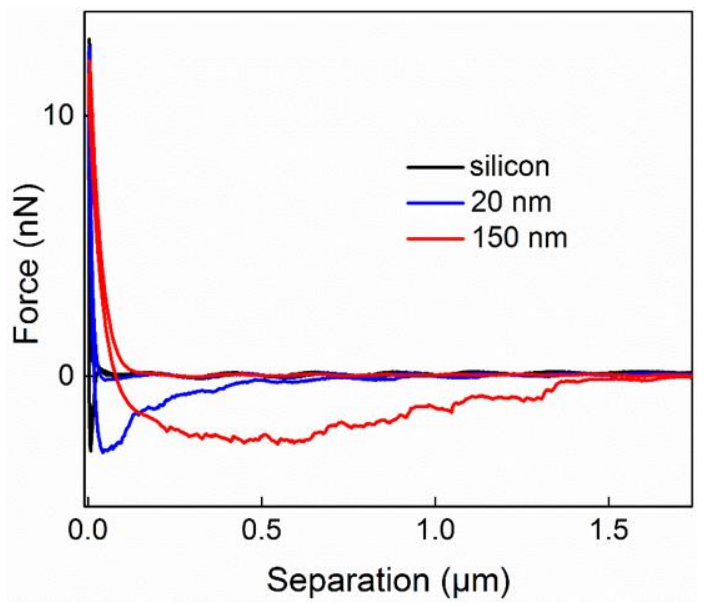

Fig. S4.4. Force-Separation curve of bare silicon substrate, $20 \mathrm{~nm}$ PNIPAM and $150 \mathrm{~nm}$ PNIPAM brush in $10 \mathrm{vol} \%$ ethanol-water mixture.

\subsection{References}

1. Ijspeert, A. J., Biorobotics: Using Robots to Emulate and Investigate Agile Locomotion. Science 2014, 346 (6206), 196-203.

2. Wu, Y.; Cai, M. R.; Pei, X. W.; Liang, Y. M.; Zhou, F., Switching Friction with Thermal-Responsive Gels. Macromolecular Rapid Communications 2013, 34 (22), 1785-1790.

3. Sun, T. L.; Wang, G. J.; Feng, L.; Liu, B. Q.; Ma, Y. M.; Jiang, L.; Zhu, D. B., Reversible Switching Between Superhydrophilicity and Superhydrophobicity. Angew Chem Int Edit 2004, 43 (3), 357-360;

4. Malham, I. B.; Bureau, L., Density Effects on Collapse, Compression, and Adhesion of Thermoresponsive Polymer Brushes. Langmuir 2010, 26 (7), 4762-4768;

5. Jones, D. M.; Smith, J. R.; Huck, W. T. S.; Alexander, C., Variable Adhesion of Micropatterned ThermoResponsive Polymer Brushes: AFM Investigations of Poly( $N$-isopropylacrylamide) Brushes Prepared by Surface-Initiated Polymerizations. Advanced Materials 2002, 14 (16), 1130-1134;

6. Benetti, E. M.; Zapotoczny, S.; Vancso, J., Tunable Thermo-Responsive Polymeric Platforms on Gold by "Photoiniferter"-based Surface Grafting. Advanced Materials 2007, 19 (2), 268-271.

7. Sui, X. F.; Chen, Q.; Hempenius, M. A.; Vancso, G. J., Probing the Collapse Dynamics of Poly $(N$ isopropylacrylamide) Brushes by AFM: Effects of Co-non-solvency and Grafting Densities. Small 2011, 7 (10), 1440-1447;

8. Li, A.; Ramakrishna, S. N.; Nalam, P. C.; Benetti, E. M.; Spencer, N. D., Stratified Polymer Grafts: Synthesis and Characterization of Layered 'Brush' and 'Gel' Structures. Adv Mater Interfaces 2014, 1 (1), 1300007;

9. de Beer, S.; Kutnyanszky, E.; Schon, P. M.; Vancso, G. J.; Muser, M. H., Solvent-Induced Immiscibility of Polymer Brushes Eliminates Dissipation Channels. Nature Communications 2014, 5, 3781.

10. Lee, H.; Lee, B. P.; Messersmith, P. B., A Reversible Wet/Dry Adhesive Inspired by Mussels and Geckos. Nature 2007, 448 (7151), 338-342;

11. Gong, J. P., Friction and Lubrication of Hydrogels - its Richness and Complexity. Soft Matter 2006, 2 (7), 544-552.

12. Milner, S. T., Polymer Brushes. Science 1991, 251 (4996), 905-914. 
13. Vyas, M. K.; Schneider, K.; Nandan, B.; Stamm, M., Switching of Friction by Binary Polymer Brushes. Soft Matter 2008, 4 (5), 1024-1032.

14. Chen, Q.; Kooij, E. S.; Sui, X. F.; Padberg, C. J.; Hempenius, M. A.; Schon, P. M.; Vancso, G. J., Collapse from the top: brushes of poly-( $N$-isopropylacrylamide) in co-non-solvent mixtures. Soft Matter 2014, 10 (17), 3134-3142.

15. Dai, H. J.; Chen, Q.; Qin, H. L.; Guan, Y.; Shen, D. Y.; Hua, Y. Q.; Tang, Y. L.; Xu, J., A TemperatureResponsive Copolymer Hydrogel in Controlled Drug Delivery. Macromolecules 2006, 39 (19), 65846589.

16. Winnik, F. M.; Ringsdorf, H.; Venzmer, J., Methanol-Water as a Co-Non-Solvent System for Poly $(N$ isopropylacrylamide). Macromolecules 1990, 23 (8), 2415-2416.

17. Wu, T.; Gong, P.; Szleifer, I.; Vlcek, P.; Subr, V.; Genzer, J., Behavior of Surface-Anchored Poly(acrylic acid) Brushes with Grafting Density Gradients on Solid Substrates: 1. Experiment. Macromolecules 2007, 40 (24), 8756-8764.

18. Jakubiak, R.; Bunning, T. J.; Vaia, R. A.; Natarajan, L. V.; Tondiglia, V. P., Electrically Switchable, One-Dimensional Polymeric Resonators from Holographic Photopolymerization: A New Approach for Active Photonic Bandgap Materials. Advanced Materials 2003, 15 (3), 241-244.

19. Shen, J. M.; Gao, F. Y.; Guan, L. P.; Su, W.; Yang, Y. J.; Li, Q. R.; Jin, Z. C., Graphene oxide-Fe $\mathrm{O}_{4}$ Nanocomposite for Combination of Dual-Drug Chemotherapy with Photothermal Therapy. Rsc Adv 2014, 4 (35), 18473-18484.

20. Wei, Q. B.; Cai, M. R.; Zhou, F.; Liu, W. M., Dramatically Tuning Friction Using Responsive Polyelectrolyte Brushes. Macromolecules 2013, 46 (23), 9368-9379;

21. Yu, Y. L.; Kieviet, B. D.; Kutnyanszky, E.; Vancso, G. J.; de Beer, S., Cosolvency-Induced Switching of the Adhesion between Poly(methyl methacrylate) Brushes. Acs Macro Lett 2015, 4 (1), 75-79;

22. de Beer, S., Switchable Friction Using Contacts of Stimulus-Responsive and Nonresponding Swollen Polymer Brushes. Langmuir 2014, 30 (27), 8085-8090.

23. Scherzinger, C.; Lindner, P.; Keerl, M.; Richtering, W., Co-non-solvency of Poly (N, Ndiethylacrylamide)(PDEAAM) and Poly ( $N$-isopropylacrylamide)(PNIPAM) Based Microgels in Water/Methanol Mixtures: Copolymer vs Core- Shell Microgel. Macromolecules 2010, 43 (16), 68296833 ;

24. Scherzinger, C.; Schwarz, A.; Bardow, A.; Leonhard, K.; Richtering, W., Co-Non-Solvency of Poly-(Nisopropyl acrylamide) (PNIPAM): Microgels Versus Linear Chains and Macrogels. Current Opinion in Colloid \& Interface Science 2014, 19 (2), 84-94;

25. Tanaka, F.; Koga, T.; Winnik, F. M., Temperature-Responsive Polymers in Mixed Solvents: Competitive Hydrogen Bonds Cause Co-Non-Solvency. Physical review letters 2008, 101 (2), 028302;

26. Wang, H.; An, Y.; Huang, N.; Ma, R. J.; Shi, L. Q., Investigation of the Co-Non-solvency Effect on Micellization Behavior of Poly(styrene-b- $N$-isopropylacrylamide). J Colloid Interf Sci 2008, 317 (2), 637-642;

27. Winnik, F. M.; Ottaviani, M. F.; Bossmann, S. H.; Pan, W.; Garcia-Garibay, M.; Turro, N. J., Co-NonSolvency of Poly( $N$-isopropylacrylamide): a Look at Spin-Labeled Polymers in Mixtures of Water and Tetrahydrofuran. Macromolecules 1993, 26 (17), 4577-4585.

28. Mukherji, D.; Kremer, K., Coil-Globule-Coil Transition of PNIPAm in Aqueous Methanol: Coupling All-Atom Simulations to Semi-Grand Canonical Coarse-Grained Reservoir. Macromolecules 2013, 46 (22), 9158-9163;

29. Mukae, K.; Sakurai, M.; Sawamura, S.; Makino, K.; Kim, S. W.; Ueda, I.; Shirahama, K., Swelling of Poly( $N$-isopropylacrylamide) Gels in Water-Alcohol (C1-C4) Mixed-Solvents. J Phys Chem-Us 1993, 97 (3), 737-741. 
30. Zhu, P. W.; Napper, D. H., Coil-to-Globule Type Transitions and Swelling of Poly $(N$ isopropylacrylamide) and Poly(acrylamide) at Latex Interfaces in Alcohol-Water Mixtures. J Colloid Interf Sci 1996, 177 (2), 343-352.

31. Liu, M. Z.; Bian, F. L.; Sheng, F. L., FTIR Study on Molecular Structure of Poly( $N$-isopropylacrylamide) in Mixed Solvent of Methanol and Water. Eur Polym J 2005, 41 (2), 283-291;

32. Mukherji, D.; Marques, C. M.; Kremer, K., Polymer Collapse in Miscible Good Solvents is a Generic Phenomenon Driven by Preferential Adsorption. Nature Communications 2014, 5, 4882.

33. Mukherji, D.; Marques, C. M.; Stuehn, T.; Kremer, K., Co-Non-Solvency: Mean-Field polymer Theory does not Describe Polymer Collapse Transition in a Mixture of two Competing Good Solvents. The Journal of Chemical Physics 2015, 142 (11), 114903.

34. Young, T. H.; Chuang, W. Y., Thermodynamic Analysis on the Co-Non-Solvency of Poly (vinyl alcohol) in Water-DMSO Mixtures through the Ternary Interaction Parameter. J Membrane Sci 2002, 210 (2), 349-359;

35. Saunders, B. R.; Crowther, H. M.; Vincent, B., Poly[(methyl methacrylate)-co-(methacrylic acid)] Microgel Particles: Swelling Control using pH, Co-Non-Solvency, and Osmotic Deswelling. Macromolecules 1997, 30 (3), 482-487.

36. Ito, Y.; Ito, T.; Takaba, H.; Nakao, S., Development of Gating Membranes that are Sensitive to the Concentration of Ethanol. J Membrane Sci 2005, 261 (1-2), 145-151.

37. Liu, L.; Song, X. L.; Ju, X. J.; Xie, R.; Liu, Z.; Chu, L. Y., Conversion of Alcoholic Concentration Variations into Mechanical Force via Core-Shell Capsules. J Phys Chem B 2012, 116 (3), 974-979.

38. Balko, S. M.; Kreer, T.; Costanzo, P. J.; Patten, T. E.; Johner, A.; Kuhl, T. L.; Marques, C. M., Polymer Brushes under High Load. Plos One 2013, 8 (3).

39. Semenov, A. N., Contribution to the Theory of Microphase Layering in Block-Copolymer Melts. Zh Eksp Teor Fiz+ 1985, 88 (4), 1242-1256;

40. Milner, S. T.; Witten, T. A.; Cates, M. E., Theory of the Grafted Polymer Brush. Macromolecules 1988, 21 (8), 2610-2619;

41. Skvortsov, A. M.; Pavlushkov, I. V.; Gorbunov, A. A.; Zhulina, Y. B.; Borisov, O. V.; Pryamitsyn, V. A., Structure of Densely Grafted Polymeric Monolayers. Polymer Science U.S.S.R. 1988, 30 (8), 17061715.

47. Biesheuvel, P. M.; de Vos, W. M.; Amoskov, V. M., Semianalytical Continuum Model for Nondilute Neutral and Charged Brushes including Finite Dtretching. Macromolecules 2008, 41 (16), 6254-6259;

48. Shim, D. F. K.; Cates, M. E., Finite Extensibility and Density Saturation Effects in the Polymer Brush. $J$ Phys-Paris 1989, 50 (24), 3535-3551.

49. Dan, N.; Tirrell, M., Effect of Bimodal Molecular-Weight Distribution on the Polymer Brush. Macromolecules 1993, 26 (24), 6467-6473;

50. de Vos, W. M.; Leermakers, F. A. M., Modeling the Dtructure of a Polydisperse Polymer Brush. Polymer 2009, 50 (1), 305-316.

51. Kooij, E. S.; Sui, X. F.; Hempenius, M. A.; Zandvliet, H. J. W.; Vancso, G. J., Probing the Thermal Collapse of Poly( $N$-isopropylacrylamide) Grafts by Quantitative in Situ Ellipsometry. J Phys Chem B 2012, 116 (30), 9261-9268.

52. Bittrich, E.; Burkert, S.; Muller, M.; Eichhorn, K. J.; Stamm, M.; Uhlmann, P., Temperature-Sensitive Swelling of Poly( $N$-isopropylacrylamide) Brushes with Low Molecular Weight and Grafting Density. Langmuir 2012, 28 (7), 3439-3448; 
53. Rahane, S. B.; Floyd, J. A.; Metters, A. T.; Kilbey, S. M., Swelling Behavior of Multiresponsive Poly(methacrylic acid)-block-Poly( $N$-isopropylacrylamide) Brushes Synthesized using Surface-Initiated Photoiniferter-Mediated Photopolymerization. Adv Funct Mater 2008, 18 (8), 1232-1240.

54. Dalkas, G.; Pagonis, K.; Bokias, G., Control of the Lower Critical Solution Temperature-type Co-NonSolvency Properties of Poly $(N$-isopropylacrylamide $)$ in Water-Dioxane Mixtures through Copolymerisation with Acrylamide. Polymer 2006, 47 (1), 243-248.

55. Yu, Y. L.; Kieviet, B. D.; Liu, F.; Siretanu, I.; Kutnyánszky, E.; Vancso, G. J.; de Beer, S., Stretching of Collapsed Polymers causes an Enhanced Dissipative Response of PNIPAM Brushes near their LCST. Soft Matter 2015

56. Zhang, J.; Chu, L. Y.; Li, Y. K.; Lee, Y. M., Dual Thermo- and pH-sensitive Poly $(N$ isopropylacrylamide-co-acrylic acid) Hydrogels with Rapid Response Behaviors. Polymer 2007, 48 (6), 1718-1728.

57. Oh, S. Y.; Bae, Y. C., Molecular Thermodynamic Analysis for Reentrant and Reentrant-Convex type Swelling Behaviors of Thermo-Sensitive Hydrogels in Mixed Solvents. Polymer 2013, 54 (9), 2308-2314.

58. Cole, M. A.; Voelcker, N. H.; Thissen, H.; Horn, R. G.; Griesser, H. J., Colloid Probe AFM Study of Thermal Collapse and Protein Interactions of Poly ( $N$-isopropylacrylamide) Coatings. Soft Matter 2010, 6 (12), 2657-2667;

59. Jhon, Y. K.; Bhat, R. R.; Jeong, C.; Rojas, O. J.; Szleifer, I.; Genzer, J., Salt-Induced Depression of Lower Critical Solution Temperature in a Surface-Grafted Neutral Thermoresponsive Polymer. Macromolecular rapid communications 2006, 27 (9), 697-701;

60. Liu, G. M.; Zhang, G. Z., Collapse and Swelling of Thermally Sensitive Poly( $N$-isopropylacrylamide) Brushes Monitored with a Quartz Crystal Microbalance. J Phys Chem B 2005, 109 (2), 743-747;

61. Ma, S. H.; Wang, D. A.; Liang, Y. M.; Sun, B. Q.; Gorb, S. N.; Zhou, F., Gecko-Inspired but Chemically Switched Friction and Adhesion on Nanofibrillar Surfaces. Small 2015, 11 (9-10), 1131-1137.

62. Singh, M. K.; Ilg, P.; Espinosa-Marzal, R. M.; Kroger, M.; Spencer, N. D., Polymer Brushes under Shear: Molecular Dynamics Simulations Compared to Experiments. Langmuir 2015, 31 (16), 4798-4805.

63. Gilanyi, T.; Varga, I.; Meszaros, R.; Filipcsei, G.; Zrinyi, M., Characterisation of Monodisperse Poly $(N$ isopropylacrylamide) Microgel Particles. Phys Chem Chem Phys 2000, 2 (9), 1973-1977.

64. Drechsler, A.; Synytska, A.; Uhlmann, P.; Elmahdy, M. M.; Stamm, M.; Kremer, F., Interaction Forces between Microsized Silica Particles and Weak Polyelectrolyte Brushes at Varying $\mathrm{pH}$ and Salt Concentration. Langmuir 2010, 26 (9), 6400-6410;

65. Willott, J. D.; Murdoch, T. J.; Webber, G. B.; Wanless, E. J., Nature of the Specific Anion Response of a Hydrophobic Weak Polyelectrolyte Brush Revealed by AFM Force Measurements. Macromolecules 2016, 49 (6), 2327-2338.

66. de Beer, S.; Kutnyanszky, E.; Muser, M. H.; Vancso, G. J., Preparation and Friction Force Microscopy Measurements of Immiscible, Opposing Polymer Brushes. Jove-J Vis Exp 2014, (94), 52285.

67. Pyun, J.; Kowalewski, T.; Matyjaszewski, K., Synthesis of Polymer Brushes using Atom Transfer Radical Polymerization. Macromolecular Rapid Communications 2003, 24 (18), 1043-1059.

68. Cummings, C.; Murata, H.; Koepsel, R.; Russell, A. J., Tailoring Enzyme Activity and Stability using Polymer-based Protein Engineering. Biomaterials 2013, 34 (30), 7437-7443.

69. Wagner, K.; Cheng, P.; Vezenov, D., Noncontact Method for Calibration of Lateral Forces in Scanning Force Microscopy. Langmuir 2011, 27 (8), 4635-4644. 
Chapter 5

\section{Effect of Lateral Deformation by Thermo-Responsive Polymer Brushes on the Measured Friction Forces}

* This chapter has been published in: Ramakrishna, S.N., Cirelli, M., Mohammad Divandari, M., Benetti, E.M.; Langmuir. 2017, 33, 4164-4171. 


\section{Contents}

Chapter 5 Effects of Lateral Deformation by Thermoresponsive Polymer Brushes on the Measured Friction Forces.................................... 95

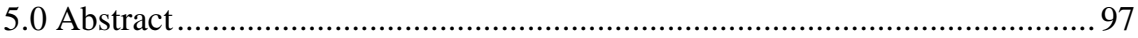

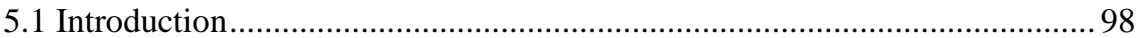

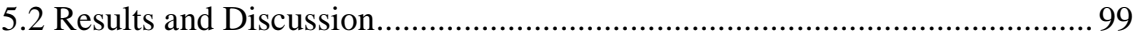

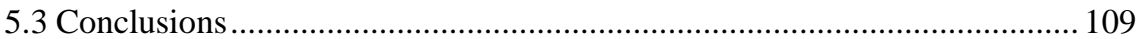

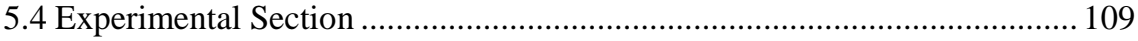

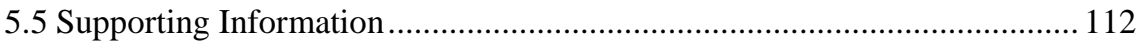

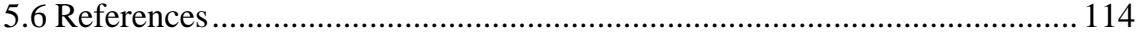




\subsection{Abstract}

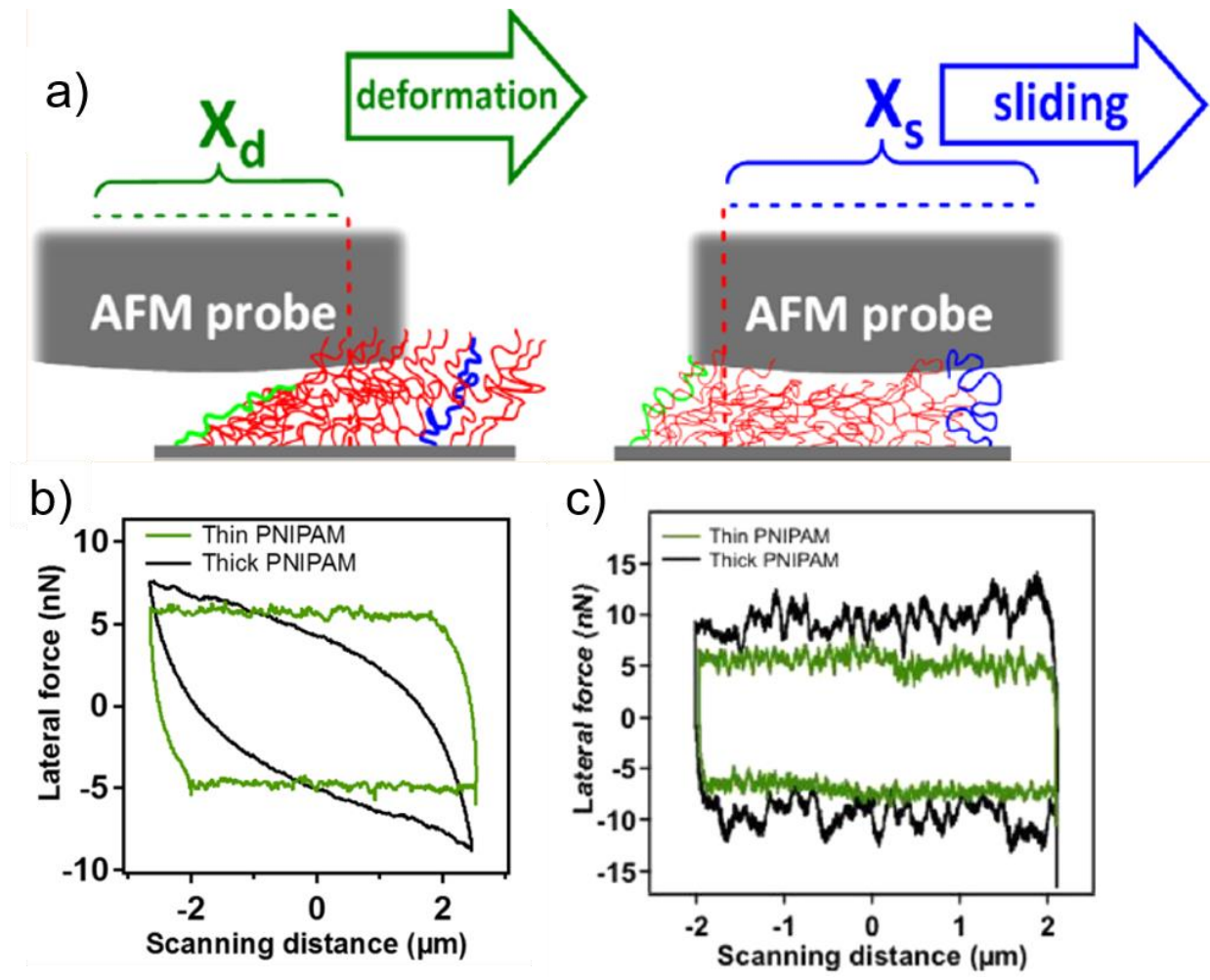

TOC Chapter 5: Schematic representation of the bursh deformation and sliding during lateral AFM force measurements is shown in a). The friction loops recorded on thin (light green lines) and thick PNIPAM brushes (black lines) in water at the temperature below LCST and above LCST, b) and c), respectively.

The nanotribological properties of hydrophilic polymer brushes are conveniently analyzed by lateral force microscopy (LFM). However, the measurement of friction for highly swollen and relatively thick polymer brushes can be strongly affected by the tendency of the compliant brush to be laterally deformed by the shearing probe. This phenomenon induces a "tilting" in the recorded friction loops, which is generated by the lateral bending and stretching of the grafts. In this study we highlight how the brush lateral deformation mainly affects the friction measurements of swollen PNIPAM brushes below lower critical solution temperature (LCST) when relatively short scanning distances are applied. Under these conditions, the energy dissipation recorded by LFM is almost uniquely determined by stretching and bending of the compliant brush back and forth along the scanning direction, and it is not correlated to dynamic friction between two sliding surfaces. In contrast, when the scanning distance applied during LFM is relevantly longer than the brush lateral deformation, sliding of the probe on the brush interface becomes dominant, and a correct measurement of dynamic friction can be accomplished. 
By increasing the temperature above the LCST, the PNIPAM brushes undergo dehydration and assume a collapsed morphology, thereby hindering their lateral deformation by scanning probe. Hence, at $40{ }^{\circ} \mathrm{C}$ in water the recorded friction loops do not show any tilting and LFM accurately describes the dynamic friction between the probe and the polymer surface.

\subsection{Introduction}

The lubricating properties of hydrophilic polymer brushes have been increasingly attracted the attention of materials scientists ${ }^{1-3}$ and tribologists ${ }^{4-10}$ during the last two decades. Due to a combination of osmotic force and conformational entropy, densely grafted, swollen brushes can act as efficient boundary lubricants within aqueous media. This distinctive feature provides to brush-coated surfaces a markedly low coefficient of friction (COF), when this is measured by micro- and nano-tribometers, ${ }^{11-16}$ surface forces apparatus (SFA) $)^{17-19}$ and atomic force microscopy (AFM). ${ }^{7,20-22}$

The frictional dissipation mechanisms as well as the effects of solvent quality on the nanotribological properties of polymer brushes were intensively studied both experimentally ${ }^{23-28}$ and by simulation. ${ }^{29-33}$ In particular, AFM-based techniques, such as lateral force microscopy (LFM), have been applied to study nanoscale friction generated between identical tribopairs, usually fabricated by grafting-to ${ }^{34}$ of surface reactive copolymers. ${ }^{25,35,36}$ Alternatively, LFM coupled to grafting-from methods, such as surfaceinitiated controlled radical polymerizations (SI-CRP), were employed to investigate the nanotribological properties of asymmetric tribopairs, concentrating on the interaction between sharp or colloidal AFM probes and relatively thick brush layers. ${ }^{37-40}$

A particular interest was devoted to the nanotribological properties of stimuliresponsive polymer brushes, and especially poly( $N$-isopropylacrylamide) (PNIPAM)based grafts, which across the lower critical solution temperature (LCST) of $30-33{ }^{\circ} \mathrm{C}$ in water undergo a transition from swollen to dehydrated. ${ }^{41,42}$ AFM methods were applied for measuring friction and nanomechanical properties with nano-Newton $(\mathrm{nN})$ resolution between a well-defined asperity and PNIPAM brushes presenting different thicknesses and grafting densities. ${ }^{6,38,39,43-45}$ Although several studies helped dissecting the influence of these and other brush parameters on the nanotribological characteristics of PNIPAM brushes, a comprehensive and unambiguous understanding of the mechanics of interaction between the AFM probe and the brush surface across its LCST is still missing.

In this work, we demonstrate that the tendency of the PNIPAM grafts to be laterally deformed by the shearing AFM probe, which varies over the temperature-induced transition, determines brush-probe interactions, while a thorough understanding of brush lateral deformability enables the correct measurement of friction by LFM. In particular, 
we address these issues by analyzing the frictional properties of PNIPAM brushes presenting different thicknesses below and above LCST.

We recently demonstrated that the measurement of friction between polymer brushes and an AFM probe includes different contributions originating from the reciprocal sliding of the two surfaces, plus the lateral bending and stretching of the tethered polymer chains. $^{38,46}$ The impact of this latter term on the measurement of friction can be conveniently visualized through the analysis of the friction "loops" recorded using LFM. $^{38,46}$ A tilted section of the loop is ascribed to the initial lateral deformation of the swollen brush, while a following, flat friction force trace is produced by the steady sliding of the probe on the polymer interface.

For PNIPAM brushes immersed in water below their LCST the lateral deformation by the compliant brush affects the dissipation recorded via the friction loops, and thus the applied scanning distance must be accordingly tuned to obtain a reliable value of COF. In contrast, above LCST lateral deformability of the collapsed brush becomes irrelevant, and sliding of the probe on the brush interface uniquely determines the frictional dissipation.

\subsection{Results and Discussion}

PNIPAM brushes were synthesized from initiator-functionalized silicon substrates by surface-initiated atom transfer radical polymerization (SI-ATRP), varying the polymerization time and the catalyst/monomer mixture in order to obtain the desired brush thickness. ${ }^{38}$ In particular, our objective is to investigate the frictional properties of relatively thin and thick PNIPAM brushes. Thus, two types of PNIPAM brushes alternatively presenting ellipsometric dry thicknesses of $11 \pm 1$ and $417 \pm 4 \mathrm{~nm}$ (Table 5.1) were synthesized (named thin PNIPAM and thick PNIPAM, respectively). Fourier transform infrared spectroscopy (FTIR) and water contact angle (CA) confirmed the successful formation of uniform PNIPAM films from initiator-functionalized silicon oxide substrates (data reported in the Supporting Information, SI). The dry thickness values recorded by VASE were additionally confirmed by AFM step-height measurements, imaging the height difference between the brush surface and areas on the substrates where the films were mechanically removed by means of a plastic tweezer (Figure 5.1) ${ }^{52} \mathrm{In}$ addition, an average PNIPAM grafting density of 0.43 chains $\mathrm{nm}-2$ was estimated by detaching the grafts via HF treatments, and subsequently analyzing the polymer sample by gel permeating chromatography (GPC; see the SIn for details).

The morphology of thin and thick PNIPAM immersed in water both below LCST $\left(25^{\circ} \mathrm{C}\right)$ and above the LCST $\left(40^{\circ} \mathrm{C}\right)$ were subsequently studied by tapping mode AFM (Figure 5.2). Below LCST, PNIPAM brushes showed smooth topographies, characteristic of swollen brushes, and presented a root mean square (RMS) roughness of $0.1 \pm 0.02$ and 
$1.5 \pm 0.5 \mathrm{~nm}$, for thin PNIPAM and thick-PNIPAM, respectively (RMS values were calculated over an area of $5 \times 5 \mu \mathrm{m}^{2}$ ).

Above the LCST, a marked increase of roughness was observed, due to the aggregation between PNIPAM grafts coupled to their vertical collapse. The formation of globular aggregates constituted by collapsed grafts was clearly observed for both thin and thick PNIPAM, and produced an increase of RMS values that reached $2 \pm 0.3$ and $8 \pm 1$ $\mathrm{nm}$, respectively (calculated over an area of $5 \times 5 \mu \mathrm{m}^{2}$ ) (Figure 5.2). ${ }^{53}$

a)

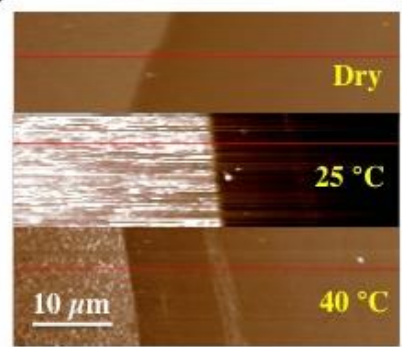

b)

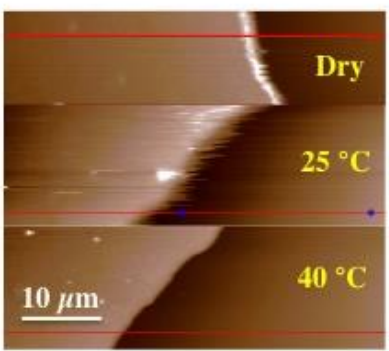

c)

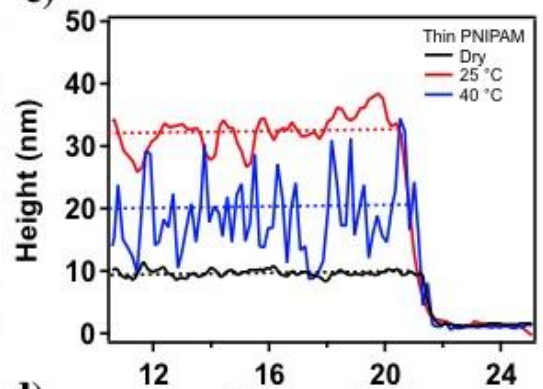

d)

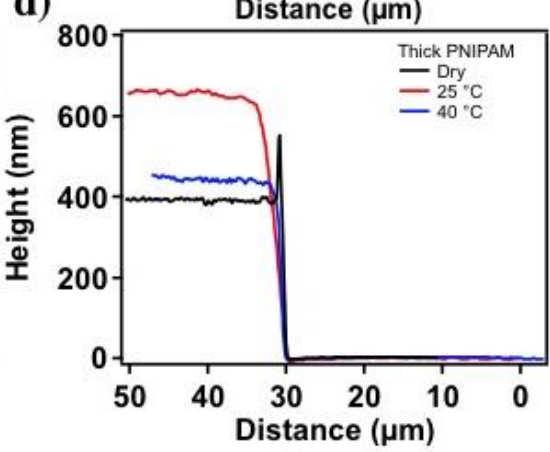

Fig. 5.1: Tapping mode AFM micrographs $(a, b)$ and corresponding section profiles $(c, d)$ recorded over the "scratched" brush and silicon interface of thin PNIPAM and thick PNIPAM. The red lines highlighted in the micrographs in a) and b) indicate where the section profiles reported in c) and d) were measured. The dotted lines in c) indicate the average values of thickness. The images, were recorded under dry conditions, and in milliQ water at 25 and $40{ }^{\circ} \mathrm{C}$. A cantilever (SNL-10, D triangular) from Bruker Corporation was used for recording all the micrographs. This presented a normal spring constant of approximately $0.06 \mathrm{~N} \cdot \mathrm{m}^{-1}$. The scanning speed was kept set at $1 \mathrm{~Hz}$. The amplitude set point and the drive amplitude were set at 0.8 and $0.3 \mathrm{~V}$, respectively.

The swelling properties of thin and thick PNIPAM in Milli-Q water were examined by VASE and AFM step-height measurements. The average thickness of PNIPAM brushes below LCST, was measured by VASE as $47 \pm 6$ and $1033 \pm 22 \mathrm{~nm}$, for thin and thick PNIPAM, respectively (Table 5.1). When compared to the corresponding dry thickness values, swelling ratios of 3.2 and 1.4 were calculated for the two polymer brush layers (calculated as the difference between swollen thickness and dry thickness, divided by dry thickness). Above the LCST, the temperature-driven collapse produced a marked 
decrease of the ellipsometric thicknesses, which at $40{ }^{\circ} \mathrm{C}$ in water reached $13 \pm 4$ and 486 $\pm 2 \mathrm{~nm}$ for thin and thick PNIPAM, respectively.

Table 5.1: VASE and AFM thickness data of thin and thick PNIPAM brushes in dry condition and in water at $25^{\circ}$ and $40^{\circ} \mathrm{C}$

\begin{tabular}{|c|c|c|c|c|c|c|}
\hline \multirow{3}{*}{ Sample } & \multicolumn{3}{|c|}{ Ellipsometry } & \multicolumn{3}{|c|}{ AFM } \\
\hline & Dry & $\begin{array}{l}\text { Water } \\
25^{\circ} \mathrm{C}\end{array}$ & $\begin{array}{l}\text { Water } \\
45^{\circ} \mathrm{C}\end{array}$ & Dry & $\begin{array}{l}\text { Water } \\
25^{\circ} \mathrm{C}\end{array}$ & $\begin{array}{l}\text { Water } \\
40^{\circ} \mathrm{C}\end{array}$ \\
\hline & $\mathbf{n m}$ & $\mathbf{n m}$ & $\mathbf{n m}$ & $\mathbf{n m}$ & $\mathbf{n m}$ & $\mathbf{n m}$ \\
\hline $\begin{array}{c}\text { Thin } \\
\text { PNIAPM }\end{array}$ & $11 \pm 1$ & $47 \pm 6$ & $13 \pm 4$ & $9 \pm 3$ & $33 \pm 8$ & $19 \pm 10$ \\
\hline $\begin{array}{c}\text { Thick } \\
\text { PNIPAM }\end{array}$ & $417 \pm 4$ & $1033 \pm 22$ & $486 \pm 2$ & $390 \pm 6$ & $655 \pm 15$ & $440 \pm 12$ \\
\hline
\end{tabular}

a)

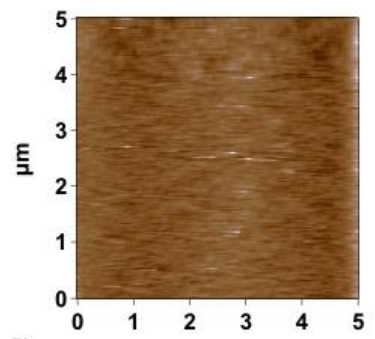

d)

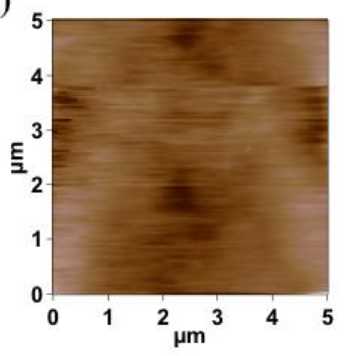

b)

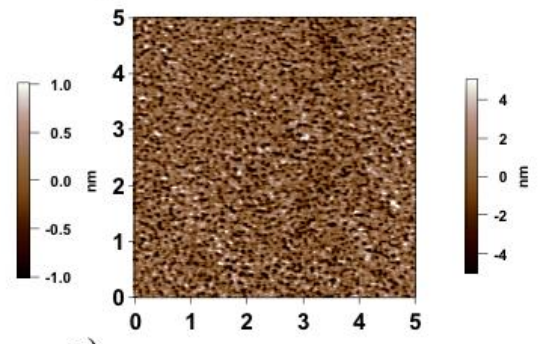

c)

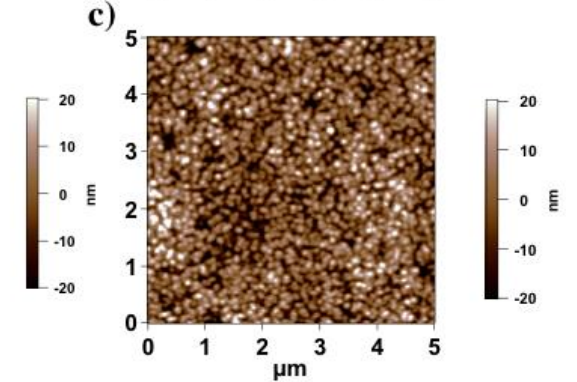

Fig. 5.2: Tapping-mode AFM micrographs of PNIPAM brushes immersed in water. (a) and (b) thin PNIPAM brushes in water at 25 and $40{ }^{\circ} \mathrm{C}$, respectively. (c) and (d) thick PNIPAM brushes in water at 25 and $40^{\circ} \mathrm{C}$, respectively. The same cantilever (SNL-10, D triangular) from Bruker Corporation was used for recording all the micrographs. This presented a normal spring constant of approximately $0.06 \mathrm{~N} \cdot \mathrm{m}^{-1}$. The scanning speed was kept set at $1 \mathrm{~Hz}$. The amplitude set point and the drive amplitude were set at 0.7 and $0.3 \mathrm{~V}$, respectively.

The thickness values obtained by VASE were subsequently confirmed by AFM stepheight measurements. As shown in Figure 5.1 and Table 5.1, thin and thick PNIPAM brushes immersed in water at $25{ }^{\circ} \mathrm{C}$ showed height values of $33 \pm 8$ and $655 \pm 15 \mathrm{~nm}$, respectively. In contrast, at $40{ }^{\circ} \mathrm{C}$ the height of the brushes dropped to $19 \pm 10$ and $440 \pm$ $12 \mathrm{~nm}$, due to the collapse of the PNIPAM grafts. The deviation of these height values 
from the swelling data obtained by VASE was probably due to the compression of the brush by the AFM tip while scanning over the brush surfaces. ${ }^{52}$ This effect resulted more marked at $25{ }^{\circ} \mathrm{C}$ in water, when the swollen and compliant brush can be compressed more easily by the AFM tip, originating an underestimate of the brush step-height.

\section{Normal and lateral AFM force measurements.}

The adhesive properties of thin and thick PNIPAM brushes, below and above LCST were examined by acquiring force-distance (F-D) curves by colloidal probe AFM (CPAFM). As shown in Figure 5.3a, at $25^{\circ} \mathrm{C}$ in water both thin and thick PNIPAM brushes showed repulsive interactions during the approach of the colloidal probe, due to the repulsion against compression exerted by swollen and densely grafted brushes. ${ }^{53}$ Under these conditions, both thin and thick brushes displayed a slight hysteresis between the approaching and the retracting F-D profiles. This phenomenon was probably due to the stretching of the grafted chains up to several hundreds of $\mathrm{nm}$ from the grafting surface during the retraction of the colloidal probe, as recently observed for similar PNIPAM brushes by Yu et al. ${ }^{54}$

Above the LCST, the dehydration of thin and thick PNIPAM brushes produced a marked steepening of the approaching F-D profiles (Figure 5.3b). In addition, the adhesive interactions recorded in the retracting F-D curve confirmed the presence of hydrophobic domains produced by the collapse of PNIAPM grafts above LCST. ${ }^{53}$ Remarkably, an order magnitude increase in the adhesion force could be observed for thick PNIPAM grafts compared to thin ones. Namely, for thin-PNIPAM brushes an average adhesion force of $0.8 \pm 0.1 \mathrm{nN}$ was recorded, whereas thick PNIPAM brushes showed a much higher value of $6.3 \pm 0.6 \mathrm{nN}$, as shown in Figure 5.3c. The higher adhesion force recorded on thick-PNIPAM brushes was due to the increased contact area between the AFM colloid and the compressed brush. In addition, the long-range interactions recorded in the retraction profiles of the F-D curves were presumably generated by the vertical stretching of long polymer grafts within thick brushes by the retracting probe.
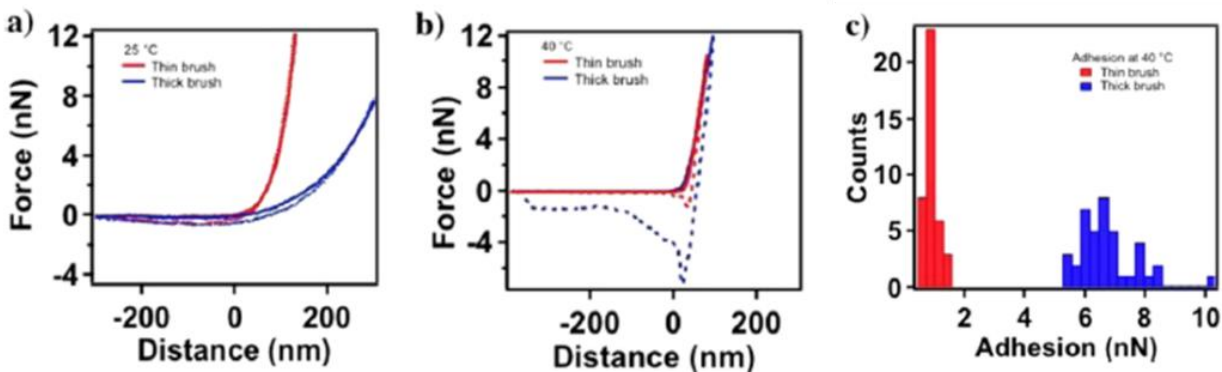

Fig. 5.3: F-D curves for thin and thick PNIPAM brushes at $25^{\circ} \mathrm{C}$ (a) and $40{ }^{\circ} \mathrm{C}$ (b). The solid lines indicate the approaching curves, while the dashed lines indicate the retracting curves. In c) the distributions of adhesion forces recorded at $40{ }^{\circ} \mathrm{C}$ for thin (red) and thick PNIPAM (blue) brushes are reported. F-D curves were collected 
at a speed of $1 \mu \mathrm{m} \cdot \mathrm{s}^{-1}$ with a cantilever having normal spring constant of $1.57 \mathrm{~N} \cdot \mathrm{m}^{-1}$ and presenting a silica colloid of $8 \mu \mathrm{m}$ radius.

The nanotribological properties of the different PNIPAM brushes were subsequently investigated by colloidal probe LFM.

a)

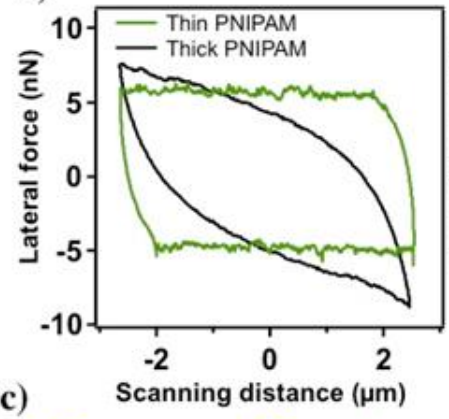

b)

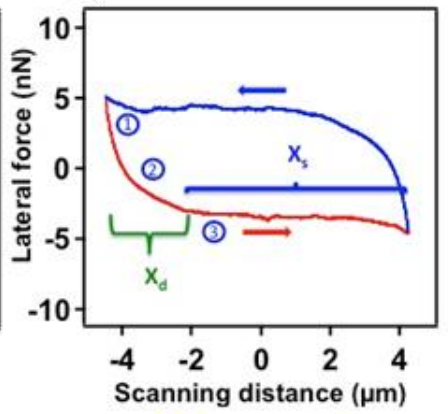

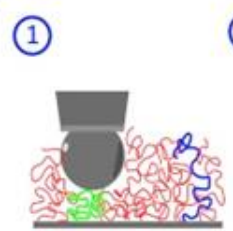

d)

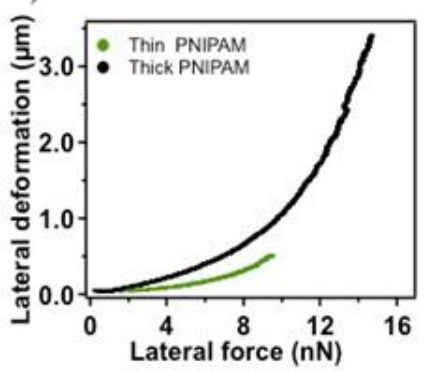

(3)

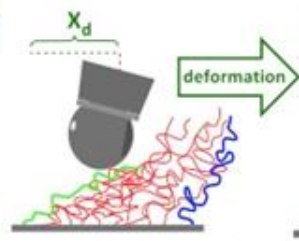

e)

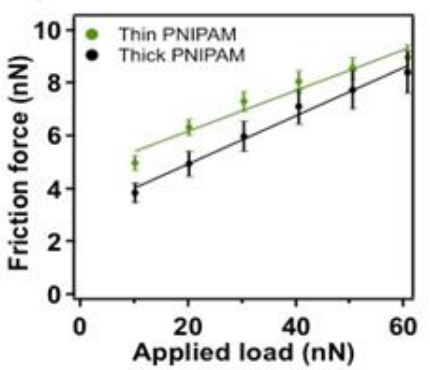

Fig. 5.4: (a) Friction loops recorded by LFM on thin and thick PNIPAM brushes immersed in water at $25^{\circ} \mathrm{C}$ for the applied load of $10 \mathrm{nN}$. In (b) a typical friction loop recorded on thick PNIPAM brushes is reported; the tilted portion (Xd) and the sliding portion (Xs) of the loop are also highlighted. A schematic representing brush deformation and sliding during scanning is reported in (c). In (d) the lateral deformation of thin and thick PNIPAM brushes is plotted against the recorded lateral force. (e) $\mathrm{F}_{\mathrm{f}}-\mathrm{L}$ profiles for thin and thick PNIPAM brushes recorded in water at $25^{\circ} \mathrm{C}$, the solid lines indicate the fit to the linear regression. In the inset, $\mathrm{F}_{\mathrm{f}}-\mathrm{L}$ profiles are reported applying the same vertical scale of Figure 5.5b, where the frictional properties of the PNIPAM brushes above LCST are plotted. The scanning distance and the scanning rate applied during CP-AFM friction measurements were $9 \mu \mathrm{m}$ and $1 \mu \mathrm{m} \cdot \mathrm{s}^{-1}$, respectively. The radius of the colloid used was $9 \mu \mathrm{m}$. The normal and torsional spring constants of the cantilever used were $0.263 \mathrm{~N} \cdot \mathrm{m}^{-1}$ and $5.52 \mathrm{E}^{-9} \mathrm{~N} \cdot \mathrm{m}$, respectively.

In Figure 5.4a, typical friction loops recorded on thin and thick PNIPAM brushes at $25^{\circ} \mathrm{C}$ in water are reported. In the swollen state PNIPAM brushes present tilted friction loops. As previously described by us, ${ }^{46,55}$ the tilted sections of the friction traces originate from the deformation (lateral bending and stretching) of swollen and compliant brushes 
by the scanning probe $\left(X_{d}\right.$ in Figure 5.4b and 5.4c). When the shear stress exerted by the probe overcomes the spring force of the deformed brush sliding finally occurs $\left(X_{\mathrm{s}}\right.$ in Figure 5.4b and 5.4c), until scanning direction reversal and the consequent deformation and sliding towards the opposite direction.

Comparing the friction loops recorded for thin and thick PNIPAM brushes, a much lower contribution of tilting was observed for the thinner brushes with respect to the thicker ones. Namely, the lateral piezo extension corresponding to the tilted portion of the friction trace, $X_{d}$ (or maximum lateral deformation) for thin PNIPAM brushes was estimated as $415 \pm 30 \mathrm{~nm}$, whereas thick PNIPAM showed a much higher $\mathrm{X}_{\mathrm{d}}$ of $3010 \pm$ $55 \mathrm{~nm}$, as shown in Figure 5.4d.

Friction-vs-load $\left(\mathrm{F}_{\mathrm{f}}-\mathrm{L}\right)$ measurements further elucidated the frictional properties of thin and thick PNIPAM brushes. As reported in Figure 5.4e, at $25^{\circ} \mathrm{C}$ the recorded friction force increased nearly linearly with the applied load. However, just a slight difference in COF was observed among thin and thick PNIPAM brushes $(0.08$ and 0.09 for thin and thick PNIPAM brushes, respectively). ${ }^{40}$

It is noteworthy to mention that although no clear adhesion could be recorded between the colloid and the swollen PNIPAM brushes, the $\mathrm{F}_{\mathrm{f}}-\mathrm{L}$ profiles measured at $25^{\circ} \mathrm{C}$ did not clearly follow Amontons' law, ${ }^{40}$ i.e., the recorded friction force was not directly proportional to the applied load. We believe that the observed slight deviation was possibly due to the interaction between the AFM colloid and the PNIPAM grafts, which was recorded as a slight but visible hysteresis between the approaching and retracting F$D$ profiles (Figure 5.3a). This interaction and the consequent stretching of the chains affected the measured lateral force and resulted in a small variation of the slope of the $\mathrm{F}_{\mathrm{f}^{-}}$ L profiles.

a)

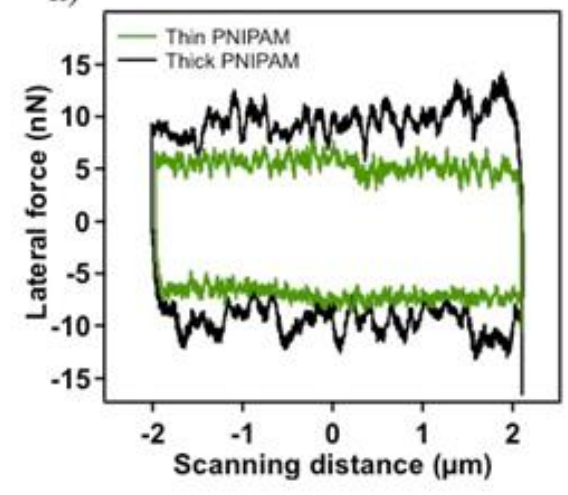

b)

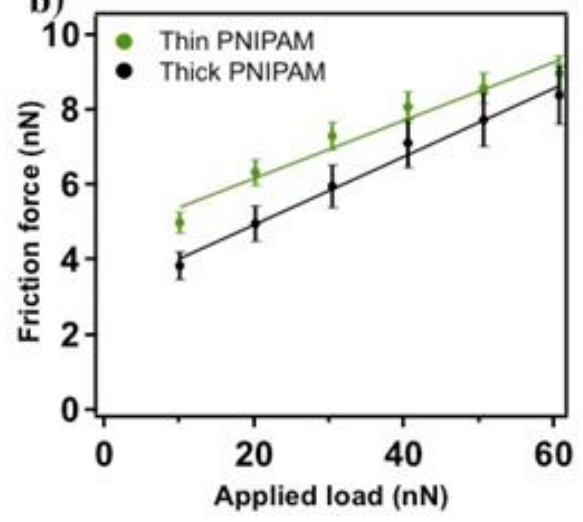

Fig. 5.5: (a) Friction loops recorded on thin- (green trace) and thick PNIPAM brushes (black trace) at $40{ }^{\circ} \mathrm{C}$ in milli-Q water by applying a normal load of $10 \mathrm{nN}$. (b) $\mathrm{F}_{\mathrm{f}}-\mathrm{L}$ profiles recorded on thin (green trace) and thick PNIPAM brushes (black trace) at $40{ }^{\circ} \mathrm{C}$ in milli-Q water. The scanning distance and scanning rate applied during LFM were $9 \mu \mathrm{m}$ and $1 \mu \mathrm{m} \cdot \mathrm{s}^{-1}$, respectively. The radius of the colloid used was $9 \mu \mathrm{m}$. The normal and torsional spring constants of the cantilever used were $0.263 \mathrm{~N} \cdot \mathrm{m}^{-1}$ and $5.52 \mathrm{E}^{-9} \mathrm{~N} \cdot \mathrm{m}$, respectively. 
At $40{ }^{\circ} \mathrm{C}$, the friction loops recorded on thin and thick PNIPAM brushes displayed very similar profiles, and no tilting effects were observed (Figure 5.5a). This result indicates that collapsed PNIPAM brushes could not be laterally deformed by the shearing probe, irrespective of their thickness.

As expected, the $\mathrm{F}_{\mathrm{f}}-\mathrm{L}$ measurements for thin and thick PNIPAM brushes at $40{ }^{\circ} \mathrm{C}$ showed relevantly higher friction compared to $25^{\circ} \mathrm{C}$. However, as displayed in Figure 5.5b, thick PNIPAM brushes presented a steeper $\mathrm{F}_{\mathrm{f}}$ - $\mathrm{L}$ profile if compared to the one recorded on thin PNIPAM analogues, with COF values of 0.9 and 0.5 for thick and thin PNIPAM brushes, respectively. The higher friction and COF observed for thick PNIPAM brushes in the collapsed state was likely due to the high adhesion force and increased surface roughness recorded above LCST for relatively thick PNIPAM brushes with respect to thinner ones.

\section{Effect of scanning distance on friction measurements.}

In order to investigate the effect of brush lateral deformation on the friction measurements and on the determination of COF, we firstly analyzed the friction loops recorded on thick PNIPAM brushes at $25{ }^{\circ} \mathrm{C}$, applying scanning distances of 1,5 and 9 $\mu \mathrm{m}$, and maintaining a constant scanning speed of $1 \mu \mathrm{m} \cdot \mathrm{s}^{-1}$. As displayed in Figure 5.6a, at the smallest scanning distance tested of $1 \mu \mathrm{m}$, the friction loop showed a completely tilted shape, as the lateral deformation of the swollen brush dominates brush-colloidal probe interaction, and sliding is never attained. Under these experimental conditions, the dissipation recorded via the friction loop (green trace in Figure 5.6a) is solely due to the back and forth bending and stretching of the swollen brushes. It is important to underling that at not slipping the apparent friction force induced by the cantilever torsion was due to the resistance by the PNIPAM grafts to bend and stretch along the lateral direction. An increase of the scanning distance to $5 \mu \mathrm{m}$ causes the brushes to be initially deformed and stretched until static friction is overcome and sliding is finally gained (brown trace in Figure 5.6a). In this case, the dissipation measured via the friction loop originates from a combination of both brush stretching and sliding of the colloidal probe. A further increase of the scanning distance until $9 \mu \mathrm{m}$, which is a considerably larger distance than the maximum lateral stretching of the grafted chains, produces a friction loop presenting a tilted section after scanning direction reversal, followed by steady sliding of the colloid over several $\mu \mathrm{m}$ of piezo displacement. Thus at relatively large scanning distances, the frictional dissipation is dominated by the sliding of the colloid over the brush surface (black trace in Figure 5.6a). 
a)

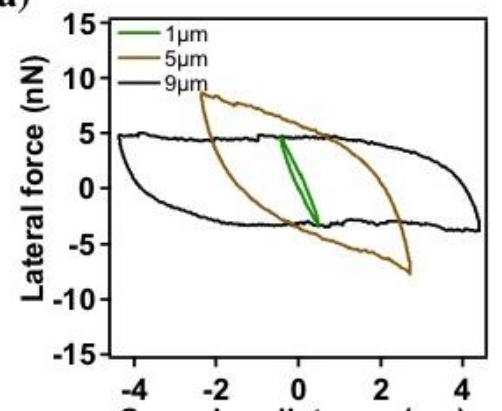

c)

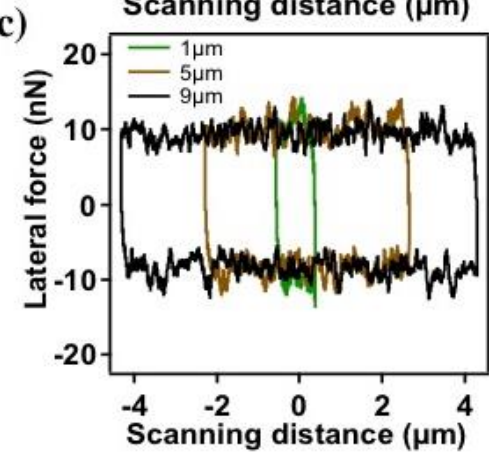

b)
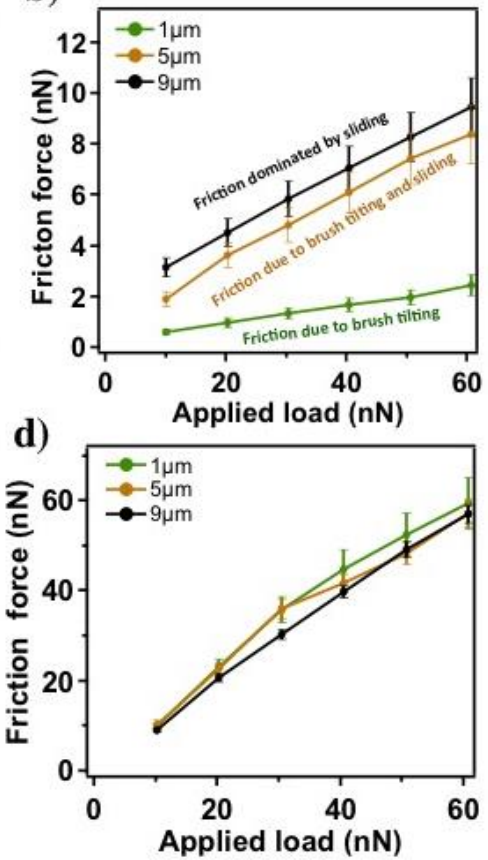

Fig. 5.6: (a) Friction loops recorded on thick PNIPAM brushes at $25{ }^{\circ} \mathrm{C}$ in water applying scanning distances of 1,5 and $9 \mu \mathrm{m}$. (b) $\mathrm{F}_{\mathrm{f}}$-L profiles recorded on thick-PNIPAM brushes at $25{ }^{\circ} \mathrm{C}$ in water applying scanning distances of 1,5 and $9 \mu \mathrm{m}$. Friction loops and $\mathrm{F}_{\mathrm{f}}-\mathrm{L}$ profiles recorded on thick PNIPAM brushes at $40{ }^{\circ} \mathrm{C}$ in water (c and d). The scanning velocity during the experiments was kept constant at $1 \mu \mathrm{m} \cdot \mathrm{s}^{-1}$. The normal and torsional spring constant of the cantilever used were $0.263 \mathrm{~N} \cdot \mathrm{m}^{-1}$ and $5.52 \mathrm{E}^{-9} \mathrm{~N} \cdot \mathrm{m}$ respectively.

When a scanning distance of $5 \mu \mathrm{m}$ is applied, a virtual increase in the recorded lateral force toward direction reversal is observed. We believe this phenomenon was due to the piling-up of the deformed grafts along the scan ing direction, which caused an increase of the lateral force experienced by the AFM probe. Since the rate at which the deformed brushes are recovering their equilibrium conformation is presumably lower compared to the applied scan rate, this topographical change by the brush surface affected the friction measurements. As a confirmation of this assumption, we laterally scanned the same brush with a sharp tip and observed an increase of the recorded height values on the edges of the Z-sensor micrographs (Figure 5.7), indicating brush piling-up towards scanning direction reversal. 


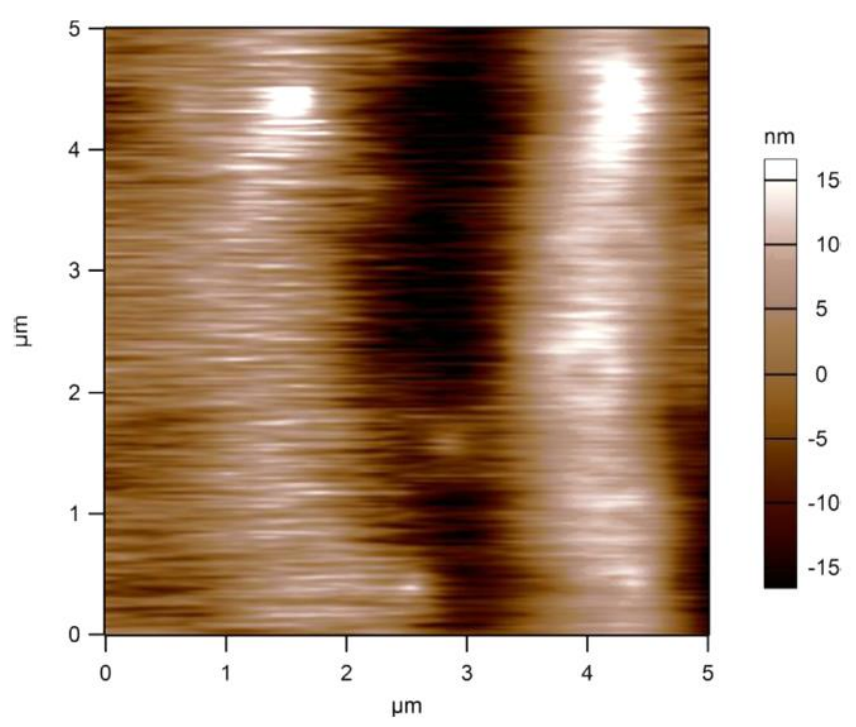

Fig. 5.7: AFM height micrograph in contact mode for thick PNIPAM brushes immersed in milli-Q water recorded at 25 and $40{ }^{\circ} \mathrm{C}$ with a sharp tip (SNL-10, applied load of about $20 \mathrm{nN}$ ).

The comparison of the $\mathrm{F}_{\mathrm{f}} \mathrm{L}$ plots recorded at the three different scanning distances studied highlights how a variation of brush-colloidal probe interactions can lead to different measurements of the COF. The lowest COF value of 0.03 was obtained in the case of $1 \mu \mathrm{m}$ of scanning distance. Across this relatively short scanning distance, the measured friction force was solely due to the energy dissipation caused by the lateral bending and stretching of the swollen brush (Figure 5.6a green trace). A marked increase in friction, with average $\mathrm{COF}$ of 0.08 was observed for $\mathrm{F}_{\mathrm{f}}-\mathrm{L}$ profiles at $5 \mu \mathrm{m}$ of scanning distance. In this particular case, the frictional energy dissipation recorded via the loops derived from the combination of brush lateral deformation and sliding (Figure 5.6a brown trace). In contrast, at the largest scanning distance of $9 \mu \mathrm{m}$ the measured friction force is mainly determined by sliding of the colloid, and an average COF of 0.1 was measured (Figure 5.6b black trace).

The effect of brush lateral deformation on the measurement of friction becomes irrelevant at $40{ }^{\circ} \mathrm{C}$, when PNIPAM brushes present a collapsed morphology. Under this condition, the friction loops do not show any tilt, and the $\mathrm{F}_{\mathrm{f}}-\mathrm{L}$ plots display a similar profile irrespective of the scanning distance applied. A constant COF of 0.9 is thus measured in all three cases, as indicated in Figure 5.6d.

Similar results are also obtained for thin PNIPAM brushes, although the occurrence of sliding was observed at a value of scanning distance relevantly shorter with respect to the more laterally deformable, thick PNIPAM brushes. As shown in Figure 5.8a, the transition from a completely tilted loop to a combination of tilting and sliding was recorded at $1.5 \mu \mathrm{m}$ of scanning distance, while at larger distances sliding of the colloidal 
probe dominated the frictional properties of thin PNIPAM brushes. In this case, the COF values of $0.04,0.12$, and 0.14 were obtained for $0.5 \mu \mathrm{m}, 1.5 \mu \mathrm{m}$, and $5 \mu \mathrm{m}$ scanning distances respectively (Figure 5.8b). Similar to the result observed for thick PNIPAM brushes, at $40{ }^{\circ} \mathrm{C}$ the lateral deformation of thin brushes was completely suppressed and no tilting was observed in the recorded friction loops (Figure 5.8c). Hence, when recorded on the thin PNIPAM brushes above the LCST, the $\mathrm{F}_{\mathrm{f}}-\mathrm{L}$ profiles measured across different scanning distances overlapped on each other and indicated a constant COF of 0.5 (Figure 5.8d).

a)

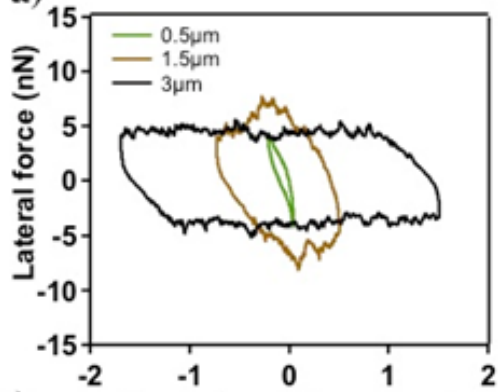

c)

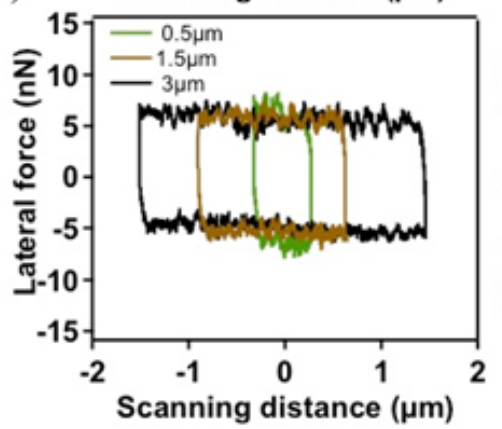

b)

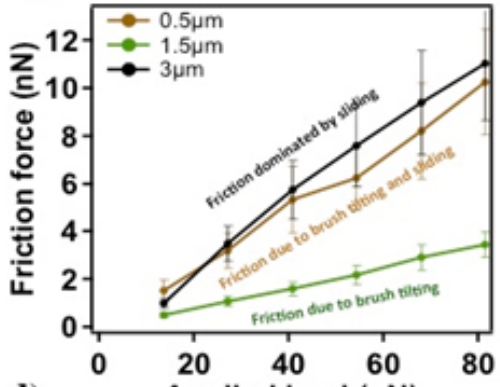

d) Applied load ( $\mathrm{nN})$

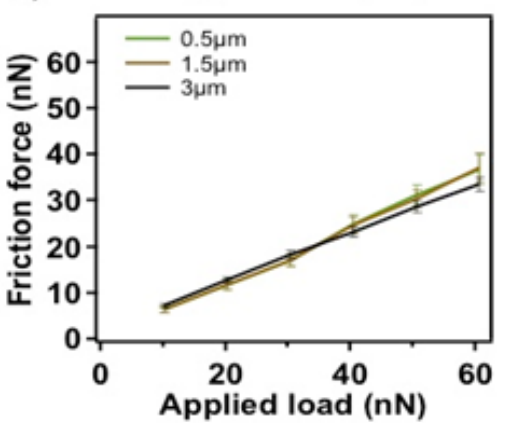

Fig. 5.8: (a) Friction loops recorded on thin PNIPAM brushes at $25^{\circ} \mathrm{C}$ in water applying scanning distances of $0.5,1.5$ and $3 \mu \mathrm{m}$. (b) $\mathrm{F}_{\mathrm{f}}-\mathrm{L}$ plots obtained for thick PNIPAM brushes at $25^{\circ} \mathrm{C}$ in water applying $0.5,1.5$ and $3 \mu \mathrm{m}$ scanning distance. Friction loops and $\mathrm{F}_{\mathrm{f}}-\mathrm{L}$ profiles recorded on thin-PNIAPM at $40^{\circ} \mathrm{C}$ are displayed in (c) and (d). The scanning velocity during the experiments was kept constant at $1 \mu \mathrm{m} \cdot \mathrm{s}^{-1}$. The normal and torsional spring constant of the cantilever used were $0.263 \mathrm{~N} \cdot \mathrm{m}^{-1}$ and $5.52 \mathrm{E}^{-9} \mathrm{~N} \cdot \mathrm{m}$ respectively.

These results further confirmed that the contribution of brush lateral deformation to the dissipation of frictional energy can strongly alter the friction and thus COF values when the scanning distance applied during LFM is shorter or comparable to the maximum lateral deformation of the measured brush $\left(\mathrm{X}_{\mathrm{d}}\right)$. This is particularly valid for highly swollen brushes, as in the case of PNIPAM immersed in water below its LCST, whereas in a bad solvent, the shearing probe can hardly laterally deform and stretch the collapsed and dehydrated brushes, and the frictional energy dissipation is mainly determined by the sliding of the probe over the brush surface. 


\subsection{Conclusions}

In this work, we investigated the effect of the lateral deformation of a swollen brush by a shearing AFM probe on the measurement of friction forces. Brush lateral deformation, comprising bending and stretching of the grafts, can be quantified through the analysis of the friction force loops recorded by LFM. The tendency of a brush to be laterally deformed depends on brush parameters, such as the grafted-chain length (i.e. brush thickness) and it is particularly relevant and interesting in the case of thermo-responsive polymer brushes, being the swelling properties of such grafts precisely tunable by varying the temperature of the medium. For both thin and thick PNIPAM brushes immersed in water at $25^{\circ} \mathrm{C}$, the friction loops display a tilted behaviour, with thicker brushes that can be laterally deformed more than thinner analogues. Thus, when relatively short scanning distances are applied for recording the friction loops, brush deformation mainly determines the frictional dissipation, while sliding of the colloid on the brush surface does not occur. Under these conditions, a relatively low COF was measured, which is uniquely originating from the bending and stretching back and forth of the swollen brushes. The application of scanning distances relevantly longer than the brush lateral deformation (expressed as $\mathrm{X}_{\mathrm{d}}$ ) produces friction loops displaying a tilted shape solely at scanning direction reversal, while sliding of the colloid on the brushes dominates the frictional dissipation recorded via the loops. In these cases, the slope of the $\mathrm{F}_{\mathrm{f}}-\mathrm{L}$ profiles as well as the derived COF values increase.

In contrast, due to brush dehydration and collapse above LCST, PNIPAM brushes cannot be laterally deformed at $40{ }^{\circ} \mathrm{C}$ in water. Tilting of the friction loops could not be observed under these particular conditions and the $\mathrm{F}_{\mathrm{f}}-\mathrm{L}$ profiles showed a constant slope irrespective of the scanning distance applied for recording the loops.

Brush lateral deformation is thus proved to play a relevant effect on the measurement of friction and COF, especially when the measured brush is immersed in a good solvent and when relatively short scanning distances are applied during LFM. Our study demonstrates that while measuring $\mathrm{COF}$, brush thickness, swelling properties and applied scanning distances are parameters to be carefully considered, as the simple recording of TMR (Trace minus Retrace) values from the friction loops may yield a conceptually wrong estimate of COF.

\subsection{Experimental section}

\section{Synthesis of PNIPAM brushes of different thicknesses.}

PNIPAM brushes of two different thicknesses were synthesized by surface-initiated atom radical polymerization (SI-ATRP) from initiator-functionalized silicon substrates (P/B <100>, Si-mat, Germany) according to the previously reported procedure. ${ }^{38}$ The 
catalyst system comprised $\mathrm{CuBr}$ (Sigma Aldrich), $\mathrm{CuBr}_{2}$ (Sigma Aldrich) and $\mathrm{N}, \mathrm{N}, \mathrm{N}^{\prime}, \mathrm{N}^{\prime \prime}, \mathrm{N}^{\prime \prime}$-pentamethyldiethylenetriamine (PMDETA) (Sigma Aldrich) in water/methanol mixtures.

The chemical composition of the so-fabricated PNIPAM brushes was confirmed by Fourier-transform infrared spectroscopy (FT-IR) using a spectrometer (BIO-RAD FTS575C FTIR equipped with a nitrogen-cooled cryogenic cadmium mercury telluride detector) with spectral resolution of $8 \mathrm{~cm}^{-1}$, applying 2048 scans for both the background spectra (performed on freshly cleaned silicon substrates) and the PNIPAM brush-modified samples. The surface-modification steps were also verified by contact angle (CA) measurements, using the sessile drop method. Both FTIR and CA characterizations are reported in the Supporting Information (Figure S5.1 and Figure S5.2).

\section{Characterization techniques}

Ellipsometry. The dry and wet thicknesses of PNIPAM brushes were measured by variable angle spectroscopic ellipsometry (VASE) using a Woolam ellipsometer (J.A. Woolam Co. U.S.) equipped with a custom-built, liquid cell with a temperature controller. $\Psi$ and $\Delta$ as a function of wavelength $(275-827 \mathrm{~nm})$ were analyzed employing the package CompleteEASE (Woollam), using bulk dielectric functions for silicon, silicon dioxide and water. The brush-underlying substrates were considered as silicon dioxide film on top of silicon substrate.

The analysis of the brush layers were performed using the Cauchy model:

$$
n=A+B \cdot \lambda^{-2}+C \cdot \lambda^{-4}
$$

where $A, B$ and $C$ represent the fitting parameters. We used $C=0$ and assumed that the polymer films are fully transparent, i.e. the refractive index is a real quantity (the imaginary part is neglected). In the case of dry PNIPAM brushes, a homogeneous layer with a refractive index expressed with a single Cauchy relation was considered. The films thickness $\mathrm{d}$ and the two Cauchy parameters $A$ and $B$ were used as fitting parameters. The dry thickness was obtained by performing the measurements at three different incident angles, namely $65^{\circ}, 70^{\circ}$ and $75^{\circ}$.

For PNIPAM brushes immersed in water at $25{ }^{\circ} \mathrm{C}$ we considered a density gradient across the film thickness employing a two-layers model to describe the optical response of the films (dense polymeric layer + graded polymeric layer) as it was previously described in detail. ${ }^{38 .}{ }^{39}$ For PNIPAM brushes immersed in water at $40{ }^{\circ} \mathrm{C}$ a single-layer model was used. ${ }^{47}$

AFM Measurements. AFM measurements were carried out using a MFP-3D (Asylum Research, an Oxford Instruments company, Santa Barbara, CA, USA) equipped with a bio heater. Height micrographs of the brush surfaces were performed in milli-Q water below and above LCST of PNIPAM $\left(25^{\circ} \mathrm{C}\right.$ and $\left.40^{\circ} \mathrm{C}\right)$ using AC mode. Cantilevers 
(SNL-10, D triangular) from Bruker Corporation, having a normal spring constant of $0.06 \mathrm{~N} \cdot \mathrm{m}^{-1}$ were used for the tapping mode imaging in liquid.

Normal and lateral force measurements were carried out in milli-Q water at 25 and $40{ }^{\circ} \mathrm{C}$, using a silica microsphere glued on a tip-less cantilever. The normal spring constant of the Au-coated, cantilever (NSC-12, Mikromash, Bulgaria) was measured by thermalnoise method ${ }^{48}$ and the torsional spring constant was estimated according to the Sader's method. ${ }^{49}$ Both the normal and the torsional spring constants of the cantilever were measured before attaching the colloidal microsphere. A silica sphere of radius $\sim 8 \mu \mathrm{m}$ (EKA chemicals AB, Kromasil R) was glued with a two component Araldite glue to the end of the tip-less cantilever by means of a home-built micromanipulator. ${ }^{50}$ The colloidal probe was treated with UV/Ozone (BioForces Nanosciences) for $30 \mathrm{~min}$ just before the measurement.

The adhesive properties of PNIPAM brushes, below and above LCST were examined by acquiring the force-vs-distance (F-D) curves (40 curves) over 3 different areas. Gaussian function was used to calculate the distribution of the adhesion force over measured 120 F-D curves.

Lateral-force calibration was done by using the "test- probe method" described by Cannara $e$ t $a l .{ }^{51}$ A freshly cleaned, smooth edge of the silicon wafer was used as a "wall" for measuring the lateral sensitivity. A test probe (cantilever glued with a silica colloidal sphere of diameter around $40 \mu \mathrm{m}$ ) was moved laterally into contact with the wall. The lateral sensitivity of the photo detector was acquired by measuring the slope of the lateral deflection-vs-piezo displacement curve. Friction loops were recorded by lateral force microscopy (LFM) scanning the cantilever laterally over the brush surfaces. From the friction loops, the true friction values were obtained by averaging trace and retrace curves. Friction coefficient (COF) values were calculated by the slope of friction vs applied load plots assuming Amontons' law:

$$
\mathrm{F}=\mu \mathrm{L}
$$

where $\mathrm{F}$ is friction force, $\mu$ is the coefficient of friction, and $\mathrm{L}$ is the applied load. 


\subsection{Supporting information}

\section{Contact Angle (CA) measurements}

\begin{tabular}{|c|c|c|c|}
\hline & $\mathrm{SiO}_{2}$ & $\begin{array}{c}\text { ATRP } \\
\text { initiator }\end{array}$ & $\begin{array}{c}\text { PNIPAM } \\
\text { brush }\end{array}$ \\
\hline Static CA & $20 \pm 5^{\circ}$ & $63 \pm 6^{\circ}$ & $54 \pm 4^{\circ}$ \\
\hline & & & \\
& & & - \\
\hline
\end{tabular}

Figure S5.1: Static water contact angle measured on bare silicon oxide surface, initiator functionalized surface and on PNIPAM coated surface.

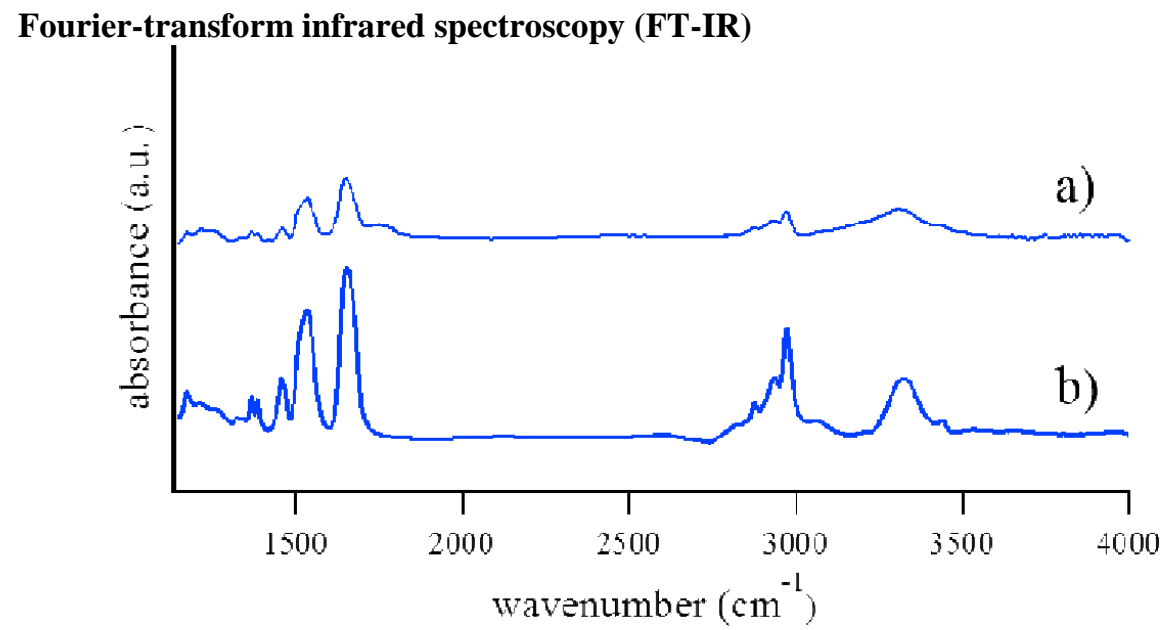

Figure S5.2. FTIR spectra of (a) thin PNIPAM films and (b) thick PNIPAM film.

\section{Gel permeation chromatography (GPC) on detached polymer brushes}

In order to estimate the grafting density of the PNIPAM brushes fabricated in this study, $110 \mathrm{~nm}$ thick PNIPAM grafts synthesized by SI-ATRP were detached from a $2.5 \mathrm{x} 4$ $\mathrm{cm}^{2}$ silicon oxide substrate by hydrofluoric acid (HF) treatment. Subsequently, the detached polymer sample was analyzed by GPC resulting in a $\mathrm{M}_{\mathrm{w}}$ of $280 \mathrm{kDa}, \mathrm{Mn}$ of 170 $\mathrm{kDa}(\mathrm{PDI} \approx 1.7)$ and thus an average grafting density of 0.43 chains $\cdot \mathrm{nm}^{-2}$.

\section{Calibration of lateral force using the test-probe method}

The torsional stiffness $\left(K_{T}\right)$ of the cantilever was calculated using Sader's method, where the unloaded resonant frequency $(f)$ and the quality factor $(Q)$ due to torsional vibrations of the cantilever are calculated by recording a power spectrum in torsional mode 
(In Asylum AR software, using cross-point panel to change the mode from 'Infast' to 'lateral'). $\mathrm{T} \mathrm{h}$ e length, $\mathrm{t} \mathrm{h}$ e width and $\mathrm{t} \mathrm{h}$ e thickness of the cantilevers were determined by scanning electron microscopy (SEM). The obtained torsional spring constant was converted to the lateral spring constant $\left(K_{L}\right)$ using the equation $K_{L}=K_{T} / h^{2}$; where $h$ indicates the length of the torsional arm (equal to the sum of the diameter of the sphere and half the thickness of the cantilever beam). A silica colloid was subsequently glued to a cantilever presenting width, thickness and spring constant comparable to the ones of the cantilever used in the LFM experiments ('test probe' used for lateral sensitivity measurement). The diameter of the colloid used for the test was always larger than the width of the cantilever (Figure S5.3).

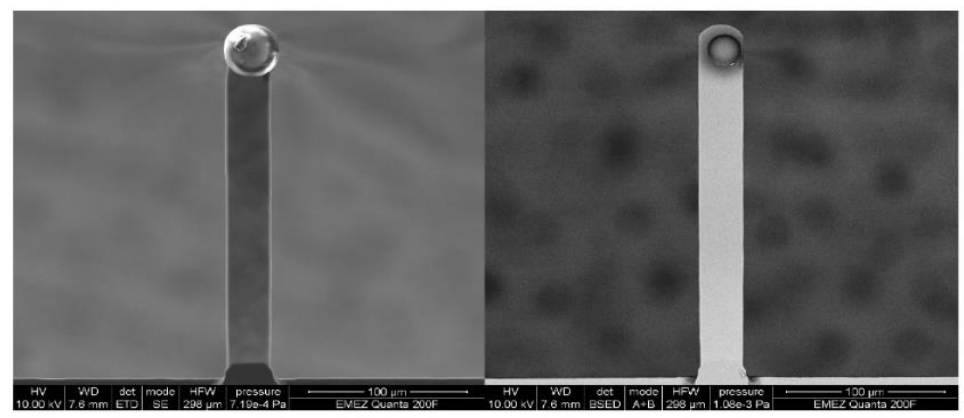

Figure S5.3. SEM images show an example of test probe and target probe.

In the next step, a silicon wafer was cut along its crystal plane $<100\rangle$ and glued to a glass slide, in such a way that the smooth edge of the wafer (along the crystal plane) was used as a vertical wall to twist the cantilever laterally (Figure S5.4). This measurement was carried out in water to account for the change in refractive index for the measurement of the lateral sensitivity.
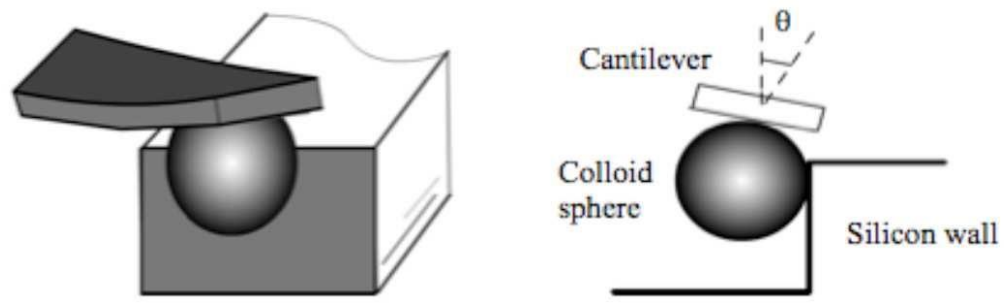

Figure S5.4. The schematic representation of the calibration method using the test probe. 


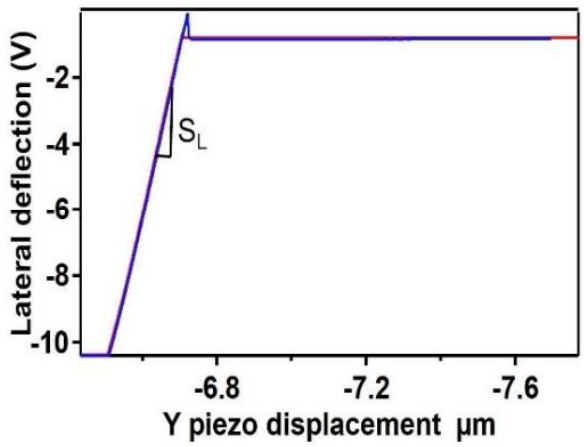

Figure S5.5. An example of lateral deflection-vs-Y piezo displacement curve recorded by moving a silica probe against a hard wall in water.

The slope of the recorded lateral deflection-vs-Y piezo displacement curve provided the lateral sensitivity of the tested cantilever.

Finally, the obtained lateral sensitivity was used to estimate the lateral sensitivity of the probe used for the LFM measurements $\left(S_{\text {lat,target }}\right)$, by introducing the correction factors for the height of the probe, the sum signal, and the in-plane bending of the cantilever, according to the following equation:

$$
S_{\text {lat, target }}=S_{\text {lat, test }} \frac{\left(\frac{T_{\text {target }}}{T_{\text {test }}}\right)\left(1+\varepsilon_{\text {test }}\right)}{\left(\frac{h_{\text {target }}}{h_{\text {test }}}\right)\left(1+\varepsilon_{\text {target }}\right)}
$$

Where $T_{\text {lat,target }}$ and $T_{\text {test }}$ are the intensities of laser signals, $h_{\text {target }} h_{\text {test }}$ are torsional arm length and $\varepsilon_{\text {test }}$ and $\varepsilon_{\text {target }}$ are the lateral in-plane bending of the target and test probes, respectively.

The measured friction signal was then converted to force with a conversion factor $(\alpha)$, defined as $\alpha=K_{L} / S_{\text {lat,target }}$; where $K_{L}$ is lateral spring constant of the cantilever used for the LFM measurements.

\subsection{References}

1. Jiang, S.; Cao, Z. Ultralow-Fouling, Functionalizable, and Hydrolyzable Zwitterionic Materials and Their Derivatives for Biological Applications. Adv. Mater. 2010, 22 (9), 920-932.

2. Navarro, M.; Benetti, E. M.; Zapotoczny, S.; Planell, J. A.; Vancso, G. J. Buried, Covalently Attached RGD Peptide Motifs in Poly- (Methacrylic Acid) Brush Layers: the Effect of Brush Structure on Cell Adhesion. Langmuir 2008, 24 (19), 10996-11002.

3. Krishnamoorthy, M.; Hakobyan, S.; Ramstedt, M.; Gautrot, J. E. Surface-Initiated Polymer Brushes in the Biomedical Field: Applications in membrane Science, Biosensing, Cell Culture, Regenerative Medicine and Antibacterial Coatings. Chem. Rev. 2014, 114 (21), 10976-11026.

4. Li, A.; Ramakrishna, S. N.; Kooij, E. S.; Espinosa-Marzal, R. M.; Spencer, N. D. Poly(Acrylamide) Films at the Solvent-Induced Glass Transition: Adhesion, Tribology, and the Influence of Crosslinking. Soft Matter 2012, 8 (35), 9092-9100. 
5. Lee, S.; Muller, M.; Ratoi-Salagean, M.; Voros, J.; Pasche, S.; De Paul, S. M.; Spikes, H. A.; Textor, M.; Spencer, N. D. Boundary Lubrication of Oxide Surfaces by Poly(L-Lysine)-G-Poly(Ethylene Glycol) (PLL-G-PEG) in Aqueous Media. Tribol. Lett. 2003, 15 (3), 231-239.

6. de Beer, S.; Kutnyanszky, E.; Scho“n, P. M.; Vancso, G. J.; Müser, M. H. Solvent-Induced Immiscibility of Polymer Brushes Eliminates Dissipation Channels. Nat. Commun. 2014, 5, 3781.

7. Rosenberg, K. J.; Goren, T.; Crockett, R.; Spencer, N. D. Load- Induced Transitions in the Lubricity of Adsorbed Poly(L-Lysine)-GDextran as a Function of Polysaccharide Chain Density. ACS Appl. Mater. Interfaces 2011, 3 (8), 3020-3025.

8. Heeb, R.; Bielecki, R. M.; Lee, S.; Spencer, N. D. Room- Temperature, Aqueous-Phase Fabrication of Poly(Methacrylic Acid) Brushes by UV-LED-Induced, Controlled Radical Polymerization with High Selectivity for Surface-Bound Species. Macromolecules 2009, 42 (22), 9124-9132.

9. Chen, M.; Briscoe, W. H.; Armes, S. P.; Klein, J. Lubrication at Physiological Pressures by Polyzwitterionic Brushes. Science 2009, 323 (5922), 1698-1701.

10. Kobayashi, M.; Takahara, A. Tribological Properties of Hydrophilic Polymer Brushes Under Wet Conditions. Chem. Rec. 2010, 10 (4), 208-216.

11. Lee, S.; Spencer, N. D. Aqueous Lubrication of Polymers: Influence of Surface Modification. Tribol. Int. 2005, 38 (11-12), 922-930.

12. Nalam, P. C.; Clasohm, J. N.; Mashaghi, A.; Spencer, N. D. Macrotribological Studies of Poly(L-Lysine)Graft-Poly(Ethylene Glycol) in Aqueous Glycerol Mixtures. Tribol. Lett. 2010, 37 (3), 541-552.

13. Müller, M.; Lee, S.; Spikes, H. A.; Spencer, N. D. The Influence of Molecular Architecture on the Macroscopic Lubrication Properties of the Brush-Like Co-Polyelectrolyte Poly(L-Lysine)- G-Poly(Ethylene Glycol) (PLL- G-PEG) Adsorbed on Oxide Surfaces. Tribol. Lett. 2003, 15 (4) , 395-405 .

14. Sakata, H.; Kobayashi, M.; Otsuka, H.; Takahara, A. Tribological Properties of Poly(Methyl Methacrylate) Brushes Prepared by Surface- Initiated Atom Transfer Radical Polymerization. Polym. J. 2005, 37 (10), 767-775.

15. Lee, S.; Muller, M.; Heeb, R.; Zürcher, S.; Tosatti, S.; Heinrich, M.; Amstad, F.; Pechmann, S.; Spencer, N. D. Self-Healing Behavior of a Polyelectrolyte-Based Lubricant Additive for Aqueous Lubrication of Oxide Materials. Tribol. Lett. 2006, 24 (3), 217-223.

16. Kobayashi, M.; Terayama, Y.; Hosaka, N.; Kaido, M.; Suzuki, A.; Yamada, N.; Torikai, N.; Ishihara, K.; Takahara, A. Friction Behavior of High-Density Poly(2-Methacryloyloxyethyl Phosphorylcholine) Brush in Aqueous Media. Soft Matter 2007, 3 (6), 740-746.

17. Drobek, T.; Spencer, N. D. Nanotribology of Surface-Grafted PEG Layers in an Aqueous Environment. Langmuir 2008, 24, 1484.

18. Raviv, U.; Giasson, S.; Kampf, N.; Gohy, J.-F.; Jeröme, R.; Klein, J. Lubrication by Charged Polymers. Nature 2003, 425 (6954), 163-165.

19. Tairy, O.; Kampf, N.; Driver, M. J.; Armes, S. P.; Klein, J. Dense, Highly Hydrated Polymer Brushes via Modified Atom-Transfer- Radical-Polymerization: Structure, Surface Interactions, and Frictional Dissipation. Macromolecules 2015, 48 (1), 140-151.

20. Müller, M. T.; Yan, X.; Lee, S.; Perry, S. S.; Spencer, N. D. Lubrication Properties of a Brushlike Copolymer as a Function of the Amount of Solvent Absorbed Within the Brush. Macromolecules 2005, 38 (13), 5706-5713.

21. Nalam, P. C.; Ramakrishna, S. N.; Espinosa-Marzal, R. M.; Spencer, N. D. Exploring Lubrication Regimes at the Nanoscale: Nanotribological Characterization of Silica and Polymer Brushes in Viscous Solvents. Langmuir 2013, 29 (32), 10149-10158. 
22. Hurley, C. R.; Leggett, G. J. Influence of the Solvent Environment on the Contact Mechanics of Tip-Sample Interactions in Friction Force Microscopy of Poly(Ethylene Terephthalate) Films. Langmuir 2006, 22 (9), 4179-4183.

23. Klein, J.; Kamiyama, Y.; Yoshizawa, H.; Israelachvili, J. N.; Fredrickson, G. H.; Pincus, P.; Fetters, L. J. Lubrication Forces Between Surfaces Bearing Polymer Brushes. Macromolecules 1993, 26 (21), 5552-5560.

24. Tadmor, R.; Janik, J.; Klein, J.; Fetters, L. J. Sliding Friction with Polymer Brushes. Phys. Rev. Lett. 2003, 91 (11), 115503.

25. Brady, M. A.; Limpoco, F. T.; Perry, S. S. Solvent-Dependent Friction Force Response of Poly(Ethylenimine)-Graft-Poly(Ethylene Glycol) Brushes Investigated by Atomic Force Microscopy. Langmuir 2009, 25 (13), 7443-7449.

26. Raviv, U.; Tadmor, R.; Klein, J. Shear and Frictional Interactions Between Adsorbed Polymer Layers in a Good Solvent. J. Phys. Chem. B 2001, 105, 8125-8134.

27. Klein, J.; Kumacheva, E.; Mahalu, D.; Perahia, D.; Fetters, L. J. Reduction of Frictional Forces Between Solid-Surfaces Bearing Polymer Brushes. Nature 1994, 370 (6491), 634-636.

28. Klein, J.; Perahia, D.; Warburg, S. Forces Between Polymer- Bearing Surfaces Undergoing Shear. Nature 1991, 352 (6331), 143-145.

29. Carrillo, J.-M. Y.; Russano, D.; Dobrynin, A. V. Friction Between Brush Layers of Charged and Neutral Bottle-Brush Macromolecules. Molecular Dynamics Simulations. Langmuir 2011, 27, 14599.

30. Grest, G. S. Interfacial Sliding of Polymer Brushes: a Molecular Dynamics Simulation. Phys. Rev. Lett. 1996, 76 (26), 4979-4982.

31. de Beer, S.; Mu“ser, M. H. Alternative Dissipation mechanisms and the Effect of the Solvent in Friction Between Polymer Brushes on Rough Surfaces. Soft Matter 2013, 9 (30), 7234-7241.

32. Singh, M. K.; Ilg, P.; Espinosa-Marzal, R. M.; Kro“ger, M.; Spencer, N. D. Polymer Brushes Under Shear: Molecular Dynamics Simulations Compared to Experiments. Langmuir 2015, 31, 4798.

33. de Beer, S.; Mu“ser, M. H. Friction in (Im-) Miscible Polymer Brush Systems and the Role of Transverse Polymer Tilting. Macromolecules 2014, 47 (21), 7666-7673.

34. Zdyrko, B.; Luzinov, I. Polymer Brushes by the "Grafting to" Method. Macromol. Rapid Commun. 2011, 32 (12), 859-869.

35. Yan, X.; Perry, S. S; Spencer, N. D; Pasche, S.; De Paul, S. M; Textor, M.; Lim, M. S. Reduction of Friction at Oxide Interfaces Upon Polymer Adsorption From Aqueous Solutions. Langmuir 2004, 20 (2), 423-428.

36. Pettersson, T.; Naderi, A; Makuška, R.; Claesson, P. M Lubrication Properties of Bottle-Brush Polyelectrolytes: an AFM Study on the Effect of Side Chain and Charge Density. Langmuir 2008, 24 (7), 3336-3347.

37. Kitano, K.; Inoue, Y.; Matsuno, R.; Takai, M.; Ishihara, K. Nanoscale Evaluation of Lubricity on WellDefined Polymer Brush Surfaces Using QCM-D and AFM. Colloids Surf., B 2009, 74 (1), 350-357.

38. Ramakrishna, S. N.; Cirelli, M.; Kooij, E. S.; Gunnewiek, M. K.; Benetti, E. M. Amplified Responsiveness of Multilayered Polymer Grafts: Synergy Between Brushes and Hydrogels. Macromolecules 2015, 48 (19), 7106-7116.

39. Chen, Q.; Kooij, E. S.; Sui, X.; Padberg, C. J.; Hempenius, M. A.; Schön, P. M.; Vancso, G. J. Collapse From the Top: Brushes of Poly-(N-Isopropylacrylamide) in Co-Nonsolvent Mixtures. Soft Matter 2014, 10 (17), 3134-3142. 
40. Zhang, Z.; Morse, A. J.; Armes, S. P.; Lewis, A. L.; Geoghegan, M.; Leggett, G. J. Effect of Brush Thickness and Solvent Composition on the Friction Force Response of Poly(2-(Methacryloyloxy)Ethylphosphorylcholine) Brushes. Langmuir 2011, 27, 2514.

41. Smidsrød, O; Guillet, J. E. Study of Polymer-Solute Interactions by Gas Chromatography. Macromolecules 1969, 2 (3), 272-277.

42. Schmitt, F. J.; Park, C.; Simon, J.; Ringsdorf, H.; Israelachvili, J. Direct Surface Force and Contact Angle Measurements of an Adsorbed Polymer with a Lower Critical Solution Temperature. Langmuir 1998, 14 (10), 2838-2845.

43. de Beer, S. Switchable Friction Using Contacts of Stimulus- Responsive and Nonresponding Swollen Polymer Brushes. Langmuir 2014, 30 (27), 8085-8090.

44. Yu, Y.; Cirelli, M.; Kieviet, B. D.; Kooij, E. S.; Vancso, G. J.; de Beer, S. Tunable Friction by Employment of Co-Non-Solvency of PNIPAM Brushes. Polymer 2016, 102, 372-378.

45. Sui, X.; Chen, Q.; Hempenius, M. A.; Vancso, G. J. Probing the Collapse Dynamics of Poly $(N$ Isopropylacrylamide) Brushes by AFM: Effects of Co-Nonsolvency and Grafting Densities. Small 2011, 7 (10), 1440-1447.

46. Li, A.; Ramakrishna, S. N.; Nalam, P. C.; Benetti, E. M.; Spencer, N. D. Stratified Polymer Grafts: Synthesis and Characterization of Layered 'Brush' and 'Gel' Structures. Adv. Mater. Interfaces 2014, 1 (1), 1300007.

47. Kooij, E. S.; Sui, X.; Hempenius, M. A.; Zandvliet, H. J. W.; Vancso, G. J. Probing the Thermal Collapse of Poly( $N$-Isopropylacrylamide) Grafts by Quantitative in Situ Ellipsometry. J. Phys. Chem. B 2012, 116 (30), 9261-9268.

48. Butt, H. J.; Jaschke, M. Calculation of Thermal Noise in Atomic-Force Microscopy. Nanotechnology 1995, $6(1), 1-7$.

49. Green, C. P.; Lioe, H.; Cleveland, J. P.; Proksch, R.; Mulvaney,P.; Sader, J. E. Normal and Torsional Spring Constants of AtomicForce Microscope Cantilevers. Rev. Sci. Instrum. 2004, 75 (6), 1988-1996.

50. Ducker, W. A.; Senden, T. J.; Pashley, R. M. Direct Measurement of Colloidal Forces Using an Atomic Force Microscope. Nature 1991, 353 (6341), 239-241.

51. Cannara, R. J.; Eglin, M.; Carpick, R. W. Lateral Force Calibration in Atomic Force Microscopy: a New Lateral Force Calibration method and General Guidelines for Optimization. Rev. Sci. Instrum. 2006, 77 (5), 053701.

52. Benetti, E. M.; Reimhult, E.; de Bruin, J.; Zapotoczny, S.; Textor, M.; Vancso, G. J. Poly(Methacrylic Acid) Grafts Grown From Designer Surfaces: the Effect of Initiator Coverage on Polymerization Kinetics, Morphology, and Properties. Macromolecules 2009, 42 (5), 1640-1647.

53. Benetti, E. M.; Zapotoczny, S.; Vancso, G. J. Tunable Thermoresponsive Polymeric Platforms on Gold by "Photoiniferter"- Based Surface Grafting. Adv. Mater. 2007, 19 (2), 268-271.

54. Yu, Y.; Kieviet, B. D.; Liu, F.; Siretanu, I.; Kutnyanszky, E.; Vancso, G. J.; de Beer, S. Stretching of Collapsed Polymers Causes an Enhanced Dissipative Response of PNIPAM Brushes Near Their LCST. Soft Matter 2015, 11 (43), 8508-8516.

55. Gunnewiek, M. K.; Ramakrishna, S. N.; di Luca, A.; Vancso, G.J.; Moroni, L.; Benetti, E. M. Stem-Cell Clinging by a Thread: AFM Measure of Polymer-Brush Lateral Deformation. Adv. Mater. Interfaces 2016, 3 (3), 1500456. 
Chapter 5

5 


\section{Chapter 6}

\section{Protective Coatings}

\section{for Complex Aluminum Substrates}

Part of this chapter is published in the final report of Marie Skłodowska-Curie ITNComplex Wetting (ITN-607861). 


\section{Contents}

Chapter 6 Protective Coatings for Aluminum Substrates

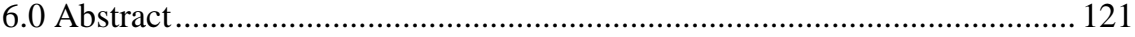

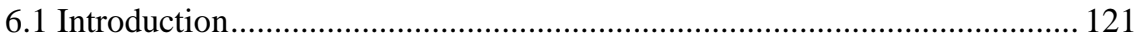

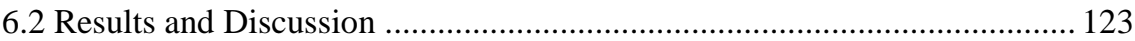

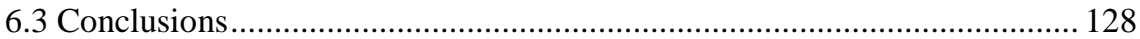

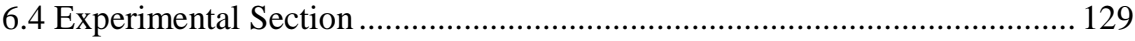

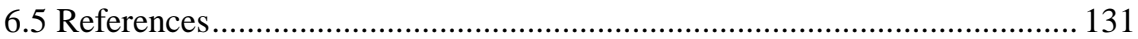




\subsection{Abstract}

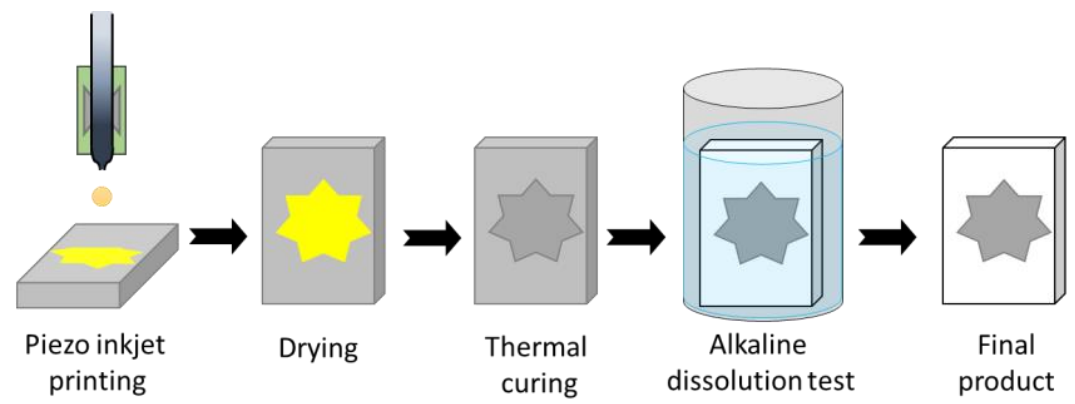

Printed

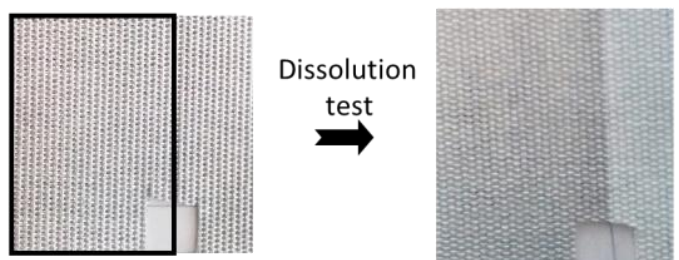

TOC Chapter 6: Schematic representation of the accelerated corrosion test for the inkjet printed protective coatings (top). Optical images of the printed samples before and after dissolution test (bottom).

This chapter presents and discusses the use of polyurethane-based films deposited by ink jet printing as protective coatings for aluminum metalized polyvinyl chloride (PVC) fabrics. It is demonstrated that ink jet printing is capable of covering porous fabrics, while the newly developed coatings are capable of sufficient anti-corrosion performance to pass the required alkaline dissolution test for one of the industrial partners involved in this project. Moreover, the spreading and evaporation processes of the ink on the porous fabrics were investigated as well as the effect a double-side printing approach on the delamination of the aluminum coating through the analysis with different microscopy techniques from macro- to nano-scale. We concluded that full surface coverage is not required to pass the corrosion test.

\subsection{Introduction}

In the past few decades, inkjet printing has evolved from an emerging technique to an established economic competitive method for the deposition of coatings with spatial control and high resolution. ${ }^{1-3}$ Besides the clear advantages of ink-jet printing, like producing patterns and gradients, compared to traditional techniques, like screen-printing and dip-coating, ${ }^{4-5}$ it is now also more frequently employed for full coating coverage.$^{6-7}$ This is mainly ascribed to the ability of inkjet printing to precisely deposit discrete dots onto surfaces so that the coating material is consumed only when needed, which can lead 
to material consumption reduction and allow for the production of diversified, smallvolume, and even personalized products. ${ }^{8}$

Following this trend, the industrial partners involved in the present project have also observed a clear shift from traditional coating methods toward printing porous substrates to replace conventional processes. The main challenge in understanding and optimizing this phenomenon is the competition between ink diffusion on the substrate and ink penetration in the pores before full coverage is achieved. ${ }^{9}$

Of particular interest to the industrial partners in this research project was assessing, in detail, the viability of inkjet printing to coat a metalized, polymer-based fabric using proprietary polyurethane-based inks prepared by TANATEX Chemicals B.V. (Ede, The Netherlands). ${ }^{10}$ The substrates supplied by Verosol (Eibergen, The Netherlands) ${ }^{11}$ consisted of microporous polyvinyl chloride (PVC) or polyethylene terephthalate (PET) fabrics coated with approximately a $100 \mathrm{~nm}$ aluminum layer deposited by physical vapour deposition (PVD). The function of the polyurethane-based coating was primarily to protect the aluminum layer from environmental influences that can cause corrosion.

Hence, full surface coverage without defects was required in order for the industrial partner to consider replacing their current roll-to-roll dip-coating process with an inkjet printing-based process. In order to evaluate the anti-corrosion performance of the various deposited coatings, they were exposed to an alkaline dissolution test, which is standard in accelerated corrosion testing. ${ }^{12-13}$ The primary objective was to identify the minimum printer passes required for the polyurethane-based ink dispersions to produce a functional anti-corrosion coating. Functional anti-corrosion coating, in this case, means that the deposited coating passed an accelerated alkaline dissolution test (see Experimental section). Moreover, the back side of the fabric was also coated since back-side coating was assumed to eventually result in full fabric coverage and, thus, reduce the chance of coating delamination. The process scheme and experimental design are shown in Figure 6.1 and 6.2, respectively.

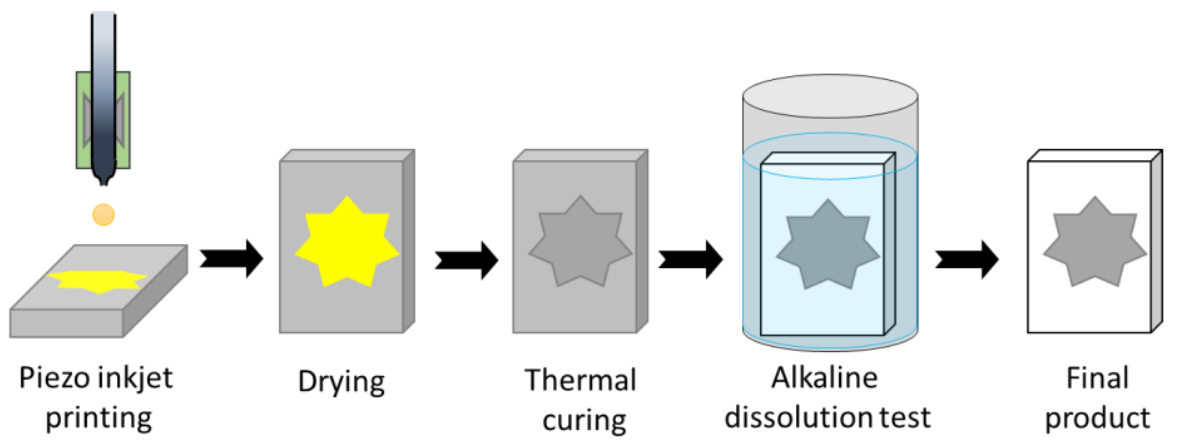

Fig. 6.1: Block diagram of the inkjet printing deposition of polyurethane-based coatings on metalized fabrics followed by an accelerated alkaline corrosion test. 
Front: Aluminum metalized side

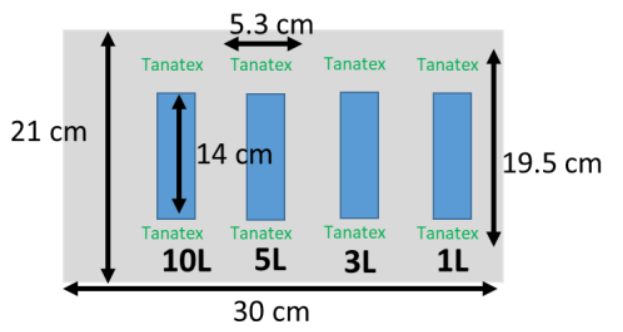

Back: PVC side

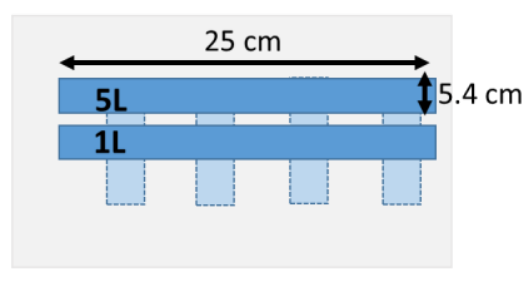

Fig. 6.2: Experimental design to evaluate the number of printed passes needed to obtain a satisfactory anticorrosion performance. The front and back sides are the metalized and not metalized sides of the fabrics, respectively.

\subsection{Results and Discussion}

In this section, we summarize the generic findings that were obtained during the course of this research with a focus on the "deposition and characterization challenges".

Firstly, the complex fluids were designed as inks suitable to be employed in the inkjet printing process (i.e. the viscosity within the range of values $2-15 \mathrm{cP}$ and the surface tension within the range of $\left.20-50 \mathrm{mN} \cdot \mathrm{m}^{-1}\right){ }^{3,5,7}$ The exact composition of the pigments and of the dispersant of all the Tanatex inks is proprietary. A generic description of the physical and chemical properties of the inks is summarized in Table 6.4. The difference between the inks was in the type and amount of the functional material and/or in the type of crosslinking agent. Ink $\mathrm{A}$ is an emulsion of an oligomeric aliphatic polyether functionalized with isocyanate groups, ink B is an emulsion with a high molecular weight (MW) polycarbonate and polyurethane, ink $\mathrm{C}$ is a non-ionic telechelic polycarbonate polyurethane dispersion with a short-chain extender, while ink D has the same functional material of the ink $\mathrm{C}$ but with a long amine as chain extender.

Various patterns were printed on the porous fabrics followed by a drying process as explained more in detail in the experimental part. Following the deposition of the various polyurethane-based inks by inkjet printing, selected fabrics were inspected with scanning electron microscope (SEM) imaging. Figure 6.3 shows top-view SEM images of a metalized PVC fabric coated with one, five, and ten layers of ink B. From Figure 6.3, it is clear that one layer of deposited ink is not sufficient to obtain full surface coverage, while five layers nearly provide full surface coverage. Complete surface coverage of the metalized PVC fabric was obtained upon the deposition of ten layers. Furthermore, from Figure 6.3, it is clear that neither splashing nor the coffee stain effect occurred. In addition, the ink wetted the surface well. The good ink substrate wettability was the same for all the inks evaluated.

Figure 6.4 shows a SEM image of the metalized side of an Al-met. PVC fabric coated with five layers of ink B, in which pore clogging (arrow I) and ink spanning of the contact 
edges (arrow II) as well as the woven yarn corners (arrow III) are clearly visible. This can be ascribed to capillary effects caused by the woven structure of the fabric and the cylindrical shape of the yarn. We note that pore clogging should be avoided as much as possible, since it can alter the optical properties of the fabric.

\section{One printed layer - Ink B}
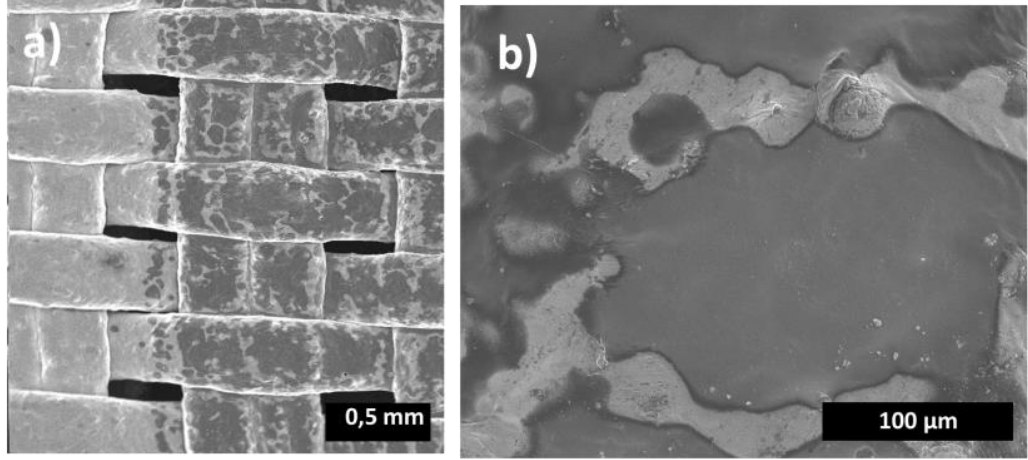

Five printed layer - Ink B
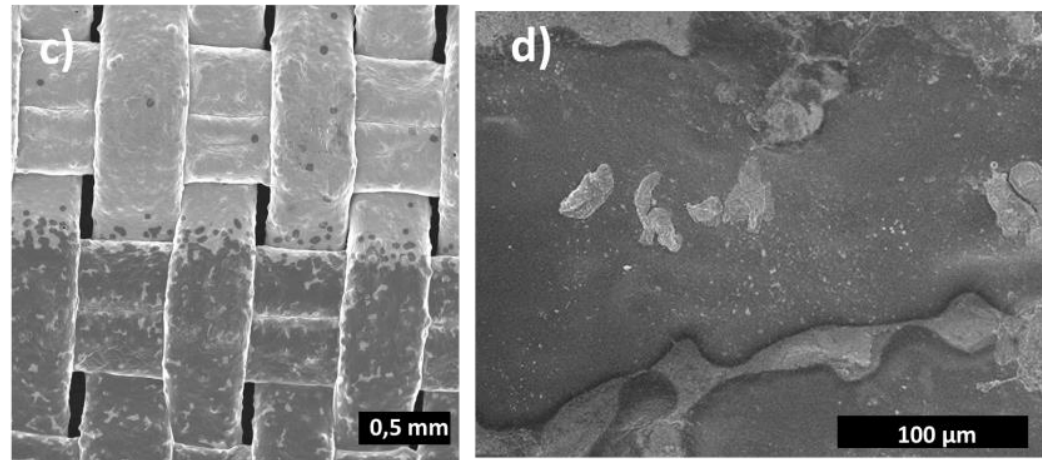

Ten printed layer - Ink B
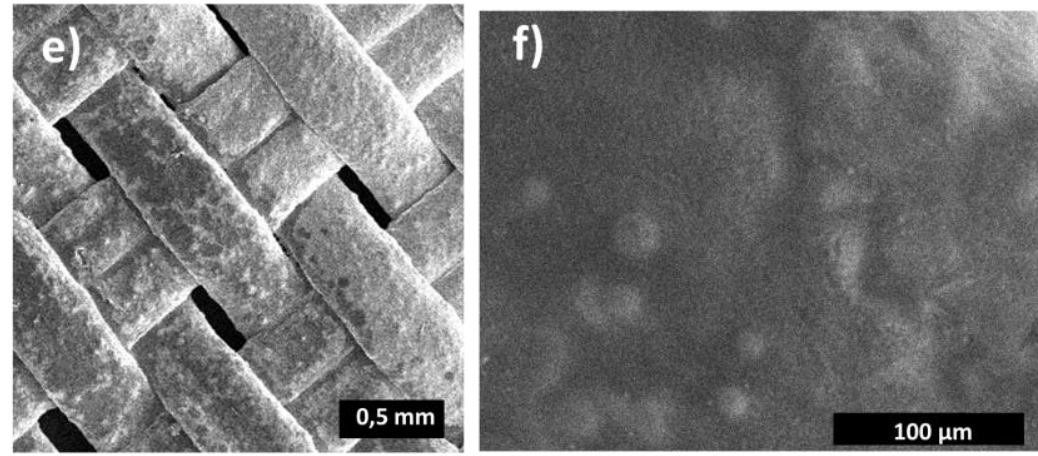

Fig. 6.4: SEM images of the metalized side of the Al-met. PVC fabrics coated with one (a) - b)), five (c) d)), and ten layers (e) - f)) of ink B, showing the surface coverage of the PVC fabric. 


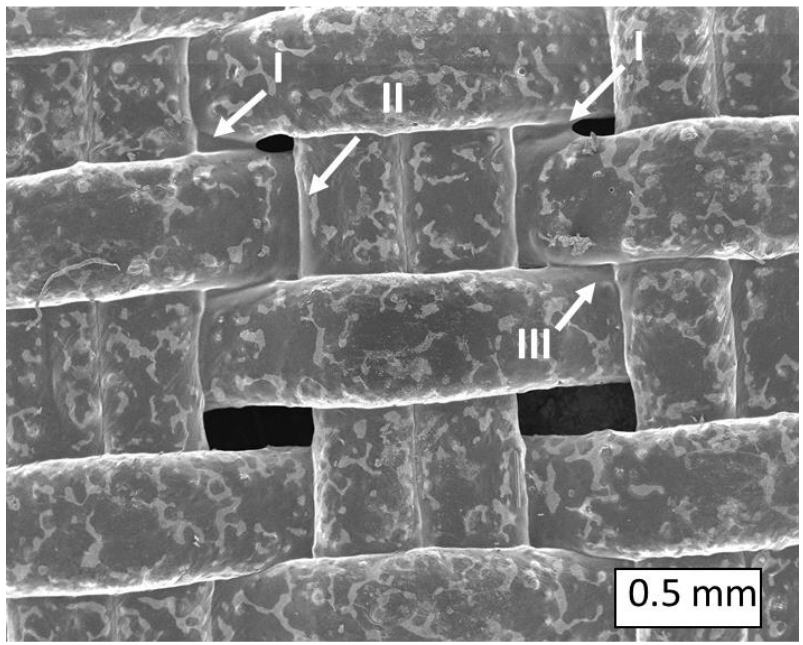

Fig. 6.4: SEM image of the metalized side of the Al-met. PVC fabrics coated with five layers of ink B, showing the clogging and reduction of pore size of the PVC fabric (arrow I), as well as the accumulation of ink in the contact edge (arrow II) and in the corners of the woven yarn (arrow III).

Following the successful deposition of the various inks using the layer-by-layer approach according to the scheme depicted in Figure 6.2, the anti-corrosion performance of the coatings obtained was qualitatively assessed by the accelerated alkaline immersion tests. To demonstrate the accelerated nature of this test, untreated metalized fabrics were first immersed in a sodium carbonate solution. This resulted in the complete disappearance of the $0.1 \mu \mathrm{m}$ deposited aluminum layer within four minutes, as can be seen in Figure 6.5.

As received After alkaline test

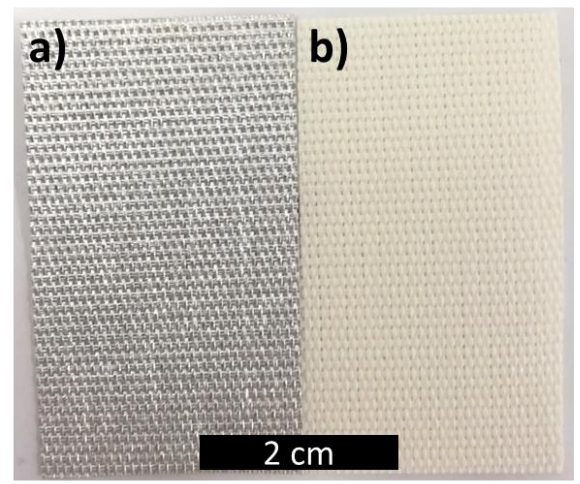

Fig. 6.5: Photographs of Al-metal. PVC samples before (A) and after (B) the accelerated alkaline immersion test.

Table 6.1 shows images of the Al-Met. PVC fabric with one and five printed layers of ink B on the front side of the fabric and five layers printed on the back side of the fabric 
before and after the alkaline immersion tests, respectively. From Table 6.1, it is evident that the deposition of one layer of ink B (with a dry thickness of the coating layer within the range of 50-100 $\mathrm{nm}$ ) at the front side of the fabric is not sufficient to prevent the corrosion of the aluminum metalized layer (compare Table 6.1 A and 6.1B). This was expected, since one printed layer did not protect a large surface area, as is shown in Table 6.1. Following the deposition of the five layers of ink B on the front side of the fabric, a sufficient corrosion protection was obtained (compare Table 6.1C and 6.1D).

Table 6.1: Visual inspection of the front side of metalized PVC fabrics coated with different number of layers of ink B on the front side of the fabric and five layers on the back of the fabric. The optical images compare the aluminum metalized side of the fabric coated with one layer on the front side and five layers on the back side of the fabric before and after the alkaline test, a) and b), respectively, and with five layers printed on the front side and on the back side of the fabric before and after the accelerated alkaline immersion test, c) and d), respectively.

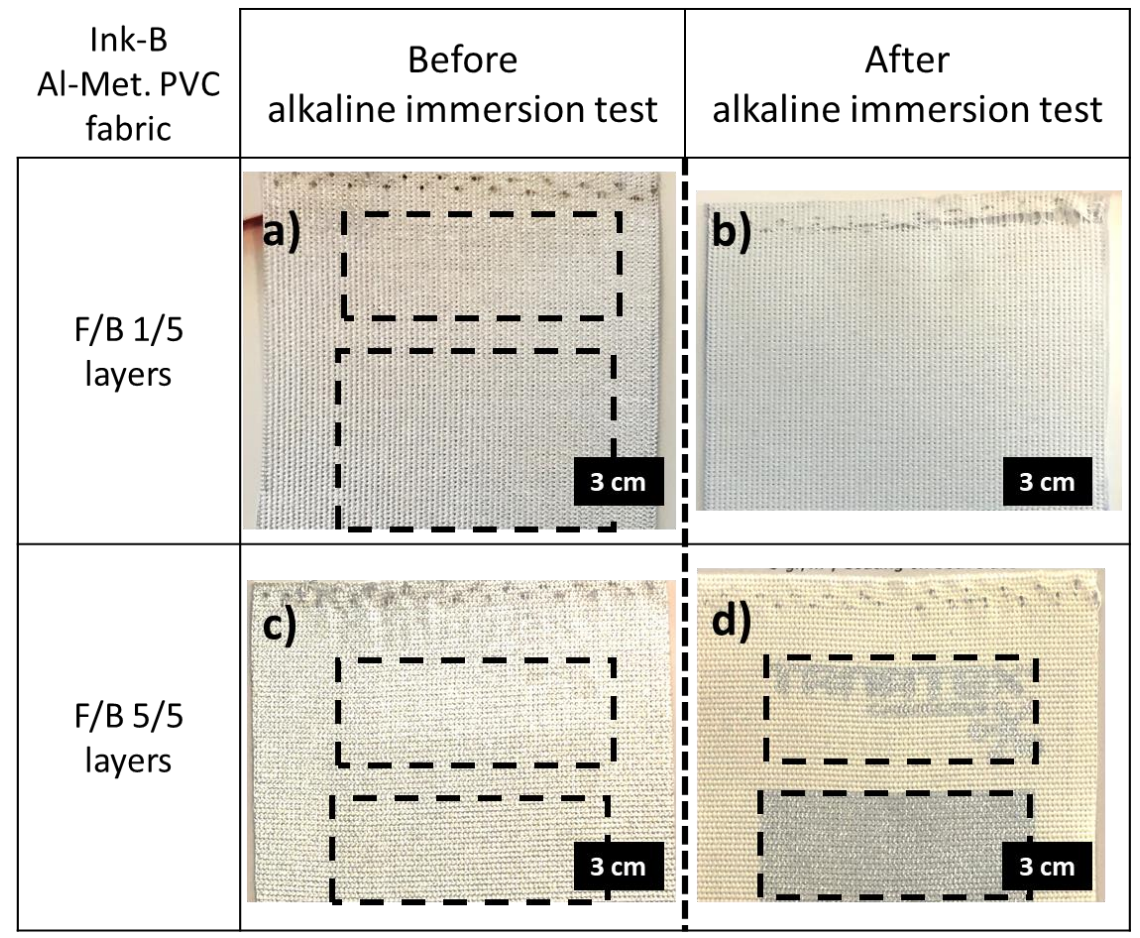

The qualitative visual assessment of the anti-corrosion performance of the various inks is presented in Table 6.2. Based on changes in color and a reflection of the aluminum layer, the anti-corrosion performance was classified as follows:

-/- the metalized layer was fully consumed.

- the metalized layer was consumed for $>50 \%$ (surface coverage) and partly changed the appearance. 
+ the metalized layer was consumed for $<50 \%$ (surface coverage) and partly changed the appearance.

$+/+$ the metalized layer did not show signs of metalized layer consumption nor did it change its appearance.

Table 6.2: Qualitative visual assessment of the anti-corrosion performance of the inkjet-printed inks through the layer-by-layer approach on the Al-met. PVC fabric.

\begin{tabular}{|c|c|c|c|c|c|c|}
\hline & lkali & $\mathrm{imm}$ & rsion test & on metalli & ed PV & bric \\
\hline Back la & t layer & 0 layer & $\begin{array}{c}1 \text { layer } \\
(50-100 \mathrm{~nm})\end{array}$ & $\begin{array}{c}3 \text { layers } \\
(100-300 \mathrm{~nm})\end{array}$ & $\begin{array}{l}5 \text { layers } \\
(500 \mathrm{~nm}\end{array}$ & $\begin{array}{c}10 \text { layers } \\
(1000 \mu \mathrm{m})\end{array}$ \\
\hline Ink-A & $5 \mathrm{~L}$ & n.d. & -- & ++ & n.d. & n.d. \\
\hline & $O L$ & -- & -- & -- & -- & + \\
\hline Ink-B & $1 \mathrm{~L}$ & -- & -- & -- & -- & ++ \\
\hline & $5 \mathrm{~L}$ & -- & -- & -- & + & ++ \\
\hline & $\mathrm{OL}$ & -- & -- & -- & + & ++ \\
\hline Ink-C & $1 \mathrm{~L}$ & -- & -- & -- & + & ++ \\
\hline & $5 \mathrm{~L}$ & -- & -- & -- & ++ & ++ \\
\hline & OL & -- & -- & -- & - & + \\
\hline Ink-D & $1 \mathrm{~L}$ & -- & -- & -- & + & ++ \\
\hline & $5 \mathrm{~L}$ & -- & -- & + & + & ++ \\
\hline & & ++ & & o Damage & & \\
\hline & & + & First s & gn of corrosio & & \\
\hline & & - & Moderate $\mathrm{d}$ & mage (less tha & $50 \%)$ & \\
\hline & & -- & Comp & etely destroye & & \\
\hline & & n.d. & & No Data & & \\
\hline
\end{tabular}

From Table 6.2, it is clear that upon increasing the number of layers on the front as well as the back side of the fabric the anti-corrosion performance increased. The best performance of the polycarbonate polyurethane-based inks was obtained from the deposition of 10 layers of ink $\mathrm{C}$ on the front side of the metalized PVC fabric, with no need to print the back side of the fabric. Alternatively, using the same total number of ink $\mathrm{C}$ layers by printing five layers on both the front and back sides of the metalized PVC fabric resulted in a qualitative pass as well. Although not investigated in detail, it is thought that this can be ascribed to a decrease in pore size after the deposition of five layers of ink, as is shown in Figure 6.4. Despite the fact that it requires the same total number of layers 
and thus the ink consumption is the same, coating of both fabric sides requires additional turning of the sample, which is an additional processing step and, as such, is not preferred.

Provided the nearly identical inkjet conditions used for the deposition of the inks, it can be assumed that similar ink volumes per $\mathrm{cm}^{2}$ were deposited for ink B and ink C. Hence, based on identical surface tensions and differences in solid content, it was expected that the amount of material deposited by ink B (solid content 43 wt.\%) was higher compared to ink C (solid content $30 \mathrm{wt} . \%$ ). Despite this, ink B required more layers in order to pass the alkaline immersion test; however more important to the assessment of ink type effects on alkaline resistance is identifying the chemical composition of the inks, ${ }^{14}$ which, for the purpose of this project, did not have to be disclosed. Next to this, we note the good performance of ink A, with three front layers and five back layers, it exhibits the lowest amount of deposited solid while outperforming the other ink types. This may be ascribed to the known chemical resistance of polyether-based polyurethanes, ${ }^{15-16}$ nevertheless, without detailed information on the exact ink composition, this conclusion is merely speculative. However, it demonstrates that, by optimizing the ink composition, the amount of ink as well as the overall number of layers required to pass the accelerated alkaline dissolution test can be significantly reduced.

Moreover, as shown in Figure 6.3, the ink surface coverage from five layers of ink was not complete. This raises the question of why some of these deposited ink layers pass the corrosion test. The answer lies in the corrosion mechanism and inhibition from applying coatings. Aluminum surfaces in the air rapidly and naturally form an aluminum oxide layer that protects the inner aluminum surface from further oxidation; however when exposed to more aggressive environments, such as the alkaline solution used in the immersion test, the oxide layer becomes soluble and its rapid dissolution occurs more quickly than the formation of a new oxide layer. ${ }^{17-18}$ This eventually leads to auto catalytic pitting corrosion in which the exposed aluminum layer and the oxide layer act as anode and cathode, respectively. ${ }^{19}$

Through the deposition of a polymer coatings, a barrier formed to prevent the penetration of corrosive species (i.e. corrosive $\mathrm{OH}^{-}$ions), thus minimizing the electrochemical reactions on the aluminum surface. ${ }^{19}$ As such, the corrosion was significantly reduced even when the surface coverage was not complete.

\subsection{Conclusions}

Inkjet printing was successfully applied to deposited polyurethane-based coatings on metallized PVC fabrics. Through a layer-by-layer approach, full surface coverage was obtained after ten printer passes. Eventually, the anti-corrosion performance of the deposited coatings was evaluated by an alkaline dissolution test, and the deposition of ten layers of ink was found sufficient to pass the test. Meanwhile, for ink C, it was 
demonstrated that the deposition of five layers on both the front and back sides of the fabric was sufficient to pass the test, meaning that a nearly full surface coverage of ink is, depending on the ink composition, already sufficient to prevent the aluminum layer from corroding.

\subsection{Experimental section}

\section{Materials and chemicals}

Chemicals. Sodium carbonate $\left(\mathrm{Na}_{2} \mathrm{CO}_{3},>99.0 \%\right)$ was purchased from Sigma Aldrich, and Milli-Q water (Advantage A-10, Millipore, Merk, Darmstadt, Germany) was used throughout the experiments.

Inks. Four different inks were obtained from TANATEX Chemicals B.V. (Ede, the Netherlands). The composition as well as the physical and chemical properties of the inks are summarized in Table 6.3. The physical properties were tailored to make the inks suitable for inkjet printing. Specifically, ink-A was an aqueous emulsion of an oligomeric aliphatic polyether functional isocyanate sulfite-blocked polyurethane, while ink-B was an aqueous emulsion of a high molecular weight polycarbonate polyurethane dispersion. Ink-C and ink-D consisted of nonionic telechelic polycarbonate polyurethane dispersions containing a short amine (ethylene diamine) and long (C36 diamine) amine-terminated chain extenders, respectively.

Table 6.3: Physical and chemical properties of the four obtained inks.

\begin{tabular}{|c|c|c|c|c|c|}
\hline \multicolumn{2}{|c|}{ Ink - TANATEX } & Ink-A & Ink-B & Ink-C & Ink-D \\
\hline \multicolumn{2}{|c|}{ Composition } & $\begin{array}{l}\text { Aqueous emulsion of } \\
\text { oligomeric aliphatic } \\
\text { polyether functional } \\
\text { isocyanate, sulphite } \\
\text { blocked }\end{array}$ & $\begin{array}{l}\text { Aqueous emulsion of } \\
\text { high MW } \\
\text { polycarbonate } \\
\text { polyurethane } \\
\text { dispersion }\end{array}$ & \begin{tabular}{|} 
Non ionic telechelic \\
polycarbonate \\
polyurethane \\
dispersion with short \\
amine chain \\
extender \\
\end{tabular} & $\begin{array}{c}\text { Non ionic telechelic } \\
\text { polycarbonate } \\
\text { polyurethane } \\
\text { dispersion with long } \\
\text { amine chain } \\
\text { extender }\end{array}$ \\
\hline \multicolumn{2}{|c|}{ Appearance } & $\begin{array}{l}\text { Colorless, } \\
\text { transparent }\end{array}$ & Opaque, turbid & $\begin{array}{l}\text { Colorless, } \\
\text { transparent }\end{array}$ & $\begin{array}{l}\text { Colorless, } \\
\text { transparent }\end{array}$ \\
\hline $\mathrm{pH}$ & (upH) & $5-6$ & $7,5-8,5$ & $5-6$ & $8,5-9,5$ \\
\hline $\begin{array}{l}\text { Surface } \\
\text { Tension }^{1}\end{array}$ & $(\mathrm{mN} / \mathrm{m})$ & 28 & 27 & 27 & 29 \\
\hline Viscosity ${ }^{2}$ & $\left(\mathrm{mPa}^{*} \mathrm{~s}\right)$ & 6 & 9 & 6 & 12 \\
\hline $\begin{array}{l}\text { Temperature } \\
\text { of Curing }\end{array}$ & $\left({ }^{\circ} \mathrm{C}\right)$ & $160^{\circ} \mathrm{C}$ & $160^{\circ} \mathrm{C}^{*}$ & $160^{\circ} \mathrm{C}$ & $160^{\circ} \mathrm{C}$ \\
\hline Solid Content ${ }^{3}$ & $(\% w / w)$ & 14 & 43 & 30 & 25 \\
\hline
\end{tabular}

1) The surface tension was measured by the pendant drop method. 2) The viscosity was determined via rheology measurements and 3) the solid content was determined via gravimetric measurements of small aliquots of the inks. 
Woven fabrics. The Verosol (Eibergen, the Netherlands) provided fabrics consisting of PVC-coated glass fiber yarns, the surfaces of which were aluminum-metalized on one side (from now on, denoted as Al-met. PVC fabric).

The thickness of the fabric was $500 \mu \mathrm{m}$, and the thickness of the PVD deposited aluminum layer was approximately $100 \mathrm{~nm}$. The reflectivity of the metalized fabric side was clearly visible in the laser confocal microscopy images shown in Figure 6.6. The pore area between the yarns was approximately $0.09 \mathrm{~mm}^{2}$, which was determined from optical microscopy and porosimetry measurements (see Figure 6.6). The samples were obtained as $30 \times 21 \mathrm{~cm}$ sheets and used as received.

\begin{tabular}{|c|c|c|}
\hline \multicolumn{2}{|l|}{ Substrates } & Al-met. PVC fabric \\
\hline \multirow{2}{*}{ Composition } & Front & Al (metalized) \\
\hline & Back & Glass fibers covered by PVC \\
\hline Thickness & (mm) & 0.50 \\
\hline Weight & $\left(\mathrm{g} / \mathrm{m}^{2}\right)$ & 400 \\
\hline Woven Structure & & 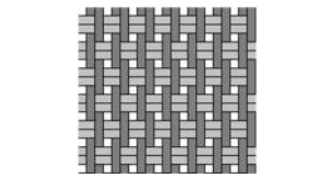 \\
\hline \multirow{2}{*}{ Pore size } & $(\mu \mathrm{m})$ & $150 \times 350$ \\
\hline & $(\mu \mathrm{m})$ & $101^{\mathrm{a}}$ \\
\hline
\end{tabular}
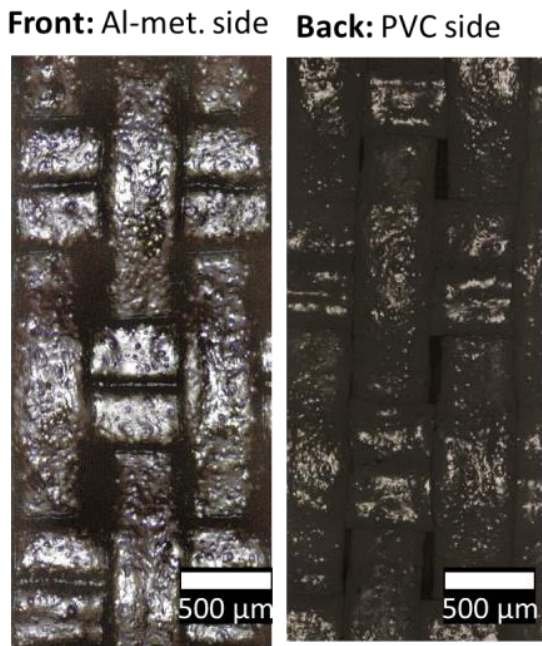

a) As determined by wetting liquid porosimetry.

Fig. 6.6: Properties of the Al-met. PVC fabric and laser confocal microscopy images of its front and back sides.

\section{Inkjet printing}

Continuous inkjet printing was carried out with a commercial Epson Stylus Pro 4880 inkjet printer (Epson America, Inc, America) equipped with a micro-piezo Epson F187000 print head consisting of eight nozzle lines (corresponding to eight channels) with 180 nozzles per line and a nozzle diameter of $10 \mu \mathrm{m}$. In operation, the firing distance between the substrate and print head was approximately $1 \mathrm{~cm}$. Each ink layer was printed at room temperature under default inkjet printing conditions that were optimized to achieve a resolution of $1440 \times 720$ dpi. First, the back side of the fabric was coated with one, three, and five layers, which was followed by evaporation of the ink solvent into the air for a few minutes and the subsequent printed patterns being thermally cured at $160{ }^{\circ} \mathrm{C}$ for 10 minutes; then, the metalized side was coated with one, three, five and ten layers of the same ink used on the back side, air dried for few minutes, and subsequently thermally cured at $160{ }^{\circ} \mathrm{C}$ for 10 minutes. 


\section{Alkaline corrosion test}

The anti-corrosion performance of the inkjet-deposited coatings was evaluated by an accelerated alkaline corrosion test, as is schematically shown in Figure 6.1. The test conditions given below are in accordance with an internal standard test (Verosol). First, the coated substrates were washed with ethanol and then air dried. Subsequently, the printed samples were immersed in an aqueous solution of $1 \mathrm{wt} \%$ sodium carbonate $(\mathrm{pH}$ of the solution approximately 11.6) at $40{ }^{\circ} \mathrm{C}$ for 30 minutes. Next, the samples were washed with copious amounts of Milli-Q water and ethanol, after which they were left to dry for visual evaluation of the anti-corrosion performance.

\section{Characterization techniques}

Optical microscopy, OM. A BX60 Olympus microscope (Olympus Europa SE \&Co, Germany) equipped with $5 \mathrm{x}, 10 \mathrm{x}$, and 20x magnification objective lenses was used to investigate the morphology of the samples before and after the printing process as well as after the alkaline corrosion test.

Laser confocal microscopy. In order to study the quality of the deposited coatings and deposited aluminum layer after the alkaline corrosion test, a laser confocal Keyence VK 9710 K microscope (LSCM, violet laser VK-9700, Keyence Corporation, Osaka, Japan) featuring a 10x magnification objective lens was used.

Scanning electron microscopy, SEM. Top-view surface electron microscopy images of the fabrics were obtained from a field-emission SEM (JSM-633OF, JEOL, Benelux) at $3 \mathrm{keV}$ accelerating voltage.

Liquid wetting porosimetry. The pore volume distribution (PVD) of the fabrics was determined by liquid porosimetry. ${ }^{20}$ The PVD was determined with an auto-porosimeter (TRI/Priston, USA) using a $0.1 \mathrm{~g} \cdot \mathrm{L}^{-1}$ of Triton X-100 (a non-ionic surfactant wetting liquid, $\cos \theta=1)$ in Milli-Q water. The chamber height was $6.4 \mathrm{~mm}$. Measurements were performed in receding mode for the range of 1 to $900 \mu \mathrm{m}$ of $2 \times 3$ pre-saturated samples cut randomly from the fabric sample. The final PVD was obtained as the average of three measurements of the different samples. The thickness of the pre-saturated fabric was measured with a caliber. The surface tension of the solution was $30 \mathrm{mN} \cdot \mathrm{m}^{-1}$ and the density was $1 \mathrm{~g} \cdot \mathrm{cm}^{-3}$. The liquid retention was studied by examining absorption/desorption hysteresis loops (i.e. a sequence of intrusion-extrusion curves) monitoring the cumulative uptake of liquid from the dry fabric.

\subsection{References}

1. Calvert, P. P. P., Inkjet printing forS materials and devices. Chemistry of Materials 2001, 13 (10), 32993305 .

2. Menard, E.; Meitl, M. A.; Sun, Y.; Park, J.-U.; Shir, D. J.-L.; Nam, Y.-S.; Jeon, S.; Rogers, J. A., Microand nanopatterning techniques for organic electronic and optoelectronic systems. Chemical Reviews 2007, 107 (4), 1117-1160. 
3. Hoath, S. D., Fundamentals of inkjet printing: the science of inkjet and droplets. John Wiley \& Sons: 2016.

4. de Gans, B. J.; Schubert, U. S., Inkjet Printing of Polymer Micro-Arrays and Libraries: Instrumentation, Requirements, and Perspectives. Macromolecular Rapid Communications 2003, 24 (11), 659-666.

5. Tekin, E.; Smith, P. J.; Schubert, U. S., Inkjet printing as a deposition and patterning tool for polymers and inorganic particles. Soft Matter 2008, 4 (4), 703-713.

6. Singh, M.; Haverinen, H. M.; Dhagat, P.; Jabbour, G. E., Inkjet printing-process and its applications. Advanced Materials 2010, 22 (6), 673-685.

7. Derby, B., Inkjet printing of functional and structural materials: fluid property requirements, feature stability, and resolution. Annual Review of Materials Research 2010, 40, 395-414.

8. Zapka, W., Handbook of Industrial Inkjet Printing: A Full System Approach. John Wiley \& Sons: 2017.

9. Complex wetting: reasearch and training, Home page, www.CoWet.eu (accessed October 2019)

10. Tanatex - Textile Processing Solutions, Home Page, https://tanatexchemicals.com (accessed October 2019)

11. Verosol, Home page, https://verosol.nl (accessed October 2019)

12. Šimor, M.; Fiala, A.; Kováčik, D.; Hlídek, P.; Wypkema, A.; Kuipers, R., Corrosion protection of a thin aluminium layer deposited on polyester. Surface and Coatings Technology 2007, 201 (18), 7802-7812.

13. Xhanari, K.; Finšgar, M., Organic corrosion inhibitors for aluminum and its alloys in chloride and alkaline solutions: a review. Arabian Journal of Chemistry 2016.

14. Kim, B. S.; Kim, B. K., Enhancement of hydrolytic stability and adhesion of waterborne polyurethanes. Journal of Applied Polymer Science 2005, 97 (5), 1961-1969.

15. Pavlova, M.; Draganova, M.; Novakov, P., Hydrolytic stability and protective properties of polyurethane oligomers based on polyester/ether/polyols. Polymer 1985, 26 (12), 1901-1905.

16. Akindoyo, J. O.; Beg, M.; Ghazali, S.; Islam, M.; Jeyaratnam, N.; Yuvaraj, A., Polyurethane types, synthesis and applications-a review. Rsc Advances 2016, 6 (115), 114453-114482.

17. Pourbaix, M., Atlas of electrochemical equilibria in aqueous solution. NACE 1974, 307.

18. Padash, R., Experimental and theoretical study of aluminium corrosion in $\mathrm{NaOH}, \mathrm{NaCl}$ and $\mathrm{HCl}$ solutions. Anti-Corrosion Methods and Materials 2018, 65 (4), 350-360.

19. Abdeen, D. H.; El Hachach, M.; Koc, M.; Atieh, M. A., A Review on the Corrosion Behaviour of Nanocoatings on Metallic Substrates. Materials 2019, 12 (2), 210.

20. Miller, B.; Tyomkin, I., Liquid porosimetry: new methodology and applications. Journal of Colloid and Interface Science 1994, 162 (1), 163-170. 
Chapter 7

\section{Grafting-To and Grafting-From Approaches to Obtain Stimuli-Responsive Patterned Fluorescent Polymer Films by Combining ATRP and Inkjet Printing}

* This chapter has been published in: (a) Tas S., Kopec M., van der Pol R., Cirelli M., de Vries I., Bolukbas D.A., Tempelman K., Benes N.E., Hempenius M.A., Vancso G.J., de Beer S.; Macromolecular Chemistry and Physics. 2019, 1800537. (b) Kopec M., Tas S., Cirelli M., van der Pol R., de Vries I., Vancso G.J., de Beer S.; ACS Applied Polymer Materials. 2019, 1, 136-140. 


\section{Contents}

\section{Chapter 7 Grafting-To and Grafting-From Approaches to Obtain}

Stimuli-Responsive Patterned Fluorescent Polymer Films by Combining ATRP and Inkjet Printing ...................................... 133

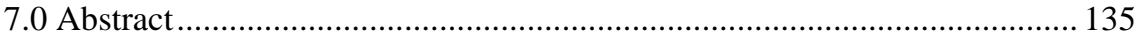

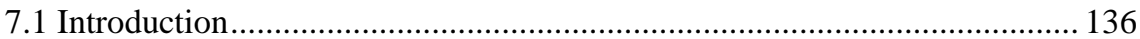

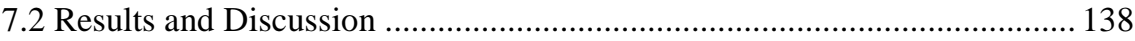

7.2.1 Preparation of Polymer Films........................................................... 138

7.2.2 Co-solvency behaviour of Fluorescein End-Tethered PMMA Grafts obtained both via GF and GT Approaches in IPA-W ................................ 144

7.2.3 Preparation of Fluorescent Patterns ................................................ 147

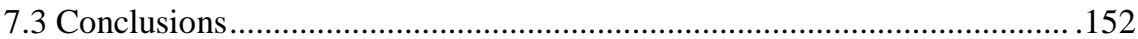

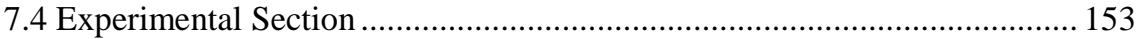

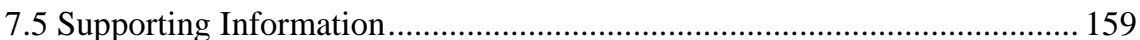

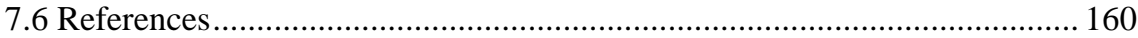




\subsection{Abstract}
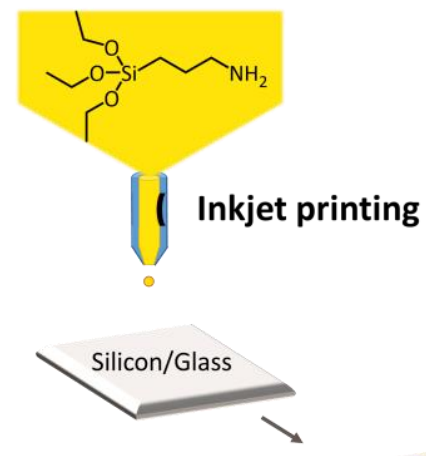

\section{Thermal curing}

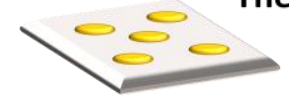

Grafting-to

1. ARGET-ATRP in sol.

2. Exchange $\mathrm{Br} / \mathrm{N}_{3}$

3. Click reaction

4. Coupling

\section{Grafting-from}

1. Coupling ATRP Ini.

2. SI-ATRP

3. Exchange $\mathrm{Br} / \mathrm{N}_{3}$

4. Click reaction
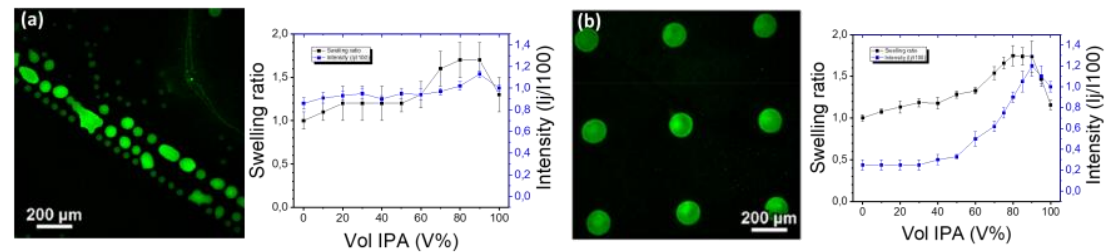

TOC of Chapter 7: Schematic representation of the fabrication GT and GF routes to prepare fluorescent labeled PMMA patterns combining inkjet printing and ATRP. Fluorescence response and solvent-dependency of the fluorescence response of the printed patterns on glass.

The preparation of alcohol-responsive fluorescent ultrathin films and patterns is presented via chemical vapour deposition (CVD) or inkjet printing (IJP) combined with the grafting-from (GF) and grafting-to (GT) deposition approaches. Firstly, we report the synthesis of a telechelic $\alpha$-carboxyl, $\omega$-fluorescein-functionalized PMMA $(\alpha$-COOH-PMMA- $\omega$-FAM $) \quad$ via activators regenerated by electron transfer atom transfer radical polymerization (ARGETATRP), bromide-azide exchange, and copper(I)-catalyzed alkyneazide cycloaddition (CuAAC) click reactions. Then, the anchoring via GT approach of the telechelic $\alpha-\mathrm{COOH}$ PMMA- $\omega$-FAM to an amine-functionalized silicon or glass substrates was obtained via dip coating. Secondly, the fabrication of the fluorescent films employing SI-ATRP, bromide-azide exchange, and $\mathrm{CuACC}$ click reactions is described. For both fabrication routes, the solvent-induced fluorescence response of the thin films was investigated varying the solvent quality in a iso-propanol/water mixture (IPA-W). Finally, fluorescent patterns were obtained via inkjet printing for both GF and GT approaches. The formulation 
and the printability of an APTES-based functional ink, as well as the surface characterization of the patterns are reported.

\subsection{Introduction}

One of particular interest surface modification approaches is the employment of surfacegrafted polymer films (e.g., single end-tethered polymer chains) as ultrathin functional nanocoatings. ${ }^{1-5}$ Surface-grafting of polymers has emerged as a powerful means of surface modification and preparation of hybrid materials, thanks to the interesting properties of polymer chains with functional moieties (e.g., recognition units, dye probes, etc.) and the stimuli-responsiveness to the different stimuli of the surrounding (such as $\mathrm{pH}$, temperature, ionic strength, pressure, and solvent). ${ }^{1-3}$

Surface-grafted polymer films can be easily obtained by various techniques. ${ }^{1-5}$ For example, reversible deactivation radical polymerizations (RDRP) enable a controlled synthesis of polymers with precisely tailored molecular weights, dispersities, chain topologies, and functionalities.$^{6-8}$ Two general approaches to fabricate surface-anchored polymer layers involve the attachment of end-functionalized polymer chains to a surface modified with reactive groups $(\mathrm{GT})^{9}$ or surface-initiated polymerization from a surface-immobilized initiator layer (GF). ${ }^{3-4,10}$ The grafting-from is a predominantly preferred approach for the single end-anchored polymer films, as it leads to higher grafting densities and thicknesses, forming a polymer brush layer., ${ }^{11-13}$ Nevertheless, a coupling of a pre-synthesized polymer to the surface allows more precise characterization of molecular weight, dispersity and chain-end functionality (CEF) of tethered chains before the immobilization. Furthermore, a relatively feasible scale-up due to the simplicity of the method makes grafting-to promising for preparation of functional polymer nano-coatings. ${ }^{9}$ However, this approach leads to lower grafting densities of the single endtethered polymer chains and the formation of a thin film where the chains assume the mushroom conformation. ${ }^{14-16}$ The choice of the grafting approach strongly influences the application and the physical and chemical properties of the polymer layer because the surface properties as well as the responsiveness of the polymer coatings are strongly affected by the grafting density of the anchored chains.

Dye labeling of polymers at specific sites in the chain is a versatile method to follow various physicochemical phenomena occurring on the nanoscale. ${ }^{17-18}$ For example, fluorescent dye-labeled polymers have been used to study polymer chain dynamics in solution, ${ }^{19-21}$ the local glass transition temperature of block copolymers ${ }^{22}$ and the structure of block copolymer micelles. ${ }^{23}$ Fluorescent dye-labeled stimuli-responsive polymer brushes have been prepared and utilized in ion $^{24-26}$ sensing and organic light-emitting diode (OLED) ${ }^{27-29}$ or in studying the degrafting kinetics of brushes. ${ }^{30}$ In this context, responsive polymer brushes were modified with fluorescent probes and widely used in the design of stimuli-responsive sensors. ${ }^{31-34}$ Chain-end labeling of polymers with a dye 
is anticipated to be a promising route to incorporate chemical and bio-sensing functionality in polymer-based surface coatings. Precise localization of chromophores in polymer nanostructures can be especially useful in light-harvesting applications, where aggregation of chromophores is often a serious limitation. ${ }^{35-37}$ Attaching a dye at the free end of a polymer chain rather than at the side group of each repeating unit may lead to a lower aggregation and prevent the self-quenching, thus the quantum efficiency of the emitting layer increases. ${ }^{35,38}$ Additionally, it can ensure a minimal interaction of the dye with the surface as well as enable further selective binding of molecules to the endfunctionalized film. ${ }^{17}$

To date, limited examples of the dye chain-end labeled polymer brushes include converting alkyl bromide end groups of polymer brushes to amines before reacting with an isothiocyanate-functionalized dye $\mathrm{e}^{39}$ and nitroxide radical exchange during polymerization. ${ }^{27}$ On the other hand, various types of click chemistry were used to modify chain-ends of polymer brushes with e.g., recognition elements, ${ }^{12,40-42} \mathrm{DNA},{ }^{43}$ and polyethylene glycol (PEG). ${ }^{44}$ Most fluorescently labeled polymer brushes have been prepared by functionalizing side chains with dye molecules as a post-polymerization step, ${ }^{24,28,30,45}$ by copolymerization with a fluorescent monomer ${ }^{26,33,46-47}$ or by selfassembly of charged fluorescent molecules on charged polymer brushes by electrostatic interactions. ${ }^{48}$ Controlling the selective immobilization of dye chain-end labeled polymer chain patterns is very important in areas such as sensing, cell adhesion, nanoelectronics, 'smart textiles', etc. ${ }^{49-50}$ For instance, microcontact printing has been employed to fabricate coatings introducing surface patterns at the stage of the initiator deposition, followed by a surface-initiated polymerization ${ }^{27,51-52}$ or to fabricate rewritable surfaces composed of fluorescent brushes as demonstrated by du Prez et al. ${ }^{53}$ Also inkjet printing has been used, either to directly prepare initiator patterns ${ }^{54}$ or to selectively etch homogenously deposited initiator layers. ${ }^{55}$ Utilizing this technique, Edmonson et al. ${ }^{56}$ described the deposition of polyelectrolytebased macroinitiators allowing a submicron patterning of subsequently grafted brushes due to phase separation in a printed drop. ${ }^{56}$ Additionally, a recent progress in photoinduced RDRP methods opened a straightforward route to photopatterning of polymer brushes by spatially controlled polymerization via a photomask. ${ }^{57-58}$ Recently, Hawker et al. ${ }^{28}$ reported fabrication of photopatterned arrays of donor-acceptor photoactive brushes into a fully-operating multicolored and white organic light-emitting diode (OLED) devices. ${ }^{28}$

In contrast, to the best of our knowledge, the combination of inkjet printing and graftingto approaches has never been used to immobilize end-labeled fluorescent films to a patterned surfaces selectively modified with (3-aminopropyl)triethoxysilane (APTES) utilizing inkjet printing.

In this Chapter, firstly we present two fabrication routes to prepare the fluorescent PMMA films on silicon and glass surfaces based on grafting-from and grafting-to approaches, as is shown in Figure 7.1. Subsequently, the surface properties and the 
responsiveness behavior of the poly(methylmethacrylate) (PMMA)-based polymer films were investigated by measuring the thickness and the fluorescence of the polymer films via atom force microscopy (AFM) and fluorescent confocal microscopy, respectively. We have demonstrated the sensitivity of the PMMA layer to the quality of the solvent mixture utilizing co-solvency effect displayed in alcohol-water mixtures (e.g., in iso-propanol/water, IPA$\mathrm{W}$, mixtures). ${ }^{59-61}$ Moreover, fluorescent patterns were fabricated on the glass and silicon surfaces via inkjet printing process applying both grafting methods. The surface as well as the fluorescent properties of the printed patterns with switchable co-solvent-induced fluorescence response were investigated via AFM and fluorescent confocal microscopy, respectively.
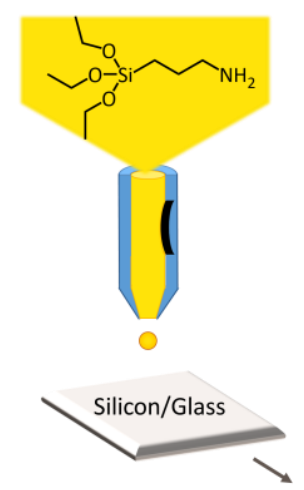

1. ARGET-ATRP in sol.

2. Exchange $\mathrm{Br} / \mathrm{N}_{3}$

3. Click reaction
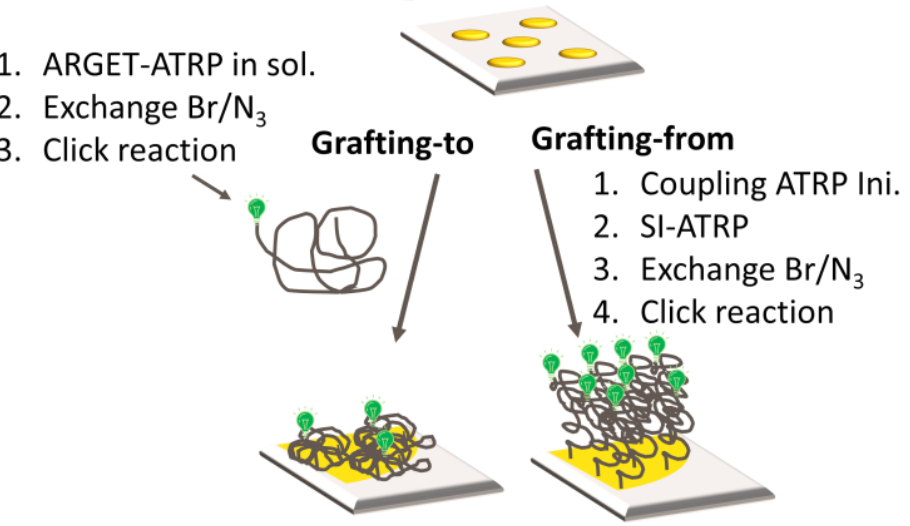

Fig. 7.1: A schematic representation of the fabrication routes to prepare fluorescent labeled PMMA patterns combining inkjet printing and ATRP employing a grafting-to and grafting-from approach.

\subsection{Results and discussion}

\subsubsection{Preparation of polymer films}

\section{a. Grafting-to approach}


Synthesis of $\alpha$-Carboxyl, $\omega$-Fluorescein-Functionalized PMMA. A telechelic PMMA functionalized, with a surface-reactive carboxyl group and a fluorescent dye, was synthesized according to Figure 7.2 combining ATRP and CuAAC click chemistry.

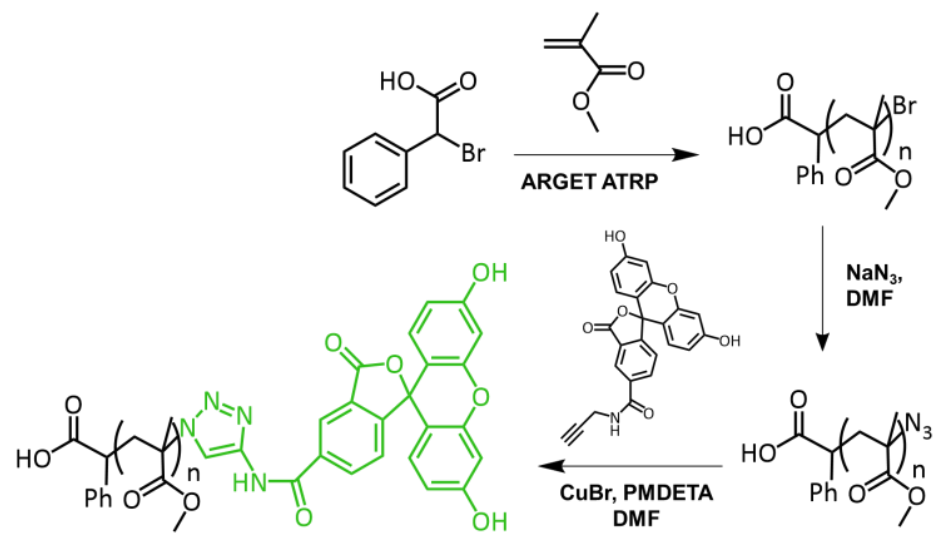

Fig. 7.2: Schematic representation of the synthesis route to $\alpha$-Carbonxyl, $\omega$-Fluorescein-functionalized PMMA (PMMA-FAM) by combination of ARGET-ATRP and CuAAC click chemistry.

The familiar combination of ATRP with CuAAC click chemistry was used as a straightforward route to prepare polymers with desired chain-end functionality. ${ }^{62}$

First, well-defined PMMA was synthesized by ARGET-ATRP. Importantly, $\alpha$ bromophenylacetic acid (BPAA) was used as an initiator to introduce carboxyl groups at the $\alpha$-chain ends. The reaction was conducted at $60{ }^{\circ} \mathrm{C}$ in anisole with $50 \mathrm{ppm}$ ( $v s$ monomer) of $\mathrm{CuBr}_{2}$ /TPMA (tris(2-pyridiylmethyl)amine) catalyst and tin(II) 2ethylhexanoate as a reducing agent. Gel permeation chromatography (GPC) measurements (Figure 7.3a) confirmed excellent control over the polymerization and formation of the well-defined PMMA $\left(M_{n \text {,theory }}=17000, M_{n, G P C}=18500, M_{w} / M_{n}=1.15\right)$.
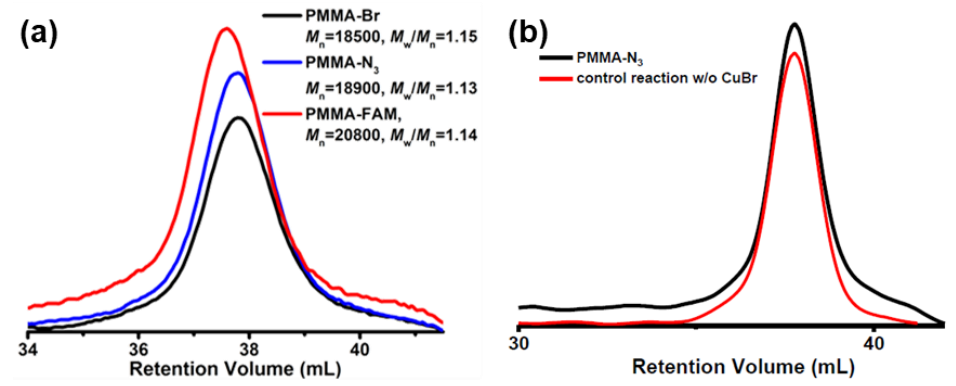

Fig. 7.3: (a) GPC traces of the as-synthesized polymer (PMMA-Br, black line), after substitution of bromide chain ends with azides (PMMA- $\mathrm{N}_{3}$, blue line) and after modification with fluorescein by click chemistry (PMMA-FAM, red line). (b) GPC traces of control click reaction performed without the addition of the $\mathrm{CuBr}$ catalyst. The curves are shifted vertically for clarity.

Next, the bromine chain-ends were transformed to azides by reacting PMMA-Br with $\mathrm{NaN}_{3}$ before employing $\mathrm{CuAAC}$ to click azides with an alkyne-functionalized fluorescein derivative (5-FAM). ${ }^{1} \mathrm{H}$ NMR measurements showed typical spectra of PMMA, although 
due to the relatively high $M_{n}$, no peaks assignable to the end groups (i.e., the initiator and the fluorescent dye) were detected (Figure S7.1). Interestingly, the chromatograms of PMMA-Br and PMMA-N $\mathrm{N}_{3}$ essentially overlapped, while a visible shift toward higher molecular weights was observed after reaction with 5-FAM (Figure 7.3a). This was likely due to the high molar mass of the dye $\left(M_{5-F A M}=413 \mathrm{~g} \cdot \mathrm{mol}^{-1}\right)$. A GPC trace of a control experiment, namely upon treating PMMA-N $\mathrm{N}_{3}$ with 5- FAM without the catalyst, did not exhibit any shift (Figure 7.3b). In all cases, dispersities remained low $\left(M_{w} / M_{n}<1.15\right)$, without any visible tailing.

To surely confirm the chain-end functionalization, the attachment of the fluorescein was followed by spectrofluorometry in 80 vol.\% IPA-W (Figure 7.4b), a good solvent for both PMMA and fluorescein. The excitation and emission spectra of the PMMA-FAM showed maxima at 488 and $524 \mathrm{~nm}$, respectively, corresponding to the free 5-FAM (Figure 7.4a). No fluorescence was observed for control samples obtained by stirring $\mathrm{PMMA}_{3} \mathrm{~N}_{3}$ in DMF with 5-FAM without $\mathrm{CuBr}$ overnight, ruling out any physical contamination of the polymer with the dye.

(a)

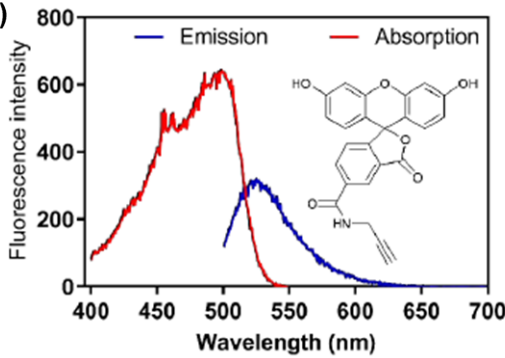

(b)

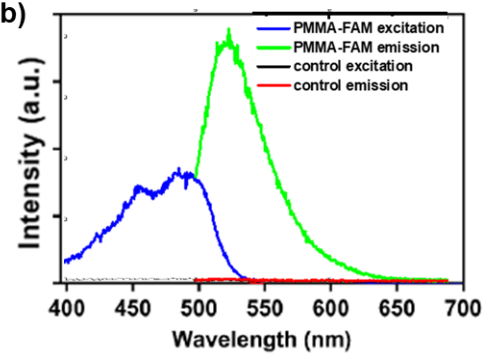

Fig. 7.4: a) and b) emission and excitation spectra of 5-FAM in co-solvency conditions (IPA-W 4/1 v/v) and of PMMA-FAM and a control polymer treated with 5-FAM dye in the absence of the catalyst, respectively.

Fluorescent grafted films (via grafting to): Dye-functionalized films were prepared by surface grafting to silica substrates previously modified with APTES via chemical vapor deposition (CVD). Simple dip coating from a DMF solution of the PMMA-FAM (1 $\mathrm{mg} \mathrm{mL} \mathrm{m}^{-1}$ ) was used to couple $\alpha$-carboxyl groups of the telechelic polymer with the amine groups of APTES. The reaction was conducted at room temperature without any additional catalyst. After $72 \mathrm{~h}$, the wafers were removed from the solution and analyzed by atomic force microscopy (AFM) to reveal a uniform film on the surface (Figure 7.5a). The thickness of the fluorescent-PMMA thin films were measured by AFM, as shown in Figure 7.5a. The average dry thickness of the film measured by AFM after gently scratching the surface using Teflon tweezers was found to be $6 \pm 2 \mathrm{~nm}$. Due to the slow diffusion of polymer chains to the surface, the coupling time of the PMMA-FAM to the CVD-made APTES-modified silicon samples was 72 hours; for shorter deposition times the film resulted in very thin films with no uniform surface coverage due to the slow 
diffusion of polymer chains to the surface, rendering AFM thickness measurements inconclusive (Figure 7.5b).

(a)

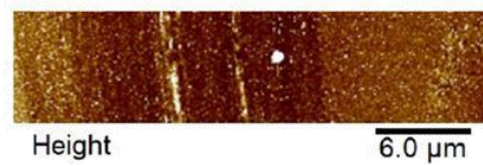

(b)

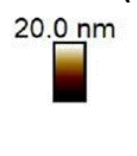

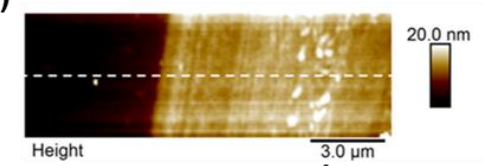

(c)

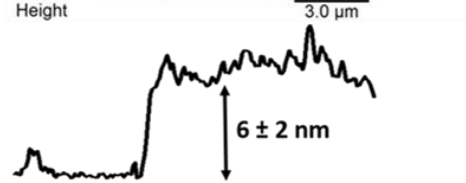

Fig. 7.5: (a) and (b) AFM height images of the PMMA-FAM films grafted to APTES-modified (via CVD) silica substrates for 48 and 72 hours, respectively. (c) Is the height profile of the dashed line of the PMMA-FAM films grafted to APTES-modified silica substrates for 72 hours.

The grafting density was then determined from the Equation 7.1:

$\sigma=\frac{N_{A} \rho h}{M_{n}}$

where $N_{A}$ is the Avogadro number, $\rho$ is polymer density $\left(1.18 \mathrm{~g} \mathrm{~cm}^{-3}\right), h$ is dry film thickness, and $M_{n}$ is the molecular weight of the dye-functionalized PMMA $\left(M_{n}=19\right.$ 000-20 000). The grafting density was calculated to be in the range of $\sigma=0.14-0.22$ $\mathrm{nm}^{-2}$.

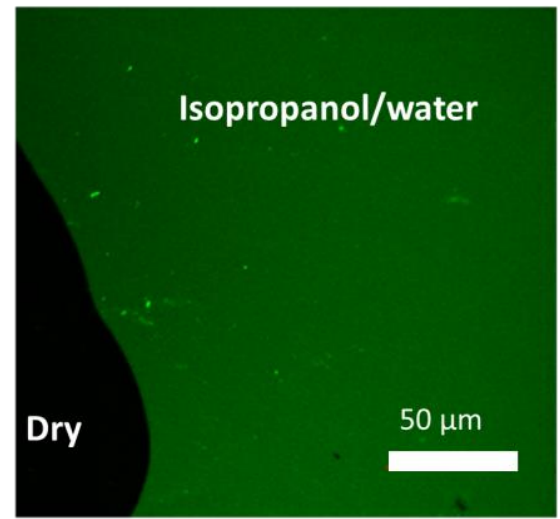

Fig. 7.6: Fluorescence microscopy image of the films grafted to APTES-modified glass surface. The green area corresponds to the film swollen in isopropanol/water mixture, while the dark area to the left shows quenched fluorescence due to the collapse of the brush upon evaporation of the solvent.

Figure 7.6 shows a plane scan of the PMMA-FAM layer recorded by confocal microscopy probing the successful modification of the silicon surfaces. An isopropanol/water mixture (4/1 v/v) was used as a solvent to ensure good solubility of the PMMA backbone as well as efficient solubilization of the hydrophilic chain-ends. ${ }^{59,63} \mathrm{~A}$ 
clear, uniform emission corresponding to the fluorescein-modified chain ends was observed. The dark area of the image corresponds to the region where the liquid already evaporated, leaving a dry film. As expected, the fluorescence was quenched due to the aggregation of the dye upon the collapse of the grafted polymer chains, and no emission was observed in this area.

\section{b. Grafting-from approach}

Fluorescent grafted films (via grafting from). The synthetic route to end-labeled PMMA brushes is shown in Figure 7.7. First, the initiator (3-(2-bromoisobutyryl) propyl) dimethylchlorosilane (BDCS) was coupled to the silicon surface by CVD. PMMA brushes were then grafted from the initiator-modified surface by SI-ATRP, $3,5,64$ utilizing a previously published procedure (step 1 in Figure 7.7). ${ }^{65-66}$

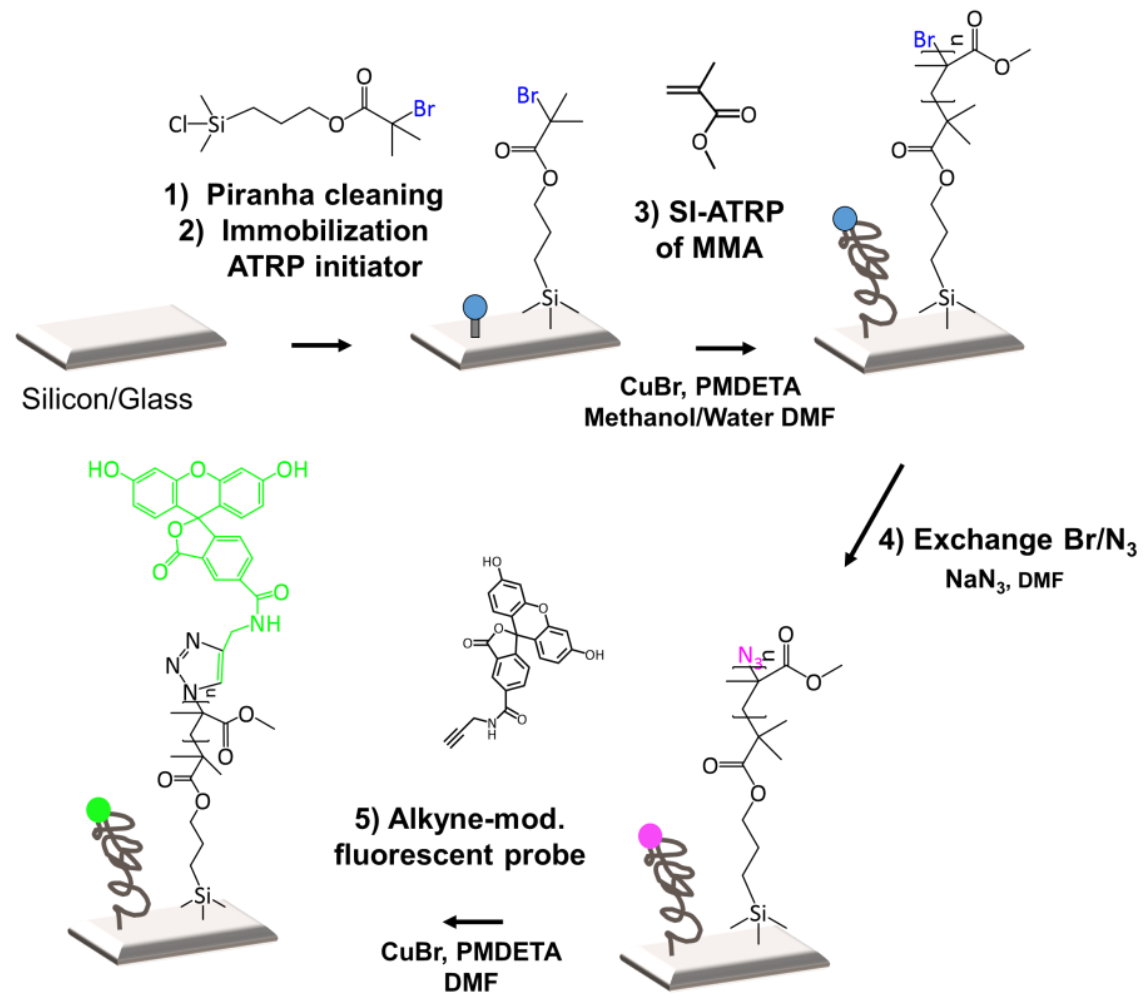

Fig. 7.7: Schematic representation of the synthetic route to $\omega$-Fluorescein-functionalized PMMA grafted onto the silicon substrate.

Polymerization was followed by measuring the thickness of samples reacted for specific time intervals via ellipsometry (Figure 7.8a). An initial short period (1 h) of fast growth was observed before the sufficient amount of deactivator $\left(\mathrm{CuBr}_{2}\right)$ build up in the system, followed by a linear increase of the brush thickness with polymerization time. 
Samples prepared in $6 \mathrm{~h}$ (i.e., $45 \mathrm{~nm}$-thick) were degrafted from the surface using tetrabutylammonium fluoride (TBAF, Figure 7.8b) and analyzed by GPC. A symmetric peak with $M_{\mathrm{n}}=25100 \mathrm{~g} \cdot \mathrm{mol}^{-1}$ and narrow molecular weight distribution $\left(M_{\mathrm{w}} / M_{\mathrm{n}}=1.11\right.$, Figure 1B) was observed, pointing out the excellent control over the surface-initiated polymerization. Moreover, the quality of degrafting was evaluated via FTIR analyzing the chemical composition of the PMMA film grafted on silicon and cleavage silicon surface. In the spectra (Figure 7.8c), before degrafting, a strong absorbance was observed at 1730 $\mathrm{cm}^{-1}$, which corresponds to double bond stretching vibration of carboxyl group $\mathrm{C}=\mathrm{O}$. The typical C-H stretching bands were observed at 3000 and $2950 \mathrm{~cm}^{-1}$. After degrafting PMMA with TBAF, the characteristic peaks of PMMA disappears in the spectra, indicating successful degrafting of PMMA polymer brushes.

(a)

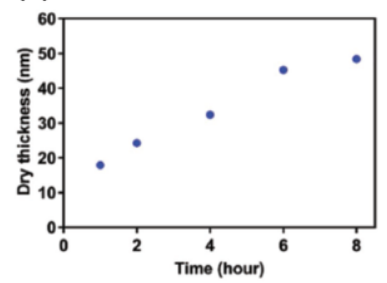

(b)

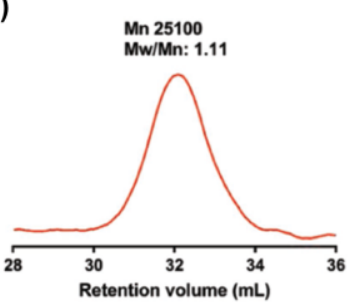

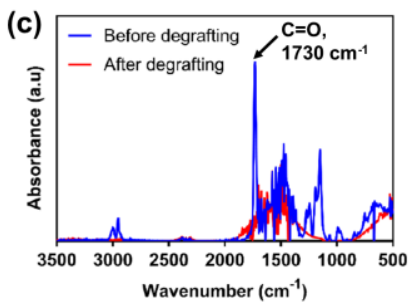

Fig. 7.8: (a) Dry thickness of the PMMA brush as a function of polymerization time. (b) GPC trace of the brushes, which were synthesized in $6 \mathrm{~h}$ and degrafted by TBAF. (c) FTIR spectrum of PMMA brushes before and after degrafting.

For an efficient chain end modification, it is critical that the bromide chain end functionality (CEF) remains high after the SI-ATRP step. Thus, to ensure that the extent of unavoidable termination events during the polymerization was kept at the lowest possible level, the reaction was stopped before reaching a plateau. Hence, a sample prepared in $2 \mathrm{~h}$ (i.e., $25 \mathrm{~nm}$-thick) was selected for further functionalization by replacing the $\mathrm{Br}$ at the PMMA chain ends by a CuAAC click reaction.

Successful coupling of the dye was verified by measuring the fluorescence of the asprepared films on glass. Figure 7.9a shows a fluorescence microscopy image of fluorescein end-functionalized PMMA brushes in an 80 vol.\% IPA-W mixture, which was a good solvent for PMMA. Since in this solvent FAM alkyne exhibits excitation and emission maxima around 486 and $524 \mathrm{~nm}$, respectively (Figure 7.4b), an excitation wavelength of $488 \mathrm{~nm}$ was applied. Clear, green emission was observed confirming efficient functionalization of the chain ends with a fluorescent probe. On the left side of the image, no fluorescent signal is visible due to partial evaporation of the isopropanolwater droplet. As a control, the fluorescence of both plain PMMA and PMMA-N 3 brushes, treated with the dye but without the catalyst, were measured (see Figure 7.9b and Figure 7.9c). Lack of fluorescence for these control surfaces shows that the dye was chemically bonded to the brushes and that its physical adsorption to the surface could be excluded. 
The fluorescence behavior of the FAM-PMMA layers was stable over time as is shown in Figure S7.3. Therefore, the changes in intensity were mainly due to the change in swelling, since measurements of the fluorescence intensity of free alkynylated fluorescein in the different solvents showed that the dye exhibits higher fluorescence intensity in water compared to $80 / 20$ vol.\% IPA-W mixture (see Figure S6),

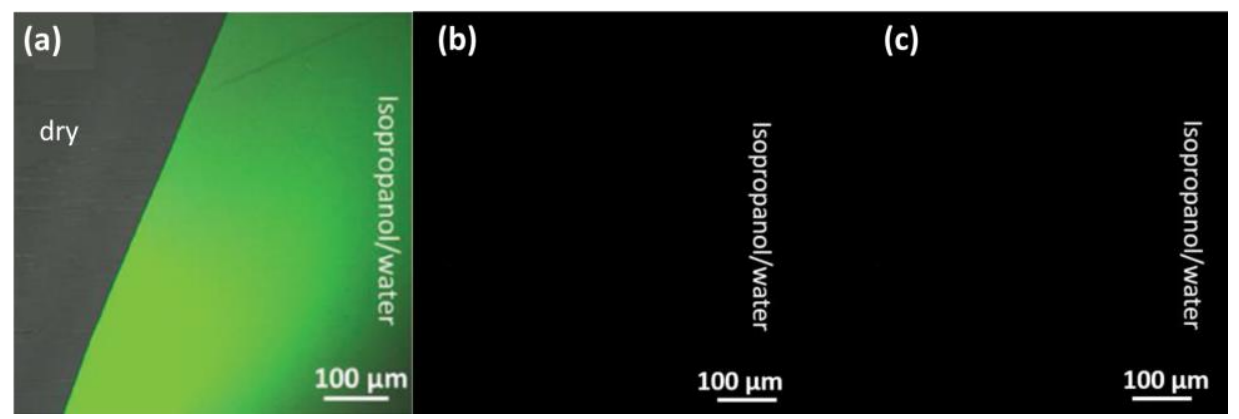

Fig. 7.9: (a) Fluorescence microscopy image of $25 \mathrm{~nm} P M M A-N_{3}$ brushes upon attachment of FAM by click chemistry. Fluorescence microscopy images of (b) $25 \mathrm{~nm}$-thick PMMA-N $\mathrm{N}_{3}$ brush treated overnight with the dye but without the copper catalyst and (c) PMMA-Br brush before further functionalization.

\subsubsection{Co-solvency behaviour of fluorescein end-tethered PMMA grafts obtained both via GF and GT approaches in IPA-water mixtures}

The co-solvency induced responsiveness of the PMMA-based grafts was investigated for the GF and GT methods examining the fluorescent intensity changes as a function of the swelling ratio of the polymer films varying the solvent composition, as illustrated in Fig. 7.10. The globule-to-random coil transition of PMMA grafts can be induced by the quality of the solvent as the PMMA chains collapsed in pure water or pure isopropanol while swelled in a mixture of these solvents (co-solvent regime conditions). When the brushes were in the collapsed state the fluorescent dye molecules self-quenched due to aggregation which resulted in lower the fluorescent intensity than when the FAM functionalized PMMA brushes swelled in the co-solvent mixture. Indeed, the fluorescence response of the FAM dye in 80 vol.\% IPA-W mixture was measured to be 20x higher than in water (Figure S7.3). In addition, the fluorescence intensity of the dye both in water and 80 vol.\% IPA-W mixture was stable over a period of $360 \mathrm{~s}$. 


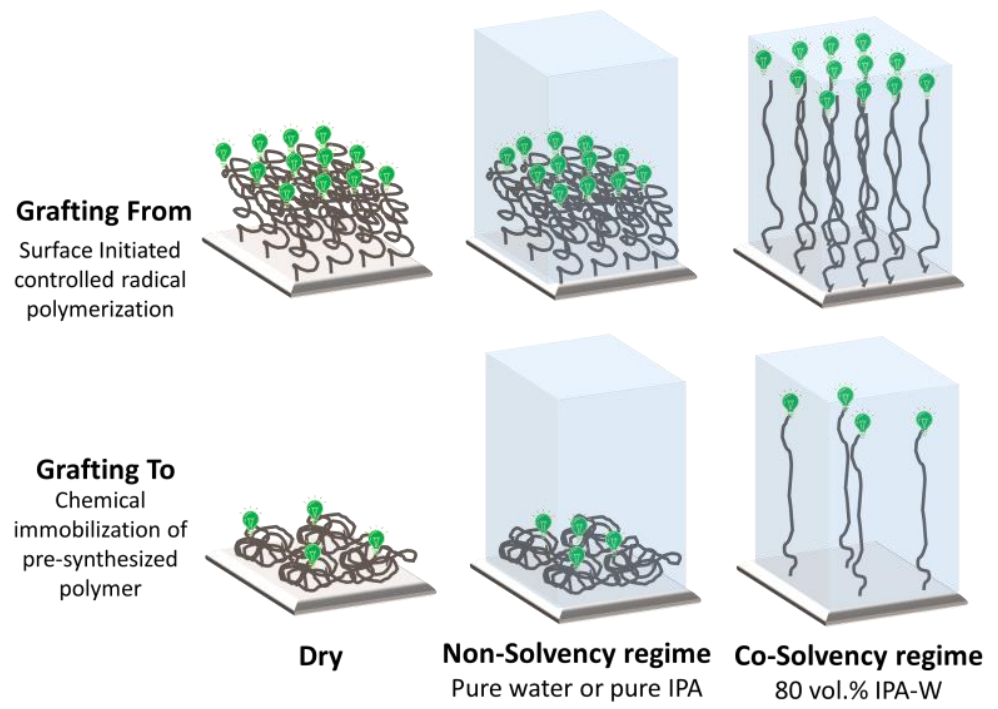

Fig. 7.10: Schematic representation of the fluorescent-labelled PMMA patterns and the solvent-induced globule-to-random coil transition for GT and GF fabrication routes.

The distinct on/off fluorescence response induced by co-solvent-stimulus of the PMMA-FAM brush layers was demonstrated for both the fabrication routes, as is shown in Figure 7.11. Furthermore, the switching of the fluorescence properties resulted reversible.

The switchable fluorescent intensity of the chain-end functionalized PMMA brushes from 80 vol.\% IPA-Water mixture and pure water. The fluorescent intensity in poor solvent conditions (in water) was consistently lower that the fluorescent intensity in good solvent conditions (80 vol.\% IPA-W mixture) because the brushes forced the fluorescent dye molecules to aggregate when ae in the collapsed state, leading to self-quenching and a weak fluorescence response.

(a)

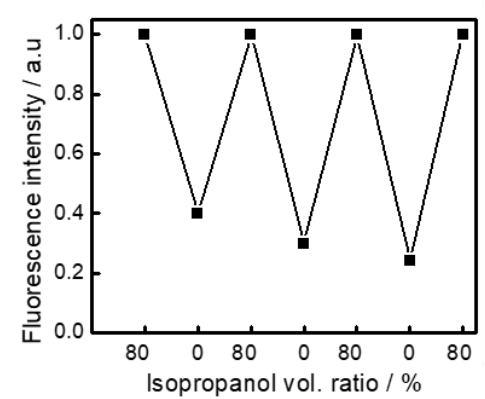

(b)

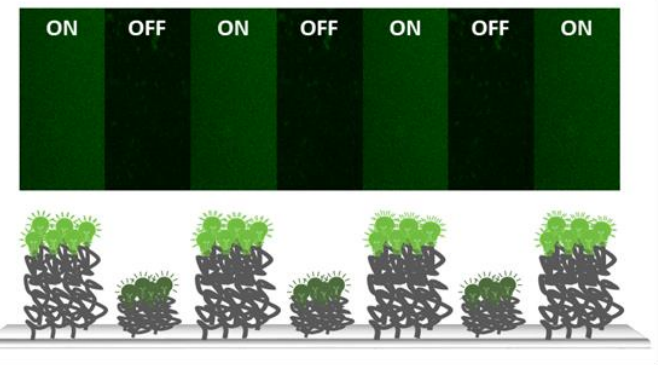

Fig. 7.11: (a) Average fluorescence intensity of the PMMA-FAM films in bad solvent conditions (pure water) and in good co-solvent conditions (80 vol.\% IPA-W) cycles. (b) Fluorescence images of the co-solventresponsive fluorescent switching of PMMA-FAM layers. 
The different fluorescence properties of the films depends on the grafting density of the end tethered chains, as is shown in Figure 7.12 and 7.13.

\section{Grafting From}

(a)

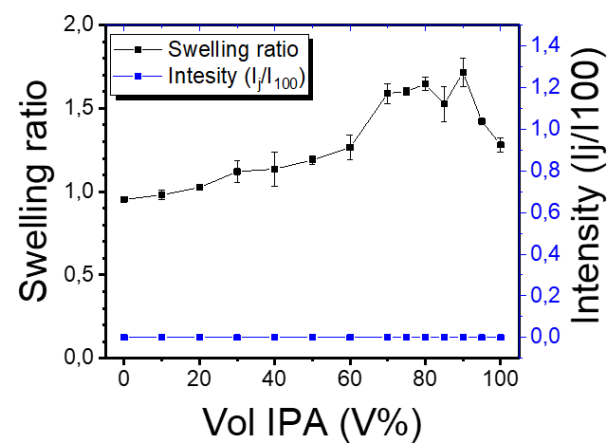

(b)

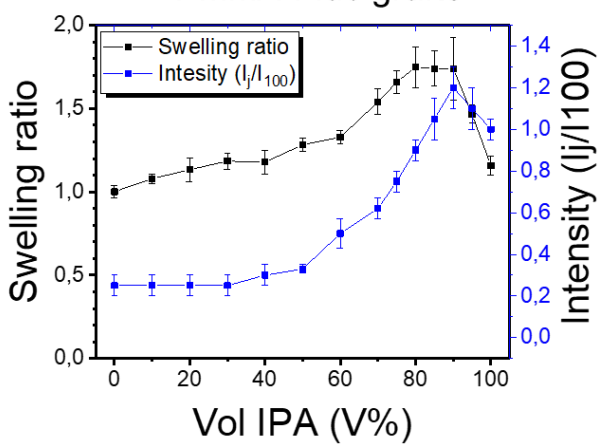

Fig. 7.12: Effect of the solvent mixture (isopropanol/water mixture) on the swelling ratio and fluorescent intensity of PMMA-Br and PMMA-FAM films grafted from APTES deposited via CVD, (a) and (b), respectively.

\section{Grafting To}

PMMA-Fluo grafts

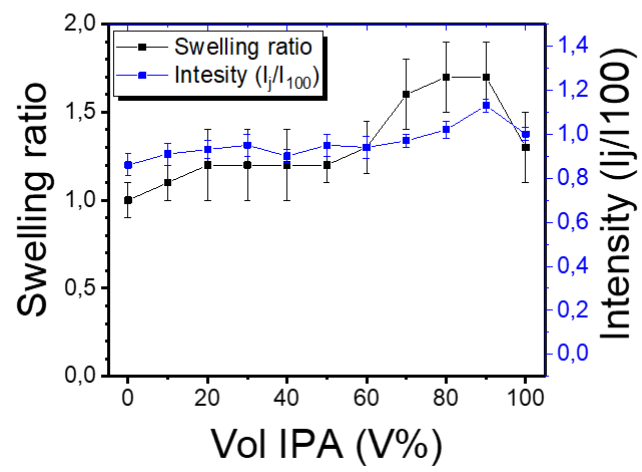

Fig. 7.13: Effect of the IPA-W mixture on the swelling ratio and fluorescent intensity of PMMA-FAM films grafted to APTES-modified glass surfaces.

The grafting density of the film obtained via GT was $0.14-0.22 \mathrm{~nm}^{-2}$, while fluorescent-functionalized chains in the GF approach were more densely packed resulting in a grafting density of $1.1 \mathrm{~nm}^{-2}$. The dye-functionalized PMMA brushes obtained via GF approach were measured to swell in GF by approximately $40 \%$ in 80 vol.\% IPA-W mixture (Figure 7.12), while the one obtained via GT deposition swelled by approximately $60 \%$ (see Figure 7.13). 


\subsubsection{Preparation of fluorescent patterns}

\section{a. Inkjet printing process.}

The fluorescent patterned surface were fabricated using glass and silicon substrates via two distinct methods: GT and GF, as is shown in Fig. 7.1. Via the inkjet printing process, APTES patterns were prepared and chemically immobilized on both silicon and glass surfaces thanks to the silane anchoring moieties. Subsequently, the fluorescent telechelic $\alpha$-COOH-PMMA- $\omega$-FAM was coupled to the amino end-functionalized printed patterns for the GT, while SI-ATRP of MMA, halogen-azide exchange, and CuACC click reactions with the fluorescent probe were performed for the GF approach.

The glass and the silicon substrates were cleaned in piranha solution and were modified by inkjet printing of APTES ink. The chemical stability over the storage, the viscosity, the surface tension, and the wettability of the ink onto the substrate are important physicochemical parameters to consider during the formulation of an ink for a stable functional ink as well as to optimize for accurate and repeatable deposition of the functional ink in Drop-on-Demand (DoD) mode. ${ }^{67}$ The fluid property requirements for the ink to be inkjet printed with piezo inkjet printed via DoD are a viscoelastic behavior (Newtonian behavior), a viscosity between 1-20 mPas and a surface tension between 20$70 \mathrm{mN} \cdot \mathrm{m}^{-1}$. Thus, the optimal formulation of the APTES-containing ink was composed by $10 \mathrm{w} \%$ of the functional compound dissolved in a mixture of ethanol and ethylene glycol (60 vol.\% ethanol/ethylene glycol mixture) in order to optimize the rheological and wetting properties (ethylene glycol was used as a cosolvent due to its high boiling point and low surface tension). ${ }^{68-69}$ As shown in Figure S7.4, the ink had a viscosity of $3.5 \pm 1$ $\mathrm{mPa} \cdot \mathrm{s}$ and the surface tension of $37 \pm 3 \mathrm{mN} \cdot \mathrm{m}^{-1}$. Moreover, the ink behaved as a Newtonian liquid (in the examined range of shear rate).

The printability of the APTES ink was evaluated in continuous (C) and in Drop-onDemand (DoD) inkjet printing modes. The droplet formation was studied in the range of frequency between $1000-100 \mathrm{~Hz}$ to test the stability and repeatability of the droplet formation, simulating the DoD approach. A stable droplet formation in DoD mode was achieved by employing a single trapezoid waveform with a pulse voltage of $52 \mathrm{~V}$ for 17 $\mu \mathrm{s}$, as shown in Figure 7.14a and $\mathbf{b}$. The time-resolved optical images of the droplet formation (Figure 7.14c) demonstrates the stable droplet formation without any satellite droplets. 


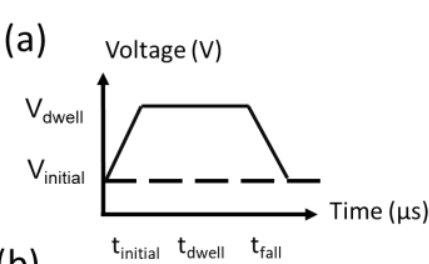

(c)

(b)

\begin{tabular}{|c|c|c|}
\hline \multicolumn{3}{|c|}{ Jetting test } \\
\hline Frequency & $\mathrm{Hz}$ & $\begin{array}{c}1000- \\
100\end{array}$ \\
\hline \multicolumn{3}{|c|}{ DoD-Printing conditions } \\
\hline $\mathrm{V}_{\text {initial }}$ & $\mathrm{V}$ & 0 \\
\hline $\mathrm{t}_{\text {initial }}$ & $\mu \mathrm{s}$ & 1 \\
\hline $\mathrm{V}_{\text {dwell }}$ & $\mathrm{V}$ & 52 \\
\hline $\mathrm{t}_{\mathrm{dwell}}$ & $\mu \mathrm{s}$ & 17 \\
\hline $\mathrm{t}_{\text {fall }}$ & $\mu \mathrm{s}$ & 1 \\
\hline Back Pressure & $\mathrm{Psi}$ & 0.2 \\
\hline
\end{tabular}
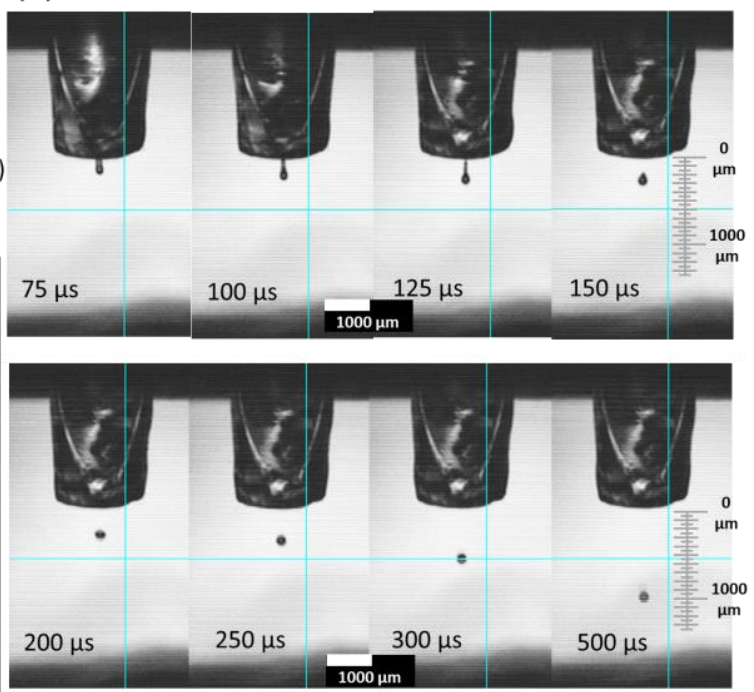

Fig. 7.14: characterization of the printability of the APTES-containing ink via piezo inkjet printing. (a) and (b) piezoelectric actuator waveform and the conditions used in this experiment for a stable DoD inkjet printing. (c) Series of high-speed photographic images of the droplet formation as it is ejected from the nozzle at strobe delay times between 75-500 $\mu$ s after the start of the piezo actuation. Characteristics of the drop: speed $2 \mathrm{~m} / \mathrm{s}$ and volume about $4 \mathrm{~nL}$.

The impact and the spreading of the ink onto the sample surfaces affect the quality and the morphology of the printed patterns which were studied via AFM and static contact angle measurements. Firstly, surface wettability is a critical parameter, which has an effect on the final sizes and shape of the printed droplets. ${ }^{68}$ The ink spread uniformly onto the glass/silicon surfaces without any formation of satellite drop formation due to the impact, as shown in Figure S7.5a. As illustrates in Figure S7.5b, the contact angle between the APTES-containing ink and glass substrate was measured to be $20 \pm 5^{\circ}$.

The printed APTES patterns were annealed in vacuum at $110{ }^{\circ} \mathrm{C}$ for $1 \mathrm{~h}$. The topography of the films was investigated via optical microscopy and AFM measurements, as shown in Figure 7.15. Various patterns were printed. The diameter of the printed droplets were around $100-120 \mu \mathrm{m}$, as shown in Figure 7.15a, which was larger than the diameter of the droplet generated by the nozzle (the diameter of the nozzle was $60 \mu \mathrm{m}$ ). The AFM imaging of the dry printed drops revealed few-micrometer-thick features of polymerized/cross-linked APTES, and a moderate coffee stain effect, as shown in Figure 7.15b and Figure 7.15c. The surface roughness values of the annealed drops were on the order of $\mathrm{R}_{\mathrm{a}}=100-150 \mathrm{~nm}$. 

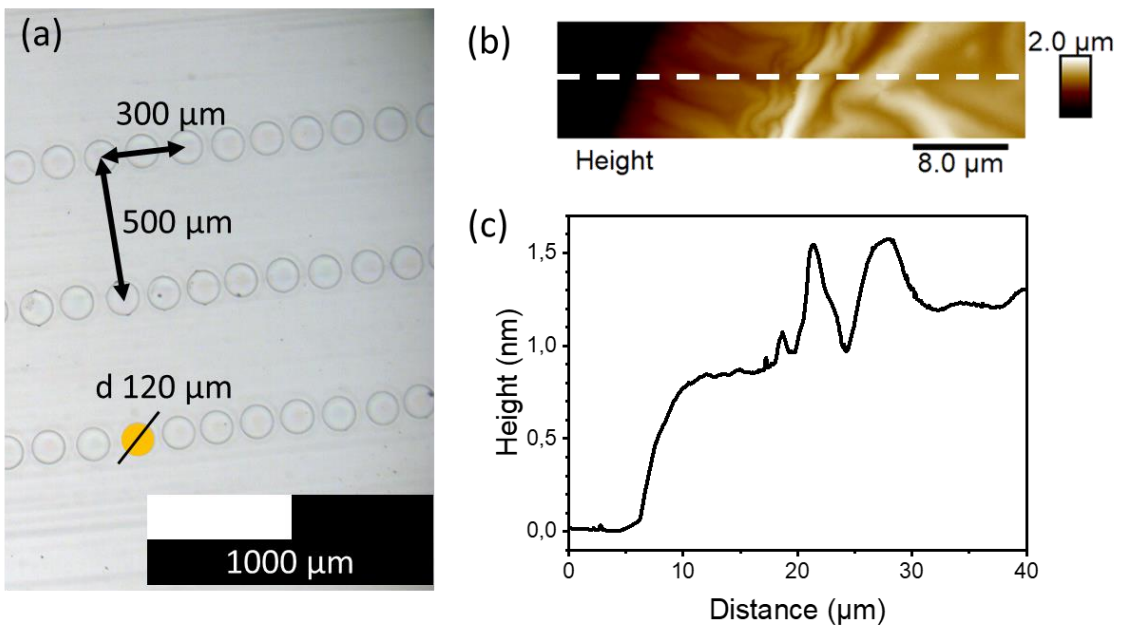

Fig. 7.15: Optical microscopy and AFM images of the ATPES-printed patterns on glass surfaces, as shown in (a) and (b) respectively. (c) Shows the step height profile of the APTES drops.

The chemical composition of the printed patterns was confirmed via FTIR microscopy analysis verifying the presence of the characteristic absorbance bands of the APTES films: amine and silane absorption bands at approximately 3200 and $1078 \mathrm{~cm}^{-1}$, respectively.

\section{b-1. Fluorescent PMMA chains grafted to the printed APTES patterns.}

As-prepared annealed printed APTES-modified surfaces were immersed in a DMF solution of $\alpha$-COOH-PMMA- $\omega$-FAM under the same conditions as described above (see Section 8.2.1.1).

Figure 7.16 presents the AFM height image of the drop after immobilization of the fluorescent telechelic PMMA and does not show a large difference from the annealed printed APTES drops (see Figure 7.15). After the immobilization of the PMMA-FAM the variation of the thickness of the drop was only of few nanometers, as demonstrated in the previous part (see Figure 7.6, PMMA-FAM grafted to APTES-modified silicon surfaces via CVD), rendering AFM thickness measurements unsuitable.

a)

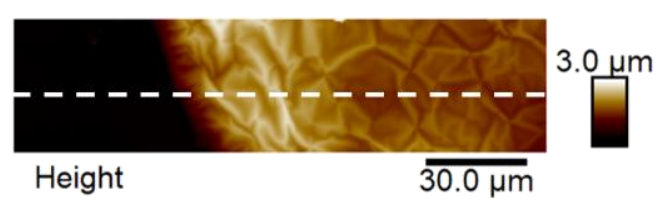

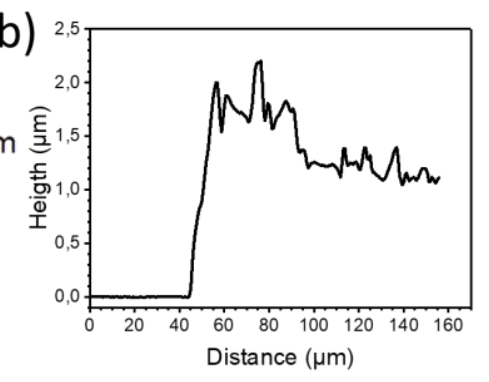

Fig. 7.16: AFM height image of the printed fluorescent patterns, GT approach. 
The successful fabrication of the fluorescent patterns was demonstrated via FTIR microscope spectroscopy and fluorescent confocal microscopy. Confocal fluorescent microscopy was used to visualize printed patterns functionalized with PMMA-FAM films in dry (Figure 7.17a) and swollen in isopropanol/water or acetone, as shown in Figure 7.17b and 7.17c respectively. Fluorescent patterns were obtained selectively at the printed spots of $\sim 120 \mu \mathrm{m}$ in diameter and with various spacing between the droplets. Most importantly, no signs of fluorescence were detected outside of the printed areas, confirming that nonspecific deposition on the nonfunctionalized surface did not occur, e.g., via physisorption. Even though the high surface roughness of the drops prevented a direct measurement of the grafting density of PMMA-FAM films, the observed uniform emission of the printed spots indicated they were successfully decorated with a thin film of PMMA-FAM.

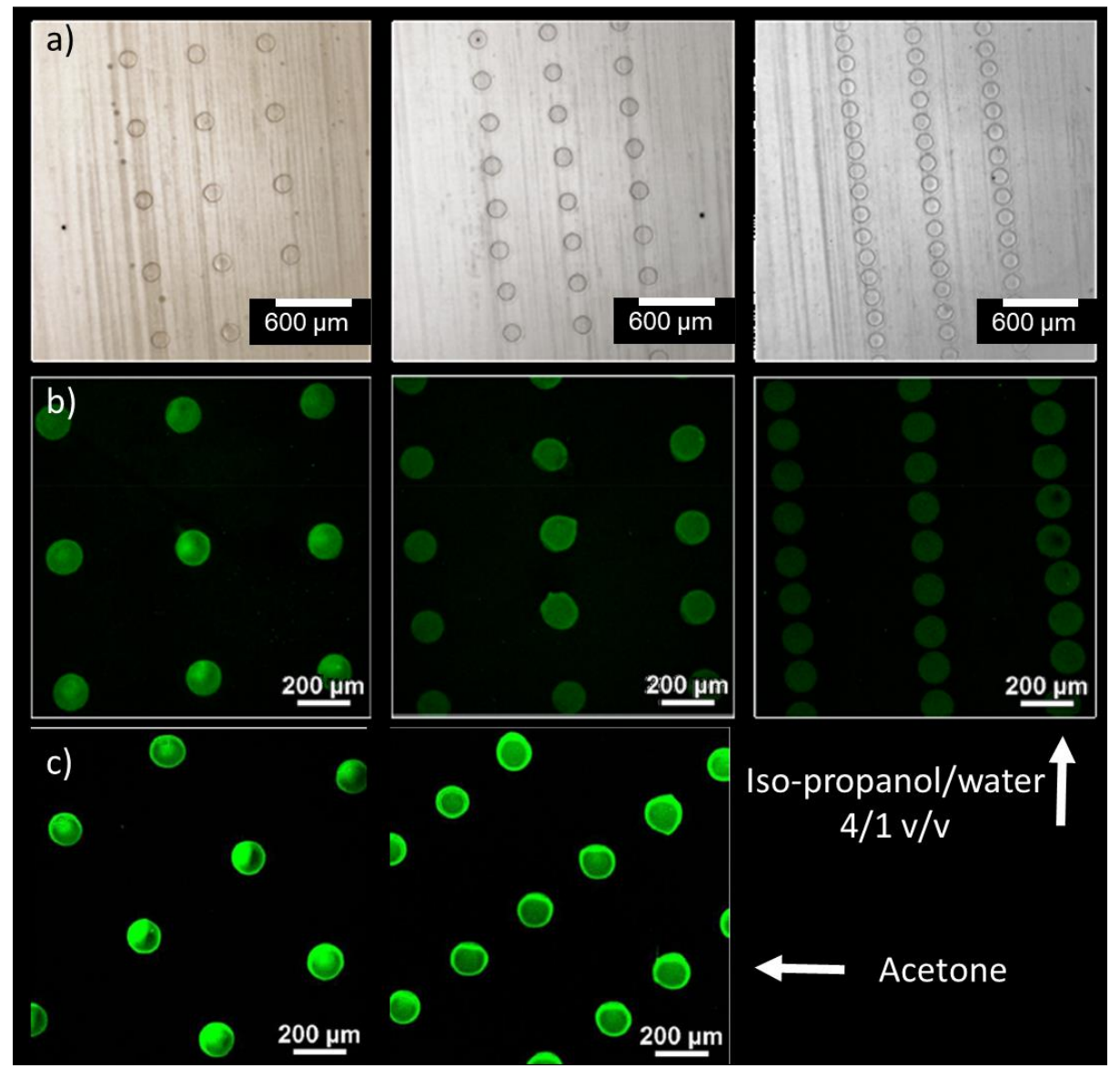

Fig. 7.17: (a) Microscopy images of glass substrate after deposition of APTES-containing ink by inkjet printing (top row); b) and c) confocal microscopy images of the same substrates upon functionalization with PMMA-FAM brushes by the grafting to method and recorded in 80 vol. $\%$ IPA-W and in pure acetone mixtures, respectively. 


\section{b-2. Synthesis of the fluorescent PMMA chains from the printed APTES patterns via}

\section{SI-ATRP}

The fluorescent end-labeled PMMA brushes were synthesized under the same conditions as described above (see Section 8.2.1.2). Briefly, the ATRP initiator $\alpha$-bromoisobutyryl bromide (BiBB) was coupled onto the amino terminated drops in a solution of toluene and tri-ethylenamine (TEA), the SI-ATRP of PMMA was performed in water/methanol mixture for $2 \mathrm{~h}$, the halogen-azide exchange reaction was conducted in DMF for $24 \mathrm{~h}$, and the coupling of the fluorescein-based dye via CuAAC was conducted in DMF for $24 \mathrm{~h}$. A detailed description of the experimental procedures can be found in the Experimental Section.

The surface morphology and the film height were evaluated via AFM, as shown in Figure 7.18a, b, and c. We noted that the FAM-modified drops showed a moderate coffee stain effect and a thickness of less than $1 \mu \mathrm{m}$. Figure 7.18d shows a fluorescence microscopy image of the fluorescent-printed patterns via the GF approach, recorded in 80 vol.\% IPA-W mixture, confirming the successful fabrication and immobilization of the dye to the printed APTES patterns.

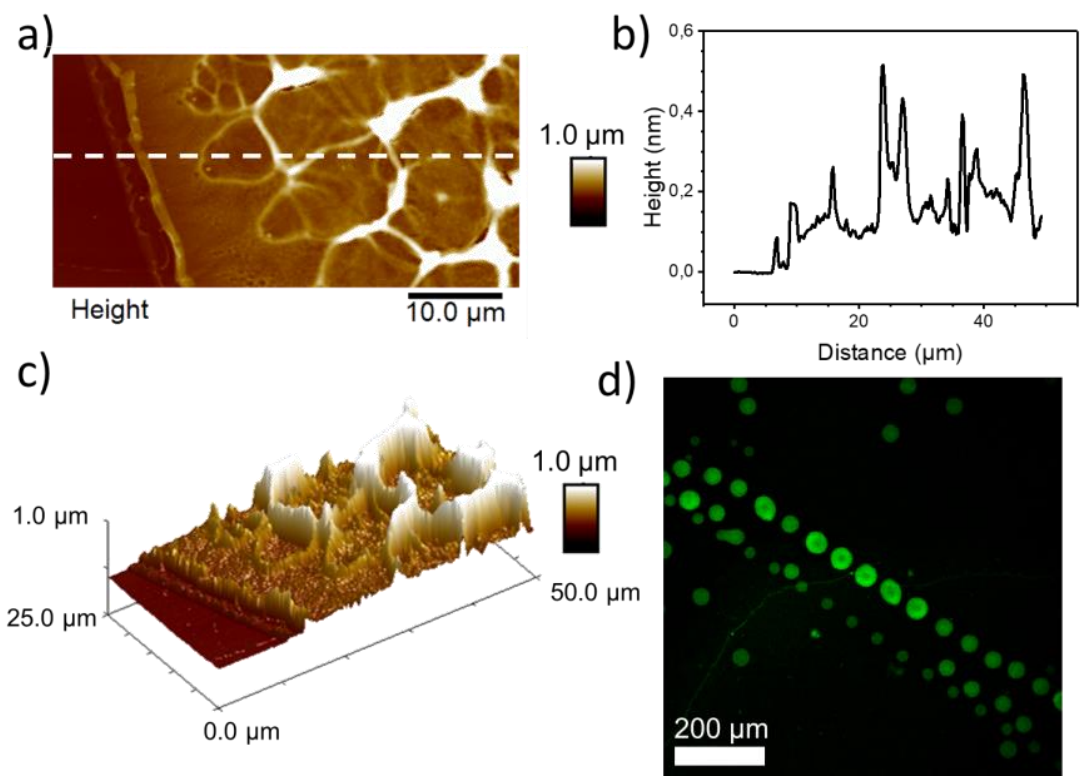

Fig. 7.18: Characterization of the fluorescent patterns obtained via the GF approach. Characterization of the surface properties via AFM microscopy: a) is a two dimensional AFM height images and b) is the height profile of the patterns corresponding to the dashed line of the printed fluorescent patterns. c) is a three dimensional AFM height images of the printed fluorescence patterns. d) is the fluorescence image of the fluorescent patterns in 80 vol\% IPA-W mixture. 


\subsection{Conclusions}

In summary, we have demonstrated two straightforward synthetic procedures to fabricate alcohol-sensitive fluorescent PMMA-based films and patterns by employing CVD and inkjet printing deposition/patterning. A clear fluorescence response of the fluorescent-modified PMMA grafts in an 80 vol.\% IPA-W mixture confirmed the successful synthetic procedures and the co-solvency effect on the fluorescence response of the functional films. Moreover, the effect of the fluorescence response was investigated as a function of the quality of the solvent demonstrating that the maximum swelling of the polymer film corresponded to the maximum of the fluorescence intensity. Switchable fluorescent response was illustrated by utilizing the co-solvency effect displayed by PMMA brushes in isopropanol-water mixtures. Fluorescent patterns were obtained via inkjet printing using the GF and GT approaches. The results demonstrated the combination of efficiency and simplicity of the grafting to method, rendering it a promising technique for preparing functional polymer nano-coatings, as is shown in Figure 7.19. Such smart macromolecular nanostructures can be employed in the design of future sensors, switchable surfaces or optoelectronic devices. Given the versatility of both ATRP (in solution and surface initiated) and click chemistry, these approaches can be viewed as a general strategy for fabrication of polymer-grafted surfaces with various functionalities readily available by simple chain-end substitution. Such functional patterned surfaces can find applications in sensing, light harvesting, or organic electronics. Further experiments are underway to extend this approach to different polymer systems as well as to elucidate the effect of grafting density on the photophysical properties of end-labeled surfaceanchored chains.
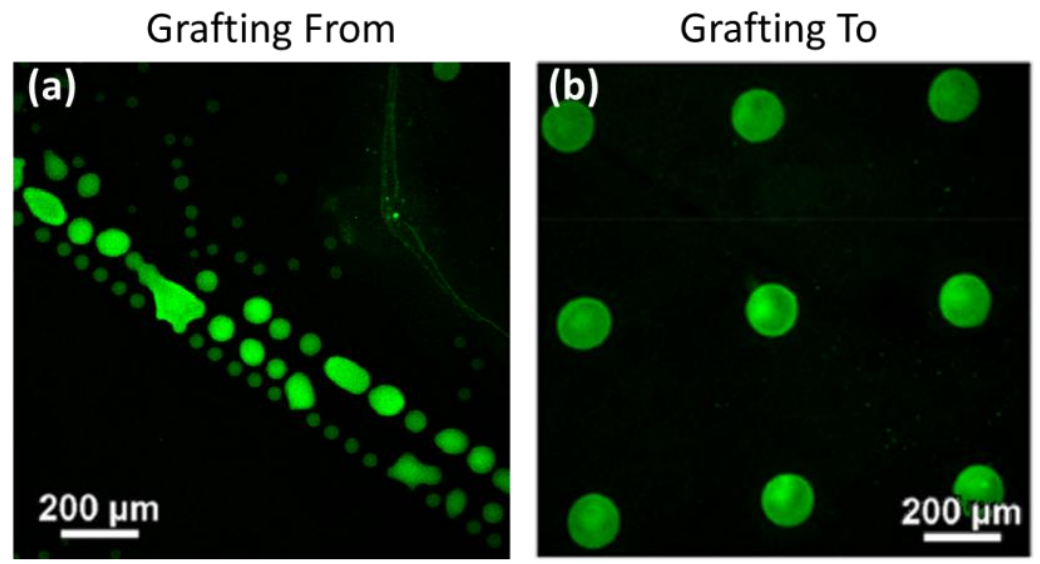

Fig. 7.19: Fluorescence confocal microscopy images of the fluorescent patterns prepared from printed APTES patterns onto a glass surface (scale bar is $200 \mu \mathrm{m}$ ) through grafting from and grafting to approaches, (a) and (b) respectively. The images were recorded in 80 vol. $\%$ IPA-W mixture. 


\subsection{Experimental section}

\section{Materials}

Methyl methacrylate (MMA, 99\%), $\alpha$-bromophenylacetic acid (BPAA, 98\%), tris(2-

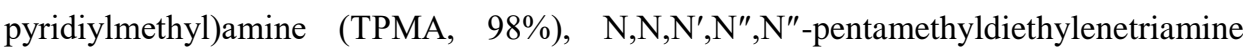
(PMDETA, 99\%), 2,2'-Bipyridyl ( $\geq 99 \%)$, tin(II) 2-ethylhexanoate ( $\mathrm{Sn}(\mathrm{EH}) 2,92.5$ $100.0 \%$ ), copper (I) bromide (CuBr, 99.999\%), copper (II) bromide $\left(\mathrm{CuBr}_{2}, 99 \%\right)$, ascorbic acid, sodium azide $\left(\mathrm{NaN}_{3},>99.5 \%\right)$, tetrabutylammonium fluoride (TBAF), solution chlorodimethylhydrosilane (98\%), allyl 2-bromo-2-methyl propionate (98\%), (3aminopropyl)triethoxysilane (APTES) were purchased from Sigma-Aldrich. MMA was passed through a column of basic alumina to remove inhibitor. Copper (I) bromide $(\mathrm{CuBr}$, Aldrich, 98\%) was stirred in excessive acetic acid and filtered till the suspension solution was light yellow, and was dried in vacuum oven at room temperature overnight. Alkynylated fluorescein (FAM alkyne, 5-isomer) was purchased from Lumiphore. All solvents were of high purity, and Milli-Q water was used in all experiments.

\section{Grafting-to}

Synthesis of $\boldsymbol{\alpha}$-carboxyl PMMA-Br by ARGET ATRP. Polymerization conditions were based on previously reported procedures. ${ }^{70-71}$ Briefly, $12.6 \mathrm{mg}$ ( $0.03 \mathrm{mmol}, 0.1 \mathrm{eq}$.) of $\mathrm{Sn}(\mathrm{EH})_{2}$ was placed in a Schlenk flask and degassed by purging with nitrogen for 30 min. MMA was degassed with nitrogen, and $10 \mathrm{~mL}$ (93.5 mmol, 300 eq.) was injected into the Schlenk flask. EBPAA (67.0 mg, $0.31 \mathrm{mmol}, 1$ eq.), $\mathrm{CuBr}_{2}(1.0 \mathrm{mg}, 0.004 \mathrm{mmol}$, 0.015 eq.), TPMA ( $4.1 \mathrm{mg}, 0.01 \mathrm{mmol}, 0.045$ eq.), dimethylformamide (DMF) $(0.5 \mathrm{~mL}$, NMR standard), and anisole ( $5 \mathrm{~mL}$ ) were degassed in a separate vial for $30 \mathrm{~min}$ and transferred to the Schlenk flask under nitrogen. The reaction was started by immersing the Schlenk flask into an oil bath and conducted for 23 hours at $60{ }^{\circ} \mathrm{C}$. Polymerization was stopped at desired monomer conversion (56\%) by exposing the reaction to air and PMMA was precipitated in methanol, filtered and dried under vacuum overnight.

Br-N $N_{3}$ exchange reaction, $\boldsymbol{\alpha}$-COOH-PMMA- $\omega-\mathbf{N}_{3} .2 \mathrm{~g}(0.11 \mathrm{mmol}, 1$ eq. $)$ of PMMA-Br was dissolved in $5 \mathrm{~mL}$ of DMF. $70.3 \mathrm{mg}$ (1.08 mmol, 10 eq.) of $\mathrm{NaN}_{3}$ was then added and reaction was stirred for 24 hours at room temperature. The polymer was precipitated in methanol, filtered and dried under vacuum overnight.

Coupling via CuACC click chemistry of PMMA-N3 with 5-FAM. $0.5 \mathrm{~g}$ of the obtained PMMA-N 3 (0.027 mmol, 1 eq.) was dissolved in $5 \mathrm{~mL}$ DMF. $33.52 \mathrm{mg}(0.008$ mmol, 3 eq.) of 5-FAM dye and $0.006 \mathrm{~mL}$ ( $0.003 \mathrm{mmol}, 1$ eq.) of PMDETA were added and the solution was degassed by purging with nitrogen for 20 minutes. Then, it was transferred to the degassed vial with $0.8 \mathrm{mg}(0.003 \mathrm{mmol}, 1 \mathrm{eq}$.) of $\mathrm{CuBr}$ and stirred for $24 \mathrm{~h}$ at room temperature. The polymer was precipitated in methanol, filtered, re-dissolved in DMF, passed through a column of neutral alumina to remove any unreacted dye, precipitated again in methanol, filtered and dried under vacuum overnight. 
Silicon and glass preparation. Silicon and glass substrates were cleaned by piranha solution $\left(\mathrm{H}_{2} \mathrm{SO}_{4}: \mathrm{H}_{2} \mathrm{O}_{2}, 3: 1 \mathrm{v} / \mathrm{v}\right)$, then rinsed extensively with water, ethanol and dried using a nitrogen stream (piranha solution reacts with organic compounds and should be handled with extreme caution).

CVD of APTES. The piranha cleaned silicon or glass substrates were placed in the desiccator including a petri dish with APTES. The desiccator was placed under vacuum for $15 \mathrm{~min}$. After $15 \mathrm{~min}$ the desiccator was fully closed. The substrates together with the APTES were left overnight under vacuum. Afterwards the substrates were rinsed, with toluene, ethanol, MilliQ water, ethanol to remove physically attached APTES and dried in a stream of $\mathrm{N}_{2}$.

Patterned surfaces by ink-jet printing. An ink solution was prepared with $2 \mathrm{~mL}$ ethylene glycol, $3 \mathrm{~mL}$ ethanol and $0.585 \mathrm{~mL}$ of APTES.

The solution was printed by inkjet printing on a piranha-cleaned glass or silicon surface.

After printing the substrates were left overnight and rinsed with ethanol, water and ethanol and placed in a vacuum oven at $110^{\circ} \mathrm{C}$ for 1 hour. A Jetlab IV (MicroFab Technologies, Plano, TX, USA) printer was used with adjustable $\mathrm{Z}$ height, $\mathrm{X}-\mathrm{Y}$ stage and controller. Drop on Demand patterning was achieved by using a sinusoidal type waveform: dwell amplitude of $52 \mathrm{~V}$ for $17 \mu$ s with a rise and fall time at $0 \mathrm{~V}$ for $1 \mu \mathrm{s}$. The nozzle used was Nozzle type MJ-B7-39-30 with $60 \mu \mathrm{m}$ orifice diameter. The drop was $100 \mu \mathrm{m}$ diameter and $120 \mu \mathrm{m}$ printed drops diameter. To follow drop deposition a CDD camera with LED light was used. The plate and head temperature were set at $23^{\circ} \mathrm{C}$. Before printing, the printer was cleaned with isopropanol, ethanol, Milli-Q water and ethanol.

Surface grafting of telechelic $\boldsymbol{\alpha - C O O H - P M M A - \omega - F A M . ~ T h e ~ s u b s t r a t e s ~}$ functionalized with APTES were added to a flask with PMMAFAM solution $\left(1 \mathrm{mg} \cdot \mathrm{mL}^{-1}\right)$ and left stirring for 72 hours. Afterwards the substrates were cleaned with DMF, ethanol, Milli-Q water, ethanol and dried in a stream of nitrogen.

\section{Grafting-from}

CVD of ATRP-initiator and synthesis of PMMA Brushes via SI-ATRP. Organosilane initiator was synthesized via hydrosilylation of allyl-2-bromomethylpropionate with dimethylchlorosilane catalyzed by choloplatinic acid forming (3(2-bromoisobutyryl) propyl)dimethylchlorosilane (BDCS), as previously reported. ${ }^{72-73} \mathrm{~A}$ monolayer of BDCS was deposited on piranha clean substrates by vapor deposition in a desiccator under vacuum for $24 \mathrm{~h}$, which was followed by atom transfer radical polymerization (ATRP) of methyl methacrylate (MMA) at room temperature for $2 \mathrm{~h}$ under nitrogen.

MMA (17.6 g, $176 \mathrm{mmol})$ and 2,2'-bipyridine (1.11 g, $7.11 \mathrm{mmol})$ were dissolved in the ATRP medium (methanol: deionized water, $13.8 \mathrm{~mL}: 3.8 \mathrm{~mL}, 430 \mathrm{mmol}: 210 \mathrm{mmol}$ ) 
and the solution was degassed before pouring into the Schlenk flask with $\mathrm{CuBr}$ (516 mg, $3.56 \mathrm{mmol}$ ) under nitrogen atmosphere. After stirring for $15 \mathrm{~min}$, the ATRP mixture was transferred to the nitrogen-filled vials with the initiator-coated substrates. After polymerization, the substrates were rinsed with ethanol and water, and dried under nitrogen.

Br-N $N_{3}$ exchange reaction of the PMMA brushes. In a round bottom flask, $97.5 \mathrm{mg}$ $(1.5 \mathrm{mmol})$ sodium azide was added to $36 \mathrm{~mL}$ of dimethylformamide (DMF). The solution was then purged for 20 min with nitrogen. Next, the solution was transferred into a flask containing PMMA brush functionalized substrates, which was beforehand sealed with a rubber septum and purged with nitrogen for $20 \mathrm{~min}$. After $24 \mathrm{~h}$ of reaction, the substrates were rinsed extensively with DMF, ethanol and water, and dried under a stream of nitrogen.

Functionalization of PMMA Brush Chain Ends with Fluorescein Via Click Chemistry. $10.7 \mathrm{mg}$ (0.26 mmol) alkynylated fluorescein, $29.2 \mathrm{mg}(0.12 \mathrm{mmol}) \mathrm{CuBr}$ and $2.5 \mu \mathrm{L}(0.12 \mathrm{mmol})$ PMDETA were added to $36 \mathrm{~mL}$ DMF and the solution was purged with nitrogen for $20 \mathrm{~min}$. For the click reaction between alkynylated fluorescein and azideterminated polymer brush, the solution was transferred into a flask containing azide chainend functionalized PMMA brush substrates, which was also purged with nitrogen for 20 min. The click reaction was performed at room temperature under nitrogen atmosphere for $24 \mathrm{~h}$. Afterwards, the substrates were rinsed extensively with DMF, ethanol, and water to remove the unreacted dye. The samples were then dried under a stream of nitrogen and kept in dark.

Degrafting of PMMA Brush Layer To determine the grafting density, PMMA brushes were degrafted from the surface using TBAF. ${ }^{65-66} \mathrm{Si}$ wafers grafted with $40-45$ nm PMMA brushes were put in flask containing $0.1 \mathrm{~m}$ TBAF in $5.5 \mathrm{~mL}$ tetrahydrofuran (THF). The samples were kept stirring at $55^{\circ} \mathrm{C}$ for $24 \mathrm{~h}$. After $24 \mathrm{~h}$, the substrates were rinsed with THF, toluene, water and ethanol, and dried under a stream of nitrogen. THF was evaporated and the polymer sample was then re-dissolved in THF for gel permeation chromatography (GPC). Before and after degrafting, dry brush thickness on the substrate was measured using ellipsometry. After degrafting, the thickness decreased to $0.3 \mathrm{~nm}$, which indicates virtually complete detachment of the brushes. The conformation of the polymer brush is mainly governed by the interplay between $M n$ and the grafting density $\left(\sigma_{\mathrm{p}}\right) .^{74}$ The grafting density is difficult to determine directly; however, it can be determined as $1.13 \mathrm{~nm}^{-2}$ by using the equation (1).

\section{Inkjet printing}

Formulation and characterization of the APTES-containing inks. The rheological properties of the inks were evaluated by rotational UDS-200 rheometer (Anton Paar Germany GmbH., Ostfidern, Germany) equipped with parallel plates geometry. For all the rheology, surface tension measurements, and inkjet printing process the ink solutions were filtered through a $0.45 \mu \mathrm{m}$ PTFE filter. The steady-shear viscosity of the inks was 
evaluated for all the inks via flow measurements (also named as steady-shear rate sweep), aiming to simulate the piezo inkjet printing process. The measurements were performed at $20{ }^{\circ} \mathrm{C}$ (controlled by a Peltier temperature controller) using a MP31 spindle (a plain plate with a diameter of $50 \mathrm{~mm}$ ). Before the flow measurements, the gap (set at $500 \mu \mathrm{m}$ ) was filled with $1 \mathrm{~mL}$ of the ink solutions and the solution was then stabilized via a preshear step of $0.5 \mathrm{~min}$. The rheological properties were measured by increasing the shear rate at constant logarithmic steps from 100 to $2400 \mathrm{~s}^{-1}$, ensuring the steady-state flow of the sample. The viscosity was calculated as the steady-state shear stress divided by the applied shear rate. The steady-shear viscosity was averaged in the range between 500$2000 \mathrm{~s}^{-1}$. The surface tension was determined by the pendant drop method with an OCA 15 (Dataphysics Instruments, Germany). The surface tension was estimated by fitting the outline of the pendant drop; the drop contour was fitted by the Young-Laplace equation considering the density of the ink to be the same as the density of the solvent. A needle $0.70 \mathrm{~mm}$ of outer diameter was used to develop droplets of about $6 \mu \mathrm{L}$ of volume and the average of the surface tension values, as well as the standard deviations were calculated with at least three different measurements.

Inkjet printing. A Jetlab IV Printer (MicroFab Technologies Inc. USA) equipped with glass nozzle MicroFab B7-39-30 having a $60 \mu \mathrm{m}$ diameter was used to deposit the ink on the sample surfaces. The printability of the inks was evaluated by studying the effect of the shape of the input waveform and of the process conditions on radius, volume, trajectory and speed of the droplets. These parameters were estimated by analyzing the high speed camera images captured by the horizontal camera at different strobe delay time in C-IJ mode. The deposition of the functional ink was achieved in DoD-IJ printing mode. The annealed APTES-printed samples were used directly for the coupling with the ATRPinitiator. Therefore, the APTES-printed samples were immersed in a solution of $40 \mathrm{ml}$ of toluene and $100 \mu \mathrm{l}$ of TEA and cooled in an ice bath. The solution was stirred, the BIBB was added drop wise and the reaction was terminated after $2 \mathrm{~h}$. The samples were washed in toluene, ethanol, and water, followed by drying in a stream of $\mathrm{N}_{2}$. The SI-ATRP of MMA, the Br- $\mathrm{N}_{3}$ exchange, and the $\mathrm{CuACC}$ of the fluorescent probe were performed as mentioned above in the Section 7.4.3.

\section{Characterization}

GPC: was performed on a Waters system (pump: Waters 515, USA, injector: Hewlett-Packard 1050 USA, detector: Waters 2414 and Waters Styragel HR3-6 columns) with DMF $50 \mathrm{mM} \mathrm{LiCl}$ as eluent. Molecular weights were calculated using PMMA calibration.

${ }^{1}$ H NMR: (Bruker Avance $400 \mathrm{MHz}$ ) was used to calculate monomer conversion in chloroform- $\mathrm{d}_{3}$.

AFM: Brush swelling and topography measurements were performed in tapping mode on a Multimode 8 (Bruker) using a NanoScope V (Veeco) controller and a JV 
vertical engage scanner. A scratch was made on the sample to reveal the bare silicon wafer in order to be able to measure the relative height between the brush and the bare silicon. The sample was freshly prepared and the same isopropanol-water mixture solutions were used as in the ellipsometry measurements. For each measurement, images were obtained over a scan size of 30.0 by $15.0 \mu \mathrm{m}$ with a scan rate of $0.300 \mathrm{~Hz}$. First, the relative height of the dry brush in atmospheric conditions was measured in tapping mode using Olympus cantilevers (silicon probe, resonance frequency around $70 \mathrm{kHz}$, force constant around 2 $\mathrm{N} \cdot \mathrm{m}^{-1}$, diameter of tip approximately $9 \mathrm{~nm}$, coated with an aluminum reflecting layer). Second, the relative heights were measured immersed in the different isopropanol-water mixtures by imaging the brush under a low normal load $(<1 \mathrm{nN})$ in contact mode using a colloid probe in a glass liquid cell (Bruker). A polystyrene colloid, $5 \mu \mathrm{m}$ in diameter, was attached onto a MikroMasch HQ line cantilever, the HQ:NSC35/Pt. By thermal noise analysis the spring constant was calculated to be $0.3 \pm 0.03 \mathrm{~N} \cdot \mathrm{m}^{-1}$ at room temperature and ambient conditions. In each obtained image, the relative height is measured at six different positions and an average is taken and used for further calculations. The amplitude set point was kept at approximately $95 \%$ of the free amplitude to minimize deformation of the polymer surface.

Fluorescence confocal microscopy: For the fluorescence measurements the Micro 40 confocal microscopy from Halcionics was used with 5x magnification. Excitation and emission spectra were measured with a PerkinElmer LS55 spectrofluorometer. Excitation and emissions slits were set to a $4.0 \mathrm{~nm}$ and $2.5 \mathrm{~nm}$ band pass, respectively. Fluorescence experiments were performed on Nikon A1 confocal microscope equipped with a piezo stage, controller, DU4 multidetector and LU4 multilaser. 60x contact objective (oil, MRD01691, $2.481 \mu \mathrm{m} \cdot \mathrm{px}^{-1}$ and 10x air objectives (MRH00101, $0408 \mu \mathrm{M} \mathrm{px}^{-1}$ ). The samples were illuminated by a $488 \mathrm{~nm}$ laser.

Spectrofluorometer: Fluorescence spectra were measured using a PerkinElmer LS55 spectrofluorometer. Excitation and emission slits were set to a $4.0 \mathrm{~nm}$ and $2.5 \mathrm{~nm}$ band pass, respectively. FAM alkyne, 5-isomer fluorescence was excited at $488 \mathrm{~nm}$. Fluorescence experiments were performed on Nikon A1 confocal microscope equipped with a piezo stage, controller, DU4 multidetector, and LU4 multilaser. 60x contact objective (oil, MRD01691, $2.481 \mu \mathrm{m} \cdot \mathrm{px}^{-1}$ and 10x air objectives (MRH00101, 0408 $\left.\mu \mathrm{M} \cdot \mathrm{px}^{-1}\right)$. The samples were illuminated by a $488 \mathrm{~nm}$ laser. Images were acquired by using a constant set of parameters for each sample. Image processing was done with Image $\mathbf{J}$ 1.52a software.

Ellipsometry: The optical experiments were performed using a Woollam variableangle spetroscopic ellipsometer (VASE) system (M2000, J.A. Woollam Co., Inc). Measurements were performed as a function of the photon energy in the range $0.8-4.5 \mathrm{eV}$ for the dry experiment and in the range 1.1-4.5 eV for the liquid experiments, a step size of $0.1 \mathrm{eV}$ (corresponds to wavelength range of 276-1550 nm and in liquid 276-1127 nm). 
Dry film thicknesses of the PMMA brushes were measured at three different angles (65, 70 , and $75^{\circ}$ ), while the experiment in liquid were conducted in a liquid cell with a fixed angle of incidence of $63^{\circ}$. The ellipsometric data were fit to determine the dry thickness of the polymer brush layer. Optical dispersion ( $n$ ) can be modelled with a Cauchy dispersion model.

$$
n(\lambda)=A+B / \lambda^{2}+C / \lambda^{4}
$$

where $n$ is the refractive index, $A, B$, and $C$ are the Cauchy parameters that can be determined by fitting the equation to measured refractive indices at known wavelengths. A three-layer model consisting of a silicon substrate, a silicon oxide layer, and a Cauchy layer (representing PMMA layer) was used to simulate the experimental data. The thickness $\mathrm{d}$ and the Cauchy parameters $A$ and $B$ were used as fitting parameters.

IR: Vacuum infrared absorbance spectrum measurements were conducted on AlphaP Bruker device. Background measurements were performed on a bare silicon wafer to act as a reference. For all the measurements, an average of 256 scans was taken.

Piezo-inkjet printer: A Jetlab 4 system printer (MicroFab Technologies, Plano, TX) was operated in drop on demand mode. Glass nozzles (MicroFab B7-39-30) with a diameter of $60 \mu \mathrm{m}$ were used. The printer was equipped with a horizontal CCD camera equipped with a LED light for drop jetting analysis and a vertical camera that allows the alignment of the print head to the sample. The nozzle was purged by jetting with pure water and ethanol between experiments. The plate and head temperature were set to $23{ }^{\circ} \mathrm{C}$. The printer was cleaned with isopropanol, ethanol and Milli-Q water before printing. The droplet formation of the inks was investigated analyzing the drop generated in continuous inkjet printing mode with firing frequency between 100-1000 Hz. Inkjet printing was set to define pre-established patterns with different configurations at room temperature. An ink solution was made with $2 \mathrm{~mL}$ ethylene glycol, $3 \mathrm{~mL}$ ethanol and 585 $\mu \mathrm{L}$ APTES.

Rheology: The rheological properties of the inks were evaluated by rotational UDS200 rheometer (Antoon Paar) equipped with parallel plates geometry. The steady-shear viscosity of the inks was evaluated for all the inks via flow measurement (also named as steady-shear rate sweep) aiming to simulate the piezo inkjet printing process. The measurements were performed at $20{ }^{\circ} \mathrm{C}$ (controlled by a Peltier temperature controller) using a MP31 spindle (a plain plate with a diameter of $50 \mathrm{~mm}$ ). Before the flow measurements, the gap (set at $500 \mu \mathrm{m}$ ) was filled with $1 \mathrm{~mL}$ of the ink solutions and the solution was then stabilized via a pre-shear step of $0.5 \mathrm{~min}$. The rheological properties were measured by increasing the shear rate at constant logarithmic steps from 0.1 to 2400 $\mathrm{s}^{-1}$, ensuring the steady flow state of the sample. The shear stress was assessed every $3 \mathrm{~s}$. The experiments were repeated three times using newly prepared solutions. The viscosity was calculated as the steady-state shear stress divided by the applied shear rate. The 
steady-shear viscosity was averaged in the range between 500-2000 $\mathrm{s}^{-1}$. All the measurements and inkjet printing processes were performed on the filtered ink solution through a $0.45 \mu \mathrm{m}$ PTFE filter.

Surface tension: The surface tension was determined by the pedant drop method with an OCA 15 (Dataphysics Instruments, Germany). The surface tension was estimated by fitting the outline of the pendant drop by the Young-Laplace equation. A needle of 0.70 $\mathrm{mm}$ of outer diameter was used to develop droplets of about $6 \mu \mathrm{L}$ of volume and the average of the surface tension values and the standard deviations were calculated with at least three different measurements.

\subsection{Supporting information}

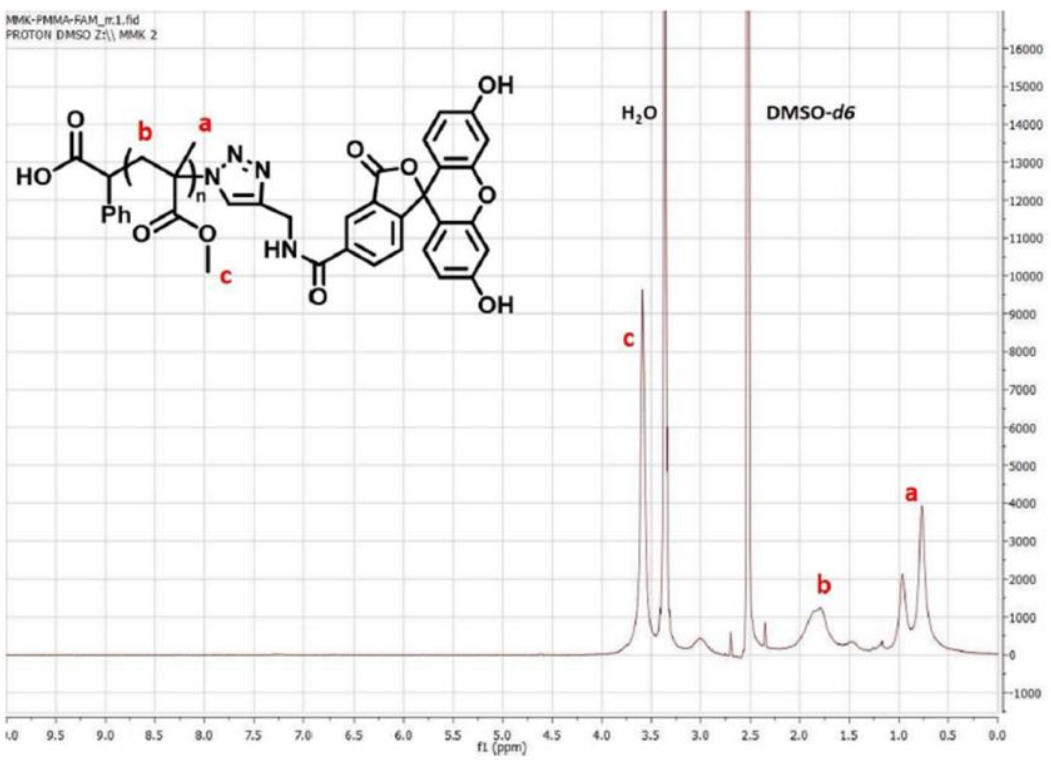

Fig. S7.1: ${ }^{1} \mathrm{H}$ NMR spectrum of the synthesized telechelic PMMA-FAM; a, b and c correspond to the $\mathrm{CH}_{3},-\mathrm{CH}_{2}$ - and $-\mathrm{OCH}_{3}$ groups, respectively. 


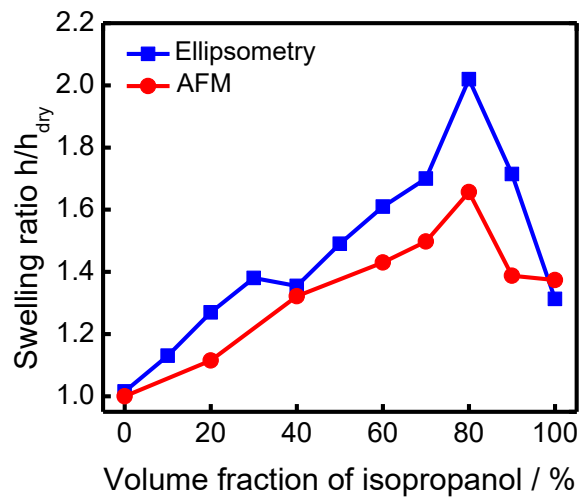

Figure S7.2: Swelling ratio of PMMA brushes obtained via SI-ATRP in different isopropanol content.

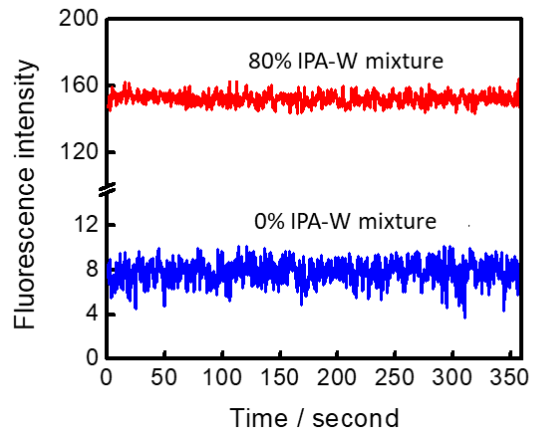

Figure S7.3: Solvent-depended fluorescence intensity of alkynylated fluorescein.

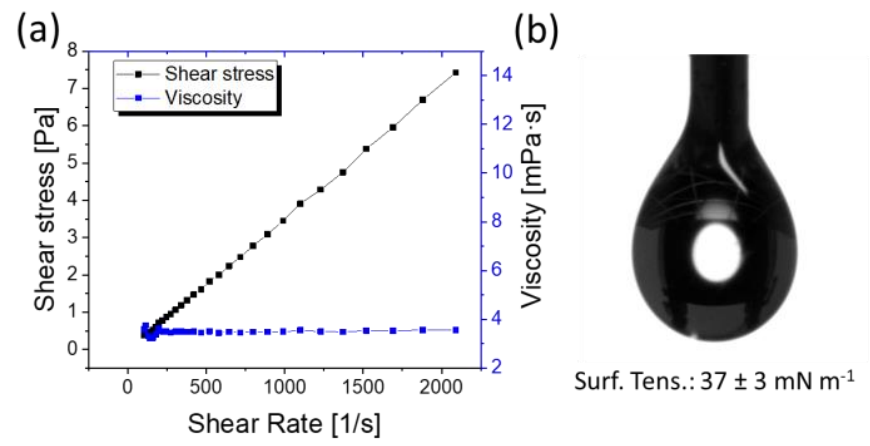

(b)

Fig. S7.4: Viscosity and surface tension of the APTES containing ink (10 w\% in ethanol-ethylene glycol mixture 3:2 v:v), (a) and (b), respectively. 


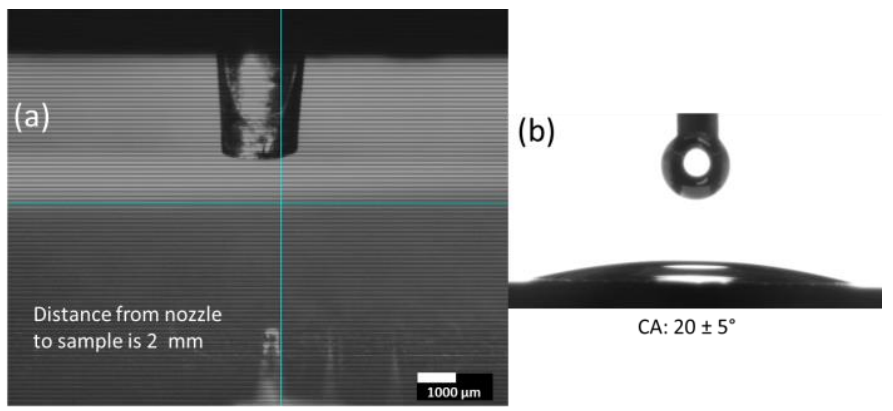

Fig S7.5: Optical micrograph of (a) the printed patterns during the inkjet printing process of the APTEScontaining ink and (b) of a spreading experiment of the ink onto silicon surface.

\subsection{References}

1. Currie, E. P. K.; Norde, W.; Cohen Stuart, M. A., Tethered polymer chains: surface chemistry and their impact on colloidal and surface properties. Advances in Colloid and Interface Science 2003, 100-102, 205-265.

2. Feng, X.; Sui, X.; Hempenius, M. A.; Vancso, G. J., Electrografting of Stimuli-Responsive, Redox Active Organometallic Polymers to Gold from Ionic Liquids. Journal of the American Chemical Society 2014, 136 (22), 7865-7868.

3. Zoppe, J. O.; Ataman, N. C.; Mocny, P.; Wang, J.; Moraes, J.; Klok, H.-A., Surface-Initiated Controlled Radical Polymerization: State-of-the-Art, Opportunities, and Challenges in Surface and Interface Engineering with Polymer Brushes. Chem. Rev. 2017, 117 (3), 1105-1318.

4. Krishnamoorthy, M.; Hakobyan, S.; Ramstedt, M.; Gautrot, J. E., Surface-Initiated Polymer Brushes in the Biomedical Field: Applications in Membrane Science, Biosensing, Cell Culture, Regenerative Medicine and Antibacterial Coatings. Chemical Reviews 2014, 114 (21), 10976-11026.

5. Hui, C. M.; Pietrasik, J.; Schmitt, M.; Mahoney, C.; Choi, J.; Bockstaller, M. R.; Matyjaszewski, K., Surface-Initiated Polymerization as an Enabling Tool for Multifunctional (Nano-)Engineered Hybrid Materials. Chemistry of Materials 2014, 26 (1), 745-762.

6. Matyjaszewski, K.; Tsarevsky, N. V., Nanostructured Functional Materials Prepared by Atom Transfer Radical Polymerization. Nature Chemistry 2009, 1 (4), 276-288.

7. Matyjaszewski, K.; Tsarevsky, N. V., Macromolecular Engineering by Atom Transfer Radical Polymerization. Journal of the American Chemical Society 2014, 136 (18), 6513-6533.

8. Ribelli, T. G.; Lorandi, F.; Fantin, M.; Matyjaszewski, K., Atom transfer radical polymerization: billion times more active catalysts and new initiation systems. Macromolecular Rapid Communications 2019, 40 (1), 1800616.

9. Zdyrko, B.; Luzinov, I., Polymer Brushes by the Grafting to Method. Macromol Rapid Commun 2011, 32 (12), 859-69.

10. Minko, S., Grafting on Solid Surfaces: Grafting to and Grafting from Methods. In Polymer Surfaces and Interfaces: Characterization, Modification and Applications, Stamm, M., Ed. Springer Berlin Heidelberg: Berlin, Heidelberg, 2008; 215-234.

11. Théophile, P.; S., M. C.; Markus, M., Synthesis and Applications of Compartmentalised Molecular Polymer Brushes. Angewandte Chemie International Edition 2018, 57 (24), 6982-6994. 
12. Nguyen, A. T.; Baggerman, J.; Paulusse, J. M. J.; Zuilhof, H.; van Rijn, C. J. M., Bioconjugation of Protein-Repellent Zwitterionic Polymer Brushes Grafted from Silicon Nitride. Langmuir 2012, 28 (1), 604-610.

13. Nguyen, A. T.; Baggerman, J.; Paulusse, J. M. J.; van Rijn, C. J. M.; Zuilhof, H., Stable Protein-Repellent Zwitterionic Polymer Brushes Grafted from Silicon Nitride. Langmuir 2011, 27 (6), 2587-2594.

14. Minko, S., Responsive polymer brushes. Journal of Macromolecular Science, Part C: Polymer Reviews 2006, 46 (4), 397-420.

15. Minko, S., Grafting on solid surfaces: Grafting to and grafting from methods. In Polymer surfaces and interfaces, Springer: 2008; 215-234.

16. Advincula, R. C., Polymer brushes. Encyclopedia of Polymer Science and Technology 2004.

17. Beija, M.; Charreyre, M. T.; Martinho, J. M. G., Dye-labelled polymer chains at specific sites: Synthesis by living/controlled polymerization. Progress in Polymer Science 2011, 36 (4), 568-602.

18. Shunjie, L.; Yanhua, C.; Haoke, Z.; Zijie, Q.; K., K. R. T.; Y., L. J. W.; Zhong, T. B., In Situ Monitoring of RAFT Polymerization by Tetraphenylethylene-Containing Agents with Aggregation-Induced Emission Characteristics. Angewandte Chemie International Edition 2018, 57 (21), 6274-6278.

19. Ingratta, M.; Hollinger, J.; Duhamel, J., A case for using randomly labeled polymers to study long-range polymer chain dynamics by fluorescence. Journal of the American Chemical Society 2008, 130 (29), 94209428.

20. Chen, S.; Duhamel, J.; Winnik, M. A., Probing End-to-End Cyclization beyond Willemski and Fixman. The Journal of Physical Chemistry B 2011, 115 (13), 3289-3302.

21. Yip, J.; Duhamel, J.; Qiu, X. P.; Winnik, F. M., Long-Range Polymer Chain Dynamics of Pyrene-Labeled Poly(N-isopropylacrylamide)s Studied by Fluorescence. Macromolecules 2011, 44 (13), 5363-5372.

22. Christie, D.; Register, R. A.; Priestley, R. D., Direct Measurement of the Local Glass Transition in SelfAssembled Copolymers with Nanometer Resolution. ACS Central Science 2018.

23. Marcelo, G.; J. V. Prazeres, T.; Charreyre, M.-T.; Martinho, J. M. G.; Farinha, J. P. S., Thermoresponsive Micelles of Phenanthrene- $\alpha$-end-labeled Poly(N-decylacrylamide-b-N,N-diethylacrylamide) in Water. Macromolecules 2010, 43 (1), 501-510.

24. Qiang, P.; Ming-Gui, X.; Koon-Gee, N.; En-Tang, K., A New Nitrite-selective Fluorescent Sensor Fabricated from Surface-initiated Atom-transfer Radical Polymerization. Chemistry Letters 2005, 34 (12), 1628-1629.

25. Sha, J.; Song, Y.; Liu, B.; Lü, C., Host-guest-recognition-based polymer brush-functionalized mesoporous silica nanoparticles loaded with conjugated polymers: A facile FRET-based ratiometric probe for $\mathrm{Hg}^{2+}$. Microporous and Mesoporous Materials 2015, 218, 137-143.

26. Wan, X.; Yao, S.; Liu, H.; Yao, Y., Selective fluorescence sensing of $\mathrm{Hg}^{2+}$ and $\mathrm{Zn}^{2+}$ ions through dual independent channels based on the site-specific functionalization of mesoporous silica nanoparticles. Journal of Materials Chemistry A 2013, 1 (35), 10505-10512.

27. Jhaveri, S. B.; Beinhoff, M.; Hawker, C. J.; Carter, K. R.; Sogah, D. Y., Chain-End Functionalized Nanopatterned Polymer Brushes Grown via in Situ Nitroxide Free Radical Exchange. ACS Nano 2008, 2 (4), 719-727.

28. Page, Z. A.; Narupai, B.; Pester, C. W.; Bou Zerdan, R.; Sokolov, A.; Laitar, D. S.; Mukhopadhyay, S.; Sprague, S.; McGrath, A. J.; Kramer, J. W.; Trefonas, P.; Hawker, C. J., Novel Strategy for Photopatterning Emissive Polymer Brushes for Organic Light Emitting Diode Applications. ACS Central Science 2017, 3 (6), 654-661.

29. Oliver, R.; Kevin, D. B.; Benjamin, V.; Lucas, S.; Martin, K.; F., A. H.; Jan, R. B.; E., D. P. F., Rewritable Polymer Brush Micropatterns Grafted by Triazolinedione Click Chemistry. Angewandte Chemie International Edition 2015, 54 (44), 13126-13129. 
30. Borozenko, O.; Godin, R.; Lau, K. L.; Mah, W.; Cosa, G.; Skene, W. G.; Giasson, S., Monitoring in RealTime the Degrafting of Covalently Attached Fluorescent Polymer Brushes Grafted to Silica SubstratesEffects of pH and Salt. Macromolecules 2011, 44 (20), 8177-8184.

31. Madsen, J.; Ducker, R. E.; Al Jaf, O.; Cartron, M. L.; Alswieleh, A. M.; Smith, C. H.; Hunter, C. N.; Armes, S. P.; Leggett, G. J., Fabrication of microstructured binary polymer brush corrals with integral $\mathrm{pH}$ sensing for studies of proton transport in model membrane systems. Chemical Science 2018, 9 (8), 2238-2251.

32. Akkilic, N.; Molenaar, R.; Claessens, M. M.; Blum, C.; de Vos, W. M., Monitoring the switching of single BSA-ATTO 488 molecules covalently end-attached to a pH-responsive PAA brush. Langmuir 2016, 32 (35), 8803-8811.

33. Wu, T.; Zou, G.; Hu, J. M.; Liu, S. Y., Fabrication of Photoswitchable and Thermotunable Multicolor Fluorescent Hybrid Silica Nanoparticles Coated with Dye-Labeled Poly(N-isopropylacrylamide) Brushes. Chemistry of Materials 2009, 21 (16), 3788-3798.

34. Johanna, B.; Johann, E.; Maarten, B. P.; Andreas, F.; S., H. W. T., Direct Correlation between Local Pressure and Fluorescence Output in Mechanoresponsive Polyelectrolyte Brushes. Angewandte Chemie International Edition 2011, 50 (41), 9629-9632.

35. Wolski, K.; Szuwarzyński, M.; Kopeć, M.; Zapotoczny, S., Ordered Photo- and Electroactive Thin Polymer Layers. European Polymer Journal 2015, 65, 155-170.

36. Kopeć, M.; Rozpędzik, A.; Łapok, Ł.; Geue, T.; Laschewsky, A.; Zapotoczny, S., Stratified Micellar Multilayers_-Toward Nanostructured Photoreactors. Chemistry of Materials 2016, 28 (7), 2219-2228.

37. Szuwarzyński, M.; Wolski, K.; Pomorska, A.; Uchacz, T.; Gut, A.; Łapok, Ł.; Zapotoczny, S., Photoactive Surface-Grafted Polymer Brushes with Phthalocyanine Bridging Groups as an Advanced Architecture for Light Harvesting. Chemistry- A European Journal 2017, 23, 11239-11243.

38. Beija, M.; Charreyre, M.-T.; Martinho, J. M., Dye-labelled polymer chains at specific sites: Synthesis by living/controlled polymerization. Progress in Polymer Science 2011, 36 (4), 568-602.

39. Matsuno, R.; Ishihara, K., The End Group Modification of Phospholipid Polymer Brush Grafted on Ferric Oxide Nanoparticles for Diagnostics. MRS Proceedings 2008, 1093, 1093-CC04-11.

40. Kuzmyn, A. R.; de los Santos Pereira, A.; Pop-Georgievski, O.; Bruns, M.; Brynda, E.; RodriguezEmmenegger, C., Exploiting end group functionalization for the design of antifouling bioactive brushes. Polymer Chemistry 2014, 5 (13), 4124-4131.

41. Parrillo, V.; Pereira, A. D.; Riedel, T.; Rodriguez-Emmenegger, C., Catalyst-free click functionalization of polymer brushes preserves antifouling properties enabling detection in blood plasma. Anal Chim Acta 2017, $971,78-87$.

42. van Andel, E.; de Bus, I.; Tijhaar, E. J.; Smulders, M. M. J.; Savelkoul, H. F. J.; Zuilhof, H., Highly Specific Binding on Antifouling Zwitterionic Polymer-Coated Microbeads as Measured by Flow Cytometry. ACS Applied Materials \& Interfaces 2017, 9 (44), 38211-38221.

43. Oh, J. S.; Wang, Y. F.; Pine, D. J.; Yi, G. R., High-Density PEO-b-DNA Brushes on Polymer Particles for Colloidal Superstructures. Chemistry of Materials 2015, 27 (24), 8337-8344.

44. Wang, C. G.; Goto, A., Solvent-Selective Reactions of Alkyl Iodide With Sodium Azide for Radical Generation and Azide Substitution and Their Application to One-Pot Synthesis of Chain-EndFunctionalized Polymers. Journal of the American Chemical Society 2017, 139 (30), 10551-10560.

45. Michał, S.; Karol, W.; Agata, P.; Tomasz, U.; Arkadiusz, G.; Łukasz, Ł.; Szczepan, Z., Photoactive Surface-Grafted Polymer Brushes with Phthalocyanine Bridging Groups as an Advanced Architecture for Light-Harvesting. Chemistry - A European Journal 2017, 23 (47), 11239-11243. 
46. Nese, A.; Lebedeva, N. V.; Sherwood, G.; Averick, S.; Li, Y.; Gao, H.; Peteanu, L.; Sheiko, S. S.; Matyjaszewski, K., pH-Responsive Fluorescent Molecular Bottlebrushes Prepared by Atom Transfer Radical Polymerization. Macromolecules 2011, 44 (15), 5905-5910.

47. Song, Y.; Lu, J.; Liu, B.; Lu, C., Temperature responsive polymer brushes grafted from graphene oxide: an efficient fluorescent sensing platform for 2,4,6-trinitrophenol. Journal of Materials Chemistry C 2016, 4 (29), 7083-7092.

48. Yang, X.; Shen, B.; Jiang, Y.; Zhao, Z.; Wang, C.; Ma, C.; Yang, B.; Lin, Q., A novel fluorescent polymer brushes film as a device for ultrasensitive detection of TNT. Journal of Materials Chemistry A 2013, 1 (4), 1201-1206.

49. Seung, R. Y.; Sang-Hoon, B.; Huajun, C.; Nicholas, D. M.; Yang, Y., Recent Progress in Materials and Devices toward Printable and Flexible Sensors. Advanced Materials 2016, 28 (22), 4415-4440.

50. Nie, Z.; Kumacheva, E., Patterning Surfaces with Functional Polymers. Nature Materials 2008, 7, 277.

51. Jones, D. M.; Smith, J. R.; Huck, W. T. S.; Alexander, C., Variable Adhesion of Micropatterned Thermoresponsive Polymer Brushes: AFM Investigations of Poly(N-isopropylacrylamide) Brushes Prepared by Surface-Initiated Polymerizations. Advanced Materials 2002, 14 (16), 1130-1134.

52. Benetti, E. M.; Zapotoczny, S.; Vancso, G. J., Tunable Thermoresponsive Polymeric Platforms on Gold by Photoiniferter -Based Surface Grafting. Advanced Materials 2007, 19 (2), 268-271.

53. Roling, O.; De Bruycker, K.; Vonhören, B.; Stricker, L.; Körsgen, M.; Arlinghaus, H. F.; Ravoo, B. J.; Du Prez, F. E., Rewritable Polymer Brush Micropatterns Grafted by Triazolinedione Click Chemistry. Angewandte Chemie International Edition 2015, 54 (44), 13126-13129.

54. Sankhe, A. Y.; Booth, B. D.; Wiker, N. J.; Kilbey, S. M., Inkjet-Printed Monolayers as Platforms for Tethered Polymers. Langmuir 2005, 21 (12), 5332-5336.

55. Emmerling, S. G. J.; Langer, L. B. N.; Pihan, S. A.; Lellig, P.; Gutmann, J. S., Patterning of a Surface Immobilized ATRP Initiator with an Inkjet Printer. Macromolecules 2010, 43 (11), 5033-5042.

56. Parry, A. V. S.; Straub, A. J.; Villar-Alvarez, E. M.; Phuengphol, T.; Nicoll, J. E. R.; W. K, X. L.; Jordan, L. M.; Moore, K. L.; Taboada, P.; Yeates, S. G.; Edmondson, S., Submicron Patterning of Polymer Brushes: An Unexpected Discovery from Inkjet Printing of Polyelectrolyte Macroinitiators. Journal of the American Chemical Society 2016, 138 (29), 9009-9012.

57. Poelma, J. E.; Fors, B. P.; Meyers, G. F.; Kramer, J. W.; Hawker, C. J., Fabrication of Complex ThreeDimensional Polymer Brush Nanostructures through Light-Mediated Living Radical Polymerization. Angewandte Chemie International Edition 2013, 125 (27), 6982-6986.

58. Discekici, E. H.; Pester, C. W.; Treat, N. J.; Lawrence, J.; Mattson, K. M.; Narupai, B.; Toumayan, E. P.; Luo, Y.; McGrath, A. J.; Clark, P. G.; Read de Alaniz, J.; Hawker, C. J., Simple Benchtop Approach to Polymer Brush Nanostructures Using Visible-Light-Mediated Metal-Free Atom Transfer Radical Polymerization. ACS Macro Letters 2016, 5 (2), 258-262.

59. Hoogenboom, R.; Becer, C. R.; Guerrero-Sanchez, C.; Hoeppener, S.; Schubert, U. S., Solubility and thermoresponsiveness of PMMA in alcohol-water solvent mixtures. Australian Journal of Chemistry 2010, 63 (8), 1173-1178.

60. Mukherji, D.; Marques, C. M.; Stuehn, T.; Kremer, K., Depleted depletion drives polymer swelling in poor solvent mixtures. Nature Communications 2017, 8 (1), 1374.

61. Yu, Y.; Kieviet, B. D.; Kutnyanszky, E.; Vancso, G. J.; de Beer, S., Cosolvency-Induced Switching of the Adhesion between Poly(methyl methacrylate) Brushes. ACS Macro Letters 2015, 4 (1), 75-79.

62. Golas, P. L.; Matyjaszewski, K., Marrying Click Chemistry with Polymerization: Expanding the Scope of Polymeric Materials. Chemical Society Reviews 2010, 39 (4), 1338-1354.

63. Yu, Y.; Kieviet, B. D.; Kutnyanszky, E.; Vancso, G. J.; de Beer, S., Cosolvency-Induced Switching of the Adhesion Between Poly(methyl methacrylate) brushes. ACS Macro Letters 2014, 4 (1), 75-79. 
64. Xie, G.; Khabibullin, A.; Pietrasik, J.; Yan, J.; Matyjaszewski, K., Polymer Brushes by Atom Transfer Radical Polymerization. Polymer and Biopolymer Brushes: For Materials Science and Biotechnology 2018, 242.

65. Patil, R. R.; Turgman-Cohen, S.; Šrogl, J. í.; Kiserow, D.; Genzer, J., On-Demand Degrafting and the Study of Molecular Weight and Grafting Density of Poly(methyl methacrylate) Brushes on Flat Silica Substrates. Langmuir 2015, 31 (8), 2372-2381.

66. Patil, R. R.; Turgman-Cohen, S.; Šrogl, J. í.; Kiserow, D.; Genzer, J., Direct Measurement of Molecular Weight and Grafting Density by Controlled and Quantitative Degrafting of Surface-Anchored Poly(methyl methacrylate). ACS Macro Letters 2015, 4 (2), 251-254.

67. Magdassi, S., Ink Requirements and Formulations Guidelines. The chemistry of inkjet inks. MAGDASSI, S. Eds 2010, 19-42.

68. Kuang, M.; Wang, L.; Song, Y., Controllable Printing Droplets for high-Resolution Patterns. Advanced Materials 2014, 26 (40), 6950-6958.

69. Parry, A. V.; Straub, A. J.; Villar-Alvarez, E. M.; Phuengphol, T.; Nicoll, J. E.; Jordan, L. M.; Moore, K. L.; Taboada, P.; Yeates, S. G.; Edmondson, S., Submicron Patterning of Polymer Brushes: an Unexpected Discovery from Inkjet Printing of Polyelectrolyte Macroinitiators. Journal of the American Chemical Society 2016, 138 (29), 9009-9012.

70. Jakubowski, W.; Min, K.; Matyjaszewski, K., Activators Regenerated by Electron Transfer for Atom Transfer Radical Polymerization of Styrene. Macromolecules 2006, 39 (1), 39-45.

71. Matyjaszewski, K.; Jakubowski, W.; Min, K.; Tang, W.; Huang, J.; Braunecker, W. A.; Tsarevsky, N. V., Diminishing Catalyst Concentration in atom Transfer Radical Polymerization with Reducing Agents. Proceedings of the National Academy of Sciences 2006, 103 (42), 15309-15314.

72. Iwata, R.; Suk-In, P.; Hoven, V. P.; Takahara, A.; Akiyoshi, K.; Iwasaki, Y., Control of Nanobiointerfaces Generated from Well-Defined Biomimetic Polymer Brushes for Protein and Cell Manipulations. Biomacromolecules 2004, 5 (6), 2308-2314.

73. Santonicola, M. G.; de Groot, G. W.; Memesa, M.; Meszyńska, A.; Vancso, G. J., Reversible pHcontrolled Switching of Poly(methacrylic acid) Grafts for Functional Biointerfaces. Langmuir 2010, 26 (22), 17513-17519.

74. Bain, E. D.; Turgman-Cohen, S.; Genzer, J., Progress in Computer Simulation of Bulk, Confined, and Surface-initiated Polymerizations. Macromolecular Theory and Simulations 2013, 22 (1), 8-30. 
Chapter 7 
Chapter 8

Printing "Smart" Inks of Redox-Responsive Organometallic Polymers on Microelectrode Arrays for Molecular Sensing

* This chapter has been published in: Cirelli M., Hao J., Bor T.C., Duvigneau J., Benson N., Akkerman R., Hempenius M.A., Vancso G.J.; ACS Applied Materials \& Interfaces. 2019, 11 (40), 37060-37068. 


\section{Contents}

\section{Chapter 8 Printing "Smart" Inks of Redox Responsive} Organometallic Polymers on Microelectrode Arrays for Molecular Sensing............................................................................... 167

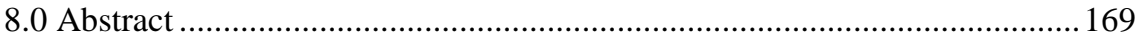

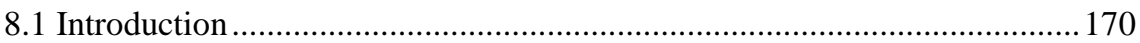

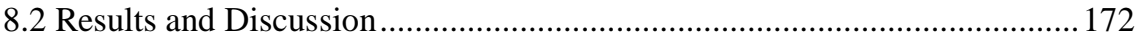

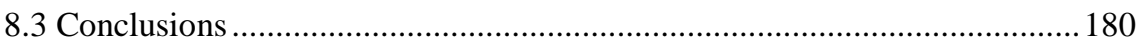

8.4 Experimental Section …................................................................... 181

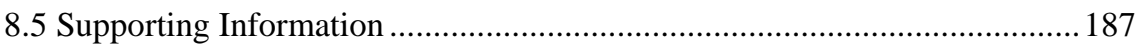

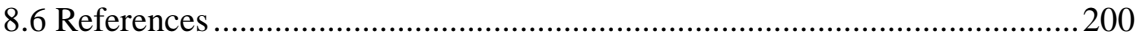




\subsection{Abstract}

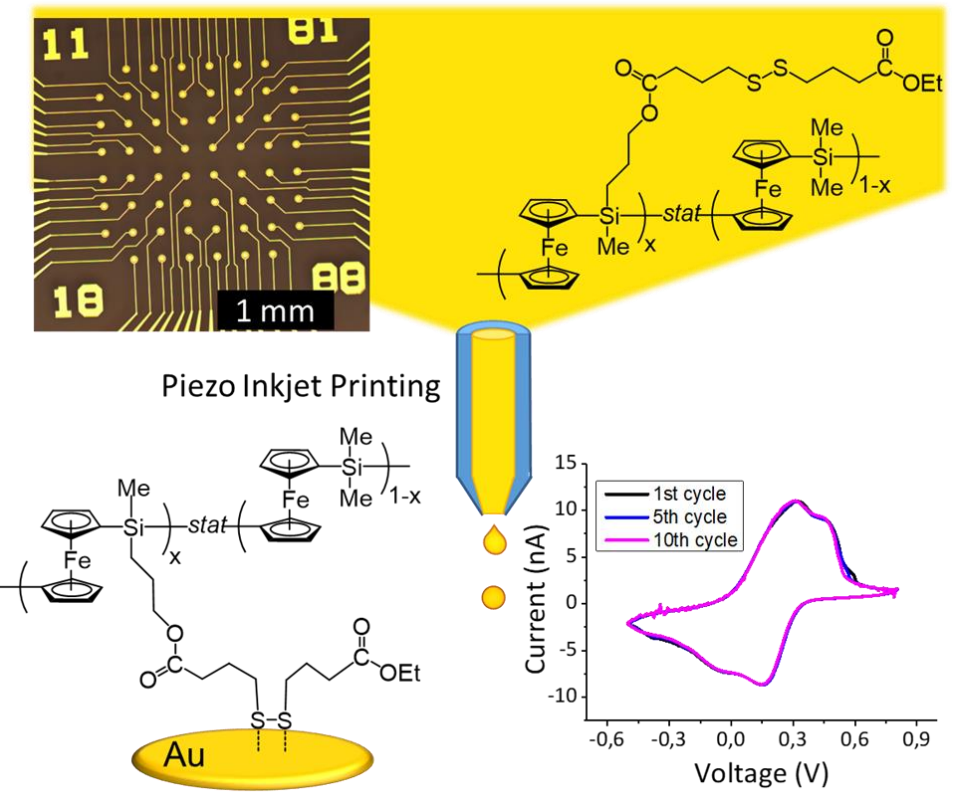

TOC Chapter 8: Printing "smart" inks of redox-responsive organometallic polymers on microelectrode arrays (MEAs) for molecular sensing; commercial MEAs were modified with a new redox-responsive disulfidemodified poly(ferrocenylsilane) (PFS) ink by using inkjet printing in the Drop-on-Demand (DoD) mode and tested as an amperometric electrochemical sensing device for ascorbic acid.

Printing arrays of responsive spots for multiplexed sensing with electrochemical readout requires new molecules and precise, high-throughput deposition of active compounds on microelectrodes with spatial control. We have designed and developed new redox-responsive polymers, featuring a poly(ferrocenylsilane) (PFS) backbone and side groups with disulfide units, which allow an efficient and stable bonding to gold (Au) substrates, using sulphur-gold coupling chemistry in a grafting-to (GT) approach. The polymer molecules can be employed for area selective molecular sensing following their deposition by high precision inkjet printing. The new PFS-derivatives, which serve as "molecular inks", were characterized by ${ }^{1} \mathrm{H}$ NMR, ${ }^{13} \mathrm{C}$ NMR and FTIR spectroscopies and by GPC. The viscosity and surface tension of the inks were assessed by rheology and pendant drop contact angle measurements, respectively. Commercial microelectrode arrays were modified using the new PFS ink by using inkjet printing in the Drop-onDemand (DoD) mode. FTIR spectroscopy, AFM and EDX-SEM confirmed a successful, spatially localized PFS modification of the individual electrodes within the sensing cells of the MEAs. The potential application of these devices to act as an electrochemical sensor array was demonstrated with a model analyte, ascorbic acid, using cyclic voltammetry and amperometric measurements. 


\subsection{Introduction}

Microelectrode arrays (MEAs) allow one to perform multiplexed parallel analysis of complex mixtures of redox active analytes with selective and specific sensing of the individual constituents in the microelectrode cells that make up the array. ${ }^{1-10}$ For a successful implementation of such devices, one could consider selective printing of specific sensing molecular inks into the individual microelectrode cells (MEs) that can exhibit a different composition from "pixel" to "pixel" within the array. ${ }^{6-10}$ The recognition inks should adhere to the ME surface and provide analyte selective signals. Printing using multiple reservoirs would be needed to render each electrochemical microcell working as a specific sensing element.

Towards achieving this objective, we describe in this contribution the first necessary steps including a) the development of a novel redox stimulus responsive molecular ink and b) the construction of single ME sensing "pixels" using one printing reservoir and a single nozzle. By doing so, we provide elements for designing devices such as "aiming" the ink to accurately hit the microelectrode with high printing accuracy. We describe how printing accuracy can be evaluated. We then test the ME device concept in a model sensing process.

Stimulus responsive (or "smart") polymers have been in the focus of recent interest due to their ability to be used in functional, integrated material constructs for wetting and friction control, sensing, monitoring and steering processes, in mechanical actuation, as self-healing biomedical platforms, and in molecular delivery. ${ }^{11-16}$ Among the many possible stimuli utilized in conjunction with polymers, electrochemical (redox) control has received relatively little attention when compared to temperature, $\mathrm{pH}$, light, and chemical stimuli. ${ }^{17-21}$ One of the reasons for the employment of the "classical" stimuli is that the range of synthetic polymers, which exhibit a reversible redox response, is rather limited. ${ }^{17,}$ 19-21

The emerging class of redox responsive organometallic poly(ferrocenylsilane)s (PFSs), which feature alternating ferrocene units and substituted bridging $\mathrm{Si}$ in the main chain, has shown a great potential to fabricate redox-sensitive molecular sensing platforms. $^{21-24}$ In PFSs, the silane units can be employed in substitution chemistry, yielding a broad range of functional macromolecules. The variations of side-group structure allows one to tune the physical and chemical properties of PFS, while the ferrocene units remain responsible for preserving the redox activity. ${ }^{22-26}$ The stimulus responsive behavior implies that changes of the redox environmental conditions trigger changes of the oxidation state of the iron atom in the ferrocene group. We note that redox chemistry of PFS, including chemical and electrochemical redox transitions, have been subject of several studies. ${ }^{18,26-29}$ Corresponding papers discuss intra- and inter-chain electron transfer during electrochemical oxidation/reduction, ${ }^{30-31}$ as well as the effect of chemical oxidizing and reducing agents, ${ }^{23}$ and redox behavior of PFS attached to the 
surface of various substrates. ${ }^{20-21}$ PFS-decorated electrodes have been used for electrochemical sensing of ascorbic acid, ${ }^{20,32-34}$ hydrogen peroxide, ${ }^{34}$ glucose ${ }^{35-36}$ and inorganic ions, ${ }^{37}$ and/or to enhance detection sensitivity, ${ }^{18,21}$ and photoconductivity. ${ }^{38}$

MEAs have been successfully utilized in many areas in biomedical applications, , 3-5 as well as in environmental sensing ${ }^{2,39-40}$ and food quality monitoring. ${ }^{9}, 40$ The reason for this interest is that MEAs enable the stimulation and recording of electrical signals of multiple MEs with low background charging and high current density. This is related to the efficient mass transport of the redox active species to, and from, the micro sized electrode surface (i.e. the electrode diameter is less than $100 \mu \mathrm{m}$ ) and the bulk solution. ${ }^{6}$, 10,41

One of the many challenges in the development of multiplexed MEA sensors is to individually and independently immobilize functional coatings onto the electrode surface of each of the MEs with a high quality of spatial control. ${ }^{7,9,42-43}$ Benefits of precise deposition of responsive functional coatings are expected to further boost the use of MEAs, for example, by fabrication of MEAs on soft materials, or functionalization of MEAs improving their performance and biocompatibility. ${ }^{44}$ This would allow in-situ multiplex sensing, cell patterning, and delivery applications. 2, 4, 9, 39-40, 43 Two main strategies can be used to chemically immobilize sensing macromolecules on different electrode surfaces encompassing the grafting-from ${ }^{16,45-47}$ (GF) or the grafting-to ${ }^{16,48}$ (GT) approaches. In this study, we decorated bare gold ME surfaces via the GT approach employing the well-known strong and stable sulfur-gold binding chemistry. ${ }^{49-50}$

Various sulfur-containing PFS macromolecules have been prepared and reported previously. For example, end-capping of living PFS chains with ethylene sulfide provided access to thiol end-functionalized PFSs. ${ }^{51}$ Post-polymerization modification using thiolene chemistry proved to be another, highly versatile approach to obtain sulfurfunctionalized PFSs. ${ }^{52}$ In this study, first a PFS random copolymer possessing a tailored amount of reactive halopropyl side groups was synthesized via platinum catalyzed ring opening polymerization of silicon-bridged ferrocenophanes. ${ }^{53-54}$ Then, disulfide moieties were attached to the haloalkyl groups by nucleophilic substitution to afford disulfidefunctionalized PFSs with a well-controlled composition capable to covalently graft to gold surfaces, as is shown in Figure 8.1.

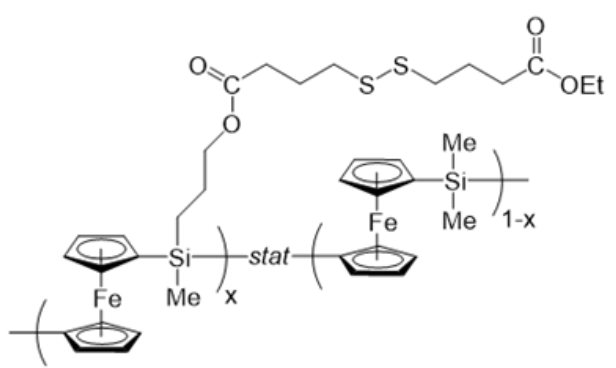

Fig. 8.1: Structure of the disulfide functionalized poly(ferrocenylsilane) copolymer used in this study. 
Molecular surface immobilization of PFS films on the electrodes investigated here was already realized via the GT approach, layer-by-layer (LbL), ${ }^{34,55}$ drop casting, ${ }^{32}$ dip coating, ${ }^{51}$ spin coating, ${ }^{56}$ and electrografting ${ }^{33}$ deposition techniques. In previous work of Vancso et al. ${ }^{55}$ the immobilization of PFS derivates in LbL fashion on MEAs was achieved by physical immobilization (i.e. electrostatic interactions) and used to fabricate a redox responsive multilayer platform for local controlled release of multiple molecular payloads from the surfaces. ${ }^{55}$ In comparison to these techniques, inkjet printing technology is a very promising, reliable and convenient way for patterning by deposition to individually decorate the MEA cells. The advantages of this technique include contactfree deposition on the electrode substrates (minimizing contaminations or alterations), no need of masks, versatile applicability and targeted delivery. ${ }^{57-61}$ The ink can be water- or organic solvent-based, and good repeatability and spatial accuracy in the deposition process has been achieved. ${ }^{62-63}$ Inkjet printing can be performed in continuous ink jet (CIJ), or in drop-on-demand (DoD-IJ) ink jet modes, respectively. In C-IJP, the ink solution is pumped through a nozzle to form a liquid jet. This mode is mainly used in high-speed graphical applications due to the high throughput of the production. In the DoD-IJ mode the droplet is formed by an acoustic pulse, generated by a piezoelectric or thermal trigger, and is printed from a reservoir through a nozzle with high placement accuracy. Employing the DoD mode minimizes the amount of ink used for the deposition (few tens of $\mathrm{pL}$ ) and thus reduces ink waste and minimizes the consumption of the functional compound.

Therefore, following a thorough characterization of the here described novel PFSbased ink, we performed printability tests and evaluated the accuracy, chemical composition, and topological and morphological features of the PFS printed patterns. The PFS modified MEAs were then tested as an ascorbic acid sensor in a case study. To this end, the stability and the electrochemical properties of the tethered PFS polymer films via cyclic voltammetry and chronoamperometry experiments were assessed.

\subsection{Results and Discussion}

As mentioned in the introduction, we present and discuss the design, and the implementation of a potentially multiplexed electrochemical sensing device. The individual sensors in a fully multiplexed instrument can each hold different responsive materials for specific sensing applications, which need to be printed onto the micro electrode surface.

\section{Synthesis of disulfide-functionalized poly(ferrocenylsilane)s, PFS 5.}

First, we discuss the synthesis of a new PFS-based redox responsive polymer which can be immobilized via sulfur-gold coupling. This new PFS-based random copolymer was designed to possess a tailored number of disulfide moieties to ensure a stable covalent attachment of the polymer to the gold surface. Disulfide modified poly(ferrocenylsilane) random copolymers were prepared in three steps, starting with the transition metal- 
catalyzed ring opening polymerization (ROP) of [1]dimethylsilaferrocenophane $\mathbf{1}$ and [1](3-chloropropyl)methylsila-ferrocenophane 2 to afford PFS 3 (Scheme 8.1).

Scheme 8.1: Synthesis of disulfide-functionalized PFS 5.

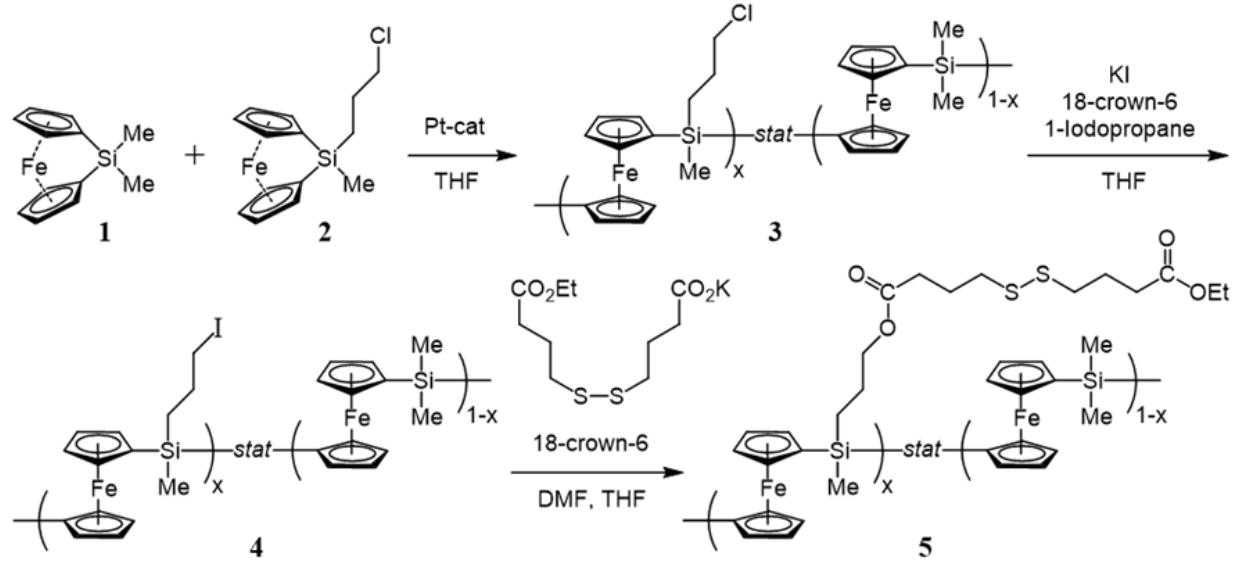

The chloropropyl moieties introduced by copolymerization of $\mathbf{1}$ with ferrocenophane 2 enable further derivatization of the formed PFS chains by nucleophilic substitution, in this work by their conversion into more reactive iodopropyl groups, yielding PFS 4 . Reaction of PFS 4 with the potassium salt of 4,4-dithiobutyric acid monoethyl ester led to disulfide-functionalized PFS 5. As transition metal catalyzed random copolymerization of ferrocenophanes has been demonstrated earlier, we anticipated that the presence of 5 mol\% ferrocenophanes $\mathbf{2}$ in the monomer mixture would lead to the incorporation of a corresponding amount of disulfide groups along the PFS chains.

The PFS copolymers 3, 4 and 5 were characterized by ${ }^{1} \mathrm{H}$ and ${ }^{13} \mathrm{C}$ NMR spectroscopy, FTIR spectroscopy and gel permeation chromatography (GPC) (see Supporting Information, SI). NMR spectroscopy confirmed the complete conversion of PFS 3 into its iodo- analogue PFS 4 through halogen exchange (Figure S8.7). In addition, the attachment of the disulfide moieties to PFS 4 occurred with quantitative conversion. By comparing the integral of the characteristic $\mathrm{CH}_{2}-\mathrm{Cl}$ triplet at $\delta=3.24 \mathrm{ppm}$ with the integral of the ferrocene signals at $\delta=4.06$ and $4.24 \mathrm{ppm}$ of PFS 3, the chloropropyl group content was determined to be approximately $7 \mathrm{~mol} \%$. A similar value was found for the amount of iodopropyl groups in the polymer 4. The integrals of the signals belonging to the methylene moieties adjacent to the disulfide groups in polymer $\mathbf{5}$ at $\delta=$ $2.45 \mathrm{ppm}$ showed a disulfide content of $5 \mathrm{~mol} \%$ in this polymer. In the FTIR spectrum of PFS 5, characteristic absorptions of the ester groups $\left(\mathrm{C}=\mathrm{O}\right.$ stretch, 1750 and $\left.1735 \mathrm{~cm}^{-1}\right)$ and ferrocene rings $\left(1182,1165\right.$, and $\left.1037 \mathrm{~cm}^{-1}\right)$ were observed.

The molar masses of the macromolecules were determined by GPC measurements. For PFS 5, $M_{\mathrm{n}}=138500 \mathrm{~g} \cdot \mathrm{mol}^{-1}, M_{\mathrm{w}}=278800 \mathrm{~g} \cdot \mathrm{mol}^{-1}, M_{\mathrm{w}} / M_{\mathrm{n}}=2.0$ were found (the values refer to data determined using PS calibration standards). For this relatively high molar mass, with an average degree of polymerization $D P_{\mathrm{n}}$ exceeding $500,5 \mathrm{~mol} \%$ 
corresponds to around 25 disulfide moieties per PFS chain, which should be sufficient for robust immobilization of these chains on gold surfaces in the sensing device.

\section{DoD piezo inkjet printing of the PFS 5 based ink.}

Following the completion of the synthesis of this new ink constituent PFS 5, our attention turned to the printing process and the printing accuracy. While this is obviously dependent on the printer device employed, some generic results will be discussed below. The first question to be tackled for a new ink is related to its printability.

Important physical properties regarding the ink encompass viscosity, density and surface tension since these quantities affect the droplet formation, and thus impact the repeatability and the accuracy of the inkjet deposition process. ${ }^{60-61,64-65}$ The requirements for a printable ink for the Pixdro LP50 inkjet printer equipped with a DMC-11610 cartridge, as used in this study, include a viscosity range between 1 and $20 \mathrm{mPa} \cdot \mathrm{s}$ and a surface tension between 25 and $50 \mathrm{mN} \cdot \mathrm{m}^{-1}$. Thus, regarding the formulation of the inks, the choice of the solvent is a key parameter and it is necessary to consider the polymer solubility, the chemical stability over time, the surface tension as well as viscosity. In our case, toluene was a good candidate, because it is a good solvent for PFS 5, with a low boiling temperature (allowing the ink to dry quickly enough to prevent smudging) and it also exhibits good wetting properties on gold.

As explained in more detail in the experimental section and in the SI, toluene based inks with 2.5, 5 and 10 wt\% PFS 5, which are referred to as ink A, ink B and ink C, respectively, were prepared and their solution properties were characterized (Table S8.1). All solutions exhibited Newtonian behavior in the tested shear rate range between 500 and $2000 \mathrm{~s}^{-1}$. The shear-viscosity values increased with an increasing solid content in the solution (between 1.8 and $11 \mathrm{mPa} \cdot \mathrm{s}$ ), and the surface tension values slightly decreased upon increasing the amount of polymer in the ink (between 28 and $26 \mathrm{mN} \cdot \mathrm{m}^{-1}$ ), as shown

in, Figure S8.9. Consequently, all tested inks exhibited a suitable viscosity for inkjet printing and their surface tension was compatible with the surface characteristics of the gold electrode surface.

We chose "ink A" as the best candidate in this work, as it provides good rheological properties (with shear viscosity of $1.8 \mathrm{mPa} \cdot \mathrm{s}$ ), ideal printability (i.e. stable, no satellite drops, repeatable droplet formation), accurate deposition, and a long time storage stability (i.e. the physical and chemical ink properties were stable for more than six months). In addition, since it has the lowest concentration of PFS 5 required to obtain a printable ink, it also allows for the deposition of the lowest amount of PFS 5 per drop, eventually enhancing control over the deposited layer thickness. In fact, as shown in Figure 8.2a from the high-speed image collection, the ink developed a stable jet with drop volumes of about $10 \mathrm{pL}$ and a velocity of $5 \mathrm{~m} \cdot \mathrm{s}^{-1}$ using the simple actuator waveform as depicted in Figure 2b. No satellite droplets formed during printing and a good accuracy and precision with a misalignment of less than $0.26^{\circ}$ was obtained, as shown in Figure 8.2c. 

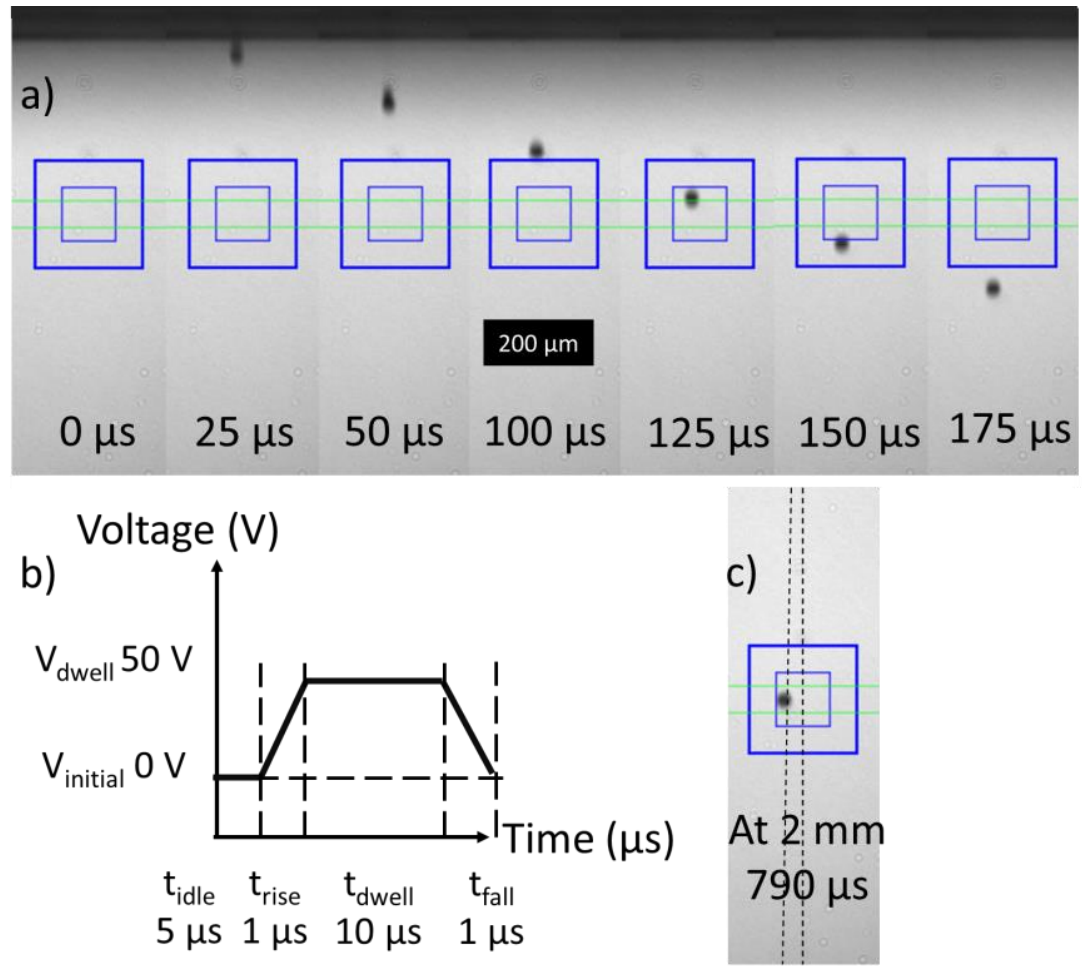

Fig. 8.2: a) Series of high-speed photographic images of "ink A" (see Experimental Section) as it is ejected from the nozzle at times between 0 to $175 \mu \mathrm{s}$ after the start of the piezo actuation. Characteristics of the drop: speed $5 \mathrm{~m} \cdot \mathrm{s}^{-1}$ and volume $10 \mathrm{pL}$. b) The piezoelectric actuator waveform (potential vs. time) used for a stable drop-on-demand inkjet printing of "ink A". c) High-speed photographic image recorded at a distance of $2 \mathrm{~mm}$ from the nozzle which corresponds to the distance between the print-head and the MAE substrate during the deposition of the ink, revealing a misalignment of $0.26^{\circ}$.

\section{Characterization of the device: surface and electrochemical properties.}

The successful deposition of PFS 5 ink via inkjet printing was confirmed by visual inspection via optical and laser confocal microscopy. Then, the successful immobilization of the ultrathin redox active polymer films onto the ME surface was verified via HRSEM-EDX, ATR-FTIR microscopy and cyclic voltammetry (CV) measurements, as shown in Figure 8.3 and in the $\mathbf{S I}$. 
a)

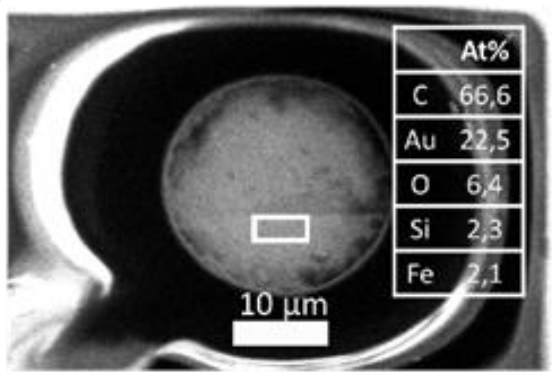

c)

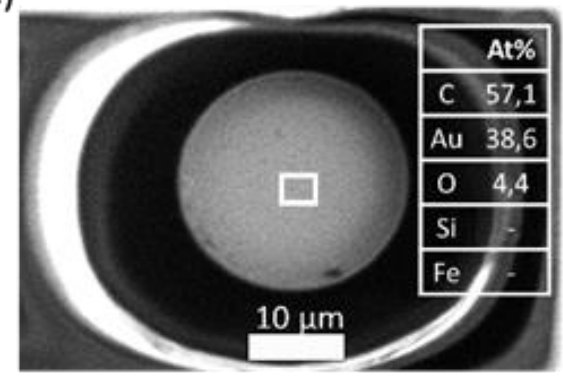

b)

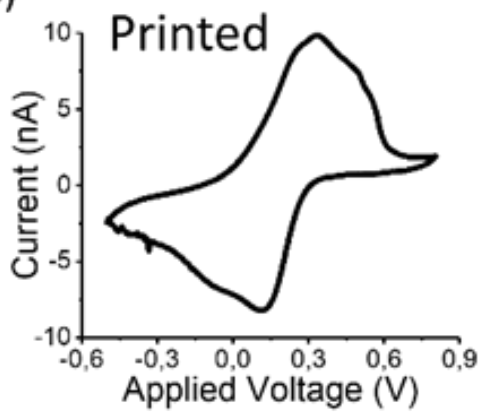

d)

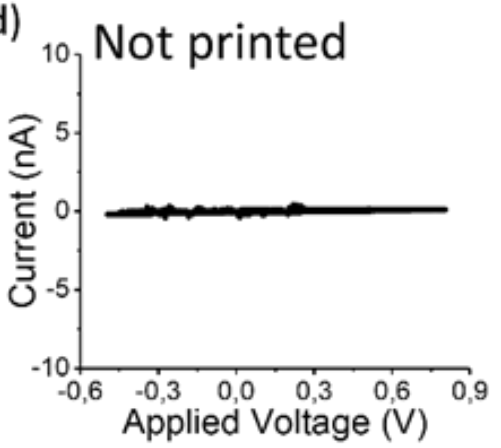

Fig. 8.3: Surface characterization of the MEs via SEM-EDX: a) and c) SEM images of PFS 5 modified and bare gold electrodes of the MEA, respectively. The insets show the elemental composition of the areas in the rectangular white boxes. b) and d) CVs of a PFS 5 modified and a bare electrode of the MEA, respectively. Scan rate $50 \mathrm{mV} \cdot \mathrm{s}^{-1}$, in $100 \mathrm{mM} \mathrm{NaClO}_{4}, \mathrm{Pt}$ (wire)-RE/CE, and potential range between -500 and $800 \mathrm{mV}$ vs Pt.

EDX analysis confirmed the presence of $\mathrm{Fe}$ and $\mathrm{Si}$ in equal atomic percentages after deposition of PFS $\mathbf{5}$ ink on the MEs, as is shown in the inset of the SEM images in Figure 8.3a and 8.3c. The presence of $S$ was not observed with EDX analysis since its concentration was below the sensitivity of the instrument. In FTIR spectra, the typical absorbance bands of ferrocene and of the ester groups were detected, proving the grafting of the disulfide modified PFSs onto the gold surface, as shown in Figure S8.14. A morphological analysis of the PFS grafts was performed by AFM, confirming a generally flat and uniform topology over the electrode surfaces. AFM surface profile measurements showed that the value for the average step height of the dry PFS 5 film was $8 \pm 3 \mathrm{~nm}$ and the average surface roughness was $1.2 \pm 0.5 \mathrm{~nm}$, as shown in Figure S8.13.

The conclusive confirmation of the successful ME surface modification was obtained $\mathrm{CV}$ measurements on the PFS 5 decorated MEs. As shown in Figure 8.3b and 8.3d, the characteristic oxidation and reduction peaks of the ferrocene moieties of PFS (between -100 and $400 \mathrm{mV}$ versus Pt electrode) are clearly present for the printed electrodes, while no current was observed for the non-printed MEs. The double-wave voltammogram, typical of PFS, indicates $p-\pi d-\pi$ electron orbital overlap due to electronic delocalization between neighboring ferrocene units in the polymer via the Si bridge. ${ }^{24-25,27,31}$ For some 
of the modified PFS 5 MEs, as shown in Figure S8.15, a single-wave voltammogram was recorded due to the (likely) instability of the positively charged intermediate in the aqueous solution, inhomogeneity of the coating, or due to different thickness of the polymer films and the resulting heterogeneity in electron transfer paths. ${ }^{8,66}$

As shown in Figure S8.15, we propose a classification of the printing quality based on the electrochemical and electrocatalytic properties of the coated MEs differentiating among well-modified, poor-modified and non-functionalized MEs, respectively. The electron transfer mechanism of the well-modified MEs was essentially reversible, while for the poorly modified electrodes the electron transfer process was more complex. Here the rate of electron transport through films and transport between the films and the electrode were rather slow (i.e. at low scan rates the process was quasi reversible, while at high scan rates it was pratically irreversible). For the well-modified electrodes, the surface coverage, $\Gamma$, of PFS 5 was estimated, according to Equation 8.1, ${ }^{8}$ to be $1.6 \times 10^{-9}$ mol ferrocene units per $\mathrm{cm}^{2}$.

$$
\Gamma=Q /(n F A)
$$

in which, $A$ is the geometric surface area of the gold working ME, $n$ is the number of electrons involved in the redox process ( 1 for ferrocene units), $F$ is the Faraday's constant (96485 C. $\mathrm{mol}^{-1}$ ) and $Q$ is the charge passed during the oxidation/reduction of ferrocene sites. The grafting value of $1.60 \times 10^{-9} \mathrm{~mol} \cdot \mathrm{cm}^{-2}$ is in the order of magnitude of values shown in literature for GT high molar mass macromolecules, and indicates the presence of a thin, uniform and relatively densely grafted PFS film on the electrode surface. ${ }^{8,20}$

In order to confirm the stability of deposited PFS 5 films on the ME, successive CV measurements were conducted. For comparison, repeated CV measurements were performed for deposited films of iodine functionalized PFS (PFS 4). Figure 8.4 shows the CVs for PFS 5 (a) and PFS 4 (b) layers on gold electrodes.

From Figure 8.4, it is clear that for PFS 5 films the oxidation and reduction peaks remained unchanged and are reproducible for more than 10 potential cycles, indicating that no PFS chains desorb from the substrate. On the other hand, as shown in Figure 8.4b, the oxidation and reduction currents for PFS 4 films decreased after each CV indicating the gradual desorption of the iodopropyl functionalized PFS 4 from the Au-ME. Hence, the introduction of a disulfide functionality along the PFS backbone is a necessity to significantly enhance the printed layer stability on gold electrodes.

Having thus established the functionalization of an individual ME by inkjet printing, we then turned our attention to testing the individual microelectrode cells for sensing applications. For this purpose, we used ascorbic acid as a standard and well-characterized redox analyte. In earlier works, we have reported and described the use of PFS sensors for ascorbic acid detection. ${ }^{20,32-34}$ The PFS films used in these studies served as electron transfer mediator in the electrocatalytic oxidation of ascorbic acid in amperometric chemical sensors. 
a) PFS 5-mod. Au-ME

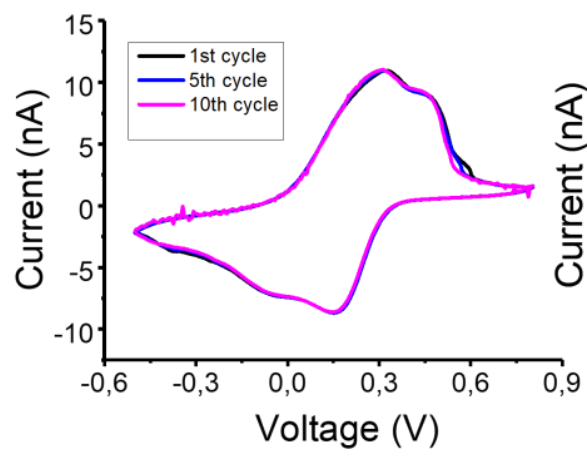

b) PFS 4-mod. Au-ME

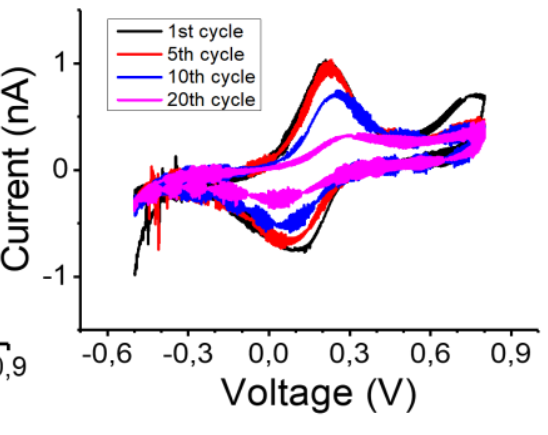

Fig. 8.4: Evaluation of the electrochemical stability of printed surface-tethered PFS 5 layers printed on gold electrodes of the MEA by repeated CV measurements. Scan rate $50 \mathrm{mV} \cdot \mathrm{s}^{-1}$, in $100 \mathrm{mM} \mathrm{NaClO}, \mathrm{Pt}($ wire)$\mathrm{RE} / \mathrm{CE}$, and potential range between -500 and $800 \mathrm{mV}$ vs Pt.

In Figure 8.5 we demonstrate the ability of the ME grafted PFS films to mediate and catalyze the electron transfer process of the ascorbic acid oxidation. Voltammograms for the bare and the modified gold MEs in presence and in absence of ascorbic acid are shown.
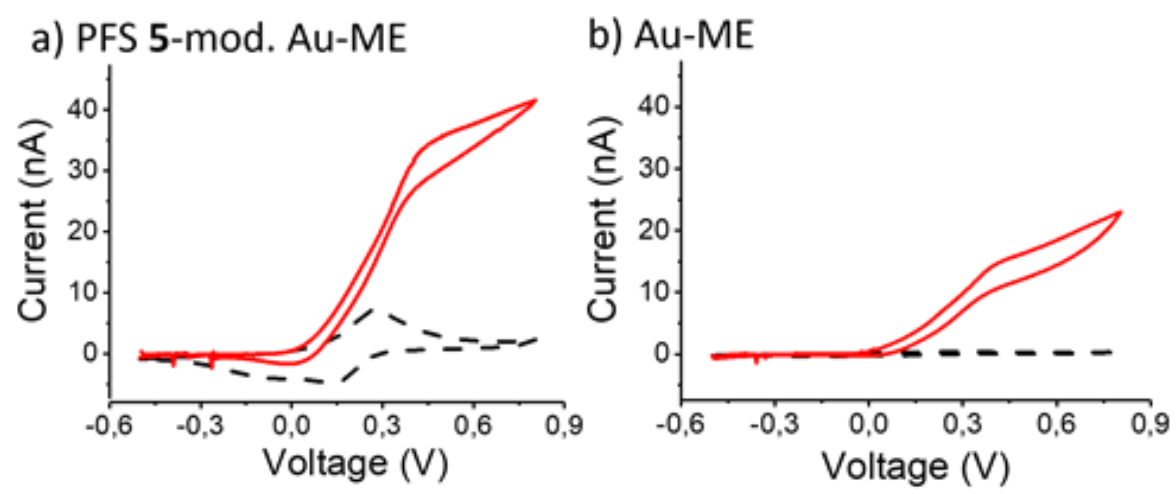

Fig. 8.5: Comparison of CVs of the electrochemical properties in the presence (red solid line) and absence (black dashed line) of $35 \mathrm{mM}$ ascorbic acid for a PFS 5 modified (a) and un-modified (b) Au-MEs, respectively. Scan rate $50 \mathrm{mV} \cdot \mathrm{s}^{-1}$, in $100 \mathrm{mM} \mathrm{NaClO}$, $\mathrm{Pt}$ (wire)-RE/CE, and potential range between -500 and $800 \mathrm{mV} v \mathrm{~s}$ Pt.

The CVs of the PFS-immobilized Au-ME show a typical, essentially reversible redox response in the absence of the analyte (Figure 5a). In the presence of $35 \mathrm{mM}$ of ascorbic acid, an enhanced anodic peak current (e.g., at $+0.4 \mathrm{~V} v s \mathrm{Pt})$ was recorded, demonstrating the electrocatalytic properties of the PFS films. In the control experiment, the CVs of the bare MEs show a typical ascorbic acid oxidation redox wave (around $+0.37 \mathrm{~V} v s \mathrm{Pt}$ ) without any enhancement of the anodic current, as shown in Figure 8.5b.

The amperometric response of a PFS modified ME to the successive additions of ascorbic acid was evaluated through chronoamperometric measurements. These 
experiments allowed us to obtain information on the detection limit (DL) quantification limit (QL) sensitivity, linearity and the limit of linearity (LOL) of the sensing pixel as shown in Figure 8.6.
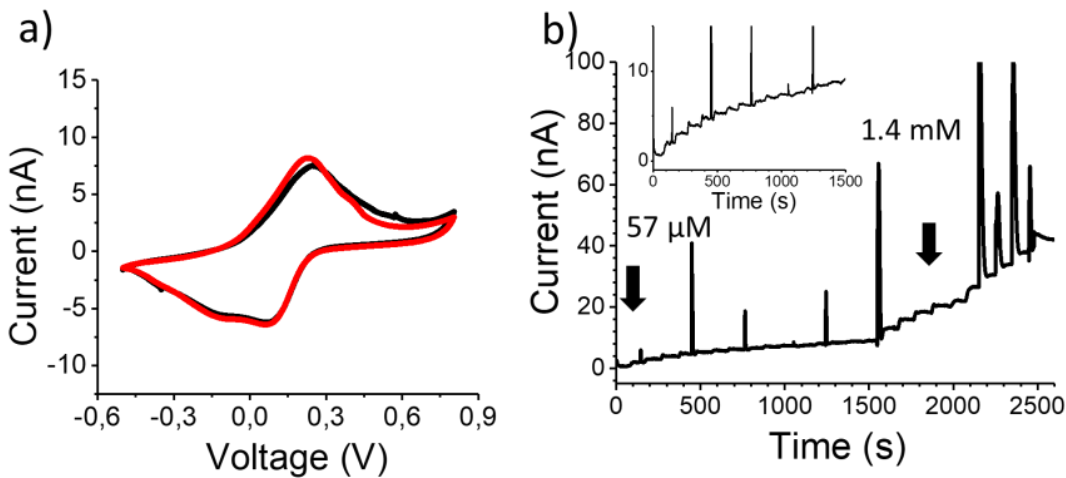

c)

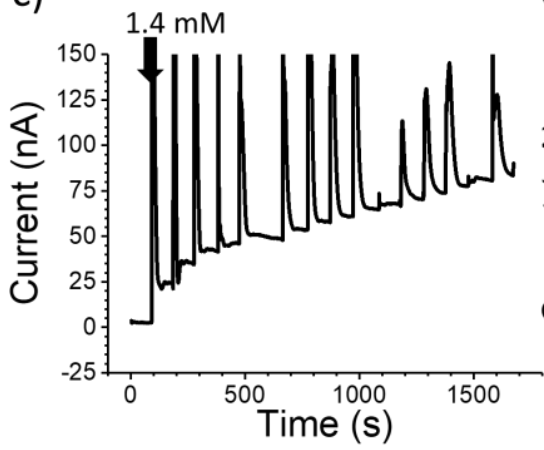

d)

Fig. 8.6: a) Cyclic voltammogram of the PFS 5 modified ME used for the amperometric experiments before and after the amperometric experiments. Scan rate $50 \mathrm{mV} \mathrm{s}^{-1}$, in $100 \mathrm{mM} \mathrm{NaClO}_{4}, \mathrm{Pt}$ (wire)-RE/CE, and potential range between -500 and $800 \mathrm{mV}$ vs Pt. Chronoamperometric response of a modified ME to the successive additions of ascorbic acid to evaluate b) the limit of detection and c) the linearity of the calibration curve. d) Is the calibration process of a PFS 5-modified ME for ascorbic acid sensing. In the inset of d), the calibration curve between $0.057-2 \mathrm{mM}$ is shown. The cell contained $2 \mathrm{~mL}$ of $100 \mathrm{mM} \mathrm{NaClO}_{4}$ aqueous solution, $\mathrm{Pt}$ (wire)-RE/CE, and the potential was set at $400 \mathrm{mV}$ vs Pt.

The change in the anodic current increase upon adding ascorbic acid in the cell solution as described by the Randles-Sevcik's relationship, ${ }^{8}$ see Figure $\mathbf{8 . 6 b}$ and $\mathbf{6 c}$. The amperometric response of PFS-decorated MEs was rapid and the anodic steady-state current was reached within few seconds after the addition of an ascorbic acid aliquot. (We note that the registered spikes of the current were due to the manual injection of the analyte in the cell via a micropipette). As shown in Figure 8.6d, calibration of the PFS modified electrode was performed and it exhibited three regimes, ${ }^{67}$ i.e. the noise, a linear and a nonlinear regime. The noise regime (up to $0.9 \mathrm{mM}$ ) is characterized by an increase of the current as a function of the ascorbic acid concentration (coefficient of linear regression $R^{2}=0.941$ ), but with low sensitivity of the modified electrode (sensitivity is 9 $\mathrm{nA} \mathrm{mM}^{-1}$, considering the slope of the linear regression), and low signal-to-noise ratio 
(below 100). Theoretically, the DL and the QL values can be defined as 3 times and 10 times the standard deviation of the blank signal, respectively. Thus, the theoretical DL and the theoretical QL values were 33 and $111 \mu \mathrm{M}$, respectively, considering $0.1 \mathrm{nA}$ as the standard deviation of the anodic current value of the solution without the analyte. However, experimentally, the anodic current value increased with a well-defined step after the addition of $57 \mu \mathrm{M}$ of ascorbic acid in the cell solution permitting us to define this concentration value as the experimental QL. The linear calibration regime, between 0.9 and $2 \mathrm{mM}$, is characterized by a linear increase of the current upon increasing the analyte concentration and a good sensitivity $\left(31.4 \mathrm{nA} \cdot \mathrm{mM}^{-1}, R^{2}=0.985\right)$. In the nonlinear regime, above the LOL of $2 \mathrm{mM}$ of ascorbic acid, a deviation from the linear regime was observed. This is caused by the slow diffusion rate of the analyte through the diffusion layer above the electroactive film with a drastic change in the sensitivity of the sensor (in the linear regime the sensitivity was $31.4 \mathrm{nA} \mathrm{mM}^{-1}$, while in the nonlinear regime the sensitivity value dropped to $1.0 \mathrm{nA} \mathrm{mM}^{-1}$ ). The stability and the reproducibility of the electrochemical properties of the PFS modified MEs were tested before and after the amperometric experiments. As shown in Figure 8.6a, there were no changes in the CVs following the amperometric experiments. As such, the good stability of the printed PFS 5 layers was confirmed once more, demonstrating their feasibility to be utilized as inks that allow advancement to the required next step, which is the development of MEAs with analyte-specific read outs for its individual MEs.

\subsection{Conclusions}

New redox-responsive polymers, i.e. PFS 5, with a PFS backbone and 5 molar percent side groups with disulfide units were successfully synthesized and exploited as molecular inks for the spatially controlled surface modification of commercial MEAs by drop on demand inkjet printing. First, PFS 5 inks in toluene with a viscosity and surface tension of $1.8 \mathrm{mPa} \cdot \mathrm{s}$ and $28 \mathrm{mN} \cdot \mathrm{m}^{-1}$ as determined by rheology and pendant drop measurements were developed. Subsequently, commercial MEAs were modified using PFS 5 based ink by printing in DoD mode with good accuracy. FTIR spectroscopy and EDX-SEM confirmed a successful, spatially localized PFS surface modification of the individual gold-coated MEs. The deposited PFS 5 layer thickness was approximately 8 $\mathrm{nm}$ as was estimated from AFM analysis, while from CV measurements a ferrocene unit grafting density of $1.60 \times 10^{-9} \mathrm{~mol} \cdot \mathrm{cm}^{-2}$ was determined, confirming the presence of an ultrathin, uniform and relative densely grafted PFS film on the electrode surface. Repeated CV measurements confirmed the redox-activity and stability of the printed PFS layers on the modified MEAs. The good layer stability was ascribed to the strong and stable bonding between the gold electrode surface and the PFS disulfide side groups. Finally, the potential of these devices to act as an electrochemical sensor array was demonstrated with a model analyte, ascorbic acid, using cyclic voltammetry and amperometric measurements. Good sensitivity and stable responses to ascorbic acid were 
achieved. To conclude, the first steps towards the multiplex "pixel based" electrochemical sensing by DoD-IJP PFS modified MEAs were successfully demonstrated. Future efforts should be directed towards the development of printed analyte specific micro electrode array "pixels".

\subsection{Experimental section}

\section{Materials.}

[1]Dimethylsilaferrocenophane $\mathbf{1}$ and ([1]3-chloropropyl)methylsilaferrocenophane 2 were synthesized according to published procedures. ${ }^{68} 4,4^{\prime}$-Dithiodibutyric acid (DTDB, 95\%), iodoethane (99\%), 1-iodopropane (99\%), hydrogen hexachloroplatinate(IV) hydrate $\left(\mathrm{H}_{2} \mathrm{PtCl}_{6} \cdot 6 \mathrm{H}_{2} \mathrm{O}, 99.9+\%\right)$, dicyclohexano-18-crown-6 (98\%), potassium iodide (KI, 99+\%), sodium chloride $(\mathrm{NaCl})$, sodium sulphate $\left(\mathrm{Na}_{2} \mathrm{SO}_{4}\right.$, anhydrous, $\left.99 \%\right)$, chloroform-d $\left(\mathrm{CDCl}_{3}, 99.8\right.$ atom \% D), toluene-d $(99.6$ atom \% D) were obtained from Sigma-Aldrich Chemie GmbH (Steinheim, Germany) and used as received. For the electrochemistry experiments, sodium perchlorate $\left(\mathrm{NaClO}_{4}\right.$, ACS Reagent, $\left.>98.0 \%\right)$ and L-ascorbic acid (ACS Reagent, 99+\%) were obtained from Sigma-Aldrich Chemie $\mathrm{GmbH}$ (Steinheim, Germany) and used as received. Toluene, dimethylformamide (DMF), dimethyl sulfoxide (DMSO), tetrahydrofuran (THF), ethanol (EtOH) and methanol $(\mathrm{MeOH})$, all of AR grade, were obtained from Biosolve Chimie SARL (Dieuze, France) and Milli-Q grade water (Millipore Coorporation) was used in all the experiments. Ultrapure nitrogen was used throughout.

\section{Synthesis and characterization of disulfide-functionalized poly(ferrocenylsilane)s,} PFS 5.

4,4'-Dithiodibutyric acid monoethyl ester. 4,4'-Dithiodibutyric acid, DTDB, was alkylated following a published procedure. ${ }^{69}$ A suspension of powdered potassium hydroxide $85 \%(1.33 \mathrm{~g}, 20 \mathrm{mmol})$ in DMSO $(13 \mathrm{~mL})$ was stirred vigorously for 30 minutes at room temperature. A solution of DTDB $(2.384 \mathrm{~g}, 9.5 \mathrm{mmol})$ was added in DMSO $(10 \mathrm{~mL})$ and the mixture was stirred for 15 minutes. After cooling in an ice-water bath, a solution of iodoethane $(2.23 \mathrm{~g}, 14.3 \mathrm{mmol})$ in DMSO $(20 \mathrm{~mL})$ was added using a dropping funnel. Stirring was continued for $2 \mathrm{~h}$ at room temperature, followed by the addition of ice-water $(150 \mathrm{~mL})$. The $\mathrm{pH}$ value of the mixture was adjusted to $\mathrm{pH} 3-4$ with dilute hydrochloric acid. The resulting solution was extracted three times with ethyl acetate $(3 \times 15 \mathrm{~mL})$ and the organic layer was washed three times with saturated aqueous sodium chloride $(3 \times 15 \mathrm{~mL})$. The solution was dried over anhydrous sodium sulfate and concentrated under reduced pressure. Purification was carried out by column chromatography using $n$-hexane/acetone (1:1 vol:vol) as eluent to obtain $1.27 \mathrm{~g}$ (4.77 mmol) of product. Yield: $50.2 \%$. 
${ }^{1} \mathbf{H}$ NMR $\left(400 \mathrm{MHz}, \mathrm{CDCl}_{3}\right) \delta: 1.25\left(\mathrm{CH}_{3}, \mathrm{t}, 3 \mathrm{H}\right) ; 2.015\left(\mathrm{O}=\mathrm{CCH}_{2} \mathrm{CH}_{2}, \mathrm{~m}, 4 \mathrm{H}\right) ; 2.42$ $\left(\mathrm{O}=\mathrm{CCH}_{2}, \mathrm{t}, 2 \mathrm{H}\right) ; 2.49\left(\mathrm{O}=\mathrm{CCH}_{2}, \mathrm{t}, 2 \mathrm{H}\right) ; 2.715\left(\mathrm{CH}_{2} \mathrm{SSCH}_{2}, \mathrm{~m}, 4 \mathrm{H}\right) ; 4.125\left(\mathrm{CH}_{2} \mathrm{CH}_{3}, \mathrm{~m}\right.$, $2 \mathrm{H})$.

${ }^{13} \mathbf{C}$ NMR (100 MHz, $\left.\mathrm{CDCl}_{3}\right) \delta: 14.22\left(\mathrm{CH}_{3}\right) ; 23.91\left(\mathrm{O}=\mathrm{CCH}_{2} \mathrm{CH}_{2}\right) ; 24.22$ $\left(\mathrm{O}=\mathrm{CCH}_{2} \mathrm{CH}_{2}\right) ; 32.36\left(\mathrm{O}=\mathrm{CCH}_{2}\right) ; 32.66\left(\mathrm{O}=\mathrm{CCH}_{2}\right) ; 37.59 \quad\left(\mathrm{CH}_{2} \mathrm{SSCH}_{2}\right) ; 37.81$ $\left(\mathrm{CH}_{2} \mathrm{SSCH}_{2}\right) ; 60.55\left(\mathrm{CH}_{2} \mathrm{CH}_{3}\right) ; 173.14(\mathrm{C}=\mathrm{O}) ; 178.53(\mathrm{C}=\mathrm{O})$.

FTIR (4000-500 $\mathrm{cm}^{-1}$, ATR-FTIR Brucker ALPHA): $3400-2800 \mathrm{~cm}^{-1}(\mathrm{O}-\mathrm{H}$ strectching vibration, $v \mathrm{O}-\mathrm{H})$, acid), 1728 and $1704 \mathrm{~cm}^{-1}(\mathrm{C}=\mathrm{O}$ stretching vibrations, $v \mathrm{C}=\mathrm{O}), 510 \mathrm{~cm}^{-1}$ (S-S stretching vibration of disulfide group, $\left.v \mathrm{~S}-\mathrm{S}\right)$.

Chloropropyl-functionalized PFS 3. In a glove box filled with prepurified $\mathrm{N}_{2}$, [1]dimethylsilaferrocenophane $\mathbf{1}\left(1.40 \mathrm{~g}, 5.78 \cdot 10^{-3} \mathrm{~mol}\right)$ and [1](3-chloropropyl)methylsilaferrocenophane 2 (93 mg, 3.05 $\left.10^{-4} \mathrm{~mol}, 5 \mathrm{~mol} \%\right)$ were dissolved in dry THF (20 mL) in a $50 \mathrm{~mL}$ 1-necked roundbottom flask. A small grain of hexachloroplatinic acid $(5 \mathrm{mg})$ was added under stirring according to the well-established procedure. ${ }^{53-54}$ Stirring was continued for $36 \mathrm{~h}$. The solution was then diluted by adding THF $(10 \mathrm{~mL})$ and the polymer was precipitated in $\mathrm{MeOH}(200 \mathrm{~mL})$. This mixture was stirred for $1 \mathrm{~h}$ to coagulate the polymer. After isolating the polymer, it was precipitated again in $\mathrm{MeOH}$, dried in a flow of $\mathrm{N}_{2}$ and further dried under vacuum ( 6 mbar, $24 \mathrm{~h}$, followed by $1 \cdot 10^{-3}$ mbar, $24 \mathrm{~h}$ ). Yield: $1.40 \mathrm{~g}$.

${ }^{1} \mathbf{H}$ NMR (400 MHz, toluene-d $\left.)_{8}\right) \delta: 0.53\left(\mathrm{SiCH}_{3}, \mathrm{~s}, 6 \mathrm{H}\right) ; 0.98\left(\mathrm{Si}_{-} \mathrm{CH}_{2}, \mathrm{~m}, 0.16 \mathrm{H}\right)$; $1.77\left(\mathrm{CH}_{2}, \mathrm{~m}, 0.16 \mathrm{H}\right) ; 3.24\left(\mathrm{CH}_{2} \mathrm{Cl}, \mathrm{t}, 0.16 \mathrm{H}\right) ; 4.06+4.24(\mathrm{Cp}, \mathrm{m}, 8 \mathrm{H})$; as shown in Figure S8.1.

${ }^{13} \mathrm{C}$ NMR $\left(100 \mathrm{MHz}\right.$, toluene-d $\left.{ }_{8}\right) \delta:-2.97\left(\mathrm{CH}_{3} \mathrm{SiCH}_{2}\right) ;-0.54\left(\mathrm{CH}_{3} \mathrm{SiCH}_{3}\right) ; 14.38$ $\left(\mathrm{SiCH}_{2}\right) ; 28.34\left(\mathrm{CH}_{2}\right) ; 48.06\left(\mathrm{CH}_{2} \mathrm{Cl}\right) ; 71.75+73.62(\mathrm{Cp})$; as shown in Figure S8.2.

FTIR (4000-500 $\left.\mathrm{cm}^{-1}\right): 1380,1361$ and $1248 \mathrm{~cm}^{-1}\left(\mathrm{SiCH}_{3}\right.$ deformation vibrations); 1450 and $1350 \mathrm{~cm}^{-1}$ (C-H bending vibrations alkanes); 1182 and $1165 \mathrm{~cm}^{-1}$ (asymmetric ring in-plane $\mathrm{C}-\mathrm{H}$ vibrations for ferrocene rings); $1037 \mathrm{~cm}^{-1}$ (asymmetric ring out-ofplane $\mathrm{C}-\mathrm{H}$ vibrations for ferrocene rings), $800-600 \mathrm{~cm}^{-1}$ (C-Cl stretching vibrations, $\nu \mathrm{C}$ $\mathrm{Cl})$.

GPC in THF: $M_{\mathrm{n}}=236702 \mathrm{~g} / \mathrm{mol}, M_{\mathrm{w}}=383590 \mathrm{~g} / \mathrm{mol}, M_{\mathrm{w}} / M_{\mathrm{n}}=1.62$.

Iodopropyl-functionalized PFS 4. PFS 3 (1.30 g) was dissolved in THF (40 mL) and dicyclohexano-18-crown-6 (1.0 g, $2.7 \mathrm{mmol})$, KI (1.0 g, $6.0 \mathrm{mmol})$ and 1iodopropane $(3 \mathrm{~mL}, 31 \mathrm{mmol})$ were added. The reaction mixture was stirred at $40{ }^{\circ} \mathrm{C}$ for 2 weeks. Following a week, some iodopropane $(2 \mathrm{~mL})$ was added. The polymer was precipitated twice in $\mathrm{MeOH}(200 \mathrm{~mL})$, dried in a flow of $\mathrm{N}_{2}$ and further dried under vacuum (6 mbar, $24 \mathrm{~h}$, followed by $\left.1 \cdot 10^{-3} \mathrm{mbar}, 24 \mathrm{~h}\right)$. Yield: $1.35 \mathrm{~g}$.

${ }^{1}$ H NMR (400 MHz, toluene-d $)_{8} \delta: 0.53\left(\mathrm{SiCH}_{3}, \mathrm{~s}, 6 \mathrm{H}\right) ; 0.95\left(\mathrm{Si}_{-} \mathrm{CH}_{2}, \mathrm{~m}, 0.16 \mathrm{H}\right)$; $1.79\left(\mathrm{CH}_{2}, \mathrm{~m}, 0.16 \mathrm{H}\right) ; 2.88\left(\mathrm{CH}_{2} \mathrm{I}, \mathrm{t}, 0.16 \mathrm{H}\right) ; 4.06+4.24(\mathrm{Cp}, \mathrm{m}, 8 \mathrm{H})$; as shown in Figure S8.3. 
${ }^{13} \mathrm{C}$ NMR $\left(100 \mathrm{MHz}\right.$, toluene-d $\left.{ }_{8}\right) \delta:-2.94\left(\mathrm{CH}_{3} \mathrm{SiCH}_{2}\right) ;-0.55\left(\mathrm{CH}_{3} \mathrm{SiCH}_{3}\right) ; 11.59$ $\left(\mathrm{SiCH}_{2}\right) ; 18.55\left(\mathrm{CH}_{2}\right) ; 29.33\left(\mathrm{CH}_{2} \mathrm{I}\right) ; 71.74+73.61(\mathrm{Cp})$; as shown in Figure S8.4.

FTIR (Bruker ALPHA, 4000-500 $\left.\mathrm{cm}^{-1}\right): 1380,1361$ and $1248 \mathrm{~cm}^{-1}\left(\mathrm{SiCH}_{3}\right.$ deformation vibrations); 1450 and $1350 \mathrm{~cm}^{-1}$ (C-H bending vibrations alkanes); 1182 and $1165 \mathrm{~cm}^{-1}$ (asymmetric ring in-plane $\mathrm{C}-\mathrm{H}$ vibrations for ferrocene rings); $1037 \mathrm{~cm}^{-1}$ (asymmetric ring out-of-plane $\mathrm{C}-\mathrm{H}$ vibrations for ferrocene rings).

GPC in THF: $M_{\mathrm{n}}=126718 \mathrm{~g} / \mathrm{mol}, M_{\mathrm{w}}=235494 \mathrm{~g} / \mathrm{mol}, M_{\mathrm{w}} / M_{\mathrm{n}}=1.86$.

Disulfide-functionalized PFS 5. 4,4-Dithiodibutyric acid monoethyl esther ( $0.352 \mathrm{~g}$, $1.32 \mathrm{mmol})$, dicyclohexano-18-crown-6 (0.50 g, $1.34 \mathrm{mmol})$ and $\mathrm{K}_{2} \mathrm{CO}_{3}(0.174 \mathrm{~g}, 1.26$ mmol) were dissolved in DMF $(10 \mathrm{~mL})$ in a $25 \mathrm{~mL}$ round bottom flask under $\mathrm{N}_{2}$. After stirring for $2 \mathrm{~h}$, the solution was taken up in a syringe and added to a solution of PFS 4 $(1.1291 \mathrm{~g})$ in THF $(20 \mathrm{~mL})$, which was also kept under a nitrogen atmosphere. The flask containing the 4,4-dithiodibutyric acid monoethyl esther was rinsed twice with DMF (2 $\mathrm{mL}$ ) which was also transferred by syringe to the PFS solution. The reaction mixture was stirred for 1 week at $20^{\circ} \mathrm{C}$ and then added dropwise to methanol $(200 \mathrm{~mL})$ to precipitate the polymer. The polymer was precipitated again from THF $(20 \mathrm{~mL})$ into MeOH $(200$ $\mathrm{mL}$ ), dried in a flow of $\mathrm{N}_{2}$ and further dried under vacuum ( 6 mbar, $24 \mathrm{~h}$, followed by $1 \cdot 10^{-3}$ mbar, $24 \mathrm{~h}$ ). Yield: $1.13 \mathrm{~g}$.

${ }^{1}$ H NMR (400 MHz, toluene-d $\left.\mathrm{d}_{8}\right) \delta: 0.53\left(\mathrm{SiCH}_{3}, \mathrm{~s}, 6 \mathrm{H}\right) ; 0.95\left(\mathrm{Si}_{-} \mathrm{CH}_{2}, \mathrm{~m}, 0.16 \mathrm{H}\right)$; $1.00\left(\mathrm{CH}_{2} \mathrm{CH}_{3}, \mathrm{t}\right) ; 1.77\left(\mathrm{SiCH}_{2} \mathrm{CH}_{2}, \mathrm{~m}, 0.16 \mathrm{H}\right) ; 1.87 \quad\left(\mathrm{O}=\mathrm{CCH}_{2} \mathrm{CH}_{2}, \mathrm{~m}\right) ; 1.92$ $\left(\mathrm{O}=\mathrm{CCH}_{2} \mathrm{CH}_{2}, \mathrm{~m}\right) ; 2.14\left(\mathrm{O}=\mathrm{CCH}_{2}, \mathrm{~m}, 0.16 \mathrm{H}\right) ; 2.23\left(\mathrm{O}=\mathrm{CCH}_{2}, \mathrm{~m}, 0.16 \mathrm{H}\right) ; 2.45$ $\left(\mathrm{CH}_{2} \mathrm{SSCH}_{2}, \mathrm{~m}, 0.24 \mathrm{H}\right) ; 4.06+4.24(\mathrm{Cp}, \mathrm{m}, 8 \mathrm{H})$; as shown in Figure $\mathbf{S 5}$.

${ }^{13} \mathrm{C}$ NMR $\left(100 \mathrm{MHz}\right.$, toluene-d $\left.{ }_{8}\right) \delta:-2.97\left(\mathrm{CH}_{3} \mathrm{SiCH}_{2}\right) ;-0.56\left(\mathrm{CH}_{3} \mathrm{SiCH}_{3}\right) ; 12.89$ $(\mathrm{SiCH}) ; 14.35\left(\mathrm{CH}_{2} \mathrm{CH}_{3}\right) ; 24.57\left(\mathrm{O}=\mathrm{CCH}_{2} \mathrm{CH}_{2}\right) ; 24.64\left(\mathrm{O}=\mathrm{CCH}_{2} \mathrm{CH}_{2}\right) ; 32.56\left(\mathrm{O}=\mathrm{CCH}_{2}\right)$; $32.65\left(\mathrm{O}=\mathrm{CCH}_{2}\right) ; 37.94\left(\mathrm{CH}_{2} \mathrm{SSCH}_{2}\right) ; 60.08\left(\mathrm{CH}_{2} \mathrm{CH}_{3}\right) ; 71.73+73.60(\mathrm{Cp}) ; 172.04$ $(\mathrm{C}=\mathrm{O}) ; 172.24(\mathrm{C}=\mathrm{O})$; as shown in Figure $\mathbf{S 6}$.

FTIR (4000-500 $\left.\mathrm{cm}^{-1}\right): 1750$ and $1735 \mathrm{~cm}^{-1}(\mathrm{C}=\mathrm{O}$ stretching vibrations of ester groups, $v \mathrm{C}=\mathrm{O}) ; 1380,1361$ and $1248 \mathrm{~cm}^{-1}\left(\mathrm{SiCH}_{3}\right.$ deformation vibrations); 1450 and $1350 \mathrm{~cm}^{-1}$ (C-H bending vibrations alkanes); 1182 and $1165 \mathrm{~cm}^{-1}$ (asymmetric ring inplane $\mathrm{C}-\mathrm{H}$ vibrations for ferrocene rings); $1037 \mathrm{~cm}^{-1}$ (asymmetric ring out-of-plane $\mathrm{C}-\mathrm{H}$ vibrations for ferrocene rings).

GPC in THF: $M_{\mathrm{n}}=138498 \mathrm{~g} / \mathrm{mol}, M_{\mathrm{w}}=278794 \mathrm{~g} / \mathrm{mol}, M_{\mathrm{w}} / M_{\mathrm{n}}=2.01$.

\section{Devices and methods.}

Microelectrode array, MEA. The MEA biochips used in this experiment were MEA 60-200-Au (Qwane Biosciences SA, Switzerland) characterized by 60 plain gold electrodes, each with $40 \mu \mathrm{m}$ diameter, a center-to-center spacing of $200 \mu \mathrm{m}$, with an insultator layer of SU-8 with a thickness of $0.7 \mu \mathrm{m}$, and an impedance of 700-900 k $\Omega$ positioned on a transparent $10 \mathrm{~mm}$ x $10 \mathrm{~mm}$ glass chip. The MEA chip had dimensions of $49 \mathrm{~mm} \times 49 \mathrm{~mm} \times 1 \mathrm{~mm}$ in PCB base material with Au contact pads. A schematic 
illustration of the MEA chip design is shown in Figure S8. The Au contact pads are used for connecting the MEA to the Autolab PGSTAT 10 electrochemical workstation (Ecochemie, Utrecht, the Netherlands) through a MEA 1060-inv-BC (Multi Channel Systems). The supporting plastic ring has an inner diameter of $26.5 \mathrm{~mm}$, an outer diameter of $30 \mathrm{~mm}$ and a height of $6 \mathrm{~mm}$.

\section{Characterization techniques}

IR: Fourier transform infrared spectra were collected with a Bruker ALPHA single attenuated total reflection (ATR-FTIR) spectrometer equipped with an ATR single reflection crystal (Bruker optik $\mathrm{GmbH}$, Ettlingen, Germany) and a Bruker Vertex V70 equipped with an Hyperion 2000 infrared microscope (utilizing a 15x magnification objective) and a Mid-band MCT detector (liquid $\mathrm{N}_{2}$ cooled with preamplifier, $12000-$ $600 \mathrm{~cm}^{-1}$ ). The spectra were collected in the range of $4000-400 \mathrm{~cm}^{-1}$ with a spectral resolution of $4 \mathrm{~cm}^{-1}$ employing at least 128 scans. Background spectra were recorded against air.

${ }^{1} \mathrm{H}$ and ${ }^{13} \mathrm{C}$ NMR: ${ }^{1} \mathrm{H}$ and ${ }^{13} \mathrm{C}$ NMR spectra were obtained on a Bruker Avance III $400 \mathrm{MHz}$ instrument at 400.1 and $100.6 \mathrm{MHz}$, respectively. ${ }^{1} \mathrm{H}$ and ${ }^{13} \mathrm{C}$ chemical shifts were based on the solvent residual signals.

GPC: GPC measurements were performed using ultrastyragel columns with pore sizes of $10^{5}, 10^{4}, 10^{3}$ and $500 \AA$ (Waters), equipped with a guard column of $500 \AA$, a 515 pump (Waters), a 1050 injector (Agilent) and a 2414 differential refractometer (Waters). All sample solutions were prepared at a concentration of $5 \mathrm{mg} \cdot \mathrm{mL}^{-1}$ and filtered through a $0.45 \mu \mathrm{m}$ PTFE filter prior to a GPC run.

Rheology: The rheological properties of the inks were evaluated using a rotational UDS-200 rheometer (Anton Paar Germany GmbH, Ostfildern, Germany) equipped with parallel plates geometry and the surface tension was determined by the pendant drop method with an OCA 15 (Dataphysics Instruments, Germany). The steady-shear viscosity of the inks was evaluated using steady-shear rate sweep measurements aiming to simulate the piezo inkjet printing process. ${ }^{70}$ The measurements were performed at $20{ }^{\circ} \mathrm{C}$ (controlled by a Peltier temperature controller) using an MP31 spindle (a plain plate with a diameter of $50 \mathrm{~mm}$ ). Prior to performing the flow measurements, the gap (set at $500 \mu \mathrm{m}$ ) was filled with $1 \mathrm{~mL}$ of the ink solutions and the solution was then stabilized via a preshear step of $0.5 \mathrm{~min}$. The rheological properties were measured by increasing the shear rate at constant logarithmic steps from 0.1 to $2400 \mathrm{~s}^{-1}$, ensuring a steady flow state of the liquid. The shear stress was assessed every $3 \mathrm{~s}$ (measuring point duration $=1 /$ shear rate). The experiments were repeated three times using newly prepared solutions. The viscosity was calculated as the steady-state shear stress divided by the applied shear rate. The steady-shear viscosity was average between 500-2000 s-1. Surface tension values were determined by fitting the outline of pendant drops; the drop contour was fitted by the Young-Laplace equation considering the density of the ink to be the same as the density of the solvent (density of toluene is $0.87 \mathrm{~g} \cdot \mathrm{mL}^{-1}$ ). A needle of $0.70 \mathrm{~mm}$ outer diameter 
was used to develop droplets of about $6 \mu \mathrm{L}$ of volume and the average of the surface tension values and the standard deviations were calculated using at least three different measurements.

Inkjet printer. A Dimatix Piezo inkjet 2831 Printer (FUJIFILM Dimatix inc., Santa Clara, Ca, USA) PIXDRO LP 50, featuring a DMP-2850 print head and a DMC 11610 cartridge (16 nozzles having a $23 \mu \mathrm{m}$ diameter, $10 \mathrm{pL}$ nominal drop volume), was used to deposit the redox active ink on the MEAs. The printer was fitted out with a horizontal CCD camera, featuring a LED light for drop jetting analysis, and with a fiducial camera that allows the alignment of the print head with respect to the sample. The 16 nozzles of the DMC 11610 cartridge can fire all simultaneously or in single mode. The droplet formation of the inks (i.e. the printability) was investigated analyzing with the PIDRO Advanced Drop Analysis software (ADA, PixDro, Meyer Burger, the Netherlands) the drop generated in continuous inkjet printing mode. The alignment of the sample to the print-head was obtained via the standard protocol employed from the PIDRO Advanced Drop Analysis software named "fiducial alignment". To increase the accuracy of the inkjet printing deposition in DoD mode, a single nozzle was selected during the optimization procedure and it was tested separately after the optimization of the driving waveform using all nozzles. This nozzle was used to align and calibrate the print head to the MEA substrate through fiducial alignment which consists of three reference marks with a size of $250 \mu \mathrm{m}$ and with a minimal tolerance of $93 \mu \mathrm{m}$ and were chosen on the borders of the Au contact pads positioned at the corner of the MEA chip setting the "mark size" to $250 \mu \mathrm{m}$. The droplet formation of the inks was investigated as a function of delay time. The drops were generated in the continuous inkjet, CIJ, printing mode and analyzed with the PIDRO Advanced Drop Analysis software (ADA, PixDro, Meyer Burger, the Netherlands). The pattern was designed and edited as BMP file.

AFM. Atomic force microscopy was performed on an MFP-3D AFM (Asylum Research, USA) in tapping mode. Images were recorded in air using commercially available Olympus silicon cantilevers (average nominal resonance frequency of $70 \mathrm{kHz}$ and spring constant of $2 \mathrm{~N} / \mathrm{m}$ ).

SEM. Surface morphology and the elemental mappings of the bonded coatings on the MEA biochip were investigated by high-resolution scanning electron microscopy (FE-SEM, JEOL, JSM-6400F, the Netherlands) equipped with energy dispersive X-Ray analyzer (EDX) at 5 and $20 \mathrm{keV}$ of accelerating voltage.

Laser confocal microscopy. The surface of the MEAs was examined using a laser confocal Keyence VK 9710 K microscope (LSCM, violet laser VK-9700, Keyence Corporation, Osaka, Japan) featuring a 10x magnification objective lens.

Electrochemical properties.To evaluate the electrochemical properties of the PFS decorated MEA biochips, an Autolab PGSTAT 10 electrochemical workstation (Ecochemie, Utrecht, the Netherlands) was used via cyclic voltammetry (CV) and chronoamperometry measurements. For the chronoamperometric experiments, the 
solution was mixed using a peristaltic pump (LKB Bromma, 2132 microperpex peristaltic pump). The electrochemical properties and the stability of the printed PFS layers were evaluated by recording CVs of the current in $100 \mathrm{mM}$ aqueous $\mathrm{NaClO}_{4}$ solution between -500 and $800 \mathrm{mV}$, using a Pt wire as a reference and counter electrode (length of $1.5 \mathrm{~cm}$ and $0.5 \mathrm{~mm}$ diameter) as a function of the number of cycles. The experiments were repeated at least 10 times in $100 \mathrm{mM}$ aqueous $\mathrm{NaClO}_{4}$ solution in the range of -500 and $800 \mathrm{mV}$ with a scan rate of $50 \mathrm{mV} \cdot \mathrm{s}^{-1}$ using the decorated PFS gold electrode as working electrode and a $\mathrm{Pt}$ wire as a reference and counter electrode. The electrocatalytic response of the modified electrodes was investigated by recording CVs of modified and bare gold electrodes of the MEA in $100 \mathrm{mM}$ aqueous $\mathrm{NaClO}_{4}$ solution and in the presence of ascorbic acid with $50 \mathrm{mV} \cdot \mathrm{s}^{-1}$ scan rate, potential range between 500 and $800 \mathrm{mV}$, and using a Pt wire as a reference and counter electrode. The chronoamperograms were measured setting the potential at $400 \mathrm{mV}$ (against Pt wire) in $2 \mathrm{~mL}$ of $100 \mathrm{mM}$ aqueous $\mathrm{NaClO}_{4}$ solution. Small aliquots of the ascorbic acid aqueous solution were added each $100 \mathrm{sec}$ to the sensing system and mixed while recording the current response. For the current, the equilibrium value was reached in $50 \mathrm{sec}$ after each addition and the average of the current was used for the calibration curve.

DoD piezo inkjet printing of the PFS 5 based ink. PFS 5 was dissolved in toluene at different concentrations, including 2.5, 5 and $10 \mathrm{w} . \%$; corresponding solutions were named as "ink A", "ink B" and "ink C", respectively. The physical and chemical properties of the inks are summarized in Table S1. The rheological properties and the surface tension of the inks were evaluated via steady-shear rate sweep and pendant drop measurements, respectively, as shown in the $\mathbf{S I}$.

PFS 5 ink was printed on MEA chips and unpatterned gold-coated wafer samples, the latter allowing investigation of the homogeneity, step height and roughness of the printed PFS films. The MEA chips, without the ring (see Figure S8), were cleaned with ethanol and dried in a stream of $\mathrm{N}_{2}$ before being printed with PFS-based inks. The images were collected via the fiducial camera and the camera is positioned on the side of printhead close to the nozzles. The image analysis was conducted at constant focus and intensity of the coax and ring lights. The alignment was considered optimal when the score of the alignment was set at least $90 \%$. The PFS printed MEA biochips were rinsed with toluene, ethanol and water, and dried in a stream of $\mathrm{N}_{2}$. Finally, the samples were annealed at room temperature in a vacuum oven overnight. The printed patterns were rinsed with toluene, ethanol and water, and then dried in a stream of $\mathrm{N}_{2}$.

\section{Characterization of the sensing device}

Surface and electrochemical properties. The surface and electrochemical properties of the PFS 5 printed MEA chips were studied investigating the chemical composition of the printed films via EDX-HR SEM and mapping ATR-FTIR microscopy measurements, the topography and morphology via laser confocal microscopy and AFM. The electrical properties of the surface-immobilized PFS chains were studied using CV. 
More detailed information on the experimental part can be found in the Supporting Information.

\subsection{Supporting Information}

\section{Synthesis and characterization of disulfide-functionalized PFS 5}

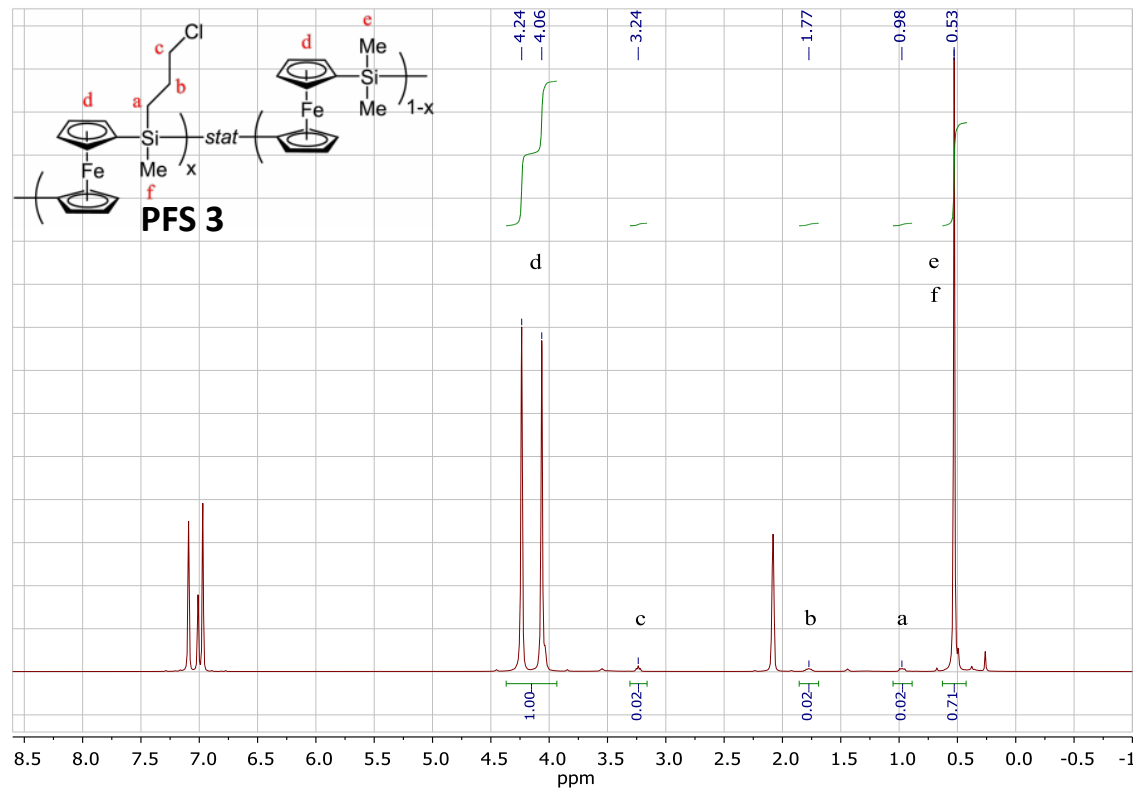

Figure S8.1: ${ }^{1} \mathrm{H}$ NMR spectrum of PFS 3 in toluene- $\mathrm{d}_{8}$.

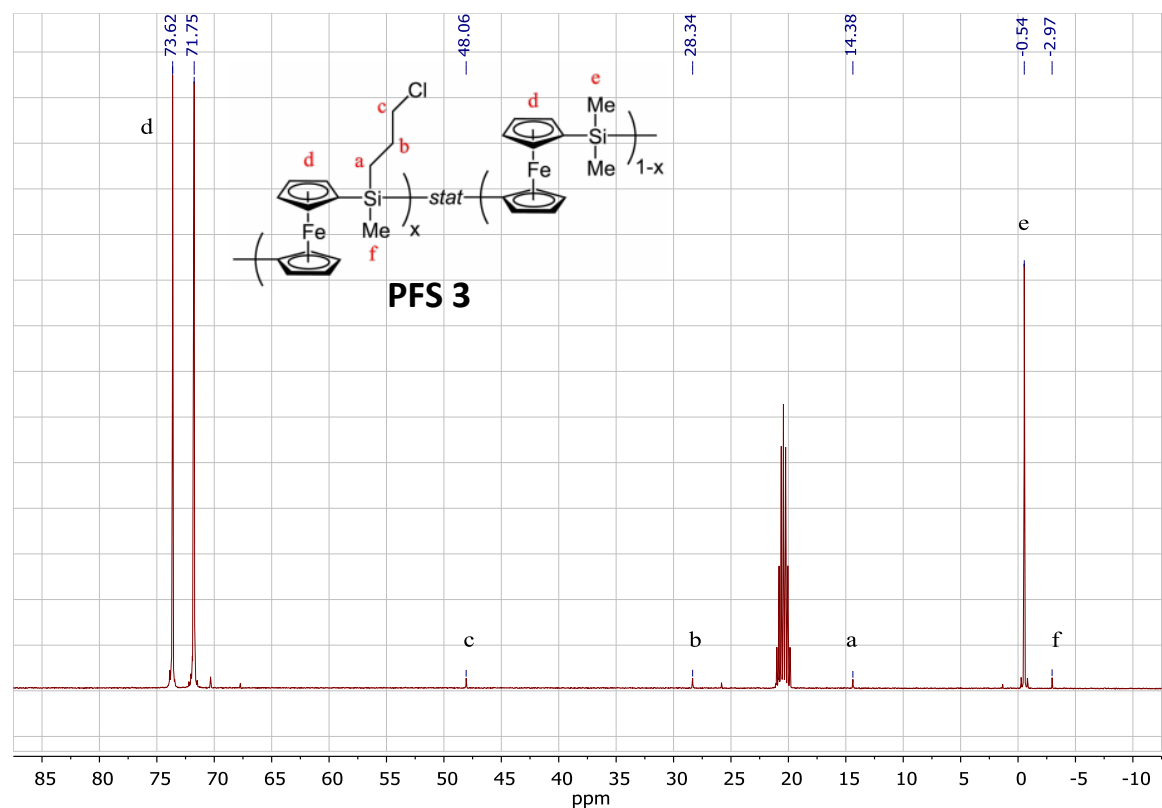

Figure S8.2: ${ }^{13} \mathrm{C}$ NMR spectrum of PFS 3 in toluene- $\mathrm{d}_{8}$. 


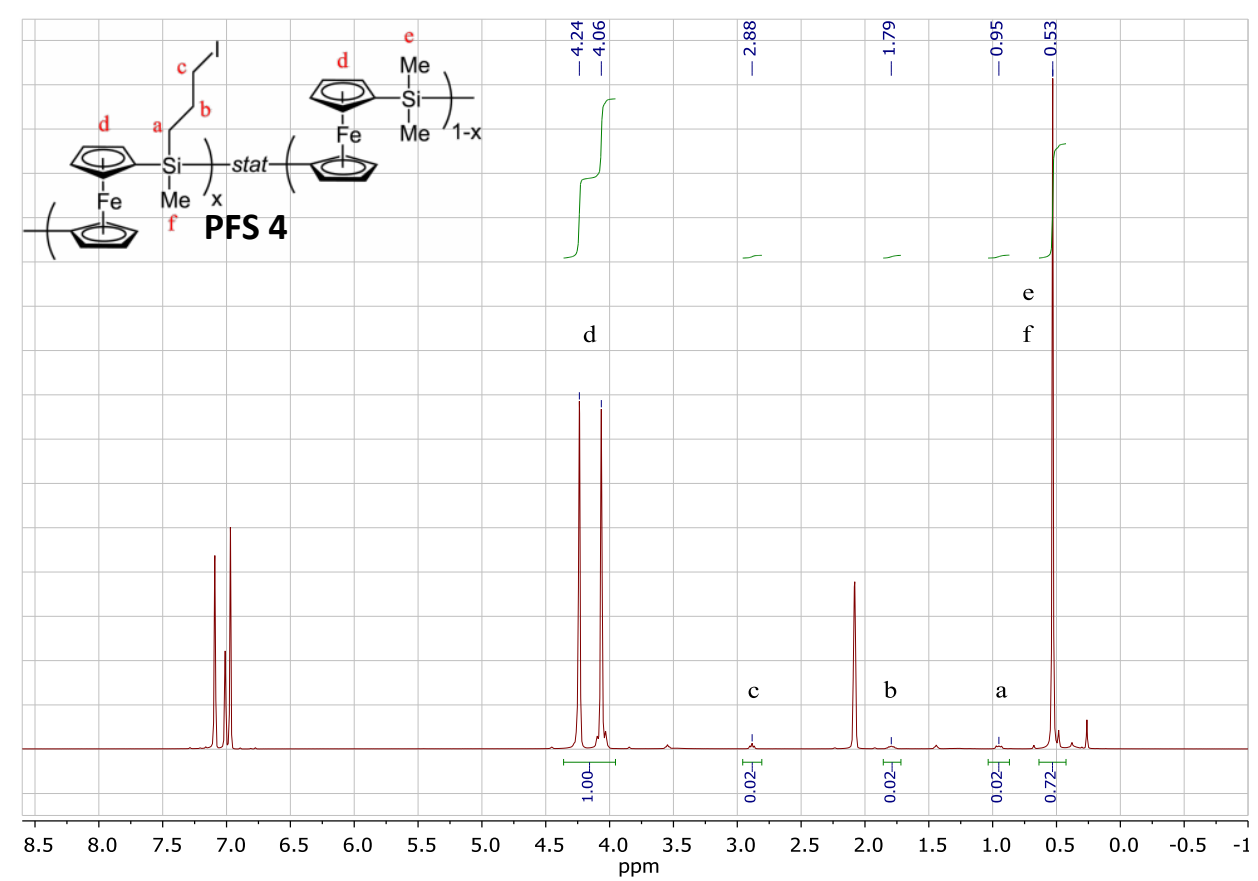

Figure S8.3: ${ }^{1} \mathrm{H}$ NMR spectrum of PFS 4 in toluene- $\mathrm{d}_{8}$.

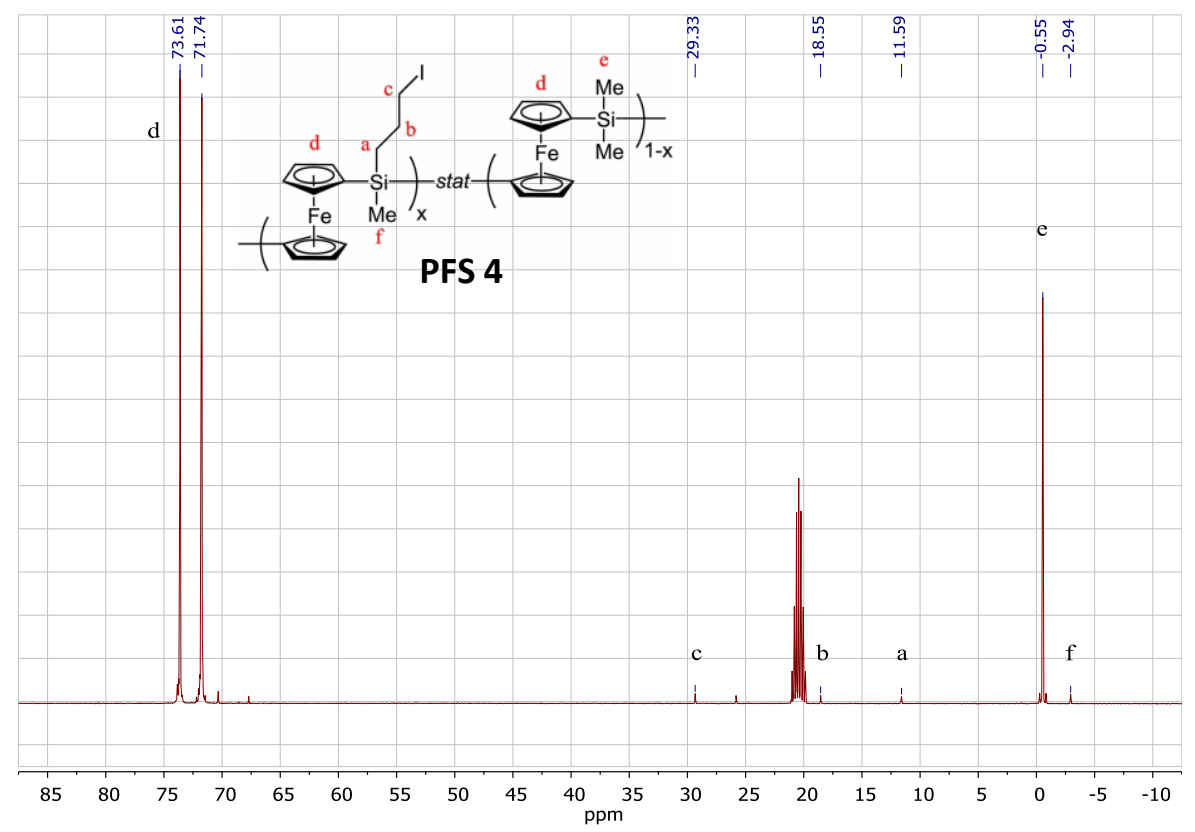

Figure S8.4: ${ }^{13} \mathrm{C}$ NMR spectrum of PFS 4 in toluene- $\mathrm{d}_{8}$. 


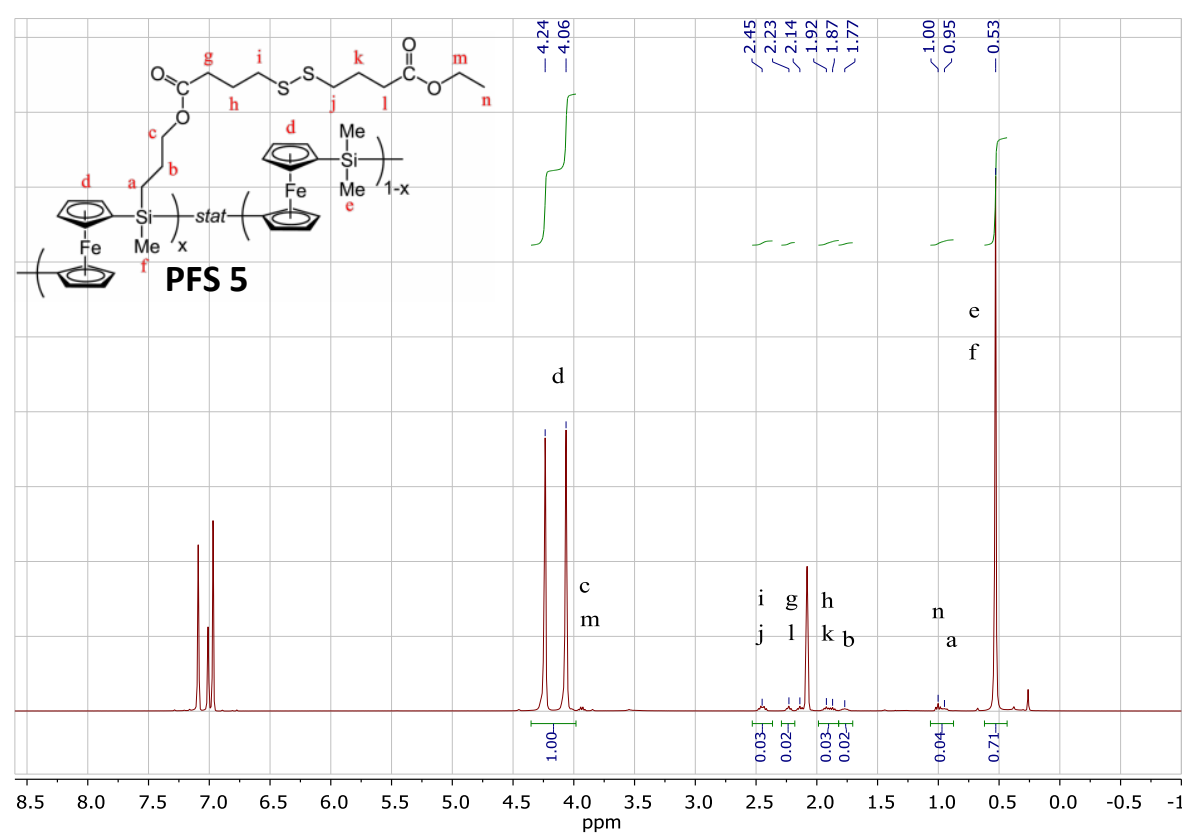

Figure S8.5: ${ }^{1} \mathrm{H}$ NMR spectrum of PFS 5 in toluene- $\mathrm{d}_{8}$.

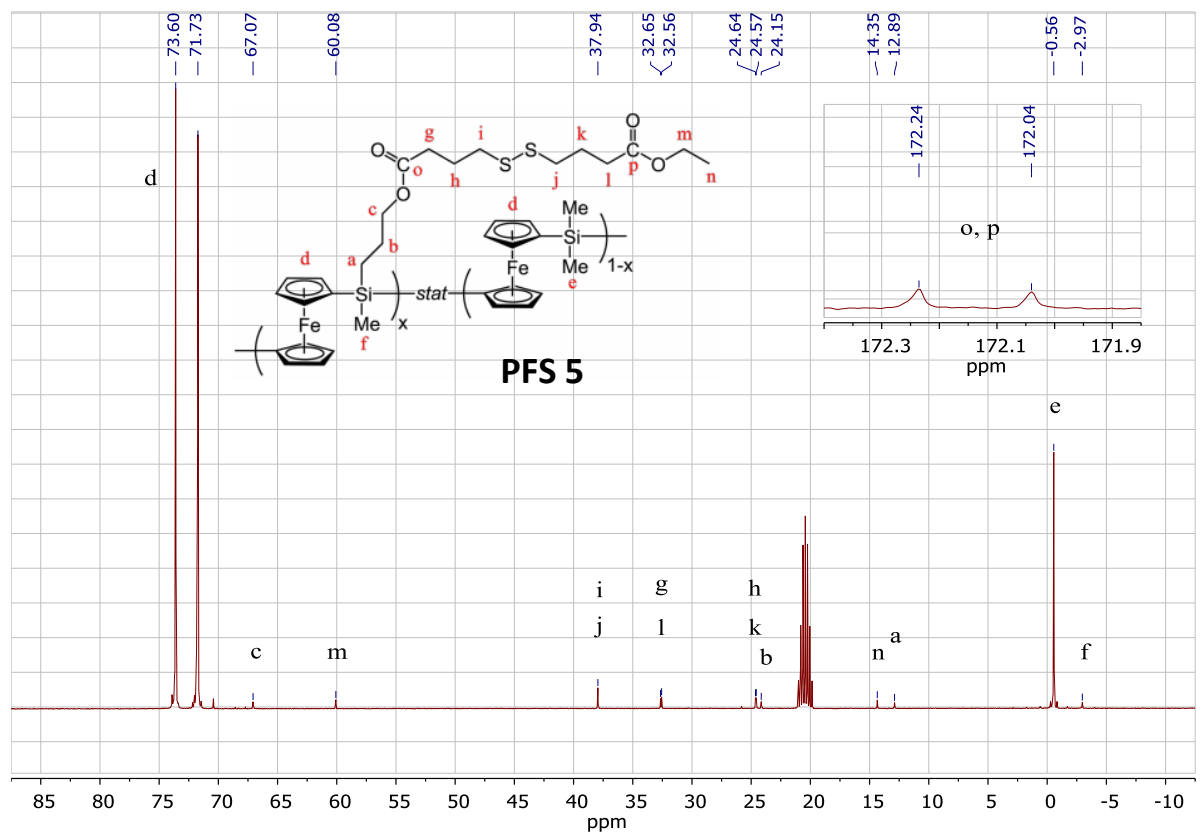

Figure S8.6: ${ }^{13} \mathrm{C}$ NMR spectrum of PFS 5 in toluene- $\mathrm{d}_{8}$.

Figure S8.7 shows zoomed-in ${ }^{1} \mathrm{H}$ NMR spectra of the respective PFS derivatives to confirm the quantitative conversion of the functional side groups from the chloro $\left(\mathrm{CH}_{2}-\right.$ $\mathrm{Cl}$ at $\delta=3.24 \mathrm{ppm})$, to the iodo $\left(\mathrm{CH}_{2}-\mathrm{I}\right.$ at $\left.\delta=2.88 \mathrm{ppm}\right)$ and to the disulfide-based ester $\left(\mathrm{CH}_{2}-\mathrm{SS}-\mathrm{CH}_{2}\right.$ at $\left.\delta=2.45 \mathrm{ppm}\right)$. 


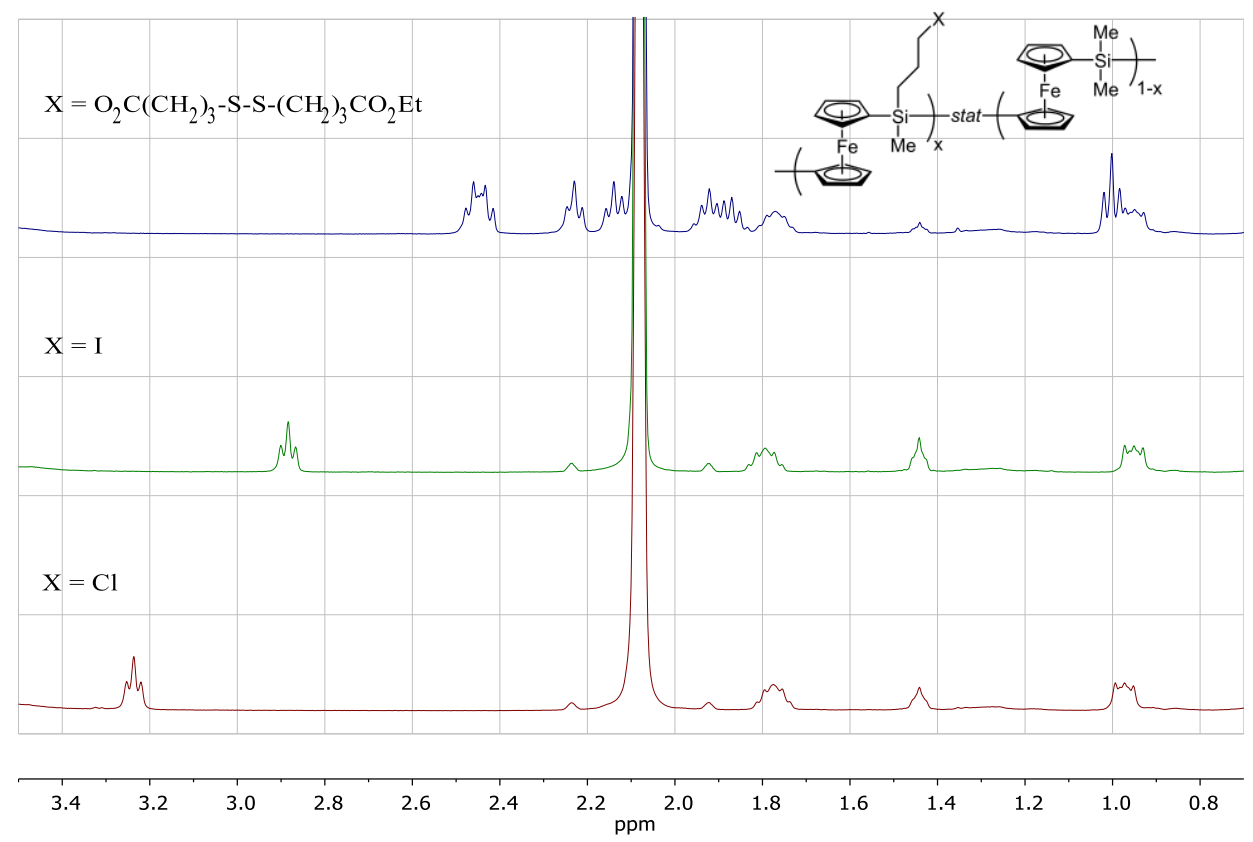

Figure S8.7: Zoom-in of the ${ }^{1} \mathrm{H}$ NMR spectra of PFS 3, 4 and 5 (in toluene- $\mathrm{d}_{8}$ ) in the region between $\delta=$ 0.7 and $3.5 \mathrm{ppm}$ demonstrating the complete conversion of chloropropyl- into iodopropyl side groups and the subsequent quantitative introduction of disulfide-bearing side groups.

a)

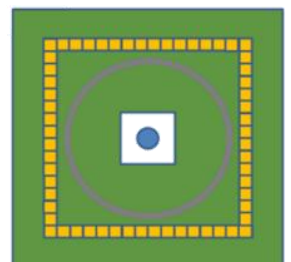

b)

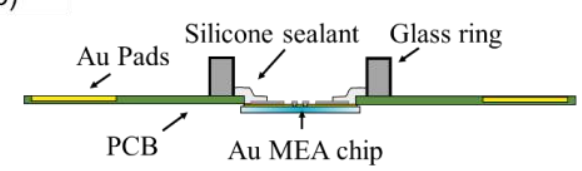

c)

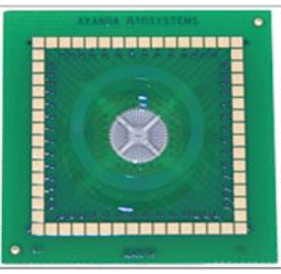

$49 \times 49 \mathrm{~mm}$

d)
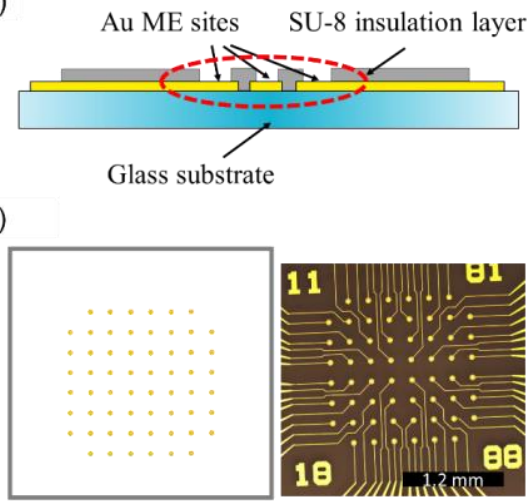

Figure S8.8: Pictures and schematic representation of the MEA 60-200-Au chip used in this study. a) and b) are top and side views of the MEA chip; c) and d) show more detailed side and top views of the electrode array of the Au MEA chip.

\section{Formulation and characterization of the redox stimuli-responsive PFS-based inks.}

Appropriate formulation of the functional ink materials will lead to a stable, wellcontrolled and repeatable inkjet process. Important physical properties of the inkjet inks include viscosity, density and surface tension since they affect the droplet formation, the repeatability and the accuracy of the inkjet deposition process. ${ }^{63,65,71}$ PFS 5 was dissolved 
in toluene at different concentrations, including 2.5, 5 and $10 \mathrm{wt} \%$; corresponding solutions were named as "ink A", "ink B" and "ink C", respectively. The physical and chemical properties of the inks are summarized in Table S8.1.

Table S8.1: Physical and chemical properties of the PFS-based inks.

\begin{tabular}{|c|c|c|c|c|c|}
\hline \multicolumn{3}{|c|}{ Formulation } & \multicolumn{3}{|c|}{ Toluene-based inks } \\
\hline \multicolumn{2}{|c|}{ Components } & Note/Function & Ink A & Ink B & Ink C \\
\hline $\begin{array}{l}\text { Functional } \\
\text { pigment }\end{array}$ & (wt $\%$ concentration) & Redox active & 2.5 & 5.0 & 10 \\
\hline Solvent & (wt $\%$ concentration) & Vehicle or carrier & 97.5 & 95.0 & 90.0 \\
\hline Density $(\rho)$ & $\left(\mathrm{kg} \mathrm{m}^{-3}\right.$ at $\left.25^{\circ} \mathrm{C}\right)$ & & 867 & 867 & 867 \\
\hline $\begin{array}{c}\text { Surface } \\
\text { tension }(\gamma)^{\mathrm{a}}\end{array}$ & $\left(\mathrm{mN} \mathrm{m}^{-1}\right.$ at $\left.20^{\circ} \mathrm{C}\right)$ & $\begin{array}{c}\text { Range } 25-50 \mathrm{mN} \\
\mathrm{m}^{-1} \text { at } 20^{\circ} \mathrm{C}\end{array}$ & 28 & 28 & 26 \\
\hline Viscosity $(\eta)^{b}$ & $\begin{array}{c}\left(\mathrm{mPa} \cdot \mathrm{s} \text { at } 20^{\circ} \mathrm{C},\right. \\
\left.\text { average } 500-2000 \mathrm{~s}^{-1}\right)\end{array}$ & $\begin{array}{c}\text { Range } 1-20 \mathrm{mPa} \cdot \mathrm{s} \text { at } \\
20^{\circ} \mathrm{C}\end{array}$ & $1.8 \pm 0.5$ & $8.1 \pm 0.5$ & $11 \pm 1$ \\
\hline $\begin{array}{c}\text { Reynolds } \\
\text { number }(\mathrm{Re})^{\mathrm{c}}\end{array}$ & - & $R e=v a \rho / \eta$ & 63.6 & 14.3 & 10.4 \\
\hline $\begin{array}{l}\text { Weber number } \\
\qquad(\mathrm{We})^{\mathrm{c}}\end{array}$ & - & $W e=v^{2} a \rho / \gamma$ & 24.5 & 24.5 & 24.5 \\
\hline $\begin{array}{l}\text { Ohnesorge } \\
\text { number }(\mathrm{Oh} \\
=1 / \mathrm{Z})^{\mathrm{c}}\end{array}$ & - & $\begin{array}{c}O h=W e^{1 / 2} / R e \\
=\eta /(a \rho \gamma)^{1 / 2}\end{array}$ & 0.08 & 0.35 & 0.48 \\
\hline Parameter Z & & $Z=1 / O h$ & 12 & 3 & 2 \\
\hline
\end{tabular}

a: pendant drop, b: steady-shear rate sweep via rotational viscometry and c: for the evaluation of $R e, W e$ and $O h$ the following was considered: $v$ is the velocity of the drop $\left(5 \mathrm{~m} \cdot \mathrm{s}^{-1}\right), a$ is the diameter of the nozzle, $\rho$ is the density of the fluid, $\eta$ is the viscosity and $\gamma$ is the surface tension. In addition, the drop diameter was $20 \mu \mathrm{m}$. 
a)

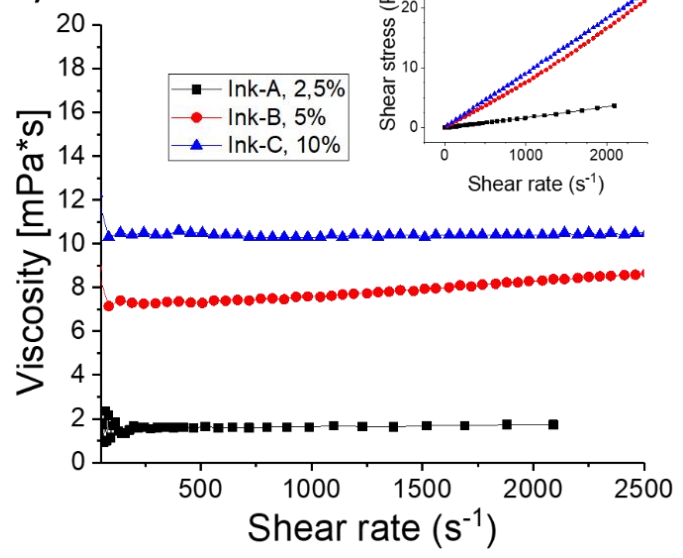

b)

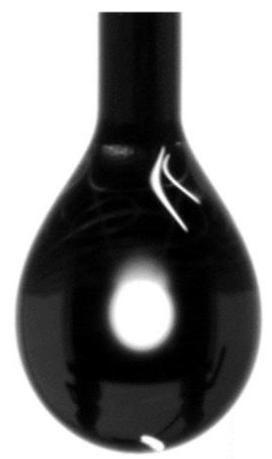

$1.4 \mathrm{~cm}$

Figure S8.9: Rheology and surface tension experiments. a) Measurements of steady-shear viscosity of the PFS based inks, the inset shows the shear stress curves. b) A typical photograph used in surface tension measurements via the pendant drop technique of "ink A".

The behavior of fluids during inkjet printing can be represented by the Reynolds number $(R e)$, the Weber number (We), and the Ohnesorge number $(O h)$, and be used to design a starting piezo waveform for a stable DoD inkjet printing process. ${ }^{63}$ Considering these physical parameters, "ink B" and "ink C" could be easily printable, while the use of "ink A", due to the low viscosity, could result in the formation of undesired satellite drops. However, the optimization of the printing conditions must be experimentally verified and tested through the evaluation of the droplet formation as a function of the inkjet printing conditions. In practice, it turned out that "ink A" was the best compromise between printability, optimization of the formulation and quality of the redox active coatings produced.

The droplet formation of the inks was investigated as a function of delay time. The drops were generated in the continuous inkjet, CIJ, printing mode and analyzed with the PIDRO Advanced Drop Analysis software (ADA, PixDro, Meyer Burger, the Netherlands). High-speed images of the drops were recorded and used to evaluate the speed of the drop, the drop volume, and the jet direction, as is shown in Figure 8.3. The effect of different types of driving waveforms (e.g., single trapezoidal shaped and bipolar pulse), waveform parameters (e.g., drive voltage, dwell time, rise time, fall time, etc.) and process parameters (e.g., the temperature of the head and the backpressure of the ink reservoir) were explored in detail, eventually leading to a robust, repeatable and precise printability of the inks. The DMC 11610 cartridge has 16 nozzles that can fire all simultaneously or in single mode. To increase the accuracy of the inkjet printing deposition in DoD mode, a single nozzle was selected during the optimization procedure and it was tested separately after the optimization of the driving waveform using all nozzles. This nozzle was used to align and calibrate the print head to the MEA substrate 
through fiducial alignment which consists of three reference marks with a size of $250 \mu \mathrm{m}$ and with a minimal tolerance of $93 \mu \mathrm{m}$.

\section{Printing and characterization of the PFS modified MEA chips.}

PFS 5 ink was printed on MEA chips and unpatterned gold-coated wafer samples, the latter allowing investigation of the homogeneity, step height and roughness of the printed PFS films. The MEA chips, without the ring (see Figure S8.8), were cleaned with ethanol and dried in a stream of $\mathrm{N}_{2}$ before being printed with PFS-based inks. The printer was aligned with the MEA via "fiducial alignment" using three reference marks on the substrate and the pattern was designed and edited as BMP file. The three reference marks were chosen on the borders of the Au contact pads positioned at the corner of the MEA chip setting the "mark size" to $250 \mu \mathrm{m}$. The images were collected via the fiducial camera which was positioned on the side of the print-head close to the nozzles. Image analysis was conducted at constant focus and intensity of the coax and ring lights. The alignment was considered optimal when the score of the alignment was set at least $90 \%$. The PFS printed MEA biochips were rinsed with toluene, ethanol, and water, and dried in a stream of $\mathrm{N}_{2}$. Finally, the samples were annealed at room temperature in a vacuum oven overnight. The printed patterns were rinsed with toluene, ethanol, and water, and then dried in a stream of $\mathrm{N}_{2}$.

Following modification of the MEA with PFS 5 by inkjet printing, the quality of the printed pattern was evaluated by visual inspection through optical microscopy and laser confocal microscopy measurements as is shown in Figure S8.10 and Figure S8.11, respectively. Figure S8.10a shows a printed pattern on a plain gold substrate. The printed pattern consisted of an 8x8 array of dots (center-to-center distance was $200 \mu \mathrm{m}$ ). Figure S8.10b shows a different PFS 5 printed pattern on the MEA chip, in which the MEs of the first row were not modifed demonstrating the high spatial control of the inkjet printing deposition. The precision and accuracy of the ink deposition on the MEA chip were evaluated by optical and laser confocal microscopes. The laser mode was used to adjust the filter and brightness for the capturing of a clear image, with a $\mathrm{Z}$-axis scanning range set to $0.020 \mu \mathrm{m}$. The images were acquired with a 10x magnification objective lens and an optical zoom of 1.0x and analyzed using VK analyzer software. The surface roughness of the electrodes (mean roughness and root mean square roughness, $R a$ and $R q$ respectively) was measured before and after the printing process. $R a$ and $R q$ were evaluated on an electrode area of $1250 \mu \mathrm{m}^{2}$. 
a)

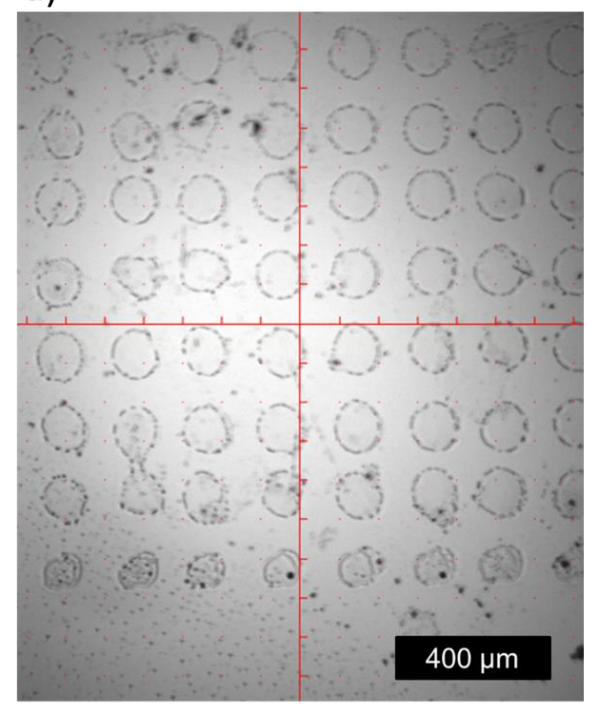

b)

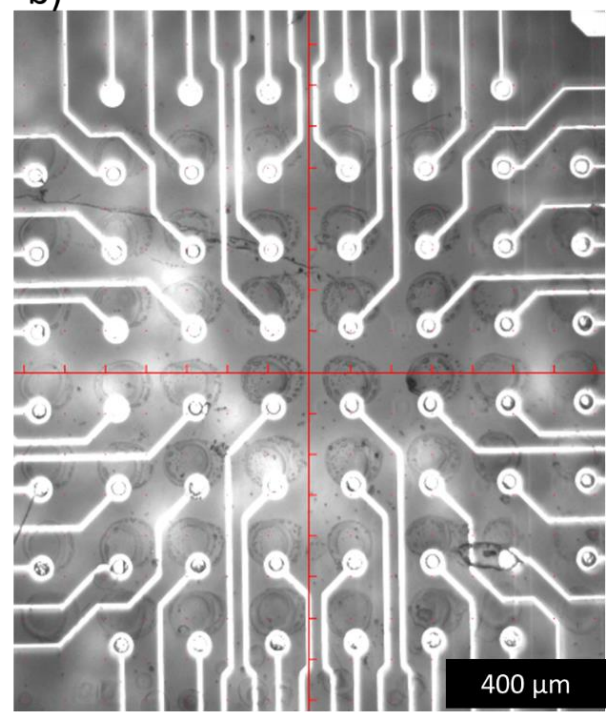

Fig. S8.10: Typical optical microscopy image of a) an ink A printed pattern on a plain gold substrate and b) of the ink A printed pattern on a MEA chip. Printing conditions: FUJIFILM Dimatix 2831 piezo inkjet printer equipped with DMC-11610 cartridge, at room temperature, firing voltage of $50 \mathrm{~V}$ for $10 \mu \mathrm{s}$, fill time of $5 \mu \mathrm{s}$, rise and fall time of $1 \mu \mathrm{s}$ and a distance of $2 \mathrm{~mm}$ of the print head from the surface.

3D surface images (laser confocal microscopy) and height images (in false color view) of the clean and ink A printed MEAs show the micro-scale topography of the samples and allow one to estimate the precision and the accuracy in the deposition of the ink. The height profile and the roughness of the PFS 5 decorated ME surfaces were determined, as shown in Figure S8.11. The as-received Au-MEA chip was characterized by an $R a$ value of $0.8 \pm 0.2 \mu \mathrm{m}$ and an $R q$ value of $1.2 \pm 0.3 \mu \mathrm{m}$, while for the "ink A" modified MEA chip the $R a$ value was $1.3 \pm 0.3 \mu \mathrm{m}$ and the $R q$ value was $1.7 \pm 0.4 \mu \mathrm{m}$. The change in the topographic properties of the samples (increase of the roughness and a change in the height profile) point towards the successful deposition of PFS 5 on the MEAs via inkjet printing. 

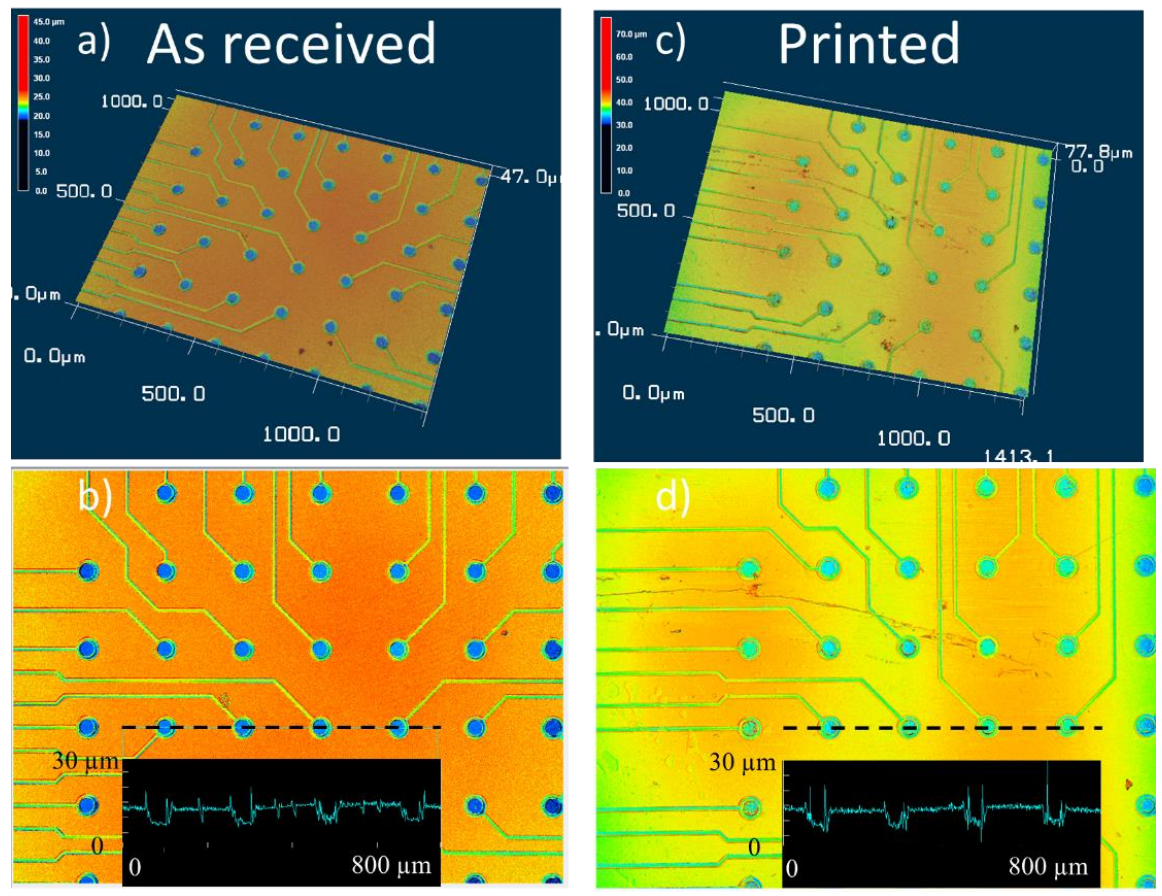

Fig. S8.11: a) and b) are representative laser confocal microscope images of the MEA chips, as received. c) and d) show laser confocal microscope images of the PFS 5 modified MEAs. a) and c) are the 3-D reconstruction surface profiles; b) and d) correspond to the height images and the height profiles of an array of MEs.

Figure S8.12 shows SEM images of the surfaces of PFS 5 films on the Au-ME of the MEA chip. The electrode exhibits a somewhat heterogeneous deposition of the redox active film. At the center and at the border of the well of the electrode there are some irregularities which one may ascribe to contamination of the MEA chip.
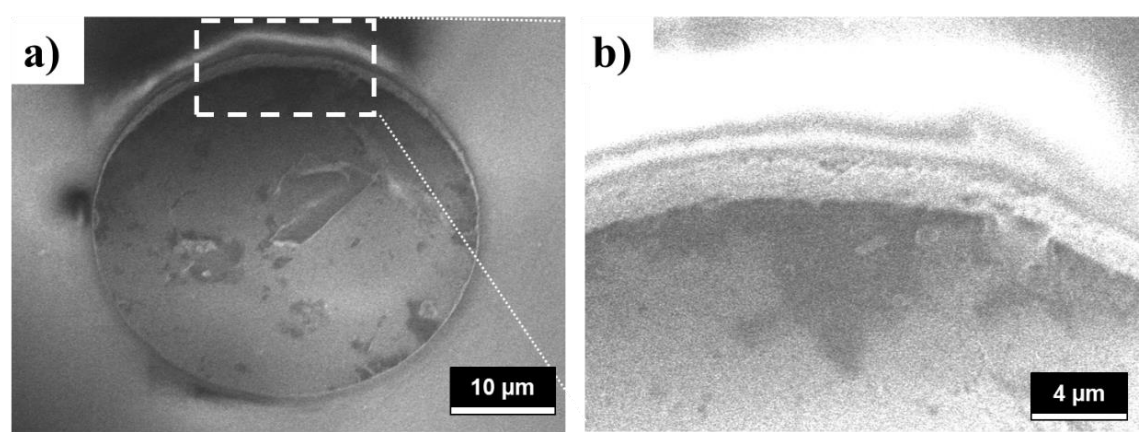

Fig. S8.12: Morphological characterization of a) the inkjet printed disulfide-modified PFS film on AuMEs with b) a higher magnification image of the border/edge.

In order to characterize the nanoscale topology of the surfaces, the roughness and the thickness of the printed layers were measured by AFM, as shown in Figure S8.13. The thickness of the PFS films tethered on gold coated surfaces was studied by scratching the 
polymer films with a Teflon tweezer and measuring the difference in the step height via tapping mode AFM. At least three different measurements of each sample were performed and averaged, resulting in a film thickness of $8 \pm 3 \mathrm{~nm}$. The topology of the polymer coatings was evaluated analysing 3 different spots with a scan area of $1 \mathrm{x} 1 \mu \mathrm{m}^{2}$. The values of the average surface roughness $(R a)$ and the root mean square average $(R q)$ were $1.2 \pm 0.5 \mathrm{~nm}$ and $1.76 \pm 0.5 \mathrm{~nm}$, respectively.
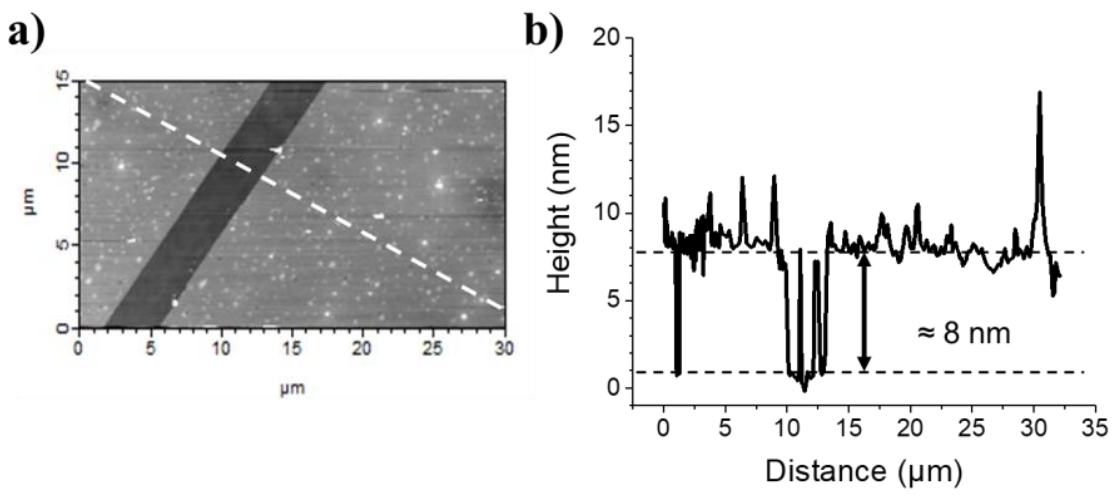

Fig. S8.13: Typical tapping mode AFM topography image a) and height profile b) of a representative dry PFS 5 film immobilized on the Au substrate over a scratch made with a Teflon tweezer.

The chemical composition of the printed MEAs was obtained by EDX-HR SEM and mapping ATR-FTIR microscopy measurements, confirming the presence of the PFS films only on the desired areas of the MEs. Figure S8.14 shows the FTIR spectra in different locations of the MEA and the specific chemical groups of PFS $\mathbf{5}$ are indicated by the highlighted regions, i.e. the $\mathrm{C}-\mathrm{H}$ stretch absorbance band of the Fc rings at 3087 $\mathrm{cm}^{-1}$, and the absorbance bands ascribed to the vibration of Fc rings at 1037 and 1165 $\mathrm{cm}^{-1}$. 

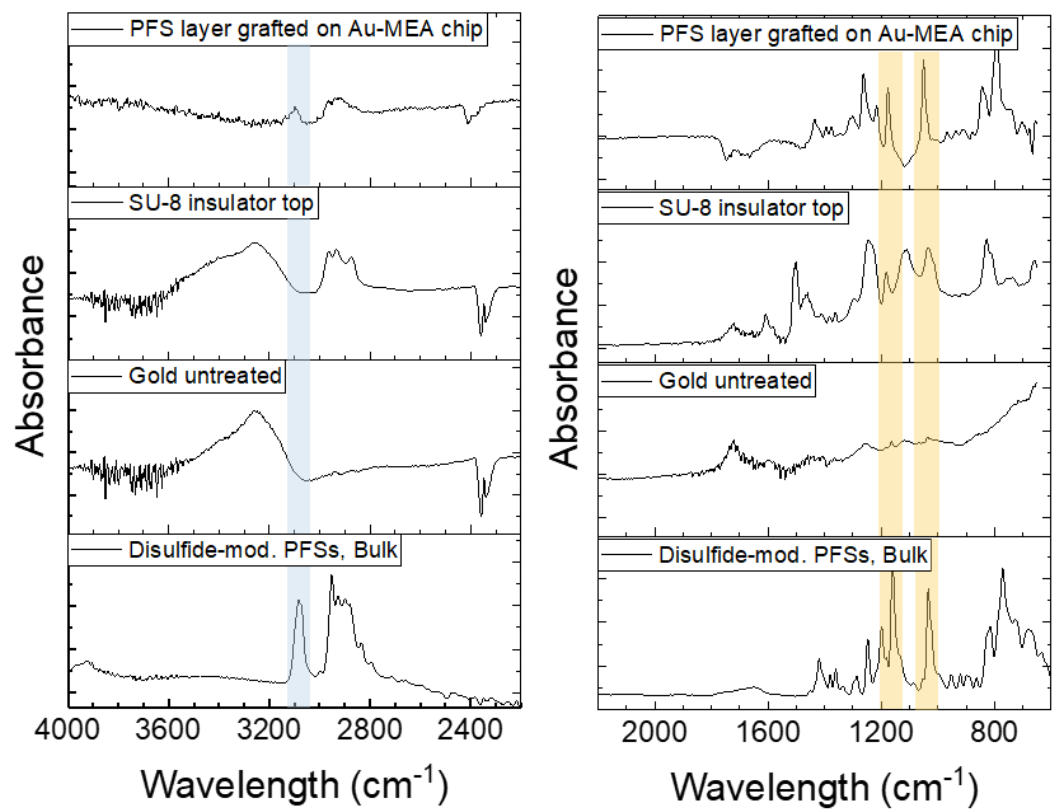

Fig. S8.14: From the bottom to the top: FTIR spectrum of bulk PFS 5, of the clean AU-ME, of the insulator (SU-8) layer and of the PFS 5 printed MEs. On the left the spectrum at high energy regions (4000$\left.2200 \mathrm{~cm}^{-1}\right)$ and on the right the FTIR spectrum in low energy regions $\left(2200-600 \mathrm{~cm}^{-1}\right)$.

Classification based on the electrochemical properties of the PFS 5 printed MEAs.

The PFS 5 modified MEs were classified based on the electrochemical properties of the grafted redox active film, as shown in Figure S8.15:

a) PFS well-modified MEs are characterized by anodic and cathodic currents exceeding +5 and $-5 \mathrm{nA}$, respectively, transferred charge values for the oxidation and the reduction of ferrocene sites of at least $10 \mathrm{nC}$ and the presence of electrocatalytic phenomena. For example, the well-modified ME 36 is shown in Figure S8.15a and S8.15d.

b) PFS poorly-modified MEs are characterized by anodic and cathodic currents in the range between +5 and $-5 \mathrm{nA}$, transferred charge values of $1 \mathrm{nC}$ and no electrocatalytic phenomena for the oxidation of ascorbic acid. Figure S8.15b and S8.15e show the redox properties of ME 54 as an example.

c) Non-modified ME, for example ME 61, where no appreciable current signal and no electrocatalytic phenomena were detected, see Figure S8.15c and S8.15f. 

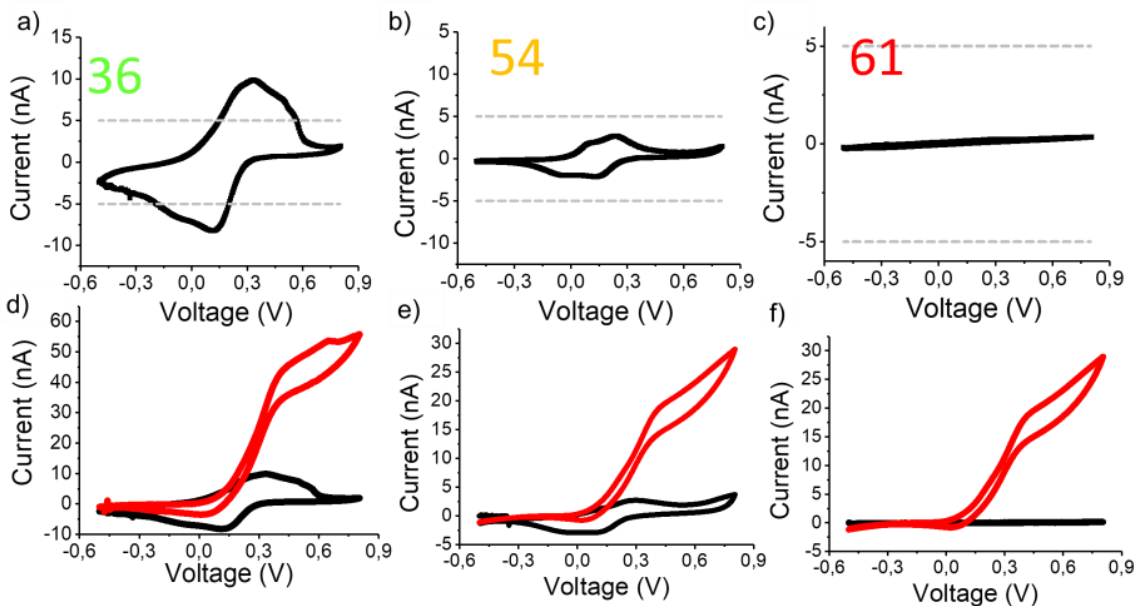

Fig. S8.15: Electrochemical properties of Au-MEs (representative for the MEA) modified with PFS 5, resulting in layers with different quality. a) and d) show the CVs of a well of a PFS 5 modified ME (number 36); a) shows the $\mathrm{CV}$ in $100 \mathrm{mM} \mathrm{NaClO}_{4}$ aqueous solution [Pt(wire)-RE/CE] and d) shows the $\mathrm{CV}$ in presence of the analyte (ascorbic acid). b) and e) feature a PFS 5 modified ME with poor modification quality (number 54) in the absence and presence of ascorbic acid, respectively. c) and f) describe a non-modified ME (number 61 ) in the absence and presence of ascorbic acid, respectively. Note the different y axis scale in a, c and d. Scan rate $50 \mathrm{mV} \cdot \mathrm{s}^{-1}$, in $100 \mathrm{mM} \mathrm{NaClO}$ and in the presence of $35 \mathrm{mM}$ of ascorbic acid, $\mathrm{Pt}($ wire)-RE/CE, and in the potential range between -500 and $800 \mathrm{mV} v s$. Pt.

The electrochemical stability of the redox active PFS films was examined by successive $\mathrm{CV}$ cycles in aqueous electrolyte solution for two representative sample (MEs number 36 and 74), as shown in the Figure S8.16. The oxidation and reduction current peaks were unchanged and reproducible after more than 10 cycles for both samples confirming that no PFS chains desorbed from the various ME surfaces owing to the stable $\mathrm{S}$-Au bond between the redox active coating and the PFS 5 modified ME surfaces.

a)

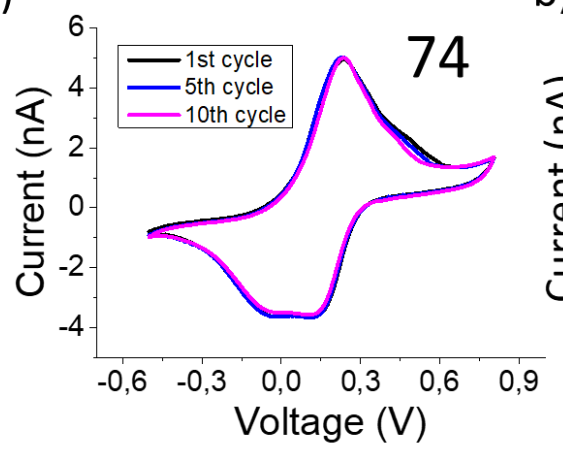

b)

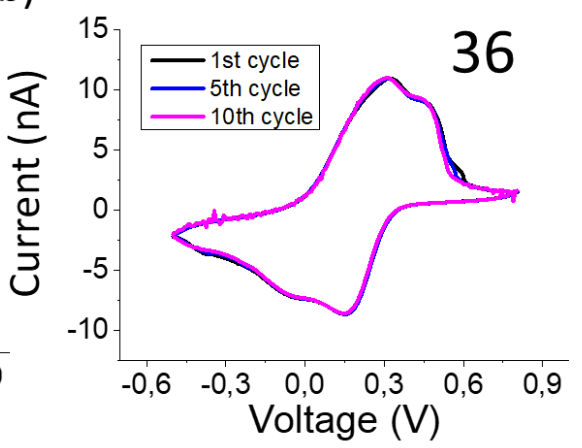

Fig. S8.16: Typical CVs of two representative printed PFS 5 films on different MEs number 74 and 36, respectively (a) and (b). Scan rate $50 \mathrm{mV} \cdot \mathrm{s}^{-1}$, in $100 \mathrm{mM} \mathrm{NaClO} 4, \mathrm{Pt}($ wire)-RE/CE, and potential range between -500 and $800 \mathrm{mV}$ vs. Pt. 
Classification based on the electrochemical properties of the PFS 5 printed MEAs.

The final evaluation of the PFS printed MEAs was carried out by a combination of visual inspection, chemical composition analysis, surface analysis, and electrochemical characterization of the functionalized MEAs.

Visual inspection of the printed MEA by optical microscopy confirmed a good accuracy of the inkjet printing process for deposition of "ink A", as shown in Figure S8.17a. All drops were deposited on the electrodes in the designed pattern with a good accuracy (few tens of microns). (We note that the deposition accuracy may be improved by a revised formulation of the redox active ink (more viscous), working in a wellcontrolled environment (no air flow) and inkjet printing at a lower distance between the print-head and the substrate.) Subsequently, the chemical composition was verified. Then, the electrochemical properties of the redox active MEs were tested as well as their performance as sensing device in absence and in presence of ascorbic acid.

As summarized in Figure S8.17b, there were 34 (63\%) well modified, 21 (39\%) poorly modified and only 4 (8\%) non-modified MEs.

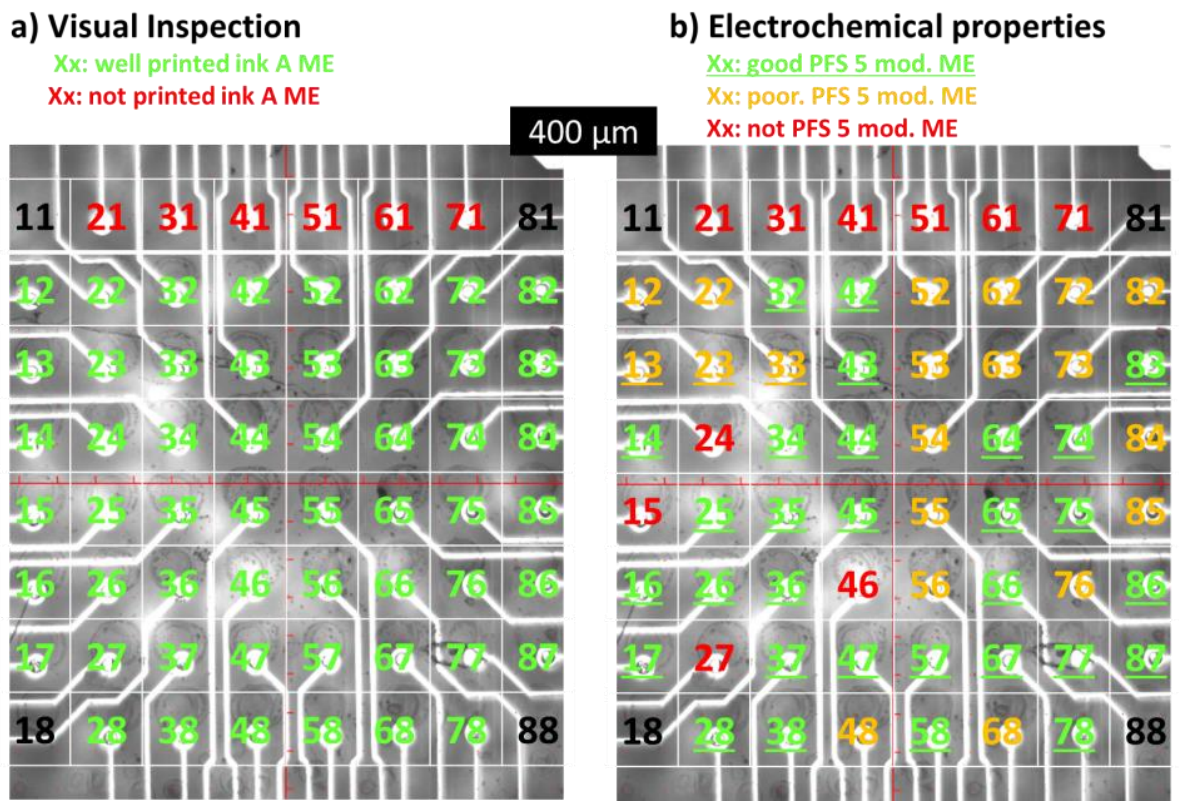

Fig. S8.17: Optical images of the printed MEA chip. On the left, a) visual inspection of the printed pattern on the MEA chip: in green the electrodes that are covered and in red the electrodes that were not functionalized on purpose. On the right, b) the classification of the electrodes after electrochemical characterization: in green the well-modified, in yellow the poorly modified and in red the non-modified MEs.

To further characterize the electroactive behavior of the redox-active films, we studied the scan rate dependence of the peak current as summarized in Figure S18. This included the assessment of the process to show whether it was diffusion or adsorption controlled. Figure S8.18a shows the effect of the scan rate on the double-wave voltammogram. As the scan rate increases, the shape of the CVs becomes more distorted 
and the current intensity increases as described by the Faraday and Randles-Sevcik equations. The values of the peak current as a function of the square root of the scan rate showed a linear relationship in the range of $10-50 \mathrm{mV} \mathrm{s}^{-1}$, as shown in the Figure $\mathbf{S 8 . 1 8 b}$ and Figure S8.18c, which is typical for diffusion controlled processes obeying Fick's law. ${ }^{8}$
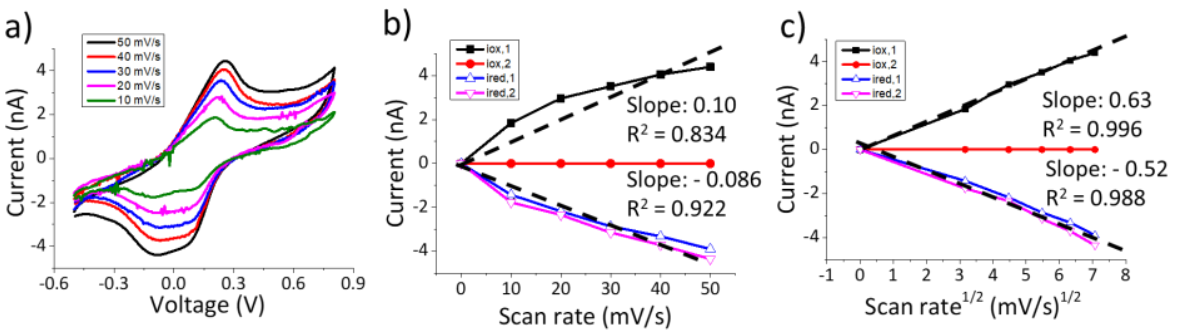

Fig. S8.18: (a) CVs of the scan rate of a typical printed PFS film immobilized on gold at different scan rates. Scan rate in the range of $10-50 \mathrm{mV} \mathrm{s}^{-1}$, in $100 \mathrm{mM} \mathrm{NaClO}_{4}, \mathrm{Pt}$ (wire)-RE/CE, and potential range between -500 and $800 \mathrm{mV} v s$. Pt. (b) Plot of peak currents vs. scan rate, and (c) plot of peak current vs. square root of the scan rate for these printed PFS films.

\subsection{References}

1. Thomas Jr, C.; Springer, P.; Loeb, G.; Berwald-Netter, Y.; Okun, L., A miniature microelectrode array to monitor the bioelectric activity of cultured cells. Experimental Cell Research 1972, 74 (1), 61-66.

2. Feeney, R.; Kounaves, S. P., Microfabricated ultramicroelectrode arrays: Developments, advances, and applications in environmental analysis. Electroanal 2000, 12 (9), 677-684.

3. Pine, J., A history of MEA development. In Advances in Network Electrophysiology, Springer: 2006; pp $3-23$.

4. Spira, M. E.; Hai, A., Multi-electrode array technologies for neuroscience and cardiology. Nature nanotechnology 2013, 8 (2), 83 .

5. Obien, M. E. J.; Deligkaris, K.; Bullmann, T.; Bakkum, D. J.; Frey, U., Revealing neuronal function through microelectrode array recordings. Frontiers in Neuroscience 2015, 8, 423.

6. Nagale, M. P.; Fritsch, I., Individually addressable, submicrometer band electrode arrays. 2. Electrochemical characterization. Analytical Chemistry 1998, 70 (14), 2908-2913.

7. Nagale, M. P.; Fritsch, I., Individually Addressable, Submicrometer Band Electrode Arrays. 1. Fabrication from Multilayered Materials. Analytical Chemistry 1998, 70 (14), 2902-2907.

8. Bard, A. J.; Faulkner, L. R., Fundamentals and applications. Electrochemical Methods 2001, 2, 482.

9. Escuder-Gilabert, L.; Peris, M., Highlights in recent applications of electronic tongues in food analysis. Analytica Chimica Acta 2010, 665 (1), 15-25.

10. Dimaki, M.; Vergani, M.; Heiskanen, A.; Kwasny, D.; Sasso, L.; Carminati, M.; Gerrard, J.; Emneus, J.; Svendsen, W., A compact microelectrode array chip with multiple measuring sites for electrochemical applications. Sensors 2014, 14 (6), 9505-9521.

11. Mendes, P. M., Stimuli-responsive surfaces for bio-applications. Chemical Society Reviews 2008, 37 (11), 2512-2529.

12. Bawa, P.; Pillay, V.; Choonara, Y. E.; Du Toit, L. C., Stimuli-responsive polymers and their applications in drug delivery. Biomedical Materials 2009, 4 (2), 022001. 
13. Roy, D.; Cambre, J. N.; Sumerlin, B. S., Future perspectives and recent advances in stimuli-responsive materials. Progress in Polymer Science 2010, 35 (12), 278-301.

14. Stuart, M. A. C.; Huck, W. T.; Genzer, J.; Müller, M.; Ober, C.; Stamm, M.; Sukhorukov, G. B.; Szleifer, I.; Tsukruk, V. V.; Urban, M., Emerging applications of stimuli-responsive polymer materials. Nature Materials 2010, 9 (2), 101.

15. Schattling, P.; Jochum, F. D.; Theato, P., Multi-stimuli responsive polymers-the all-in-one talents. Polymer Chemistry 2014, 5 (1), 25-36.

16. Xie, G.; Khabibullin, A.; Pietrasik, J.; Yan, J.; Matyjaszewski, K., Polymer Brushes by Atom Transfer Radical Polymerization. Polymer and Biopolymer Brushes: For Materials Science and Biotechnology 2018, 2, 242.

17. Whittell, G. R.; Manners, I., Metallopolymers: New multifunctional materials. Advanced Materials 2007, 19 (21), 3439-3468.

18. Song, J.; Vancso, G. J., Responsive organometallic polymer grafts: electrochemical switching of surface properties and current mediation behavior. Langmuir 2011, 27 (11), 6822-6829.

19. Nakahata, M.; Takashima, Y.; Yamaguchi, H.; Harada, A., Redox-responsive self-healing materials formed from host-guest polymers. Nature Communications 2011, 2, 511.

20. Sui, X.; Feng, X.; Song, J.; Hempenius, M. A.; Vancso, G. J., Electrochemical sensing by surfaceimmobilized poly (ferrocenylsilane) grafts. Journal of Materials Chemistry 2012, 22 (22), 11261-11267.

21. Feng, X.; Zhang, K.; Hempenius, M. A.; Vancso, G. J., Organometallic polymers for electrode decoration in sensing applications. RSC Advances 2015, 5 (129), 106355-106376.

22. Nguyen, P.; Gómez-Elipe, P.; Manners, I., Organometallic polymers with transition metals in the main chain. Chemical Reviews 1999, 99 (6), 1515-1548.

23. Giannotti, M.; Lv, H.; Ma, Y.; Steenvoorden, M.; Overweg, A.; Roerdink, M.; Hempenius, M. A.; Vancso, G. J., Stimulus responsive poly (ferrocenylsilanes): redox chemistry of iron in the main chain. Journal of Inorganic and Organometallic Polymers and Materials 2005, 15 (4), 527-540.

24. Hailes, R. L.; Oliver, A. M.; Gwyther, J.; Whittell, G. R.; Manners, I., Polyferrocenylsilanes: synthesis, properties, and applications. Chemical Society Reviews 2016, 45 (19), 5358-5407.

25. Foucher, D.; Ziembinski, R.; Petersen, R.; Pudelski, J.; Edwards, M.; Ni, Y.; Massey, J.; Jaeger, C. R.; Vancso, G. J.; Manners, I., Synthesis, characterization, and properties of high molecular weight unsymmetrically substituted poly (ferrocenylsilanes). Macromolecules 1994, 27 (14), 3992-3999.

26. Zou, S.; Ma, Y.; Hempenius, M. A.; Schönherr, H.; Vancso, G. J., Grafting of single, stimuli-responsive poly (ferrocenylsilane) polymer chains to gold surfaces. Langmuir 2004, 20 (15), 6278-6287.

27. Nguyen, M. T.; Diaz, A. F.; Dement'ev, V. V.; Pannell, K. H., Electrochemical and electrochromic properties of poly (dialkylsilyleneferrocenylene) films. Chemistry of Materials 1994, 6 (7), 952-954.

28. Rulkens, R.; Ni, Y.; Manners, I., Living anionic ring-opening polymerization of silicon-bridged [1] ferrocenophanes: synthesis and characterization of poly (ferrocenylsilane)-polysiloxane block copolymers. Journal of the American Chemical Society 1994, 116 (26), 12121-12122.

29. Péter, M.; Lammertink, R. G.; Hempenius, M. A.; Vancso, G. J., Electrochemistry of surface-grafted stimulus-responsive monolayers of poly (ferrocenyldimethylsilane) on gold. Langmuir 2005, 21 (11), $5115-5123$.

30. Foucher, D. A.; Honeyman, C. H.; Nelson, J. M.; Tang, B. Z.; Manners, I., Organometallic ferrocenyl polymers displaying tunable cooperative interactions between transition metal centers. Angewandte Chemie International Edition In English 1993, 32 (12), 1709-1711.

31. Rulkens, R.; Lough, A. J.; Manners, I.; Lovelace, S. R.; Grant, C.; Geiger, W. E., Linear oligo (ferrocenyldimethylsilanes) with between two and nine ferrocene units: Electrochemical and structural models for poly (ferrocenylsilane) high polymers. Journal of the American Chemical Society 1996, 118 (50), 12683-12695. 
32. Cui, K.; Song, Y.; Wang, L., Electrochemical and electrocatalytic behaviors of poly (ferrocenylsilane)/DNA modified glass carbon electrode. Electrochemistry Communications 2008, 10 (11), 1712-1715.

33. Feng, X.; Sui, X.; Hempenius, M. A.; Vancso, G. J., Electrografting of stimuli-responsive, redox active organometallic polymers to gold from ionic liquids. Journal of the American Chemical Society 2014, 136 (22), 7865-7868.

34. Feng, X.; Cumurcu, A.; Sui, X.; Song, J.; Hempenius, M. A.; Vancso, G. J., Covalent layer-by-layer assembly of redox-active polymer multilayers. Langmuir 2013, 29 (24), 7257-7265.

35. Hendry, S.; Cardosi, M.; Turner, A.; Neuse, E., Polyferrocenes as mediators in amperometric biosensors for glucose. Analytica Chimica Acta 1993, 281 (3), 453-459.

36. Lee, J.; Ahn, H.; Choi, I.; Boese, M.; Park, M. J., Enhanced Charge Transport in Enzyme-Wired Organometallic Block Copolymers for Bioenergy and Biosensors. Macromolecules 2012, 45 (7), 3121 3128 .

37. Chen, C.; Song, Y.; Wang, L., A Novel Sensor Based on Layer-by-Layer Hybridized Phosphomolybdate and Poly (ferrocenylsilane) on a Cysteamine Modified Gold Electrode. Electroanalysis: An International Journal Devoted to Fundamental and Practical Aspects of Electroanalysis 2008, 20 (23), 2543-2548.

38. Cyr, P. W.; Tzolov, M.; Manners, I.; Sargent, E. H., Photooxidation and photoconductivity of polyferrocenylsilane thin films. Macromolecular Chemistry and Physics 2003, 204 (7), 915-921.

39. Xu, Z.; Dong, Q.; Otieno, B.; Liu, Y.; Williams, I.; Cai, D.; Li, Y.; Lei, Y.; Li, B., Real-time in situ sensing of multiple water quality related parameters using micro-electrode array (MEA) fabricated by inkjet-printing technology (IPT). Sensors and Actuators B: Chemical 2016, 237, 1108-1119.

40. Rapini, R.; Marrazza, G., Electrochemical aptasensors for contaminants detection in food and environment: Recent advances. Bioelectrochemistry 2017, 118, 47-61.

41. Huang, X. J.; O'Mahony, A. M.; Compton, R. G., Microelectrode arrays for electrochemistry: approaches to fabrication. Small 2009, 5 (7), 776-788.

42. Wang, J., Modified electrodes for electrochemical sensors. Electroanal 1991, 3 (4-5), 255-259.

43. Falconnet, D.; Csucs, G.; Grandin, H. M.; Textor, M., Surface engineering approaches to micropattern surfaces for cell-based assays. Biomaterials 2006, 27 (16), 3044-3063.

44. Adly, N.; Weidlich, S.; Seyock, S.; Brings, F.; Yakushenko, A.; Offenhäusser, A.; Wolfrum, B., Printed microelectrode arrays on soft materials: from PDMS to hydrogels. npj Flexible Electronics 2018, 2 (1), 15.

45. Edmondson, S.; Osborne, V. L.; Huck, W. T., Polymer brushes via surface-initiated polymerizations. Chemical Society Reviews 2004, 33 (1), 14-22.

46. Barbey, R. R. R., Polymer brushes via surface-initiated controlled radical polymerization: synthesis, characterization, properties, and applications. Chemical Reviews 2009, 109 (11), 5437-5527.

47. Zoppe, J. O.; Ataman, N. C.; Mocny, P.; Wang, J.; Moraes, J.; Klok, H.-A., Surface-initiated controlled radical polymerization: state-of-the-art, opportunities, and challenges in surface and interface engineering with polymer brushes. Chemical Reviews 2017, 117 (3), 1105-1318.

48. Zdyrko, B.; Luzinov, I., Polymer brushes by the "grafting to" method. Macromolecular Rapid Communications 2011, 32 (12), 859-869.

49. Love, J. C.; Estroff, L. A.; Kriebel, J. K.; Nuzzo, R. G.; Whitesides, G. M., Self-assembled monolayers of thiolates on metals as a form of nanotechnology. Chemical Reviews 2005, 105 (4), 1103-1170.

50. Pensa, E.; Cortes, E.; Corthey, G.; Carro, P.; Vericat, C.; Fonticelli, M. H.; Benitez, G.; Rubert, A. A.; Salvarezza, R. C., The chemistry of the sulfur-gold interface: in search of a unified model. Accounts of Chemical Research 2012, 45 (8), 1183-1192. 
51. Péter, M.; Lammertink, R. H.; Hempenius, M.; Julius Vancso, G.; Os, M.; Beulen, M. J.; Reinhoudt, D., Synthesis, characterization and thin film formation of end-functionalized organometallic polymers. Chemical Communications 1999, (4), 359-360.

52. Chabanne, L.; Pfirrmann, S.; Lunn, D. J.; Manners, I., Controlled thiol-ene post-polymerization reactions on polyferrocenylsilane homopolymers and block copolymers. Polymer Chemistry 2013, 4 (7), 2353 2360

53. Gómez-Elipe, P.; Macdonald, P. M.; Manners, I., Architectural Control in the Transition-MetalCatalyzed Ring-Opening Polymerization of Silicon-Bridged [1] Ferrocenophanes. Angewandte Chemie International Edition in English 1997, 36 (7), 762-764.

54. Hempenius, M. A.; Brito, F. F.; Vancso, G. J., Synthesis and Characterization of Anionic and Cationic Poly(ferrocenylsilane) Polyelectrolytes. Macromolecules 2003, 36 (17), 6683-6688.

55. Song, J.; Jańczewski, D.; Ma, Y.; van Ingen, L.; Sim, C. E.; Goh, Q.; Xu, J.; Vancso, G. J., Electrochemically controlled release of molecular guests from redox responsive polymeric multilayers and devices. European Polymer Journal 2013, 49 (9), 2477-2484.

56. Lammertink, R. G.; Hempenius, M. A.; Vancso, G. J.; Shin, K.; Rafailovich, M. H.; Sokolov, J., Morphology and Surface Relief Structures of Asymmetric Poly (styrene-b lock-ferrocenylsilane) Thin Films. Macromolecules 2001, 34 (4), 942-950.

57. Calvert, P. P. P., Inkjet printing for materials and devices. Chemistry of Materials 2001, 13 (10), 32993305 .

58. De Gans, B. J.; Duineveld, P. C.; Schubert, U. S., Inkjet printing of polymers: state of the art and future developments. Advanced Materials 2004, 16 (3), 203-213.

59. Geissler, M.; Xia, Y., Patterning: Principles and some new developments. Advanced Materials 2004, 16 (15), 1249-1269.

60. Li, J.; Rossignol, F.; Macdonald, J., Inkjet printing for biosensor fabrication: combining chemistry and technology for advanced manufacturing. Lab on a Chip 2015, 15 (12), 2538-2558.

61. Moya, A.; Gabriel, G.; Villa, R.; del Campo, F. J., Inkjet-printed electrochemical sensors. Current Opinion in Electrochemistry 2017, 3 (1), 29-39.

62. Derby, B., Inkjet printing of functional and structural materials: fluid property requirements, feature stability, and resolution. Annual Review of Materials Research 2010, 40, 395-414.

63. Hoath, S. D., Fundamentals of inkjet printing: the science of inkjet and droplets. John Wiley \& Sons: 2016.

64. Avila-Zárraga, J. G.; Martínez, R., Efficient methylation of carboxylic acids with potassium hydroxide/methyl sulfoxide and iodomethane. Synthetic Communications 2001, 31 (14), 2177-2183.

65. Wijshoff, H., The dynamics of the piezo inkjet printhead operation. Physics Reports 2010, 491 (4-5), 77177.

66. Komuro, N.; Takaki, S.; Suzuki, K.; Citterio, D., Inkjet printed (bio) chemical sensing devices. Analytical and Bioanalytical Chemistry 2013, 405 (17), 5785-5805.

67. Evans, D. H., One-electron and two-electron transfers in electrochemistry and homogeneous solution reactions. Chemical Reviews 2008, 108 (7), 2113-2144.

68. Hempenius, M. A.; Brito, F. F.; Vancso, G. J., Synthesis and Characterization of Anionic and Cationic Poly(ferrocenylsilane) Polyelectrolytes. Macromolecules 2003, 36, 17, 6683-6688.

69. O’Hare, D., Biosensors and sensor systems. In Body Sensor Networks, Springer: 2014; pp 55-115.

70. McKinley, G. H.; Renardy, M., Wolfgang von Ohnesorge. Physics of Fluids 2011, 23, 12, 127101.

71. Shlomo, M., The Chemistry of Inkjet Inks. World Scientific: 2009. 
Chapter 9

Synthesis and Design of Bio-Inspired ATRP Functional Macro-Initiators Applicable to a Broad Range of Surfaces

We attempt to develop a platform that can be universally applied as engineered molecular adhesives. In this Chapter, we describe recent findings of our ongoing research aiming at catechol-based designer molecular adhesives. 


\section{Contents}

Chapter 9 Synthesis and Design of Bio-Inspired ATRP Functional Macro-Initiators Applicable to a Broad Range of Surfaces...................................................................................................... 205

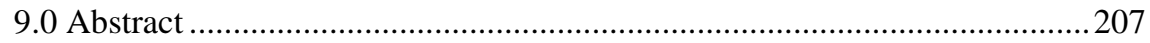

9.1 Mussel-Inspired ATRP Functional Macroinitiators ..................................208

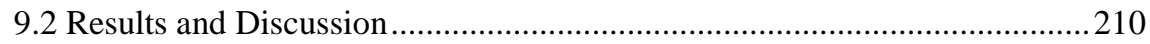

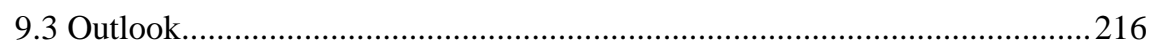

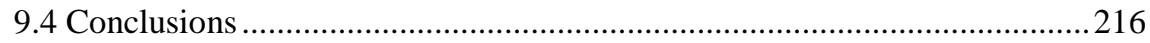

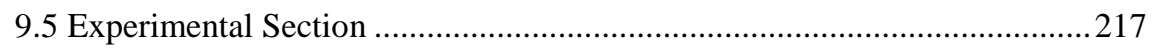

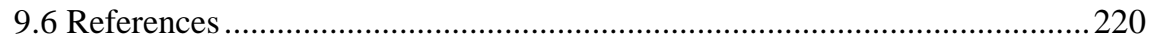




\subsection{Abstract}

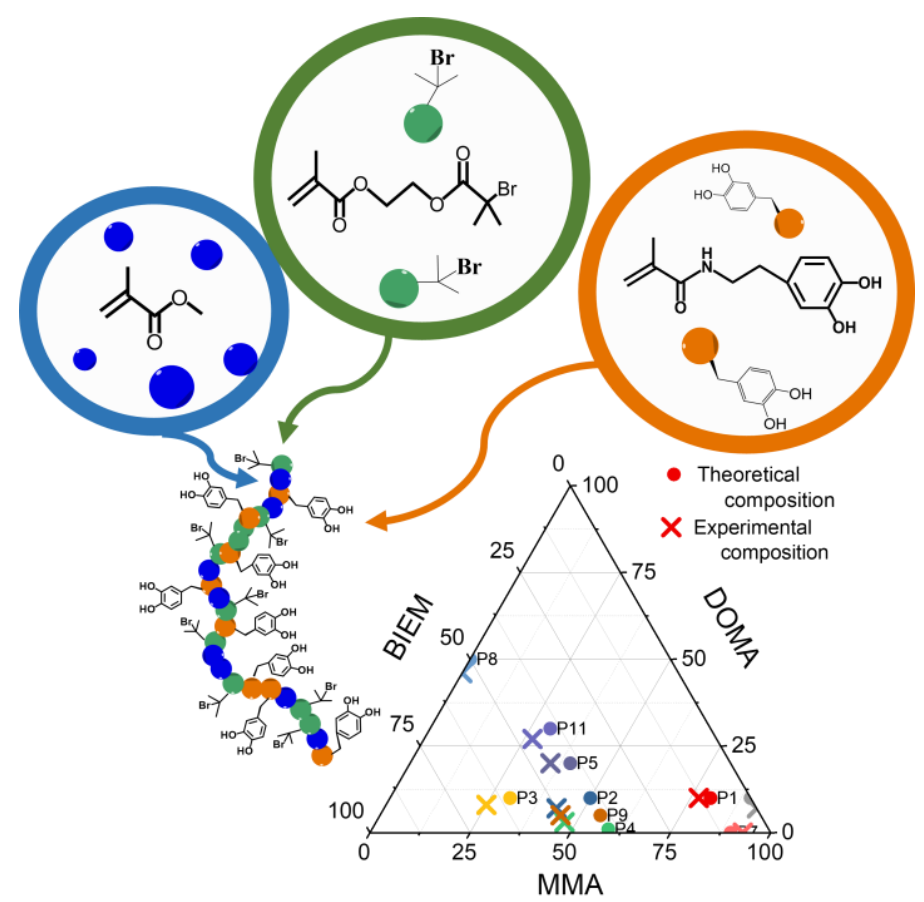

TOC Chapter 9: A schematic representation of the atom transfer radical polymerization (ATRP) macroinitiators containing anchoring groups, ATRP initiating groups and solubilizing groups and the agreement between the composition of the feed and the composition of the macro-initiator.

The controlled deposition of functional coatings on a wide range of substrates via inkjet printing broadens their potential application, for which of particular interest is a development of polymer brushes grown from large and complex surfaces. Achieving these objectives, a quick and reliable deposition of surface-tethered initiator layers is needed. In this Chapter, the synthesis and deposition of a catechol containing musselinspired macro-initiator (MI) layer is proposed and discussed. With the tailor-made MIs developed here, the substrate independent rapid deposition of stable initiator layers comes within reach, and combined with less air-sensitive atom transfer radical polymerization (ATRP) methods, it allows one to perform large-scale surface modification with polymer brushes at an industrial level.

\subsection{Mussel-inspired ATRP functional macroinitiators}

Surface initiated controlled radical polymerization (SI-CRP) allows for the growth of polymer brushes with controlled composition and brush densities. ${ }^{1-5}$ As presented and discussed in previous Chapters, the atom transfer radical polymerization (ATRP) is one of the most versatile and robust techniques to synthesize polymer brush films with 
complex and various architectures for numerous applications. ${ }^{6-9}$ Prior to the brush growth from surface initiated polymerization (SIP), a stable and surface active initiator layer must be deposited onto the surface. ${ }^{4-5,10}$ Preceeding the selection of a proper initiator, the grafting density, brush thickness, and the surface attachment mechanism must be considered.

Typically, small molecule initiators are characterized by having a singular strong substrate-dependent covalent anchoring point (e.g., chlorosilane for oxides or thiol-based for gold and for some noble metals). Often, the anchoring point is susceptible to debinding, which can lead to unstable initiator active coupling layers. ${ }^{11-12}$ In addition, the surface derivatization of these small initiator molecules is often a tedious, costly, and time-consuming process. ${ }^{11}$ Macro-initiators (MIs) were recently reported in the open literature as broadly applicable alternatives to small molecule initiators for the synthesis of single-end tethered polymer chains., ${ }^{4}$ 10, 13-14

In order to functionalize a diverse variety of substrates and subsequently grow stable polymer brush layers onto them, robust and versatile anchoring layers must be designed and developed. Often, specific noncovalent reversible interactions (e.g. H-bonds, electrostatic interactions, metal-ligand complexation, hydrophobic and $\pi$ - $\pi$ stacking interactions) can be utilized to achieve the adhesion required to bond to various materials. ${ }^{4}, 10,15-16$ In addition, multiple non-covalent reversible anchoring points can strongly attach macromolecules, and homogenous polymer films can be obtained by a statistical distribution of the anchoring component situated along the backbone of the polymer. ${ }^{4,10,13-15}$ Moreover, the copolymer structure affects the surface properties of the coating, as well as the adhesion of the macroinitiator to the surface of interest and the homogeneity of the polymer brush layer grafted from the initiated surface. ${ }^{4,10}$

In particular, catechol chemistry to prepare adhesive groups has drawn much scientific attention as a universal, efficient, reversible, and stable anchoring functionality that can be exploited for the fabrication of polymer brush films from various substrates, as is shown in Figure 9.1. . $^{10,16-28}$

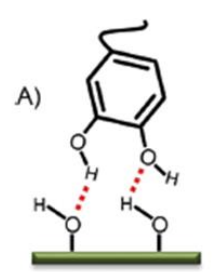

C)

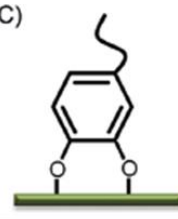

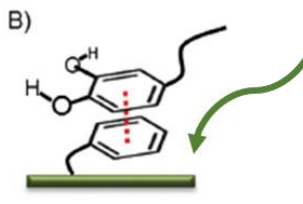

D)

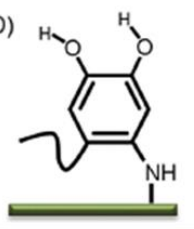

Material surfaces

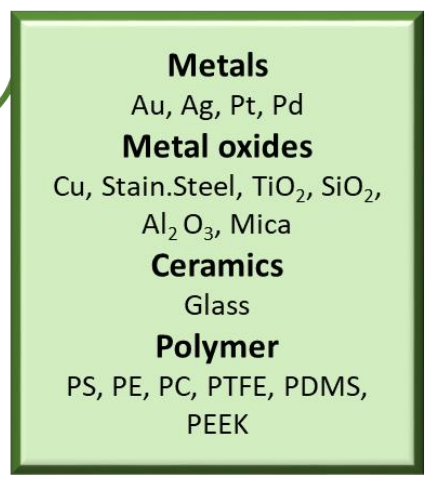


Fig. 9.1: Schematic representation of the main catechol-surface interactions and the possible material: (A) hydrogen bonds, (B) $\pi$ - $\pi$ stacking, (C) coordination, and (D) an example of covalent bonding via Michael-type addition with an amino-functionalized surface. Modified from ${ }^{29} 30$

Catechol-derivates can bind to versatile material surfaces, including hydrophobic, metal and metal oxide, and polymer materials. ${ }^{16}$ For example, Wang et al. ${ }^{24}$ reported on the SIP of $N$-isopropylacrylamide (NIPAM) from two components of ATRP-MIs anchored on various substrates (like the surface of titanium). ${ }^{24}$ Moreover, Wei et al. ${ }^{25}$ reported on the random copolymerization of two different anchoring moieties (i.e. dopamine and pyrene-containing monomers) and an ATRP initiating moiety that can be exploited for the fabrication of polymer brushes from various substrates. ${ }^{25}$

In this Chapter, we present the design and synthesis of a series of ATRP functional MIs that contain anchoring (i.e. catechol-based), solubilizing (i.e. methyl metacrylatebased), and ATRP initiating moieties (i.e. alkyl ternary bromide) (see Figure 9.2).

Because of their known adherence to a broad range of substrates, ${ }^{17,}{ }^{21}$ dopamine methacrylamide (DOMA) was selected as the anchoring group. ${ }^{20,25,28}$ Halogenated alkanes are widely used as ATRP initiators, ${ }^{3,}$ 6-9 and specifically, 2-(2bromoisobutyryloxy)ethyl methacrylate (BIEM) was considered a good vinyl-bearing ATRP functional monomer. As a third monomer, methyl methacrylate (MMA) was selected in order to tune the physical and chemical properties of the MI, i.e. solubility of the copolymers as well as to "dilute" the functional monomers within one chain to obtain the desired molar ratios. It is thought that the latter provides control over the final brush density as well as allows for a reduction in the overall consumption of more costly monomers, which are important from an economical point of view. In addition, selecting MMA as the third monomer ensured that all the copolymer building blocks had similar reactivities in free radical polymerization (FRP), which is desired for the synthesis of statistical copolymers. ${ }^{31-32}$

In the following sections, we present the successful synthesis of ATRP MIs with various molar ratios of initiator, solubilizing, and anchoring moieties. Subsequently, the catechol adhesive functionality in respect to silicon as a model substrate as well as the surface activity of the ATRP initiator moieties are presented and discussed.

\subsection{Results and discussion}

\section{Synthesis of the macro-initiators via free radical polymerization, FRP.}

Various copolymers with different molar ratios of BIEM, MMA, and DOMA were synthesized via free radical polymerization (FRP) in DMF with AIBN as initiator, as is shown in Figure 9.2. 


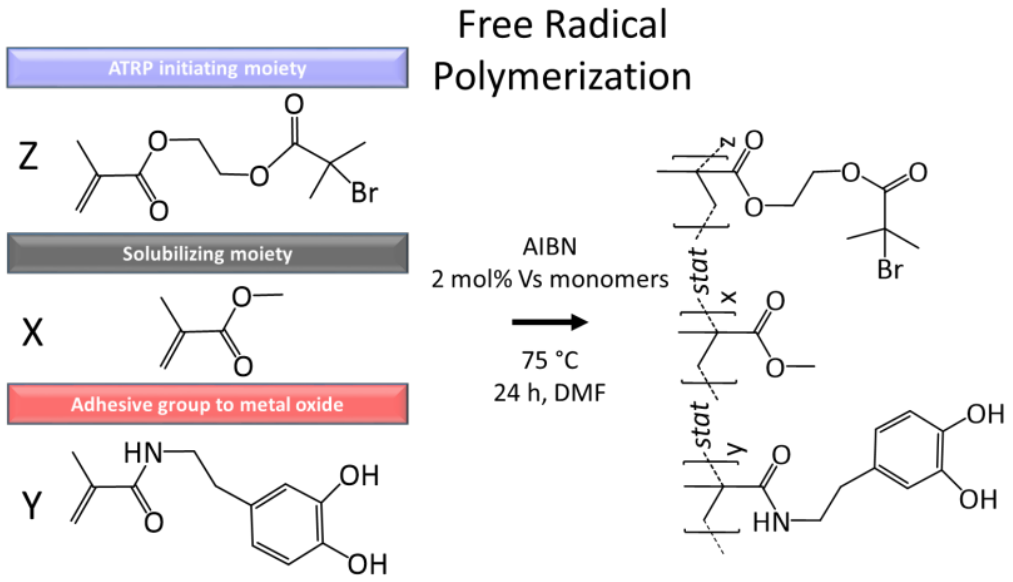

Fig. 9.2: Schematic representation of the free radical copolymerization of the three building blocks: DOMA as an anchoring group, MMA as solubilizing agent, and BIEM as an ATRP initiating moiety.

The chemical composition of the copolymers was confirmed by ${ }^{1} \mathrm{H}$ NMR and FTIR spectroscopy measurements, and the results are shown in Figure 9.3. The ${ }^{1} \mathrm{H}$ NMR spectra were used to calculate the molar ratio of the respective monomers in the copolymers. The name of each copolymer is denoted by $S_{x} A_{y} I_{z}$, while the MMA, DOMA, and BIEM groups are respectively denoted by $\mathrm{S}, \mathrm{A}$, and I. Here $\mathrm{x}, \mathrm{y}$, and $\mathrm{z}$ represent the respective molar ratios of the MMA, DOMA, and BIEM moieties.

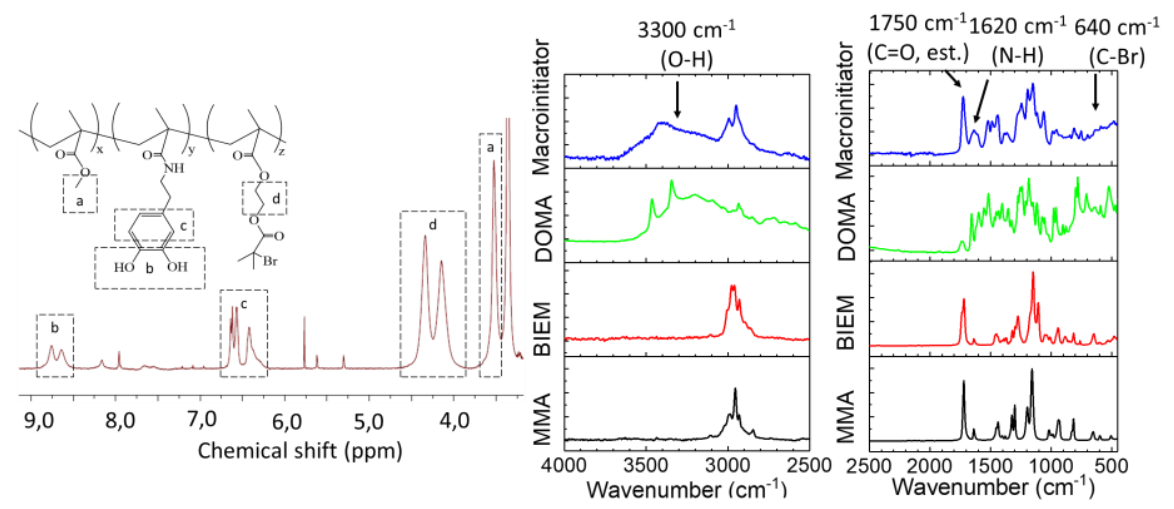

Fig. 9.3: ${ }^{1} \mathrm{H}$ NMR and FTIR spectra of a $\mathrm{S}_{2} \mathrm{~A}_{1} \mathrm{I}_{2}$ macro-initiator containing $40 \mathrm{~mol} \%$ of MMA, $20 \mathrm{~mol} \%$ of DOMA, and $40 \mathrm{~mol} \%$ of BIEM.

Figure 9.4 shows that there is a clear relationship between the molar composition of the ATRP MIs and the molar feed ratios of the reactants in the free radical copolymerization. In addition, the $\mathrm{M}_{\mathrm{w}}$ and the PDI values, as determined by GPC measurements relative to PMMA, are also shown. The $\mathrm{M}_{\mathrm{w}}$ values of the copolymers ranged between $11.7 \mathrm{kDa}$ and $136 \mathrm{kDa}$, and the PDI values were between 1.2 and 2.5 . It should be noted that the PDIs of the lower $\mathrm{M}_{\mathrm{w}}$ copolymers (i.e. $\mathrm{S}_{3} \mathrm{~A}_{1} \mathrm{I}_{6}, \mathrm{~S}_{59} \mathrm{~A}_{1} \mathrm{I}_{40}, \mathrm{~S}_{9} \mathrm{~A}_{1}$, 
$\mathrm{S}_{9} \mathrm{I}_{1}, \mathrm{~S}_{11} \mathrm{~A}_{11} \mathrm{I}_{8}$, and $\mathrm{S}_{3} \mathrm{~A}_{3} \mathrm{I}_{4}$ macromolecules with $\mathrm{M}_{\mathrm{w}}<30 \mathrm{kDa}$ ) were below 1.5, which is close to the minimum expected PDI for polymers obtained by FRP.

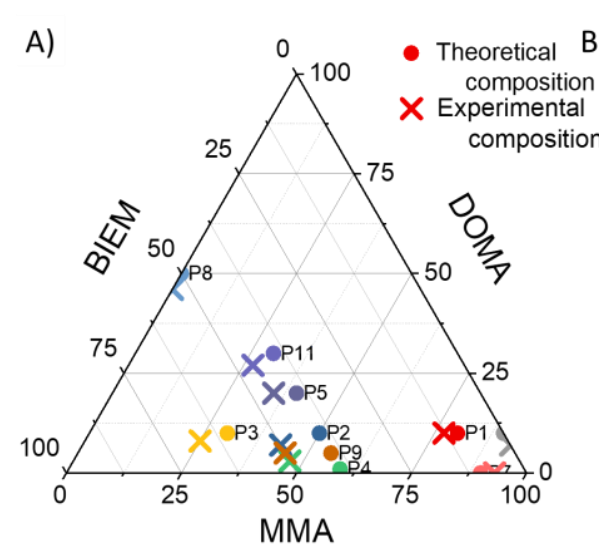

\begin{tabular}{|c|c|c|}
\hline & MW & \multirow{2}{*}{ PDI } \\
\cline { 2 - 2 } & $\mathrm{kDa}$ & \\
\hline $\mathrm{S}_{8} \mathrm{~A}_{1} \mathrm{I}_{1}$ & 75 & 2,0 \\
\hline $\mathrm{S}_{5} \mathrm{~A}_{1} \mathrm{I}_{4}$ & 45 & 1,5 \\
\hline $\mathrm{S}_{3} \mathrm{~A}_{1} \mathrm{I}_{6}$ & 23 & 1,4 \\
\hline $\mathrm{S}_{49} \mathrm{~A}_{1} \mathrm{I}_{40}$ & 12 & 1,2 \\
\hline $\mathrm{S}_{2} \mathrm{~A}_{1} \mathrm{I}_{2}$ & 91 & 2,4 \\
\hline $\mathrm{S}_{9} \mathrm{~A}_{1}$ & 26 & 1,3 \\
\hline $\mathrm{S}_{9} \mathrm{I}_{1}$ & 25 & 1,4 \\
\hline $\mathrm{A}_{1} \mathrm{I}_{1}$ & 136 & 2,5 \\
\hline $\mathrm{S}_{11} \mathrm{~A}_{1} \mathrm{I}_{8}$ & 26 & 1,4 \\
\hline $\mathrm{S}_{2} \mathrm{~A}_{1} \mathrm{I}_{2}$ & 55 & 1,6 \\
\hline $\mathrm{S}_{3} \mathrm{~A}_{3} \mathrm{I}_{4}$ & 34 & 1,5 \\
\hline
\end{tabular}

Fig. 9.4: Comparison between the feed (solid circles) and the macro-initiator molar composition (cross), as determined by ${ }^{1} \mathrm{H}$ NMR spectroscopy (A). In (B), the weight average molar mass (Mw) and polydispersity index values (PDI) of the macro-initiators as determined by GPC are shown.

The solubility of the ATRP MIs was strongly affected by their chemical composition. MMA-rich copolymers precipitated in aqueous solutions, whereas MMA-poor copolymers demanded more polar conditions. In addition, copolymers with DOMA ratios exceeding $40 \mathrm{~mol} \%$ may become completely insoluble due to self-crosslinking caused by electrostatic interactions and/or intra- and inter-molecular crosslinking between the catechol side chains. ${ }^{20}$

Although ${ }^{1} \mathrm{H}$ NMR confirmed the incorporation of the ATRP functional moieties in the ATRP MIs, it was of pivotal importance to prove the activity of the incorporated ATRP initiator units. This was confirmed by the successful synthesis of macromolecular bottlebrushes with polyacrylonitrile (PAN) side chains via solution ATRP of acrylonitrile (AN) initiated by the macroinitiator. ${ }^{33-34}$

Following the successful synthesis of the MIs, their surface anchoring capabilities were investigated, as presented and discussed in the next section.

\section{Modification of silicon surfaces by drop casting of ATRP-MIs.}

In order to evaluate the capability of the MIs to be used as a versatile platform for the fabrication of polymer brush films, two species with 1 mol\% and $30 \mathrm{~mol} \%$ DOMA content, $\mathrm{S}_{59} \mathrm{~A}_{1} \mathrm{I}_{40}$, and $\mathrm{S}_{3} \mathrm{~A}_{3} \mathrm{I}_{4}$, respectively, were deposited on silicon surfaces as model substrates, as is shown in Figure 9.5. 


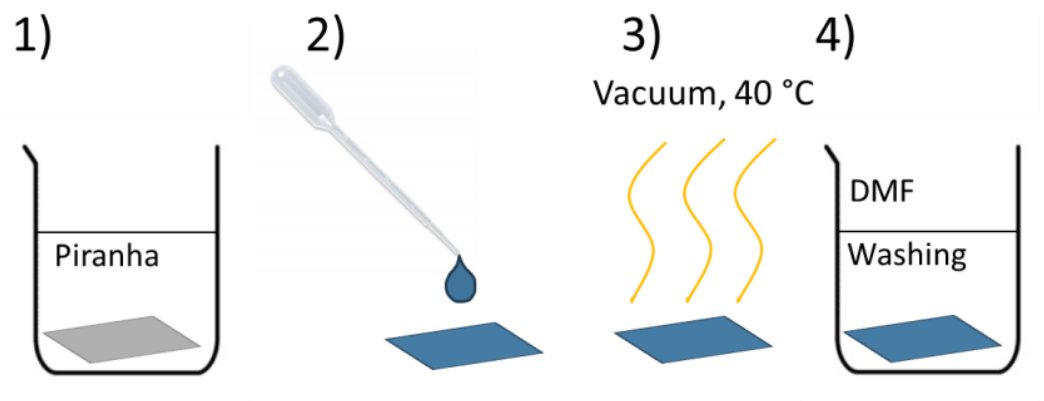

Fig. 9.5: Scheme of the drop casting of macro-initiators on silicon surfaces as model substrates.

DMF was a common solvent for all ATRP MIs we used. However, its high boiling point made it necessary to vacuum dry the drop casted films at $40{ }^{\circ} \mathrm{C}$ for 1.5 hours. Figure 9.6 shows typical optical microscopy images of the casted and extensively washed films of $\mathrm{S}_{59} \mathrm{~A}_{1} \mathrm{I}_{40}$, and $\mathrm{S}_{3} \mathrm{~A}_{3} \mathrm{I}_{4}$ on silicon substrates. The thickness of the as-casted films was approximately $1 \mu \mathrm{m}$, indicating the deposition of a significant amount of nonspecificly bound material. The static water contact angle for the drop casted $\mathrm{S}_{59} \mathrm{~A}_{1} \mathrm{I}_{40}$, and $\mathrm{S}_{3} \mathrm{~A}_{3} \mathrm{I}_{4}$ films was approximately $70^{\circ}$, which is consistent with values reported by Wei et al. ${ }^{25}$ and provided a reference value for the water contact angle of immobilized DOMA- and BIEM-containing copolymers on silicon surfaces.

The clearly visible black spots in the as-casted films (see Figure 9.6 A and B) can be identified as defects ascribed to i) possible self-agglomeration via self-crosslinking between catechol moieties of the the MIs or ii) nonoptimal deposition and drying conditions used during drop casting.

Following the extensive washing of the MI modified surfaces with DMF, the nonbound MI molecules were removed. From Figure 9.6C, it is clear from the visibility of the scratch that for the MI containing 1 mol\% DOMA, most of the material was removed. This was consistent with the absence of color on the surface as well as with the decrease in the static water contact angle to $54^{\circ}$. From Figure 9.6D, it is evident that a relatively homogenous macroinitiator film was bonded to the silicon substrate. From the color of the residual film (i.e. grey-blueish color), a thickness of 60 to $90 \mathrm{~nm}$ was estimated. This qualitative observation was consistent with a static water contact angle of approximately $70^{\circ}$, which was similar to the static contact angle of the as-deposited film.

Figure 9.7 shows the FTIR spectra of the $\mathrm{S}_{59} \mathrm{~A}_{1} \mathrm{I}_{40}$, and $\mathrm{S}_{3} \mathrm{~A}_{3} \mathrm{I}_{4}$ MI layers on the silicon substrates after washing. From Figure 9.7A, the absence of clear absorption bands is consistent with the previous visual observations and confirms that most of the $\mathrm{S}_{59} \mathrm{~A}_{1} \mathrm{I}_{40}$ was removed from the surface by washing. Due to the presence of a thin and non-uniform film as well as an insufficient number of acquired spectra, the intensity of the FTIR spectra is relatively low, the signal-to-noise ratio is relatively high, and the presence of an intense absorbance peak of $\mathrm{CO}_{2}$ is well visible. In Figure 9.7B, the clearly visible 
absorbance band around $1730 \mathrm{~cm}^{-1}$ is ascribed to the carbonyl groups of the ester moieties in the ATRP MI. Furthermore, the absorbance band around $1625 \mathrm{~cm}^{-1}$ can be attributed to the amide bonds present in DOMA moieties.

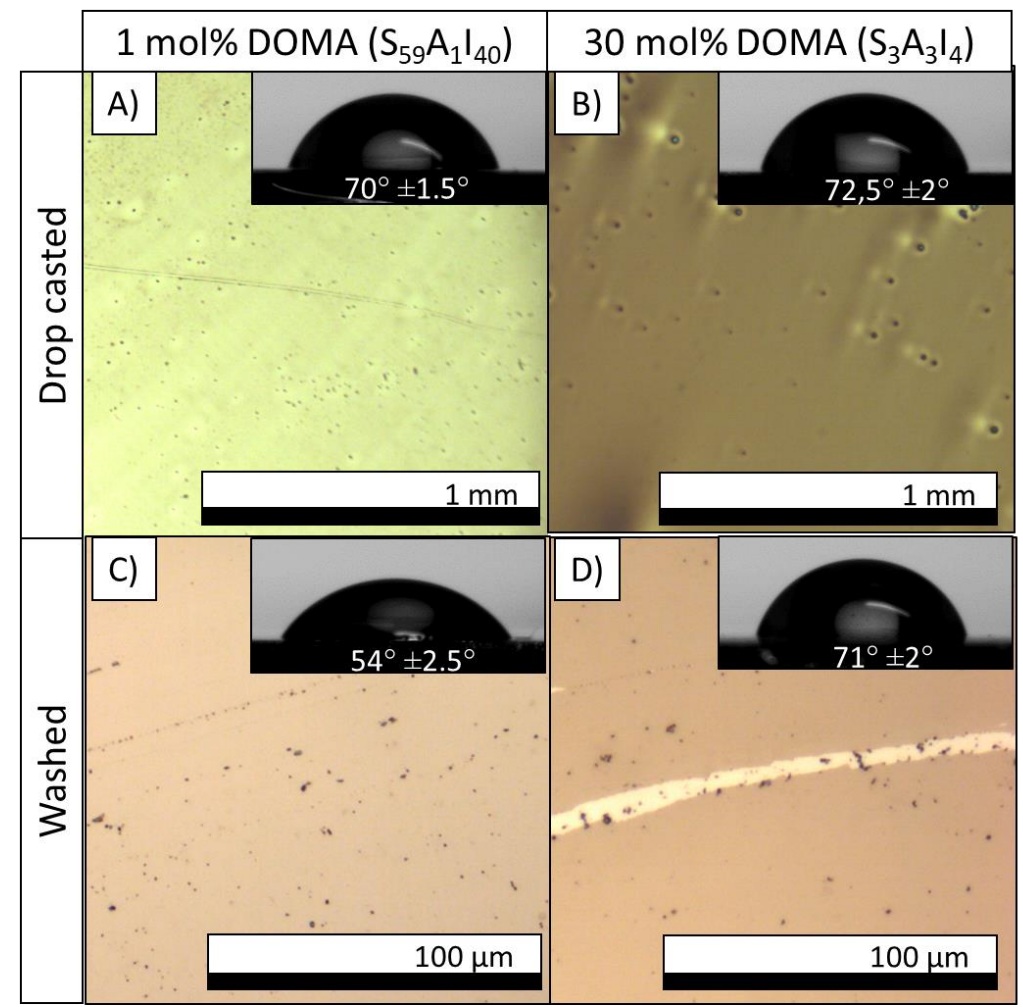

Fig. 9.6: A) and B) are optical images of the as-casted $S_{59} A_{1} I_{40}$, and $S_{3} A_{3} I_{4}$ macro-initiator films onto the silicon substrates, respectively. C) and D) are optical microscopy images of the extensively washed $\mathrm{S}_{59} \mathrm{~A}_{1} \mathrm{I}_{40}$, and $\mathrm{S}_{3} \mathrm{~A}_{3} \mathrm{I}_{4}$ macro-initiators films, respectively. The insets are images of representative static water contact angle measurements.
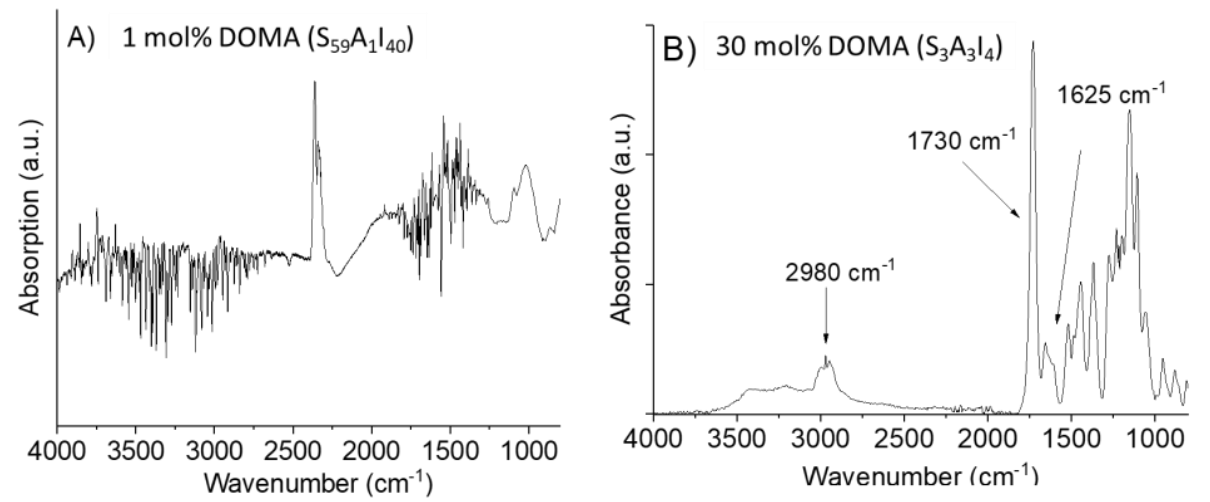

Fig. 9.7: FTIR spectra of the casted and extensively washed $S_{59} A_{1} I_{40}$, (A) and $S_{3} A_{3} I_{4}$ (B) macro-initiator layers on the silicon substrates. 


\section{SMI-ATRP of AN brushes from MIs deposited on silicon substrates.}

Following the successful immobilization of the $\mathrm{S}_{3} \mathrm{~A}_{3} \mathrm{I}_{4}$ ATRP MI layer, PAN brushes were grown via surface-initiated ATRP, as is schematically shown in Figure 9.8.

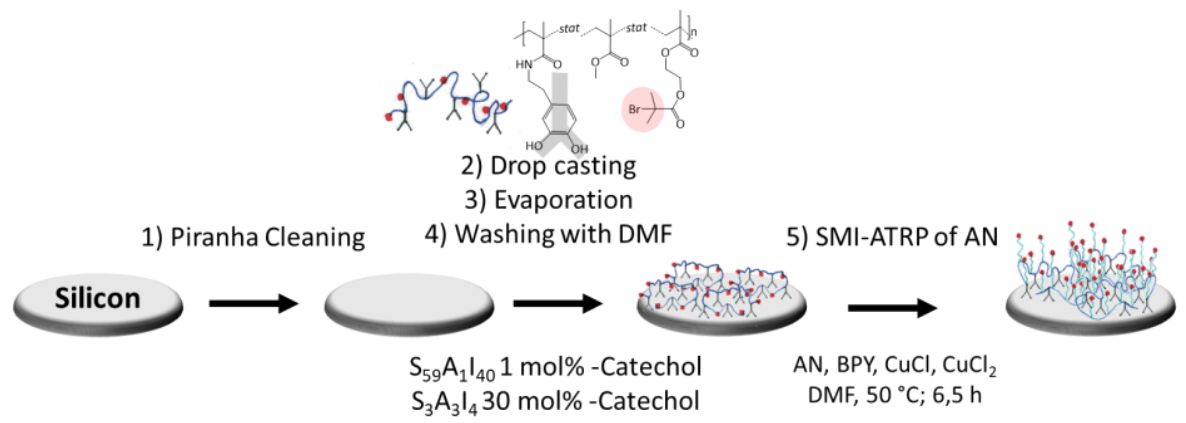

Fig. 9.8: Scheme of the surface-initiated ATRP of PAN brushes grown from macro-initiator layers deposited by drop casting on silicon surfaces.

Figure 9.9 shows the FTIR spectra of the PAN brush layers grown from the $\mathrm{S}_{59} \mathrm{~A}_{1} \mathrm{I}_{40}$, and $\mathrm{S}_{3} \mathrm{~A}_{3} \mathrm{I}_{4}$ ATRP MI layers. The FTIR spectrum of the PAN film grafted from $\mathrm{S}_{3} \mathrm{~A}_{3} \mathrm{I}_{4}$ immobilized onto silicon substrates (Figure 9B) shows the characteristic nitrile absorption band at $2244 \mathrm{~cm}^{-1}$, which confirms successful PAN brush synthesis. On the other hand, Figure 9A shows a small absorption band at $2244 \mathrm{~cm}^{-1}$ close to the noise level of the FTIR spectrum, which, as expected, confirmed the presence of minor amounts of PAN on the $\mathrm{S}_{59} \mathrm{~A}_{1} \mathrm{I}_{40}$-modified surface. In addition, the values of the static water contact angles for PAN grown from $\mathrm{S}_{59} \mathrm{~A}_{1} \mathrm{I}_{40}$, and $\mathrm{S}_{3} \mathrm{~A}_{3} \mathrm{I}_{4}$ layers were $\sim 49^{\circ}$ and $\sim 59^{\circ}$, respectively. The latter value is consistent with literature for PAN films values reported by Lin et al. ${ }^{35}$
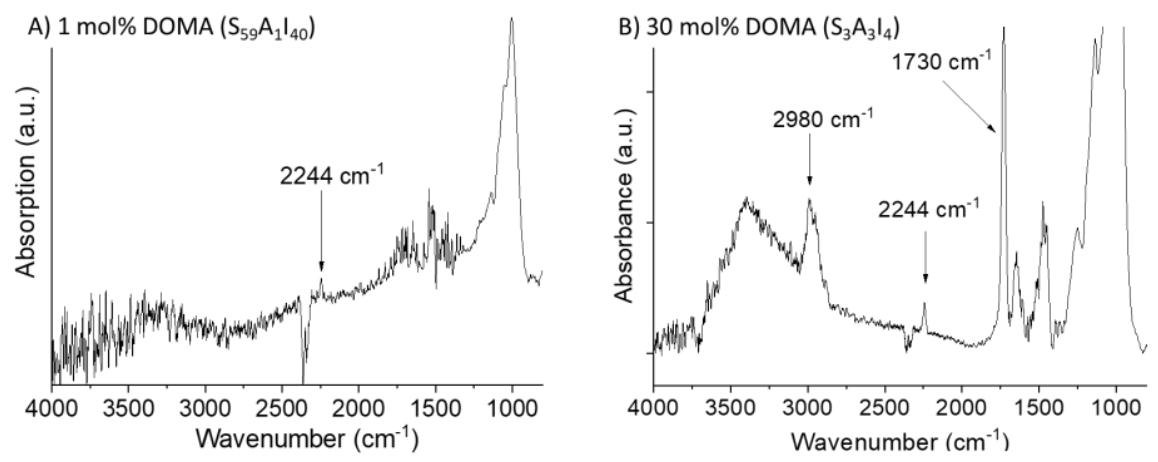

Fig. 9.9: FTIR transmission spectra of the single end-tethered $P A N$ chains grown from the extensively washed, drop casted $\mathrm{S}_{59} \mathrm{~A}_{1} \mathrm{I}_{40}$, (A) and $\mathrm{S}_{3} \mathrm{~A}_{3} \mathrm{I}_{4}($ B) ATRP macro-initiators on silicon oxide surfaces.

Based on these qualitative observations, it is clear that $\mathrm{S}_{3} \mathrm{~A}_{3} \mathrm{I}_{4}$ resulted in a suitable ATRP MI that was easily immobilized on silicon oxide surfaces and used to fabricate 
functional brush-based coatings. Future steps should be taken to ensure a more detailed characterization of the deposited layers with surface-sensitive techniques (e.g., AFM, XPS, or SEM-EDX) that have proper lateral and in-depth resolution in order to more thoroughly identify the MI bonding mechanism and final brush layer compositions.

Furthermore, another specific aim is to optimize the MI composition so that the stable anchoring layer can be readily obtained at a lower DOMA concentration for a broader range of substrates. In addition, future efforts should be directed toward understanding the relationship between initiator concentration in the MI and eventual brush density.

\subsection{Outlook}

The results presented in this Chapter have demonstrated the feasibility of using a tailor-made three component macro-initiators as versatile and readily applicable coupling layers between substrates and brushes of interest. Future work should be directed towards the detailed characterization of the deposited MI layers on various substrates in order to test the grafting density and anchoring stability. Macroinitiators can be used to modify most material surfaces. However, future research should consider studies of the layer stability for the different substrates with subsequent optimization of the composition of the adhesive moieties in the ATRP MIs. The nanomechanical properties of different catechol containing copolymers, with various amount of catechol moieties, can be investigated using catechol-modified AFM tips in AFM force-distance experiments on different material surfaces.

Moreover, depending on the application, various coatings can be grafted enhancing the surface properties of the material or fabrication of smart surfaces. For this purpose GF and GT approaches may be employed. The deposition of the MI layers is not only limited to drop-casting, and it is predicted that other application techniques could possibly be employed (e.g., inkjet printing). Thus, an exchange of the solubilizing monomer for a more hydrophilic one could play a key role in the processability of the macroinitiator polymer as well as of the molecular bottlebrush coatings.

\subsection{Conclusions}

Firstly, a DOMA monomer was synthesized from methacrylic anhydride and dopamine with good yield and good purity as confirmed by FTIR and ${ }^{1} \mathrm{H}$ NMR spectroscopy.

Subsequently, various ATRP-MI containing different ratios of DOMA, BIEM, and MMA groups were prepared by free radical polymerization as demonstrated by FTIR and ${ }^{1} \mathrm{H}$ NMR spectroscopy. The results confirmed that within the molar ratios selected, a good control over copolymer composition can be achieved with respect to the feed composition. The $\mathrm{M}_{\mathrm{w}}$ of the copolymers was in the range between $12 \mathrm{kDa}$ and $140 \mathrm{kDa}$, and the PDI 
was the range between 1.2 and 2.5, as determined by GPC. Interestingly, the DOMA concentration had a severe effect on the copolymer solubility because the ATRP MIs were poorly soluble in DMF or DMSO for DOMA concentrations exceeding $20 \mathrm{~mol} \%$.

The activity of the ATRP initiating sites of the macromolecules was probed by synthesizing molecular bottlebrushes with side chains of polyacrylonitrile (PAN). The bottlebrush macromolecules were characterized by GPC, FTIR and ${ }^{1} \mathrm{H}$ NMR.

The adhesive moieties were also tested by grafting the ATRP-MIs on a model surface (silicon) followed by SI-ATRP of AN. From this experiment, we have concluded that the ATRP-macroinitiators we have obtained can be used as SI-ATRP initiating sites for e.g. AN monomer. However, the chemical stability of the MI layer is strongly affected by the DOMA amount of the macroinitiators, as is shown from the water static contact angle and FTIR measurements. Additionally, the third component had a key role to avoid the crosslinking reactions between the DOMA moieties assuring the stability of the free polymer chains in solution. Thus, a structure optimization for best performance must still be tackled.

\subsection{Experimental section}

\section{Materials}

Methyl methacrylate (MMA, Sigma-Aldrich 99\%), acrylonitrile (AN, SigmaAldrich, $>99 \%$ ) were purified by passing over a column of aluminum oxide to remove the inhibitors. 2-(2-Bromoisobutyryloxy)ethyl methacrylate (BIEM, Sigma-Aldrich, 95\%), dopamine hydrochloride (Sigma-Aldrich), methacrylic anhydride (Sigma-Aldrich, $94 \%$ ), sodium tetraborate decahydrate $\left(\mathrm{Na}_{2} \mathrm{~B}_{4} \mathrm{O}_{7} \cdot 10 \mathrm{H}_{2} \mathrm{O}\right.$, Sigma-Aldrich, $\left.\geqslant 99,5\right)$, sodium bicarbonate $\left(\mathrm{NaHCO}_{3}\right.$, J.T. Baker, $\left.\geqslant 99 \%\right)$, magnesium sulfate $\left(\mathrm{MgSO}_{4}\right.$, SigmaAldrich, $\geqslant 99.5 \%)$, sulfuric acid $\left(\mathrm{H}_{2} \mathrm{SO}_{4}\right.$, Biosolve, 95-97\%), hydrogen peroxide $\left(\mathrm{H}_{2} \mathrm{O}_{2}\right.$, Merck, 30\%), hydrochloric acid fuming ( $\mathrm{HCl}$, Sigma-Aldrich, 37\%), sodium hydroxide $(\mathrm{NaOH}$, Merck, $\geqslant 98 \%)$ copper(I)chloride (CuCl, Sigma-Aldrich, $\geqslant 99 \%)$, copper(II)chloride $\left(\mathrm{CuCl}_{2}\right.$, Sigma-Aldrich, 99\%), 2,2'-bipyridyne (bpy, Sigma-Aldrich, 97\%), 2,2'-azobis(2-methylpropionitrile) (AIBN, Sigma-Aldrich, 98\%) was recrystallized before use. $\mathrm{CuCl}$ was purified by dispersion within glacial acetic acid three times for 1.5 hours. Subsequent washing ethanol and ethyl acetate (two times each) resulted in a greyish slurry. The product was allowed to dry in vacuum at room temperature overnight and stored under nitrogen conditions. Dimethyl sulfoxide- $\mathrm{d}_{6}$ (DMSO-d ${ }_{6}, \quad$ Sigma-Aldrich, $\left.99.9 \%\right)$, chloroform-d $\left(\mathrm{CDCl}_{3}\right.$, Cambridge Isotope Laboratories). Tetrahydrofuran (THF, Biosolve, $\geqslant 99.8 \%$ ), ethyl acetate (EtOAc, SigmaAldrich, 99.8\%), $n$-hexane (Biosolve, $\geqslant 95 \%$ ), $N, N$-Dimethylformamide (DMF, Merck, Emsure) were used as received and MilliQ water was obtained from a MilliQ Advantage A 10 purification system (Millipore, Billerica, Ma, USA). 
Dopamine methacrylate (DOMA): The catechol functionalized monomer was synthesized according to Messersmith's method with some modifications. ${ }^{28}$ Buffer salts sodium borate $(5 \mathrm{~g})$ and sodium bicarbonate $(2 \mathrm{~g})$ were dissolved in $50 \mathrm{~mL}$ milli-Q water and purged with dry nitrogen for 20 minutes. $2 \mathrm{~g}$ dopamine- $\mathrm{HCl}(10.56 \mathrm{mmol})$ was added to the oxygen free solution and the $\mathrm{pH}$ was adjusted to above 8 with addition of $1.0 \mathrm{M}$ $\mathrm{NaOH}$. A total of $2.0 \mathrm{~mL}$ methacrylate anhydride $(2.09 \mathrm{mmol})$ was mixed with $20 \mathrm{ml}$ THF and added drop-wise into the above solution. The reaction was allowed to proceed for $6 \mathrm{~h}$ under gentle stirring at room temperature under continuous purging with nitrogen gas. After completing the reaction, the solution was acidified with $1.0 \mathrm{M} \mathrm{HCl}$ to around $\mathrm{pH}$ 2. The solution turned opaque as a consequence of the presence of the insoluble DOMA monomer. The organic phase was extracted three times with $50 \mathrm{~mL}$ ethyl acetate from the aqueous phase and dried over anhydrous $\mathrm{MgSO}_{4}$. The extract was precipitated in $200 \mathrm{~mL} n$-hexane to obtain a gray slurry. The DOMA containing slurry was washed for the second time with ethyl acetate and precipitated in hexane to remove excess reactants. The final product was allowed to dry in vacuum oven overnight at room temperature, and stored under nitrogen conditions.

Copolymerization via FRP: Copolymerization of methyl methacrylate (MMA), dopamine methacrylamide (DOMA) and 2-(2-bromoisobutyryloxy)ethyl methacrylate (BIEM) was conducted by a free radical process following a modified procedure of Yang et al. ${ }^{36}$ The polymerization was carried out at $75{ }^{\circ} \mathrm{C}$ for 24 hours. Different ratios of monomers with a total of $2.0 \mathrm{mmol}, 2 \mathrm{~mol} \%$ AIBN were dissolved in $2 \mathrm{ml} \mathrm{DMF}$ and added to a $20 \mathrm{ml}$ glass vial. After purging with argon for 1 hour, the reaction was started by heating the mixture in an oil bath at $75{ }^{\circ} \mathrm{C}$. The purging outlet was removed while maintaining an argon overpressure as evaporation of the MMA monomer was noted during the bubbling of argon in the solution and the FRP (likely due to its boiling point of $101{ }^{\circ} \mathrm{C}$ being too close to the polymerization temperature of $75^{\circ} \mathrm{C}$ ). The reaction mixture was allowed to cool down and the reaction was stopped by exposing the mixture to oxygen. Three precipitation methods were used to separate the polymer from reactants and solvent. $\mathrm{S}_{8} \mathrm{~A}_{1} \mathrm{I}_{1}, \mathrm{~A}_{1} \mathrm{I}_{1}, \mathrm{~S}_{2} \mathrm{~A}_{1} \mathrm{I}_{2}$, and $\mathrm{S}_{3} \mathrm{~A}_{3} \mathrm{I}_{4}$ were precipitated by adding the mixture to $\mathrm{n}$ hexane resulting in two phases. By slowly adding dichloromethane, the two mixtures became miscible and the polymer precipitated. $\mathrm{S}_{5} \mathrm{~A}_{1} \mathrm{I}_{4}, \mathrm{~S}_{3} \mathrm{~A}_{1} \mathrm{I}_{6}, \mathrm{~S}_{59} \mathrm{~A}_{1} \mathrm{I}_{40} \mathrm{~S}_{2} \mathrm{~A}_{1} \mathrm{I}_{2}, \mathrm{~S}_{9} \mathrm{~A}_{1}, \mathrm{~S}_{9} \mathrm{I}_{1}$, and $\mathrm{S}_{11} \mathrm{~A}_{1} \mathrm{I}_{8}$, were precipitated in excess water. After filtering using Whatman nr. 4 paper filter, the samples were placed in a vacuum oven at room temperature overnight in order to evaporate remaining solvents.

Synthesis of PAN-based molecular bottlebrushes: A round bottom flask containing the monomer acrylonitrile (AN, $7.2 \mathrm{ml}, 88 \mathrm{mmol}$ ), the ligand 2,2'-bipyridyne (bpy, $0.0336 \mathrm{gr}, 0.217 \mathrm{mmol}$ ), an internal standard dioxane (200 $\mu \mathrm{l})$ and solvent DMF (14 $\mathrm{ml}$ ) was used in the reaction. The reaction mixture was stirred and deoxygenated by 
flushing with argon for at least 30 minutes. The second flask, containing $\mathrm{CuCl}(9.8 \mathrm{mg}$, $0.098 \mathrm{mmol})$ and $\mathrm{CuCl}_{2}(1.3 \mathrm{mg}, 0.0094 \mathrm{mmol})$ was purged with argon for at least 30 minutes. Next, the monomer/ligand solution was transferred to the second flask, containing $\mathrm{CuCl}$ and $\mathrm{CuCl}_{2}$ (under argon atmosphere) using a syringe. The syringe was purged with argon three times before material transfer. The mixture was stirred for 30 min in order to dissolve the $\mathrm{CuCl}$ and $\mathrm{CuCl}_{2}$ which obtained a deep red-brown color. The ATRP MI (14 mg) was dissolved in $1.0 \mathrm{ml}$ DMF and transferred to a round-bottom flask. After deoxygenating for 30 minutes, the reaction mixture was added to the MI solution and placed in an oil bath at $50{ }^{\circ} \mathrm{C}$ to start the polymerization. The polymerization was allowed to proceed for 20 hours and was stopped by exposing the solution to oxygen. Samples before and after polymerization were taken for monomer conversion measurements by ${ }^{1} \mathrm{H}-\mathrm{NMR}$. The so obtained molecular bottlebrush was precipitated in an excess amount of water and filtered using Whatman filter papers nr. 4 with subsequent washing for 3 times. The solvents residues were evaporated in vacuum overnight.

Anchoring of the ATRP macroinitiators to the silicon surface: Silicon substrates were cut using a diamond tipped cutter in samples of approximately $1 \times 1 \mathrm{~cm}^{2}$. Subsequent cleaning and activation was done by piranha solution $\left(\mathrm{H}_{2} \mathrm{SO}_{4}: \mathrm{H}_{2} \mathrm{O}_{2}=3: 1\right.$, v/v) for 20 minutes and extensively rinsed with water, ethanol and dried under a stream of nitrogen. DMF solutions containing 2 w.\% ATRP MI were produced. Once the polymer was dissolved, the solutions were drop casted on clean and activated substrates. A loading of $40 \mathrm{mg}$ per sample was the target amount (i.e. corresponding to 3 droplets of solution). In order to evaporate the DMF at a higher rate, the samples were dried in vacuum at $40{ }^{\circ} \mathrm{C}$. The samples showed a nicely covered layer with some color variation. The physisorbed polymer was washed of by resolving the polymer in a DMF solution. The solution was kept in motion by a shaking table which speeded up the resolving process. The resolving process differed from a period of one day until a few weeks.

\section{Devices and methods}

Contact angle: Static water contact angle measurements were carried out on an optical contact angle device equipped with an electronic syringe unit (OCA15, Dataphysics, Germany). Contact angle values of the modified substrates at each reaction step were measured, and at least three samples were assessed for each step.

FT-IR: FT-IR were performed using an ALPHA FTIR spectrometer from Bruker with a spectral resolution of $4 \mathrm{~cm}^{-1}$ and averaged over 64 scans. The FTIR was used with an ATR module for all products that were not immobilized on silicon oxide surfaces, for immobilized samples an universal transmission module was used. Bare silicon oxide was used for background measurements for the immobilized samples on silicon oxide. The data was processed by means of OPUS software. 
GPC: GPC measurements were performed using ultrastyragel columns with pore sizes of $10^{5}, 10^{4}, 10^{3}$ and $500 \AA$ (Waters), equipped with a guard column of $500 \AA$, a 515 pump (Waters), a 1050 injector (Agilent) and a 2414 differential refractometer (Waters). All polymers were dissolved in a $0.1 \mathrm{M} \mathrm{LiCl}$ DMF solution with a concentration of 5 $\mathrm{mg} / \mathrm{ml}$. The solutions were filtered before use in order to remove dust or any crosslinked polymer.

${ }^{1}$ H NMR: ${ }^{1} \mathrm{H}$ NMR spectra were obtained on a Bruker Avance III $400 \mathrm{MHz}$ instrument at 400.1 MHz. Poorly dissolvable polymers were left in the deuterated solvents on a shaker table prior to the measurements. 1,4-Dioxane was added to the solutions as an internal standard, approximately $10 \mathrm{~mol} \%$ of the reactant. The data was processed using MestReNova (v10). ${ }^{1} \mathrm{H}$ chemical shifts were based on the solvent residual signals.

\subsection{References}

1. Braunecker, W. A. W. W. A., Controlled/living radical polymerization: Features, developments, and perspectives. Progress in Polymer Science 2007, 32 (1), 93-146.

2. Shipp, D. A., Reversible-deactivation radical polymerizations. Polymer Reviews 2011, 51 (2), 99-103.

3. Matyjaszewski, K., Atom Transfer Radical Polymerization (ATRP): Current Status and Future Perspectives. Macromolecules 2012, 45 (10), 4015-4039.

4. Zoppe, J. O.; Ataman, N. C.; Mocny, P.; Wang, J.; Moraes, J.; Klok, H.-A., Surface-initiated controlled radical polymerization: state-of-the-art, opportunities, and challenges in surface and interface engineering with polymer brushes. Chemical Reviews 2017, 117 (3), 1105-1318.

5. Barbey, R. R. R., Polymer brushes via surface-initiated controlled radical polymerization: synthesis, characterization, properties, and applications. Chemical Reviews 2009, 109 (11), 5437-5527.

6. Matyjaszewski, K., Advanced materials by atom transfer radical polymerization. Advanced Materials 2018, 30 (23), 1706441.

7. Matyjaszewski, K.; Xia, J., Atom transfer radical polymerization. Chemical Reviews 2001, 101 (9), 2921 2990 .

8. Ribelli, T. G.; Lorandi, F.; Fantin, M.; Matyjaszewski, K., Atom transfer radical polymerization: billion times more active catalysts and new initiation systems. Macromolecular Rapid Communications 2019, $40(1), 1800616$.

9. Edmondson, S.; Osborne, V. L.; Huck, W. T., Polymer brushes via surface-initiated polymerizations. Chemical Society Reviews 2004, 33 (1), 14-22.

10. Minko, S., Grafting on solid surfaces:"Grafting to" and "grafting from" methods. In Polymer Surfaces and Interfaces, Springer: 2008; pp 215-234.

11. Husseman, M.; Malmström, E. E.; McNamara, M.; Mate, M.; Mecerreyes, D.; Benoit, D. G.; Hedrick, J. L.; Mansky, P.; Huang, E.; Russell, T. P., Controlled synthesis of polymer brushes by "living” free radical polymerization techniques. Macromolecules 1999, 32 (5), 1424-1431.

12. Vericat, C.; Vela, M.; Benitez, G.; Carro, P.; Salvarezza, R., Self-assembled monolayers of thiols and dithiols on gold: new challenges for a well-known system. Chemical Society Reviews 2010, 39 (5), 1805 1834

13. Liu, Y.; Klep, V.; Zdyrko, B.; Luzinov, I., Polymer grafting via ATRP initiated from macroinitiator synthesized on surface. Langmuir 2004, 20 (16), 6710-6718.

14. Li, X.; Prukop, S. L.; Biswal, S. L.; Verduzco, R., Surface Properties of Bottlebrush Polymer Thin Films. Macromolecules 2012, 45 (17), 7118-7127.

15. Heinzmann, C.; Weder, C.; de Espinosa, L. M., Supramolecular polymer adhesives: advanced materials inspired by nature. Chemical Society Reviews 2016, 45 (2), 342-358. 
16. Lee, H.; Dellatore, S. M.; Miller, W. M.; Messersmith, P. B., Mussel-inspired surface chemistry for multifunctional coatings. Science 2007, 318 (5849), 426-430.

17. Faure, E.; Falentin-Daudré, C.; Jérôme, C.; Lyskawa, J.; Fournier, D.; Woisel, P.; Detrembleur, C., Catechols as versatile platforms in polymer chemistry. Progress in Polymer Science 2013, 38 (1), 236270.

18. Moulay, S., Dopa/catechol-tethered polymers: Bioadhesives and biomimetic adhesive materials. Polymer Reviews 2014, 54 (3), 436-513.

19. Olofsson, K.; Granskog, V.; Cai, Y.; Hult, A.; Malkoch, M., Activated dopamine derivatives as primers for adhesive-patch fixation of bone fractures. RSC Advances 2016, 6 (31), 26398-26405.

20. Patil, N.; Jerome, C.; Detrembleur, C., Recent advances in the synthesis of catechol-derived (bio) polymers for applications in energy storage and environment. Progress in Polymer Science 2018, 82, 3491.

21. Sedó, J.; Saiz - Poseu, J.; Busqué, F.; Ruiz - Molina, D., Catechol - based biomimetic functional materials. Advanced Materials 2013, 25 (5), 653-701.

22. Kord Forooshani, P.; Lee, B. P., Recent approaches in designing bioadhesive materials inspired by mussel adhesive protein. Journal of Polymer Science Part A: Polymer Chemistry 2017, 55 (1), 9-33.

23. Hu, H.; Yu, B.; Ye, Q.; Gu, Y.; Zhou, F., Modification of carbon nanotubes with a nanothin polydopamine layer and polydimethylamino-ethyl methacrylate brushes. Carbon 2010, 48 (8), 2347 2353.

24. Wang, X.; Ye, Q.; Gao, T.; Liu, J.; Zhou, F., Self-assembly of catecholic macroinitiator on various substrates and surface-initiated polymerization. Langmuir 2012, 28 (5), 2574-2581.

25. Wei, Q.; Wang, X.; Zhou, F., A versatile macro-initiator with dual functional anchoring groups for surface-initiated atom transfer radical polymerization on various substrates. Polymer Chemistry 2012, 3 (8), 2129-2137.

26. Zhao, Y.; Wu, Y.; Wang, L.; Zhang, M.; Chen, X.; Liu, M.; Fan, J.; Liu, J.; Zhou, F.; Wang, Z., Bioinspired reversible underwater adhesive. Nature Communications 2017, 8 (1), 2218.

27. Fan, X.; Lin, L.; Dalsin, J. L.; Messersmith, P. B., Biomimetic anchor for surface-initiated polymerization from metal substrates. Journal of the American Chemical Society 2005, 127 (45), 15843-15847.

28. Lee, H.; Lee, B. P.; Messersmith, P. B., A reversible wet/dry adhesive inspired by mussels and geckos. Nature 2007, 448 (7151), 338.

29. Saiz - Poseu, J.; Mancebo - Aracil, J.; Nador, F.; Busqué, F.; Ruiz - Molina, D., The Chemistry behind Catechol - Based Adhesion. Angewandte Chemie International Edition 2019, 58 (3), 696-714.

30. Krogsgaard, M.; Nue, V.; Birkedal, H., Mussel - Inspired Materials: Self - Healing through Coordination Chemistry. Chemistry - A European Journal 2016, 22 (3), 844-857.

31. Rusu, M.; Rusu, M.; Popa, M.; Delaite, C.; Riess, G., New radio-opaque acrylic bone cements. I. The synthesis of bromine containing methacrylates. Polymer Bulletin 2007, 59 (1), 25-30.

32. Stepuk, A.; Halter, J. G.; Schaetz, A.; Grass, R. N.; Stark, W. J., Mussel-inspired load bearing metalpolymer glues. Chemical Communications 2012, 48 (50), 6238-6240.

33. Willems, M. H. M. Design and synthesis of catechol-containing ATRP macro-initiator polymers as a platform for metal oxide surface functionalization. University of Twente, 2019.

34. Xie, G.; Martinez, M. R.; Olszewski, M.; Sheiko, S. S.; Matyjaszewski, K., Molecular Bottlebrushes as Novel Materials. Biomacromolecules 2019, 20 (1), 27-54.

35. Lin, W.-C.; Liu, T.-Y.; Yang, M.-C., Hemocompatibility of polyacrylonitrile dialysis membrane immobilized with chitosan and heparin conjugate. Biomaterials 2004, 25 (10), 1947-1957.

36. Yang, J.; Bos, I.; Pranger, W.; Stuiver, A.; Velders, A. H.; Stuart, M. A. C.; Kamperman, M., A clear coat from a water soluble precursor: a bioinspired paint concept. Journal of Materials Chemistry A 2016, 4 (18), 6868-6877. 


\section{Summary}

This Thesis describes the preparation and characterization of addressable functional and stimuli-responsive polymer architectures and their versatile application as anticorrosion, adhesive, tunable friction, antifouling, drug delivery, and chemical sensing coatings fo advanced surface engineering applications. Moreover, we have combined inkjet printing and various chemical immobilization approaches (e.g., grafting to and grafting from) to fabricate functional and smart patterns on several materials.

In Chapter 1, a short introduction to the topics related to this Thesis and the motivation of the research are presented.

Subsequently, a general introduction to the main topic of this work is presented in Chapter 2. Firstly, an overview of the reversible deactivation-activation radical polymerization (RDRP) techniques used to synthesize new macromolecules is presented. Among the RDRP techniques, the atom transfer radical polymerization (ATRP) process and its evolutions are highlighted. Then, the most relevant strategies to chemically graft polymer chains to a surface are discussed by introducing the most important design factors. Therefore, we present inkjet printing as a patterning technique. We described the fundamentals of the process and the challenges of this deposition/patterning technique focusing on the ink formulation, droplet formation, impact, spreading, wetting, and evaporation of the ink. Among the chemically tethered polymer surfaces, the physical and chemical properties of single end-attached polymer chains, as well as their main factors are discussed. Finally, a few typical examples of anticorrosion, as well as stimuliresponsive, such as temperature-, solvent-, redox-, and multi stimuli-responsive coatings are presented.

The stimuli-responsive properties of PNIPAM, which is one of the most studied multi-responsive polymers, are investigated in Chapter 3. The temperature- and solventinduced transition of single end-tethered PNIPAM layers with different grafting densities were investigated via neutron reflectivity (NR) measurements. The control and synthesis of different grafting densities of the PNIPAM grafts were obtained controlling the ratio between active and inactive ATRP initiator compounds coupled onto the surface followed 
by SI-ATRP of NIPAM. Moreover, the PNIPAM layers were characterized via AFM and ellipsometry analysis. The "from the top" mechanism of co-nonsolvency conformational transition of PNIPAM brushes in 3:1 water:ethanol mixture was demonstrated.

Furthermore, also the tribomechanical properties of PNIPAM were investigated in Chapter 4 and in Chapter 5. In Chapter 4, we explored the tunable friction by cononsolvency of PNIPAM brushes. The PNIPAM brushes are swollen in pure solvent but adopt a collapsed conformational state when immersed in certain solvent-co-solvent mixtures. The friction measurements performed via AFM have demonstrated that the friction was low due to the high osmotic pressure, while the friction increased when the PNIPAM collapsed (with a mixture of $30 \%$ volume fraction of ethanol in water). Surprisingly, the maximum friction was observed in $10 \%$ volume fraction of ethanol in water which does not correspond to the maximum of the shrinking of the PNIPAM brush layer. Then, in Chapter 5, we investigated also the tribomechanical properties above and below the lower critical solution temperature (LCST) of two different PNIPAM brush layers: one thin (dry thickness was around $10 \mathrm{~nm}$ ) and one thick (dry thickness higher than $410 \mathrm{~nm}$ ) brush layers. The tribomechanical properties were investigated by recording friction loops using lateral force microscopy, LFM. In this study, we highlight how the brush lateral deformation mainly affects the friction measurements of swollen PNIPAM brushes (temperature below LCST) when relatively short scanning distances are applied. In contrast, when the scanning distance of the friction loop measurements is longer than the brush lateral deformation, sliding of the probe on the brush interface becomes dominant, and correct measurement of dynamic friction can be accomplished. When the PNIPAM brushes undergo dehydration and assume a collapsed morphology (temperature above LCST) the friction loops do not show any tilting due to the hindering of their lateral deformation. Thus, the friction loops accurately describe the dynamic friction between the colloidal AFM probe and the polymer brush coating surface.

Up to this point, the polymer coatings were deposited over the entire surface of the material. As results of a joint project with Tanatex and Verosol, i.e. members of the Marie Skłodowska-Curie Initial Training Network "Complex wetting phenomena", a better indepth understanding of the inkjet printing process has been achieved allowing the development of various reactive, functional and smart coatings described in the second part of the thesis. The formulation, the droplet formation, as well as the interaction of the polyurethane-based complex fluid with the material surface and the evaporation of the ink are investigated. The work described in Chapter $\mathbf{6}$ focuses on the formulation and application of anti-corrosive polyurethane-based coatings for aluminum and its alloys. Various inks were prepared with suitable physical and chemical properties for piezoinkjet printing allowing the patterning of anti-corrosion coatings on porous aluminum metalized textile substrates. The anti-corrosion performance of the coatings was verified with a model alkaline dissolution experiment. The quality of the coated materials was 
investigated via optical, laser confocal and scanning electron microscopy. It is shown that the surface coverage and the chemical composition of the coating play an important role in the protective properties of the coatings.

Co-solvent-responsiveness properties of PMMA films in water/iso-propanol mixture were studied in Chapter 7. Firstly, two synthetic routes for the fabrication of fluorescent PMMA-containing brush coatings were presented based on grafting to and grafting from approaches. The silanization over the entire surface area of the substrates was performed for both approaches, however in grafting from the PMMA brushes were synthesized via SI-ATRP, followed by halogen-azide exchange and Copper(I)-catalyzed alkyneazide cycloaddition (CuAAC) click chemistry for coupling the fluorescent probe. In grafting to, the fluorescent telechelic PMMA was coupled to the amine functionalized surface after it was synthesized in solution via activators regenerated by electron transfer atom transfer radical polymerization (ARGET-ATRP) using a carboxyl terminated ATRP initiator. Comparing the two methods, the films obtained via grafting from were thicker and with a higher grafting density than the brush layers obtained via grafting to. However, both approaches were robust and the resulting fluorescent films were macroscopically homogenous. Secondly, the co-solvency behavior of the PMMA films was tested in isopropanol-water mixture analyzing the swelling and the fluorescent behavior via AFM and fluorescent confocal measurements, respectively. The PMMA containing fluorescent films collapsed when immersed in pure solvents resulting in low fluorescent intensity, while the PMMA segments swelled when immersed in $80 \%$ volume fraction of isopropanol in water increasing the fluorescence of the film. In the last part of this Chapter, numerous amino-reactive (i.e. APTES modified) patterns were inkjet printed on glass and silicon substrates. The APTES-containing ink was prepared in a mixture of ethanol and glycol (3/2 v/v). The inkjet printability, as well as the droplet formation were optimized. The APTES-patterns were few-micrometer-thick. Finally, both approaches were employed and the fabrication of the fluorescent patterns was confirmed by fluorescent confocal microscopy.

The inkjet printing process was also used for the project described in Chapter 8. An electrochemical sensing device for the detection of a model analyte (e.g., ascorbic acid) was fabricated by printing a new redox-responsive poly(ferrocenylsilane) (PFS) with disulfide side groups capable to covalently bind on gold surfaces. The PFS was synthesized via Platinum-catalyzed ring opening polymerization (ROP), followed by a halogen exchange reaction and a nucleophilic substitution reaction with the disulfidecontaining compound. Then, suitable inks for piezo inkjet printing process were prepared with different amounts of the functional compound in toluene, followed by the deposition on commercial microelectrode arrays (MEAs) consisting of 60 micro planar gold electrodes with different patterns via inkjet printing. The surface properties of the redoxactive films were characterized and the sensing device was tested as an amperometric 
sensor for ascorbic acid demonstrating the enhanced sensitivity due to the PFS coating. The limit of detection of the PFS-modified MEAs was measured to be lower than $60 \mu \mathrm{M}$ and the limit of linearity between 0.9 and $2 \mathrm{mM}$ of ascorbic acid in $100 \mathrm{mM}$ sodium perchlorate solution.

Intrigued by the versatility of the inkjet printing process, in Chapter 9 a series of catechol-containing ATRP macroinitiators were synthesized via free radical polymerization (FRP). After the synthesis of a suitable catechol-based acrylic monomer, dopamine methacrylamide (DOMA), a three components copolymerization via FRP was performed. The three monomers were DOMA as adhesive group, 2-(2bromoisobutyryloxy)ethyl methacrylate (BIEM) as ATRP initiating group, and the methyl methacrylate (MMA) monomer as solubilizing agent. Good control over the chemical composition in the ATRP macroinitiators was obtained with respect to the feed composition. Then, firstly the activity of the ATRP moieties was confirmed synthesizing molecular bottle brushes with poly(acrylonitrile) (PAN) side chains and then the adhesive properties of the catechol-derivatives were verified by grafting the ATRP-macroinitiator on silicon oxide followed by the SI-ATRP of the AN. The solubilizing component is an important factor for the processability of the polymer solution. Inks containing the ATRPmacroinitiator in DMF were prepared and inkjet printed on silicon surfaces create fluorescent patterns. Preliminary results indicate the chemical immobilization of the ATRP macroinitiator layer. These findings inspire our continuous effort to develop new catechol coupling chemistry based strategies that allows the wide spread utilization of readily applicable designer functional and stimuli-responsive coatings on nearly any surface. 


\section{Samenvatting}

Dit proefschrift beschrijft de fabricatie en karakterisatie van functionele en op stimuli reagerende polymeer systemen en hun veelzijdige toepassing. Met als voorbeeld coatings voor geavanceerde toepassingen als anticorrosie, hechting, instelbare wrijving, antifouling, medicijnafgifte en chemische detectie. Bovendien hebben we inkjetprinters en verschillende routes van chemische immobilisatie gecombineerd (bijv. "grafting to" en "grafting from") om functionele en slimme patronen op verschillende materialen te fabriceren.

In Hoofdstuk 1 wordt een korte inleiding van de onderwerpen gerelateerd aan dit proefschrift en de motivatie van het uitgevoerde onderzoek gegeven.

In Hoofdstuk 2 wordt een algemene inleiding gegeven van het hoofdonderwerp van dit proefschrift. Allereerst wordt er een overzicht gegeven van de "reversible deactivation-activation radical polymerization" (RDRP) technieken welke gebruikt werden om nieuwe macromoleculen te synthetiseren. Binnen de bekende RDRP-technieken werd er voor al gekeken naar de ontwikkeling van "atom transfer radical polymerization" (ATRP) processen. Hierna worden de meest relevante strategieën om polymeerketens chemisch te graften op een oppervlak en de daarbij behorende meest belangrijke desing factoren besproken. Verder wordt inkjetprinten als een patroonvormings techniek geïntroduceerd. De basis van dit proces en de uitdagingen van deze depositie/patroonvormingstechniek, met name gericht op de inktformulering, druppelvorming, impact, spreiding, bevochtiging en verdamping van de inkt, worden beschreven. Van de chemisch behandelde polymeeroppervlakken worden zowel de fysische en chemische eigenschappen van de eind gebonden polymeerketens, als ook de belangrijkste factoren besproken. Ten slotte worden er een paar typische voorbeelden van anticorrosie en stimuli responsieve coatings, 
zoals temperatuur-, oplosmiddel-, redox- en multi-stimuli-responsieve coatings beschreven.

De stimuli-responsieve eigenschappen van "poly $(N$-isopropyl acrylamide" (PNIPAM), welke een van de meest bestudeerde multi-responsieve polymeren is, wordt onderzocht in Hoofdstuk 3. De door temperatuur en oplosmiddel veroorzaakte overgangen van eind-geïmmobiliseerde PNIPAM-lagen met verschillende grafting dichtheden werden onderzocht via neutronenreflectiviteit (NR) metingen. De gecontrolleerde synthese van de PNIPAM-grafts met verschillende grafting dichtheden werd verkregen door de ratio tussen actieve en inactieve ATRP-initiator verbindingen welke gekoppeld zijn aan het oppervlak te variëren gevolgd door de "surface initiated atom transfer radical polymerization" (SI-ATRP) van NIPAM. De PNIPAM-lagen werden gekarakteriseerd met "atomic force microscopy" (AFM) en ellipsometrieanalyses. Het "van boven af" mechanisme van de conformatie overgang in PNIPAM-borstels door co-non-oplosbaarheid in een 3:1 water:ethanol mengsel werd aangetoond.

Tevens werden in Hoofdstuk 4 en Hoofdstuk 5 de tribo-mechanische eigenschappen van PNIPAM onderzocht. In Hoofdstuk 4 hebben we de veranderende wrijving door de co-non-oplosbaarheid van PNIPAM-borstels onderzocht. De PNIPAM-borstels zijn gezwollen in het pure oplosmiddel, maar slaan neer in een mengsel van de twee oplosmiddelen. De wrijvingsmetingen welke uitgevoerd zijn met de AFM hebben aangetoond dat de wrijving laag is als gevolg van een hoge osmotische druk en dat de wrijving toeneemt als de PNIPAM borstels neerslaan (in een mengsel van $30 \%$ volumefractie ethanol in water). Verrassenderwijs werd de maximale wrijving waargenomen in $10 \%$ volumefractie ethanol in water wat niet overeenkomt met het maximum van het krimpen van de PNIPAM-borstellaag. In Hoofdstuk 5 werden vervolgens de eigenschappen boven en onder de lage kritieke oplossingstemperatuur (LCST) van twee verschillende PNIPAM-borstellagen onderzocht: één dunne (droge dikte was ongeveer $10 \mathrm{~nm}$ ) en één dikke (droge dikte hoger dan $410 \mathrm{~nm}$ ) borstellaag. De tribomechanische eigenschappen werden onderzocht door wrijvingslussen te onderzoeken met behulp van lateral force microscopy, LFM. In deze studie werd onderzocht hoe de laterale vervorming van de borstel de wrijvingsmetingen van gezwollen PNIPAM-borstels (temperatuur onder LCST) beïnvloedt wanneer er een aantal relatief korte scanafstanden worden gebruikt. Echter, wanneer de scan afstand tijdens de wrijvingslus metingen langer zijn dan 
de laterale vervorming van de borstel, werd het glijden van de colloïdale AFMprobe op het borsteloppervlak dominant en kon daardoor een correcte meting van de dynamische wrijving worden verkregen. Wanneer de PNIPAM-borstels dehydrateren en een ingestorte morfologie aannemen (temperatuur boven LCST), vertonen de wrijvingslussen geen tilt vanwege het belemmeren van hun laterale vervorming. Hieruit blijkt dat de wrijvingslussen nauwkeurig de dynamische wrijving beschrijven tussen de colloïdale AFM-probe en het polymeerborstel coatings oppervlak.

Tot dit punt werden de polymeercoatings gedeponeerd over het gehele oppervlak van het materiaal. Door een samenwerking met Tanatex en Verosol, leden van het Marie Skłodowska-Curie Initial Training Network "Complex wetting phenomena", is er meer diepgang verkregen in het inkjetprintproces, waardoor de ontwikkeling van verschillende reactieve, functionele en slimme coatings zoals beschreven in het tweede deel van dit proefschrift mogelijk werd. De formulering, de druppelvorming, net als de interactie van op polyurethaan gebaseerde complexe vloeistoffen (inkt) met het materiaaloppervlak en de verdamping van de inkt zijn onderzocht. Het werk beschreven in Hoofdstuk 6 richt zich op de formulering en toepassing van corrosie werende coatings op basis van polyurethaan voor aluminium en zijn legeringen. Verschillende inkten werden geprepareerd met geschikte fysische en chemische eigenschappen voor het piëzo-inkjetprinten van patronen van anticorrosieve coatings op poreuze aluminium gemetalliseerde textielsubstraten. De anti-corrosieprestaties van de coatings werden getest met een model oplossingsexperiment in een alkalisch medium. De kwaliteit van de gecoate materialen werd onderzocht via optische, laserconfocale en scanning elektronenmicroscopie. Er is aangetoond dat de oppervlaktebedekking en de chemische samenstelling van de coatings een belangrijke rol spelen bij de beschermende eigenschappen van de coatings.

Hoofdstuk 7 richt zich op de Co-oplosmiddel-reactiviteitseigenschappen van PMMA-films in een water / iso-propanolmengsel. Ten eerste, werden twee synthetische routes gepresenteerd voor de fabricage van fluorescerende PMMAbevattende borstelcoatings op basis van grafting from en grafting to. De silanisatie over het gehele oppervlakte van de substraten is gebruikt voor beide grafting methodes, waarbij in de grafting from methode de PMMA-borstels gesynthetiseerd werden via SI-ATRP, gevolgd door halogeen-azide-uitwisseling en Copper(I)-catalyzed alkyneazide cycloaddition (CuAAC) klik-chemie voor het koppelen van de fluorescerende probe. In de "grafting to" methode, werd de 
fluorescerende telechelische PMMA gekoppeld aan een met amine gefuctionaliseerde oppervlak, nadat het in een oplossing was gesynthetiseerd via een Activator Regenerated Electron Transfer Atom Transfer Radical Polymerization (ARGET-ATRP) met behulp van een carboxyl-getermineerde ATRP-initiator. Van deze twee methodes is de film verkregen via de grafting from methode dikker en heeft deze een hogere grafting dichtheid dan de film verkregen via grafting to. Beide methodes waren echter robuust en de fluorescerende films waren macroscopisch homogeen. Ten tweede werd het cosolvabiliteitsgedrag van de PMMA-films getest in een mengsel van isopropanol en water waarna het zwellings- en fluorescentiegedrag geanalyseerd werd via respectievelijk AFM en fluorescentie metingen. De PMMA bevattende fluorescerende films slaan neer wanneer ze werden ondergedompeld in pure oplosmiddelen, wat resulteerde in een lage fluorescentie-intensiteit, en de PMMA-segmenten zwellen wanneer ze werden ondergedompeld in $80 \%$ volumefractie van isopropanol in water, waardoor de fluorescentie van de film werd verhoogd. In het laatste deel van dit hoofdstuk werden talrijke aminoreactieve (d.w.z. APTES gemodificeerde) patronen door middel van inkjet printen op glas en siliciumsubstraten geprint. De APTES-bevattende inkt werd bereid in een mengsel van ethanol en glycol (3/2 v/v). De inkjet printbaarheid en de druppelvorming werden geoptimaliseerd. De APTES-patronen waren enkele micrometers dik. Uiteindelijk werden beide methodes toegepast en werd de fabricage van de fluorescerende patronen bevestigd door fluorescerende confocale microscopie.

Voor het project in Hoofdstuk 8 werd ook het inkjet-printproces gebruikt. Door het printen van een nieuw redox responsieve poly (ferrocenylsilaan) (PFS) met disulfide zijgroepen die in staat zijn om covalent te binden op goud oppervlakten, was het mogelijk om een elektrochemisch detectieapparaat voor de detectie van een modelanalyte (bijv. ascorbinezuur) te produceren. De PFS werd gesynthetiseerd via platina-gekatalyseerde ringopeningspolymerisatie (ROP), gevolgd door een halogeenuitwisselingsreactie en een nucleofiele substitutiereactie met een disulfide-bevattende verbinding. Vervolgens werden geschikte inkten voor het piëzo-inkjetprintproces bereid met verschillende hoeveelheden van de functionelecomponent in tolueen, deze inkten werden gedeponeerd op commerciële micro-elektrode-arrays (MEA's) bestaande uit 60 micro-vlakke goud elektroden met verschillende patronen via inkjet-printen. De oppervlakte-eigenschappen van de redox-actieve films werden gekarakteriseerd, 
en het detectieapparaat werd getest als een amperometrische sensor voor ascorbinezuur. De verhoogde gevoeligheid door de aangebrachte PFS-coating werd aangetoond. De detectielimiet van de PFS-gemodificeerde MEA's werd gemeten en deze bleek lager te zijn dan $60 \mu \mathrm{M}$ en de lineariteitslimiet lag tussen de 0.9 en $2 \mathrm{mM}$ ascorbinezuur in $100 \mathrm{mM}$ natriumperchloraat oplossing.

Geïntrigeerd door de veelzijdigheid van het inkjetprintproces, werden in Hoofdstuk 9 een reeks van catechol bevattende ATRP-macro-initiators gesynthetiseerd via vrije radicalen polymerisatie (FRP). Na de synthese van een geschikt, op catechol gebaseerde acrylmonomeer, namelijk dopamine methacrylamide (DOMA), werd een drie-componenten copolymerisatie uitgevoerd via FRP. De drie monomeren waren DOMA als hechtgroep, 2- (2broomisobutyryloxy) ethylmethacrylaat (BIEM) als ATRP-initiërende groep en het methylmethacrylaat (MMA) -monomeer als oplosbaar makend middel. Goede controle over de chemische samenstelling van de ATRP-macro-initiators werd verkregen met betrekking tot de monomeer begin samenstelling. Eerst werd de activiteit van de ATRP-groepen bevestigd doormiddel van het synthetiseren van moleculaire "bottlebrushes" met poly(acrylonitril) (PAN) zijketens. Vervolgens werden de hechtingseigenschappen van de catecholderivaten bevestigd doormiddel van het graften van de ATRP-macro-initiator op siliciumoxide gevolgd door de SI-ATRP van AN. De oplosbaar makende component is een belangrijke factor voor de verwerkbaarheid van de polymeeroplossing. De inkten, bestaande uit een ATRP-macro-initiator in DMF, werden bereid en geprint doormiddel van inkjetprinten op silicium oppervlakken om fluorescerende patronen te creëeren. De voorlopige resultaten geven de chemische immobilisatie van de ATRP-macro-initiatorlaag aan. Deze bevindingen inspireren ons om nieuwe op catechol-koppelingschemie gebaseerde strategieën te blijven ontwikkelen die het brede gebruik van gemakkelijk toepasbare functionele en stimuli-responsieve coatings op vrijwel elk oppervlak mogelijk maken. 
Samenvatting 


\section{Acknowledgements}

"Nothing is possible without a great team". Many people have contributed over these four years and I would like to express my gratitude to the many mentors, colleagues, and friends that have made this Ph.D. so unique and beautiful!

First of all, I would like to express my gratitude to my promoters Prof. Julius G. Vancso and Prof. Remko Akkerman for giving me the opportunity to do my Ph.D. and to return to the University of Twente. Dear Julius, it was not easy at the beginning because my enthusiasm and my eagerness to collaborate and get involved in new scientific projects. Then, as the best friendship starts, a "breakthrough" meeting gave us an opportunity to understand each other and to build the team that makes all of this possible. You challenged me, pushed to my limits, and taught me how to meet a deadline and stay focused on the target. Dear Remko, we did not spend a lot of time, but all our meetings were scientifically inspiring. Moreover, I felt always supported thanks to your good mood, patience, and passion, even in the darkest moments of the project.

Next to my promoters, I would like to thank my supervisors Dr. Joost Duvigneau and Dr. Ton Bor. Thank you so much, Joost, to find the time for our weekly meetings and to address me to dr. Niels Benson for a fantastic collaboration with the University of Duisburg-Essen. You spent an awful amount of time with the correction of my writings. Ton, together with Remko, you were always available and ready to support me. I wish that your super 3-D printer will start to work on super interesting projects!

Further, I would like to thank my committee members Prof. Frieder Mugele, Prof. Xiaofeng Sui, Prof. Rob Lammertink, Prof. Roberto Simonutti, and Prof. Pascal Jonkheijm, for their effort and time they invested in reading my Thesis.

Prof. Sissi de Beer, I can never fully express my gratitude to for your tireless support, optimism, dedication, passion, and the trust to carry out my suggestions. Thanks to your excellent ideas, we could shape really interesting projects. Moreover, I appreciate all the time spent talking about life and hobbies, during our coffee breaks, gym moments, or dinners. And obviously, thank also to Tom for trying to convert me in a beer-man with the fantastic Lazy Jane beer. By the way, I have still not tried your super-hyper-master grill skills. I wish that one day I can taste it. Thank to Prof. Mark Hempenius for his 
support and the transfer of his knowledge on the synthesis of the various polymers over my time in MTP.

I am honored to have been part of the Marie Skłodowska-Curie initial training network: "Complex wetting phenomena" (MC-ITN-CoWet) for the financial support and allowing me to meet inspiring and passionate professors, scientists, and colleagues. I enjoyed to participating in the CoWet-events and through this experience became a better person, as well as a better scientist. In particular, I would like to thank my project-partners Sebastiaan Kolmschot and Laura Berns from Tanatex Chemicals, and Robert Kuipers from Verosol. Also my heartfelt thanks to many other friends and colleagues: Ehsanul (and Rodica), Aleksandra, Pedro, Yingdi, Abhijeet, Thanasis, Dorota, etc.

My deepest gratitude to all the professors, scientists, and researchers for trusting me in doing science and to share their knowledge with me and to whom this PhDvwork also belongs: Prof. Stefan Kooij, Dr. Roberto Quintana, Prof. Niels Benson, and Dr. Edmondo Benetti, Dr. Yunlong Yu, and Dr. Orsolya Czakkel.

I would like to thank my formal students Chris Aarsten, Sergio, and Mathies, as well as all the unofficial students/friends over all my Ph.D. You were always able to bust again my motivation, my interest, and desired to do science with your table-soccer breaks, your beer breaks, your questions, your doubts, your smiles, your problems, or your Friday's experiment. Good luck for your future and remember: "play hard, work harder, and party the hardiest!"

During these four years, I never felt alone thanks to my Enschede family composed of colleagues and friends which I admire. Thank you so much my fantastic Greek friends: Pantelis and Niko. Pante, thank you so much for taking the time and helping me with your super AFM skills. Maybe, one day, you will have another chance to beat me in the carting (one time I can let you win!). Niko (our DJ King Nick), I wish all the best for your family and your future! Liuuuu! The time we spent in Everesstraat was lovely: the fights on the political future of the world, history, economics, and science, to motivate each other during the tricky periods of the respective lives, and for our fantastic parties. I miss all of it! Many thanks to the various post-docs of the MTP group (Maciek, Olivier, Sinem), not only for the great science that you have taught me, but also for the fun during the unforgivable moments that we have shared over the years: the "rubber-band" concerts, the picnic on the river in Deventer, the tango lessons, the overnight experiments in AFM room, the weddings. Everything was great! Together with the Zoetebier's family (Bram, Basia, Casper, and the newborn Oscar), I was always able always to completely refresh my mind and switch off the "thicking" working brain. I will never forget the flirting tips of Casper: flowers, dance, show your moves, and impress her with the help of your friends.

As I have already mentioned, during my Ph.D., I was "managing" also a "psychotherapeutic coffee bar" in my office with a lot of hustle and bustle. This was only 
possible thanks to the "huge" patience, kindness, and welcoming nature of my office mates Yan, Kaihuan, Amrutha, and Lucie.

I extend my gratitude to Afrodite, Maike Wiemann, Maike Brand, Minmin, and all the other friends I have met in Enschede.

Thanks to my housemates (Ines and Ozlem) to understand me when I was often cold and distant during the high-stress periods and for keeping a warm, peaceful, and superwell organized house.

The (current and "old") MTPers for all the legendary moments that we have shared during the working hours, as well as the after-work time. Thanks to Hubert, Annerieke, Andreas, Paola, Maria Teresa, William, Roland, Mark, Lehani, for the great memories.

I am thankful to Elio \& Zuzanna, Jacopo, Daniele, Anna, Luca, and Francesco (the wonderful Italian mafia) that I have met in Enshede for their company and the PES tournament!!!

I am also grateful to my all-time friends who are not so nearby: Miao Miao, Alberto, Miriam, Fabietto, Giacomo, Lorenzo. All the time I was coming back to Italy, they were always available to meet me with a good aperol spritz or a negroni!

My formal and unofficial paranymphs: Michel, Ramon, Gyorgos, Clemens, and Lucie. Michuu, always there for everything, always willing to help, and always available for a good dinner. You are like a brother to me. Thanks. Ramy, thank you for all the coollime time, to let me discover my dark side of gambling, and make even the hateful gym enjoyable. Gyorgy, good luck for your Ph.D. You have a bright future in front of you if you follow your dreams! Thanks to Clemens (and Sabine) for all the time spent explainining and teach me all the instrument and the tools to discover and do science, as well as to save me from all the dutch burocracy. Thanks to Lucie for her superb communication skills (she spent so much awful time to check and re-check my writing that I will be always greateful), for her critical thinking, and her desire to travel. Guys, you were my crew, always and always willing to help me and understand me. With you on my side, I could fulfill my goals and ambitions. We spent so much time together, we shared so many experience and moments that I will miss you like "the" Hell!!!

I would like to thank my beloved family. You have unconditionally supported me to follow my dreams. This is priceless!!!!

To finish my Ph.D. would not be possible without my "Routine": infinite coffee break (my own coffee machine), the breakfast moment at the Beagel and Beans,Starbucks (thank you so much Pam) and at the Canteen (Thank you so much Carola), the dinners at Poco Mucho, Happy Italy, Tao, Dominos, and Fellini, the cinema, Holland Casino. "Happiness is a way of travel, it is not a destination". Looking back over the last four years, I feel that it has been the most happy, challenging, intense, busy, pleasant, crazy, and inspiring period of my life.

Grazie. 


\section{Publications}

\section{Journals}

- Yu Y., Cirelli M., Li P., Ding Z., Yuan Y., de Beer S., Vancso G.J., Zhang S., Enhanced Stability of Poly(3-sulfopropyl methacrylate potassium) Brushes Coated on Artificial Implants in Combatting Bacterial Infections, Industrial \& Engineering Chemistry Research, 2019, 58 (47), 21459-21465

- $\quad$ Cirelli M., Hao J., Bor T. C., Duvigneau J., Benson N., Akkerman R., Hempenius M.A., Vancso G.J.. Printing "Smart” Inks of Redox Responsive Organometallic breathing pores on command redox-responsive Polymers on Microelectrode Arrays for Molecular Sensing, ACS Applied Materials \& Interfaces, 2019, 11 (40), 37060-37068.

- Guo S., Quintana R., Cirelli M., Toa Z.S.D., Arjunan Vasantha V., Kooij, E.S., Jańczewski D., Vancso G.J.. Brush Swelling and Attachment Strength of Barnacle Adhesion Protein on Zwitterionic Polymer Films as a Function of Macromolecular Structure, Langmuir, 2019, 35 (24), 8085-8094.

- Kopeć M., Tas S., Cirelli M., van der Pol R., de Vries I., Vancso G.J., de Beer S.. Fluorescent Patterns by Selective Grafting of a Telechelic Polymer, Applied Polymer Materials, 2019, 1 (2), 136-140.

- Tas S., Kopeć M., van der Pol R., Cirelli M., de Vries I., Bölükbas D.A., Tempelman K., Benes N.E., Hempenius M.A., Vancso G.J., de Beer S.. Chain End-Functionalized Polymer Brushes with Switchable Fluorescence Response, Macromolecular Chemistry and Physics, 2019, 220 (5), 1022-1352.

- Ramakrishna S.N., Cirelli M., Divandari M., Benetti E.M.. Effects of Lateral Deformation by Thermoresponsive Polymer Brushes on the Measured Friction Forces, Langmuir, 2017, 33 (17), 4164-4171.

- Yu Y., Cirelli M., Kieviet B.D., Kooij E.S., Vancso G.J., de Beer S.. Tunable Friction by Employment of Co-Non-Solvency of PNIPAM Brushes, Polymer, 2016, 102, 372-378.

- Ramakrishna S.N., Cirelli M., Kooij E.S., Gunnewiek M.K., Benetti E.M.. Amplified Responsiveness of Multilayered Polymer Grafts: Synergy Between Brushes and Hydrogels, Macromolecules, 2015, 48 (19), 7106-7116. 


\section{To be Submitted}

- $\quad$ Czakkel O., Cirelli M., Duvigneau J., Vancso G.J.. Swelling and Collapse of PNIPAM brushes in Response to Temperature and Co-Non-Solvents Unveiled by Neutron Reflectivity.

- $\quad$ Horst J.R., Brio Perez M., Cohen R., Cirelli M., Elshof M.G., Andreski A., Hempenius M.A., Benes N.E., Vancso G.J., Damen C., de Beer S.. Poly(methyl acrylate) Brushes that Swell in Acetone Vapor for Gas Sensing Applications. 